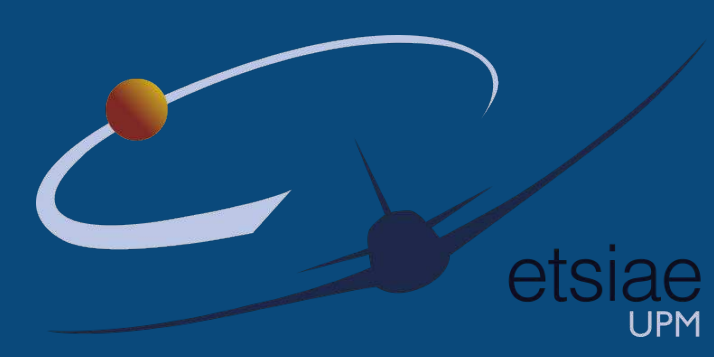

\title{
A high-order discontinuous Galerkin multiphase flow solver for industrial applications
}

\author{
Doctoral Thesis \\ By \\ Juan Manzanero Torrico \\ Aeronautical Engineer
}

January 2020 

Departamento de Matemática Aplicada a la Ingeniería Aeroespacial Escuela Técnica Superior de Ingeniería Aeronáutica y del Espacio

\section{A high-order discontinuous Galerkin multiphase flow solver for industrial applications}

by

Juan Manzanero Torrico

Aeronautical Engineer

Advisors:

Gonzalo Rubio Calzado

Ph.D. in Aeronautical Engineering

Esteban Ferrer Vaccarezza

Ph.D. in Engineering

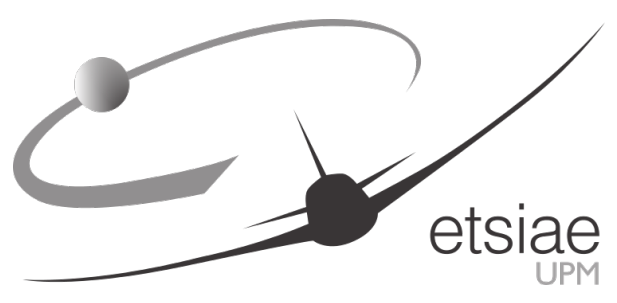

Madrid, January 2020 



\section{POLITÉCNICA}

El tribunal nombrado por el Sr. Rector Magnífico de la Universidad Politécnica de Madrid, el día de de $20 \ldots . .$.

Presidente:

Vocal:

Vocal:

Vocal:

Secretario:

Suplente:

Suplente:

Realizado el acto de defensa y lectura de la Tesis el día de de $20 \ldots$ en la E.T.S.I./Facultad.

Calificación.

EL PRESIDENTE

LOS VOCALES 

A Elisa, Rosi, Juanjo, y a Máximo. 



\title{
A high-order discontinuous Galerkin multiphase flow solver for industrial applications
}

\begin{abstract}
This thesis investigates models and simulation techniques to solve multiphase flows. Applications for multiphase flows encompasses chemical and process technology, power generation, nuclear reactor safety, or oil and gas. We concentrate on developing models that allow for varying flow morphologies (e.g. both a polydisperse bubbly flow and a fully separated flow) as those present in crude oil transport in pipes. Additionally, we want to retain most chemical phenomena as the phase separation of immiscible fluids, i.e., the coalescence and growth of stable separated phases from an initially mixed state, as well as other fundamental properties such as phases (mass) conservation.

We model multiphase phenomena using phase field methods. Phase field (or diffuse interface) methods, as opposed to the sharp interface methods, consider a finite, yet thin, thickness of the interface that separates the two fluids. Therefore, the thermodynamic properties (e.g. the density) vary smoothly across the interface. From the available phase field methods, we solve the Cahn-Hilliard equation, which naturally retains the phase separation phenomena from the minimization of a free-energy, and additionally is phase-conserving.

The Cahn-Hilliard equation is coupled with the incompressible Navier-Stokes equations. This is a two-way coupling, since the Cahn-Hilliard governs the density of the Navier-Stokes flow, and also introduces capillary forces at the interfaces. Then, the Navier-Stokes equations modify the Cahn-Hilliard equation to transport the phases with its velocity field. To obtain an efficient model, we relax the incompressibility constraint with an artificial compressibility model, that hyperbolizes the system providing an evolution equation for the pressure. In this thesis, we construct the approximation of two multiphase models: an provably stable two-phase NS/CH system, and a (non provably stable) three-phase NS/CH system.

We approximate the models with the high-order Discontinuous Galerkin Spectral Element Method (DGSEM). The DGSEM is chosen since the schemes are arbitrarily high-order accurate, they handle unstructured curvilinear meshes, and they have been widely used to solve hyperbolic/parabolic systems. From the different DGSEM variants, we use the DGSEM with Gauss-Lobatto points, since it allows us to construct schemes that are provably stable. The parabolic systems studied in this thesis involve non-linear terms that can easily make the scheme unstable. We include a thourough
\end{abstract}


study of the errors incurred to construct schemes that are provably stable.

To study the stability of the proposed schemes, we first study two simplified cases: provably stable approximations of the incompressible Navier-Stokes equations with variable density and artificial compressibility, and the standalone version (i.e. without fluid motion) of the Cahn-Hilliard equations. Then, we combine the findings to construct a provably stable approximation of the two-phases incompressible NavierStokes/Cahn-Hilliard system. Finally, we also construct a three-phase solver from the provably stable incompressible Navier-Stokes solver. We have found that the solvers developed in this thesis are robust and they can solve multiphase problems with very high Reynolds numbers and density ratios.

In the final part of the thesis, we also study the numerical errors incurred in turbulent solutions of the compressible Navier-Stokes equations, when the grid resolution is not enough to capture the small eddies present in the flow. From this study, we develop a novel Smagorinsky-SVV LES model that suits high-order DG schemes and has improved the solutions obtained with the standard Smagorinsky model.

Keywords: multiphase flow, Cahn-Hilliard, Navier-Stokes, computational fluid dynamics, high order, discontinuous Galerkin, SBP-SAT, entropy stability 


\section{A high-order discontinuous Galerkin multiphase flow solver for industrial applications}

\section{Resumen}

En esta tesis se han investigado modelos y técnicas de simulación para la resolución de flujos multifase. Tecnologías químicas y de procesos, generación de energía, seguridad de reactores nucleares, o la industria de petróleo son sólo algunas de las aplicaciones relacionadas con los flujos multifase. En particular, el estudio se concentra en el desarrollo de modelos que permiten la resolución con flujos de morfología variable (e.g. tanto flujos con burbujas dispersas como fases totalmente separadas), como los presentes en el transporte del crudo de petróleo en tuberías. Además, se desea mantener fenómenos químicos como la separación de fases en fluidos inmiscibles, es decir, la coalescencia y crecimiento de fases estables desde un estado de mezcla, junto a otras propiedades fundamentales como la conservación de las fases (de la masa) en el modelo.

Se ha utilizado un modelo de campo de fase (o interfaz difusa), que a diferencia de los modelos de interfaz delgada, consideran una interfaz fina pero cuyo ancho es finito. Así, las propiedades termodinámicas (e.g. la densidad) varían de forma suave a través de la interfaz. En concreto, en esta tesis se ha adoptado el modelo de CahnHilliard, que retiene el fenómeno de separación de fases de forma natural a través de la minimización de una función de energía libre, y además conserva las fases.

La ecuación de Cahn-Hilliard se acopla con las ecuaciones de Navier-Stokes incompresibles. Este acoplamiento ocurre en doble sentido, ya que la ecuación de CahnHilliard modifica la densidad que interviene en las ecuaciones de Navier-Stokes, y además introduce fuerzas de tensión capilar en la interfaz. Por el otro lado, las ecuaciones de Navier-Stokes intervienen en la ecuación de Cahn-Hilliard ya que transportan las diferentes fases con su campo de velocidades. Para simplificar el modelo, se ha relajado la condición de incompresibilidad para adoptar un modelo de compresibilidad artificial, el cual hiperboliza el sistema de ecuaciones en derivadas parciales a través de una ecuación de evolución adicional para la presión. En esta tesis, se han construído esquemas numéricos para dos modelos multifase: un modelo estable de dos fases NS/CH, y otro modelo (sin garantías sobre su estabilidad) de tres fases $\mathrm{NS} / \mathrm{CH}$.

La aproximación numérica de los modelos se realiza mediante un método de alto orden Galerkin discontinuo de elementos espectrales (DGSEM). Entre las ventajas 
del método, destacan la posibilidad de elegir el orden del esquema, el uso de mallas no estructuradas con elementos curvilineos, y su uso habitual por otros autores para resolver sistemas hiperbólicos/parabólicos como los encontrados en los modelos multifase de esta tesis. En particular, se utiliza el DGSEM basado en puntos de Gauss-Lobatto, ya que permiten construir esquemas estables. Los sistemas de tipo parabólico estudiados en esta tesis contienen términos no lineales que pueden desestabilizar el esquema numérico con facilidad si no se realiza un detallado estudio de los errores. Esto último, junto con el uso de la versión GL del DGSEM, permiten construir esquemas estables y robustos para su uso industrial.

En primer lugar se estudian dos casos simplificados: las aproximaciones estables de las ecuaciones de Navier-Stokes incompresibles con densidad variable y compresibilidad artificial, junto con las de la ecuación de Cahn-Hilliard (desacoplada de NS). Una vez entendido su funcionamiento aislado, se combinan para dar lugar al esquema estable del modelo de dos fases Navier-Stokes incompresible/Cahn-Hilliard. Por último, también se ha diseñado un esquema para un modelo de tres fases que utiliza el esquema estable de las ecuaciones de Navier-Stokes incompresibles, combinado con las ecuaciones de Cahn-Hilliard para tres fases. Los solvers desarrollados en esta tesis han resultado robustos y capaces de resolver problemas multifases a muy alto número de Reynolds y con alto ratio de densidades.

En la última parte de la tesis, se realiza un estudio del comportamiento de los errores numéricos en soluciones turbulentas de las ecuaciones de Navier-Stokes compresibles, cuando la resolución de la malla computacional es incapaz de resolver las pequeñas escalas presentes en el flujo. A partir de este estudio, se ha desarrollado un modelo LES Smagorinsky con viscosidad espectral. Este modelo está especialmente diseñado para modelos de alto orden, el cual obtiene mejores resultados comparado con el modelo de Smagorinsky tradicional.

Keywords: flujo multifase, Cahn-Hilliard, Navier-Stokes, dinámica de fluidos computacional, alto orden, Galerkin discontinuo, estabilidad no-lineal 


\section{Acknowledgements}

I want to thank my supervisors Gonzalo and Esteban for their help. You have taught, guided, and helped me to make my decisions and to define my goals. Your spirit and ambition for scientific research have been contagious, and I hope that you are proud of what we have achieved together. Thank you.

A special mention to my professional father, Eusebio. Thanks for investing on me and on my formation, for your support, for making possible my assistance to international conferences, internships and projects, and for the good times.

David, you have been the cornerstone of this thesis. I could not have reached my goals without you. This work is also yours. As Gonzalo says "Juan is very productive when you are around". I have learned tons with you, and I appreciate your closeness, your hospitality, your readiness, and, above all, your friendship.

A special mention to Javi for being very attentive with me, and for the good environment he creates in the group. Thanks to Miguel, Jesús, Álex, Andrés, Jorge, Iván, Lucas, Jose, Bartolo, Marta, Tere, Mariola, Carlos, Sole, Juan Ángel, Fernando, Mario, Vicky, Binghua, Yimzhu, Wojtek, Gerasimos, Diego and Joju, thanks for your support and the good times.

Thanks to my family for their unconditional support. To Elisa, Juanjo, and Rosi. Also special thanks to my grandfather Máximo.

Thanks to my flatmates Alejandro, Guille, and to Jaime for giving me the best time in Madrid, and making our home perfect to finish a doctoral thesis. Thanks to all my lifetime friends and my friends for life.

Thanks to music for being the perfect escape valve. To Esteban for being my personal drummer, to Iván, Eddie, Buddy, Jose, and the aerospace school music club.

Lastly, thanks to Repsol for your trust on our group for the development of your software tools, to Ángel Rivero, Susana Gómez, and Gabriel Rucabado for their personal involvement. Thanks to Gustaaf Jacobs (San Diego State University) for his hospitality. Finally, I acknowledge the Universidad Politécnica de Madrid (www.upm.es) for providing computing resources on Magerit Supercomputer. 



\section{Agradecimientos}

Quiero agradecer a mis tutores Gonzalo y Esteban por su inestimable ayuda. Desde el primer día me he sentido muy arropado por vosotros. Me habéis enseñado, guiado, y ayudado a tomar decisiones y definir mi rumbo. Vuestro espíritu y ambición por el mundo de la investigación me han resultado muy contagiosas, y espero haceros sentir orgullosos de lo que hemos conseguido juntos. Gracias.

Una mención especial para mi padre profesional, Eusebio. Gracias por apostar por mí, por invertir en mi formación, por tu apoyo, por llevarme a congresos, estancias, y proyectos, y por las risas. Te debo mucho Eusebio, muchas gracias.

David, has sido la piedra angular de esta tesis, no tengas duda que no hubiera logrado mis objetivos sin tí. Este trabajo también es tuyo. Como dice Gonzalo "Juan es muy productivo cuando estás cerca". He aprendido mucho contigo y estoy muy agradecido por tu cercanía, tu hospitalidad, y tu disponibilidad.

Quiero dedicar un comentario a Javi por ser tan atento conmigo, y por su buen rollo contagioso que hace del departamento un lugar especial. Gracias a Miguel y Jesús por la procastinación, al pull-boy team, Jorge, Iván, y Lucas, a la gente del Ghetto, Álex y Andrés por haberme ayudado mucho, y a los demás, Jose, Bartolo, Marta, Tere, Mariola, Carlos, Sole, Juan Ángel, Fernando, Mario, Vicky, Binghua, Yimzhu, Wojtek, Gerasimos, y a Joju, muchas gracias por vuestro apoyo y por los buenos momentos. Y por supuesto, a Diego.

Gracias a mi familia por su apoyo incondicional. A Elisa, a Juanjo y a Rosi. A mis abuelos Paulina, Horacio, Virginia, y con especial cariño a mi abuelo Máximo, que seguro se siente muy orgulloso.

Quiero agradecer a mis compañeros de piso Alejandro, Guille, y a Jaime por hacer de Madrid mi casa, y proporcionarme un ambiente ideal para el desarrollo de una tesis doctoral. Muchas gracias a mis amigos de toda la vida, y mis amigos para toda la vida, a Dani Juárez, Marina, Emma, Rodri, Sara, Ángela, Ñackas, Rufo, Txema, Viti, Chepi, David, Jandro, Houdini, Laura, Aisa, Christian, Edu, y a Naman.

Gracias a la música por ser la válvula de escape perfecta. A Esteban por ser mi batería de confianza, a Iván, que me ha contagiado su energía y sus ganas, y que espero que nunca renuncie a su sueño musical, a Eddie, Buddy, Jose Pastor, y al club de música aeroespacial.

Gracias por último a Repsol por confiar en nuestro grupo para el desarrollo de sus herramientas, a Ángel Rivero, Susana Gómez, y Gabriel Rucabado por involucrarse personalmente en el proyecto, al Centro de Supercomputación y Visualización 
de Madrid (CeSViMa) por haber proporcionado recursos informáticos en el Supercomputador Magerit, y a Gustaaf Jacobs (San Diego State University) por su hospitalidad. 


\section{Contents}

Contents

Nomenclature

xix

List of Figures

Xxiii

List of Tables

xxxi

1 Introduction 1

1.1 Motivation . . . . . . . . . . . . . . . . 1

1.2 High-order methods: the Discontinuous Galerkin Spectral Element Method

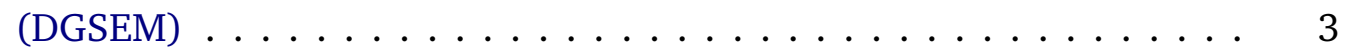

1.3 Multiphase flows $\ldots \ldots \ldots \ldots \ldots \ldots \ldots$

1.4 Objectives and methodology $\ldots \ldots \ldots \ldots \ldots \ldots$

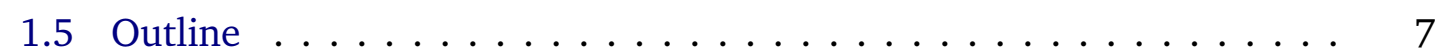

1.6 Highlights and novel contributions ............. 8

1.7 Notation . . . . . . . . . . . . . . . . . . . . . . 11

I Background: discontinuous Galerkin schemes and entropystability

2 The DGSEM $\quad 19$

2.1 Introduction . . . . . . . . . . . . . . . . . . . . 19 
2.2 Differential geometry and curvilinear elements . . . . . . . . . 20

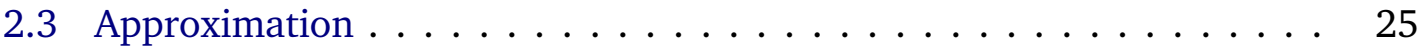

2.4 Approximation of integrals through quadratures . . . . . . . . . . . . . 29

2.5 DG Approximation of conservation laws . . . . . . . . . . . . . . 32

2.6 Approximation of non-conservative systems . . . . . . . . . . . 37

2.7 Summary of DG approximations . . . . . . . . . . . . . . . . . . . . 39

2.8 Discretization example: the compressible Navier-Stokes equations . . . 39

3 Entropy stability: a unified treatment of DGSEM stability for conservative and non-conservative systems 43

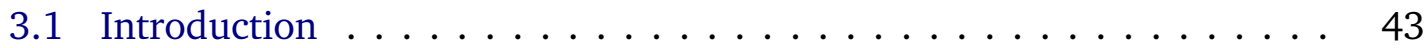

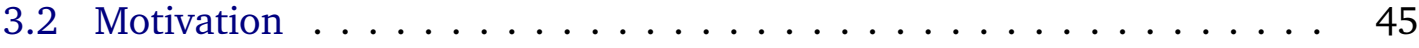

3.3 A simple finite difference example . . . . . . . . . . . . . . . . . . 49

3.4 Increasing the complexity: DGSEM discretization of the one dimensional variable speed advection equation . . . . . . . . . . . 53

3.5 Entropy analysis in general non-linear three-dimensional conservation laws . . . . . . . . . . . . . . . . . . . . . . . 59

3.6 Entropy-stability for non-conservative systems . . . . . . . . . . . 66

\section{Development of high-order DG entropy-stable and industrial} solvers

4 Entropy-stable approximation of the iNSE with variable density and artificial compressibility

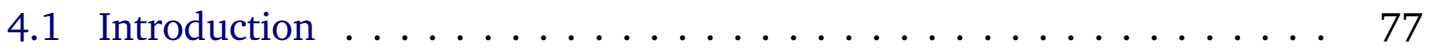

4.2 Governing equations . . . . . . . . . . . . . . . . . . 79

4.3 Entropy analysis . . . . . . . . . . . . . . . . . . . . . 80

4.4 Discontinuous Galerkin approximation . . . . . . . . . . . . . . 86

4.5 Stability analysis . . . . . . . . . . . . . . . . . 95

4.6 Numerical experiments . . . . . . . . . . . . . . . . . . . . . . . . 106

4.7 Summary and conclusions . . . . . . . . . . . . . . . . . . . . . 119 
5 The Cahn-Hilliard equation (CHE) $\quad 121$

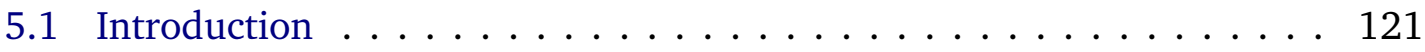

5.2 Cahn-Hilliard equation $\ldots \ldots \ldots \ldots \ldots$

5.3 Continuous free-energy stability . . . . . . . . . . . . . . . . . . . . . . 124

5.4 Discontinuous Galerkin spectral element approximation of the CahnHilliard equation . . . . . . . . . . . . . . . . 126

5.5 Semi-discrete stability analysis . . . . . . . . . . . . . . . . . . . . . 128

5.6 Fully-discrete stability using an IMEX time integrator . . . . . . . . . . . 132

5.7 Numerical experiments . . . . . . . . . . . . . . . . . . . . . . . . . . . 142

5.8 Summary . . . . . . . . . . . . . . . . . . . . . 150

6 Entropy-stable two-phase iNS/CH solver 153

6.1 Introduction . . . . . . . . . . . . . . . . . . . . . 153

6.2 Governing equations . . . . . . . . . . . . . . . . 155

6.3 Entropy analysis of the iNS/CH system . . . . . . . . . . . . . . . 160

6.4 Space and time discretization . . . . . . . . . . . . . . . 166

6.5 Semi-discrete stability analysis . . . . . . . . . . . . . . . . . . 177

6.6 Numerical experiments . . . . . . . . . . . . . . . . . . . . . . . . 185

6.7 Summary and conclusions . . . . . . . . . . . . . . . . . . . . . . . 199

\section{Development of a three-phase solver for industrial applica-} tions

7 DG approximation of a three-phase model 205

7.1 Introduction . . . . . . . . . . . . . . . . . . . . . 205

7.2 Model description . . . . . . . . . . . . . . . . . . . . . . . 206

7.3 Discontinuous Galerkin approximation . . . . . . . . . . . . . 211

7.4 Numerical experiments . . . . . . . . . . . . . . . . . . . . . . . . 214

7.5 Conclusions . . . . . . . . . . . . . . . . . . . . . . . . 219

8 A 3-phases multiphase solver for the O\&G industry 221 


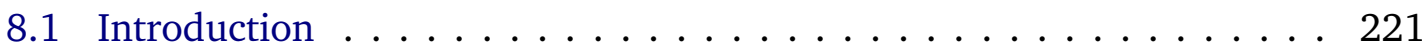

8.2 Governing equations . . . . . . . . . . . . . . . . . . . . 223

8.3 Discontinuous Galerkin method and IMEX scheme . . . . . . . . . . 226

8.4 Two-phase simulations . . . . . . . . . . . . . . . . . . . . . 232

8.5 Three-phase simulations . . . . . . . . . . . . . . . 238

8.6 Conclusions . . . . . . . . . . . . . . . . . . . . . . . 248

IV Accuracy of entropy-stable DG schemes for under-resolved turbulent simulations

9 Insights on the accuracy of the ES-DG in turbulent flows 253

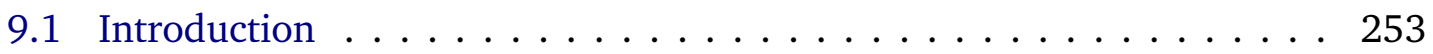

9.2 Methodology . . . . . . . . . . . . . . . . . . . . 256

9.3 Numerical Results . . . . . . . . . . . . . . . . . . . . . . . . . 267

9.4 Design of a Smagorisnky-SVV scheme . . . . . . . . . . . . . . . 275

9.5 Conclusions . . . . . . . . . . . . . . . . . . . . . . 280

$\begin{array}{ll}\text { Concluding remarks and future work } & 283\end{array}$

$\begin{array}{lr}\text { Appendices } & 287\end{array}$

$\begin{array}{ll}\text { A Additional proofs } & \mathbf{2 8 9}\end{array}$

A.1 Entropy-stability with non-conservative terms . . . . . . . . . . . . 289

B HORSES3D: A High Order (DG) Spectral Element Solver 295

B.1 Strong Scalability . . . . . . . . . . . . . . . . . . . . . . . . 296

$\begin{array}{ll}\text { Bibliography } & 301\end{array}$ 


\section{Nomenclature}

\begin{tabular}{|c|c|c|c|}
\hline \multicolumn{2}{|c|}{ Acronyms } & \multirow{2}{*}{$\begin{array}{l}\text { IP } \\
\mathrm{L}\end{array}$} & \multirow{2}{*}{$\begin{array}{l}\text { Interior Penalty } \\
\text { Left }\end{array}$} \\
\hline ACM & Compressibility & & \\
\hline & Method & $\mathrm{LU}$ & Lower-Upper decomposi- \\
\hline $\mathrm{Ba}$ & Back & & tion \\
\hline \multirow[t]{2}{*}{$\mathrm{BDF}-J$} & \multirow{2}{*}{$\begin{array}{l}J \text {-th order Backward Dif- } \\
\text { ferentiation Formula }\end{array}$} & NSE & Navier-Stokes Equations \\
\hline & & O\& G & Oil and Gas \\
\hline Bo & Bottom & PBT & Physical Boundary Terms \\
\hline BR1 & Bassi-Rebay 1 method & $\mathrm{R}$ & Right \\
\hline CFL & Courant-Friedrichs-Lewy & RK & Runge-Kutta \\
\hline DG & Discontinuous Galerkin & SAT & Simultaneous-approximation- \\
\hline \multirow[t]{2}{*}{ DGSEM } & \multirow{2}{*}{$\begin{array}{l}\text { Discontinuous Galerkin } \\
\text { Spectral Element Method }\end{array}$} & & \\
\hline & & SBP & Summation-by-parts \\
\hline \multirow{2}{*}{ DNS } & \multirow{2}{*}{$\begin{array}{l}\text { Direct Numerical Simula- } \\
\text { tion }\end{array}$} & SVV & Spectral Vanishing Viscosity \\
\hline & & $\mathrm{T}$ & Top \\
\hline ERS & Exact Riemann Solver & $\mathrm{TGV}$ & Taylor-Green Vortex \\
\hline $\mathrm{F}$ & Front & \multicolumn{2}{|c|}{ Greek Symbols } \\
\hline GL & Gauss-Lobatto & $\beta_{i}$ & Penalty parameters \\
\hline IBT & Interior Boundary Terms & $\delta_{i j}$ & Kronecker's delta \\
\hline iLES & $\begin{array}{l}\text { implicit Large Eddy Simula- } \\
\text { tion }\end{array}$ & $\overline{\overline{\boldsymbol{\sigma}}}$ & $\begin{array}{l}\text { Interface tension coeffi- } \\
\text { cients tensor }\end{array}$ \\
\hline \multirow{3}{*}{$\begin{array}{l}\text { IMEX } \\
\text { iNS/CH }\end{array}$} & \multirow{3}{*}{$\begin{array}{l}\text { IMplicit-EXplicit } \\
\text { incompressible Navier- } \\
\text { Stokes/Cahn-Hilliard }\end{array}$} & $\epsilon$ & Rate of dissipation of the ki- \\
\hline & & & netic energy \\
\hline & & $\eta$ & Molecular viscosity \\
\hline
\end{tabular}




\begin{tabular}{|c|c|c|c|}
\hline$\eta$ & Viscosity of phase $i$ & $\vec{\nabla}$ & Nabla operator \\
\hline$\kappa$ & Thermal conductivity & $\vec{\nabla}_{\xi}$ & Reference space nabla \\
\hline$\kappa_{\beta i}$ & $\begin{array}{l}\text { Coefficient of the penalty } \\
\text { parameter }\end{array}$ & $\vec{\xi}$ & $\begin{array}{l}\text { Element local coordinates } \\
\vec{\xi}=(\xi, \eta, \zeta)\end{array}$ \\
\hline \multirow{3}{*}{$\begin{array}{l}\mu \\
\mu_{i}\end{array}$} & Chemical potential & $\vec{\xi}_{f}$ & \multirow{2}{*}{$\begin{array}{l}\text { Face local } \\
\vec{\xi}_{f}=(\hat{\xi}, \hat{\eta})\end{array}$} \\
\hline & Chemical potential of phase & & \\
\hline & $i$ & $\overline{\bar{\tau}}$ & Viscous tensor \\
\hline$\Omega$ & Physical domain & & Strain tensor \\
\hline$\omega$ & Temporal frequency & $\theta_{w}=\theta_{12}^{w}$ & Wall contact angle between \\
\hline \multirow[t]{2}{*}{$\Pi$} & \multirow{2}{*}{$\begin{array}{l}\text { IMEX non-linear terms re- } \\
\text { mainder }\end{array}$} & & phases 1 and 2 \\
\hline & & $\tilde{\rho}$ & Limited density \\
\hline$\Psi$ & Entropy potential & $\Upsilon$ & $\Upsilon$ operator \\
\hline$\rho e$ & Total energy $\rho e=\frac{p}{\gamma-1}+\frac{1}{2} \rho v_{\text {tot }}^{2}$ & $\varepsilon$ & Interface thickness \\
\hline$\rho h$ & Total enthalpy $\rho h=\rho e+p$ & $\zeta$ & Enstrophy \\
\hline$\rho$ & Density & $f_{i}$ & free-energy \\
\hline$\rho_{0}$ & $\begin{array}{l}\text { Reference density (for the } \\
\text { ACM) }\end{array}$ & & derivatives of phase $i$ \\
\hline$\rho_{i}$ & Density of phase $i$ & \multicolumn{2}{|c|}{ Roman Symbols } \\
\hline \multirow[t]{2}{*}{$\sigma=\sigma_{12}$} & \multirow{2}{*}{$\begin{array}{l}\text { Interface tension coeffi- } \\
\text { cient between phases } 1 \text { and } \\
2\end{array}$} & $\overline{\mathscr{E}}$ & Total entropy \\
\hline & & $\overline{\mathscr{F}}$ & Total free-energy \\
\hline \multirow[t]{2}{*}{$\sigma_{i j}$} & \multirow{2}{*}{$\begin{array}{l}\text { Interface tension coeffi- } \\
\text { cient between phases } i \text { and } \\
j\end{array}$} & $\underline{\mathbf{B}}$ & $\begin{array}{l}\text { Viscous fluxes coefficients } \\
\text { block matrix }\end{array}$ \\
\hline & & $\underline{\mathbf{m}}$ & Metrics block matrix \\
\hline$\Sigma_{i}$ & Spreading factors & $\Delta t$ & Time-step size \\
\hline \multirow[t]{2}{*}{$\vec{\lambda}_{m}$} & \multirow{2}{*}{$\begin{array}{l}\text { Non-conservative } \\
\text { variables }\end{array}$} & Fr & Froude number \\
\hline & & $\hat{k}$ & Non-dimensional \\
\hline \multirow[t]{2}{*}{$\overrightarrow{\boldsymbol{\phi}}_{m}$} & \multirow{2}{*}{$\begin{array}{l}\text { Non-conservative terms co- } \\
\text { efficients }\end{array}$} & & $\mathrm{n}$ \\
\hline & & $\hat{n}$ & Reference element normal \\
\hline \multirow[t]{2}{*}{$\mu_{\Sigma}$} & \multirow{2}{*}{$\begin{array}{l}\text { Chemical potentials di- } \\
\text { vided by the spreading fac- } \\
\text { tors }\end{array}$} & $\mathbb{D}(\overrightarrow{\mathbf{F}})^{\#}$ & $\begin{array}{l}\text { vector } \\
\text { Two-point divergence of } \overrightarrow{\mathbf{F}}^{\#}\end{array}$ \\
\hline & & $\mathbb{D}_{\text {grad }}^{\text {DM }}$ & De-aliased metrics gradi- \\
\hline$\phi, \vec{\varphi}$ & Test functions & & ent \\
\hline
\end{tabular}




\begin{tabular}{|c|c|c|c|}
\hline $\mathscr{E}$ & Mathematical entropy & $\vec{f}_{e}^{\mathscr{E}}$ & Inviscid entropy flux \\
\hline $\mathscr{E}^{S}$ & Surface entropy & $\vec{f}_{v}^{\mathscr{E}}$ & Viscous entropy flux \\
\hline $\mathscr{E}_{i}^{S}$ & Interior faces numerical en- & $\vec{g}$ & Gravity acceleration \\
\hline & tropy & $\vec{H}^{\mathscr{E}}$ & Time contraction surface \\
\hline $\mathscr{F}$ & Free-energy & & term \\
\hline $\mathscr{F}^{S}$ & Surface free-energy & $\vec{n}$ & Normal vector \\
\hline $\mathscr{K}$ & Kinetic energy & $\vec{S}_{f}(\hat{\xi}, \hat{\eta})$ & Parametric surface function \\
\hline \multirow[t]{2}{*}{$\mathscr{P}$} & \multirow{2}{*}{$\begin{array}{l}\text { Artificial } \\
\text { energy }\end{array}$} & $\vec{u}$ & Velocity $\vec{u}=(u, v, w)$ \\
\hline & & $\vec{X}$ & Transfinite mapping \\
\hline$L(\mathbf{q})$ & $\begin{array}{l}\text { Operator for a general state } \\
\text { vector time derivative }\end{array}$ & $\vec{x}$ & $\begin{array}{l}\text { Spatial coordinates } \vec{x}= \\
(x, y, z)\end{array}$ \\
\hline \multirow[t]{2}{*}{$\partial \Omega, \partial E$} & \multirow{2}{*}{$\begin{array}{l}\text { Boundary of a domain/ele- } \\
\text { ment }\end{array}$} & $a$ & Advection speed \\
\hline & & $c$ & Concentration \\
\hline $\operatorname{Re}$ & Reynolds number & \multirow[t]{2}{*}{$c_{0}$} & \multirow{2}{*}{$\begin{array}{l}\text { Artificial compressibility } \\
\text { sound speed }\end{array}$} \\
\hline $\mathrm{T}$ & Rotation matrix & & \\
\hline$\underline{h}$ & Mass matrix & $c_{i}$ & Concentration of phase $i$ \\
\hline $\overrightarrow{\mathbf{f}}$ & $\begin{array}{l}\text { Fluxes of a conservation } \\
\text { law }\end{array}$ & $C_{S}=0.2$ & $\begin{array}{l}\text { Smagorinsky model con- } \\
\text { stant }\end{array}$ \\
\hline \multirow[t]{2}{*}{$\overrightarrow{\mathbf{F}}^{\mathrm{EC}}$} & \multirow{2}{*}{$\begin{array}{l}\text { Two-point entropy con- } \\
\text { serving flux }\end{array}$} & $D_{i j}$ & Derivative matrix $D_{i j}=l_{i}^{\prime}\left(\xi_{j}\right)$ \\
\hline & & $E$ & \multirow{2}{*}{$\begin{array}{l}\text { The reference element } E= \\
{[-1,1]^{3}}\end{array}$} \\
\hline $\overrightarrow{\mathbf{f}}_{e}(\mathbf{q})$ & Inviscid (Euler) flux & & \\
\hline $\overrightarrow{\mathbf{f}}_{v}(\mathbf{q}, \vec{\nabla} \mathbf{w})$ & Diffusive (viscous) flux & $e$ & A mesh element \\
\hline $\overrightarrow{\mathbf{g}}=\vec{\nabla} \mathbf{w}$ & Gradient block vector & $F$ & $\begin{array}{l}\text { The reference face } \\
F=[-1,1]^{2}\end{array}$ \\
\hline \multirow{2}{*}{$\begin{array}{l}\mathbf{q} \\
s(\mathbf{q})\end{array}$} & State vector & \multirow{2}{*}{$f_{0}$} & Chemical free-energy \\
\hline & Source term & & Boundary free-energy \\
\hline $\mathbf{w}$ & Gradient/entropy variables & $h$ & Element size \\
\hline$\vec{a}^{i}$ & Contravariant basis & $J$ & Transfinite mapping Jaco- \\
\hline$\vec{a}_{i}$ & Covariant basis & & bian \\
\hline \multirow[t]{2}{*}{$\vec{e}_{g}$} & \multirow{2}{*}{$\begin{array}{l}\text { Unit vector in the gravity } \\
\text { direction }\end{array}$} & $J_{f}$ & Surface Jacobian \\
\hline & & $k$ & Spatial wave-number \\
\hline $\begin{array}{l}\vec{e}_{i} \\
\vec{f}_{\mathscr{E}}\end{array}$ & $\begin{array}{l}\text { Unit space vectors } \\
\vec{f}_{\mathscr{E}} \quad \vec{f}_{\mathscr{E}} \quad \vec{f}_{\mathscr{E}}\end{array}$ & $K_{0}$ & IMEX coefficient that se- \\
\hline & Entropy flux $f^{\mathscr{E}}=f_{e}^{\mathscr{E}}+f_{v}^{\mathscr{E}}$ & & lects the first order scheme \\
\hline
\end{tabular}




\begin{tabular}{|c|c|c|c|}
\hline$L_{k}(\xi)$ & Legendre polynomials & $w_{i}$ & Quadrature weights \\
\hline$M_{0}$ & Mobility & & \\
\hline$N$ & Polynomial order & \multicolumn{2}{|c|}{ Superscripts } \\
\hline$N_{c}$ & $\begin{array}{l}\text { Number of non-conservative } \\
\text { terms }\end{array}$ & $\hat{x}^{i}, \hat{\xi}^{i}$ & $\begin{array}{l}\text { Unit vector along a direc- } \\
\text { tion }\end{array}$ \\
\hline$N_{E}$ & Number of equations & $\vec{F}^{\diamond}$ & Diamond flux \\
\hline$p$ & Pressure & $\vec{F}^{\star}$ & Numerical flux \\
\hline$p_{c}$ & Capillary pressure & $\tilde{c}$ & Limited concentration \\
\hline$p_{s}$ & Static pressure & $\tilde{f}^{i}$ & $\underset{\sim}{\text { Contravariant }}$ \\
\hline \multirow[t]{2}{*}{$S_{0}$} & IMEX scheme stabilization & & $\tilde{f}^{i}=J \vec{a}^{i} \cdot \vec{f}$ \\
\hline & coefficient & $u^{n}$ & Value of $u$ in $t=t_{n}$ \\
\hline$T$ & Temperature & $u^{\theta}$ & Value of $u$ for the IMEX \\
\hline$t$ & Time & & state \\
\hline$t_{F}$ & Final time & \multirow{2}{*}{\multicolumn{2}{|c|}{ Subscripts }} \\
\hline$t_{F}$ & Final time & & \\
\hline$t_{\mathrm{CH}}$ & $\begin{array}{l}\text { Chemical characteristic } \\
\text { time }\end{array}$ & $Q_{f}$ & $\begin{array}{l}\text { Projection of } Q \text { to the face } \\
f\end{array}$ \\
\hline$v_{s, i j}$ & $\begin{array}{l}\text { Slip velocity between } \\
\text { phases } i \text { and } j\end{array}$ & $Q_{i j k}$ & Nodal values of $Q(\vec{\xi})$ \\
\hline \multirow[t]{2}{*}{$v_{s, i}$} & Superficial velocity of & \multicolumn{2}{|c|}{ Other Symbols } \\
\hline & phase $i$ & $\{\{u\}\}$ & Average $\{\{u\}\}=\frac{1}{2}\left(u_{L}+u_{R}\right)$ \\
\hline$v_{\text {tot }}$ & Total velocity $v_{\text {tot }}^{2}=u^{2}+v^{2}+w^{2}$ & $\llbracket u \rrbracket$ & Jump $\llbracket u \rrbracket=u_{R}-u_{L}$ \\
\hline
\end{tabular}




\section{List of Figures}

2.1 Geometrical transformations in the DG method. First, the domain is divided into non-overlapping hexahedral elements that form the computational mesh. Then, different elements are transformed to the reference element with the inverse of the transfinite mapping (2.1) . . .

2.2 Representation of the local coordinates at the six faces. The faces with $\xi^{i}=-1$ have been represented in blue, and the faces with $\xi^{i}=1 \mathrm{in}$ red. The normal vector to the reference element faces $\hat{n}$ is also represented

2.3 Representation of the interpolation nodes $\left(\xi_{i}, \eta_{j}, \zeta_{k}\right)$ in the reference element (left), and their physical space position $(x, y, z)_{i j k}$ after the application of the mapping (2.1) (right). A polynomial order $N=3$, and Gauss-Lobatto points are considered for this example . . . . . . . .

3.1 Number of test crashed before a given time with a random initial condition. The scheme was not constructed pursuing an entropy inequality, whereas the entropy-stable scheme (not represented) did not crash in any of the presented simulations . . . . . . . . . . . .

4.1 Polynomial order $N$ convergence study using the manufactured solution (4.118) on a $4^{3}$ Cartesian grid. We consider three schemes: split-form DG with average of the product (one average) and product of the averages (two averages) in the momentum, and standard DG. All three schemes show spectral convergence behavior. The uneven rate of convergence is an effect of the BR1 scheme . . . . . . . . . . 108

4.2 $\mathrm{L}^{2}$ error of the Kovasznay test case solution with an $8^{2}$ mesh, for different polynomial orders. We use the split-form scheme with the product of the averages, and the residual threshold in the time integration is $10^{-9} 110$ 
4.3 $\mathrm{L}^{2}$ error of the Kovasznay test case solution with an $8^{2}$ mesh, for different values of the artificial compressibility sound speed $c_{0}$. We use the split-form scheme with the product of the averages, and the residual threshold in the time integration is $10^{-9}$. Two polynomial orders are considered: $N=5$ and $N=7 \ldots \ldots \ldots \ldots \ldots$

4.4 Lid driven cavity: streamlines of the solution for the three Reynolds numbers considered. . . . . . . . . . . . . . . . .

4.5 Evolution of the entropy time derivative for the four schemes considered 114

4.6 Rayleigh-Taylor instability with $\mathrm{Re}=1000$ : density contours. The artificial compressibility sound speed is $\rho_{0} c_{0}^{2}=5 \cdot 10^{3} \ldots \ldots \ldots$

4.7 Rayleigh-Taylor instability with $\mathrm{Re}=1000$ : effect of the artificial compressibility $M_{0}$ parameter. We studied the temporal convergence comparing the solution with three different values: $\rho_{0} c_{0}^{2}=200$ (blue), $10^{3}$ (red), $5 \cdot 10^{3}$ (black). We see that $5 \cdot 10^{3}$ represents a converged value in the well-resolved regions, while where the flow is under-resolved, the solution is highly dependent on $c_{0} \ldots \ldots \ldots \ldots$

4.8 Rayleigh-Taylor instability with $\mathrm{Re}=5000$ : density contours. The artificial compressibility sound speed is $\rho_{0} c_{0}^{2}=5 \cdot 10^{3} \ldots \ldots \ldots$

4.9 Rayleigh-Taylor instability with $\mathrm{Re}=5000$ : time evolution of the entropy (4.12) for the split-form scheme (with the product of the averages in momentum) and the standard DG scheme. We find that not only the standard scheme is unstable and crashes, but also that the amplitude of the oscillations in the under-resolved stages are higher. Although the entropy of the under-resolved split form solution is oscillatory, it is more robust $\ldots \ldots \ldots \ldots \ldots \ldots \ldots \ldots$

5.1 Graphical representation of the elliptical isoline $\Pi^{\star}=0$ for several $S_{0}$ values (indicated by the contour labels). The box $[0,1]^{2}$ has also been represented. A sufficient condition for positiveness is that the pair $\left(C^{n}, C^{n+1}\right)$ stays inside the ellipse for a given $S_{0}$ value. In 5.1(b) we have represented for each $S_{0}$ the interval $\left[1-C_{s}, C_{s}\right]^{2}$ in which the non-linear terms are stable, which corresponds to the formula $C_{s}=\frac{1}{2}\left(1+\sqrt{\frac{2 S_{0}+1}{3}}\right)$

5.2 Polynomial order convergence study without and with interface stabilization. We show that the convergence is faster and smoother with interface stabilization, and that the convergence rate is exponential only with interface stabilization . . . . . . . . . . . . . . . . . 143 
5.3 Temporal convergence study. The dashed line represents the theoretical linear convergence rate. We confirm that the scheme is first order accurate in time, as designed . . . . . . . . . . . . . . . . . . . . 144

5.4 Spatial convergence study for different polynomial orders. We vary the mesh spacing from $\Delta x=0.125$ (16x16 mesh) to $\Delta x=0.03125$ (64x64 mesh). Dashed lines represent the theoretical convergence rate, $\Delta x^{N+1}$, for each polynomial . . . . . . . . . . . . . . . . . . . . . . 145

5.5 Four meshes used for the spinodal decomposition test. The distorted mesh has straight faces, while the curved mesh edges are curvilinear . 146

5.6 Evolution of the free-energy $\overline{\mathscr{F}}^{N}$ for the "T" domain. We consider two $K_{0}$ cases: Crank-Nicolson $\left(K_{0}=1 / 2\right)$ and backward Euler $\left(K_{0}=1\right)$. We find that the solution depends on $S_{0}$ only if we use the Crank-Nicolson scheme, producing an unstable scheme if $S_{0}=0$ (dashed line), and a different solution for $S_{0}=2$ (dot-dash line in the unstructured mesh). For the rest of the cases (solid lines), the solution agrees with Jokisaari et al. [2017], represented with circles . . . . . . . . . . . . . . . 147

5.7 Representation of the final equilibrium solution in the curved and unstructured mesh. We find that the impact from the mesh on the solution is minimal, since interfaces are not aligned with the elements faces .

5.8 Free-energy evolution comparison of the distorted (solid line), curved (dashed line), and unstructured (dash-dot line) meshes with the Cartesian mesh. We find that, despite the initial and final values being approximately the same, in the evolution the errors are maintained low (of order $10^{-3}$ ) for the same number of degrees of freedom . . . . . . .

5.9 Representation of the mesh used for the three dimensional spinodal decomposition simulation . . . . . . . . . . . . . . . . . 150

5.10 Evolution of the phases with time for the three dimensional spinoidal decomposition. Blue and red contours represent the equilibrium phases $C=0$ and $C=1$ respectively $\ldots \ldots \ldots \ldots \ldots$

5.11 Evolution of the free-energy $\overline{\mathscr{F}}^{N}$ with time for the three-dimensional spinodal decomposition simulation . . . . . . . . . . . . . . 151 
6.1 Exponential convergence of the manufactured solution (6.114) as the polynomial order increases. We represent the $\mathrm{L}^{2}$ errors in concentration, $x$ - and $y$-momentum, and pressure. The polynomial order ranges from 3 to 10, and we integrate in time until $t_{F}=0.1$ with two time step sizes: $\Delta t=10^{-4}$ and $10^{-5}$. All physical parameters are given in Table

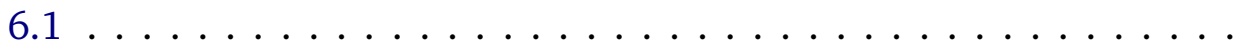

6.2 Random chosen initial condition: evolution of the entropy time derivative $\overline{\mathscr{E}}_{t}^{N}$ for three polynomial orders $N=2,3,4$. We consider three schemes: Gauss-Lobatto points with the ERS and central fluxes, and Gauss points with the ERS. For all the polynomial orders, using GaussLobatto points is an entropy preserving scheme. The dissipation is a result of physical viscosity and chemical potential for central fluxes, to which the dissipation by the exact Riemann solver can added. On the contrary, the Gauss approximation entropy time derivative is not always negative, which numerically diverges as a result $\ldots \ldots \ldots \ldots$

6.3 Random initial condition: evolution of the entropy remainder (6.116) for three polynomial orders $N=2,3,4$. We consider three schemes: Gauss-Lobatto points with the ERS and central fluxes, and Gauss points with the ERS. The remainder studies the dissipation introduced by the scheme without considering physical viscosity and chemical potential. Thus, the Gauss-Lobatto scheme with the ERS is dissipative (always negative), the Gauss-Lobatto scheme with central fluxes is conserving (always zero), and the scheme with Gauss points is unstable and crashes190

6.4 Random initial condition: we run 100 simulations modifying the random numbers sequence, of which 30 crashed with Gauss points. In this figure, the physical time in which the simulation diverged is represented. Although the other test passed the final time $t_{F}=10.0$, their entropy time derivative was found to be positive in several time-steps. None of the 100 simulations crashed with Gauss-Lobatto . . . . . . .

6.5 Rising bubble Test 1: position of the bubble and flow configuration at the final time $t=3.0 \ldots \ldots \ldots \ldots \ldots \ldots$

6.6 Rising bubble Test 1: evolution of the bubble center of gravity and rise velocity. The dots represent the reference solution from Hysing et al. [2009], while the solid line represents the solution obtained in this work195

6.7 Rising bubble Test 2: different snapshots showing the position of the bubble and the flow configuration . . . . . . . . . . . . . 196 
6.8 Rising bubble Test 2: evolution of the bubble center of gravity and rise velocity. The dots represent the reference solution from Hysing et al. [2009], while the solid line represents the solution obtained in this work197

6.9 Evolution of the physical mode that configures the annular flow. We have represented fluid 2 (i.e. $c \leqslant 0.5$ ), colored with vertical velocity, $u$. We see that during early times, there is an unstable physical mode that triggers the annular flow. When fluid 2 rises above the interface, it is dragged and accelerated by the quicker fluid 1 . When it is confined below the interface, it is slowed down by the wall. This evolves nonlinearly to the final state $\ldots \ldots \ldots \ldots \ldots \ldots$

6.10 Annular flow simulation. We present four view angles to the simulation at $t=4.0 \mathrm{~s}$. In the front view, the mesh cross section is also represented 199

7.1 Convention used to designate the wall contact angle with respect to two-phase $i$ and $j$. Note that $\theta_{i j}^{w}+\theta_{j i}^{w}=\pi \ldots \ldots \ldots 210$

7.2 Convergence study: $\mathrm{L}^{2}$ errors as a function of the polynomial order $N$ for the manufactured solution $(7.36) \ldots \ldots \ldots 215$

7.3 Captive bubble simulation. Phase 1 is white, Phase 2 is black, and Phase

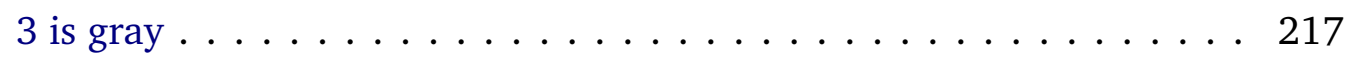

7.4 Wall contact angle simulation . . . . . . . . . . . . . . 218

8.1 Configuration of a layered inflow. The position of the two interfaces is provided by the values $y_{12}$ and $y_{23} \ldots \ldots \ldots \ldots$

8.2 Two-phase solver: polynomial order convergence study of the manufactured solution (8.37). We represent the $\mathrm{L}^{2}$ errors in concentration, $x$-and $y$-momentum, and pressure. The polynomial order ranges from 2 to 10 , and we integrate in time until $t_{F}=0.1$ with two time step sizes: $\Delta t=10^{-4}$ and $10^{-5}$. All physical parameters are given in Table $8.1 \ldots$

8.3 Two-phase solver: flow pattern map of the two-phase flow in a horizontal pipe with a diameter of 1 meter. The data has been extracted from Taitel and Dukler $[1976] \ldots \ldots \ldots \ldots \ldots$

8.4 Two-phase solver: results of test cases 1-4 (organised top to bottom) at $t=4 \mathrm{~s}$. Stratified, Slug, Dispersed Bubble and Annular Flow regimes can be seen . . . . . . . . . . . . . . . . . 236 
8.5 Two-phase solver: representation of the density contour in a pipe cross section. The four regimes (stratified, slug, dispersed bubble, and annular) are represented, along with the cross section of the mesh . . . . . .

8.6 Two-phase solver: maximum value of the concentration $c_{1}$ residual $\left(c_{1, t}\right)$ with time, for the four pipe flow regimes studied. We compare the standard DG scheme with Gauss points (solid, red line) to split-form DG (dashed, black), and we see that the standard/Gauss DG scheme is stable only for the smooth stratified flow regime. Otherwise, the standard/Gauss DG scheme crashes after some iterations, while the split-form DG is always stable for the simulations presented here . . . . . . . .

8.7 Three-phase solver: polynomial order convergence study of the manufactured solution (8.38). We represent the $\mathrm{L}^{2}$ errors of the two concentrations $c_{1}$ and $c_{2}, x$ - and $y$-momentum, and pressure. The polynomial order ranges from 2 to 10 , and we integrate in time until $t_{F}=0.1$ with two time step sizes: $\Delta t=10^{-4}$ and $10^{-5}$. All physical parameters are given in Table $8.5 \ldots \ldots \ldots \ldots \ldots \ldots \ldots$

8.8 Three-phase solver: snapshots of the fluid evolution of the three-phase channel flow. We have represented the heavy fluid (Phase 2) in red, the light fluid (Phase 3 ) in black, and the medium density fluid (Phase 1)

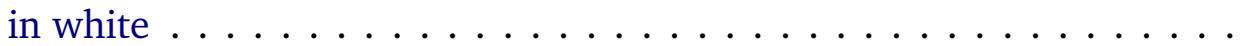

8.9 Three-phase solver: three-phase annular flow simulation: the fluid configuration is represented in $t=3$. The heavy fluid (Phase 3 ) is represented in blue, while the light fluid (Phase 1) is represented in gray245

8.10 Three-phase solver: computational mesh for the T-pipe domain, with 1700 elements . . . . . . . . . . . . . . . . . . . . 246

8.11 Three-phase solver: evolution of phase two (blue) and phase three (gray) for the first 4.5 seconds $\ldots \ldots \ldots \ldots \ldots \ldots$

8.12 Three-phase solver: representation of the three-phase at the final time

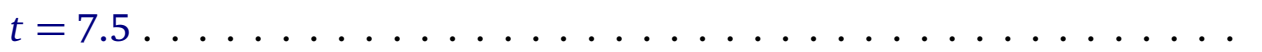

8.13 Three-phase solver: total velocity contour at the final time $t=7.5 \quad$. . 250

9.1 Representation of the power law kernel (9.29) for several $P_{S V V}$ values. The higher the kernel power coefficient $P_{S V V}$, the higher the filtering performed on the viscous tensor . . . . . . . . . . . . . . 265 
9.2 Dispersion-dissipation errors in the advection equation. Figures depict the eigenvalues computed with an energy conserving DG (i.e. with central fluxes, $\lambda=0$ ) and polynomial order $N=7 \ldots \ldots 268$

9.3 Kinetic energy spectra of the inviscid Taylor-Green vortex problem (with Mach number $M_{0}=0.1$ ) in $t=14$, alongside the theoretical Kolmogorov's solution $k^{-5 / 3}$ Pope [2001]. This result has been computed with the energy conserving DG scheme introduced in Gassner et al. [2016a]. We find that the lack of dissipation leads to energy accumulation in high wave-numbers, where the solution is severely under-resolved269

9.4 Dispersion and dissipation errors with $\lambda=1.0 \ldots \ldots \ldots$

9.5 Diffusion of the different mode sets, and the effect of the Riemann solver parameter $\lambda$. This representation allows us to follow the different bifurcation points that change the behaviour of the scheme dissipation. The colours represent the different mode groups identified in Figure 9.2(a). The precise values of $\operatorname{Im}(\omega)$ for the cases studied in the TGV (next section) are $0.7038(\lambda=0.1), 17.27(\lambda=1), 0.4065(\lambda=10) 271$

9.6 Inviscid Taylor-Green vortex problem $\left(M_{0}=0.1\right)$. The configuration is a periodic box with $32^{3}$ elements and polynomial order $N=3$. For the interfaces, we have used Roe Riemann solver with the lambda stabilisation $\lambda \operatorname{diss}(u, \phi)$ as defined in (9.19). We have considered the values $\lambda=0.1,1$, and 10 , whose results show clear parallelism with von Neumann analysis performed in Figure $9.5 \ldots \ldots \ldots$

9.7 Von Neumann dissipation curves using SVV with upwind $(\lambda=1)$. Particularly, the effect of the SVV kernel power, $P_{S V V}$, is studied, where we have also included the particular cases with $P_{S V V}=0$ (standard second order derivative), and the inviscid limit (Euler) . . . . . . . . .

9.8 Inviscid Taylor-Green vortex problem (with Mach number $M_{0}=0.1$ ) with $4^{3}$ elements and polynomial order $N=8$. We have used the SVV operator as defined in (9.30), where we set the SVV viscosity to $\eta_{S V V}=$ 0.005 (equivalent to a $R e_{S V V}=200$ ), and we vary the kernel power $P_{S V V}$. Note that the major difference between the standard NavierStokes discretization $\left(P_{S V V}=0\right)$ and the $P_{S V V}=0.1$ scheme is on the low wave-numbers . . . . . . . . . . . . . . . . . . . . 275 
9.9 Kinetic energy spectra in $t=8$ with the proposed Smagorinsky-SVV strategy for two polynomial orders. Both cases were computed using a $8^{3}$ Cartesian mesh, solving the inviscid Taylor-Green vortex problem with Mach number $M_{0}=0.1$. Different values of the SVV kernel power $P_{S V V}$ were studied $\ldots \ldots \ldots \ldots \ldots \ldots$

9.10 Numerical viscosity introduced by the Smagorinsky-SVV strategy. Both cases were computed using a $8^{3}$ Cartesian mesh. Different values of the SVV kernel power $P_{S V V}$ were studied. Two effects are regarded: the capability of the SVV to remove the dissipation in the laminar region of the TGV problem $(t<3)$, and the lack of dissipation presented by the SVV with high kernel power coefficients . . . . . . . . . .

9.11 Inviscid Taylor-Green vortex problem $\left(M_{0}=0.1\right)$ kinetic energy spectra in $t=8$ and $t=14$ with the Smagorinsky-SVV strategy using two polynomial orders $(N=4$ and $N=8)$. Both cases were computed using a $8^{3}$ Cartesian mesh, and the SVV kernel power is $P_{S V V}=0.1 \ldots$

9.12 Channel flow statistics as a function of wall distance $y^{+}=y u_{\tau} / v$. We consider three scheme: the Smagorinksy-SVV LES configuration (abbreviated as SVV+LES), the Smagorinsky LES model without SVV (LES), and the implicit LES (iLES). Dashed lines represent the law of the wall, in Figure 9.12(a), and DNS solutions from Kim et al. [1987], in Figure 9.12 (b) . . . . . . . . . . . . . . . . . . . . . 280

B.1 Strong OpenMP scalability test . . . . . . . . . . . . . . . . . . . . . 297

B.2 Strong scalability solving the TGV problem with a $32^{3}$ mesh . . . . . . 298

B.3 Strong scalability solving the TGV problem with a $64^{3}$ mesh . . . . . . 299 
2.1 Different DG discretizations for conservative and non-conservative systems ......................... 40

3.1 Summary of the continuous (cont.) and discrete (disc.) entropy analyses performed for the conservative system $\ldots \ldots \ldots \ldots \ldots$

3.2 Summary of the entropy analysis performed for the non-conservative system. The stability of the standard DG scheme assumes a non-conservative system compatible with Property 1 (3.109) and Property 2 (3.110) . . 74

4.1 Manufactured solution (4.118) convergence analysis: we use $4^{3}, 6^{3}, 8^{3}$, $12^{3}$ and $16^{3}$ meshes, and $N=2,3,4$ and 5 . We select the split-form DG with the average of the momentum, and run all simulations until $t_{F}=10$ using the explicit RK3 scheme with $C F L=0.5 \ldots \ldots$. . . . 109

4.2 Lid driven cavity with $\operatorname{Re}=1000 \ldots \ldots \ldots \ldots \ldots$. . . . . . . . 112

4.3 Lid driven cavity with $\operatorname{Re}=5000 \ldots \ldots \ldots \ldots \ldots \ldots$

4.4 Lid driven cavity with $\operatorname{Re}=7500 \ldots \ldots \ldots \ldots$. . . . . . . . . 113

6.1 List of the parameter values used with the manufactured solution (6.114)186

6.2 Manufactured solution (6.114) convergence analysis: we use $4^{2}, 8^{2}$, and $16^{2}$ meshes, and $N=2,3,4$ and 5. The final time is $t_{F}=0.1$, and we use the IMEX BDF2 scheme with $\Delta t=5 \cdot 10^{-5} \ldots \ldots \ldots$

6.3 Physical parameters for the random initial condition test problem . . 188

6.4 Parameters of the Static bubble test problem . . . . . . . . . . . . . . 192

6.5 Results of the Static bubble test problem . . . . . . . . . . . . . . . . . 192

6.6 Physical parameters of the Rising bubble test problem . . . . . . . . 193 
6.7 Physical parameters of the annular flow . . . . . . . . . . . . . . . 195

7.1 List of the parameter values used with the manufactured solution (7.36) 215

7.2 Captive bubble simulation: interface tension coefficient values and equilibrium angles studied . . . . . . . . . . . . . . . . . 216

7.3 Captive bubble simulation: physical and numerical parameters . . . . 216

7.4 Wall contact angle simulation: physical and numerical parameters . . 218

7.5 Wall contact angle simulation: wall contact angles specified for the three tests studied . . . . . . . . . . . . . . . . . . . . . . . 219

8.1 List of the parameter values used with the manufactured solution (8.37) 232

8.2 Two-phase solver: manufactured solution (8.37) convergence analysis: we use five meshes with $4^{2}, 6^{2}, 8^{2}, 12^{2}$ and $16^{2}$ meshes, and $N=2,3,4$ and 5. The final time is $t_{F}=0.1$, and we use the IMEX scheme with $\Delta t=5 \cdot 10^{-5} \ldots \ldots \ldots \ldots \ldots \ldots \ldots \ldots$

8.3 Two-phase solver: superficial velocities (in $\mathrm{m} / \mathrm{s}$ ) and theoretical flow regime in horizontal pipe test case . . . . . . . . . . . . . . . 235

8.4 Two-phase solver: physical parameters of the pipe flow . . . . . . . . 236

8.5 Three-phase solver: list of the parameter values used with the manufactured solution $(8.38) \ldots \ldots$. . . . . . . . . . . . 239

8.6 Three-phase solver: manufactured solution (8.38) convergence analysis: we use $4^{3}, 8^{3}$, and $16^{3}$ meshes, and $N=2,3,4$ and 5 . The final time is $t_{F}=0.1$, and we use the IMEX scheme with $\Delta t=5 \cdot 10^{-5} \ldots 240$

8.7 Three-phase solver: list of the parameter values used for the twodimensional three-phase channel . . . . . . . . . . . . . . . . . . 241

8.8 Three-phase solver: list of the parameter values used for the threephase annular flow . . . . . . . . . . . . . . . . . . . 242

8.9 Three-phase solver: list of the parameter values used for the threephase "T" simulation . . . . . . . . . . . . . . . . . . . . . . . . 244

9.1 Numerical fluxes used in this chapter for advective and diffusive terms 258

9.2 Summary of dissipative mechanisms: $\hat{k}$ denotes the non-dimensional wave-number, $\hat{k}=k h /(N+1) \ldots \ldots \ldots \ldots$ 


\section{Chapter 1}

\section{Introduction}

\section{Contents}

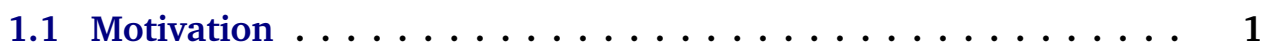

1.2 High-order methods: the Discontinuous Galerkin Spectral Element Method (DGSEM) . . . . . . . . . . . . . 3

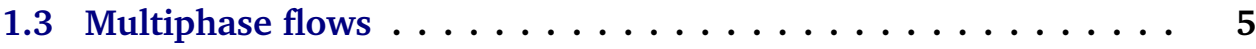

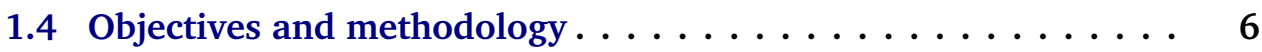

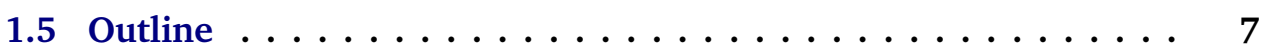

1.6 Highlights and novel contributions $\ldots \ldots \ldots \ldots \ldots$

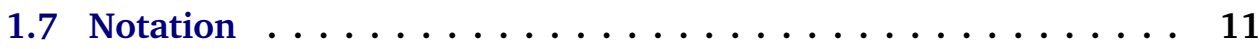

\subsection{Motivation}

Physical phenomena are often described with mathematical models. A mathematical model is commonly represented by a system of equations, complemented with the problem geometry, and the initial and boundary data. The vast majority of mathematical models studied for scientific and industrial applications are non-linear sets of partial differential equations, in complex three-dimensional domains, with arbitrary initial and boundary data, which do not admit an analytical solution. In the light of the complexity of the original analytical problem, a numerical approximation of the mathematical model is solved instead.

The simulation of complex phenomena is highly dependent on the maturity of the computational resources and the numerical algorithms. Although the equations that govern these computations date since 1840, the required computing power has only become available in the last 20 to 25 years. In recent decades, numerical modeling has 
been an essential component of engineering design and analysis, which complements wind-tunnel and experimental measurements. This is certainly true for the computational fluid dynamics (CFD), which has grown into the ability to solve flow fields that involve complex physical processes, in sophisticated geometries. While wind-tunnel and laboratory experiments will always have a role in the design process, the reproduction of these tests using CFD offers advantages in terms of cost and setup time. This allows to efficiently explore a large design space, the use of parametric geometries, and to perform shape optimizations that might unattainable and unaffordable to reproduce experimentally. As a point of reference, the license for commercial CFD tools can range from 10 to 100 thousand of dollars.

However, there are two main concerns with numerical modeling. The first is related with the algorithm robustness, and the second with its accuracy. First: Will I obtain a solution?. In other words, if the algorithm is capable to converge and provide a solution that the user can study. Second: Can I trust that solution?. Numerical modeling approximates the mathematical model, which itself represents an approximation of natural phenomena. This transforms an irresolvable equation into a very large number of simpler equations that can be easily solved with a computer, but it is not always clear if the approximation represents the real experiment with fidelity.

The simulation of fluid flow in the CFD paradigm requires one to solve convection dominated problems. The most extended, established, and trusted method has been the Finite Volume (FV) method Toro [2013]; Eymard et al. [2000]; LeVeque et al. [2002]. The FV method divides the geometry into smaller elements, where the solution is approximated by a constant value that represents the mean average in the element. Then, the fluid dynamics equations are written in integral form for each of the volumes. The FV method offers simplicity, the highest geometrical flexibility to date, the use of broadly shaped (e.g. arbitrary polyhedral) elements, and a vast number of trusted and very good documented implementations available. The main limitation of the low-order FV method, however, is that the approximation of the solution by a constant value in each cell requires a very large number of cells, or the inclusion of additional models, to accurately solve complex problems.

In an attempt to overcome the limitations of the FV method, higher-order approximations where the solution is non-constant in each cell have been developed. An example is a spectral method. The spectral method considers a single cell where the solution of the differential equation is written as a sum of global basis functions (e.g. Fourier or Chebyshev polynomials) and then to find the coefficients from the restriction of the PDE to the discrete ansatz. Although the application of spectral methods is limited, as they can only solve domains that can be mapped to a cube, they are still used for physical understanding as they represent the methods with the most inherent 
(per number of degrees of freedom) accuracy to date.

In an attempt to generalize Spectral methods to arbitrary geometries, the Cell average Flux-Corrected Transport (FCT) method Giannakouros and Karniadakis [1994], the penalty method Funaro and Gottlieb [1991]; Hesthaven and Gottlieb [1996] or the staggered grid method Kopriva [1998] were developed.

To date, one of the methods that provide the best trade-off between geometrical flexibility and accuracy is the Discontinuous Galerkin (DG) method. DG methods were first introduced by Reed and Hill Reed and Hill [1973] to solve the neutron transport equation, and were then generalized to elliptic and parabolic equations Baker [1977]; Bassi and Rebay [1997]. The DG method is suitable for solving a variety of problems, such as electromagnetic Bohm et al. [2018], compressible Bassi et al. [1997], incompressible Karniadakis and Sherwin [1999], and multiphase fluid dynamic problems Klieber and Riviere [2006].

\subsection{High-order methods: the Discontinuous Galerkin Spectral Element Method (DGSEM)}

The DG method combines features from the finite volume, the finite element, and the finite difference methods. As in the vast majority of numerical methods, the DG method divides the domain into non-overlapping elements that compose the computational mesh. For the DG method, as in the finite element and the finite volume methods, this mesh can be fully unstructured, so that the location and orientation of the different elements is arbitrary and they do not follow any regular pattern. Then, as in the finite element method, the solution is approximated in each element by a (non-constant) polynomial function.

The DG method is fully flexible in the polynomial order $N$ of the functions used, so that higher polynomial orders leads to a higher accuracy for a given mesh, but the complexity of the algorithm and its implementation is independent on $N$. The equations are approximated by writing a weak form that uses the Galerkin method, where the test functions belong to the same functional space as the solution. The latter leads to the characteristic feature of DG methods: since the test functions are linearly independent polynomials within each element, the solution will be discontinuous across the inter-elements interfaces. The inter-element coupling is performed through the replacement of the multi-valued interface solutions by a single-valued approximation that depends on both states, in the same fashion as in the finite volume method (e.g. the use of a Riemann solver). Finally, as in the finite element Method the weak form 
integrals are approximated using Gauss quadrature rules.

From the brief description of DG methods, we can infer some properties that make DG methods interesting for both academic and industrial perspectives:

- Arbitrary order of accuracy. The exactness of the approximation can be controlled changing the polynomial order $N$ of the solution. Furthermore, for smooth solutions the formal order of convergence is $N+1$ Canuto et al. [2007].

- Low dispersive and dissipative errors. One of the attractive properties of the high-order DG setting is its low numerical dissipation for well-resolved scales, and its high numerical dissipation for badly-resolved scales Hesthaven and Warburton [2007]. In other words, if the solution is well resolved the errors are small, while an under-resolved simulation triggers numerical diffusion that acts on the small scales.

- Use of unstructured meshes with curvilinear elements, that allow the representation of arbitrarily complex geometries Kopriva [2006]. The use of curvilinear elements at the boundaries increases the accuracy of the geometry representation.

- Adaptivity: to locally increase the accuracy in badly-resolved regions. In DG methods, there are three commonly adopted methods: h-adaption (i.e. local refinement of the mesh elements), p-adaption (i.e. local increases of the polynomial order), and r-adaption (i.e. relocation of the existing mesh points) Hartmann and Houston [2002]; Hartmann [2006]; Rueda-Ramírez et al. [2019a].

- Robusness. DG methods can be constructed provably stable Gassner [2013], which makes them suitable to compute under-resolved solutions (e.g. turbulence), and for the use in industrial applications. These enhancements make DG methods very robust.

- Efficient parallelization. The DG method has intensive in-volume operations, and then communication between the adjacent element to get the interface solution. This architecture of the scheme can easily take advantage of sequential and distributed memory parallelization, as the operations are highly localized (i.e. an element does not need to communicate with all the mesh elements, only its neighbours). Examples of open source DG implementations for large scale applications are Cantwell et al. [2015]; Krais et al. [2019]. 


\subsection{Multiphase flows}

Multiphase flows contain two or more immiscible fluids that occupy a region in space. The study of multiphase flows is of broad interest, from both scientific and industrial perspectives. In particular, the oil and gas industry investigates multiphase flows of immiscible fluids (e.g. oil and water). Without mixing, the typical flow configuration is the segregation of the fluids, separated by a thin interfacial region that behaves like a permeable membrane. At the interface of two dissimilar fluids, the forces acting on the molecules of each of these fluids are not the same as within each phase and generate interfacial tension.

Among the various techniques to approach the solution of immiscible multiphase flows, one finds two broad categories: sharp and diffuse interface models. In the former, the interface is considered infinitely thin, and acts as a physical boundary condition where the fluids are coupled and balanced by the interfacial forces Sussman et al. [2007]. An example of a sharp interface model is the level-set method Olsson and Kreiss [2005], where the interface is tracked by an additional variable advected by the flow velocity field.

Diffuse interface, or phase field approaches, however, regularize the problem with the introduction of an interface with non-zero thickness and a smooth transition of thermodynamic variables between both fluids. This time, interfacial forces (which in the sharp interface approach are delta functions applied at the interface) are transformed to body forces whose effect is concentrated at the interface Lowengrub and Truskinovsky [1998]. One of the biggest advantages of the phase field method is that an explicit tracking of the interface is not needed, and it is replaced by additional variables that continuously describe the relative concentration of the phases.

In this thesis, we adopt a Cahn-Hilliard based phase field method Cahn and Hilliard [1958, 1959]. Cahn-Hilliard models were originally developed to describe nearcritical behavior of binary mixtures and they are popular for three main reasons: they are conservative (i.e. the volume occupied by each phase does not change in time), they can be constructed consistent (i.e. if one phase is not present initially, it remains absent in future times), and they retain the phase separation phenomena. The latter is critical for the simulation of immiscible fluids: from an initially mixed state (which for the Cahn-Hilliard equation is a metastable state), the equilibrium phases can coalesce and separate to create and destruct new interfaces until a new equilibrium state is reached with a lower energy associated. The Cahn-Hilliard type models are the only ones to date that naturally incorporate the three properties previously enumerated. Different fields have taken advantage of these features, e.g., image processing Bertozzi et al. [2006], multimaterial structural mechanical analyses Zhou and Wang 
[2007], and biological models Khain and Sander [2008], among others.

In addition to a model that describes the evolution of the thermodynamic properties of the flow, the mathematical model is complete with the evolution equations for the flow field. We couple the Cahn-Hilliard equation with the incompressible Navier-Stokes equations. In this thesis, to solve for the pressure we adopt an artificial compressibility model that represents an approximation to incompressible flows. We choose the incompressible limit of the Navier-Stokes because we aim to solve the flow of liquids or very low Mach number gases. The coupling of the Cahn-Hilliard and the incompressible Navier-Stokes equations to solve multiphase flows has seen an increasing popularity, of which a review can be found in Hosseini et al. [2017].

\subsection{Objectives and methodology}

In this work, we develop a mathematical model, a robust DG approximation, and the implementation of a multiphase flow solver. The flows considered here have some level of phase or component separation at a scale well above the molecular level. The goal of this work is to develop a robust solver, capable of solving high-Reynolds number turbulent flows, with high density ratio, and in complex three-dimensional geometries.

In this thesis, the robustness follows from the concept of entropy-stability. For non-linear systems of partial differential equations, the existence of a scalar mathematical entropy that is bounded allows us to ensure that the physical flow quantities are bounded in time. Following this idea, we orient our efforts towards constructing numerical schemes that are entropy-stable also discretely. To construct a robust scheme, the impact of all the terms in the mathematical model has to be understood.

In the road map to construct an entropy-stable two-phase solver, we first study two simplificated cases: the fluid flow engine, without a multiphase model in Chapter 4, and the standalone version of the multiphase model in Chapter 5. Then, these two models are combined in Chapter 6 to derive a robust two-phase multiphase solver. Additionally, in Chapter 8 we develop a discontinuous Galerkin approximation for the solution of three-phase flows that focuses on the simulation of oil and gas transport in pipes.

The robustness of the method is desirable since it prevents the simulations from crashing. However, robustness is only one face of the coin. The fidelity of the numerical method representing the underlying physics must also be considered, to confirm that the numerical scheme can be trusted. A robust numerical method can compute a solution under challenging conditions. However, although stability gives physical 
consistency in some sense, it does not ensure that the solution is physically correct. It is possible to show that the scheme is accurate for smooth solutions (e.g. using the method of manufactured solutions), however, when the resolution is not enough to accurately represent the physics (i.e. an under-resolved solution) the numerical errors incurred play an important role. This is specially important when solving high Reynolds number turbulent flows. In the absence of a turbulence model (known as the implicit Large Eddy Simulation, iLES, approach), is the numerical errors the ones that control the formation and dissipation of the small eddies that can not be accurately solved. Therefore, we dedicate a last chapter on the study of the dissipative mechanisms available in the discontinuous Galerkin method, applied to the solution of turbulent flows.

\subsection{Outline}

This thesis is divided in four parts:

- Part I provides backgrounds on discontinuous Galerkin schemes and entropystability. We first describe the implementation of the discontinuous Galerkin method for general conservation laws and non-conservative systems in Chapter 2 . Then, we derive the construction of entropy-stable schemes for the general systems in Chapter 3.

- Part II contains novel discontinuous Galerkin schemes for five different sets of equations. We construct an entropy-stable approximation of the incompressible Navier-Stokes equations with artificial compressibility and variable density in Chapter 4, a free-energy stable approximation of the Cahn-Hilliard equation in Chapter 5, and an entropy-stable model and its numerical approximation of a two-phase incompressible Navier-Stokes/Cahn-Hilliard system in Chapter 6.

- Part III is dedicated to a discontinuous Galerkin approximation of a three-phase Cahn-Hilliard model in Chapter 7, and a three-phase flow solver for industrial applications in Chapter 8.

- Part IV gives insights on the behavior of numerical errors in under-resolved turbulent solutions. In Chapter 9, we develop and test a novel turbulence LES model that combines the Spectral Vanishing Viscosity method with the standard Smagorinsky LES model. 


\subsection{Highlights and novel contributions}

This thesis features chapters that have been published or are under-review in scientific journals. Precisely, the sharper stability analysis of the linear advection equation presented in Sec. (3.4.2) has been published in Journal of Scientific Computing Manzanero et al. [2018a]. The entropy-stable approximation of the incompressible Navier-Stokes equations of Chapter 4 and the free-energy stable approximation of the Cahn-Hilliard equations have been published in Journal of Computational Physics Manzanero et al. [2020a,b]. The entropy-stable two-phase solver of Chapter 6 has been accepted in Journal of Computational Physics Manzanero et al. [2020a,c]. The DG approximation of the three-phase Cahn-Hilliard model is currently under-review in Computers \& Fluids, and the novel Smagorinsky-SVV LES model has been published in Computers \& Fluids Manzanero et al. [2020d].

\subsubsection{List of Publications}

The following publications and dissemination activities were carried out during the development of this thesis:

\section{Journal Publications}

- Manzanero, J.; Rubio, G.; Ferrer, E.; Valero, E.; Kopriva, D. A. (2018). Insights on aliasing driven instabilities for advection equations with application to Gauss-Lobatto discontinuous Galerkin methods. Journal of Scientific Computing, 75(3), 1262-1281.

- Manzanero, J.; Rubio, G.; Ferrer, E.; Valero, E. (2018). Dispersion-dissipation analysis for advection problems with nonconstant coefficients: Applications to discontinuous Galerkin formulations. SIAM Journal on Scientific Computing, 40(2), A747-A768.

- Manzanero, J.; Rueda-Ramírez, A. M.; Rubio, G.; Ferrer, E. (2018). The Bassi Rebay 1 scheme is a special case of the Symmetric Interior Penalty formulation for discontinuous Galerkin discretisations with Gauss-Lobatto points. Journal of Computational Physics, 363, 1-10.

- Rueda-Ramírez, A. M.; Manzanero, J.; Ferrer, E.; Rubio, G.; Valero, E. (2019). A $p$-multigrid strategy with anisotropic $p$-adaptation based on truncation errors for high-order discontinuous Galerkin methods. Journal of Computational Physics, 378, 209-233. 
- Manzanero, J.; Rubio, G.; Kopriva, D. A; Ferrer, E.; Valero, E. (2019). A free-energy stable nodal discontinuous Galerkin approximation with summation-by-parts property for the Cahn-Hilliard equation. Journal of Computational Physics, 2019, 109072.

- Manzanero, J., Rubio, G., Kopriva, D. A., Ferrer, E., Valero, E. (2020). An entropy-stable discontinuous Galerkin approximation for the incompressible Navier-Stokes equations with variable density and artificial compressibility. Journal of Computational Physics, 408, 109241.

- Manzanero, J., Ferrer, E., Rubio, G., Valero, E. (2020). Design of a Smagorinsky Spectral Vanishing Viscosity turbulence model for discontinuous Galerkin methods. Computers \& Fluids, 200, 104440.

- Manzanero, J., Rubio, G., Kopriva, D. A., Ferrer, E., Valero, E. Entropy-stable discontinuous Galerkin approximation with summation-by-parts property for the incompressible Navier-Stokes/Cahn-Hilliard system. Accepted in Journal of Computational Physics.

\section{Book Chapters}

- Ferrer, E., Manzanero, J., Rueda-Ramírez, A. M., Rubio, G., Valero, E. Implicit large eddy simulations for NACA0012 airfoils using compressible and incompressible discontinuous Galerkin solvers. Proceedings of the International Conference on Spectral and High-Order Methods (ICOSAHOM 2018). London, UK.

- Manzanero, J.; Redondo, C.; Rubio, G.; Ferrer, E.; Valero, E.; Gómez-Álvarez, S.; Rivero-Jiménez, A. A high-order discontinuous Galerkin solver for multiphase flows. Proceedings of the International Conference on Spectral and HighOrder Methods (ICOSAHOM 2018). London, UK.

- Gómez-Álvarez, S., Rivero-Jiménez, A., Rubio, G., Manzanero, J., Redondo, C. (2019). Novel Coupled Cahn-Hilliard Navier-Stokes Solver for the Evaluation of Oil and Gas Multiphase Flow. In BHR 19th International Conference on Multiphase Production Technology. BHR Group.

\section{Participation in International Conferences}

- Manzanero, J.; Rubio, G.; Ferrer, E.; Díaz-Ojeda, H.R.; Valero, E. Dispersiondiffusion analysis for advection problems with non-constant coefficients: appli- 
cations to discontinuous Galerkin formulations. 19h International Conference on Finite Elements in Flow Problems (FEF 2017). Rome, Italy.

- Manzanero, J.; Ferrer, E.; Rubio, G.; Valero, E. Towards robust approaches for Large Eddy Simulations in high order discontinuous Galerkin methods. 7th European conference on Computational Fluid Dynamics (ECFD-7 2018). Glasgow, UK.

- Ferrer, E.; Manzanero, J.; Rueda-Ramírez, A. M.; Rubio, G.; Valero, E. Towards robust Large Eddy Simulations using discontinuous Galerkin methods. International Conference on Spectral and High-Order Methods (ICOSAHOM 2018). London, UK.

- Manzanero, J.; Rubio, G.; Kopriva, D.A.; Ferrer, E.; Valero, E. A free-energy stable nodal discontinuous Galerkin approximation with summation-by-parts property for the Cahn-Hilliard equation. VII European workshop on high order nonlinear numerical methods for evolutionary PDEs: theory and applications (HONOM 2019). Madrid, Spain.

- Ferrer, E.; Manzanero, J.; Rueda-Ramírez, A. M.; Rubio, G.; Valero, E. Implicit large eddy simulations for airfoils using compressible and incompressible discontinuous Galerkin solvers. VII European workshop on high order nonlinear numerical methods for evolutionary PDEs: theory and applications (HONOM 2019). Madrid, Spain.

- Manzanero, J.; Redondo, C.; Rubio, G.; Ferrer, E.; Valero, E.; Gómez-Álvarez, S.; Rivero, A. A high-order discontinuous Galerkin solver for multiphase flows. VII European workshop on high order nonlinear numerical methods for evolutionary PDEs: theory and applications (HONOM 2019). Madrid, Spain.

- Manzanero, J.; Rubio, G.; Kopriva, D.A.; Ferrer, E.; Valero, E. A free-energy stable nodal discontinuous Galerkin approximation with summation-by-parts property for the Cahn-Hilliard equation. North American High-Order Methods Conference (NAHOMCon 2019). San Diego, USA.

Additionally, other parts of this thesis are currently under review for publication in international Journals:

- Manzanero, J., Redondo, C., Rubio, G., Ferrer, E., Rivero-Jiménez, A. A discontinuous Galerkin approximation for a wall-bounded consistent three-component Cahn-Hilliard flow model. Submitted to Computers \& Fluids. 


\subsection{Notation}

Some of the proofs and derivations included in this work are technical. The following notation helps to simplify the description and handling of a system of partial differential equations. As an example, we consider the compressible Euler equations. The Euler fluid dynamics equations form a hyperbolic system that describes the evolution of five variables: density $\rho$, momentum $\rho \vec{u}$, and energy $\rho e$. We call these variables the state variables, which depend on the three spatial coordinates $\vec{x}=(x, y, z)=\left(x_{1}, x_{2}, x_{3}\right)$ and the time $t$ (e.g. $\left.\rho=\rho(\vec{x}, t)\right)$. We write the continuity, momentum, and energy equations, as

$$
\begin{aligned}
\rho_{t}+\vec{\nabla} \cdot(\rho \vec{u}) & =0, \\
(\rho \vec{u})_{t}+\vec{\nabla} \cdot(\rho \vec{u} \vec{u}) & =-\vec{\nabla} p, \\
(\rho e)_{t}+\vec{\nabla} \cdot(\rho h \vec{u}) & =0,
\end{aligned}
$$

where the density $\rho$, pressure $p$, energy $\rho e$, enthalpy $\rho h$, and Temperature $T$ are related assuming the ideal gas law,

$$
\rho e=\frac{p}{\gamma-1}+\frac{1}{2} \rho\left(u^{2}+v^{2}+w^{2}\right), \quad \rho h=\rho e+p, \quad p=\rho R T .
$$

In (1.1), $\vec{u}=(u, v, w)$ is the velocity field, and $\vec{\nabla}=(\partial / \partial x, \partial / \partial y, \partial / \partial z)$ is the gradient operator. The system of equations can be written in a vector fashion,

$$
\left(\begin{array}{c}
\rho \\
\rho \vec{u} \\
\rho e
\end{array}\right)_{t}+\frac{\partial}{\partial x}\left(\begin{array}{c}
\rho u \\
\rho u \vec{u} \\
\rho h u
\end{array}\right)+\frac{\partial}{\partial y}\left(\begin{array}{c}
\rho v \\
\rho v \vec{u} \\
\rho h v
\end{array}\right)+\frac{\partial}{\partial z}\left(\begin{array}{c}
\rho w \\
\rho w \vec{u} \\
\rho h w
\end{array}\right)=\left(\begin{array}{c}
0 \\
\overrightarrow{0} \\
0
\end{array}\right),
$$

The first vector, with a time derivative, is called the state vector, while the other three vectors are called fluxes.

We follow Gassner et al. [2018] and introduce a notation to systematically define vectors of different nature:

- Space vectors, which belong to $\mathbb{R}^{3}$, are represented with an arrow on top (e.g. the velocity $\vec{u}=(u, v, w)$, or the gradient operator $\vec{\nabla})$,

$$
\vec{u}=u \vec{e}_{1}+v \vec{e}_{2}+w \vec{e}_{3}=\sum_{i=1}^{3} u_{i} \vec{e}_{i}
$$

with $\vec{e}_{i}$ the unit space vectors. 
- State vectors, which belong to $\mathbb{R}^{N_{\mathrm{E}}}$ where $N_{\mathrm{E}}$ is the number of equations $\left(N_{\mathrm{E}}=5\right.$ for the Euler equations), combine the five state variables and they use a bold symbol. For example, the state vector is

$$
\mathbf{q}=(\rho, \rho \vec{u}, \rho e)=\rho \mathbf{e}_{1}+\rho u \mathbf{e}_{2}+\rho v \mathbf{e}_{3}+\rho w \mathbf{e}_{4}+\rho e \mathbf{e}_{5}=\sum_{i}^{N_{\mathrm{E}}} q^{(i)} \mathbf{e}_{i},
$$

while the flux along a direction $i$ is $\mathbf{f}_{i}=\left(\rho u_{i}, \rho u_{i} \vec{u}, \rho h u_{i}\right)$.

This notation allows us to write the system (1.3) as

$$
\mathbf{q}_{t}+\mathbf{f}_{1, x}+\mathbf{f}_{2, y}+\mathbf{f}_{3, z}=\mathbf{q}_{t}+\mathbf{f}_{x}+\mathbf{g}_{y}+\mathbf{h}_{z}=\mathbf{0},
$$

where the three spatial coordinates of the fluxes are

$$
\mathbf{f}=\mathbf{f}_{1}=\left(\begin{array}{c}
\rho u \\
\rho u \vec{u} \\
\rho h u
\end{array}\right), \quad \mathbf{g}=\mathbf{f}_{2}=\left(\begin{array}{c}
\rho v \\
\rho v \vec{u} \\
\rho h v
\end{array}\right), \mathbf{h}=\mathbf{f}_{3}=\left(\begin{array}{c}
\rho w \\
\rho w \vec{u} \\
\rho h w
\end{array}\right) .
$$

We define block vectors as the result of inlining three state vectors (for example, for the fluxes). Block vectors belong to $\mathbb{R}^{3 x N_{\mathrm{E}}}$ and are represented with a bold symbol and an arrow, $\overrightarrow{\mathbf{f}}$,

$$
\overrightarrow{\mathbf{f}}=\left(\begin{array}{c}
\mathbf{f}_{1} \\
\mathbf{f}_{2} \\
\mathbf{f}_{3}
\end{array}\right)=\left(\begin{array}{l}
\mathbf{f} \\
\mathbf{g} \\
\mathbf{h}
\end{array}\right) \text {. }
$$

Operators between space, state, and block vectors can be defined. Precisely,

1. The product between two block vectors is a scalar,

$$
\overrightarrow{\mathbf{f}}^{T} \overrightarrow{\mathbf{g}}=\sum_{i=1}^{3} \mathbf{f}_{i}^{T} \mathbf{g}_{i}
$$

2. The product between a space vector and a block vector is a state vector (and the gradient operator produces the divergence of the flux),

$$
\vec{v} \cdot \overrightarrow{\mathbf{f}}=\sum_{i=1}^{3} v_{i} \mathbf{f}_{i}, \quad \vec{\nabla} \cdot \overrightarrow{\mathbf{f}}=\sum_{i=1}^{3} \frac{\partial \mathbf{f}_{i}}{\partial x_{i}} .
$$

3. The product between a state vector and a block vector is a space vector,

$$
\mathbf{q}^{T} \overrightarrow{\mathbf{f}}=\sum_{i=1}^{3} \mathbf{q}^{T} \mathbf{f}_{i} \vec{e}_{i}
$$


4. The product between a space vector and a state vector is a block vector (and in particular the gradient operator gives the gradient of the state vector),

$$
\vec{v} \mathbf{q}=\left(\begin{array}{c}
v_{1} \mathbf{q} \\
v_{2} \mathbf{q} \\
v_{3} \mathbf{q}
\end{array}\right), \quad \vec{\nabla} \mathbf{q}=\left(\begin{array}{c}
\mathbf{q}_{x} \\
\mathbf{q}_{y} \\
\mathbf{q}_{z}
\end{array}\right)
$$

We lastly define the operator $\Upsilon$ that transforms a $N_{\mathrm{E}} \times 3$ (state-space) matrix into a $3 N_{\mathrm{E}} \times 1$ block vector,

$$
\overrightarrow{\mathbf{f}}=\Upsilon\left(\begin{array}{ccc}
f^{(1)} & g^{(1)} & h^{(1)} \\
f^{(2)} & g^{(2)} & h^{(2)} \\
f^{(3)} & g^{(3)} & h^{(3)} \\
\vdots & \vdots & \vdots \\
f^{\left(N_{\mathrm{E}}\right)} & g^{\left(N_{\mathrm{E}}\right)} & h^{\left(N_{\mathrm{E}}\right)}
\end{array}\right)=\left(\begin{array}{c}
\mathbf{f} \\
\mathbf{g} \\
\mathbf{h}
\end{array}\right)
$$

Block vectors make it possible to represent the system (1.3) in a compact form,

$$
\mathbf{q}_{t}+\vec{\nabla} \cdot \overrightarrow{\mathbf{f}}=0 \text {. }
$$

Finally, we define three matrices of different nature,

- State matrices $\left(N_{\mathrm{E}} \times N_{\mathrm{E}}\right)$ : applied to a state vector give another state vector. We represent state matrices with a bold symbol and with an underline. For the example of the Euler equations $\left(N_{\mathrm{E}}=5\right)$, a matrix that rotates the velocities to an auxiliary frame $\left(\vec{n}, \vec{t}_{1}, \vec{t}_{2}\right)$, but leaves density and energy unaltered, is

$$
\underline{\mathrm{T}}=\left(\begin{array}{ccccc}
1 & 0 & 0 & 0 & 0 \\
0 & n_{x} & n_{y} & n_{z} & 0 \\
0 & t_{1, x} & t_{1, y} & t_{1, z} & 0 \\
0 & t_{2, x} & t_{2, y} & t_{2, z} & 0 \\
0 & 0 & 0 & 0 & 1
\end{array}\right), \quad\left(\begin{array}{c}
\rho \\
\rho u_{n} \\
\rho v_{t 1} \\
\rho v_{t 2} \\
\rho e
\end{array}\right)=\underline{\mathrm{T}}\left(\begin{array}{c}
\rho \\
\rho u \\
\rho v \\
\rho w \\
\rho e
\end{array}\right) .
$$

- Space matrices $(3 \times 3)$ : space matrices (tensors) applied to a space vector return a space vector. They are represented with a double overline. For example, a matrix that (transposed) rotates a component of the flux to an auxiliary frame $\left(\vec{a}^{1}, \vec{a}^{2}, \vec{a}^{3}\right)$ is

$$
\overline{\bar{m}}=\left(\vec{a}^{1}, \vec{a}^{2}, \vec{a}^{3}\right), \quad \overrightarrow{\tilde{f}}=\overline{\bar{m}}^{T} \vec{f} .
$$


Tensors have defined an inner product that returns a scalar,

$$
\overline{\bar{s}} \cdot \overline{\bar{t}}=\sum_{i, j=1}^{3} s_{i j} t_{i j}
$$

and space vectors have an outer product that returns a tensor,

$$
\vec{u} \vec{v}=\left(u_{1}, u_{2}, u_{3}\right)^{T}\left(v_{1}, v_{2}, v_{3}\right)=\left(\begin{array}{lll}
u_{1} v_{1} & u_{1} v_{2} & u_{1} v_{3} \\
u_{2} v_{1} & u_{2} v_{2} & u_{2} v_{3} \\
u_{3} v_{1} & u_{3} v_{2} & u_{3} v_{3}
\end{array}\right) .
$$

- Block matrices $\left(3 N_{\mathrm{E}} \times 3 N_{\mathrm{E}}\right)$ : they multiply to a block vector to get a block vector. There are two ways to construct a block matrix:

1. From nine state matrices $\underline{B}_{i j}$,

$$
\underline{\mathbf{B}}=\left(\begin{array}{lll}
\underline{B}_{11} & \underline{B}_{12} & \underline{B}_{13} \\
\underline{B}_{21} & \underline{B}_{22} & \underline{B}_{23} \\
\underline{B}_{31} & \underline{B}_{32} & \underline{B}_{33}
\end{array}\right)
$$

so that the operation $\overrightarrow{\mathbf{g}}=\underline{\mathbf{B}} \overrightarrow{\mathbf{f}}$ is

$$
\mathbf{g}_{i}=\underline{B}_{i 1} \mathbf{f}_{1}+\underline{B}_{i 2} \mathbf{f}_{2}+\underline{B}_{i 3} \mathbf{f}_{3} .
$$

An example is the definition of the viscous flux in the Navier-Stokes equations, which is linear in the gradients of the state vector,

$$
\begin{aligned}
& \mathbf{f}_{v, 1}=\left(\begin{array}{c}
0 \\
\frac{2}{3} \eta\left(2 u_{x}-v_{y}-w_{z}\right) \\
\eta\left(u_{y}+v_{x}\right) \\
\eta\left(u_{z}+w_{x}\right) \\
f_{v, 1}^{(2)} u+f_{v, 1}^{(3)} v+f_{v, 1}^{(4)} w+\kappa T_{x}
\end{array}\right), \mathbf{f}_{v, 2}=\left(\begin{array}{c}
0 \\
0 \\
\eta\left(u_{y}+v_{x}\right) \\
\eta\left(v_{z}\right) \\
\frac{2}{3} \eta\left(2 v_{y}-u_{x}-w_{z}\right) \\
\eta\left(v_{z}+w_{y}\right) \\
\mathbf{f}_{v, 3}^{(2)} u+f_{v, 2}^{(3)} v+f_{v, 2}^{(4)} w+\kappa T_{y}
\end{array}\right), \\
&\left(\begin{array}{c}
\frac{2}{3} \eta\left(2 w_{z}-u_{x}-v_{y}\right) \\
f_{v, 3}^{(2)} u+f_{v, 3}^{(3)} v+f_{v, 3}^{(4)} w+\kappa T_{z}
\end{array}\right)
\end{aligned}
$$

In (1.21), $\eta$ is the viscosity and $\kappa$ is the thermal conductivity. Viscous fluxes can be written compactly using a block matrix,

$$
\overrightarrow{\mathbf{f}}_{v}=\underline{\mathbf{B}} \vec{\nabla} \mathbf{q}, \quad \mathbf{f}_{v, i}=\underline{B}_{i 1} \mathbf{q}_{x}+\underline{B}_{i 2} \mathbf{q}_{y}+\underline{B}_{i 3} \mathbf{q}_{z},
$$


where, for instance, we provide the matrix $\underline{B}_{12}$,

$$
\underline{B}_{12}=\eta\left(\begin{array}{ccccc}
0 & 0 & 0 & 0 & 0 \\
\frac{2 v}{3 \rho} & 0 & -\frac{2}{3 \rho} & 0 & 0 \\
-\frac{u}{\rho} & \frac{1}{\rho} & 0 & 0 & 0 \\
0 & 0 & 0 & 0 & 0 \\
-\frac{u v}{3 \rho} & \frac{v}{\rho} & -\frac{2 u}{3 \rho} & 0 & 0
\end{array}\right) .
$$

The rest of the matrices can be found in Rueda-Ramírez [2019].

2. From a space matrix: this particular block matrix configuration permits one to perform the same space operation to all state variables simultaneously. If (1.16) defines a rotation for a space vector, then, to perform the same rotation to all five components of a block vector, we construct the block matrix

$$
\underline{\mathbf{m}}=\left(\begin{array}{ccc}
\overline{\bar{m}}_{11} \underline{I}_{N_{\mathrm{E}}} & \overline{\bar{m}}_{12} \underline{I}_{N_{\mathrm{E}}} & \overline{\bar{m}}_{13} \underline{I}_{N_{\mathrm{E}}} \\
\overline{\bar{m}}_{21} \underline{I}_{N_{\mathrm{E}}} & \overline{\bar{m}}_{22} \underline{I}_{N_{\mathrm{E}}} & \overline{\bar{m}}_{23} \underline{I}_{N_{\mathrm{E}}} \\
\overline{\bar{m}}_{31} \underline{I}_{N_{\mathrm{E}}} & \overline{\bar{m}}_{32} \underline{I}_{N_{\mathrm{E}}} & \overline{\bar{m}}_{33} \underline{I}_{N_{\mathrm{E}}}
\end{array}\right), \quad \overrightarrow{\tilde{\mathbf{f}}}=\underline{\mathbf{m} \mathbf{f}}, \quad \tilde{\mathbf{f}}_{i}=\underline{m}_{i 1} \mathbf{f}_{1}+\underline{m}_{i 2} \mathbf{f}_{3}+\underline{m}_{i 3} \mathbf{f}_{3},
$$

where $\underline{I}_{N_{\mathrm{E}}}$ is the $N_{\mathrm{E}} \times N_{\mathrm{E}}$ identity matrix.

The DG method approximates weak-forms of the equations. We define the integral of a function $f$ in the domain $\Omega$ as

$$
\langle f\rangle=\int_{\Omega} f \mathrm{~d} \vec{x}
$$

and the inner product of two functions $f$ and $g$ as

$$
\langle f, g\rangle=\int_{\Omega} f g \mathrm{~d} \vec{x},
$$

being $\|f\|^{2}=\langle f, f\rangle$ the $\mathrm{L}^{2}$ norm of a function. Lastly, we define two more operators to be widely used across this work. Given two states $u_{L}$ and $u_{R}$, we define the average and jump operators as

$$
\{\{u\}\}=\frac{u_{L}+u_{R}}{2}, \llbracket u \rrbracket=u_{R}-u_{L}
$$

which satisfy the algebraic identities,

$$
\llbracket u v \rrbracket=\{\{u\}\} \llbracket v \rrbracket+\llbracket u \rrbracket\{\{v\}\}, \llbracket u^{2} \rrbracket=2\{\{u\}\} \llbracket u \rrbracket .
$$





\title{
Part I \\ Background: discontinuous Galerkin schemes and entropy-stability
}

\begin{abstract}
Summary In this part we first derive the DG discretization for a general conservation law, and for non-conservative systems in Chapter 2. This chapter is meant as a foundation for Part II, where the specific solvers presented in this thesis are constructed. In Chapter 3, we review the concept of entropy-stability to obtain general conditions for the discrete entropy-stability of conservation laws and non-conservative systems. Entropy-stability is the key feature of the schemes presented in this work, as it enhances the robustness of the algorithm and its implementations. The entropy-stability enables the use of the method for challenging and industrial applications. Almost everything covered in this part is a literature review. However, in Chapter 3 we present a novel entropy analysis for advection equations with non-zero advection speeds in Sec. 3.4.2, and a small extension of the proof presented in Bohm et al. [2018] to guarantee the entropy-stability with non-conservative terms that involve the gradient of scalar fields.
\end{abstract}





\section{Chapter 2}

\section{The Discontinuous Galerkin Spectral Element Method (DGSEM)}

\section{Contents}

2.1 Introduction . . . . . . . . . . . . . . . . . . 19

2.2 Differential geometry and curvilinear elements . . . . . 20

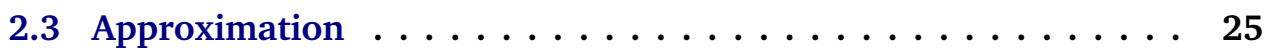

2.4 Approximation of integrals through quadratures $\ldots \ldots \ldots 29$

2.5 DG Approximation of conservation laws . . . . . . . . . . . 32

2.6 Approximation of non-conservative systems $\ldots \ldots \ldots \ldots$

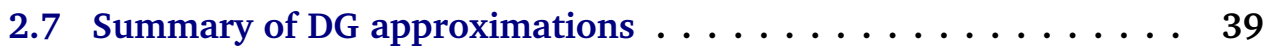

2.8 Discretization example: the compressible Navier-Stokes equations 39

\subsection{Introduction}

In this chapter we present the construction of high-order Discontinuous Galerkin (DG) schemes. Prior to describing the discrete setting, we first introduce in Sec. 2.2 the geometrical transformations that are used to transform the geometry and the operators of a hexahedral element with shaped faces from a cube, called the reference element. Then, in Sec. 2.3 we describe the approximation of functions, and in Sec. 2.4 the approximation of integrals by quadratures. We finally show the approximation of conservation laws in Sec. 2.5, and the approximation of non-conservative systems in Sec. 2.6. A summary is provided in Sec. 2.7. As a final example, we provide the 


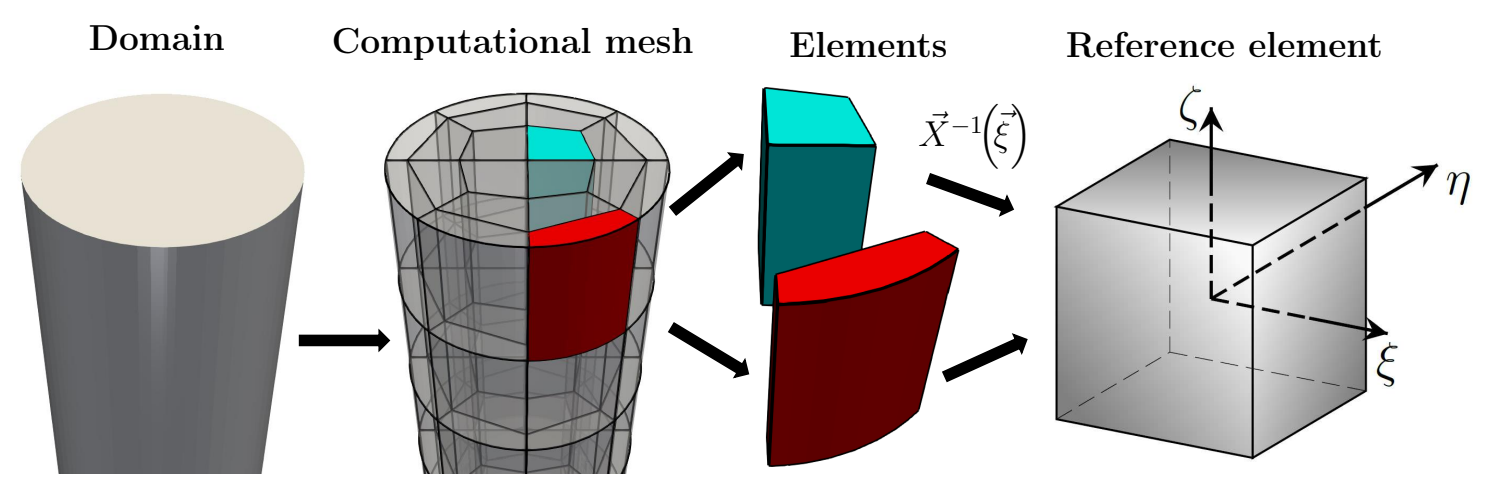

Figure 2.1: Geometrical transformations in the DG method. First, the domain is divided into non-overlapping hexahedral elements that form the computational mesh. Then, different elements are transformed to the reference element with the inverse of the transfinite mapping (2.1)

entropy-stable discretization of the compressible Navier-Stokes presented in Gassner et al. [2018] in Sec. 2.8.

\subsection{Differential geometry and curvilinear elements}

The physical domain $\Omega$ is tessellated with non-overlapping hexahedral elements, $e$, which are geometrically transformed from a reference element $E=[-1,1]^{3}$. The coordinates associated with the reference element $E$ define the computational space in which we will approximate the solutions. In one dimension, the reference element is a line (with two end-points), in two dimensions a square (with four edges), and in three dimensions a cube (with six planar faces).

The coordinate transformation is performed using a transfinite mapping $\vec{X}$ that relates the physical coordinates, $\vec{x}=\left(x^{1}, x^{2}, x^{3}\right)=(x, y, z)=x \hat{x}+y \hat{y}+z \hat{z}$, to the local reference coordinates, $\vec{\xi}=\left(\xi^{1}, \xi^{2}, \xi^{3}\right)=(\xi, \eta, \zeta)=\xi \hat{\xi}+\eta \hat{\eta}+\zeta \hat{\zeta}$, through

$$
\begin{aligned}
\vec{X}: \vec{\xi} & \in E=[-1,1]^{3} \subset \mathbb{R}^{3} \longrightarrow \vec{x} \\
(\xi, \eta, \zeta) & \vec{x}=(x, y, z)=\vec{X}(\xi, \eta, \zeta)
\end{aligned}
$$

The space vectors $\hat{x}_{i}$ and $\hat{\xi}^{i}$ are unit vectors in the three Cartesian directions of the physical and reference coordinates, respectively. Each of the elements $e$ that define the domain $\Omega$ have their own transfinite mapping function, all from the same reference element $E$, to their physical space subdomain.

In the reference element, each of the local coordinates is defined in $[-1,1]$, which in three dimensions defines six surface boundaries. These boundaries are also writ- 
ten in a parametric fashion in two new local variables $(\hat{\xi}, \hat{\eta})$ on the reference face $F=[-1,1]^{2}$. The reference coordinates at each of the six faces are given by a transformation,

$$
\begin{aligned}
\vec{\xi}_{f}: F=[-1,1]^{2} \subset \mathbb{R}^{2} & \longrightarrow E=[-1,1]^{3} \subset \mathbb{R}^{3} \\
(\hat{\xi}, \hat{\eta}) & \longrightarrow \vec{\xi}_{f}=\vec{\xi}_{f}(\hat{\xi}, \hat{\eta})=\left(\xi_{f}, \eta_{f}, \zeta_{f}\right)
\end{aligned}
$$

For each of the six faces of the reference element (see Fig. 2.2), the transformations are

$$
\begin{array}{rrrl}
\text { Left } & \vec{\xi}_{S_{\mathrm{L}}}: F \in \mathbb{R}^{2} & \longrightarrow & E \in \mathbb{R}^{3} \\
(\xi=-1) & (\hat{\xi}, \hat{\eta}) & & \vec{\xi}_{S_{\mathrm{L}}}=\vec{\xi}_{S_{\mathrm{L}}}(\hat{\xi}, \hat{\eta})=(-1, \hat{\xi}, \hat{\eta}) \\
\text { Right } & \vec{\xi}_{S_{\mathrm{R}}}: F \in \mathbb{R}^{2} & \longrightarrow & E \in \mathbb{R}^{3} \\
(\xi=1) & (\hat{\xi}, \hat{\eta}) & & \vec{\xi}_{S_{\mathrm{R}}}=\vec{\xi}_{S_{\mathrm{R}}}(\hat{\xi}, \hat{\eta})=(1, \hat{\xi}, \hat{\eta}) \\
\text { Front } & \vec{\xi}_{S_{\mathrm{F}}}: F \in \mathbb{R}^{2} & \longrightarrow & E \in \mathbb{R}^{3} \\
(\eta=-1) & (\hat{\xi}, \hat{\eta}) & & \vec{\xi}_{S_{\mathrm{F}}}=\vec{\xi}_{S_{\mathrm{F}}}(\hat{\xi}, \hat{\eta})=(\hat{\xi},-1, \hat{\eta}) \\
\text { Back } & \vec{\xi}_{S_{\mathrm{Ba}}}: F \in \mathbb{R}^{2} & \longrightarrow & E \in \mathbb{R}^{3} \\
(\eta=1) & (\hat{\xi}, \hat{\eta}) & & \vec{\xi}_{S_{\mathrm{Ba}}}=\vec{\xi}_{S_{\mathrm{Ba}}}(\hat{\xi}, \hat{\eta})=(\hat{\xi}, 1, \hat{\eta}) \\
\text { Bottom } & \vec{\xi}_{S_{\mathrm{Bo}}}: F \in \mathbb{R}^{2} & \longrightarrow & E \in \mathbb{R}^{3} \\
(\zeta=-1) & (\hat{\xi}, \hat{\eta}) & & \vec{\xi}_{S_{\mathrm{Bo}}}=\vec{\xi}_{S_{\mathrm{Bo}}}(\hat{\xi}, \hat{\eta})=(\hat{\xi}, \hat{\eta},-1) \\
\text { Top } & \vec{\xi}_{S_{\mathrm{T}}}: F \in \mathbb{R}^{2} & \longrightarrow & E \in \mathbb{R}^{3} \\
(\zeta=1) & (\hat{\xi}, \hat{\eta}) & & \vec{\xi}_{S_{\mathrm{T}}}=\vec{\xi}_{S_{\mathrm{T}}}(\hat{\xi}, \hat{\eta})=(\hat{\xi}, \hat{\eta}, 1)
\end{array}
$$

We get the coordinates of each face applying (2.3) to the element mapping $\vec{X}$, which gives a parametric function on $(\hat{\xi}, \hat{\eta})$,

$$
\vec{S}_{f}=\vec{S}_{f}(\hat{\xi}, \hat{\eta})=\vec{X}\left(\vec{\xi}_{f}(\hat{\xi}, \hat{\eta})\right) \text {. }
$$

For a valid mapping, the function $\vec{X}$ (and all the six surface functions $\vec{S}_{f}$ ) is bijective in $E=[-1,1]^{3}$. To construct the element transfinite mapping, however, we start from the face functions $\vec{S}_{f}$. When building the mesh, the information available are the six boundary functions from the element definition in the mesh. There are two ways to construct a face,

1. If the face is a quadrilateral, the surface is constructed with a transfinite submapping that blends the coordinates of the four corners $\left(\left\{\vec{x}_{i}\right\}_{i=1}^{4}\right.$, with $\vec{x}_{i} \neq \vec{x}_{j}$ if $i \neq j)$,

$$
\begin{aligned}
\vec{S}^{\left\{\vec{x}_{i}\right\}}(\hat{\xi}, \hat{\eta})= & \frac{1}{4} \vec{x}_{1}(1-\hat{\xi})(1-\hat{\eta})+\frac{1}{4} \vec{x}_{2}(1+\hat{\xi})(1-\hat{\eta}) \\
& +\frac{1}{4} \vec{x}_{3}(1+\hat{\xi})(1+\hat{\eta})+\frac{1}{4} \vec{x}_{4}(1-\hat{\xi})(1+\hat{\eta}) .
\end{aligned}
$$




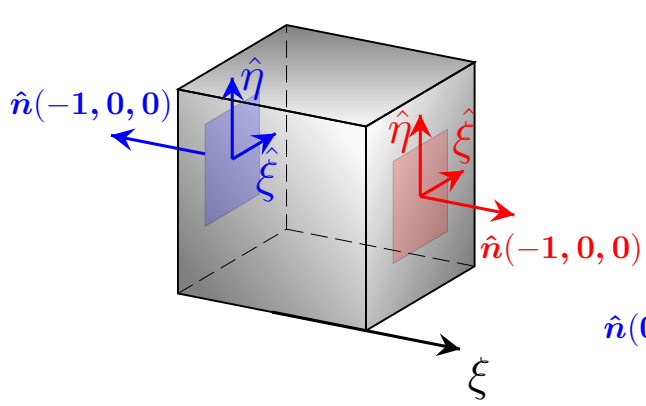

(a) $\xi= \pm 1$ (Left/Right)

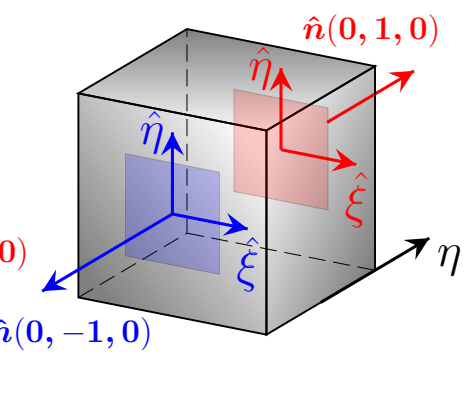

(b) $\eta= \pm 1$ (Front/Back)

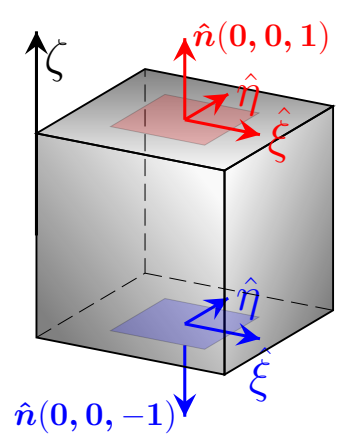

(c) $\zeta= \pm 1($ Bottom/Top)

Figure 2.2: Representation of the local coordinates at the six faces. The faces with $\xi^{i}=-1$ have been represented in blue, and the faces with $\xi^{i}=1$ in red. The normal vector to the reference element faces $\hat{n}$ is also represented

2. For a face of arbitrary geometry, a valid analytical parametric expression (or a polynomial approximation) of the surface,

$$
\vec{S}(\hat{\xi}, \hat{\eta}), \quad\left\|\vec{S}_{\hat{\xi}} \times \vec{S}_{\hat{\eta}}\right\| \neq 0 \quad \forall(\hat{\xi}, \hat{\eta}) \in F=[-1,1]^{2}
$$

has to be known.

When the parametric expression of the six surfaces that define an element are known, they are blended to construct the transfinite mapping for the element,

$$
\begin{aligned}
\vec{X}= & \frac{1}{2} \vec{S}_{\mathrm{L}}(\eta, \zeta)(1-\xi)+\frac{1}{2} \vec{S}_{\mathrm{R}}(\eta, \zeta)(\xi+1)+\frac{1}{2} \vec{S}_{\mathrm{F}}(\xi, \zeta)(1-\eta)+\frac{1}{2} \vec{S}_{\mathrm{Ba}}(\xi, \zeta)(\eta+1) \\
& +\frac{1}{2} \vec{S}_{\mathrm{Bo}}(\xi, \eta)(1-\zeta)+\frac{1}{2} \vec{S}_{\mathrm{T}}(\xi, \eta)(\zeta+1)-\frac{1}{4} \vec{S}_{\mathrm{Bo}}(\xi,-1)(1-\eta)(1-\zeta) \\
& -\frac{1}{4} \vec{S}_{\mathrm{Bo}}(1, \eta)(1+\xi)(1-\zeta)-\frac{1}{4} \vec{S}_{\mathrm{Bo}}(\xi, 1)(\eta+1)(1-\zeta)-\frac{1}{4} \vec{S}_{\mathrm{Bo}}(-1, \eta)(1-\xi)(1-\zeta) \\
& -\frac{1}{4} \vec{S}_{\mathrm{T}}(\xi,-1)(1-\eta)(1+\zeta)-\frac{1}{4} \vec{S}_{\mathrm{T}}(1, \eta)(1+\xi)(1+\zeta)-\frac{1}{4} \vec{S}_{\mathrm{T}}(\xi, 1)(\eta+1)(1+\zeta) \\
& -\frac{1}{4} \vec{S}_{\mathrm{T}}(-1, \eta)(1-\xi)(1+\zeta)-\frac{1}{4} \vec{S}_{\mathrm{L}}(-1, \zeta)(1-\xi)(1-\eta)-\frac{1}{4} \vec{S}_{\mathrm{L}}(1, \zeta)(1-\xi)(1+\eta) \\
& -\frac{1}{4} \vec{S}_{\mathrm{R}}(-1, \zeta)(1+\xi)(1-\eta)-\frac{1}{4} \vec{S}_{\mathrm{R}}(1, \zeta)(1+\xi)(1+\eta) \\
& +\frac{1}{8} \vec{S}_{\mathrm{Bo}}(-1,-1)(1-\xi)(1-\eta)(1-\zeta)+\frac{1}{8} \vec{S}_{\mathrm{Bo}}(1,-1)(1+\xi)(1-\eta)(1-\zeta) \\
& +\frac{1}{8} \vec{S}_{\mathrm{Bo}}(-1,1)(1-\xi)(1+\eta)(1-\zeta)+\frac{1}{8} \vec{S}_{\mathrm{Bo}}(1,1)(1+\xi)(1+\eta)(1-\zeta) \\
& +\frac{1}{8} \vec{S}_{\mathrm{T}}(-1,-1)(1-\xi)(1-\eta)(1+\zeta)+\frac{1}{8} \vec{S}_{\mathrm{T}}(1,-1)(1+\xi)(1-\eta)(1+\zeta) \\
& +\frac{1}{8} \vec{S}_{\mathrm{T}}(-1,1)(1-\xi)(1+\eta)(1+\zeta)+\frac{1}{8} \vec{S}_{\mathrm{T}}(1,1)(1+\xi)(1+\eta)(1+\zeta) .
\end{aligned}
$$


Note that if all the surface functions $\vec{S}_{i}$ are order $N$ polynomials, the resulting mapping is also an order $N$ polynomial.

\subsubsection{Element differential geometry}

From the transformation (2.1) one can define three covariant basis vectors as the derivative of the mapping with respect to the local coordinates,

$$
\vec{a}_{i}=\frac{\partial \vec{X}}{\partial \xi^{i}}, \quad i=1,2,3,
$$

and three contravariant basis vectors, the derivatives of the inverse transformation $\vec{X}^{-1}$ give

$$
\vec{a}^{i}=\vec{\nabla} \xi^{i}=\frac{1}{J}\left(\vec{a}_{j} \times \vec{a}_{k}\right), \quad(i, j, k) \text { cyclic, }
$$

where

$$
J=\vec{a}_{1} \cdot\left(\vec{a}_{2} \times \vec{a}_{3}\right)
$$

is the Jacobian of the mapping $\vec{X}$. A valid mapping satisfies that $J \neq 0$ in $E$. The contravariant vectors $\vec{a}^{i}$ define a normal direction to the $\xi^{i}=$ const. surfaces, which point towards increasing values of $\xi^{i}$. Thus, they represent the inward normal direction for the $\xi^{i}=-1$ boundary faces (left, front, and bottom), and the outward normal direction for the $\xi^{i}=1$ boundary faces (right, back, and top).

An important property is that the contravariant coordinate vectors satisfy the metric identities Kopriva [2006],

$$
\sum_{i=1}^{3} \frac{\partial\left(J a_{n}^{i}\right)}{\partial \xi^{i}}=0, n=1,2,3,
$$

where $a_{n}^{i}$ is the $n$-th Cartesian component of the contravariant vector $\vec{a}^{i}$.

The volume weighted contravariant basis $J \vec{a}^{i}$ are used to transform differential operators from physical $(\vec{\nabla})$ to reference $\left(\vec{\nabla}_{\xi}\right)$ space. The divergence of a vector is Gassner et al. [2018]

$$
\vec{\nabla} \cdot \vec{f}=\frac{1}{J} \vec{\nabla}_{\xi} \cdot\left(\overline{\bar{m}}^{T} \vec{f}\right)=\frac{1}{J} \vec{\nabla}_{\xi} \cdot(\overrightarrow{\tilde{f}})
$$

where

$$
\overline{\bar{m}}=\left(J \vec{a}^{\xi}, J \vec{a}^{\eta}, J \vec{a}^{\zeta}\right),
$$

and $\overrightarrow{\tilde{f}}=\underline{m}^{T} \vec{f}$ are called contravariant vectors,

$$
\overrightarrow{\tilde{f}}=\left(\tilde{f} \xi, \tilde{f}^{\eta}, \tilde{f}^{\zeta}\right)=\underline{m}^{T} \vec{f}, \quad \tilde{f}^{i}=J \vec{a}^{i} \cdot \vec{f} .
$$


Note that contravariant vectors are the components of the vector $\vec{f}$ in the contravariant basis. The gradient of a scalar is Gassner et al. [2018]

$$
\vec{\nabla} u=\frac{1}{J} \overline{\bar{m}} \vec{\nabla}_{\xi} u=\frac{1}{J}\left(J \vec{a}^{1} \frac{\partial u}{\partial \xi}+J \vec{a}^{2} \frac{\partial u}{\partial \eta}+J \vec{a}^{3} \frac{\partial u}{\partial \zeta}\right)
$$

We are interested in solving a system of PDEs, which eventually will lead to taking the divergence of a block vector $\overrightarrow{\mathbf{f}}$, and the gradient of a state vector $\mathbf{q}$. We use (1.24) to write the divergence of an entire block vector compactly. Thus, we define the block matrix $\underline{\mathbf{m}}$,

$$
\underline{\mathbf{m}}=\left(\begin{array}{ccc}
J a_{1}^{1} \underline{I}_{N_{\mathrm{E}}} & J a_{1}^{2} \underline{I}_{N_{\mathrm{E}}} & J a_{1}^{3} \underline{I}_{N_{\mathrm{E}}} \\
J a_{2}^{1} \underline{I}_{N_{\mathrm{E}}} & J a_{2}^{2} \underline{I}_{N_{\mathrm{E}}} & J a_{2}^{3} \underline{I}_{N_{\mathrm{E}}} \\
J a_{3}^{1} \underline{I}_{N_{\mathrm{E}}} & J a_{3}^{2} \underline{I}_{N_{\mathrm{E}}} & J a_{3}^{3} \underline{I}_{N_{\mathrm{E}}}
\end{array}\right),
$$

which allows us to write (2.12) for all the state variables simultaneously,

$$
\vec{\nabla} \cdot \overrightarrow{\mathbf{f}}=\frac{1}{J} \vec{\nabla}_{\xi} \cdot\left(\underline{\mathbf{m}}^{T} \overrightarrow{\mathbf{f}}\right)=\frac{1}{J} \vec{\nabla}_{\xi} \cdot \overrightarrow{\tilde{\mathbf{f}}}
$$

with $\overrightarrow{\tilde{f}}$ being the block vector of the contravariant fluxes,

$$
\overrightarrow{\tilde{\mathbf{f}}}=\underline{\mathbf{m}}^{T} \overrightarrow{\mathbf{f}}, \quad \tilde{\mathbf{f}}^{i}=J \vec{a}^{i} \cdot \overrightarrow{\mathbf{f}} .
$$

Similarly, we use the matrix (2.16) to extend the gradient (2.15) of an entire state vector,

$$
\vec{\nabla} \mathbf{q}=\frac{1}{J} \underline{\mathbf{m}} \vec{\nabla}_{\xi} \mathbf{q}
$$

\subsubsection{Face differential geometry}

At the faces, we will need the normal direction, the surface Jacobian, and the face differential of the physical space element, $e$. For the reference element, the normal vector $\hat{n}$ is simply $\hat{n}= \pm \hat{\xi}^{i}$ for $\xi^{i}= \pm 1$ (see Fig. 2.2). For the physical element, from the surface parametric expressions, we can compute

$$
\mathrm{d} \vec{S}_{f}= \pm \vec{S}_{\hat{\xi}} \times \vec{S}_{\hat{\eta}} \mathrm{d} \hat{\xi} \mathrm{d} \hat{\eta}= \pm \vec{S}_{\hat{\xi}} \times \vec{S}_{\hat{\eta}} \mathrm{d} \hat{S}
$$

where the sign has to be manually adjusted to get an outward normal vector to $E$ (given by the use of the right hand rule on each face coordinate system in Fig. 2.2). Using the chain rule for the transformation (2.4), the surface differential can be related 
to the element mapping metric terms $J \vec{a}^{i}$. Precisely, for the six faces,

$$
\begin{aligned}
& \mathrm{d} \vec{S}_{\mathrm{L}}=-\vec{X}_{\eta} \times \vec{X}_{\zeta} \mathrm{d} \hat{S}=-\vec{a}_{\eta} \times \vec{a}_{\zeta} \mathrm{d} \hat{S}=-J \vec{a}^{\xi} \mathrm{d} \hat{S}, \\
& \mathrm{~d} \vec{S}_{\mathrm{R}}=\vec{X}_{\eta} \times \vec{X}_{\zeta} \mathrm{d} \hat{S}=\vec{a}_{\eta} \times \vec{a}_{\zeta} \mathrm{d} \hat{S}=J \vec{a}^{\xi} \mathrm{d} \hat{S}, \\
& \mathrm{~d} \vec{S}_{\mathrm{F}}=\vec{X}_{\xi} \times \vec{X}_{\zeta} \mathrm{d} \hat{S}=\vec{a}_{\xi} \times \vec{a}_{\zeta} \mathrm{d} \hat{S}=-J \vec{a}^{\eta} \mathrm{d} \hat{S}, \\
& \mathrm{~d} \vec{S}_{\mathrm{B} a}=-\vec{X}_{\xi} \times \vec{X}_{\zeta} \mathrm{d} \hat{S}=-\vec{a}_{\xi} \times \vec{a}_{\zeta} \mathrm{d} \hat{S}=J \vec{a}^{\eta} \mathrm{d} \hat{S}, \\
& \mathrm{~d} \vec{S}_{\mathrm{T}}=-\vec{X}_{\xi} \times \vec{X}_{\eta} \mathrm{d} \hat{S}=-\vec{a}_{\xi} \times \vec{a}_{\eta} \mathrm{d} \hat{S}=-J \vec{a}^{\zeta} \mathrm{d} \hat{S}, \\
& \mathrm{~d} \vec{S}_{\mathrm{B} o}=\vec{X}_{\xi} \times \vec{X}_{\eta} \mathrm{d} \hat{S}=\vec{a}_{\xi} \times \vec{a}_{\eta} \mathrm{d} \hat{S}=J \vec{a}^{\zeta} \mathrm{d} \hat{S},
\end{aligned}
$$

we see systematically that the surface differential for a face $\xi^{i}= \pm 1$ is computed from the metric term $J \vec{a}^{i}$ evaluated at the face, and with changed sign for $\xi^{i}=-1$. This makes sense since contravariant vectors $\left(\vec{a}^{i}=\vec{\nabla} \xi^{i}\right)$ represent a normal direction to the isolines $\xi^{i}=$ const. Thus, we can compute surface metric terms from the projection of the element metric terms to the faces. We finally define the surface Jacobian and normal vector as

$$
\mathrm{d} \vec{S}^{i}= \pm J \vec{a}^{i} \mathrm{~d} \hat{S}=J_{f} \vec{n}^{i} \mathrm{~d} \hat{S}, \quad J_{f}=\left|J \vec{a}^{i}\right|, \quad \vec{n}^{i}= \pm \frac{J \vec{a}^{i}}{\left|J \vec{a}^{i}\right|}
$$

and, moreover, the correct contravariant vector $\pm J \vec{a}^{i}$ can be automatically selected from metric terms $\overline{\bar{m}}$ (2.16) and the reference space normal vector $\hat{n}$ (represented in Fig. 2.2),

$$
\mathrm{d} \vec{S}=\overline{\bar{m}} \hat{n} \mathrm{~d} \hat{S}
$$

\subsection{Approximation}

As explained in Sec. 2.2, the domain $\Omega$ is divided into non-overlapping elements $e$, which are geometrically transformed from the reference element $E=[-1,1]^{3}$. In each element $e$, the DGSEM approximates a continuous function by a polynomial of order $N$ written in the reference element $E$ coordinates. We call this operation $\mathrm{I}^{N}\left(\left.\bullet\right|_{e}\right)$,

$$
\left.q(\vec{x}, t)\right|_{e} \approx \mathrm{I}^{N}\left(\left.q\right|_{e}\right)=Q^{e}(\vec{\xi}, t)
$$

In this work, we refer to continuous functions with small case letters (e.g. q), and its polynomial approximation with upper case letters (e.g. Q). If a symbol lacks an upper case (e.g. some Greek letters), whether an equation considers the continuous function or the polynomial approximation should be taken in context. Lastly, the superscript $e$ that indicates which element is considered will be dropped, and an arbitrary element is assumed. 


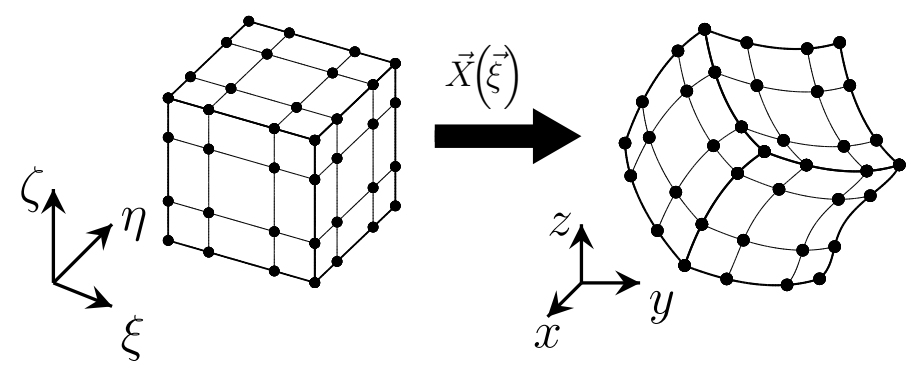

Figure 2.3: Representation of the interpolation nodes $\left(\xi_{i}, \eta_{j}, \zeta_{k}\right)$ in the reference element (left), and their physical space position $(x, y, z)_{i j k}$ after the application of the mapping (2.1) (right). A polynomial order $N=3$, and Gauss-Lobatto points are considered for this example

Among the different DG variants, we use the nodal DGSEM, where the polynomial interpolation is performed so that the continuous function and its polynomial approximation match at a set of $N+1$ nodes, $\left\{\xi_{i}, \eta_{j}, \zeta_{k}\right\}_{i, j, k=0}^{N}$,

$$
q\left(\vec{X}\left(\xi_{i}, \eta_{j}, \zeta_{k}\right)\right)=Q\left(\xi_{i}, \eta_{j}, \zeta_{k}\right)=Q_{i j k}, \quad i, j, k=0, \ldots, N
$$

The coefficients $Q_{i j k}$ are called nodal values, as they represent the value of the solution at the interpolation nodes (see Fig. 2.3). In the vast majority of the DGSEM approximations, one of two sets of nodes have been used: Gauss and Gauss-Lobatto points Kopriva [2009]. The order $N$ polynomial that can be evaluated at $(N+1)^{3}$ points $\left(\xi_{i}, \eta_{j}, \zeta_{k} ; Q_{i, j, k}\right)$ is unique. We use a tensor product Lagrange polynomials representation to construct the polynomial,

$$
Q(\vec{\xi})=\sum_{i, j, k=0}^{N} Q_{i j k} l_{i}(\xi) l_{j}(\eta) l_{k}(\zeta), l_{i}(\xi)=\prod_{\substack{j=0 \\ j \neq i}}^{N} \frac{\xi-\xi_{j}}{\xi_{j}-\xi_{i}}
$$

Note that since the Lagrange polynomials satisfy the cardinal property, $l_{i}\left(\xi_{j}\right)=\delta_{i j}$ (with $\delta_{i j}$ the Kronecker delta), the property (2.25) is satisfied automatically by construction.

When a function is also time-dependent, the coefficients will be time dependent,

$$
Q(\vec{\xi}, t)=\sum_{i, j, k=0}^{N} Q_{i j k}(t) l_{i}(\xi) l_{j}(\eta) l_{k}(\zeta), Q_{i j k}(t)=q\left(\vec{X}\left(\xi_{i}, \eta_{j}, \zeta_{k}\right), t\right)
$$

Moreover, any function of the solution, $f(q)$, is approximated also following (2.25),

$$
f\left(q\left(\vec{X}\left(\xi_{i}, \eta_{j}, \zeta_{k}\right)\right)\right)=F\left(\xi_{i}, \eta_{j}, \zeta_{k}\right)=F\left(Q_{i j k}\right)=F_{i j k}, \quad i, j, k=0, \ldots, N .
$$


The approximations of derivatives use the derivative of the Lagrange polynomials. A partial derivative (e.g. with respect to $\eta$ ) is

$$
Q_{\eta}=\sum_{i, j, k=0}^{N} Q_{i j k} l_{i}(\xi) l_{j}^{\prime}(\eta) l_{k}(\zeta)
$$

More importantly one needs the derivatives evaluated at the interpolation nodes. After using the cardinal property in the two variables that are not differentiated, we write the nodal values of the local gradient of a polynomial as

$$
\begin{aligned}
\vec{\nabla}_{\xi} Q_{i j k} & =\sum_{m=0}^{N}\left(Q_{m j k} l_{m}^{\prime}\left(\xi_{i}\right), Q_{i m k} l_{m}^{\prime}\left(\eta_{j}\right), Q_{i j m} l_{m}^{\prime}\left(\zeta_{k}\right)\right) \\
& =\sum_{m=0}^{N}\left(D_{i m} Q_{m j k}, D_{j m} Q_{i m k}, D_{k m} Q_{i j m}\right),
\end{aligned}
$$

where the matrix containing the nodal values of the lagrange polynomials derivative is called the derivative matrix, $D_{i j}=l_{i}^{\prime}\left(\xi_{j}\right)$.

It is important to remark that differentiation and interpolation do not commute Kopriva [2009],

$$
\mathrm{I}^{N}\left(q^{\prime}\right) \neq \mathrm{I}^{N}(q)^{\prime}, \quad \mathrm{I}^{N}\left(U^{2}\right)^{\prime} \neq \mathrm{I}^{N}\left(2 U U^{\prime}\right)
$$

The lack of commutativity is a result of the aliasing errors incurred Hesthaven and Warburton [2007]; Manzanero et al. [2018a]; Kopriva [2009]; Kopriva and Gassner [2014]. The aliasing errors are briefly discussed in Chapter 3.

We now describe the approximation of the geometry and the metric terms. The transfinite mapping $\vec{X}(\vec{\xi})$ is approximated using the interpolation operator $\mathrm{I}^{N}(2.25)$,

$$
\vec{X}=\sum_{i, j, k=0}^{N} \vec{X}\left(\xi_{i}, \eta_{k}, \zeta_{k}\right) l_{i}(\xi) l_{j}(\eta) l_{k}(\zeta)=\sum_{i, j, k=0}^{N} \vec{X}_{i j k} l_{i}(\xi) l_{j}(\eta) l_{k}(\zeta) .
$$

In (2.32) we assumed without loss of generality that the mapping itself is an order $N$ polynomial (i.e. all the surface functions are order $N$ polynomials) only to avoid using an additional symbol for the discrete mapping (i.e. the continuous and discrete mapping coincide). The covariant basis is computed from the direct differentiation of (2.32) using (2.30) without incurring any error,

$$
\left(\vec{a}_{1}, \vec{a}_{2}, \vec{a}_{3}\right)_{i j k}=\sum_{i, j, k=0}^{N}\left(D_{i m} \vec{X}_{m j k}, D_{j m} \vec{X}_{i m k}, D_{k m} \vec{X}_{i j m}\right)
$$

and the Jacobian is approximated from its definition (2.10),

$$
\mathscr{J}=\mathrm{I}^{N}\left(\vec{a}_{1} \cdot\left(\vec{a}_{2} \times \vec{a}_{3}\right)\right)
$$


The contravariant basis, however, cannot be approximated directly from its definition (2.9),

$$
\mathscr{J} \vec{a}^{i} \neq \mathrm{I}^{N}\left(\vec{a}_{j} \times \vec{a}_{k}\right)
$$

Since the contravariant basis is an order $2 N$ polynomial, and given the fact that interpolation and derivatives do not commute, the direct discrete version of the contravariant basis (2.35) does not satisfy the (discrete) metric identities (2.11) Kopriva [2006]. We adopt the curl form presented in Kopriva [2006],

$$
\mathscr{J} a_{n}^{i}=-\hat{x}_{i} \cdot \vec{\nabla}_{\xi} \times\left(\mathrm{I}^{N}\left(X_{l} \vec{\nabla}_{\xi} X_{m}\right)\right), \quad i=1,2,3, \quad n=1,2,3, \quad(n, m, l) \text { cyclic, }
$$

that gives an approximation of the contravariant basis that satisfies the discrete metric identities,

$$
\sum_{i=1}^{3} \frac{\partial\left(\mathscr{J} a_{n}^{i}\right)}{\partial \xi^{i}}=0, \quad n=1,2,3
$$

by construction. With the discrete contravariant basis we compute the approximation of the divergence,

$$
J \vec{\nabla} \cdot \overrightarrow{\mathbf{f}}_{i j k} \approx \vec{\nabla}_{\xi} \cdot \overrightarrow{\widetilde{\mathbf{F}}}_{i j k}=\sum_{m=0}^{N}\left(D_{i m} \tilde{\mathbf{F}}_{m j k}^{\xi}+D_{j m} \tilde{\mathbf{F}}_{i m k}^{\eta}+D_{k m} \tilde{\mathbf{F}}_{i j m}^{\zeta}\right), \quad \tilde{\mathbf{F}}_{i j k}^{i}=\mathscr{J} \vec{a}_{i j k}^{i} \cdot \overrightarrow{\mathbf{F}}_{i j k},
$$

and gradient,

$$
J \vec{\nabla} \mathbf{q}_{i j k} \approx \underline{\mathbf{M}} \vec{\nabla}_{\xi} \mathbf{Q}=\sum_{m=0}^{N}\left(\mathscr{J} \vec{a}_{i j k}^{\xi} D_{i m} \mathbf{Q}_{m j k}+\mathscr{J} \vec{a}_{i j k}^{\eta} D_{j m} \mathbf{Q}_{i m k}+\mathscr{J} \vec{a}_{i j k}^{\zeta} D_{k m} \mathbf{Q}_{i j m}\right)
$$

An important aspect of the approximation is the projection of the solution, mapping, and metric terms to the faces. On the face, the solution is approximated by an order $N$ polynomial in the reference face coordinates $(\hat{\xi}, \hat{\eta})$,

$$
Q_{f}(\hat{\xi}, \hat{\eta})=\sum_{i, j=0}^{N} Q_{i j} l_{i}(\hat{\xi}) l_{j}(\hat{\eta})
$$

Given the relation (2.3), we can write the nodal degrees of freedom on the face as

$$
Q_{i j}^{f}=\sum_{m, n, l=0}^{N} Q_{m n l} l_{m}\left(\xi_{f}\left(\hat{\xi}_{i}, \hat{\eta}_{j}\right)\right) l_{n}\left(\eta_{f}\left(\hat{\xi}_{i}, \hat{\eta}_{j}\right)\right) l_{l}\left(\zeta_{f}\left(\hat{\xi}_{i}, \hat{\eta}_{j}\right)\right)
$$

If the polynomial order and interpolation nodes used for the element are maintained for the face, the cardinal property can be applied to two of the coordinates, and then a one-dimensional projection in the remaining coordinate is performed. 
Finally, the metric terms at the face are computed from the projection of the element metric terms,

$$
\mathrm{d} \vec{S}_{i}= \pm \mathscr{J} \vec{a}^{i} \mathrm{~d} \hat{S}=\mathscr{J}_{f}^{i} \vec{n}^{i} \mathrm{~d} \hat{S}
$$

where

$$
\mathscr{J} \vec{a}_{i j}^{i}=\sum_{m, n, l=0}^{N} \mathscr{J} \vec{a}_{m n l}^{i} l_{m}\left(\xi_{f}\left(\hat{\xi}_{i}, \hat{\eta}_{j}\right)\right) l_{n}\left(\eta_{f}\left(\hat{\xi}_{i}, \hat{\eta}_{j}\right)\right) l_{l}\left(\zeta_{f}\left(\hat{\xi}_{i}, \hat{\eta}_{j}\right)\right) .
$$

Then, the normal vector and surface Jacobian are computed from (2.22).

\subsection{Approximation of integrals through quadratures}

We describe how to compute approximations to integrals. In the reference space $E$, integrals are computed using quadrature rules based on a set of Gauss-Lobatto quadrature weights $\left\{w_{i}\right\}_{i=0}^{N}$ associated to the interpolation nodes,

$$
\int_{E} F \mathrm{~d} \vec{\xi} \approx \int_{E, N} F \mathrm{~d} \vec{\xi}=\sum_{i, j, k=0}^{N} w_{i} w_{j} w_{k} F_{i j k} .
$$

We define the three-dimensional quadrature weights $w_{i j k}=w_{i} w_{j} w_{k}$. The integral in the physical elements is approximated by multiplying by the Jacobian,

$$
\int_{e} F \mathrm{~d} \vec{x} \approx \int_{e, N} F \mathrm{~d} \vec{x}=\int_{E, N} \mathscr{J} F \mathrm{~d} \vec{\xi}=\sum_{i, j, k=0}^{N} w_{i j k} \mathscr{J}_{i j k} F_{i j k}=\langle\mathscr{J} F\rangle_{E, N} .
$$

The quadrature is exact for order up to $2 N \pm 1$ ( +1 for Gauss, -1 for Gauss-Lobatto) polynomials.

Following similar arguments, we define the inner product of two functions as the integral of their product in $e$,

$$
\langle F, G\rangle_{e}=\langle J F, G\rangle_{E} \approx\langle\mathscr{J} F, G\rangle_{E, N}=\int_{E, N} \mathscr{J} F G \mathrm{~d} \vec{\xi}=\sum_{i, j, k=0}^{N} w_{i j k} \mathscr{J}_{i j k} F_{i j k} G_{i j k},
$$

and the quadrature of a single function is $\langle\mathscr{J} F, 1\rangle_{E, N}=\langle\mathscr{J} F\rangle_{E, N}$.

Surface integrals extend to all six faces of an element. Applying a two-dimensional quadrature on the reference face $F=[-1,1]^{2}$,

$$
\begin{aligned}
\int_{\partial e} \vec{F} \cdot \mathrm{d} \vec{S} & \approx \int_{\partial e, N} \vec{F} \cdot \mathrm{d} \vec{S}=\sum_{f=1}^{6} \int_{f, N} \vec{F}_{f} \cdot \mathrm{d} \vec{S}^{f}=\sum_{f=1}^{6} \int_{F, N} \vec{F}_{f} \cdot \vec{n}^{f} \mathscr{J}_{f} \mathrm{~d} \hat{S} \\
& =\sum_{f=1}^{6} \sum_{i, j=0}^{N} w_{i j} \mathscr{J}_{i j}^{f} \vec{F}_{i j}^{f} \cdot \vec{n}_{i j}^{f} .
\end{aligned}
$$


We get the same result when the integrals are computed from the contravariant components of $\vec{F}$. We integrate the contravariant flux over the boundary of the reference element $\partial E$, where $\hat{n}$ is the reference element normal vector (see Fig. 2.2),

$$
\begin{aligned}
\int_{\partial E} \overrightarrow{\tilde{F}} \cdot \hat{n} \mathrm{~d} \hat{S} \approx & \int_{\partial E, N} \overrightarrow{\tilde{F}} \cdot \hat{n} \mathrm{~d} \hat{S}=\left.\int_{F, N} \tilde{F}^{1}(\xi, \hat{\xi}, \hat{\eta}) \mathrm{d} \hat{S}\right|_{\xi=-1} ^{\xi=1} \\
& +\left.\int_{F, N} \tilde{F}^{2}(\hat{\xi}, \eta, \hat{\eta}) \mathrm{d} \hat{S}\right|_{\eta=-1} ^{\eta=1}+\left.\int_{F, N} \tilde{F}^{3}(\hat{\xi}, \hat{\eta}, \zeta) \mathrm{d} \hat{S}\right|_{\zeta=-1} ^{\zeta=1} \\
= & \sum_{i, j=0}^{N} w_{i j}\left(\tilde{F}_{i j}^{1, \mathrm{R}}-\tilde{F}_{i j}^{1, \mathrm{~L}}+\tilde{F}_{i j}^{2, \mathrm{Ba}}-\tilde{F}_{i j}^{2, \mathrm{~F}}+\tilde{F}_{i j}^{3, \mathrm{~T}}-\tilde{F}_{i j}^{3, \mathrm{Bo}}\right)
\end{aligned}
$$

Both (2.47) and (2.48) are algebraically equivalent as a result of (2.18) and (2.23),

$$
\int_{\partial e, N} \vec{F} \cdot \mathrm{d} \vec{S}=\int_{\partial E, N} \vec{F} \overline{\bar{m}} \hat{n} \mathrm{~d} \hat{S}=\int_{\partial E, N}\left(\overline{\bar{m}}^{T} \vec{F}\right) \cdot \hat{n} \mathrm{~d} \hat{S}=\int_{\partial E, N} \overrightarrow{\tilde{F}} \cdot \hat{n} \mathrm{~d} \hat{S} .
$$

\subsubsection{Gauss-Lobatto points and the SBP-SAT property}

Gauss-Lobatto points produce a special and relevant variant of the DGSEM. As opposed to Gauss points, they include the end points: $\xi_{0}=-1$ and $\xi_{N}=1$. This property is crucial for the stability, since a projection to the surface from the volume is not necessary, and one just simply selects the appropriate degree of freedom from the volume. Mathematically, $l_{i}(-1)=\delta_{i 0}$ and $l_{i}(1)=\delta_{i N}$. The Gauss-Lobatto boundary projection operator (2.41) for the six faces of the reference element is then simplified to

$$
Q_{i j}^{\mathrm{L}}=Q_{0 i j}, \quad Q_{i j}^{\mathrm{R}}=Q_{N i j}, \quad Q_{i j}^{\mathrm{F}}=Q_{i 0 j}, \quad Q_{i j}^{\mathrm{Ba}}=Q_{i N j}, \quad Q_{i j}^{\mathrm{Bo}}=Q_{i j 0}, \quad Q_{i j}^{\mathrm{T}}=Q_{i j N} .
$$

An important property that can be extracted from (2.50), is that polynomial interpolation, and projection to boundary commute when using Gauss-Lobatto points,

$$
\mathrm{I}^{N}(U V)_{f}=\mathrm{I}^{N}(U)_{f} \mathrm{I}^{N}(V)_{f}
$$

Property (2.51) is known as the Simultaneous-Approximation-Term (SAT) property, and it is the cornerstone of discrete stability.

Although the order of accuracy of the quadratures is lower than that found in Gauss points $(2 N-1$ vs $2 N+1)$, only the Gauss-Lobatto points satisfy a discrete version of the Gauss law for order $N$ polynomials,

$$
\int_{\Omega} u \vec{\nabla} \cdot \vec{v} \mathrm{~d} \vec{x}=\int_{\partial \Omega} u \vec{v} \cdot \vec{n} \mathrm{~d} \vec{S}-\int_{\Omega} \vec{v} \cdot \vec{\nabla} u \mathrm{~d} \vec{x}
$$


Given a scalar polynomial $U$, and a vector polynomial $\vec{V}$, we consider the following integral,

$$
\begin{aligned}
\left\langle U, \vec{\nabla}_{\xi} \cdot \overrightarrow{\tilde{V}}\right\rangle_{E, N}= & \int_{F, N}\left(\int_{-1, N}^{1} U \sum_{i=0}^{N} \tilde{V}_{i}^{1}(\eta, \zeta) l_{i}^{\prime}(\xi) \mathrm{d} \xi\right) \mathrm{d} \eta \mathrm{d} \zeta \\
& +\int_{F, N}\left(\int_{-1, N}^{1} U \sum_{j=0}^{N} \tilde{V}_{j}^{2}(\xi, \zeta) l_{j}^{\prime}(\eta) \mathrm{d} \eta\right) \mathrm{d} \xi \mathrm{d} \zeta \\
& +\int_{F, N}\left(\int_{-1, N}^{1} U \sum_{k=0}^{N} \tilde{V}_{k}^{3}(\xi, \eta) l_{k}^{\prime}(\zeta) \mathrm{d} \zeta\right) \mathrm{d} \xi \mathrm{d} \eta
\end{aligned}
$$

where

$$
\begin{aligned}
& \tilde{V}_{i}^{1}(\eta, \zeta)=\sum_{j k=0}^{N} \tilde{V}_{i j k}^{1} l_{j}(\eta) l_{k}(\zeta), \\
& \tilde{V}_{j}^{2}(\xi, \zeta)=\sum_{i k=0}^{N} \tilde{V}_{i j k}^{2} l_{i}(\xi) l_{k}(\zeta), \\
& \tilde{V}_{k}^{3}(\xi, \eta)=\sum_{i, j=0}^{N} \tilde{V}_{i j k}^{3} l_{i}(\xi) l_{j}(\eta) .
\end{aligned}
$$

The inner integrals are all polynomials of order $2 N-1$ in their arguments, since the product $l_{n}\left(\xi^{i}\right) l_{m}^{\prime}\left(\xi^{i}\right)$ is computed. Thus, the quadrature is computed exactly, and we can write (e.g. for the first one),

$$
\int_{-1, N}^{1} U \sum_{i=0}^{N} \tilde{V}_{i}^{1}(\eta, \zeta) l_{i}^{\prime}(\xi) \mathrm{d} \xi=\left.U \tilde{V}^{1}\right|_{\xi=-1} ^{\xi=1}-\int_{-1, N}^{1} U_{\xi} \tilde{V}^{1} \mathrm{~d} \xi .
$$

Repeating similar operations for the other two directions, we get,

$$
\begin{aligned}
\langle U, \vec{\nabla} \xi \overrightarrow{\tilde{V}}\rangle_{E, N}= & \int_{F, N}\left(\left.\left(U \tilde{V}^{1}\right)\right|_{\xi=-1} ^{\xi=1} \mathrm{~d} \eta \mathrm{d} \zeta+\left.\left(U \tilde{V}^{2}\right)\right|_{\eta=-1} ^{\eta=1} \mathrm{~d} \xi \mathrm{d} \zeta+\left.\left(U \tilde{V}^{3}\right)\right|_{\zeta=-1} ^{\zeta=1} \mathrm{~d} \xi \mathrm{d} \eta\right) \\
& -\left\langle U_{\xi}, \tilde{V}^{1}\right\rangle_{E, N}-\left\langle U_{\eta}, \tilde{V}^{2}\right\rangle_{E, N}-\left\langle U_{\zeta}, \tilde{V}^{3}\right\rangle_{E, N} \\
& =\int_{\partial E, N} U \overrightarrow{\tilde{V}} \cdot \hat{n} \mathrm{~d} \hat{S}-\langle\vec{\nabla} \xi U, \overrightarrow{\tilde{V}}\rangle_{E, N} .
\end{aligned}
$$

Eq. (2.56) is the discrete version of the Gauss law (2.52), also known as summationby-parts simultaneous-approximation-term (SBP-SAT) property. Although Gauss points' quadrature is more exact than Gauss-Lobatto, is not satisfying the SAT property (2.51) which prevents Gauss points to satisfy a discrete Gauss law. If we introduce an additional scalar polynomial $W$, and consider Gauss points,

$$
\left\langle U, \vec{\nabla}_{\xi} \cdot(W \overrightarrow{\tilde{V}})\right\rangle_{E, N}=\int_{\partial E, N} U(W \overrightarrow{\tilde{V}})_{f} \cdot \hat{n} \mathrm{~d} \hat{S}-\langle W \overrightarrow{\tilde{V}}, U\rangle_{E, N}
$$


the resulting boundary integral does not contain the product $U W \overrightarrow{\vec{V}}$, since,

$$
\int_{\partial E, N} U(W \overrightarrow{\tilde{V}})_{f} \cdot \hat{n} \mathrm{~d} \hat{S} \stackrel{\text { Gauss }}{\neq} \int_{\partial E, N} U W \overrightarrow{\tilde{V}} \cdot \vec{n} \mathrm{~d} \hat{S} .
$$

We conclude that Gauss-Lobatto points are a special form of the DGSEM with three attractive properties,

- No projection to the faces is required (2.50).

- Projection and multiplication commute (2.51).

- Satisfies a discrete version of the Gauss law (2.56).

\subsection{DG Approximation of conservation laws}

We derive the DGSEM approximation of a partial differential equation written as a conservation law,

$$
\mathbf{q}_{t}+\vec{\nabla} \cdot \overrightarrow{\mathbf{f}}_{e}(\mathbf{q})=\vec{\nabla} \cdot \overrightarrow{\mathbf{f}}_{v}(\mathbf{q}, \vec{\nabla} \mathbf{w})+\mathbf{s}(\mathbf{q}), \quad \mathbf{w}=\mathbf{w}(\mathbf{q}),
$$

where $\mathbf{q}$ is the state vector, $\overrightarrow{\mathbf{f}}_{e}(\mathbf{q})$ are the (generally non-linear) inviscid fluxes (which only depend on the state vector) $\overrightarrow{\mathbf{f}}_{v}(\mathbf{q}, \vec{\nabla} \mathbf{w})$ are the viscous fluxes, which depend on both the state and in the gradient of a set of gradient variables $\mathbf{w}$, and $\mathbf{s}(\mathbf{q})$ is a lower order source term. The auxiliary gradient variables set is introduced because often the gradients of the state vector are not the best variables in which to write the viscous fluxes (see Gassner et al. [2018]). Note that the particular case where the gradient variables are the state vector is recovered with $\mathbf{w}=\mathbf{q}$, thus, (2.59) represents a more general form of the PDE.

The nodal DG scheme is derived in 7 steps:

1. Cast a $n$-th order system as a system of $n$ first order equations by introducing auxiliary variables.

2. Tessellate the domain $\Omega$ in non-overlapping hexahedral elements $e$. Write the equations in the reference space for an element $e$.

3. Multiply the equation by an arbitrary polynomial $\phi$ inside the element and integrate in the reference element $E$.

4. Use the Gauss law for the volume integrals that involve the nabla operator. 
5. Replace the exact solution by its polynomial approximation, and the integrals by quadratures.

6. Connect discontinuous solutions in the resulting boundary integrals with a numerical flux.

We first cast the PDE as a system of two first order equations. In this particular example (2.59), the viscous (or diffusive) fluxes are second order. So, we define the gradients $\overrightarrow{\mathbf{g}}=\vec{\nabla} \mathbf{w}$, and write (2.59) as a system of two first order equations,

$$
\begin{aligned}
\mathbf{q}_{t}+\vec{\nabla} \cdot \overrightarrow{\mathbf{f}}_{e}(\mathbf{q}) & =\vec{\nabla} \cdot \overrightarrow{\mathbf{f}}_{v}(\mathbf{q}, \overrightarrow{\mathbf{g}})+\mathbf{s}(\mathbf{q}), \\
\overrightarrow{\mathbf{g}} & =\vec{\nabla} \mathbf{w} .
\end{aligned}
$$

Next, we write the system (2.60) in the reference space for an element $e$. We use the transformation for the divergence (2.17), and for the gradient (2.19) to get,

$$
\begin{aligned}
J \mathbf{q}_{t}+\vec{\nabla}_{\xi} \cdot \overrightarrow{\tilde{\mathbf{f}}}_{e} & =\vec{\nabla}_{\xi} \cdot \overrightarrow{\tilde{\mathbf{f}}}_{v}+J \mathbf{s}, \\
J \overrightarrow{\mathbf{g}} & =\underline{\mathbf{M}} \vec{\nabla}_{\xi} \mathbf{w},
\end{aligned}
$$

with the definition of contravariant vectors given in (2.18). Now we multiply the first equation by an arbitrary state vector $\boldsymbol{\phi}$, and the second equation by an arbitrary block vector $\vec{\varphi}$, and integrate in the reference element $E$,

$$
\begin{aligned}
& \left\langle J \mathbf{q}_{t}, \boldsymbol{\phi}\right\rangle_{E}+\left\langle\vec{\nabla}_{\xi} \cdot \overrightarrow{\tilde{\mathbf{f}}}_{e}, \boldsymbol{\phi}\right\rangle_{E}=\left\langle\vec{\nabla}_{\xi} \cdot \overrightarrow{\tilde{\mathbf{f}}}_{v}, \boldsymbol{\phi}\right\rangle_{E}+\langle J \mathbf{s}, \boldsymbol{\phi}\rangle_{E}, \\
& \langle J \overrightarrow{\mathbf{g}}, \vec{\varphi}\rangle_{E}=\left\langle\underline{\mathbf{M}} \vec{\nabla}_{\xi} \mathbf{w}, \vec{\varphi}\right\rangle_{E}=\left\langle\vec{\nabla}_{\xi} \mathbf{w}, \underline{\mathbf{M}}^{T} \vec{\varphi}\right\rangle_{E}=\left\langle\vec{\nabla} \vec{\xi}_{\xi} \mathbf{w}, \overrightarrow{\tilde{\varphi}}\right\rangle_{E} .
\end{aligned}
$$

In the second equation, we have moved the metric terms to the test function, and defined the contravariant test function $\overrightarrow{\tilde{\varphi}}=\underline{\mathbf{M}}^{T} \vec{\varphi}$. We now integrate by parts all volume integrals that include a gradient or divergence operator,

$$
\begin{aligned}
& \left\langle J \mathbf{q}_{t}, \boldsymbol{\phi}\right\rangle_{E}+\int_{\partial E} \boldsymbol{\phi}^{T}\left(\overrightarrow{\tilde{\mathbf{f}}}_{e}-\overrightarrow{\tilde{\mathbf{f}}}_{v}\right) \cdot \hat{n} \mathrm{~d} \hat{S}-\left\langle\overrightarrow{\tilde{\mathbf{f}}}_{e}, \vec{\nabla}_{\xi} \boldsymbol{\phi}\right\rangle_{E}=-\left\langle\overrightarrow{\tilde{\mathbf{f}}}_{v}, \vec{\nabla}_{\xi} \boldsymbol{\phi}\right\rangle_{E}+\langle J \mathbf{s}, \boldsymbol{\phi}\rangle_{E}, \\
& \langle J \overrightarrow{\mathbf{g}}, \vec{\varphi}\rangle_{E}=\int_{\partial E} \mathbf{w}^{T} \overrightarrow{\tilde{\varphi}} \cdot \hat{n} \mathrm{~d} \hat{S}-\left\langle\mathbf{w}, \vec{\nabla}_{\xi} \cdot \overrightarrow{\tilde{\varphi}}\right\rangle_{E} .
\end{aligned}
$$

The latest step is followed by the restriction of the test functions to order $N$ polynomials (for simplicity, the symbols are maintained), the replacement of the exact solution by its numerical approximation, and the approximation of the integrals by quadratures,

$$
\begin{aligned}
& \left\langle\mathscr{J} \mathbf{Q}_{t}, \boldsymbol{\phi}\right\rangle_{E, N}+\int_{\partial E, N} \boldsymbol{\phi}^{T}\left(\overrightarrow{\tilde{\mathbf{F}}}_{e}-\overrightarrow{\tilde{\mathbf{F}}}_{v}\right) \cdot \hat{n} \mathrm{~d} \hat{S}-\left\langle\overrightarrow{\tilde{\mathbf{F}}}_{e}, \vec{\nabla}_{\xi} \boldsymbol{\phi}\right\rangle_{E, N}=-\left\langle\overrightarrow{\tilde{\mathbf{F}}}_{v}, \vec{\nabla}_{\xi} \boldsymbol{\phi}\right\rangle_{E, N}+\langle\mathscr{J} \mathbf{S}, \boldsymbol{\phi}\rangle_{E}, \\
& \langle\mathscr{J} \overrightarrow{\mathbf{G}}, \vec{\varphi}\rangle_{E, N}=\int_{\partial E, N} \mathbf{W}^{T} \overrightarrow{\tilde{\varphi}} \cdot \hat{n} \mathrm{~d} \hat{S}-\left\langle\mathbf{W}, \vec{\nabla}_{\xi} \cdot \overrightarrow{\tilde{\varphi}}\right\rangle_{E, N} \cdot
\end{aligned}
$$


To this point, we have not made any comment about the continuity of the numerical solution across elements. The solution is made from order $N$ polynomials in each element, without any continuity constraint across the inter-element faces. That, combined with the use of independent test functions in each element (i.e. the test functions from different elements are not connected in any way), isolates the weak forms (2.64) from different elements, and the numerical solution is discontinuous at the interfaces. The way the DGSEM couples the solution between adjacent elements is through the surface integrals in (2.64). Because it makes also possible to get conservative schemes (i.e. the total amount of state vector only depends on physical boundary exchanges), we replace the surface integral arguments by a uniquely defined numerical flux that depends on the face state $\left(\mathbf{Q}_{L}, \mathbf{Q}_{R}\right)$ and gradients $\left(\overrightarrow{\mathbf{G}}_{L}, \overrightarrow{\mathbf{G}}_{R}\right)$ projection from the adjacent elements ,

$$
\overrightarrow{\mathbf{F}}_{e} \cdot \vec{n} \approx \overrightarrow{\mathbf{F}}_{e}^{\star}\left(\mathbf{Q}_{L}, \mathbf{Q}_{R}\right) \cdot \vec{n}, \quad \overrightarrow{\mathbf{F}}_{v} \cdot \vec{n} \approx \overrightarrow{\mathbf{F}}_{v}^{\star}\left(\mathbf{Q}_{L}, \mathbf{Q}_{R}, \overrightarrow{\mathbf{G}}_{L}, \overrightarrow{\mathbf{G}}_{R}\right) \cdot \vec{n}, \quad \mathbf{W} \approx \mathbf{W}^{\star}\left(\mathbf{Q}_{L}, \mathbf{Q}_{R}\right) .
$$

This feature is shared with finite volume methods. Indeed, for the inviscid fluxes, a suitable and extended option is to borrow exact and numerical Riemann solvers from finite volume methods. Inserting the numerical fluxes (2.65) in (2.64), we get the DG approximation of the original PDE (2.59),

$$
\begin{aligned}
& \left\langle\mathscr{J} \mathbf{Q}_{t}, \boldsymbol{\phi}\right\rangle_{E, N}+\int_{\partial E, N} \boldsymbol{\phi}^{T}\left(\overrightarrow{\tilde{\mathbf{F}}}_{e}^{\star}-\overrightarrow{\tilde{\mathbf{F}}}_{v}^{\star}\right) \cdot \hat{n} \mathrm{~d} \hat{S}=\left\langle\overrightarrow{\tilde{\mathbf{F}}}_{e}-\overrightarrow{\tilde{\mathbf{F}}}_{v}, \vec{\nabla}_{\xi} \boldsymbol{\phi}\right\rangle_{E, N}+\langle\mathscr{J} \mathbf{S}, \boldsymbol{\phi}\rangle_{E, N}, \\
& \langle\mathscr{J} \overrightarrow{\mathbf{G}}, \vec{\varphi}\rangle_{E, N}=\int_{\partial E, N} \mathbf{W}^{\star, T} \overrightarrow{\tilde{\varphi}} \cdot \hat{n} \mathrm{~d} \hat{S}-\left\langle\mathbf{W}, \vec{\nabla}_{\xi} \cdot \overrightarrow{\tilde{\varphi}}\right\rangle_{E, N} \cdot
\end{aligned}
$$

The implementation requires the nodal coefficients of the state vector time derivative and of the gradients. These are found when $(N+1)^{3}$ linearly independent test functions are introduced. The easiest set of linearly independent test functions is the tensor product Lagrange polynomials. Thus, we replace $\phi$ and $\vec{\varphi}$ by $l_{i}(\xi) l_{j}(\eta) l_{k}(\zeta)$,

$$
\begin{aligned}
\mathscr{J}_{i j k} \frac{\mathrm{d} \mathbf{Q}_{i j k}}{\mathrm{~d} t}+ & \left.\frac{l_{i}\left(\xi_{f}\right)}{w_{i}}\left(\overrightarrow{\widetilde{\mathbf{F}}}_{e}^{\star, 1}-\overrightarrow{\tilde{\mathbf{F}}}_{v}^{\star, 1}\right)_{j k}^{f}\right|_{f=\mathrm{L}} ^{\mathrm{R}}+\left.\frac{l_{j}\left(\eta_{f}\right)}{w_{j}}\left(\overrightarrow{\widetilde{\mathbf{F}}}_{e}^{\star, 2}-\overrightarrow{\tilde{\mathbf{F}}}_{v}^{\star, 2}\right)_{i k}^{f}\right|_{f=\mathrm{F}} ^{\mathrm{Ba}}+\left.\frac{l_{k}\left(\zeta_{f}\right)}{w_{k}}\left(\overrightarrow{\widetilde{\mathbf{F}}}_{e}^{\star, 3}-\overrightarrow{\widetilde{\mathbf{F}}}_{v}^{\star, 3}\right)_{i j}^{f}\right|_{f=\mathrm{Bo}} ^{\mathrm{T}} \\
= & \sum_{m=0}^{N}\left(\frac{w_{m}}{w_{i}} D_{m i}\left(\tilde{\mathbf{F}}_{e, m j k}^{1}-\tilde{\mathbf{F}}_{v, m j k}^{1}\right)+\frac{w_{m}}{w_{j}} D_{m j}\left(\tilde{\mathbf{F}}_{e, i m k}^{2}-\tilde{\mathbf{F}}_{v, i m k}^{2}\right)\right. \\
& \left.\quad+\frac{w_{m}}{w_{k}} D_{m k}\left(\tilde{\mathbf{F}}_{e, i j m}^{3}-\tilde{\mathbf{F}}_{v, i j m}^{3}\right)\right)+\mathscr{J}_{i j k} \mathbf{S}_{i j k} \\
\mathscr{J}_{i j k} \overrightarrow{\mathbf{G}}_{i j k}= & \left.\frac{l_{i}\left(\xi_{f}\right)}{w_{i}}\left(\mathscr{J} \vec{a}^{1} \mathbf{W}^{\star}\right)_{j k}^{f}\right|_{f=\mathrm{L}} ^{\mathrm{R}}+\left.\frac{l_{j}\left(\eta_{f}\right)}{w_{j}}\left(\mathscr{J} \vec{a}^{2} \mathbf{W}^{\star}\right)_{i k}^{f}\right|_{f=\mathrm{F}} ^{\mathrm{Ba}}+\left.\frac{l_{k}\left(\zeta_{f}\right)}{w_{k}}\left(\mathscr{J} \vec{a}^{3} \mathbf{W}^{\star}\right)_{i j}^{f}\right|_{f=\mathrm{B}} ^{\mathrm{T}} \\
& -\sum_{m=0}^{N}\left(\mathscr{J} \vec{a}_{i j k}^{1} \frac{w_{m}}{w_{i}} D_{m i} \mathbf{W}_{m j k}+\mathscr{J} \vec{a}_{i j k}^{2} \frac{w_{m}}{w_{j}} D_{m j} \mathbf{W}_{i m k}+\mathscr{J} \vec{a}_{i j k}^{3} \frac{w_{m}}{w_{k}} D_{m k} \mathbf{W}_{i j m}\right) .
\end{aligned}
$$


The approximation is completed with the definition of the numerical fluxes, and the application of physical boundary conditions. In the DGSEM, physical boundary conditions are applied through the numerical flux at the physical boundaries.

Finally, for a complete parallelism with the continuous system, many authors consider a strong-weak form. We get the strong-weak standard DG scheme applying the discrete Gauss law to the volume terms in (2.66),

$$
\begin{aligned}
& \left\langle\mathscr{J} \mathbf{Q}_{t}, \boldsymbol{\phi}\right\rangle_{E, N}+\int_{\partial E, N} \boldsymbol{\phi}^{T}\left(\overrightarrow{\tilde{\mathbf{F}}}_{e}^{\star}-\overrightarrow{\tilde{\mathbf{F}}}_{e}-\overrightarrow{\widetilde{\mathbf{F}}}_{v}^{\star}+\overrightarrow{\tilde{\mathbf{F}}}_{v}\right) \cdot \hat{n} \mathrm{~d} \hat{S}+\left\langle\vec{\nabla}_{\xi} \cdot\left(\overrightarrow{\tilde{\mathbf{F}}}_{e}-\overrightarrow{\tilde{\mathbf{F}}}_{v}\right), \boldsymbol{\phi}\right\rangle_{E, N}=\langle\mathscr{J} \mathbf{S}, \boldsymbol{\phi}\rangle_{E, N}, \\
& \langle\mathscr{J} \overrightarrow{\mathbf{G}}, \vec{\varphi}\rangle_{E, N}=\int_{\partial E, N} \mathbf{W}^{\star, T} \overrightarrow{\tilde{\varphi}} \cdot \hat{n} \mathrm{~d} \hat{S}-\left\langle\mathbf{W}, \vec{\nabla}_{\xi} \cdot \overrightarrow{\tilde{\varphi}}\right\rangle_{E, N} \cdot
\end{aligned}
$$

\subsubsection{Split-form schemes and the two-point divergence}

The split-form is an effective technique to control the aliasing errors (de-aliasing) and it is the key mechanism employed in this work to achieve robustness. Although we will provide more details in the subsequent chapters of this work, we define and describe in this section the split-form operator for the DGSEM.

The necessity of split-forms rest on the incapability of the scheme to satisfy a discrete product rule Kopriva [2009],

$$
\vec{\nabla}_{\xi} \mathrm{I}^{N}(U V) \neq V \vec{\nabla}_{\xi} U+U \vec{\nabla}_{\xi} V
$$

As a result, all the following different discretizations constructed as a linear blending of conservative and non-conservative forms are valid approximations of $\vec{\nabla}_{\xi}(U V)$

$$
\begin{aligned}
\vec{\nabla}_{\xi}(U V) \approx V \vec{\nabla}_{\xi} U+U \vec{\nabla}_{\xi} V & \approx \frac{1}{2} \vec{\nabla}_{\xi}(U V)+\frac{1}{2} V \vec{\nabla}_{\xi} U+\frac{1}{2} U \vec{\nabla}_{\xi} V \\
& \approx \alpha \vec{\nabla}_{\xi}(U V)+(1-\alpha)\left(V \vec{\nabla}_{\xi} U+U \vec{\nabla}_{\xi} V\right),
\end{aligned}
$$

with $\alpha \in \mathbb{R}$.

This is the basic idea behind split-forms, but in practice the de-aliasing is performed using the two-point divergence operator Carpenter et al. [2014]. First, because the two-point flux divergence enables additional discretizations that cannot be expressed as a split-form (see Gassner et al. [2016a]). Second, because sometimes two or more splittings are needed to ensure a complete removal of the aliasing errors (for instance, metric terms also need de-aliasing), which are all automatically performed efficiently with the two-point flux. Third, because often more than one 
split-form is available for a given set of equations, which become easily selected and changed solely with the choice of the two-point flux.

The two-point divergence is introduced in the weak-strong form (2.68), and it is only defined for Gauss-Lobatto points (and very recently for Gauss points in Chan et al. [2019]). In the weak-strong form, the inviscid volume terms compute the divergence of the non-linear inviscid flux $\vec{\nabla}_{\xi} \cdot \overrightarrow{\vec{F}}_{e}$. In the standard DG method, it is approximated using (2.38). In the two-point divergence, it is approximated using

$$
\begin{aligned}
\vec{\nabla}_{\xi} \cdot \overrightarrow{\widetilde{\mathbf{F}}}_{e} \approx \mathbb{D}\left(\overrightarrow{\widetilde{\mathbf{F}}}_{e}\right)^{\#}(\xi, \eta, \zeta)=2 \sum_{m=0}^{N} & l_{m}^{\prime}(\xi) \tilde{\mathbf{F}}_{e}^{1, \#}\left(\xi, \eta, \zeta ; \xi_{m}, \eta, \zeta\right) \\
+ & l_{m}^{\prime}(\eta) \tilde{\mathbf{F}}_{e}^{2, \#}\left(\xi, \eta, \zeta ; \xi, \eta_{m}, \zeta\right) \\
& +l_{m}^{\prime}(\zeta) \tilde{\mathbf{F}}_{e}^{3, \#}\left(\xi, \eta, \zeta ; \xi, \eta, \zeta_{m}\right)
\end{aligned}
$$

The contravariant numerical volume flux is constructed from a two-point flux function $\overrightarrow{\mathbf{F}}_{e}^{\#}(\cdot, \cdot)$ that is a function of two states Gassner et al. [2016a]; Tadmor [2003],

$$
\tilde{\mathbf{F}}_{e}^{l, \#}(\xi, \eta, \zeta ; \alpha, \beta, \gamma) \equiv \overrightarrow{\mathbf{F}}_{e}^{\#}(\mathbf{Q}(\xi, \eta, \zeta), \mathbf{Q}(\alpha, \beta, \gamma)) \cdot \frac{1}{2}\left(\mathscr{J} \vec{a}^{l}(\xi, \eta, \zeta)+\mathscr{J} \vec{a}^{l}(\alpha, \beta, \gamma)\right),
$$

with $l=1,2,3$. At a node, we write this contravariant flux as

$$
\left(\tilde{\mathbf{F}}_{e}^{l, \#}\right)_{(i m) j k} \equiv \overrightarrow{\mathbf{F}}_{e}^{\#}\left(\mathbf{Q}_{i j k}, \mathbf{Q}_{m j k}\right) \cdot \frac{1}{2}\left(\mathscr{J} \vec{a}_{i j k}^{l}+\mathscr{J} \vec{a}_{m j k}^{l}\right), \quad l=1,2,3 .
$$

Eq. (2.73) can be simplified if we define the two-point average

$$
\{\{u\}\}_{(i m) j k}=\frac{u_{i j k}+u_{m j k}}{2},
$$

that leads to the definition of the divergence of the two-point flux

$$
\begin{aligned}
\mathbb{D}\left(\overrightarrow{\tilde{\mathbf{F}}}_{e}\right)_{i j k}^{\#}=2 \sum_{m=0}^{N} & D_{i m} \overrightarrow{\mathbf{F}}_{e}^{\#}\left(Q_{i j k}, Q_{m j k}\right) \cdot\left\{\left\{\mathscr{J} \vec{a}^{1}\right\}\right\}_{(i m) j k} \\
+ & D_{j m} \overrightarrow{\mathbf{F}}_{e}^{\#}\left(Q_{i j k}, Q_{i m k}\right) \cdot\left\{\left\{\mathscr{J} \vec{a}^{2}\right\}\right\}_{i(j m) k} \\
+ & D_{k m} \overrightarrow{\mathbf{F}}_{e}^{\#}\left(Q_{i j k}, Q_{i j m}\right) \cdot\left\{\left\{\mathscr{J} \vec{a}^{3}\right\}\right\}_{i j(k m)}
\end{aligned}
$$

To conclude, a DG scheme that uses a two-point divergence for the inviscid fluxes is

$$
\begin{aligned}
\left\langle\mathscr{J} \mathbf{Q}_{t}, \boldsymbol{\phi}\right\rangle_{E, N} & +\int_{\partial E, N} \boldsymbol{\phi}^{T}\left(\overrightarrow{\tilde{\mathbf{F}}}_{e}^{\star}-\overrightarrow{\tilde{\mathbf{F}}}_{e}-\overrightarrow{\widetilde{\mathbf{F}}}_{v}^{\star}+\overrightarrow{\mathbf{F}}_{v}\right) \cdot \hat{n} \mathrm{~d} \hat{S}+\left\langle\mathbb{D}\left(\overrightarrow{\tilde{\mathbf{F}}}_{e}\right)^{\#}, \boldsymbol{\phi}\right\rangle_{E, N} \\
& =\left\langle\vec{\nabla}_{\xi} \cdot \overrightarrow{\tilde{\mathbf{F}}}_{v}, \boldsymbol{\phi}\right\rangle_{E, N}+\langle\mathscr{J} \mathbf{S}, \boldsymbol{\phi}\rangle_{E, N}, \\
\langle\mathscr{J} \overrightarrow{\mathbf{G}}, \vec{\varphi}\rangle_{E, N} & =\int_{\partial E, N} \mathbf{W}^{\star, T} \overrightarrow{\tilde{\varphi}} \cdot \hat{n} \mathrm{~d} \hat{S}-\left\langle\mathbf{W}, \vec{\nabla}_{\xi} \cdot \overrightarrow{\tilde{\varphi}}\right\rangle_{E, N} \cdot
\end{aligned}
$$




\subsection{Approximation of non-conservative systems}

We also describe the extension of the DG discretization to non-conservative systems. The extension is relevant in this work because the multiphase solver derived in Chapter 6 includes non-conservative terms. We follow Bohm et al. [2018] and consider an extended version of the advection-diffusion equation with $N_{c}$ non-conservative terms,

$$
\mathbf{q}_{t}+\vec{\nabla} \cdot \overrightarrow{\mathbf{f}}_{e}(\mathbf{q})+\sum_{m=1}^{N_{c}} \overrightarrow{\boldsymbol{\phi}}_{m}(\mathbf{q}) \cdot \vec{\nabla} \lambda_{m}(\mathbf{q})=\vec{\nabla} \cdot \overrightarrow{\mathbf{f}}_{v}(\mathbf{q}, \vec{\nabla} \mathbf{w})+\mathbf{s}(\mathbf{q}),
$$

Where $\overrightarrow{\boldsymbol{\phi}}_{m}$ are the (non-linear) coefficients of a set of non-conservative terms, proportional to the gradient of another (non-linear) coefficients $\lambda_{m}$.

We describe now the particularities to handle non-conservative terms, whereas all the terms involved in the conservative system (2.59) follow the same steps used in Sec. 2.5. We first transform the non-conservative terms to the reference element,

$$
J \overrightarrow{\boldsymbol{\phi}}_{m} \cdot \vec{\nabla} \lambda_{m}=\overrightarrow{\boldsymbol{\phi}}_{m} \cdot \overline{\bar{m}} \vec{\nabla}_{\xi} \lambda_{m}=\left(\underline{\mathbf{m}}^{T} \overrightarrow{\boldsymbol{\phi}}_{m}\right) \cdot \vec{\nabla}_{\xi} \lambda_{m}=\overrightarrow{\tilde{\boldsymbol{\phi}}}_{m} \cdot \vec{\nabla}_{\xi} \lambda_{m}
$$

Next, we construct a weak form in the reference element $E$, and perform integration by parts,

$$
\left\langle\overrightarrow{\tilde{\boldsymbol{\phi}}}_{m} \cdot \vec{\nabla}_{\xi} \lambda_{m}, \boldsymbol{\phi}\right\rangle_{E}=\int_{\partial E} \boldsymbol{\phi}^{T}\left(\overrightarrow{\tilde{\boldsymbol{\phi}}}_{m} \lambda_{m}\right) \cdot \hat{n} \mathrm{~d} \hat{S}-\left\langle\lambda_{m}, \vec{\nabla}_{\xi} \cdot\left(\boldsymbol{\phi}^{T} \overrightarrow{\tilde{\boldsymbol{\phi}}}_{m}\right)\right\rangle_{E} .
$$

Finally, we replace the solution by the polynomial approximation, and the integrals by quadratures,

$$
\left\langle\overrightarrow{\tilde{\boldsymbol{\phi}}}_{m} \cdot \vec{\nabla}_{\xi} \lambda_{m}, \boldsymbol{\phi}\right\rangle_{E} \approx \int_{\partial E, N} \boldsymbol{\phi}^{T}\left(\overrightarrow{\tilde{\boldsymbol{\Phi}}}_{m} \Lambda_{m}\right) \cdot \hat{n} \mathrm{~d} \hat{S}-\left\langle\Lambda_{m}, \vec{\nabla}_{\xi} \cdot\left(\boldsymbol{\phi}^{T} \overrightarrow{\tilde{\boldsymbol{\Phi}}}_{m}\right)\right\rangle_{E, N}
$$

Similar to the conservative system, the boundary quadrature quantities are not uniquely defined as a result of the inter-element discontinuities. In the conservative system, we replaced the boundary quantities by a uniquely defined numerical flux (e.g. $\overrightarrow{\mathbf{F}}_{e}^{\star}$ ), motivated by the need to construct a conservative scheme. Non-conservative terms, however, do not conserve the quantities and therefore there is no motivation to perform such an inter-element coupling. For stability, we will later show that we need to couple some quantities, and not couple others, which yields a non uniquely defined flux. Following Bohm et al. [2018], we replace the boundary quantities by a diamond flux which depends on the two states, but is not uniquely defined at the boundary,

$$
\left(\overrightarrow{\boldsymbol{\Phi}}_{m} \Lambda_{m}\right)^{\diamond}=\left(\overrightarrow{\boldsymbol{\Phi}}_{m} \Lambda_{m}\right)^{\diamond}\left(\mathbf{Q}_{L}, \mathbf{Q}_{R}\right)
$$


As opposed to the numerical flux, the diamond flux is not constrained to be single valued at the face, and its value can jump from one side to the other. We insert (2.81) in (2.80), and use the discrete Gauss law to get the discrete version of non-conservative terms,

$$
\left\langle\overrightarrow{\tilde{\boldsymbol{\phi}}}_{m} \cdot \vec{\nabla}_{\xi} \lambda_{m}, \boldsymbol{\phi}\right\rangle_{E} \approx \int_{\partial E, N} \boldsymbol{\phi}^{T}\left(\left(\overrightarrow{\tilde{\boldsymbol{\Phi}}}_{m} \Lambda_{m}\right)^{\diamond}-\left(\overrightarrow{\tilde{\boldsymbol{\Phi}}}_{m} \Lambda_{m}\right)\right) \cdot \hat{n} \mathrm{~d} \hat{S}+\left\langle\boldsymbol{\phi}, \overrightarrow{\tilde{\boldsymbol{\Phi}}}_{m} \cdot \vec{\nabla}_{\xi} \Lambda_{m}\right\rangle_{E, N} .
$$

Finally, we include the discrete version of non-conservative terms (2.82) in the discrete version of the conservative system (2.66) to get a standard (i.e. without split-forms) DG discretization of the non-conservative system (2.77),

$$
\begin{aligned}
\left\langle\mathscr{J} \mathbf{Q}_{t}, \boldsymbol{\phi}\right\rangle_{E, N} & +\int_{\partial E, N} \boldsymbol{\phi}^{T}\left(\overrightarrow{\tilde{\mathbf{F}}}_{e}^{\star}-\overrightarrow{\tilde{\mathbf{F}}}_{e}+\sum_{m=1}^{N_{c}}\left(\overrightarrow{\tilde{\mathbf{\Phi}}}_{m} \Lambda_{m}\right)^{\diamond}-\left(\overrightarrow{\tilde{\mathbf{\Phi}}}_{m} \Lambda_{m}\right)-\overrightarrow{\tilde{\mathbf{F}}}_{v}^{\star}+\overrightarrow{\tilde{\mathbf{F}}}_{v}\right) \cdot \hat{n} \mathrm{~d} \hat{S} \\
& +\left\langle\vec{\nabla}_{\xi} \cdot\left(\overrightarrow{\tilde{\mathbf{F}}}_{e}-\overrightarrow{\tilde{\mathbf{F}}}_{v}\right), \boldsymbol{\phi}\right\rangle_{E, N}+\sum_{m=1}^{N_{c}}\left\langle\overrightarrow{\tilde{\mathbf{\Phi}}}_{m} \cdot \vec{\nabla}_{\xi} \Lambda_{m}, \boldsymbol{\phi}\right\rangle_{E, N}=\langle\mathscr{J} \mathbf{S}, \boldsymbol{\phi}\rangle_{E, N}, \\
\langle\mathscr{J} \overrightarrow{\mathbf{G}}, \vec{\varphi}\rangle_{E, N} & =\int_{\partial E, N} \mathbf{W}^{\star, T} \overrightarrow{\tilde{\varphi}} \cdot \hat{n} \mathrm{~d} \hat{S}-\left\langle\mathbf{W}, \vec{\nabla}{ }_{\xi} \cdot \overrightarrow{\tilde{\varphi}}\right\rangle_{E, N} \cdot
\end{aligned}
$$

Additionally, it is possible to consider a split-form scheme of the non-conservative system that uses a two-point divergence. Thus, as in the conservative system in Sec. 2.5, we replace the standard divergence operator (2.38) by the two-point divergence (2.75), and we replace the gradient in the non-conservative term by the split-form of the gradient $\mathbb{D}_{\text {grad }}^{\mathrm{DM}}$ presented in Bohm et al. [2018], whose sole purpose is to de-aliase the metric terms,

$$
\begin{aligned}
& \left(\overrightarrow{\tilde{\boldsymbol{\Phi}}}_{m} \cdot \vec{\nabla}_{\xi} \Lambda_{m}\right)_{i j k} \approx\left(\overrightarrow{\tilde{\boldsymbol{\Phi}}}_{m} \cdot \mathbb{D}_{\text {grad }}^{\mathrm{DM}} \Lambda_{m}\right)_{i j k}=\frac{1}{2}\left(\overrightarrow{\tilde{\boldsymbol{\Phi}}}_{m} \cdot \vec{\nabla}_{\xi} \Lambda_{m}\right)_{i j k}+\frac{1}{2}\left(\overrightarrow{\boldsymbol{\Phi}}_{m} \cdot \vec{\nabla}_{\xi} \cdot\left(\overline{\bar{M}} \Lambda_{m}\right)\right)_{i j k} \\
& =\overrightarrow{\boldsymbol{\Phi}}_{m, i j k} \cdot \sum_{n=0}^{N}\left(D_{i n}\left\{\left\{\mathscr{J} \vec{a}^{1}\right\}\right\}_{(i n) j k} \Lambda_{m, n j k}+D_{j n}\left\{\left\{\mathscr{J} \vec{a}^{2}\right\}\right\}_{i(j n) k} \Lambda_{m, i n k}+D_{k n}\left\{\left\{\mathscr{J} \vec{a}^{3}\right\}\right\}_{i j(k n)} \Lambda_{m, i j n}\right) .
\end{aligned}
$$

With the two-point divergence, and the de-aliased gradient metrics, we construct a split-form scheme for non-conservative systems,

$$
\begin{aligned}
\left\langle\mathscr{J} \mathbf{Q}_{t}, \boldsymbol{\phi}\right\rangle_{E, N} & +\int_{\partial E, N} \boldsymbol{\phi}^{T}\left(\overrightarrow{\tilde{\mathbf{F}}}_{e}^{\star}-\overrightarrow{\widetilde{\mathbf{F}}}_{e}+\sum_{m=1}^{N_{c}}\left(\overrightarrow{\tilde{\boldsymbol{\Phi}}}_{m} \Lambda_{m}\right)^{\diamond}-\left(\overrightarrow{\tilde{\boldsymbol{\Phi}}}_{m} \Lambda_{m}\right)-\overrightarrow{\widetilde{\mathbf{F}}}_{v}^{\star}+\overrightarrow{\widetilde{\mathbf{F}}}_{v}\right) \cdot \hat{n} \mathrm{~d} \hat{S} \\
& +\left\langle\mathbb{D}\left(\overrightarrow{\widetilde{\mathbf{F}}}_{e}\right)^{\#}, \boldsymbol{\phi}\right\rangle_{E, N}+\sum_{m=1}^{N_{c}}\left\langle\overrightarrow{\tilde{\boldsymbol{\Phi}}}_{m} \cdot \mathbb{D}_{\text {grad }}^{\mathrm{DM}} \Lambda_{m}, \boldsymbol{\phi}\right\rangle_{E, N}=\left\langle\vec{\nabla}_{\xi} \cdot \overrightarrow{\widetilde{\mathbf{F}}}_{v}, \boldsymbol{\phi}\right\rangle_{E, N}+\langle\mathscr{J} \mathbf{S}, \boldsymbol{\phi}\rangle_{E, N}, \\
\langle\mathscr{J} \overrightarrow{\mathbf{G}}, \vec{\varphi}\rangle_{E, N} & =\int_{\partial E, N} \mathbf{W}^{\star, T} \overrightarrow{\tilde{\varphi}} \cdot \hat{n} \mathrm{~d} \hat{S}-\left\langle\mathbf{W}, \vec{\nabla}_{\xi} \cdot \overrightarrow{\tilde{\varphi}}\right\rangle_{E, N} \cdot
\end{aligned}
$$


We have written two approximations of a non-conservative system: the standard DG version, and the split-form approximation. Usually the split-form scheme is advantageous since it is more robust Bohm et al. [2018], but it might not be the case. In particular, we will see that for the two-phase solver presented in Chapter 6 both the standard and split-form schemes are robust. Nonetheless, both schemes are presented for completeness.

\subsection{Summary of DG approximations}

In this chapter we have introduced the discontinuous Galerkin approximation of general non-linear advection-diffusion systems, both conservative and non-conservative. We have derived the standard scheme, which can be written in both weak and weakstrong forms, and the split-form schemes with the use of the two-point divergence. The solution of second order parabolic systems recast the original PDE as a system of two first order equations, where the auxiliary variable $\overrightarrow{\mathbf{g}}=\vec{\nabla} \mathbf{q}$ computes the gradient of the state vector, which are introduced in the viscous fluxes $\overrightarrow{\mathbf{f}}_{v}(\mathbf{q}, \overrightarrow{\mathbf{g}})$. The choice of the gradient variables is not unique, and we also consider here the use of an auxiliary set of gradient variables $\overrightarrow{\mathbf{g}}=\vec{\nabla} \mathbf{w}(\mathbf{q})$. We summarize the different schemes in Table 2.1.

In Chapters 4, 5, and 6 we present novel split-form DG schemes for the incompressible Navier-Stokes equations with variable density and artificial compressibility, the Cahn-Hilliard equation, and the multiphase iNS/CH system, respectively.

\subsection{Discretization example: the compressible Navier-Stokes equations}

The compressible Navier-Stokes equations govern the evolution of the state vector $\mathbf{q}=(\rho, \rho \vec{u}, \rho e)$, where $\rho$ is the density, $\vec{u}$ is the velocity, and $\rho e$ is the energy. The inviscid fluxes were given in (1.7), the viscous fluxes in (1.21), and we use the gradient variables $\mathbf{w}$,

$$
\mathbf{w}=\mathbf{w}(\mathbf{q})=(\rho, \vec{u}, T) .
$$

We use the split-form scheme (2.76). For the compressible Navier-Stokes equations there are several split-forms available. A review can be found in Gassner et al. [2016a]. One of the most popular choices, and the one used in this work, is the kinetic 
Table 2.1: Different DG discretizations for conservative and non-conservative systems

Conservative system

\begin{tabular}{|c|c|c|}
\hline PDE & & $\mathbf{q}_{t}+\vec{\nabla} \cdot \overrightarrow{\mathbf{f}}_{e}(\mathbf{q})=\vec{\nabla} \cdot \overrightarrow{\mathbf{f}}_{v}(\mathbf{q}, \vec{\nabla} \mathbf{w})$ \\
\hline DG & $\begin{array}{l}\text { Standard } \\
\text { (weak) }\end{array}$ & $\left\langle\mathscr{J} \mathbf{Q}_{t}, \boldsymbol{\phi}\right\rangle_{E, N}+\int_{\partial E, N} \boldsymbol{\phi}^{T}\left(\overrightarrow{\widetilde{\mathbf{F}}}_{e}^{\star}-\overrightarrow{\widetilde{\mathbf{F}}}_{v}^{\star}\right) \cdot \hat{n} \mathrm{~d} \hat{S}=\left\langle\overrightarrow{\widetilde{\mathbf{F}}}_{e}-\overrightarrow{\widetilde{\mathbf{F}}}_{v}, \vec{\nabla}_{\xi} \boldsymbol{\phi}\right\rangle_{E, N}$ \\
\hline & $\begin{array}{l}\text { Standard } \\
\text { (strong) }\end{array}$ & $\left\langle\mathscr{J} \mathbf{Q}_{t}, \boldsymbol{\phi}\right\rangle_{E, N}+\int_{\partial E, N} \boldsymbol{\phi}^{T}\left(\overrightarrow{\mathbf{F}}_{e}^{\star}-\overrightarrow{\widetilde{\mathbf{F}}}_{e}-\overrightarrow{\widetilde{\mathbf{F}}}_{v}^{\star}+\overrightarrow{\widetilde{\mathbf{F}}}_{v}\right) \cdot \hat{n} \mathrm{~d} \hat{S}$ \\
\hline & Split-form & $\begin{array}{l}+\left\langle\vec{\nabla}_{\xi} \cdot\left(\tilde{\mathbf{F}}_{e}-\tilde{\mathbf{F}}_{v}\right), \boldsymbol{\phi}\right\rangle_{E, N}=0 \\
\left\langle\mathscr{J} \mathbf{Q}_{t}, \boldsymbol{\phi}\right\rangle_{E, N}+\int_{\partial E, N} \boldsymbol{\phi}^{T}\left(\overrightarrow{\mathbf{F}}_{e}^{\star}-\overrightarrow{\widetilde{\mathbf{F}}}_{e}-\overrightarrow{\widetilde{\mathbf{F}}}_{v}^{\star}+\overrightarrow{\mathbf{F}}_{v}\right) \cdot \hat{n} \mathrm{~d} \hat{S} \\
+\left\langle\mathbb{D}\left(\overrightarrow{\widetilde{\mathbf{F}}}_{e}\right)^{\#}, \boldsymbol{\phi}\right\rangle_{E N}=\left\langle\vec{\nabla}_{\xi} \cdot \overrightarrow{\widetilde{\mathbf{F}}}_{v}, \boldsymbol{\phi}\right\rangle_{E, N}\end{array}$ \\
\hline
\end{tabular}

Non-conservative system

\begin{tabular}{|c|c|c|}
\hline PDE & & $\mathbf{q}_{t}+\vec{\nabla} \cdot \overrightarrow{\mathbf{f}}_{e}(\mathbf{q})+\sum_{m=1}^{N_{c}} \overrightarrow{\boldsymbol{\phi}}_{m}(\mathbf{q}) \cdot \vec{\nabla} \lambda_{m}(\mathbf{q})=\vec{\nabla} \cdot \overrightarrow{\mathbf{f}}_{v}(\mathbf{q}, \vec{\nabla} \mathbf{w})$ \\
\hline DG & $\begin{array}{l}\text { Standard } \\
\text { (strong) }\end{array}$ & $\begin{array}{l}\left\langle\mathscr{g} \mathbf{Q}_{t}, \boldsymbol{\phi}\right\rangle_{E, N}+\left\langle\vec{\nabla}_{\xi} \cdot\left(\overrightarrow{\widetilde{\mathbf{F}}}_{e}-\overrightarrow{\widetilde{\mathbf{F}}}_{v}\right), \boldsymbol{\phi}\right\rangle_{E, N}+\sum_{m=1}^{N_{c}}\left\langle\overrightarrow{\widetilde{\boldsymbol{\Phi}}}_{m} \cdot \vec{\nabla}_{\xi} \Lambda_{m}, \boldsymbol{\phi}\right\rangle_{E, N} \\
+\int_{\partial F N} \boldsymbol{\phi}^{T}\left(\overrightarrow{\widetilde{\mathbf{F}}}_{e}^{\star}-\overrightarrow{\mathbf{F}}_{e}+\sum_{m=1}^{N_{c}}\left(\overrightarrow{\tilde{\boldsymbol{\Phi}}}_{m} \Lambda_{m}\right)^{\diamond}-\left(\overrightarrow{\tilde{\boldsymbol{\Phi}}}_{m} \Lambda_{m}\right)-\overrightarrow{\widetilde{\mathbf{F}}}_{v}^{\star}+\overrightarrow{\widetilde{\mathbf{F}}}_{v}\right) \cdot \hat{n} \mathrm{~d} \hat{S}=0\end{array}$ \\
\hline & Split-form & $\begin{array}{l}\left\langle\mathscr{J} \mathbf{Q}_{t}, \boldsymbol{\phi}\right\rangle_{E, N}+\left\langle\mathbb{D}\left(\overrightarrow{\widetilde{\mathbf{F}}}_{e}\right)^{\#}, \boldsymbol{\phi}\right\rangle_{E, N}+\sum_{m=1}^{N_{c}}\left\langle\overrightarrow{\tilde{\mathbf{\Phi}}}_{m} \cdot \mathbb{D}_{\text {grad }}^{\mathrm{DM}} \Lambda_{m}, \boldsymbol{\phi}\right\rangle_{E, N}-\left\langle\vec{\nabla}_{\xi} \cdot \overrightarrow{\widetilde{\mathbf{F}}}_{v}, \boldsymbol{\phi}\right\rangle_{E, N} \\
+\int_{\partial E, N} \boldsymbol{\phi}^{T}\left(\overrightarrow{\widetilde{\mathbf{F}}}_{e}^{\star}-\overrightarrow{\widetilde{\mathbf{F}}}_{e}+\sum_{m=1}^{N_{c}}\left(\tilde{\widetilde{\boldsymbol{\Phi}}}_{m} \Lambda_{m}\right)^{\diamond}-\left(\overrightarrow{\tilde{\boldsymbol{\Phi}}}_{m} \Lambda_{m}\right)-\overrightarrow{\widetilde{\mathbf{F}}}_{v}^{\star}+\overrightarrow{\widetilde{\mathbf{F}}}_{v}\right) \cdot \hat{n} \mathrm{~d} \hat{S}=0\end{array}$ \\
\hline
\end{tabular}

Gradients

$\begin{array}{ll}\text { State vector } & \langle\mathscr{G} \overrightarrow{\mathbf{G}}, \vec{\varphi}\rangle_{E, N}=\int_{\partial E, N} \mathbf{Q}^{\star, T} \overrightarrow{\tilde{\varphi}} \cdot \hat{n} \mathrm{~d} \hat{S}-\left\langle\mathbf{Q}, \vec{\nabla}_{\xi} \cdot \overrightarrow{\tilde{\varphi}}\right\rangle_{E, N} \\ \mathbf{q} & \\ \text { Auxiliary set } & \langle\mathscr{G} \overrightarrow{\mathbf{G}}, \vec{\varphi}\rangle_{E, N}=\int_{\partial E, N} \mathbf{W}^{\star, T} \vec{\varphi} \cdot \hat{n} \mathrm{~d} \hat{S}-\left\langle\mathbf{W}, \vec{\nabla}_{\xi} \cdot \vec{\varphi}\right\rangle_{E, N} \\ \mathbf{w}(\mathbf{q}) & \end{array}$ 
energy preserving scheme by Pirozzoli Pirozzoli [2011],

$$
\mathbf{F}_{\mathrm{PI}}^{\mathrm{EC}}=\left(\begin{array}{c}
\{\{\rho\}\}\{\{u\}\} \\
\{\{\rho\}\}\{\{u\}\}^{2}+\{\{p\}\} \\
\{\{\rho\}\}\{\{u\}\}\{\{v\}\} \\
\{\{\rho\}\}\{\{u\}\}\{\{w\}\} \\
\{\{\rho\}\}\{\{u\}\}\{\{h\}\}
\end{array}\right), \mathrm{G}_{\mathrm{PI}}^{\mathrm{EC}}=\left(\begin{array}{c}
\{\{\rho\}\}\{\{v\}\} \\
\{\{\rho\}\}\{\{v\}\}\{\{u\}\} \\
\{\{\rho\}\}\{\{v\}\}^{2}+\{\{p\}\} \\
\{\{\rho\}\}\{\{v\}\}\{\{w\}\} \\
\{\{\rho\}\}\{\{v\}\}\{\{h\}\}
\end{array}\right), \mathrm{H}_{\mathrm{PI}}^{\mathrm{EC}}=\left(\begin{array}{c}
\{\{\rho\}\}\{\{w\}\} \\
\{\{\rho\}\}\{\{w\}\}\{\{u\}\} \\
\{\{\rho\}\}\{\{w\}\}\{\{v\}\} \\
\{\{\rho\}\}\{\{w\}\}^{2}+\{\{p\}\} \\
\{\{\rho\}\}\{\{w\}\}\{\{h\}\}
\end{array}\right) .
$$

For the inviscid Riemann solver, the most popular option is to use an approximate Riemann solver that introduces some amount of numerical dissipation at the interelement interfaces. Common choices are Roe's Riemann solver Roe [1981], Laxfriedrich's Lax [1954], or other experimental and attractive options as a lower dissipation version of Roe's Riemann solver Oßwald et al. [2016].

For the viscous numerical fluxes, we consider the Bassi-Rebay 1 scheme (BR1), which takes the average of the solution and the viscous fluxes at the inter-element boundaries,

$$
\mathbf{W}^{\star}=\{\{\mathbf{W}\}\}, \quad \overrightarrow{\mathbf{F}}_{v}^{\star}=\left\{\left\{\overrightarrow{\mathbf{F}}_{v}(\mathbf{Q}, \overrightarrow{\mathbf{G}})\right\}\right\},
$$

and the symmetric interior penalty (IP), which uses the local gradient,

$$
\vec{\nabla} \mathbf{W}=\underline{\mathbf{M}} \vec{\nabla}_{\xi} \mathbf{W}
$$

for the approximation of the inter-element fluxes, with added numerical stabilization,

$$
\mathbf{W}^{\star}=\{\{\mathbf{W}\}\}, \quad \overrightarrow{\mathbf{F}}_{v}^{\star}=\left\{\left\{\overrightarrow{\mathbf{F}}_{v}(\mathbf{Q}, \vec{\nabla} \mathbf{W})\right\}\right\}-\beta \eta\left(\mathbf{Q}_{L} \vec{n}_{L}+\mathbf{Q}_{R} \vec{n}_{R}\right),
$$

where the penalty parameter $\beta$ depends on the mesh geometry, and the polynomial order. An estimation for $\beta$ can be found in Shahbazi et al. [2007]; Ferrer and Willden [2011].

The numerical fluxes and the two-point flux complete the discretization of the compressible Navier-Stokes equations using a split-form DG scheme. 



\section{Chapter 3}

\section{Entropy stability: a unified treatment of DGSEM stability for conservative and non-conservative systems}

\section{Contents}

3.1 Introduction $\ldots \ldots \ldots \ldots \ldots \ldots \ldots \ldots \ldots \ldots \ldots \ldots \ldots$

3.2 Motivation . . . . . . . . . . . . . . . . 45

3.3 A simple finite difference example . . . . . . . . . . . . . . . 49

3.4 Increasing the complexity: DGSEM discretization of the one dimensional variable speed advection equation $\ldots \ldots \ldots \ldots 3$

3.5 Entropy analysis in general non-linear three-dimensional conservation laws . . . . . . . . . . . . . . 59

3.6 Entropy-stability for non-conservative systems $\ldots \ldots \ldots 6$

\subsection{Introduction}

In the early stages of the development of high-order discontinuous Galerkin methods, robustness issues impeded their use for challenging and industrial applications. In the discretization of non-linear terms (e.g. the momentum $\vec{\nabla} \cdot(\rho \vec{u} \vec{u})$ of the NavierStokes equations) the method introduces aliasing errors that might de-stabilize the solution and lead to numerical divergence Manzanero et al. [2018a]. Aliasing errors become critical when the solution is under-resolved, i.e. when the wave-number of 
the solution is comparable to the mesh wave-number, whereas their impact in smooth solutions is less significant as non-linear terms are computed with more accuracy. In high-order methods, challenging applications such as computing under-resolved or iLES (for implicit Large Eddy Simulation) turbulent flows or the use of coarser grids to solve very large domains are often under-resolved, and a standard scheme without a de-aliasing technique is prone to crash.

In the past, the robusness has been enhanced in different ways. Firstly, the choice of the numerical fluxes was studied. Numerical fluxes belong to the discretization, and some (limited) amount of numerical dissipation can be introduced with an appropriate choice of the star function. Examples are using an exact solution of the Riemann problem, an approximate Riemann solver (e.g. Roe or Lax-Friedrichs) Toro [2013], or the use of the interior penalty method or other dissipative schemes Arnold et al. [2002]; Ferrer [2017] for viscous fluxes. Among the advantages of the numerical flux dissipation, we highlight that the dissipation is mostly concentrated in high wavenumbers and that it vanishes in smooth continuous solutions, which helps to maintain high-order accuracy Hesthaven and Warburton [2007]. However, as mentioned, the amount of numerical dissipation achievable through the numerical fluxes is limited Manzanero et al. [2020d], so this approach often needs to be combined with other stabilization techniques.

Secondly, artificial viscosity methods stabilize the solution by adding localized viscosity in the critical under-resolved areas Persson and Peraire [2006]. The viscosity should be non-constant and depends on the smoothness of the solution in each element, vanishing when the flow is smooth. There are applications where the use of artificial viscosity is more or less mandatory (e.g. shock capturing), but the use of artificial viscosity to damp instabilities caused by numerical errors is discouraged Tadmor and Zhong [2006].

The current trend in high-order methods encourages to avoid incurring non-linear aliasing errors instead of camouflaging them with artificial viscosity. Entropy-stability offers a systematic strategy to remove aliasing errors following physical arguments. Firstly, an entropy analysis is performed to the continuous PDE. If the continuous equations satisfy an entropy inequality (i.e. one can find a mathematical entropy that is bounded), it means that the continuous solution is bounded and it cannot diverge. To clarify, the mathematical entropy represents a global quantity that can bound the norm of the solution. With that in mind, if a numerical scheme crashes it says that it is not being consistent with the underlying physics in some way.

The entropy stability of a numerical approximation implies that the scheme follows a discrete version of the entropy bound. In the past, it has been achieved with two techniques: over-integration and split-forms. Over-integration consists in the exact 
computation of the non-linear terms increasing their order of accuracy in the computation (e.g. taking $M=2 N$ integration points). However, the application of this technique is limited (e.g. it is never possible to integrate the quotients of variables with the Gauss quadrature rule, as those found in the compressible Navier-Stokes equations), and it might result in an expensive computation if many integration points are needed (e.g. very high polynomial orders $N>8$ ).

Split-forms, however, are an alternative that has been successful to stabilize the compressible Navier-Stokes equations Gassner et al. [2018], the shallow water equations Gassner et al. [2016b], or the magneto-hydrodynamics equations Bohm et al. [2018], among others. In this work we present stable schemes for the incompressible Navier-Stokes equations with variable density and artificial compressibility, the CahnHilliard equation, and the multiphase incompressible Navier-Stokes/Cahn-Hilliard system. They key ingredient for the construction of discrete entropy-stable schemes is the discrete Gauss law. For the DGSEM, the discrete Gauss law, or SBP-SAT property, is only satisfied (without doing any further modification to the framework Chan et al. [2019]) by Gauss-Lobatto points, and it allows us to follow the steps performed in the continuous entropy analysis discretely. As a result, we find that the discrete entropy is also bounded, so that the scheme cannot crash. Unless specified otherwise, and as in the vast majority of the DGSEM literature, all the entropy analyses performed in this work are semi-discrete, where only the discretization errors incurred in the DGSEM spatial discretization are considered, and an exact time integration is assumed. The fully-discrete stability, with the additional errors incurred by the discrete time integration, is not guaranteed. However, for explicit solvers we have not experienced any numerical divergence once the CFL number is chosen appropriately.

This chapter is organized as follows: a global perspective into entropy-stability is given in Sec. 3.2. Then, we start with a simple one-dimensional finite difference scheme of a constant speed advection equation in Sec. 3.3 to stress the main characteristics of the entropy-stability. Next, we continue with the DGSEM analysis of a variable coefficient advection equation in Sec. 3.4. Finally, in Sec. 3.5 and Sec. 3.6 we consider the entropy analysis of the DGSEM scheme for general non-linear conservative and non-conservative systems.

\subsection{Motivation}

Let the general system of PDEs,

$$
\mathbf{q}_{t}+\mathscr{L}(\mathbf{q})=0
$$


where $\mathscr{L}(\mathbf{q})$ is an operator applied to the state vector $\mathbf{q}$. Eq. (3.1) is conservative if the volume integral of the state vector is conserved with time,

$$
\int_{\Omega} \mathbf{q} \mathrm{d} \vec{x}=\text { const. }
$$

which is true for the conservative system with $\mathscr{L}(\mathbf{q})=\vec{\nabla} \cdot \overrightarrow{\mathbf{f}}$ and with no influx from the boundaries, for example. The conservation of the state vector with time is a relevant property, however, it does not say that the state vector is bounded. Although the total state is conserved, it can be at the expense of the state reaching very large values in one region, and very small values in other parts of the domain, so that the total amount is conserved. We expect the state vector is to be bounded (i.e. if we can say something about the largest value it can take), since it provides us with important information: if the continuous solution is bounded in time, we should construct an approximation whose solution is also bounded in time. In other words, the simulations should not explode, because that is not what the physics is telling us.

To gather additional information on the boundedness of the state vector, one can perform a linear stability analysis. The state vector is written as the sum of a known solution of the PDE plus a small perturbation, $\mathbf{q}=\mathbf{q}_{0}+\mathbf{q}^{\prime}$. Under the assumption of a small perturbation $\left\|\mathbf{q}^{\prime}\right\| \ll 1$, one can linearize the PDE to get the evolution equation for the perturbation Schmid et al. [2002],

$$
\mathbf{q}_{t}^{\prime}+\left.\frac{\partial \mathscr{L}}{\partial \mathbf{q}}\right|_{\mathbf{q}_{0}} \mathbf{q}^{\prime}+\mathscr{O}\left(\left\|\mathbf{q}^{\prime}\right\|^{2}\right)=0
$$

and neglect second order terms $\mathscr{O}\left(\left\|\mathbf{q}^{\prime}\right\|^{2}\right)$. Linear stability theory studies the energy of the solution of the linearized problem,

$$
\bar{E}=\frac{1}{2}\left\|\mathbf{q}^{\prime}\right\|^{2}=\int_{\Omega} \frac{1}{2}\left(\mathbf{q}^{\prime}\right)^{T} \mathbf{q}^{\prime} \mathrm{d} \vec{x}
$$

which is found by multiplying (3.3) by the perturbation $\mathbf{q}^{\prime}$ and integrating over $\Omega$,

$$
\bar{E}_{t}=\frac{\mathrm{d}}{\mathrm{d} t} \int_{\Omega} \frac{1}{2}\left(\mathbf{q}^{\prime}\right)^{T} \mathbf{q}^{\prime} \mathrm{d} \vec{x}=\int_{\Omega}\left(\mathbf{q}^{\prime}\right)^{T} \mathbf{q}_{t}^{\prime} \mathrm{d} \vec{x}=-\int_{\Omega}\left(\mathbf{q}^{\prime}\right)^{T}\left(\frac{\partial \mathscr{L}}{\partial \mathbf{q}}\right)_{\mathbf{q}_{0}} \mathbf{q}^{\prime} \mathrm{d} \vec{x}=\mathscr{R}\left(\mathbf{q}_{0}, \mathbf{q}^{\prime}\right) .
$$

From the study of the linearized operator $\partial \mathscr{L} / \partial \mathbf{q}$, we find the linear stability of the original PDE (3.1) around the state $\mathbf{q}_{0}$. If $\mathscr{R}$ represents a negative semi-definite form, then the energy does not increase and the linearized PDE (3.3) is stable, while the energy grows uncontrollably and the linearized system is unstable otherwise.

In particular, if the operator $\mathscr{L}(\mathbf{q})$ is linear in the state vector $\mathbf{q}$ (e.g. the advection equation $\mathscr{L}=\underline{\mathbf{A}} \vec{\nabla} \mathbf{q})$, then the linear stability provides us with precise information with 
the stability of the system, since the original system and the linearized system coincide (and the operator $\mathscr{R}$ does not depend on the equilibrium state $\mathbf{q}_{0}$ ). Therefore, for linear equations, one performs the energy analysis to find if the energy of the system is bounded or grows, because it represents the original PDE with fidelity.

For non-linear equations, however, the linear stability theory is not enough to confirm that the state vector is bounded. Firstly, the right-hand side depends on the equilibrium state $\mathbf{q}_{0}$, and one cannot evaluate all the possible states to see if all of them represent a stable equilibrium point. Second, because the linear system being unstable does not mean that the real non-linear system is unstable. The linear system being unstable means that the perturbation grows uncontrollably, up to one point where the assumptions made for the perturbation $\left(\left\|\mathbf{q}^{\prime}\right\| \ll 1\right)$ are no longer valid. When this happens, the linearized system no longer represents the original PDE. When the perturbation reaches large values, the non-linear terms neglected in (3.3) are important, and a non-linear mechanism can be triggered to stabilize the solution. Therefore, the linear stability theory is not sufficient to assess the boundedness of the solution for non-linear PDEs.

The entropy-stability theory studies the original PDE without linearization. Thus, we find a set of non-linear coefficients $\mathbf{w}$ called entropy variables that transform the PDE to a scalar entropy inequality,

$$
\left\langle\mathbf{q}_{t}, \mathbf{w}\right\rangle+\langle\mathscr{L}, \mathbf{w}\rangle=\overline{\mathscr{E}}_{t}+\int_{\partial \Omega} \vec{f}^{\mathscr{E}} \cdot \mathrm{d} \vec{S}+\mathscr{D}=0, \quad \mathscr{D} \geqslant 0,
$$

where $\overline{\mathscr{E}}$ is the mathematical entropy, $\vec{f}^{\mathscr{E}}$ is the entropy flux, and $\mathscr{D}$ represents a physical dissipation. The boundary term only depends on the boundary conditions imposition, which we assume stable,

$$
\int_{\partial \Omega} \vec{f}^{\mathscr{E}} \cdot \mathrm{d} \vec{S} \geqslant 0
$$

If the PDE satisfies (3.6) for an appropriate set $(\mathscr{E}, \mathbf{w})$, then the mathematical entropy is bounded,

$$
\overline{\mathscr{E}}(\mathbf{q}(t)) \leqslant \overline{\mathscr{E}}\left(\mathbf{q}\left(t_{0}\right)\right)=K .
$$

To guarantee the stability, the mathematical entropy should bound the solution, i.e., a bounded entropy should imply that the state vector is also bounded. In Merriam [1989], two sufficient conditions for a valid mathematical entropy were given: if the entropy is positive and its Hessian matrix is positive definite for all the state vector values, then the entropy represents a norm of the solution and is bounded. The latter is shown using Taylor's theorem up to order two around a constant state $\overline{\mathbf{q}}$

$$
\overline{\mathscr{E}}(\mathbf{q})=\int_{\Omega}\left(\mathscr{E}(\overline{\mathbf{q}})+\mathscr{E}_{\overline{\mathbf{q}}}(\mathbf{q}-\overline{\mathbf{q}})+\frac{1}{2}(\mathbf{q}-\overline{\mathbf{q}})^{T} \mathscr{E}_{\overline{\mathbf{q}} \overline{\mathbf{q}}}^{* *}(\mathbf{q}-\overline{\mathbf{q}})\right) \mathrm{d} \vec{x},
$$


where $\overline{\mathscr{E}}_{\overline{\mathbf{q}} \overline{\mathbf{q}}}^{* *}$ is the Hessian matrix evaluated in a point in between $\mathbf{q}$ and $\overline{\mathbf{q}}$ following Taylor's theorem. We choose the reference state $\overline{\mathbf{q}}$ as the average of $\mathbf{q}$ in $\Omega$,

$$
\overline{\mathbf{q}}=\frac{1}{|\Omega|} \int_{\Omega} \mathbf{q} \mathrm{d} \vec{x}
$$

so that the second term in the integral of (3.9) cancels, and the entropy is

$$
\overline{\mathscr{E}}(\mathbf{q})=\overline{\mathscr{E}}(\overline{\mathbf{q}})+\int_{\Omega} \frac{1}{2}(\mathbf{q}-\overline{\mathbf{q}})^{T} \mathscr{E}_{\overline{\mathbf{q}} \overline{\mathbf{q}}}^{* *}(\mathbf{q}-\overline{\mathbf{q}}) \mathrm{d} \vec{x} \geqslant \int_{\Omega} \frac{1}{2}(\mathbf{q}-\overline{\mathbf{q}})^{T} \mathscr{E}_{\overline{\mathbf{q}} \overline{\mathbf{q}}}^{* *}(\mathbf{q}-\overline{\mathbf{q}}) \mathrm{d} \vec{x}
$$

since the entropy is positive for all $\overline{\mathbf{q}}$ (first condition). Then, we confirm that the solution is bounded, since the norm,

$$
\int_{\Omega} \frac{1}{2}(\mathbf{q}-\overline{\mathbf{q}})^{T} \mathscr{E}_{\overline{\mathbf{q}} \overline{\mathbf{q}}}^{* *}(\mathbf{q}-\overline{\mathbf{q}}) \mathrm{d} \vec{x} \leqslant \overline{\mathscr{E}}(\mathbf{q}) \leqslant K
$$

is bounded. Eq. (3.12) represents a valid norm since the Hessian matrix $\mathscr{E}_{\bar{q} \bar{q}}^{* *}$ is positive definite. Comparing to the linear stability analysis, no manipulations and simplifications have been made to the original PDE, and the statement (3.12) remains valid for all time and for all values of the state vector. Furthermore, the entropy-stability ensures that all the state variables are bounded individually.

The entropy-stability provides a general framework for the study of the stability of non-linear PDEs. For a given PDE, one finds a mathematical entropy $\overline{\mathscr{E}}$ that contracts the PDE to an entropy inequality (3.6) and if possible, that satisfies the two sufficient conditions (positive, and positive definite Hessian matrix). The mathematical entropy is not unique nevertheless Gassner et al. [2016a], as for the example of the compressible Navier-Stokes one can use the kinetic energy, or the thermodynamic entropy. For linear systems, both the entropy and energy stability analyses coincide (since the linearized and original systems match), and in those studied in this thesis we will adopt the entropy analysis only to keep a sharper parallelism with the study of the non-linear PDEs that follow.

The ultimate goal is not only to show that the continuous PDE satisfies an entropy inequality, but to achieve an approximation that follows a discrete version of the entropy inequality. The latter produces a remarkable enhancement in the robustness of the implementation, to the point that some schemes can be bulletproof (i.e. they do not crash under any conditions, even severely high Reynolds numbers).

As a point of reference, in Fig. (3.1) we represent the number of test crashed (30\% of the total number) with a scheme that was not constructed following a discrete entropy inequality. Details on the system of equations solved and the configuration of the test can be found in Chapter 6. None of the simulations crashed with the 


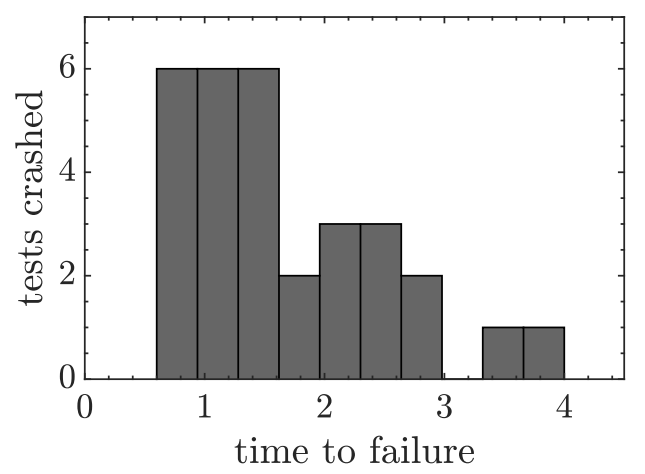

Figure 3.1: Number of test crashed before a given time with a random initial condition. The scheme was not constructed pursuing an entropy inequality, whereas the entropystable scheme (not represented) did not crash in any of the presented simulations

entropy-stable version of the approximation. This enhancement in the robustness makes worthwile to perform a detailed study of the PDE and its approximation, so that the approximation of all the terms involved is perfectly balanced to satisfy an entropy inequality.

\subsection{A simple finite difference example}

In this section, we introduce the procedure used to derive entropy-stable schemes with a simple equation, and with a simple finite difference scheme. We consider the onedimensional constant speed advection equation with periodic boundary conditions,

$$
u_{t}+a u_{x}=0, \quad u(0)=u(1), \quad 0 \leq x \leq 1, \quad a \in \mathbb{R}
$$

To construct an entropy-stable scheme, we first perform a continuous entropy analysis, and then construct a scheme by following the continuous steps discretely. We perform the continuous entropy analysis by multiplying (3.13) by the solution $u(x, t)$,

$$
u u_{t}+a u u_{x}=\left(\frac{1}{2} u^{2}\right)_{t}+\left(\frac{1}{2} a u^{2}\right)_{x}=0
$$

Eq. (3.14) is an entropy equation, since it represents a conservation law for a positive quantity $\mathscr{E}=\frac{1}{2} u^{2}$.

We integrate over the domain $x \in[0,1]$, and apply the periodic boundary condi- 
tions,

$$
\frac{\mathrm{d}}{\mathrm{d} t} \int_{0}^{1} \frac{1}{2} u^{2} \mathrm{~d} x+\int_{0}^{1}\left(\frac{1}{2} a u^{2}\right)_{x} \mathrm{~d} x=\frac{\mathrm{d}}{\mathrm{d} t} \int_{0}^{1} \frac{1}{2} u^{2} \mathrm{~d} x+\left.\frac{1}{2} a u^{2}\right|_{0} ^{1}=\frac{\mathrm{d}}{\mathrm{d} t} \int_{0}^{1} \frac{1}{2} u^{2} \mathrm{~d} x=0
$$

we find that the time derivative of the total entropy,

$$
\overline{\mathscr{E}}_{t}=0, \quad \overline{\mathscr{E}}(t)=\int_{0}^{1} \frac{1}{2} u(x, t)^{2} \mathrm{~d} x=\frac{1}{2}\langle u, u\rangle
$$

is zero, which implies that the entropy is conserved,

$$
\overline{\mathscr{E}}(t)=\overline{\mathscr{E}}(0) .
$$

Although (3.16) is an energy, we adopt the more general terminology entropy to refer to a positive quantity computed from the solution. The entropy (3.16) acts as an $\mathrm{L}^{2}$ norm of the solution,

$$
\frac{1}{2}\|u\|^{2}=\frac{1}{2} \int_{0}^{1} u^{2} \mathrm{~d} x=\frac{1}{2}\langle u, u\rangle=\overline{\mathscr{E}}(t)=\overline{\mathscr{E}}(0)=K,
$$

which bounds the solution.

Now we will show how a scheme can be constructed with entropy stability in mind. We consider a finite difference scheme, where the domain is divided into $N$ intervals, with spacing $\Delta x=1 / N$, and the solution is approximated by the grid point values $u_{i}(t)=u_{i}(i \Delta x)(i=0, \ldots, N)$. We write the advection equation (3.13) for each grid point value,

$$
\frac{\mathrm{d} u_{i}}{\mathrm{~d} t}+a\left(\frac{\mathrm{d} u}{\mathrm{~d} x}\right)_{i}=0, \quad i=0, \ldots, N-1 .
$$

We automatically enforce the periodic boundary conditions, so that $u_{N}=u_{0}$, and for convenience we can periodically extend the solution $u_{i+N}=u_{i}$.

We are left with the choice of the approximation of the derivative. From all the different choices, we will choose the discretization only with entropy stability in mind. Three popular approximations are

$$
\begin{array}{r}
\text { central }:\left(\frac{\mathrm{d} u}{\mathrm{~d} x}\right)_{x}^{c}=\frac{u_{i+1}-u_{i-1}}{2 \Delta x}, \\
\text { backward }:\left(\frac{\mathrm{d} u}{\mathrm{~d} x}\right)_{x}^{b}=\frac{u_{i}-u_{i-1}}{\Delta x}, \\
\text { forward }:\left(\frac{\mathrm{d} u}{\mathrm{~d} x}\right)_{x}^{f}=\frac{u_{i+1}-u_{i}}{\Delta x},
\end{array}
$$


For $a>0$, is is known that the central scheme is neutrally stable, that the backward scheme (known as upwind) is stable, and that the forward scheme is unstable. The entropy analysis will give us that information and guide our discretization choices.

We start studying the central scheme. Following the continuous entropy analysis, we construct a discrete entropy law by multiplying the scheme by the solution $u_{i}$ and applying the chain rule in time,

$$
u_{i} \frac{\mathrm{d} u_{i}}{\mathrm{~d} t}+a u_{i} \frac{u_{i+1}-u_{i-1}}{2 \Delta x}=\frac{\mathrm{d}}{\mathrm{d} t}\left(\frac{1}{2} u_{i}^{2}\right)+a u_{i} \frac{u_{i+1}-u_{i-1}}{2 \Delta x}=0 .
$$

In the continuous entropy analysis we integrated the resulting equation over the domain. For the discrete entropy analysis, we multiply by the mesh spacing $\Delta x$ and sum for all the mesh solutions (which represents the use of a quadrature rule to approximate the integral),

$$
\frac{\mathrm{d}}{\mathrm{d} t}\left(\sum_{i=0}^{N-1} \frac{1}{2} \Delta x u_{i}^{2}\right)+\frac{a}{2} \sum_{i=0}^{N-1}\left(u_{i+1} u_{i}-u_{i} u_{i-1}\right)=0 .
$$

As in the continuous entropy analysis, the terms associated to the advective terms are zero, since,

$$
\sum_{i=0}^{N-1} u_{i+1} u_{i}-\sum_{i=0}^{N-1} u_{i} u_{i-1}=\sum_{\hat{i}=1}^{N} u_{\hat{i}} u_{\hat{i}-1}-\sum_{i=0}^{N-1} u_{i} u_{i-1}=u_{N} u_{N-1}-u_{0} u_{-1}=0 .
$$

The periodical extension property $u_{i+N}=u_{i}$ was used in the last identity.

As a result, the central derivative approximation produces an entropy conserving scheme. We define the discrete entropy as

$$
\overline{\mathscr{E}}=\sum_{i=0}^{N-1} \frac{1}{2} \Delta x u_{i}^{2}=\frac{1}{4} \Delta x u_{0}^{2}+\sum_{i=1}^{N-1} \frac{1}{2} \Delta x u_{i}^{2}+\frac{1}{4} \Delta x u_{N}^{2}
$$

which is the result of integrating $\frac{1}{2} u^{2}$ with the trapezoid rule. As the discrete entropy is conserved, the discrete solution is bounded,

$$
\frac{1}{2} \Delta x \max _{i}\left\{u_{i}^{2}\right\} \leqslant \overline{\mathscr{E}}=\overline{\mathscr{E}}(0)=K
$$

Eq. (3.25) is a very strong statement. It says that none of the grid point values can exceed a given value, controlled through the initial condition. Thus, we can be confident that, given an arbitrary initial condition, the scheme will not crash under any circumstances, meaning that the solution does not blow up, at least semi-discretely. 
We now study the backward scheme for $a>0$ (upwind). Similarly to (3.3), we multiply the upwind scheme by the solution $u_{i}$, by $\Delta x$, and sum all the mesh components,

$$
\sum_{i=0}^{N-1} \Delta x u_{i} \frac{\mathrm{d} u_{i}}{\mathrm{~d} t}+\sum_{i=0}^{N-1} a u_{i}\left(u_{i}-u_{i-1}\right)=\frac{\mathrm{d}}{\mathrm{d} t}\left(\sum_{i=0}^{N-1} \frac{1}{2} \Delta x u_{i}^{2}\right)+\sum_{i=0}^{N-1} a u_{i}\left(u_{i}-u_{i-1}\right)=0 .
$$

Now the terms associated to the spatial discretization using an upwind method do not cancel. We can perform some manipulations on them,

$$
\begin{aligned}
\sum_{i=0}^{N-1} a u_{i}\left(u_{i}-u_{i-1}\right) & =\frac{1}{2} \sum_{i=0}^{N-1} a\left(u_{i}-u_{i-1}+u_{i}+u_{i-1}\right)\left(u_{i}-u_{i-1}\right) \\
& =\frac{1}{2} \sum_{i=0}^{N-1} a\left(u_{i}-u_{i-1}\right)^{2}+\frac{1}{2} \sum_{i=0}^{N-1} u_{i}^{2}-\frac{1}{2} \sum_{i=0}^{N-1} u_{i-1}^{2} \\
& =\frac{1}{2} \sum_{i=0}^{N-1} a\left(u_{i}-u_{i-1}\right)^{2} \geqslant 0
\end{aligned}
$$

to show that it is always a positive quantity. Note that the two individual sums are identical due to the periodicity property $u_{i+N}=u_{i}$. Thus, the discrete entropy equation for the upwind scheme (with $a>0$ ) is

$$
\overline{\mathscr{E}}_{t}=-a \sum_{i=0}^{N-1}\left(u_{i}-u_{i-1}\right)^{2} \leqslant 0, \quad \frac{1}{2} \Delta x \max _{i}\left\{u_{i}^{2}\right\} \leqslant \overline{\mathscr{E}}(t) \leqslant \overline{\mathscr{E}}(0)
$$

The upwind scheme gives a entropy dissipation law: its value is always inferior to the entropy of the initial condition, which confirms that the upwind scheme introduces stable numerical dissipation.

Finally, we follow the same steps for the forward scheme to find that

$$
\overline{\mathscr{E}}_{t}=a \sum_{i=0}^{N-1}\left(u_{i}-u_{i-1}\right)^{2} \geqslant 0, \quad \overline{\mathscr{E}}(t) \geqslant \overline{\mathscr{E}}(0)
$$

For the forward scheme, the entropy grows uncontrollably and it is not provably stable.

Thus, when designing a scheme for the advection equation, and among all the methods studied, one would have two practical options:

1. An entropy conserving scheme with central differences.

2. An entropy-stable scheme with backward (upwind) differences. 
Choosing one or another depends on other arguments, e.g. accuracy. Typically, entropystable schemes are more accurate when the equation has non-linear terms, since they prevent energy accumulation at high wave-numbers as a result of energy transfer from low to high wave-numbers due to non-linearities Manzanero et al. [2020d]; Flad and Gassner [2017]. From the point of view of stability, however, both are valid implementations that are consistent with the continuous entropy conservation law.

The concept of continuous and discrete entropy stability has been applied to a simple equation (constant speed one-dimensional advection) and to a simple scheme (first order finite difference scheme). The goal is to apply the same procedure to more complex three-dimensional, and non-linear system of equations, and to high-order discontinuous Galerkin discretizations in particular.

\subsection{Increasing the complexity: DGSEM discretization of the one dimensional variable speed advection equation}

The case of the variable speed advection equation was studied in Kopriva and Gassner [2014]. The equation is

$$
u_{t}+(a u)_{x}=0, \quad 0 \leqslant x \leqslant 1, u(0)=u(1) .
$$

In the search for an entropy-stable approximation of (3.30), we first perform the continuous entropy analysis. We construct a weak form by multiplying (3.30) by the solution, $u(x, t)$, and integrating over $\Omega=[0,1]$

$$
\left\langle u_{t}, u\right\rangle+\left\langle(a u)_{x}, u\right\rangle=\left\langle\left(\frac{1}{2} u^{2}\right)_{t}\right\rangle+\left\langle(a u)_{x}, u\right\rangle=\left\langle\mathscr{E}_{t}\right\rangle+\left\langle(a u)_{x}, u\right\rangle
$$

so that we have the evolution of a positive quantity with positive definite Hessian,

$$
\mathscr{E}=\frac{1}{2} u^{2}, \quad \overline{\mathscr{E}}=\langle\mathscr{E}\rangle=\frac{1}{2}\langle u, u\rangle=\frac{1}{2}\|u\|^{2} \geqslant 0
$$

which can serve as an entropy, and we only need to see whether we can transform the spatial terms into a conservation law,

$$
\left\langle(a u)_{x}, u\right\rangle \stackrel{?}{=}\left\langle f_{x}^{\mathscr{E}}\right\rangle=\int_{\partial \Omega} f^{\mathscr{E}} \vec{n}=\left.f^{\mathscr{E}}\right|_{0} ^{1},
$$

for an entropy flux $f^{\mathscr{E}}$ that is evaluated at the boundaries. We use the product rule for one half of $(a u)_{x}$,

$$
\left\langle(a u)_{x}, u\right\rangle=\frac{1}{2}\left\langle(a u)_{x}, u\right\rangle+\frac{1}{2}\left\langle a u_{x}, u\right\rangle+\frac{1}{2}\left\langle a_{x} u, u\right\rangle
$$


and then the Gauss law on the first term in (3.34) to get

$$
\begin{aligned}
\left\langle(a u)_{x}, u\right\rangle & =\left.\frac{1}{2} a u^{2}\right|_{0} ^{1}-\frac{1}{2}\left\langle a u, u_{x}\right\rangle+\frac{1}{2}\left\langle a u_{x}, u\right\rangle+\frac{1}{2}\left\langle a_{x} u, u\right\rangle \\
& =\left.f^{\mathscr{E}}\right|_{0} ^{1}+\frac{1}{2}\left\langle a_{x} u, u\right\rangle .
\end{aligned}
$$

We see that we get the surface integral of the entropy flux,

$$
f^{\mathscr{E}}=\frac{1}{2} a u^{2}
$$

plus a lower order source term that can be bounded,

$$
\min _{x \in[0,1]}\left\{a_{x}\right\} \overline{\mathscr{E}}=\frac{1}{2} \min _{x \in[0,1]}\left\{a_{x}\right\}\langle u, u\rangle \leqslant \frac{1}{2}\left\langle a_{x} u, u\right\rangle \leqslant \frac{1}{2} \max _{x \in[0,1]}\left\{a_{x}\right\}\langle u, u\rangle=\max _{x \in[0,1]}\left\{a_{x}\right\} \overline{\mathscr{E}}
$$

This lower order source term is a particular result in the advection equation that does not translate to other non-linear systems studied in this work. Thus, the continuous equation (3.30) satisfies the following entropy equation,

$$
\overline{\mathscr{E}}_{t}+\left.f^{\mathscr{E}}\right|_{0} ^{1}=-\frac{1}{2}\left\langle a_{x} u, u\right\rangle,
$$

which including the periodic boundary conditions $f^{\mathscr{E}}(0)=f^{\mathscr{E}}(1)$ and the bound for the lower order term,

$$
\overline{\mathscr{E}}_{t} \leqslant-\min _{x \in[0,1]}\left\{a_{x}\right\} \overline{\mathscr{E}}, \quad \overline{\mathscr{E}}(t) \leqslant \overline{\mathscr{E}}(0) \exp \left(-\min _{x \in[0,1]}\left\{a_{x}\right\} t\right)
$$

Note that the bound requires the existence of the derivative of $a(x)$, so, $a(x)$ has to be at least continuous.

Prior to get a discrete version of (3.39), we summarize the operations made to the original equation (3.30) to get the entropy equation, and see whether they can be mimicked discretely in the DGSEM-GL framework:

- The use of the product rule in (3.34). The DG scheme cannot reproduce this property discretely as differentiation and interpolation do not commute. We need to construct the scheme so that the use of the product rule is no longer needed.

- The use of the Gauss law in (3.35). The DG scheme with Gauss-Lobatto points satisfies a discrete version of the Gauss law, and it can be reproduced discretely, 
We now construct a DG scheme for (3.30). We divide the domain $\Omega=[0,1]$ into $M$ equally-sized elements $e^{m}=\left[x_{m}, x_{m+1}\right]$. Then, we use a simple affine mapping from physical to computational space,

$$
x=X^{m}(\xi)=x_{m}+\frac{1}{2}\left(x_{m+1}-x_{m}\right)(\xi+1)=x_{m}+\frac{\Delta x}{2}(\xi+1),
$$

which transforms (3.30) to

$$
\frac{\Delta x}{2} u_{t}+(a u)_{\xi}=0
$$

We construct a weak form in $e^{m}$ by multiplying by an arbitrary $N$ order polynomial test function $\phi$, and integrating over $e^{m}$. Then we integrate the flux $(a u)_{\xi}$ by parts, and replace the continuous solution by its polynomial approximation, integrals by quadratures, and the interface flux by a Riemann solver. Lastly, we apply the discrete Gauss law on the volume term to get a weak-strong form,

$$
\frac{\Delta x}{2}\left\langle U_{t}, \phi\right\rangle_{E, N}+\left.\phi\left((A U)^{\star}-(A U)\right)\right|_{-1} ^{1}+\left\langle(A U)_{\xi}, \phi\right\rangle_{E, N}=0 .
$$

From the continuous study, we can infer that (3.42) represents a DG discretization that is not provably stable. The continuous analysis required to use the product rule on $(A U)_{\xi}$, which is not available in the DG framework. Instead of solving (3.42), we modify the scheme to the split-form scheme that results after the application of the product rule (3.34),

$$
(A U)_{\xi} \approx \frac{1}{2}(A U)_{\xi}+\frac{1}{2} A U_{\xi}+\frac{1}{2} A_{\xi} U
$$

so that the scheme is now,

$$
\frac{\Delta x}{2}\left\langle U_{t}, \phi\right\rangle_{E, N}+\left.\phi\left((A U)^{\star}-(A U)\right)\right|_{-1} ^{1}+\left\langle\frac{1}{2}(A U)_{\xi}+\frac{1}{2} A U_{\xi}+\frac{1}{2} A_{\xi} U, \phi\right\rangle_{E, N}=0
$$

The approx. sign in (3.43) means that they are different representations of the flux, but that both converge to the exact flux derivative as the grid is refined (i.e. flux consistency).

Now all the steps performed in the continuous analysis are valid discretely. We replace the test function by the solution $\phi=U$, apply the chain rule in time for the entropy time derivative, and apply the discrete Gauss law to the first term in the volume integral exactly as done in the continuous setting (3.35), to get the discrete surface integral of the entropy flux,

$$
\frac{\mathrm{d}}{\mathrm{d} t}\left(\frac{\Delta x}{4}\langle U, U\rangle_{E, N}\right)+\left.U\left((A U)^{\star}-(A U)\right)\right|_{-1} ^{1}+\left.F^{\mathscr{E}}\right|_{-1} ^{1}=-\left\langle\frac{1}{2} A_{\xi} U, U\right\rangle_{E, N} .
$$


The entropy analysis is completed with the addition of the entropy balances of all elements. At the inter-element boundaries, we have to consider the exchanges between adjacent elements. We define the face $f^{m}$ as that shared by elements $e^{m}$ and $e^{m+1}$. Since periodic boundaries are used, we can define $e^{0}=e^{M}$ and $e^{M+1}=e^{1}$ for simplicity. At each face $f^{m}$, we have the positive contribution from the element at the left (evaluated at $\xi=1$ ), and the negative contribution from the element at the right (evaluated at $\xi=-1$ ). As a result, the sum over all elements is

$$
\frac{\mathrm{d}}{\mathrm{d} t} \sum_{e}\left(\frac{\Delta x}{4}\langle U, U\rangle_{E, N}\right)+\left.\sum_{f} U\left((A U)^{\star}-\frac{1}{2}(A U)\right)\right|_{R} ^{L}=-\sum_{e}\left\langle\frac{1}{2} A_{\xi} U, U\right\rangle_{E, N} .
$$

The study of the boundary terms is completed with the Riemann solver. One can use central fluxes,

$$
(A U)^{\star}=A_{f}\{\{U\}\}=A_{f} \frac{U_{L}+U_{R}}{2},
$$

to get an entropy conserving scheme (ignoring the lower order source term),

$$
\mathrm{BT}_{f}=-A_{f} \llbracket U \rrbracket\{\{U\}\}+\frac{1}{2} A_{f} \llbracket U^{2} \rrbracket=0, \llbracket U \rrbracket=U_{R}-U_{L},
$$

where we have assumed that the advection speed $a(x)$ is continuous (which implies that its discrete version using Gauss-Lobatto points it is also continuous), and we have used the algebraic relation between the jump and average operators (1.28),

$$
\llbracket U^{2} \rrbracket=\{\{U\}\} \llbracket U \rrbracket+\llbracket U \rrbracket\{\{U\}\}=2 \llbracket U \rrbracket\{\{U\}\} .
$$

As mentioned in the introduction to this chapter, the numerical flux can provide numerical dissipation if it is provided with interface dissipation. In the simple finite difference scheme we have analyzed the effect of using the upwind approximation, which translates also to DG schemes. The upwind scheme is

$$
(A U)^{\star}=A_{f}\{\{U\}\}-\left|A_{f}\right| \llbracket U \rrbracket=A_{f} \frac{U_{L}+U_{R}}{2}-\left|A_{f}\right|\left(U_{R}-U_{L}\right),
$$

so that the correct upwind side is picked automatically: if $A_{f}>0,(A U)^{\star}=A_{f} U_{L}$ (backward scheme) and $A_{f} U_{R}$ otherwise (forward scheme). Introducing (3.50) into the boundary terms,

$$
\mathrm{BT}_{f}=-A_{f} \llbracket U \rrbracket\{\{U\}\}+\left|A_{f}\right| \llbracket U \rrbracket^{2}+\frac{1}{2} A_{f} \llbracket U^{2} \rrbracket=\left|A_{f}\right| \llbracket U \rrbracket^{2} \geqslant 0,
$$

which inserted into the entropy balance (3.46),

$\overline{\mathscr{E}}_{t}=-\sum_{f}\left|A_{f}\right| \llbracket U \rrbracket^{2}-\sum_{e}\left\langle\frac{1}{2} A_{\xi} U, U\right\rangle_{E, N} \leqslant-\sum_{e}\left\langle\frac{1}{2} A_{\xi} U, U\right\rangle_{E, N}, \quad \overline{\mathscr{E}}=\sum_{e} \frac{\Delta x}{4}\langle U, U\rangle_{E, N}$, 
converts the entropy equality in an entropy inequality, identically as in the finite difference scheme with upwind difference (3.28). Using the bound for $A_{\xi}$ given in (3.37) the continuous setting, we get the discrete version of (3.39),

$$
\overline{\mathscr{E}}_{t} \leqslant-\frac{2}{\Delta x} \min _{e}\left\{A_{\xi}\right\} \overline{\mathscr{E}}, \quad \overline{\mathscr{E}}(t) \leqslant \overline{\mathscr{E}}(0) \exp \left(-\frac{2}{\Delta x} \min _{e}\left\{A_{\xi}\right\} t\right)
$$

\subsubsection{The need for a two-point divergence approximation}

The procedure to get an entropy-stable scheme for the variable speed advection equation has been to:

1. Perform a continuous entropy analysis.

2. Identify in the continuous analysis which operations used the product rule.

3. Construct the numerical scheme after the application of the product rule.

4. Follow the continuous steps, that required only the use of the discrete Gauss law.

Although this simple equation requires one splitting, this process can become tedious when three-dimensional and non-linear equations are considered. Additionally, in three-dimensions the metric terms also need an additional splitting. The two-point divergence operator encapsulates the splitting process into a single and simple operation. The one-dimensional version of the two-point divergence (2.75) for the splitform advection equation is

$$
\mathbb{D}(A U)_{i}^{A U}=2 \sum_{m=0}^{N} D_{i m} F^{A U}\left(U_{i}, U_{m}\right), F^{A U}=\{\{A\}\}\{\{U\}\}=\frac{1}{4}\left(A_{i}+A_{m}\right)\left(U_{i}+U_{m}\right),
$$

which leads to using the product rule for half of the total flux $A U$ (we call this operation to perform one splitting),

$$
\begin{aligned}
\mathbb{D}(A U)_{i}^{A U} & =\frac{1}{2} \sum_{m=0}^{N} D_{i m}\left(A_{i} U_{i}+A_{i} U_{m}+A_{m} U_{i}+A_{m} U_{m}\right) \\
& =\frac{1}{2}\left(A U_{\xi}\right)_{i}+\frac{1}{2}\left(A_{\xi} U\right)_{i}+\frac{1}{2}(A U)_{\xi, i} .
\end{aligned}
$$

In practice we first find the two-point volume flux rather than finding which splitform is consistent with the continuous entropy analysis. Then, one can check whether the two-point divergence is equivalent to a split-form, or not. 


\subsubsection{A novel sharper bound for non-zero advection speeds}

We present a novel sharper estimation to both the continuous (3.39) and discrete (3.53) bounds for the case when $a(x)$ is non-zero (and as it is continuous, it does not change it sign) Manzanero et al. [2018a]. The motivation behind finding a sharper bound rests in the fact that the original bound (3.39) allows the entropy to grow exponentially if at some point $a_{x}<0$. We tested the discrete bound (3.53) by numerical experiments with the particular choice of the advection speed,

$$
a(x)=1+\varepsilon \cos (2 \pi x), \quad \epsilon<1,
$$

and found that the split-form scheme crashes, where the rate of entropy growth was consistent with the discrete bound (3.53), which is in accordance with the theoretical estimates. However, we also tried the standard scheme and surprisingly, it did not crashed. In these simulations we used the central scheme, to avoid biasing the results with extra numerical dissipation. The fact that the standard scheme did not crash even under severely under-resolved initial conditions, led us to find a sharper energy estimate for the advection equation, which is valid only for non-zero advection speeds.

Without loss of generality we study the case $a(x)>0$ for simplicity. We consider the advection equation (3.30), and now instead of multiplying the equation by the solution $u$, it is multiplied by $a u$,

$$
\left\langle u_{t}, a u\right\rangle+\left\langle(a u)_{x}, a u\right\rangle=\left\langle\left(\frac{1}{2} a u^{2}\right)_{t}\right\rangle+\left.\frac{1}{2} a^{2} u^{2}\right|_{0} ^{1}=\overline{\mathscr{E}}_{t}+\left.f^{\mathscr{E}}\right|_{0} ^{1}=\overline{\mathscr{E}}_{t}=0 .
$$

With this choice, we get an entropy equation without any lower order source term which might lead to instabilities. The resulting entropy is now,

$$
\overline{\mathscr{E}}=\left\langle\frac{1}{2} a u^{2}\right\rangle=\int_{0}^{1} \frac{1}{2} a u^{2} \mathrm{~d} x \geqslant 0,
$$

which is always positive since $a(x)>0$. As a result, we find that although the original bound predicts an entropy growth, the reality is that the solution stays bounded and the entropy is conserved. The DG version of the scheme also satisfies the entropy equality/inequality, since we only have made use of the discrete Gauss rule to get the continuous entropy bound. We write the discrete (weak-strong form) version of (3.57),

$$
\left\langle U_{t}, A U\right\rangle_{E, N}+\left.\sum_{f} A U\left((A U)^{\star}-(A U)\right)\right|_{0} ^{1}+\left\langle(A U)_{\xi}, A U\right\rangle_{E, N}=0,
$$

and use the discrete Gauss law on (half of the volume terms) to cancel the volume 
terms,

$$
\begin{aligned}
\left\langle(A U)_{\xi}, A U\right\rangle_{E, N} & =\frac{1}{2}\left\langle(A U)_{\xi}, A U\right\rangle_{E, N}+\frac{1}{2}\left\langle(A U)_{\xi}, A U\right\rangle_{E, N} \\
& =\left.\frac{1}{2} A^{2} U^{2}\right|_{0} ^{1}-\frac{1}{2}\left\langle A U,(A U)_{\xi}\right\rangle_{E, N}+\frac{1}{2}\left\langle(A U)_{\xi}, A U\right\rangle_{E, N}=\left.\frac{1}{2} A^{2} U^{2}\right|_{0} ^{1} .
\end{aligned}
$$

As a result, we find the discrete entropy law (note that boundary terms are the same as in the original analysis, but multiplied by the constant speed which is a constant at the boundary) mimics the continuous entropy law,

$$
\overline{\mathscr{E}}_{t} \leqslant 0,
$$

where again, an entropy conserving (equality) scheme is constructed with central fluxes, and an entropy-stable (inequality) scheme with upwind.

Using this sharper bound, it is possible to estimate how the aliasing errors incurred in other discretizations differ from the standard scheme. Precisely, in Manzanero et al. [2018a] we show that for the general split-form,

$$
(A U)_{\xi} \approx(1-\alpha)(A U)_{\xi}+\alpha\left(A U_{\xi}+A_{\xi} U\right)
$$

the entropy grows as

$$
\overline{\mathscr{E}}(t) \leqslant \overline{\mathscr{E}}(0) \exp \left(-2 \alpha \frac{2}{\Delta x} \min _{e}\left\{A_{\xi}\right\} t\right),
$$

which is consistent with the original bound (3.53) when $\alpha=1 / 2$, and with the sharper bound when $\alpha=0$. In any other case, the dissipation grows with a rate computed from (3.63).

\subsection{Entropy analysis in general non-linear three-dimensional conservation laws}

We now describe the entropy analysis of a general three-dimensional conservation law. Examples of the equations that can be studied with this analysis are the compressible NSE Gassner et al. [2018], the incompressible NSE (see Chapter 4), and the CahnHilliard equation (see Chapter 5).

In this section we consider a general three-dimensional non-linear advectiondiffusion equation,

$$
\begin{aligned}
\mathbf{q}_{t}+\vec{\nabla} \cdot \overrightarrow{\mathbf{f}}_{e}(\mathbf{q}) & =\vec{\nabla} \cdot \overrightarrow{\mathbf{f}}_{v}(\mathbf{q}, \vec{\nabla} \mathbf{w}), \text { in } \Omega \subset \mathbb{R}^{3}, \\
\mathbf{w} & =\mathbf{w}(\mathbf{q}),
\end{aligned}
$$


where $\mathbf{q}(\vec{x}, t)$ is the state vector, $\overrightarrow{\mathbf{f}}_{e}$ are the inviscid fluxes, which depend non-linearly on the state vector, and $\overrightarrow{\mathbf{f}}_{v}$ are the viscous fluxes. We will suppose the viscous fluxes to depend linearly on the gradient of a set of gradient variables $\mathbf{w}$, which are called entropy variables,

$$
\overrightarrow{\mathbf{f}}_{v}(\mathbf{q}, \vec{\nabla} \mathbf{w})=\underline{\mathbf{B}}(\mathbf{q}) \vec{\nabla} \mathbf{w} \text {. }
$$

In (3.65), $\underline{\mathbf{B}}(\mathbf{q})$ are the linear coefficients, which can also depend non-linearly on the state. We assume that the viscous flux coefficients form a symmetric positive semidefinite matrix,

$$
\underline{\mathbf{B}}=\underline{\mathbf{B}}^{T}, \underline{B}_{i j}=\underline{B}_{j i}^{T}, \quad \overrightarrow{\mathbf{k}}^{T} \underline{\mathbf{B}} \overrightarrow{\mathbf{k}} \geqslant 0, \quad \sum_{i=1}^{3} \sum_{j=1}^{3} \mathbf{k}_{i}^{T} \underline{B}_{i j} \mathbf{k}_{j} \geqslant 0 . \quad \forall \overrightarrow{\mathbf{k}} \in \mathbb{R}^{N_{\mathrm{E}} \times 3} .
$$

The stability analysis rests on the existence of a pair of volume and surface mathematical entropies $\mathscr{E}(\mathbf{q}, \vec{\nabla} \mathbf{q}), \mathscr{E}^{S}(\mathbf{q})$, which produce a total entropy $\overline{\mathscr{E}}$,

$$
\overline{\mathscr{E}}(\mathbf{q}, \vec{\nabla} \mathbf{q})=\langle\mathscr{E}(\mathbf{q}, \vec{\nabla} \mathbf{q})\rangle+\int_{\partial \Omega} \mathscr{E}^{S}(\mathbf{q}) \mathrm{d} S .
$$

In this thesis, we have extended the entropy (3.67) to a more general definition, where the entropy depends on the state vector and its gradient. As a result of including the dependency with the gradients, we need to include a surface entropy $\mathscr{E}^{S}(\mathbf{q})$. The particular case where the entropy only depends on the state vector, has $\mathscr{E}^{S}=0$,

$$
\overline{\mathscr{E}}(\mathbf{q})=\langle\mathscr{E}(\mathbf{q})\rangle .
$$

The mathematical entropy satisfies two properties:

1. Positivity,

$$
\mathscr{E}(\mathbf{q}, \vec{\nabla} \mathbf{q}) \geqslant 0, \quad \forall \mathbf{q}
$$

2. and convexity, from the positive definiteness of the Hessian matrix,

$$
\mathbf{k}^{T} \frac{\partial^{2} \mathscr{E}}{\partial \mathbf{q}^{2}} \mathbf{k} \geqslant 0, \quad \forall \mathbf{k} \in \mathbb{R}^{N_{\mathrm{E}}}
$$

With the definition of the mathematical entropy, (3.68), we revisit three properties that make the original equation (3.64) be entropy-stable:

1. There exists a set of entropy variables $\mathbf{w}$ that contract the state vector time derivative to the time derivative of the total entropy,

$$
\left\langle\mathbf{q}_{t}, \mathbf{w}\right\rangle_{E, N}=\left\langle\mathscr{E}_{t}\right\rangle+\frac{\mathrm{d}}{\mathrm{d} t} \int_{\partial \Omega} \mathscr{E}^{S} \mathrm{~d} S=\overline{\mathscr{E}}_{t}
$$


2. The same entropy variables $\mathbf{w}$ also contract inviscid fluxes,

$$
\left\langle\vec{\nabla} \cdot \overrightarrow{\mathbf{f}}_{e}, \mathbf{w}\right\rangle=\int_{\partial \Omega} \vec{f}_{e}^{\mathscr{E}} \cdot \mathrm{d} \vec{S},
$$

where $\vec{f}_{e}^{\mathscr{E}}$ is the inviscid entropy flux.

3. The viscous fluxes can be linearly spanned by the gradient of the entropy variables, $\vec{\nabla} \mathbf{w}$, and they are always dissipative when multiplied by the entropy variables w,

$$
\left\langle\vec{\nabla} \cdot \overrightarrow{\mathbf{f}}_{v}, \mathbf{w}\right\rangle=\int_{\partial \Omega} \mathbf{w}^{T} \overrightarrow{\mathbf{f}}_{v} \cdot \mathrm{d} \vec{S}-\left\langle\overrightarrow{\mathbf{f}}_{v}, \vec{\nabla} \mathbf{w}\right\rangle=\int_{\partial \Omega} \vec{f}_{v}^{\mathscr{E}} \cdot \mathrm{d} \vec{S}-\left\langle\overrightarrow{\mathbf{f}}_{v}, \vec{\nabla} \mathbf{w}\right\rangle,
$$

with

$$
\vec{f}_{v}^{\mathscr{E}}=\mathbf{w}^{T} \overrightarrow{\mathbf{f}}_{v}, \quad\left\langle\overrightarrow{\mathbf{f}}_{v}, \vec{\nabla} \mathbf{w}\right\rangle=\langle\underline{\mathbf{B}} \vec{\nabla} \mathbf{w}, \vec{\nabla} \mathbf{w}\rangle \geqslant 0 .
$$

Before continuing, it is necessary to clarify some aspects of the contraction of the time coefficients and the surface entropy. Eq. (3.71) represents the general version of the time coefficient contraction for the most general definition of the entropy (3.67). In (3.71), the product with the entropy variables produces the time derivative of the volume entropy, and an additional surface entropy term $\mathscr{E}^{S}$. The addition of the surface entropy is only needed if the entropy depends on the gradient of the state vector $\vec{\nabla} \mathbf{q}$, which in other systems (e.g. the compressible NSE) is not the case. Therefore, if $\mathscr{E}(\mathbf{q})$, then $\mathscr{E}^{S}=0$, and the entropy variables can be computed as the entropy partial derivatives with respect to the state vector,

$$
\mathbf{w}=\frac{\partial \mathscr{E}(\mathbf{q})}{\partial \mathbf{q}}, \mathbf{q}_{t}^{T} \mathbf{w}=\mathbf{q}_{t}^{T} \frac{\partial \mathscr{E}(\mathbf{q})}{\partial \mathbf{q}}=\mathscr{E}_{t}
$$

For some of the systems studied in this work, the entropy depends on the gradients of the state (e.g. the free-energy of the Cahn-Hilliard equation, or the pressure stabilization term of the Laplacian artificial compressibility method).

To get the entropy evolution equation, we multiply (3.64) by the entropy variables $\mathbf{w}$ and integrate over the domain $\Omega$,

$$
\left\langle\mathbf{q}_{t}, \mathbf{w}\right\rangle+\left\langle\vec{\nabla} \cdot \overrightarrow{\mathbf{f}}_{e}, \mathbf{w}\right\rangle=\left\langle\vec{\nabla} \cdot \overrightarrow{\mathbf{f}}_{v}, \mathbf{w}\right\rangle=\int_{\partial \Omega} \mathbf{w}^{T} \overrightarrow{\mathbf{f}}_{v} \cdot \mathrm{d} \vec{S}-\left\langle\overrightarrow{\mathbf{f}}_{v}, \vec{\nabla} \mathbf{w}\right\rangle .
$$

We assume the existence of a mathematical entropy $\mathscr{E}(\mathbf{q}, \vec{\nabla} \mathbf{q})$ that satisfies the three properties (3.71), (3.72) and (3.74) which transform (3.76) to the entropy equation,

$$
\overline{\mathscr{E}}_{t}+\int_{\partial \Omega} \vec{f}^{\mathscr{E}} \cdot \mathrm{d} \vec{S}=-\langle\underline{\mathbf{B}} \vec{\nabla} \mathbf{w}, \vec{\nabla} \mathbf{w}\rangle \leqslant 0, \quad \vec{f}^{\mathscr{E}}=\vec{f}_{e}^{\mathscr{E}}+\vec{f}_{v}^{\mathscr{E}} .
$$


The conservative system (3.64) is entropy-stable as the entropy decreases in time as a result for viscous terms, and it is bounded by the initial condition and the boundary data,

$$
\begin{aligned}
\overline{\mathscr{E}}(t) & =\overline{\mathscr{E}}(0)-\int_{0}^{t}\left(\int_{\partial \Omega} \vec{f}^{\mathscr{E}} \cdot \mathrm{d} \vec{S}\right) \mathrm{d} t-\int_{0}^{t}\langle\underline{\mathbf{B}} \vec{\nabla} \mathbf{w}, \vec{\nabla} \mathbf{w}\rangle \mathrm{d} t \\
& \leqslant \overline{\mathscr{E}}(0)-\int_{0}^{t}\left(\int_{\partial \Omega} \vec{f}^{\mathscr{E}} \cdot \mathrm{d} \vec{S}\right) \mathrm{d} t .
\end{aligned}
$$

The discrete entropy-stability of the DG scheme needs the approximation to satisfy a discrete version of (3.71), (3.72) and (3.74). We consider the DG weak-strong form that uses the two-point divergence (2.76),

$$
\begin{aligned}
& \left\langle\mathscr{J} \mathbf{Q}_{t}, \boldsymbol{\phi}\right\rangle_{E, N}+\int_{\partial E, N} \boldsymbol{\phi}^{T}\left(\overrightarrow{\tilde{\mathbf{F}}}_{e}^{\star}-\overrightarrow{\tilde{\mathbf{F}}}_{e}-\overrightarrow{\tilde{\mathbf{F}}}_{v}^{\star}+\overrightarrow{\tilde{\mathbf{F}}}_{v}\right) \cdot \hat{n} \mathrm{~d} \hat{S}+\left\langle\mathbb{D}\left(\overrightarrow{\mathbf{F}}_{e}\right)^{\mathrm{EC}}, \boldsymbol{\phi}\right\rangle_{E, N}=\left\langle\vec{\nabla}_{\xi} \cdot \overrightarrow{\tilde{\mathbf{F}}}_{v}, \boldsymbol{\phi}\right\rangle_{E, N}, \\
& \langle\mathscr{J} \overrightarrow{\mathbf{G}}, \vec{\varphi}\rangle_{E, N}=\int_{\partial E, N} \mathbf{W}^{\star, T} \overrightarrow{\tilde{\varphi}} \cdot \hat{n} \mathrm{~d} \hat{S}-\left\langle\mathbf{W}, \vec{\nabla}_{\xi} \cdot \overrightarrow{\tilde{\varphi}}\right\rangle_{E, N},
\end{aligned}
$$

where the two-point divergence uses an entropy conserving two-point volume flux $\overrightarrow{\mathbf{F}}^{\mathrm{EC}}\left(\mathbf{Q}_{L}, \mathbf{Q}_{R}\right)$, to be defined. Note that we have also replaced the state vector $\mathbf{Q}$ by the entropy variables $\mathbf{W}$ in the definition of the gradient given in the second weak form.

The stability analysis follows its continuous version. We replace the test functions by $\phi=\mathrm{W}$ and $\vec{\varphi}=\overrightarrow{\mathrm{F}}_{v}$,

$$
\begin{aligned}
& \left\langle\mathscr{J} \mathbf{Q}_{t}, \mathbf{W}\right\rangle_{E, N}+\int_{\partial E, N} \mathbf{W}^{T}\left(\overrightarrow{\widetilde{\mathbf{F}}}_{e}^{\star}-\overrightarrow{\widetilde{\mathbf{F}}}_{e}-\overrightarrow{\widetilde{\mathbf{F}}}_{v}^{\star}+\overrightarrow{\widetilde{\mathbf{F}}}_{v}\right) \cdot \hat{n} \mathrm{~d} \hat{S}+\left\langle\mathbb{D}\left(\overrightarrow{\mathbf{F}}_{e}\right)^{\mathrm{EC}}, \mathbf{W}\right\rangle_{E, N}=\left\langle\vec{\nabla}_{\xi} \cdot \overrightarrow{\tilde{\mathbf{F}}}_{v}, \mathbf{W}\right\rangle_{E, N}, \\
& \left\langle\mathscr{J} \overrightarrow{\mathbf{G}}, \overrightarrow{\mathbf{F}}_{v}\right\rangle_{E, N}=\int_{\partial E, N} \mathbf{W}^{\star, T} \overrightarrow{\tilde{\mathbf{F}}}_{v} \cdot \hat{n} \mathrm{~d} \hat{S}-\left\langle\mathbf{W}, \vec{\nabla}_{\xi} \cdot \overrightarrow{\widetilde{\mathbf{F}}}_{v}\right\rangle_{E, N},
\end{aligned}
$$

and replace the last term from the second equation in the last term of the first equation,

$$
\begin{aligned}
\left\langle\mathscr{J} \mathbf{Q}_{t}, \mathbf{W}\right\rangle_{E, N} & +\int_{\partial E, N}\left(\mathbf{W}^{T}\left(\overrightarrow{\tilde{\mathbf{F}}}_{e}^{\star}-\overrightarrow{\tilde{\mathbf{F}}}_{e}-\overrightarrow{\tilde{\mathbf{F}}}_{v}^{\star}+\overrightarrow{\widetilde{\mathbf{F}}}_{v}\right)-\mathbf{W}^{\star, T} \overrightarrow{\tilde{\mathbf{F}}}_{v}\right) \cdot \hat{n} \mathrm{~d} \hat{S} \\
& +\left\langle\mathbb{D}\left(\overrightarrow{\mathbf{F}}_{e}\right)^{\mathrm{EC}}, \mathbf{W}\right\rangle_{E, N}=-\left\langle\mathscr{J} \overrightarrow{\mathbf{G}}, \overrightarrow{\mathbf{F}}_{v}\right\rangle_{E, N},
\end{aligned}
$$

so that we get a single equation. As a result, we get three volume quadratures, which represent the discrete version of (3.76). The surface quadrature will be shaped later to the discrete version of the entropy flux $\vec{f}^{\mathscr{E}}$ surface integral. As the three volume quadratures represent a discrete version of the terms involved in the entropy equation (3.76), we convert them guided by the continuous analysis (3.71), (3.72) and (3.74). 


\subsubsection{Contraction of the time coefficients}

The general case where the mathematical entropy depends on the state gradient $\mathscr{E}(\mathbf{q}, \vec{\nabla} \mathbf{q})$ needs special considerations that depend on the equations to be solved. We can prove the particular case where $\mathscr{E}(\mathbf{q})$, and the entropy variables are computed from partial derivatives (3.75). Since the chain rule in time holds in the semi-discrete version,

$$
\left\langle\mathscr{J} \mathbf{Q}_{t}, \mathbf{W}\right\rangle_{E, N}=\sum_{i j k=0}^{N} w_{i j k} \mathscr{J}_{i j k} \mathbf{W}_{i j k}^{T} \mathbf{Q}_{t}=\sum_{i j k=0}^{N} w_{i j k} \mathscr{J}_{i j k} \frac{\mathrm{d} \mathscr{E}_{i j k}}{\mathrm{~d} t}=\frac{\mathrm{d}}{\mathrm{d} t}\langle\mathscr{J} \mathscr{E}\rangle_{E, N}=\overline{\mathscr{E}}_{t}^{E, N}
$$

For the general entropy that depends on the gradients, we will assume in this chapter that we are able to write a discrete version of (3.71)

$$
\left\langle\mathscr{J} \mathbf{Q}_{t}, \mathbf{W}\right\rangle_{E, N}=\left\langle\mathscr{J} \mathscr{E}_{t}\right\rangle_{E, N}+\int_{\partial e, N} \vec{H}^{\mathscr{E}} \cdot \mathrm{d} \vec{S}
$$

where $\vec{H}^{\mathscr{E}}$ is a surface term in the time contraction that should be shaped to the surface entropy $\mathscr{E}^{S}$, and an additional numerical entropy of the inter-element faces $\mathscr{E}_{i} S$. To do so, we add (3.83) for all elements,

$$
\sum_{e}\left\langle\mathscr{J} \mathbf{Q}_{t}, \mathbf{W}\right\rangle_{E, N}=\sum_{e}\left\langle\mathscr{J} \mathscr{E}_{t}\right\rangle_{E, N}+\sum_{\substack{\text { interior } \\ \text { faces }}} \int_{f, N} \llbracket-\vec{H}^{\mathscr{E}} \rrbracket \cdot \vec{n}_{L} \mathrm{~d} S+\sum_{\substack{\text { boundary } \\ \text { faces }}} \int_{f, N} \vec{H}^{\mathscr{E}} \cdot \mathrm{d} \vec{S} .
$$

In (3.84), we include the boundary integrals from all elements, which translates to boundary integrals in all the faces of the mesh. We distinguish interior faces, where the two elements that share the faces contribute,

$$
\sum_{\substack{\text { interior } \\ \text { faces }}} \int_{f, N}\left(\vec{H}_{L}^{\mathscr{E}} \cdot \vec{n}_{L}+\vec{H}_{R}^{\mathscr{E}} \cdot \vec{n}_{R}\right) \mathrm{d} S=\sum_{\substack{\text { interior } \\ \text { faces }}} \int_{f, N}\left(\vec{H}_{L}^{\mathscr{E}}-\vec{H}_{R}^{\mathscr{E}}\right) \cdot \vec{n}_{L} \mathrm{~d} S=\sum_{\substack{\text { interior } \\ \text { faces }}} \int_{f, N} \llbracket-\vec{H}^{\mathscr{E}} \rrbracket \cdot \vec{n}_{L} \mathrm{~d} S \text {. }
$$

We have selected one of the normal vectors (e.g. $\vec{n}_{L}=-\vec{n}_{R}$ ), which produces the jump of the flux (with opposed sign). For the physical boundary faces, we just write the boundary flux.

We assume that the discretization is constructed so that the interior contribution of the surface term in the time contraction $\vec{H}^{\mathscr{E}}$ can be transformed to

$$
\sum_{\substack{\text { interior } \\ \text { faces }}} \int_{f, N} \llbracket-\vec{H}^{\mathscr{E}} \rrbracket \cdot \vec{n}_{L} \mathrm{~d} S=\frac{\mathrm{d}}{\mathrm{d} t} \sum_{\substack{\text { interior } \\ \text { faces }}} \int_{f, N} \mathscr{E}_{i}^{S} \mathrm{~d} S, \quad \mathscr{E}_{i}^{S}(\mathbf{q}) \geqslant 0,
$$

and that the surface term associated to the time contraction $\vec{H}^{\mathscr{E}}$ is transformed to the time derivative of the surface entropy $\mathscr{E}^{S}$,

$$
\sum_{\substack{\text { boundary } \\ \text { faces }}} \int_{f, N} \vec{H}^{\mathscr{E}} \cdot \mathrm{d} \vec{S}=\frac{\mathrm{d}}{\mathrm{d} t} \sum_{\substack{\text { boundary } \\ \text { faces }}} \int_{f, N} \mathscr{E}^{S} \mathrm{~d} S .
$$


We replace (3.86) and (3.87) in (3.84), so that the contraction of time coefficients is

$$
\begin{aligned}
\sum_{e}\left\langle\mathscr{J} \mathbf{Q}_{t}, \mathbf{W}\right\rangle_{E, N} & =\sum_{e}\langle\mathscr{J} \mathscr{E}\rangle_{E, N}+\sum_{\substack{\text { interior } \\
\text { faces }}} \int_{f, N} \llbracket-\vec{H}^{\mathscr{E}} \rrbracket \cdot \vec{n}_{L} \mathrm{~d} S+\sum_{\substack{\text { boundary } \\
\text { faces }}} \int_{f, N} \vec{H}^{\mathscr{E}} \cdot \mathrm{d} \vec{S} \\
& =\frac{\mathrm{d}}{\mathrm{d} t}\left(\sum_{e}\langle\mathscr{J} \mathscr{E}\rangle_{E, N}+\sum_{\substack{\text { interior } \\
\text { faces }}} \int_{f, N} \mathscr{E}_{i} S \mathrm{~d} S+\sum_{\substack{\text { boundary } \\
\text { faces }}} \int_{f, N} \mathscr{E}^{S} \mathrm{~d} S\right)=\overline{\mathscr{E}}_{t} N,
\end{aligned}
$$

with the discrete total entropy $\overline{\mathscr{E}}^{N}$,

$$
\overline{\mathscr{E}}^{N}=\sum_{e}\langle\mathscr{E}\rangle_{E, N}+\sum_{\substack{\text { interior } \\ \text { faces }}} \int_{f, N} \mathscr{E}_{i}^{S} \mathrm{~d} S+\sum_{\substack{\text { boundary } \\ \text { faces }}} \int_{f, N} \mathscr{E}^{S} \mathrm{~d} S \geqslant 0
$$

\subsubsection{Contraction of the inviscid fluxes}

In Gassner et al. [2016a] the authors prove that the entropy variables contract the inviscid fluxes discretely,

$$
\left\langle\mathbb{D}\left(\overrightarrow{\mathbf{F}}_{e}\right)^{\mathrm{EC}}, \mathbf{W}\right\rangle_{E, N}=\int_{\partial E, N} \overrightarrow{\tilde{F}}_{e}^{\mathscr{E}} \cdot \hat{n} \mathrm{~d} \hat{S}
$$

if the entropy conserving two-point volume flux satisfies Tadmor's jump condition, Gassner et al. [2016a]; Tadmor [2003]; Fisher and Carpenter [2013],

$$
\llbracket \mathbf{W}^{T} \rrbracket \overrightarrow{\mathbf{F}}_{e}^{e c}\left(\mathbf{Q}_{L}, \mathbf{Q}_{R}\right)-\llbracket \mathbf{W}^{T} \overrightarrow{\mathbf{F}}_{e} \rrbracket+\llbracket \vec{F}_{e}^{\mathscr{E}} \rrbracket=0 .
$$

This is the procedure we follow to find an appropriate split-form. We write Tadmor's jump condition for the specific entropy variables and entropy flux given by the continuous analysis and search for a two-point volume flux. Then, the scheme is automatically entropy-stable as it satisfies (3.90). Eq. (3.90) is the discrete version of (3.72).

\subsubsection{Positive semi-definiteness of the viscous fluxes}

The last quadrature of (3.81) is the discrete version of (3.74). Discretely, since viscous fluxes are pointwise positive definite,

$$
\overrightarrow{\mathbf{G}}_{i j k}^{T} \overrightarrow{\mathbf{F}}_{v, i j k}=\overrightarrow{\mathbf{G}}_{i j k}^{T} \underline{\mathbf{B}}_{i j k} \overrightarrow{\mathbf{G}}_{i j k} \geqslant 0,
$$

then,

$$
\left\langle\mathscr{J} \overrightarrow{\mathbf{G}}, \overrightarrow{\mathbf{F}}_{v}\right\rangle_{E, N}=\langle\mathscr{J} \overrightarrow{\mathbf{G}}, \underline{\mathbf{B}} \overrightarrow{\mathbf{G}}\rangle_{E, N} \geqslant 0 .
$$




\subsubsection{Stability of the boundary terms}

With (3.83), (3.90) and (3.93), and adding the entropy equation (3.81) for all the elements, we get

$$
\begin{aligned}
& \overline{\mathscr{E}}_{t}^{N}+\sum_{e} \int_{\partial E, N}\left(\overrightarrow{\tilde{F}}_{e}^{\mathscr{E}}+\mathbf{W}^{T}\left(\overrightarrow{\widetilde{\mathbf{F}}}_{e}^{\star}-\overrightarrow{\widetilde{\mathbf{F}}}_{e}-\overrightarrow{\widetilde{\mathbf{F}}}_{v}^{\star}+\overrightarrow{\widetilde{\mathbf{F}}}_{v}\right)-\mathbf{W}^{\star, T} \overrightarrow{\overrightarrow{\mathbf{F}}}_{v}\right) \cdot \hat{n} \mathrm{~d} \hat{S} \\
& =-\sum_{e}\langle\mathscr{J} \overrightarrow{\mathbf{G}}, \underline{\mathbf{B}} \overrightarrow{\mathbf{G}}\rangle_{E, N} \leqslant 0,
\end{aligned}
$$

which is similar to the result in the continuous analysis (3.77), but now the boundary integral features more terms, and does not depend only on the physical boundary data, but the inter-element data.

To study interchanges between adjacent elements, the surface integral has to be written in the physical space using (2.49),

$$
\begin{aligned}
& \int_{\partial E, N}\left(\overrightarrow{\tilde{F}}_{e}^{\mathscr{E}}+\mathbf{W}^{T}\left(\overrightarrow{\tilde{\mathbf{F}}}_{e}^{\star}-\overrightarrow{\tilde{\mathbf{F}}}_{e}-\overrightarrow{\tilde{\mathbf{F}}}_{v}^{\star}+\overrightarrow{\tilde{\mathbf{F}}}_{v}\right)-\mathbf{W}^{\star, T} \overrightarrow{\tilde{\mathbf{F}}}_{v}\right) \cdot \hat{n} \mathrm{~d} \hat{S} \\
& =\int_{\partial e, N}\left(\overrightarrow{\tilde{F}}_{e}^{\mathscr{E}}+\mathbf{W}^{T}\left(\overrightarrow{\mathbf{F}}_{e}^{\star}-\overrightarrow{\mathbf{F}}_{e}-\overrightarrow{\mathbf{F}}_{v}^{\star}+\overrightarrow{\mathbf{F}}_{v}\right)-\mathbf{W}^{\star, T} \overrightarrow{\mathbf{F}}_{v}\right) \cdot \vec{n} \mathrm{~d} S .
\end{aligned}
$$

We write interior and physical boundary faces separately. At interior faces, given the relation between outward normal vectors in adjacent cells $\vec{n}_{L}=-\vec{n}_{R}$, and given that the numerical fluxes are unique for both elements, we can write relate the integral for interior faces as

$$
\mathrm{IBT}=\sum_{\substack{\text { interior } \\ \text { faces }}} \int_{f, N}\left(\llbracket \mathbf{W}^{T} \overrightarrow{\mathbf{F}}_{e} \rrbracket-\llbracket \vec{F}_{e}^{\mathscr{E}} \rrbracket-\llbracket \mathbf{W}^{T} \rrbracket \overrightarrow{\mathbf{F}}_{e}^{\star}-\llbracket \mathbf{W}^{T} \overrightarrow{\mathbf{F}}_{v} \rrbracket+\llbracket \mathbf{W}^{T} \rrbracket \overrightarrow{\mathbf{F}}_{v}^{\star}+\mathbf{W}^{\star, T} \llbracket \overrightarrow{\mathbf{F}}_{v} \rrbracket\right) \cdot \vec{n}_{L} \mathrm{~d} S .
$$

A systematic way to cancel the interior boundary terms to get an entropy conserving scheme is to use the two-point volume flux as inviscid numerical flux, $\overrightarrow{\mathbf{F}}_{e}^{\star}=\overrightarrow{\mathbf{F}}_{e}^{\mathrm{EC}}$, and to use the Bassi-Rebay 1 scheme (BR1) for viscous fluxes, $\mathbf{W}^{\star}=\{\{\mathbf{W}\}\}, \overrightarrow{\mathbf{F}}_{v}^{\star}=\left\{\left\{\overrightarrow{\mathbf{F}}_{v}\right\}\right\}$,

$$
\begin{aligned}
\mathrm{IBT} & =\sum_{\substack{\text { interior } \\
\text { faces }}} \int_{f, N}\left(\llbracket \mathbf{W}^{T} \overrightarrow{\mathbf{F}}_{e} \rrbracket-\llbracket \vec{F}^{\mathscr{E}} \rrbracket-\llbracket \mathbf{W}^{T} \rrbracket \overrightarrow{\mathbf{F}}_{e}^{\mathrm{EC}}-\llbracket \mathbf{W}^{T} \overrightarrow{\mathbf{F}}_{v} \rrbracket+\llbracket \mathbf{W}^{T} \rrbracket\left\{\left\{\overrightarrow{\mathbf{F}}_{v}\right\}\right\}+\left\{\left\{\mathbf{W}^{T}\right\}\right\} \llbracket \overrightarrow{\mathbf{F}}_{v} \rrbracket\right) \cdot \vec{n}_{L} \mathrm{~d} S \\
& =0 .
\end{aligned}
$$

The inviscid term contributions cancel out because of Tadmor's jump condition (3.91), and the viscous terms vanish because of the algebraic relatonship between the average and jump operator (1.27). 
Obtaining an entropy-stable scheme, i.e. IBT $\geqslant 0$ depends on the particular physics to be solved. We assume that both options are available, an entropy conserving option with IBT $=0$, and an entropy-stable scheme with IBT $\geqslant 0$.

Physical boundary terms, on the contrary, depend only on the element to which the face belongs,

$$
\mathrm{PBT}=\sum_{\substack{\text { boundary } \\ \text { faces }}} \int_{f, N}\left(\vec{F}_{e}^{\mathscr{E}}+\mathbf{W}^{T}\left(\overrightarrow{\mathbf{F}}_{e}^{\star}-\overrightarrow{\mathbf{F}}_{e}-\overrightarrow{\mathbf{F}}_{v}^{\star}+\overrightarrow{\mathbf{F}}_{v}\right)-\mathbf{W}^{\star, T} \overrightarrow{\mathbf{F}}_{v}\right) \cdot \vec{n} \mathrm{~d} S .
$$

Later in this work, we will provide entropy conserving and entropy-stable approximations to solid walls.

The entropy equation results after making use of the definitions (3.96) and (3.98), in (3.94),

$$
\overline{\mathscr{E}}_{t}^{N}+\mathrm{PBT}=-\mathrm{IBT}-\sum_{e}\langle\mathscr{J} \overrightarrow{\mathbf{G}}, \underline{\mathbf{B}} \overrightarrow{\mathbf{G}}\rangle_{E, N} \leqslant-\sum_{e}\langle\mathscr{J} \overrightarrow{\mathbf{G}}, \underline{\mathbf{B}} \overrightarrow{\mathbf{G}}\rangle_{E, N} \leqslant 0
$$

which represents the discrete version of (3.77). The first inequality can be replaced by an equality if one uses a two-point volume flux that satisfies Tadmor's jump condition (3.91) and the BR1 scheme as numerical fluxes (3.97), to fully match the continuous entropy equation (3.77) discretely.

We conclude that the DG approximation (3.79) of (3.64) is entropy-stable if the two-point volume flux satisfies Tadmor's jump condition (3.91), and if appropriate choices are used for the numerical fluxes. A summary of the entropy analyses performed for conservative systems can be found in Table 3.1.

\subsection{Entropy-stability for non-conservative systems}

Non-conservative systems can also satisfy an entropy law. Given the study of conservative systems in Sec. 3.5, we only need to assess how to show the stability when non-conservative terms are included,

$$
\mathbf{q}_{t}+\vec{\nabla} \cdot \overrightarrow{\mathbf{f}}_{e}+\sum_{m=1}^{N_{c}} \overrightarrow{\boldsymbol{\phi}}_{m} \cdot \vec{\nabla} \lambda_{m}=\vec{\nabla} \cdot \overrightarrow{\mathbf{f}}_{v}
$$

Similarly to (3.76), we construct a weak form of (3.100) after the multiplication by a set of entropy variables $\mathbf{w}$ and the integration over $\Omega$,

$$
\left\langle\mathbf{q}_{t}, \mathbf{w}\right\rangle+\left\langle\vec{\nabla} \cdot \overrightarrow{\mathbf{f}}_{e}, \mathbf{w}\right\rangle+\sum_{m=1}^{N_{c}}\left\langle\overrightarrow{\boldsymbol{\phi}}_{m} \cdot \vec{\nabla} \lambda_{m}, \mathbf{w}\right\rangle-\int_{\partial \Omega} \mathbf{w}^{T} \overrightarrow{\mathbf{f}}_{v} \cdot \mathrm{d} \vec{S}=-\left\langle\overrightarrow{\mathbf{f}}_{v}, \vec{\nabla} \mathbf{w}\right\rangle
$$


Table 3.1: Summary of the continuous (cont.) and discrete (disc.) entropy analyses performed for the conservative system

Weak forms

Cont. $\quad\left\langle\mathbf{q}_{t}, \mathbf{w}\right\rangle+\left\langle\vec{\nabla} \cdot \overrightarrow{\mathbf{f}}_{e}\right\rangle-\int_{\partial \Omega} \mathbf{w}^{T} \overrightarrow{\mathbf{f}}_{v} \cdot \mathrm{d} \vec{S}=-\left\langle\vec{\nabla} \mathbf{w}, \overrightarrow{\mathbf{f}}_{v}\right\rangle$

Disc. $\quad\left\langle\mathbf{Q}_{t}, \mathbf{W}\right\rangle_{E, N}+\int_{\partial e, N}\left(\mathbf{W}^{T}\left(\overrightarrow{\mathbf{F}}_{e}^{\star}-\overrightarrow{\mathbf{F}}_{e}-\overrightarrow{\mathbf{F}}_{v}^{\star}+\overrightarrow{\mathbf{F}}_{v}\right)+\mathbf{W}^{\star, T} \overrightarrow{\mathbf{F}}_{v}\right) \cdot \mathrm{d} \vec{S}$

$$
+\left\langle\mathbb{D}\left(\overrightarrow{\mathbf{F}}_{e}\right)^{\mathrm{EC}}, \mathbf{W}\right\rangle_{E, N}=-\left\langle\mathscr{J} \overrightarrow{\mathbf{G}}, \overrightarrow{\mathbf{F}}_{v}\right\rangle_{E, N}
$$

Entropy equation

Cont. $\quad \overline{\mathscr{E}}_{t}+\int_{\partial \Omega}\left(\vec{f}_{e}^{\mathscr{E}}+\vec{f}_{v}^{\mathscr{E}}\right) \cdot \mathrm{d} \vec{S}=-\langle\mathscr{J} \vec{\nabla} \mathbf{w}, \underline{\mathbf{B}} \vec{\nabla} \mathbf{w}\rangle \leqslant 0$

Disc. $\quad \overline{\mathscr{E}}_{t}^{N}+\mathrm{PBT}=-\mathrm{IBT}-\sum_{e}\langle\mathscr{J} \overrightarrow{\mathbf{G}}, \underline{\mathbf{B}} \overrightarrow{\mathbf{G}}\rangle_{E, N} \leqslant-\sum_{e}\langle\mathscr{J} \overrightarrow{\mathbf{G}}, \underline{\mathbf{B}} \overrightarrow{\mathbf{G}}\rangle_{E, N} \leqslant 0$

$$
\begin{gathered}
\mathrm{IBT}=\sum_{\substack{\text { interior } \\
\text { faces }}} \int_{f, N}\left(\llbracket \mathbf{W}^{T} \overrightarrow{\mathbf{F}}_{e} \rrbracket-\llbracket \vec{F}_{e}^{\mathscr{E}} \rrbracket-\llbracket \mathbf{W}^{T} \rrbracket \overrightarrow{\mathbf{F}}_{e}^{\star}\right) \cdot \vec{n}_{L} \mathrm{~d} S \\
\quad+\int_{f, N}\left(-\llbracket \mathbf{W}^{T} \overrightarrow{\mathbf{F}}_{v} \rrbracket+\llbracket \mathbf{W}^{T} \rrbracket \overrightarrow{\mathbf{F}}_{v}^{\star}+\mathbf{W}^{\star, T} \llbracket \overrightarrow{\mathbf{F}}_{v} \rrbracket\right) \cdot \vec{n}_{L} \mathrm{~d} S \geqslant 0 \\
\mathrm{PBT}=\sum_{\substack{\text { boundary } \\
\text { faces }}} \int_{f, N}\left(\vec{F}_{t}^{\mathscr{E}}+\vec{F}_{e}^{\mathscr{E}}+\mathbf{W}^{T}\left(\overrightarrow{\mathbf{F}}_{e}^{\star}-\overrightarrow{\mathbf{F}}_{e}-\overrightarrow{\mathbf{F}}_{v}^{\star}+\overrightarrow{\mathbf{F}}_{v}\right)-\mathbf{W}^{\star, T} \overrightarrow{\mathbf{F}}_{v}\right) \cdot \vec{n} \mathrm{~d} S \\
\sum_{\text {interior }} \int_{f, N} \llbracket-\vec{H}^{\mathscr{E}} \rrbracket \cdot \mathrm{d} \vec{S}=\sum_{\text {interior }} \int_{f, N} \int_{\text {faces }} \mathscr{E}_{i}^{S} \mathrm{~d} S \sum_{\substack{\text { boundary } \\
\text { faces }}} \int_{f, N} \vec{H}^{\mathscr{E}} \cdot \mathrm{d} \vec{S}=\frac{\mathrm{d}}{\mathrm{d} t} \sum_{\text {boundary }} \int_{f, N} \int_{\text {faces }} \mathscr{E}^{S} \mathrm{~d} S
\end{gathered}
$$

\section{Continuous}

$\begin{array}{ll}\text { Time } & \left\langle\mathbf{q}_{t}, \mathbf{w}\right\rangle=\overline{\mathscr{E}}_{t} \\ \text { coeffs. }\end{array}$

Inviscid

fluxes

$\left\langle\vec{\nabla} \cdot \vec{f}_{e}, \mathbf{w}\right\rangle=\int_{\partial \Omega} \vec{f}_{e}^{\mathscr{E}} \cdot \mathrm{d} \vec{S}$

Viscous

fluxes $-\left\langle\overrightarrow{\mathbf{f}}_{v}, \vec{\nabla} \mathbf{w}\right\rangle=-\langle\underline{\mathbf{B}} \vec{\nabla} \mathbf{w}, \vec{\nabla} \mathbf{w}\rangle \leqslant 0$

Discrete

$\sum_{e}\left\langle\mathscr{J} \mathbf{Q}_{t}, \mathbf{W}\right\rangle_{E, N}=\overline{\mathscr{E}}_{t}^{N}$

$\left\langle\mathbb{D}\left(\overrightarrow{\mathbf{F}}_{e}\right)^{\mathrm{EC}}, \mathbf{W}\right\rangle_{E, N}=\int_{\partial e, N} \vec{F}_{e}^{\mathscr{E}} \cdot \mathrm{d} \vec{S}$

$-\left\langle\mathscr{J} \overrightarrow{\mathbf{G}}, \overrightarrow{\mathbf{F}_{v}}\right\rangle_{E, N}=-\langle\mathscr{J} \overrightarrow{\mathbf{G}}, \underline{\mathbf{B}} \overrightarrow{\mathbf{G}}\rangle_{E, N} \leqslant 0$ 
We assume the existence of a positive mathematical entropy $\mathscr{E}(\mathbf{q}, \vec{\nabla} \mathbf{q})$ that defines a set of entropy variables that contract the state vector time derivative (3.71), and that the positive definiteness of the viscous fluxes (3.74) holds. Then we only need to study the modifications required for the contraction of the inviscid and nonconservative terms to complete the entopy-stability analysis. Note that the study of the time derivatives and viscous fluxes is identical to the conservative system.

The entropy variables now must contract both inviscid fluxes and non-conservative terms simultaneously. Thus, the inviscid terms contraction (3.72) is transformed to

$$
\left\langle\vec{\nabla} \cdot \overrightarrow{\mathbf{f}}_{e}, \mathbf{w}\right\rangle+\sum_{m=1}^{N_{c}}\left\langle\overrightarrow{\boldsymbol{\phi}}_{m} \cdot \vec{\nabla} \lambda_{m}, \mathbf{w}\right\rangle=\int_{\partial \Omega} \vec{f}_{e}^{\mathscr{E}} \cdot \mathrm{d} \vec{S} .
$$

Then, if the entropy and entropy variables satisfy the time coefficients contraction (3.71), the new condition (3.102), and the viscous fluxes are positive definite (3.74), the entropy equation of the conservative system (3.77) holds for the non-conservative system.

We now study the discrete approximation. We copy the DG scheme for nonconservative systems (2.83) here and we replace the test functions by $\phi=\mathrm{W}$ and $\vec{\varphi}=\overrightarrow{\mathbf{F}}_{v}$

$$
\begin{aligned}
\left\langle\mathscr{J} \mathbf{Q}_{t}, \boldsymbol{W}\right\rangle_{E, N} & +\int_{\partial E, N}\left(\boldsymbol{W}^{T}\left(\overrightarrow{\overrightarrow{\mathbf{F}}}_{e}^{\star}-\overrightarrow{\widetilde{\mathbf{F}}}_{e}+\sum_{m=1}^{N_{c}}\left(\left(\overrightarrow{\tilde{\mathbf{\Phi}}}_{m} \Lambda_{m}\right)^{\diamond}-\left(\overrightarrow{\tilde{\mathbf{\Phi}}}_{m} \Lambda_{m}\right)\right)-\overrightarrow{\overrightarrow{\mathbf{F}}}_{v}^{\star}+\overrightarrow{\overrightarrow{\mathbf{F}}}_{v}\right)-\mathbf{W}^{\star, T} \overrightarrow{\overrightarrow{\mathbf{F}}}_{v}\right) \cdot \hat{n} \mathrm{~d} \hat{\boldsymbol{S}} \\
& +\left\langle\vec{\nabla}_{\xi} \cdot \overrightarrow{\tilde{\mathbf{F}}}_{e}, \boldsymbol{W}\right\rangle_{E, N}+\sum_{m=1}^{N_{c}}\left\langle\overrightarrow{\tilde{\mathbf{\Phi}}}_{m} \cdot \vec{\nabla}_{\xi} \Lambda_{m}, W\right\rangle_{E, N}=-\left\langle\mathscr{J} \overrightarrow{\mathbf{G}}, \overrightarrow{\mathbf{F}}_{v}\right\rangle_{E, N} \cdot
\end{aligned}
$$

As in the continuous analysis, the contraction of the time derivative coefficients (3.83), and the viscous fluxes (3.93) are identical to the conservative system. Therefore, we only discuss the discrete equivalent of the inviscid and non-conservative terms simultaneous contraction (3.102) and the new version of the interior and physical boundary terms.

First, for the invsicid and non-conservative terms contraction, unlike in the conservative system, we will distinguish two cases: the general case where the split-form scheme is required, and a particular case valid for some specific systems where the standard DG is stable by itself. The study of the particular case is of interest since the scheme and its implementation are simpler.

1. The general case: if the proof for (3.102) requires the use of the product rule or other mathematical operations without an equivalent in the discrete setting, 
then the discrete approximation needs the split-form scheme (2.85), that satisfies,

$$
\left\langle\mathbb{D}\left(\overrightarrow{\mathbf{F}}_{e}\right)^{\mathrm{EC}}, \mathbf{W}\right\rangle_{E, N}+\sum_{m=1}^{N_{c}}\left\langle\overrightarrow{\tilde{\boldsymbol{\Phi}}}_{m} \cdot \mathbb{D}_{\text {grad }}^{\mathrm{DM}} \Lambda_{m}, \mathbf{W}\right\rangle_{E, N}=\int_{\partial E, N} \overrightarrow{\tilde{F}}_{e}^{\mathscr{E}} \cdot \hat{n} \mathrm{~d} \hat{S} .
$$

2. The particular case: if the non-conservative terms represent an actual splitting of the conservative part, the demonstration of (3.102) might not require the use of the product rule. In that case, the standard DG scheme (2.83) is automatically entropy-stable without the need for split-forms,

$$
\left\langle\vec{\nabla}_{\xi} \cdot \overrightarrow{\tilde{\mathbf{F}}}_{e}, \mathbf{W}\right\rangle_{E, N}+\sum_{m=1}^{N_{c}}\left\langle\overrightarrow{\tilde{\boldsymbol{\Phi}}}_{m} \cdot \vec{\nabla}_{\xi} \Lambda_{m}, \mathbf{W}\right\rangle_{E, N}=\int_{\partial e, N} \overrightarrow{\tilde{F}}_{e}^{E} \cdot \hat{n} \mathrm{~d} \hat{S} .
$$

Either way, the resulting discrete entropy equation is (3.99), where the boundary terms are modified to include the non-conservative terms in Sec. 3.6.3

\subsubsection{Entropy-stability of general non-conservative systems}

Since the rest of the analysis (3.102) is identical to the conservative system, and the newly introduced non-conservative terms are contracted together with the conservative inviscid terms, the same entropy equation of the conservative system (3.77) results for the non-conservative system (2.77). Then, under the assumption of the simultaneous contraction of inviscid fluxes and non-conservative terms (3.102) and all the rest made for the conservative system, the continuous non-conservative system is entropy-stable.

The entropy-stability of the discrete DG system needs the use of the split-form scheme (2.85), which needs an appropriate choice for the entropy-conserving twopoint volume flux. The inclusion of the non-conservative terms modifies Tadmor's jump condition to

$$
\llbracket \mathbf{W}^{T} \rrbracket \overrightarrow{\mathbf{F}}_{e}^{\mathrm{EC}}\left(\mathbf{Q}_{L}, \mathbf{Q}_{R}\right)=\llbracket \Psi \rrbracket-\sum_{m=1}^{N_{c}} \llbracket \mathbf{W}^{T} \overrightarrow{\boldsymbol{\Phi}}_{m} \rrbracket\left\{\left\{\Lambda_{m}\right\}\right\},
$$

where $\Psi$ is the potential. In this work we use an extension of the potential presented in Bohm et al. [2018]; Liu et al. [2018] to include the gradient non-conservative terms,

$$
\Psi=\mathbf{W}^{T} \overrightarrow{\mathbf{F}}_{e}+\sum_{m=1}^{N_{c}} \mathbf{W}^{T} \overrightarrow{\boldsymbol{\Phi}}_{m} \Lambda_{m}-\vec{F}_{e}^{\mathscr{E}}
$$


A two-point volume flux that satisfies the extended Tadmor's jump condition (3.106) contracts the inviscid fluxes and non-conservative terms to the boundary integral of the entropy flux,

$$
\left\langle\mathbb{D}\left(\overrightarrow{\mathbf{F}}_{e}\right)^{\mathrm{EC}}, \mathbf{W}\right\rangle_{E, N}+\sum_{m=1}^{N_{c}}\left\langle\overrightarrow{\tilde{\boldsymbol{\Phi}}}_{m} \cdot \mathbb{D}_{\text {grad }}^{\mathrm{DM}} \Lambda_{m}, \mathbf{W}\right\rangle_{E, N}=\int_{\partial E, N} \overrightarrow{\tilde{F}}_{e}^{E} \cdot \hat{n} \mathrm{~d} \hat{S},
$$

which represents the discrete version of (3.102). The proof for (3.108) is not immediate, and can be found in Appendix A.1.

\subsubsection{Entropy-stability of a particular non-conservative system}

The non-conservative system stability rests on the same assumptions made on its conservative counterpart, where the contraction of the inviscid conservative terms by the entropy variables now also includes the non-conservative terms (3.102). The continuous and discrete proof for a general non-conservative system was done in Sec. 3.6.1.

The problem with the general analysis is that we do not know a-priori which operations are needed (e.g. multiple applications of the product rule) to show the inviscid conservative and non-conservative terms contraction (3.102). In this section, we consider an equivalent but simpler analysis that is valid for some non-conservative system, where we can know for certain that the only property needed to show (3.102) is the Gauss law (which holds discretely).

We require the system to satisfy two properties:

Property 1 The non-conservative term gradients $\lambda_{m}$ are the entropy variables,

$$
\lambda_{m}=w_{m}
$$

which implies also that $N_{c}=N_{\mathrm{E}}$.

Property 2 If inviscid fluxes, $\overrightarrow{\mathbf{f}}_{e}$, and non-conservative term coefficients, $\overrightarrow{\boldsymbol{\phi}}_{m}$, satisfy

$$
\mathbf{e}_{m}^{T} \overrightarrow{\mathbf{f}}_{e}=\mathbf{w}^{T} \overrightarrow{\boldsymbol{\phi}}_{m}, \quad m=1, \ldots, N_{E}=N_{c},
$$

where $\mathbf{e}_{m}$ is the state unit vector along the $m$-th state variable.

A non-conservative system that satisfies Prop. 1 (3.109) and Prop. 2 (3.110) has its inviscid fluxes and non-conservative terms automatically contracted by the entropy variables, and the entropy flux is computed as

$$
\vec{f}_{e}^{\mathscr{E}}=\mathbf{w}^{T} \overrightarrow{\mathbf{f}}_{e} .
$$


We prove the inviscid terms contraction for a system satisfying Prop. 1 (3.109) and Prop. 2 (3.110) in the continuous setting. To do so, we write the first term of (3.102) as

$$
\begin{aligned}
\left\langle\vec{\nabla} \cdot \overrightarrow{\mathbf{f}}_{e}, \mathbf{w}\right\rangle & =\int_{\partial \Omega} \mathbf{w}^{T} \overrightarrow{\mathbf{f}}_{e} \cdot \mathrm{d} \vec{S}-\left\langle\overrightarrow{\mathbf{f}}_{e}, \vec{\nabla} \mathbf{w}\right\rangle=\int_{\partial \Omega} \mathbf{w}^{T} \overrightarrow{\mathbf{f}}_{e} \cdot \mathrm{d} \vec{S}-\sum_{m=1}^{N_{E}}\left\langle\mathbf{e}_{m}^{T} \overrightarrow{\mathbf{f}}_{e}, \vec{\nabla} w_{m}\right\rangle \\
& =\int_{\partial \Omega} \vec{f}_{e}^{\mathscr{E}} \cdot \mathrm{d} \vec{S}-\sum_{m=1}^{N_{E}}\left\langle\mathbf{w}, \overrightarrow{\boldsymbol{\phi}}_{m} \cdot \vec{\nabla} w_{m}\right\rangle=\int_{\partial \Omega} \vec{f}_{e}^{\mathscr{E}} \cdot \mathrm{d} \vec{S}-\sum_{m=1}^{N_{c}}\left\langle\mathbf{w}, \overrightarrow{\boldsymbol{\phi}}_{m} \cdot \vec{\nabla} \lambda_{m}\right\rangle,
\end{aligned}
$$

to find that the inviscid and non-conservative terms contraction (3.102) is satisfied automatically. In the first equality we used the Gauss law, in the second equality we wrote the second scalar product as the sum of the product of the five state components. In the third equality we used Prop. 2 (3.110), and in the last equality we used Prop. 1 (3.109).

We now show that the DG scheme satisfies a discrete version of (3.102). To do so, we follow the recently made steps for the continuous analysis (3.112),

$$
\begin{aligned}
\left\langle\vec{\nabla}_{\xi} \cdot \overrightarrow{\tilde{\mathbf{F}}}_{e}, \mathbf{W}\right\rangle_{E, N} & =\int_{\partial e, N} \mathbf{W}^{T} \overrightarrow{\mathbf{F}}_{e} \cdot \vec{n} \mathrm{~d} S-\left\langle\overrightarrow{\tilde{\mathbf{F}}}_{e}, \vec{\nabla}_{\xi} \mathbf{W}\right\rangle_{E, N} \\
& =\int_{\partial e, N} \mathbf{W}^{T} \overrightarrow{\mathbf{F}}_{e} \cdot \vec{n} \mathrm{~d} S-\sum_{m=1}^{N_{\mathrm{E}}}\left\langle\mathbf{e}_{m}^{T} \overrightarrow{\tilde{\mathbf{F}}}_{e}, \vec{\nabla}_{\xi} W_{m}\right\rangle_{E, N} \\
& =\int_{\partial e, N} \vec{F}_{e}^{\mathscr{E}} \cdot \vec{n} \mathrm{~d} S-\sum_{m=1}^{N_{\mathrm{E}}}\left\langle\mathbf{W}^{T} \overrightarrow{\tilde{\mathbf{\Phi}}}_{m}, \vec{\nabla}_{\xi} W_{m}\right\rangle_{E, N} \\
& =\int_{\partial e, N} \vec{F}_{e}^{\mathscr{E}} \cdot \vec{n} \mathrm{~d} S-\sum_{m=1}^{N_{c}}\left\langle\mathbf{W}^{T} \overrightarrow{\tilde{\boldsymbol{\Phi}}}_{m}, \vec{\nabla}_{\xi} \Lambda_{m}\right\rangle_{E, N}
\end{aligned}
$$

The first identity uses the discrete Gauss law, in the second equality we explicitly wrote the scalar product as the sum of the components. The third equality uses Prop. 2 (3.110) for the volume quadrature and the definition of the entropy flux (3.111) for the surface quadrature. The last equality uses Property 1 (3.109). Note that Prop. 2 (3.110) holds for the contravariant fluxes,

$$
\mathbf{e}_{m}^{T} \overrightarrow{\tilde{\mathbf{F}}}_{e}=\mathbf{e}_{m}^{T}\left(\underline{\mathbf{M}}^{T} \overrightarrow{\mathbf{F}}_{e}\right)=\overline{\bar{M}}^{T}\left(\mathbf{e}_{m}^{T} \overrightarrow{\mathbf{F}}_{e}\right)=\overline{\bar{M}}^{T}\left(\mathbf{W}^{T} \overrightarrow{\boldsymbol{\Phi}}_{m}\right)=\mathbf{W}^{T}\left(\underline{\mathbf{M}}^{T} \overrightarrow{\boldsymbol{\Phi}}_{m}\right)=\mathbf{W}^{T} \overrightarrow{\tilde{\boldsymbol{\Phi}}}_{m} .
$$

In (3.114), space and state multiplications commute since the metric terms matrix (2.16) is built from identity matrices state-wise.

Eq. (3.113) proves (3.105), and confirms that the standard DG scheme is entropystable under the conditions in Prop. 1 (3.109), Prop. 2 (3.110), and those made for the rest of the terms shared with conservative systems (i.e. time derivative contraction, and viscous fluxes positive definiteness). 


\subsubsection{Interior and physical boundary terms}

The interior boundary terms IBT extend (3.96) with the diamond fluxes of non-conservative terms (2.81),

$$
\begin{aligned}
\text { IBT }= & \sum_{\substack{\text { interior } \\
\text { faces }}} \int_{f, N}\left(\llbracket \mathbf{W}^{T} \overrightarrow{\mathbf{F}}_{e} \rrbracket-\llbracket \vec{F}_{e}^{\mathscr{E}} \rrbracket-\llbracket \mathbf{W}^{T} \rrbracket \overrightarrow{\mathbf{F}}_{e}^{\star}+\sum_{m=1}^{N_{c}}\left(\llbracket \mathbf{W}^{T} \overrightarrow{\boldsymbol{\Phi}}_{m} \Lambda_{m} \rrbracket-\llbracket \mathbf{W}^{T}\left(\overrightarrow{\boldsymbol{\Phi}}_{m} \Lambda_{m}\right)^{\diamond} \rrbracket\right)\right. \\
& \left.-\llbracket \mathbf{W}^{T} \overrightarrow{\mathbf{F}}_{v} \rrbracket+\llbracket \mathbf{W}^{T} \rrbracket \overrightarrow{\mathbf{F}}_{v}^{\star}+\mathbf{W}^{\star, T} \llbracket \overrightarrow{\mathbf{F}}_{v} \rrbracket\right) \cdot \vec{n}_{L} \mathrm{~d} S,
\end{aligned}
$$

which need to be positive for stability (or zero for an entropy conserving scheme).

Unlike the numerical fluxes (e.g. $\overrightarrow{\mathbf{F}}_{e}^{\star}$ ), the diamond fluxes are not taken out of the jump operator since they differ from one side to the other. A systematic way to construct an entropy conserving scheme uses central fluxes,

$$
\overrightarrow{\mathbf{F}}_{e}^{\star}=\overrightarrow{\mathbf{F}}_{e}^{\mathrm{EC}}, \quad\left(\overrightarrow{\mathbf{\Phi}}_{m} \Lambda_{m}\right)^{\diamond}=\overrightarrow{\mathbf{\Phi}}_{m}\left\{\left\{\Lambda_{m}\right\}\right\}, \quad \overrightarrow{\mathbf{F}}_{v}^{\star}=\left\{\left\{\overrightarrow{\mathbf{F}}_{v}\right\}\right\}, \quad \mathbf{W}^{\star}=\{\{\mathbf{W}\}\} .
$$

With these choices, the argument of the inviscid interior boundary terms are zero (for the viscous fluxes we have already proven that they do not contribute to IBT in (3.97)),

$$
\begin{aligned}
& \llbracket \mathbf{W}^{T} \overrightarrow{\mathbf{F}}_{e} \rrbracket-\llbracket \vec{F}_{e}^{\mathscr{E}} \rrbracket-\llbracket \mathbf{W}^{T} \rrbracket \overrightarrow{\mathbf{F}}_{e}^{\mathrm{EC}}+\sum_{m=1}^{N_{c}}\left(\llbracket \mathbf{W}^{T} \overrightarrow{\boldsymbol{\Phi}}_{m} \Lambda_{m} \rrbracket-\llbracket \mathbf{W}^{T}\left(\overrightarrow{\boldsymbol{\Phi}}_{m} \Lambda_{m}\right)^{\diamond} \rrbracket\right) \\
& =\llbracket \mathbf{W}^{T} \overrightarrow{\mathbf{F}}_{e} \rrbracket-\llbracket \vec{F}^{\mathscr{E}} \rrbracket-\llbracket \mathbf{W}^{T} \overrightarrow{\mathbf{F}}_{e}+\sum_{m=1}^{N_{c}} \mathbf{W}^{T} \overrightarrow{\boldsymbol{\Phi}}_{m} \Lambda_{m}-\vec{F}^{\mathscr{E}} \rrbracket+\sum_{n=1}^{N_{c}} \llbracket \mathbf{W}^{T} \overrightarrow{\boldsymbol{\Phi}}_{m} \rrbracket\left\{\left\{\Lambda_{m}\right\}\right\} \\
& \quad+\sum_{m=1}^{N_{c}} \llbracket \mathbf{W}^{T} \overrightarrow{\mathbf{\Phi}}_{m} \Lambda_{m} \rrbracket-\sum_{m=1}^{N_{c}} \llbracket \mathbf{W}^{T} \overrightarrow{\mathbf{\Phi}}_{m} \rrbracket\left\{\left\{\Lambda_{m}\right\}\right\}=0 .
\end{aligned}
$$

Later in this work, we will show how to construct an entropy-stable scheme with dissipation at the inter-element faces.

The physical boundary terms PBT are also extended with non-conservative terms,

$$
\mathrm{PBT}=\sum_{\substack{\text { boundary } \\ \text { faces }}} \int_{f, N}\left(\vec{F}_{e}^{\mathscr{E}}+\mathbf{W}^{T}\left(\overrightarrow{\mathbf{F}}_{e}^{\star}-\overrightarrow{\mathbf{F}}_{e}+\sum_{m=1}^{N_{c}}\left(\left(\overrightarrow{\tilde{\mathbf{\Phi}}}_{m} \Lambda_{m}\right)^{\diamond}-\left(\overrightarrow{\tilde{\mathbf{\Phi}}}_{m} \Lambda_{m}\right)\right)-\overrightarrow{\mathbf{F}}_{v}^{\star}+\overrightarrow{\mathbf{F}}_{v}\right)-\mathbf{W}^{\star, T} \overrightarrow{\mathbf{F}}_{v}\right) \cdot \vec{n} \mathrm{~d} S .
$$

The entropy analysis for non-conservative systems is applied to the entropy-stable multiphase solver studied in Chapter 6. We will first present the general non-conservative system that requires the use of split-forms, and then study the particular case where the standard DG scheme is stable.

The discrete entropy law is (3.99), with IBT in (3.115), and PBT in (3.118). 


\subsubsection{Summary: entropy-stability of non-conservative systems}

We have addressed the entropy-stability of non-conservative systems. First, a continuous analysis has been performed, which shows that the entropy equation is the same as in a conservative system, assuming that the entropy variables contract both inviscid conservative fluxes and non-conservative terms simultaneously. Additionally, we have defined a novel particular case of non-conservative systems which satisfy two properties (Prop. 1 (3.109) and Prop. 2 (3.110)) that allow a simpler entropy analysis.

Second, we have studied the stability of the discrete DG approximation, confirming that it is also stable. Most parts of the analysis (time derivative coefficients and viscous fluxes) are shared with the conservative system, and the difference rests on the simultaneous contraction of inviscid conservative fluxes and non-conservative terms. We distinguished two cases: if the system satisfies Prop. 1 (3.109) and Prop. 2 (3.110), then the standard DG scheme is stable. Otherwise, we need to use a split-form scheme that satisfies an extended version of Tadmor's jump condition (3.106). Both interior and physical boundary terms have been augmented with the non-conservative terms, where the numerical and diamond fluxes have been assumed to provide a stable approximation IBT $\geqslant 0$. A systematic way to get a conserving approximation (IBT $=0$ ) is provided with central fluxes (3.116). We finish this section with a summary of the analyses performed in Table 3.2 . 
Table 3.2: Summary of the entropy analysis performed for the non-conservative system. The stability of the standard DG scheme assumes a non-conservative system compatible with Property 1 (3.109) and Property 2 (3.110)

\section{Weak forms}

\begin{tabular}{|c|c|}
\hline Cont. & $\left\langle\mathbf{q}_{t}, \mathbf{w}\right\rangle+\left\langle\vec{\nabla} \cdot \overrightarrow{\mathbf{f}}_{e}, \mathbf{w}\right\rangle+\sum_{m=1}^{5}\left\langle\overrightarrow{\boldsymbol{\phi}}_{m} \cdot \vec{\nabla} \lambda_{m}, \mathbf{w}\right\rangle-\int_{\partial \Omega} \mathbf{w}^{T} \overrightarrow{\mathbf{f}}_{v} \cdot \mathrm{d} \vec{S}=-\left\langle\overrightarrow{\mathbf{f}}_{v}, \vec{\nabla} \mathbf{w}\right\rangle$ \\
\hline $\begin{array}{l}\text { Disc. } \\
\text { (std) }\end{array}$ & $\left\langle\mathbf{Q}_{t}, \mathbf{W}\right\rangle_{E, N}+\int_{\partial e, N}\left(\mathbf{W}^{T}\left(\overrightarrow{\mathbf{F}}_{e}^{\star}-\overrightarrow{\mathbf{F}}_{e}+\sum_{m=1}^{N_{c}}\left(\left(\overrightarrow{\tilde{\mathbf{\Phi}}}_{m} \Lambda_{m}\right)^{\diamond}-\left(\overrightarrow{\tilde{\mathbf{\Phi}}}_{m} \Lambda_{m}\right)\right)-\overrightarrow{\mathbf{F}}_{v}^{\star}+\overrightarrow{\mathbf{F}}_{v}\right)+\mathbf{W}^{\star, T} \overrightarrow{\mathbf{F}}_{v}\right) \cdot \mathrm{d} \vec{S}$ \\
\hline$s c$ & $\begin{aligned}\left\langle\mathbf{Q}_{t}, \mathbf{W}\right\rangle_{E, N} & +\int_{\partial e, N}\left(\mathbf{W}^{T}\left(\overrightarrow{\mathbf{F}}_{e}^{\star}-\overrightarrow{\mathbf{F}}_{e}+\sum_{m=1}^{N_{c}}\left(\left(\overrightarrow{\tilde{\mathbf{\Phi}}}_{m} \Lambda_{m}\right)^{\diamond}-\left(\overrightarrow{\tilde{\mathbf{\Phi}}}_{m} \Lambda_{m}\right)\right)-\overrightarrow{\mathbf{F}}_{v}^{\star}+\overrightarrow{\mathbf{F}}_{v}\right)+\mathbf{W}^{\star, T} \overrightarrow{\mathbf{F}}_{v}\right) \cdot \mathrm{d} \vec{S} \\
& +\left\langle\mathbb{D}\left(\overrightarrow{\mathbf{F}}_{e}\right)^{\mathrm{EC}}, \mathbf{W}\right\rangle_{E, N}+\sum_{m=1}^{N_{c}}\left\langle\mathbf{W}^{T}, \overrightarrow{\tilde{\mathbf{\Phi}}}_{m} \cdot \mathbb{D}_{\text {grad }}^{\mathrm{DM}} \Lambda_{m}\right\rangle_{E, N}=-\left\langle\mathscr{J} \overrightarrow{\mathbf{G}}, \overrightarrow{\mathbf{F}}_{v}\right\rangle_{E, N}\end{aligned}$ \\
\hline
\end{tabular}

Entropy equation

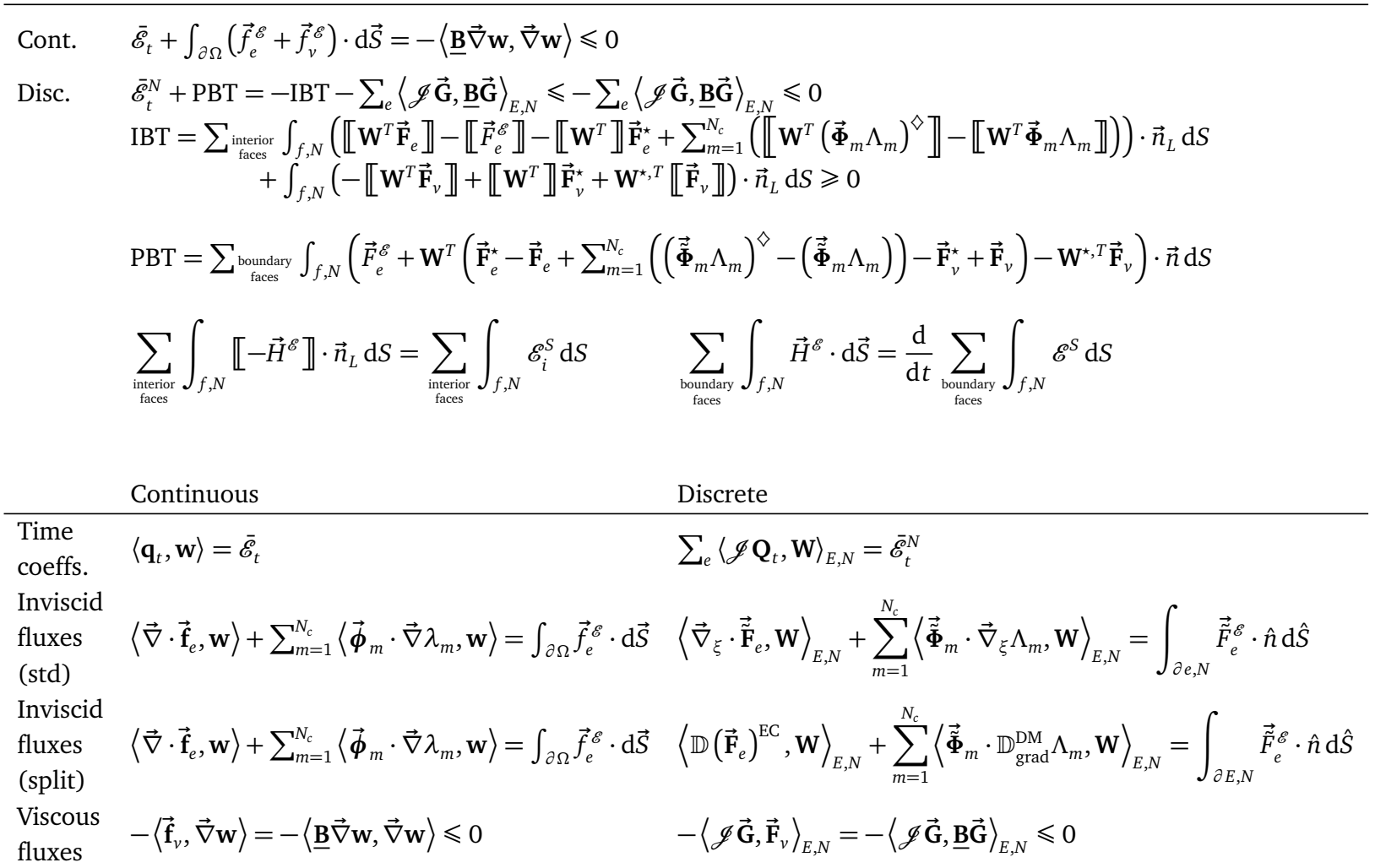




\title{
Part II \\ Development of high-order DG entropy-stable and industrial solvers
}

\begin{abstract}
Summary We construct, study and implement three entropy-stable solvers: one each for the incompressible Navier-Stokes equations with variable density and artificial compressibility, the Cahn-Hilliard equation, and the two-phase incompressible Navier-Stokes/Cahn-Hilliard system. We use the DG scheme and the entropystability proofs for the general conservation law and non-conservative systems presented in Part I. In this part, the novelty is the study (both continuous and discrete) of the entropy-stability of the incompressible Navier-Stokes equations with variable density and artificial compressibility in Chapter 4, and the semi-discrete and fullydiscrete study of the DGSEM-GL discretization of the Cahn-Hilliard equation in Chapter 5. Furthermore, in Chapter 6 we present a new multiphase model that combines the Cahn-Hilliard equation with the advective terms in conservative form, a skewsymmetric version of the momentum equation with a specific form of the capillary pressure, and the artificial compressibility model. The resulting equations are mass and entropy preserving. We also present the corresponding DGSEM-GL scheme, and the semi-discrete stability analysis.
\end{abstract}





\section{Chapter 4}

\section{Entropy-stable approximation of the incompressible Navier-Stokes equations (iNSE) with variable density and artificial compressibility}

\section{Contents}

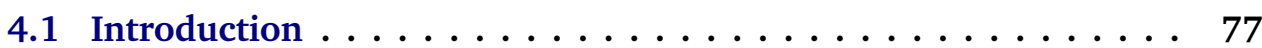

4.2 Governing equations . . . . . . . . . . . . 79

4.3 Entropy analysis $\ldots \ldots \ldots \ldots \ldots \ldots \ldots \ldots$

4.4 Discontinuous Galerkin approximation . . . . . . . . 86

4.5 Stability analysis . . . . . . . . . . . . . . 95

4.6 Numerical experiments . . . . . . . . . . . . . . . . 106

4.7 Summary and conclusions . . . . . . . . . . . . . . 119

\subsection{Introduction}

We provide an entropy-stable approximation to solve the incompressible Navier-Stokes Equations (NSE) with varying density. In the context of this thesis, the method presented herein can be used as a fluid flow engine for interface-tracking multiphase flow models (e.g. VOF Hirt and Nichols [1981]; Nichols et al. [1980], level-set Sussman et al. [1994]; Adalsteinsson and Sethian [1995], or phase-field models Manzanero 
et al. [2020c]; Jacqmin [1999]; Badalassi et al. [2003]). Precisely, we have coupled this model with the Cahn-Hilliard equation to construct two- and three-phase multiphase solvers in Chapter 8.

Among the different incompressible NSE models, we use the artificial compressibility method Shen [1997], which converts the elliptic problem into a hyperbolic system of equations, at the expense of a non divergence-free velocity field. However, it allows one to avoid constructing an approximation that satisfies the inf-sup condition Karniadakis and Sherwin [1999]; Ferrer [2017]; Babuška [1973]; Brezzi [1974]; Ferrer and Willden [2012]; Ferrer et al. [2014]. The artificial compressibility model is commonly combined with dual time-stepping, which solves an inner pseudo-time step loop until velocity divergence errors are lower than a selected threshold, then performs the physical time marching Cox et al. [2016]. In this work, we only address the spatial discretization. However, the method presented herein could be complemented with the pseudo-time step to control divergence errors. Nonetheless, we have found that solving the incompressible NSE with artificial compressibility can achieve satisfactory results even in transient simulations.

We present a nodal Discontinuous Galerkin (DG) Spectral Element Method (DGSEM) for the incompressible Navier-Stokes equations with varying density and artificial compressibility. In particular, this work uses the Gauss-Lobatto version of the DGSEM, which makes it possible to construct entropy stable schemes using the summation-byparts simultaneous-approximation-term (SBP-SAT) property and two-point entropy conserving fluxes. Moreover, it handles arbitrary three dimensional curvilinear hexahedral meshes while maintaining high order, spectral accuracy and entropy stability.

We present a novel entropy analysis for the incompressible NSE with artificial compressibility and variable density, where the traditional kinetic energy is complemented with an artificial compressibility energy that forms the mathematical entropy. The entropy conservation law is then mimicked semi-discretely, i.e. only considering spatial discretization errors. The approximation uses a split-form DG Gassner et al. [2016a, 2018], with the exact Riemann solver Bassi et al. [2017], and the Bassi-Rebay 1 (BR1) Bassi and Rebay [1997] to compute inter-element boundary fluxes. We complete the analysis with a stability study of solid wall boundary conditions. As a result, the numerical scheme is entropy-stable and parameter-free.

The rest of this chapter is organized as follows: In Sec. 4.2 we introduce the incompressible NSE with variable density and artificial compressibility, whose entropy analysis is performed in Sec. 4.3. In Sec. 4.4 we describe the split-form DG scheme, and in Sec. 4.5 we study its entropy-stability. Lastly, we perform numerical experiments in Sec. 4.6. A convergence study using manufactured solutions in Sec. 4.6.1, the solution of the Kovasznay flow problem in Sec. 4.6.2, a lid driven cavity in Sec. 
4.6.3, the inviscid three-dimensional Taylor-Green vortex problem in Sec. 4.6.4, and the Rayleigh-Taylor instability in Sec. 4.6.5.

\subsection{Governing equations}

Given the velocity $\vec{u}(\vec{x}, t)=\left(u_{1}, u_{2}, u_{3}\right)=(u, v, w)$, pressure $p(\vec{x}, t)$, and density $\rho(\vec{x}, t)$ fields, the incompressible Navier-Stokes Equations (NSE) solve the momentum equations,

$$
(\rho \vec{u})_{t}+\nabla \cdot(\rho \vec{u} \vec{u})=-\nabla p+\nabla \cdot\left(\frac{1}{\operatorname{Re}}\left(\nabla \vec{u}^{T}+\nabla \vec{u}\right)\right)+\frac{1}{F^{2}} \rho \vec{e}_{g},
$$

with Re and Fr being the Reynolds and Froude numbers, respectively, and $\vec{e}_{g}$ being a unit vector in the gravity direction.

The artificial compressibility method Shen [1997] introduces an equation for the time evolution of the pressure. In this work, we consider two approaches, the time derivative of the pressure,

$$
p_{t}+\rho_{0} c_{0}^{2} \nabla \cdot \vec{u}=0
$$

where $\rho_{0}$ is a reference density, and $c_{0}$ is the artificial compressibility model sound speed, and the time derivative of the pressure Laplacian,

$$
-\vec{\nabla}^{2} p_{t}+\rho_{0} c_{0}^{2} \vec{\nabla} \cdot \vec{u},\left.\quad \vec{\nabla} p_{t} \cdot \vec{n}\right|_{\partial \Omega}=0 .
$$

Both (4.2) and (4.3) will be studied and their entropy-stability will be addressed. However, we only provide implementation and numerical validation of the first model (4.2).

We consider a transport equation for the density, which we allow to vary spatially,

$$
\rho_{t}+\nabla \cdot(\rho \vec{u})=0
$$

Gathering (4.1)-(4.4), we regard the incompressible NSE with artificial compressibility as a parabolic system,

$$
\mathrm{L}(\mathbf{q})_{t}+\sum_{i=1}^{3} \frac{\partial \mathbf{f}_{e, i}(\mathbf{q})}{\partial x_{i}}=\sum_{i=1}^{3} \frac{\partial \mathbf{f}_{v, i}(\mathbf{q}, \nabla \mathbf{q})}{\partial x_{i}}+\mathbf{s}(\mathbf{q})
$$


with the state vector $\mathbf{q}=(\rho, \rho \vec{u}, p)$, inviscid fluxes $\mathbf{f}_{e, i}(\mathbf{q})$,

$$
\mathbf{f}_{e, 1}=\mathbf{f}_{e}=\left(\begin{array}{c}
\rho u \\
\rho u^{2}+p \\
\rho u v \\
\rho u w \\
\rho_{0} c_{0}^{2} u
\end{array}\right), \mathbf{f}_{e, 2}=\mathbf{g}_{e}=\left(\begin{array}{c}
\rho v \\
\rho u v \\
\rho v^{2}+p \\
\rho v w \\
\rho_{0} c_{0}^{2} v
\end{array}\right), \mathbf{f}_{e, 3}=\mathbf{h}_{e}=\left(\begin{array}{c}
\rho w \\
\rho u w \\
\rho v w \\
\rho w^{2}+p \\
\rho_{0} c_{0}^{2} w
\end{array}\right)
$$

viscous fluxes $\mathbf{f}_{v, i}(\mathbf{q}, \nabla \mathbf{q})$,

$$
\mathbf{f}_{v, 1}=\mathbf{f}_{v}=\left(\begin{array}{c}
0 \\
\tau_{11} \\
\tau_{21} \\
\tau_{31} \\
0
\end{array}\right), \mathbf{f}_{v, 2}=\mathbf{g}_{v}=\left(\begin{array}{c}
0 \\
\tau_{12} \\
\tau_{22} \\
\tau_{32} \\
0
\end{array}\right), \mathbf{f}_{v, 3}=\mathbf{h}_{v}=\left(\begin{array}{c}
0 \\
\tau_{13} \\
\tau_{23} \\
\tau_{33} \\
0
\end{array}\right)
$$

and source term $\mathbf{s}(\mathbf{q})=\left(0, \frac{1}{\mathrm{Fr}^{2}} \rho \vec{e}_{g}, 0\right)$. The operator $\mathrm{L}(\mathbf{q})$ is added to account for both artificial compressibility models,

$$
\mathrm{L}(\mathbf{q})_{\mathrm{AC} 1}=\mathbf{q}, \quad \mathrm{L}(\mathbf{q})_{\mathrm{AC} 2}=\left(\rho, \rho \vec{u},-\vec{\nabla}^{2} p\right) .
$$

In (4.7), we used the viscous tensor $\overline{\bar{\tau}}$

$$
\tau_{i j}=\frac{2}{R e} \overline{\bar{s}}_{i j}
$$

where

$$
\overline{\bar{s}}_{i j}=\frac{1}{2}\left(\frac{\partial u_{i}}{\partial x_{j}}+\frac{\partial u_{j}}{\partial x_{i}}\right)
$$

are the components of the strain tensor, $\overline{\bar{s}}$.

Using the block vector notation described in Sec. 1.7, we write the system in compact form,

$$
\mathrm{L}(\mathbf{q})_{t}+\vec{\nabla} \cdot \overrightarrow{\mathbf{f}}_{e}(\mathbf{q})=\vec{\nabla} \cdot \overrightarrow{\mathbf{f}}_{v}(\mathbf{q}, \vec{\nabla} \mathbf{q})+\mathbf{s}(\mathbf{q})
$$

\subsection{Entropy analysis}

The incompressible Navier-Stokes equations is a conservative non-linear system. Therefore, the entropy analysis presented in this section is a particularization of the general stability analysis presented in Sec. 3.5. As described in Sec. 3.5, the stability analysis 
rests on the existence of a scalar mathematical entropy $\mathscr{E}(\mathbf{q}, \vec{\nabla} \mathbf{q})$ that contracts the time coefficients (3.71), inviscid fluxes (3.72), and so that the viscous fluxes are positive definite (3.74). Then, the mathematical entropy satisfies the evolution equation (3.77), which implies that the entropy remains bounded by the initial and boundary data. Eq. (3.77) assumes the absence of lower order source terms (e.g. gravity) in the original equations.

The incompressible NSE with artificial compressibility (4.11), with inviscid (4.6) and viscous (4.7) fluxes admits the mathematical entropy

$$
\mathscr{E}(\mathbf{q})_{\mathrm{AC} 1}=\frac{1}{2} \rho\left(u^{2}+v^{2}+w^{2}\right)+\frac{1}{2 \rho_{0} c_{0}^{2}} p^{2}=\mathscr{K}+\mathscr{P}_{\mathrm{AC} 1},
$$

for the first artificial compressibility model (4.3), and

$$
\mathscr{E}(\mathbf{q}, \vec{\nabla} \mathbf{q})_{\mathrm{AC} 2}=\frac{1}{2} \rho\left(u^{2}+v^{2}+w^{2}\right)+\frac{1}{2 \rho_{0} c_{0}^{2}}|\vec{\nabla} p|^{2}=\mathscr{K}+\mathscr{P}_{\mathrm{AC} 2}
$$

for the second artificial compressibility model. Both are the sum of the kinetic energy,

$$
\mathscr{K}=\frac{1}{2} \rho\left(u^{2}+v^{2}+w^{2}\right),
$$

and additional energy terms due to artificial compressibility effects,

$$
\mathscr{P}_{\mathrm{AC} 1}(\mathbf{q})=\frac{1}{2 \rho_{0} c_{0}^{2}} p^{2}, \quad \mathscr{P}_{\mathrm{AC} 2}(\mathbf{q}, \vec{\nabla} \mathbf{q})=\frac{1}{2 \rho_{0} c_{0}^{2}}|\vec{\nabla} p|^{2}
$$

Note that the artificial compressibility effects vanish for very large values of $c_{0}$ (the incompressible limit). In general, when we refer to the entropy $\mathscr{E}$ without specifying an artificial compressibility model, means that the statement is valid for both.

The entropy for the incompressible NSE with the first artificial compressibility model is independent of the state vector gradient $\vec{\nabla} \mathbf{q}$. Therefore, the entropy variables can be computed directly from the entropy using the chain rule (3.75), and the surface entropy is $\mathscr{E}_{\mathrm{AC} 1}^{S}=0$. The entropy variables for the entropy (4.12) are

$$
\mathbf{w}=\frac{\partial \mathscr{E}}{\partial \mathbf{q}}=\left(-\frac{1}{2} v_{t o t}^{2}, u, v, w, \frac{1}{\rho_{0} c_{0}^{2}} p\right),
$$

where $v_{\text {tot }}=\left(u^{2}+v^{2}+w^{2}\right)^{1 / 2}$ is the total velocity. For the second artificial compressibility model, we will show that the same entropy variables are suitable to contract the various terms to get an entropy equation, and we will get its surface entropy $\mathscr{E}_{\mathrm{AC} 2}$.

We now show that the entropy (4.12) satisfies the positivity and convexity properties. By construction, we see that the entropy (4.12) is positive if the density remains positive, i.e.

$$
\mathscr{E}(\mathbf{q})_{\mathrm{AC} 1} \geqslant 0 \text { if } \rho(\vec{x} ; t) \geqslant 0
$$


Furthermore, the Hessian matrix of the entropy with respect to the state vector is

$$
\frac{\partial^{2} \mathscr{E}_{\mathrm{AC} 1}}{\partial \mathbf{q}^{2}}=\frac{\partial \mathbf{w}}{\partial \mathbf{q}}=\left(\begin{array}{ccccc}
v_{t o t}^{2} / \rho & -u / \rho & -v / \rho & -w / \rho & 0 \\
-u / \rho & 1 / \rho & 0 & 0 & 0 \\
-v / \rho & 0 & 1 / \rho & 0 & 0 \\
-w / \rho & 0 & 0 & 1 / \rho & 0 \\
0 & 0 & 0 & 0 & \frac{1}{\rho_{0} c_{0}^{2}}
\end{array}\right)
$$

which is also positive semi-definite if the density $\rho(\vec{x} ; t)$ remains positive, since its eigenvalues are $\lambda_{1,2}=\frac{1}{\rho}, \lambda_{3}=\frac{1+v_{\text {tot }}^{2}}{\rho}, \lambda_{4}=\frac{1}{\rho_{0} c_{0}^{2}}$, and $\lambda_{5}=0$. Since the entropy of the second artificial compressibility model shares the kinetic energy and the differing artificial compressibility energy is also positive, we confirm that is also a valid entropy. The density positivity condition is usually satisfied automatically for smooth initial conditions. Otherwise, in Sec. 4.6.5 we describe a conservative density clipping method used in the solution of the Rayleigh-Taylor instability.

Next, we evaluate the three properties for the entropy-stabilty of the conservative incompressible NSE system: contraction of time coefficients (3.71), contraction of inviscid fluxes (3.72), and positive definiteness of viscous fluxes (3.74). Only the contraction of the time coefficients depends on the artificial compressibility model, while the proof for the other two properties is shared between both models.

\subsubsection{Contraction of the time coefficients}

\section{First artificial compressibility model}

We next show that the entropy (4.12) satisfies the time coefficients contraction property (3.71). Although this property is satisfied automatically with the partial derivative definition (4.16) (see (3.71)), it is confirmed here by computing the product of the state vector time derivative with the entropy variables,

$$
\begin{aligned}
\mathbf{w}^{T} \mathbf{q}_{t}= & -\frac{1}{2}\left(u^{2}+v^{2}+w^{2}\right) \rho_{t}+u(\rho u)_{t}+v(\rho v)_{t}+w(\rho w)_{t}+\frac{1}{\rho_{0} c_{0}^{2}} p(p)_{t} \\
= & \left(-\frac{1}{2} u^{2} \rho_{t}+u(\rho u)_{t}\right)+\left(-\frac{1}{2} v^{2} \rho_{t}+v(\rho v)_{t}\right) \\
& +\left(-\frac{1}{2} w^{2} \rho_{t}+w(\rho w)_{t}\right)+\left(\frac{1}{2 \rho_{0} c_{0}^{2}} p^{2}\right)_{t} .
\end{aligned}
$$


To get the time derivative of the kinetic energy, we manipulate each of the three velocity components $u_{j}$ contributions as

$$
\begin{aligned}
-\frac{1}{2} u_{j}^{2} \rho_{t}+u_{j}\left(\rho u_{j}\right)_{t} & =-\frac{1}{2} u_{j}^{2} \rho_{t}+\frac{1}{2} u_{j}\left(\rho u_{j}\right)_{t}+\frac{1}{2} u_{j}\left(\rho u_{j}\right)_{t} \\
& =-\frac{1}{2} u_{j}^{2} \rho_{t}+\frac{1}{2} \rho_{t} u_{j}^{2}+\frac{1}{2} \rho u_{j} u_{j, t}+\left(\frac{1}{2} \rho u_{j}^{2}\right)_{t}-\frac{1}{2} \rho u_{j} u_{j, t} \\
& =\left(\frac{1}{2} \rho u_{j}^{2}\right)_{t} .
\end{aligned}
$$

Hence, we have constructed the particular version of the time coefficients contraction (3.71) for the incompressible NSE with the first artificial compressibility model,

$$
\left\langle\mathbf{q}_{t}, \mathbf{w}\right\rangle=\left\langle\left(\frac{1}{2} \rho\left(u^{2}+v^{2}+w^{2}\right)+\frac{1}{2 \rho_{0} c_{0}^{2}} p^{2}\right)_{t}\right\rangle=\left\langle\mathscr{E}_{\mathrm{AC} 1, t}\right\rangle=\overline{\mathscr{E}}_{\mathrm{AC} 1, t},
$$

with the surface entropy $\mathscr{E}_{\mathrm{AC} 1}^{S}=0$.

\section{Second artificial compressibility model}

We compute the time coefficients for the second artificial compressibility model, where only the terms associated to the pressure change,

$$
\begin{aligned}
\left\langle\mathrm{L}_{\mathrm{AC} 2}(\mathbf{q})_{t}, \mathbf{w}\right\rangle & =\left\langle-\frac{1}{2} v_{t o t}, \rho_{t}\right\rangle+\left\langle u,(\rho u)_{t}\right\rangle+\left\langle v,(\rho v)_{t}\right\rangle+\left\langle w,(\rho w)_{t}\right\rangle-\frac{1}{\rho_{0} c_{0}^{2}}\left\langle p, \vec{\nabla}^{2} p_{t}\right\rangle \\
& =\left\langle\mathscr{K}_{t}\right\rangle-\int_{\partial \Omega} \frac{1}{\rho_{0} c_{0}^{2}} p \vec{\nabla} p_{t} \cdot \mathrm{d} \vec{S}+\left\langle\frac{1}{\rho_{0} c_{0}^{2}} \vec{\nabla} p, \vec{\nabla} p_{t}\right\rangle \\
& =\frac{\mathrm{d}}{\mathrm{d} t}\left\langle\mathscr{K}+\frac{1}{2 \rho_{0} c_{0}^{2}}|\vec{\nabla} p|^{2}\right\rangle=\frac{\mathrm{d}}{\mathrm{d} t}\left\langle\mathscr{K}+\mathscr{P}_{\mathrm{AC} 2}\right\rangle=\overline{\mathscr{E}}_{\mathrm{AC} 2, \mathrm{t}} .
\end{aligned}
$$

In the second identity we used the Gauss law, in the third identity we removed the surface integral, as it is cancelled by the physical boundary condition (4.3), and finally we used the chain rule in time. Thus, we derive the time coefficient contraction (4.12) of the second artificial compressibility model, in which we get zero surface entropy $\mathscr{E}_{\mathrm{AC} 2}^{S}=0$.

\section{Summary}

The contraction of the time coefficients leads to the same result for the two artificial compressibility models, which we can simply write as

$$
\left\langle\mathrm{L}(\mathbf{q})_{t}, \mathbf{w}\right\rangle=\overline{\mathscr{E}}_{t} .
$$




\subsubsection{Contraction of the inviscid fluxes}

The contraction of the inviscid fluxes rests on the existence of an entropy flux, $\vec{f}_{e}^{\mathscr{E}}$, that satisfies (3.72),

$$
\left\langle\vec{\nabla} \cdot \overrightarrow{\mathbf{f}}_{e}, \mathbf{w}\right\rangle=\left\langle\vec{\nabla} \cdot \vec{f}^{\mathscr{E}}\right\rangle=\int_{\partial \Omega} \vec{f}_{e}^{\mathscr{E}} \cdot \mathrm{d} \vec{S}
$$

We write the left hand side of (4.24) for the incompressible NSE,

$$
\left\langle\vec{\nabla} \cdot \overrightarrow{\mathbf{f}}_{e}, \mathbf{w}\right\rangle=\left\langle\vec{\nabla} \cdot(\rho \vec{u}),-\frac{1}{2} v_{t o t}^{2}\right\rangle+\langle\vec{\nabla} \cdot(\rho \vec{u} \vec{u}), \vec{u}\rangle+\langle\vec{\nabla} p, \vec{u}\rangle+\left\langle\rho_{0} c_{0}^{2} \vec{\nabla} \cdot \vec{u}, \frac{1}{\rho_{0} c_{0}^{2}} p\right\rangle .
$$

We use the product rule in the momentum (which will not hold discretely, so we will need to use split-forms) for the half of the second sum of the right hand side of (4.25),

$$
\vec{\nabla} \cdot(\rho \vec{u} \vec{u})=\frac{1}{2} \vec{u} \vec{\nabla} \cdot(\rho \vec{u})+\frac{1}{2} \rho \vec{u} \vec{\nabla} \vec{u}+\frac{1}{2} \vec{\nabla} \cdot(\rho \vec{u} \vec{u}),
$$

so that (4.25) is

$$
\begin{aligned}
\left\langle\vec{\nabla} \cdot \overrightarrow{\mathbf{f}}_{e}, \mathbf{w}\right\rangle= & -\frac{1}{2}\left\langle\vec{\nabla} \cdot(\rho \vec{u}), v_{\text {tot }}^{2}\right\rangle+\frac{1}{2}\langle\vec{u} \vec{\nabla} \cdot(\rho \vec{u}), \vec{u}\rangle+\frac{1}{2}\langle\rho \vec{u} \vec{\nabla} \vec{u}, \vec{u}\rangle \\
& +\frac{1}{2}\langle\vec{\nabla} \cdot(\rho \vec{u} \vec{u}), \vec{u}\rangle+\langle\vec{\nabla} p, \vec{u}\rangle+\langle\vec{\nabla} \cdot \vec{u}, p\rangle \\
= & -\frac{1}{2}\langle\vec{\nabla} \cdot(\rho \vec{u}), \vec{u} \cdot \vec{u}\rangle+\frac{1}{2}\langle\vec{\nabla} \cdot(\rho \vec{u}), \vec{u} \cdot \vec{u}\rangle+\frac{1}{2}\langle\vec{\nabla} \vec{u}, \rho \vec{u} \vec{u}\rangle \\
& +\frac{1}{2}\langle\vec{\nabla} \cdot(\rho \vec{u} \vec{u}), \vec{u}\rangle+\langle\vec{\nabla} p, \vec{u}\rangle+\langle\vec{\nabla} \cdot \vec{u}, p\rangle \\
= & \left\langle\vec{\nabla} \cdot\left(\frac{1}{2} \rho \vec{u} \vec{u} \cdot \vec{u}\right)\right\rangle+\langle\vec{\nabla} \cdot(p \vec{u})\rangle=\int_{\partial \Omega}\left(\frac{1}{2} \rho \vec{u} \cdot \vec{u}+p\right) \vec{u} \cdot \mathrm{d} \vec{S}
\end{aligned}
$$

We therefore get the entropy flux,

$$
\vec{f}_{e}^{\mathscr{E}}=\left(\frac{1}{2} \rho \vec{u} \cdot \vec{u}+p\right) \vec{u}=\left(\frac{1}{2} \rho\left(u^{2}+v^{2}+w^{2}\right)+p\right) \vec{u} .
$$

In the third equality of (4.27), we have used the Gauss law for third/fourth, and fifth/sixth integrals, while the first two terms cancel each other.

We note that despite the entropy (4.12) is particular to the incompressible NavierStokes equations with artificial compressibility, the entropy flux is shared with the incompressible NSE without artificial compressibility.

\subsubsection{Positive semi-definiteness of the viscous fluxes}

Finally, we show that viscous fluxes and entropy variables satisfy (3.74). To do so, we re-write the viscous fluxes as a function of the entropy vector $\mathbf{w}$ (instead of the state 
vector $\mathbf{q}$ ), which in general (for both compressible and incompressible formulations) can be linearly spanned in the gradient of the entropy variables,

$$
\mathbf{f}_{v, i}(\mathbf{q}, \vec{\nabla} \mathbf{w})=\sum_{j=1}^{3} \underline{B}_{i j}(\mathbf{w}) \frac{\partial \mathbf{w}}{\partial x_{j}},
$$

or, using the block matrix representation introduced in (1.22),

$$
\overrightarrow{\mathbf{f}}_{v}(\mathbf{q}, \vec{\nabla} \mathbf{w})=\underline{\mathbf{B}}(\mathbf{q}) \vec{\nabla} \mathbf{w}
$$

In general, the coefficients $\underline{B}_{i j}(\mathbf{q})$ are non-linear and depend on the state vector. In particular to the incompressible NSE, the matrices $\underline{B}_{i j}$ are constant (i.e. they do not depend on $\mathbf{w}$ ),

$$
\begin{aligned}
& \underline{B}_{11}=\frac{1}{\operatorname{Re}}\left(\begin{array}{ccccc}
0 & 0 & 0 & 0 & 0 \\
0 & 2 & 0 & 0 & 0 \\
0 & 0 & 1 & 0 & 0 \\
0 & 0 & 0 & 1 & 0 \\
0 & 0 & 0 & 0 & 0
\end{array}\right), \underline{B}_{12}=\frac{1}{\operatorname{Re}}\left(\begin{array}{ccccc}
0 & 0 & 0 & 0 & 0 \\
0 & 0 & 0 & 0 & 0 \\
0 & 1 & 0 & 0 & 0 \\
0 & 0 & 0 & 0 & 0 \\
0 & 0 & 0 & 0 & 0
\end{array}\right), \underline{B}_{13}=\frac{1}{\operatorname{Re}}\left(\begin{array}{ccccc}
0 & 0 & 0 & 0 & 0 \\
0 & 0 & 0 & 0 & 0 \\
0 & 0 & 0 & 0 & 0 \\
0 & 1 & 0 & 0 & 0 \\
0 & 0 & 0 & 0 & 0
\end{array}\right) \text {, } \\
& \underline{B}_{21}=\frac{1}{\operatorname{Re}}\left(\begin{array}{ccccc}
0 & 0 & 0 & 0 & 0 \\
0 & 0 & 1 & 0 & 0 \\
0 & 0 & 0 & 0 & 0 \\
0 & 0 & 0 & 0 & 0 \\
0 & 0 & 0 & 0 & 0
\end{array}\right), \underline{B}_{22}=\frac{1}{\operatorname{Re}}\left(\begin{array}{ccccc}
0 & 0 & 0 & 0 & 0 \\
0 & 1 & 0 & 0 & 0 \\
0 & 0 & 2 & 0 & 0 \\
0 & 0 & 0 & 1 & 0 \\
0 & 0 & 0 & 0 & 0
\end{array}\right), \underline{B}_{23}=\frac{1}{\operatorname{Re}}\left(\begin{array}{ccccc}
0 & 0 & 0 & 0 & 0 \\
0 & 0 & 0 & 0 & 0 \\
0 & 0 & 0 & 0 & 0 \\
0 & 0 & 1 & 0 & 0 \\
0 & 0 & 0 & 0 & 0
\end{array}\right),
\end{aligned}
$$

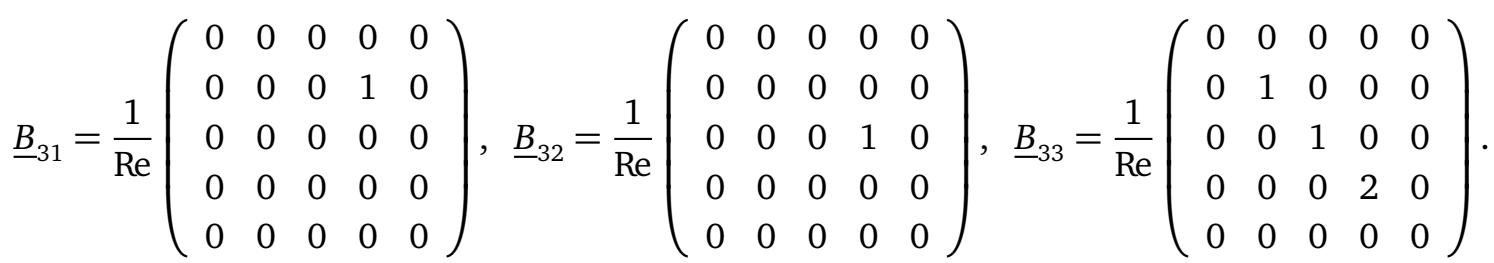

As described in Sec. 3.5, the viscous flux matrix $\underline{\mathbf{B}}$ has to be a symmetric positive definite matrix, (3.66). For the matrices given in (4.31), the symmetry is immediate, and the positive definiteness is

$$
\begin{aligned}
\sum_{i, j=1}^{3}\left(\frac{\partial \mathbf{w}}{\partial x_{i}}\right)^{T} \underline{B}_{i j}\left(\frac{\partial \mathbf{w}}{\partial x_{j}}\right)= & \frac{1}{\operatorname{Re}}\left(2 u_{x}^{2}+v_{x}^{2}+w_{x}^{2}+u_{y}^{2}+2 v_{y}^{2}+w_{y}^{2}\right. \\
& \left.\quad+u_{z}^{2}+v_{z}^{2}+2 w_{z}^{2}+2 u_{y} v_{x}+2 u_{z} w_{x}+2 v_{z} w_{y}\right) \\
= & \frac{1}{\operatorname{Re}}\left(2 u_{x}^{2}+2 v_{y}^{2}+2 w_{z}^{2}+\left(u_{y}+v_{x}\right)^{2}+\left(u_{z}+w_{x}\right)^{2}+\left(v_{z}+w_{y}\right)^{2}\right) \geqslant 0,
\end{aligned}
$$


thus proving (3.66). The last identity (4.32) is commonly represented as

$$
\left\langle\overrightarrow{\mathbf{f}}_{v}, \vec{\nabla} \mathbf{w}\right\rangle=\frac{2}{\operatorname{Re}}\langle\overline{\bar{s}}, \overline{\bar{s}}\rangle=\frac{2}{\operatorname{Re}}\left\langle|\overline{\bar{s}}|^{2}\right\rangle \geqslant 0,
$$

where $\overline{\bar{s}}$ is the strain tensor (4.10). The viscous entropy flux $\vec{f}_{v}^{\mathscr{E}}(3.74)$ is

$$
\vec{f}_{v}^{\mathscr{E}}=\mathbf{w}^{T} \overrightarrow{\mathbf{f}}_{v}=-\overline{\bar{\tau}} \cdot \vec{u} .
$$

Hence, we can write the entropy equation (3.77) in its particular form for the incompressible NSE with artificial compressibility (ignoring lower order terms), as

$$
\left\langle\left(\frac{1}{2} \rho v_{\text {tot }}^{2}+\frac{1}{2 \rho_{0} c_{0}^{2}} p^{2}\right)_{t}\right\rangle+\int_{\partial \Omega}\left(\left(\frac{1}{2} \rho v_{\text {tot }}^{2}+p\right) \vec{u}-\vec{u} \cdot \overline{\bar{\tau}}\right) \cdot \mathrm{d} \vec{S}=-\frac{2}{\operatorname{Re}}\left\langle|\overline{\bar{S}}|^{2}\right\rangle \leqslant 0 .
$$

To the best of our knowledge, this (here and in its associated journal publication Manzanero et al. [2020a]) is the first time that this entropy analysis has been performed for this set of equations.

The boundary integral is studied here for free- and no-slip wall boundary conditions. In this continuous analysis, neither contributes to the entropy, since in free-slip walls we set $\vec{u} \cdot \vec{n}=0$ and $\overline{\bar{\tau}} \cdot \vec{n}=0$, while in no-slip walls is $\vec{u}=0$. Therefore,

$$
\frac{\mathrm{d}}{\mathrm{d} t} \int_{\Omega}\left(\frac{1}{2} \rho v_{\text {tot }}^{2}+\frac{1}{2 \rho_{0} c_{0}^{2}} p^{2}\right) \mathrm{d} \vec{x}=-\int_{\Omega} \frac{2}{\operatorname{Re}}|\bar{s}|^{2} \mathrm{~d} \vec{x} \leqslant 0,
$$

so that the entropy is conserved when the flow is inviscid, and dissipated otherwise.

\subsection{Discontinuous Galerkin approximation}

In this section, we construct an entropy-stable DG scheme that satisfies a semi-discrete version of the bound (4.36) using split-forms and the SBP-SAT property.

The construction of the scheme follows the steps described in Sec. (2.5). To transform the incompressible NSE (4.11) into reference space, it is first written as a first order system, defining the auxiliary variable $\overrightarrow{\mathbf{g}}=\vec{\nabla} \mathbf{w}$ so that

$$
\begin{aligned}
\mathrm{L}(\mathbf{q})_{t}+\vec{\nabla} \cdot \overrightarrow{\mathbf{f}}_{e}(\mathbf{q}) & =\vec{\nabla} \cdot \overrightarrow{\mathbf{f}}_{v}(\overrightarrow{\mathbf{g}})+\mathbf{s}(\mathbf{q}), \\
\overrightarrow{\mathbf{g}} & =\vec{\nabla} \mathbf{w} .
\end{aligned}
$$

Recall that the incompressible NSE viscous fluxes depend only on the gradient of the entropy variables, $\overrightarrow{\mathbf{g}}$. We consider the split-form DG approximation derived in (2.76), 


$$
\begin{aligned}
\left\langle\mathscr{J} \mathrm{L}(\mathbf{Q})_{t}, \boldsymbol{\phi}\right\rangle_{E, N} & +\int_{\partial e, N} \boldsymbol{\phi}^{T}\left(\overrightarrow{\mathbf{F}}_{e}^{\star}-\overrightarrow{\mathbf{F}}_{e}\right) \cdot \vec{n} \mathrm{~d} S+\left\langle\mathbb{D}\left(\overrightarrow{\widetilde{\mathbf{F}}}_{e}\right)^{e c}, \boldsymbol{\phi}\right\rangle_{E, N} \\
& =\int_{\partial e, N} \boldsymbol{\phi}^{T}\left(\overrightarrow{\mathbf{F}}_{v}^{\star}-\overrightarrow{\mathbf{F}}_{v}\right) \cdot \vec{n} \mathrm{~d} S+\left\langle\vec{\nabla}_{\xi} \cdot \overrightarrow{\tilde{\mathbf{F}}}_{v}, \boldsymbol{\phi}\right\rangle_{E, N}+\langle\mathscr{J} \mathbf{S}, \boldsymbol{\phi}\rangle_{E, N}, \\
\langle\mathscr{J} \overrightarrow{\mathbf{G}}, \vec{\varphi}\rangle_{E, N} & =\int_{\partial e, N} \mathbf{W}^{\star, T}(\vec{\varphi} \cdot \vec{n}) \mathrm{d} S-\left\langle\mathbf{W}, \vec{\nabla}_{\xi} \cdot \overrightarrow{\tilde{\varphi}}\right\rangle_{E, N} .
\end{aligned}
$$

For the first artificial compressibility model the time coefficients operator is $\mathrm{L}(\mathbf{Q})=\mathbf{Q}$, and the approximation of the time coefficients is done as usual. For the second artificial compressibility model, we will provide the specific details in Sec. 4.4.1.

Two-point fluxes are also defined in terms of averages of the two states. We construct the two-point entropy conserving version of the fluxes, $\overrightarrow{\mathbf{F}}_{e}^{e c}$, for the incompressible NSE using the general formula

$$
\mathbf{F}_{e}^{e c}=\left(\begin{array}{c}
\tilde{\rho u} \\
\tilde{\rho u}\{\{u\}\}+\{\{p\}\} \\
\tilde{\rho u}\{\{v\}\} \\
\tilde{\rho u}\{\{w\}\} \\
\rho_{0} c_{0}^{2}\{\{u\}\}
\end{array}\right), \mathbf{G}_{e}^{e c}=\left(\begin{array}{c}
\tilde{\rho v} \\
\tilde{\rho v}\{\{u\}\} \\
\tilde{\rho v}\{\{v\}\}+\{\{p\}\} \\
\tilde{\rho v}\{\{w\}\} \\
\rho_{0} c_{0}^{2}\{\{v\}\}
\end{array}\right), \mathbf{H}_{e}^{e c}=\left(\begin{array}{c}
\tilde{\rho w} \\
\tilde{\rho w}\{\{u\}\} \\
\tilde{\rho w}\{\{v\}\} \\
\tilde{\rho w}\{\{w\}\}+\{\{p\}\} \\
\rho_{0} c_{0}^{2}\{\{w\}\}
\end{array}\right) .
$$

We consider two options for the approximation of the momentum $\rho \tilde{u}_{i}, i=1,2,3$. In the first choice, we take the average of the product, while in the second we take the product of the averages,

$$
\rho \tilde{u}_{i}^{(1)}=\left\{\left\{\rho u_{i}\right\}\right\} \text {, or } \rho \tilde{u}_{i}^{(2)}=\{\{\rho\}\}\left\{\left\{u_{i}\right\}\right\} .
$$

Finally, we note that the two-point flux form of the equation is algebraically equivalent to the approximation of a split-form of the original equations that averages conservative and non-conservative forms of the original PDEs, with an extra splitting on the metric terms. This has been studied in Sec. 4.4.4.

The approximation is completed with the definition of the numerical fluxes $\overrightarrow{\mathbf{F}}_{e}^{\star}, \overrightarrow{\mathbf{F}}_{v}^{\star}$, and $\mathbf{W}^{\star}$, which are described in Sec. 4.4.2, and the physical boundary conditions in Sec. 4.4.3. Finally, (4.38) is integrated in time using a third order low-storage explicit Runge-Kutta RK3 scheme Williamson [1980].

\subsubsection{Second artificial compressibility discrete time coefficients}

For the first artificial compressibility model, the time coefficients are simply $\left\langle\mathscr{J} \mathbf{Q}_{t}, \boldsymbol{\phi}\right\rangle_{E, N}$. 
For the second artificial compressibility model, the first four equations, which correspond to $(\rho, \rho \vec{u})_{t}$, we proceed as usual (treating time coefficients as source terms). The pressure approximation, however, needs special treatment for stability. We take the auxiliary variable $\vec{G}_{p} \approx \vec{\nabla} p$ from the gradient vector $\overrightarrow{\mathbf{G}}$, so that the approximation of the time derivative pressure Laplacian follows that of a viscous (second order derivatives) flux,

$$
-\left\langle J \vec{\nabla}^{2} p_{t}, \phi\right\rangle_{E} \approx\left\langle\overrightarrow{\tilde{G}}_{p, t}, \vec{\nabla}_{\xi} \phi\right\rangle_{E, N}-\int_{\partial E, N} \phi \overrightarrow{\tilde{G}}_{p, t}^{\star} \cdot \hat{n} \mathrm{~d} \hat{S} .
$$

The numerical flux $\vec{G}_{p, t}^{\star}$ will be defined in Sec. 4.4.2 and in Sec. 4.4.3.

\subsubsection{Numerical fluxes}

The approximation (4.38) is completed with the numerical fluxes $\mathbf{F}_{e}^{\star}, \mathbf{F}_{v}^{\star}$, and $\mathbf{W}^{\star}$, and the boundary conditions, the first of which we describe in this section.

For the inviscid fluxes we use the exact Riemann solver derived in Bassi et al. [2017]. Using the rotational invariance property of the flux Toro [2013], we write the normal flux as

$$
\overrightarrow{\mathbf{F}}_{e} \cdot \vec{n}=\underline{\mathrm{T}}^{T} \mathbf{F}_{e}(\underline{\mathrm{T}} \mathbf{Q})=\underline{\mathrm{T}}^{T} \mathbf{F}_{e}\left(\mathbf{Q}_{n}\right), \quad \underline{\mathrm{T}}=\left(\begin{array}{ccccc}
1 & 0 & 0 & 0 & 0 \\
0 & n_{x} & n_{y} & n_{z} & 0 \\
0 & t_{1, x} & t_{1, y} & t_{1, z} & 0 \\
0 & t_{2, x} & t_{2, y} & t_{2, z} & 0 \\
0 & 0 & 0 & 0 & 1
\end{array}\right),
$$

where $\underline{\mathrm{T}}$ is a rotation matrix that only affects velocities, $\vec{n}=\left(n_{x}, n_{y}, n_{z}\right)$ is the normal unit vector to the face, and $\vec{t}_{1}$ and $\vec{t}_{2}$ are two tangent unit vectors to the face. Recall that $\mathbf{F}_{e}$ is the $x$-component of the inviscid flux $\overrightarrow{\mathbf{F}}_{e}=\left(\mathbf{F}_{e}, \mathbf{G}_{e}, \mathbf{H}_{e}\right)$ (4.6). When the rotation matrix T multiplies the state vector $\mathbf{Q}$, we get the rotated state vector $\mathbf{Q}_{n}$,

$$
\mathbf{Q}_{n}=\underline{\mathrm{T}} \mathbf{Q}=\left(\rho, \rho U_{n}, \rho V_{t 1}, \rho V_{t 2}, P\right),
$$

where $U_{n}=\vec{U} \cdot \vec{n}$ is the normal velocity, and $V_{t i}=\vec{U} \cdot \vec{t}_{i}(i=1,2)$ are the two tangent velocities. Taken in context, $\rho$ refers to the polynomial approximation of the density. Note that the reference system rotation does not affect the total speed

$$
V_{t o t}^{2}=U^{2}+V^{2}+W^{2}=U_{n}^{2}+V_{t 1}^{2}+V_{t 2}^{2}
$$

The rotational invariance allows us to transform the 3D Riemann problem into a 
one dimensional problem for the rotated state vector,

$$
\frac{\partial \mathbf{Q}_{n}}{\partial t}+\frac{\partial \mathbf{F}_{e}\left(\mathbf{Q}_{n}\right)}{\partial x}=0, \quad \mathbf{Q}_{n}(x, 0)= \begin{cases}\left(\rho_{L}, \rho_{L} U_{n L}, \rho_{L} V_{t 1 L}, \rho_{L} V_{t 2 L}, P_{L}\right) & \text { if } \quad x \leqslant 0, \\ \left(\rho_{R}, \rho_{R} U_{n R}, \rho_{R} V_{t 1 R}, \rho_{R} V_{t 2 R}, P_{R}\right) & \text { if } \quad x>0,\end{cases}
$$

whose exact solution is (the details can be found in Bassi et al. [2017]),

$$
\mathbf{Q}_{n}^{\star}=\left(\begin{array}{c}
\rho^{\star} \\
\rho^{\star} U_{n}^{\star} \\
\rho^{\star} V_{t 1}^{\star} \\
\rho^{\star} V_{t 2}^{\star} \\
P^{\star}
\end{array}\right), \quad \mathbf{F}_{e}\left(\mathbf{Q}_{n}^{\star}\right)=\left(\begin{array}{c}
\rho^{\star} U_{n}^{\star} \\
\rho^{\star}\left(U_{n}^{\star}\right)^{2}+P^{\star} \\
\rho^{\star} U_{n}^{\star} V_{t 1}^{\star} \\
\rho^{\star} U_{n}^{\star} V_{t 2}^{\star} \\
\rho_{0} c_{0}^{2} U_{n}^{\star}
\end{array}\right),
$$

The star region solution is

$$
\begin{aligned}
& U_{n}^{\star}=\frac{P_{L}-P_{R}+\rho_{L} U_{n L} \lambda_{L}^{+}-\rho_{R} U_{n R} \lambda_{R}^{-}}{\rho_{L} \lambda_{L}^{+}-\rho_{R} \lambda_{R}^{-}}, P^{\star}=P_{L}+\rho_{L} \lambda_{L}^{+}\left(U_{n L}-U_{n}^{\star}\right), \\
& \rho^{\star}=\left\{\begin{array}{lll}
\rho_{L}^{\star} & \text { if } \quad U_{n}^{\star} \geqslant 0 \\
\rho_{R}^{\star} & \text { if } \quad U_{n}^{\star}<0
\end{array}, \quad \rho_{L}^{\star}=\frac{\rho_{L} \lambda_{L}^{+}}{U_{n}^{\star}-\lambda_{L}^{-}}, \quad \rho_{R}^{\star}=\frac{\rho_{R} \lambda_{R}^{-}}{U_{n}^{\star}-\lambda_{R}^{+}}, \quad V_{t i}^{\star}=\left\{\begin{array}{ccc}
V_{t i L} & \text { if } & U_{n}^{\star} \geqslant 0 \\
V_{t i R} & \text { if } & U_{n}^{\star}<0
\end{array}\right. \text { ， }\right.
\end{aligned}
$$

and the eigenvalues are

$$
\lambda_{L}^{ \pm}=\frac{U_{n L} \pm a_{L}}{2}, \lambda_{R}^{ \pm}=\frac{U_{n R} \pm a_{R}}{2}, a=\sqrt{U_{n}^{2}+\frac{4 \rho_{0} c_{0}^{2}}{\rho}} .
$$

Note that in this model, eigenvalues with the positive superscript, $\lambda_{L R}^{+}$, are always positive, and eigenvalues with negative superscript, $\lambda_{L R}^{-}$, are always negative (i.e. the flow is always subsonic). Following (4.42), we multiply $\mathbf{F}_{e}\left(\mathbf{Q}_{n}^{\star}\right)$ in (4.46) by the transposed rotation matrix $\underline{\mathrm{T}}^{T}$ to get the numerical flux $\overrightarrow{\mathrm{F}}_{e}^{\star} \cdot \vec{n}$.

For viscous fluxes we use the Bassi-Rebay 1 (BR1) scheme, which takes the average between adjacent elements for both entropy variables and fluxes,

$$
\mathbf{W}^{\star}=\left\{\{\mathbf{W}\}, \quad \overrightarrow{\mathbf{F}}_{v}^{\star}=\left\{\left\{\overrightarrow{\mathbf{F}}_{v}\right\}\right\} .\right.
$$

Finally, for the second artificial compressibility model, we use the BR1 scheme with interface stabilization,

$$
\vec{G}_{p}^{\star}=\left\{\left\{\vec{G}_{p}\right\}\right\}-\beta_{p}\left(P_{L} \vec{n}_{L}+P_{R} \vec{n}_{R}\right),
$$

where $\beta_{p}$ is a non-negative constant. Since we do not present any numerical experiment on this second artificial compressibility model, we cannot provide a precise 
estimate on $\beta_{p}$, but one can use the estimate presented in Manzanero et al. [2018b], which has been used for other similar discretizations in this work. For stability, however, it suffices that the constant is non-negative.

\subsubsection{Boundary conditions}

The full approximation is completed with the addition of boundary conditions. Here, we show how to impose free- and no-slip wall boundary conditions.

\section{Inviscid flux}

The inviscid flux is responsible for enforcing the normal velocity $u_{n}=0$, and does not control tangential velocities nor viscous stresses. Thus, the procedure for both freeand no-slip wall boundary conditions is identical.

We consider two ways to enforce the wall boundary condition through the numerical flux $\overrightarrow{\mathbf{F}}_{e}^{\star}$. The first one, directly computes the wall numerical flux. The second one, sets an artificial reflection state to be used as the external state for the exact Riemann solver.

If we do not want to use the exact Riemann solver at the boundaries, we prescribe the zero normal velocity through the numerical flux $\left(U_{n}^{\star}=0\right)$, and we take the external pressure from the interior $\left(P^{\star}=P\right)$, giving

$$
\overrightarrow{\mathbf{F}}_{e}^{\star} \cdot \vec{n}=\left(\begin{array}{c}
\rho U_{n} \\
\rho U_{n} \vec{U}+P \vec{n} \\
\rho_{0} c_{0}^{2} U_{n}
\end{array}\right)^{\star}=\left(\begin{array}{c}
0 \\
P \vec{n} \\
0
\end{array}\right) .
$$

Otherwise, we use the exact Riemann solver at the physical boundaries. To do so, we construct an external state $\mathbf{Q}_{n}^{e}$ (rotated using (4.43)) from the interior state $\mathbf{Q}_{n}^{i}$ but changing the sign of the normal velocity,

$$
\mathbf{Q}_{n}^{i}=\left(\begin{array}{c}
\rho \\
\rho U_{n} \\
\rho V_{t 1} \\
\rho V_{t 2} \\
P
\end{array}\right), \quad \mathbf{Q}_{n}^{e}=\left(\begin{array}{c}
\rho \\
-\rho U_{n} \\
\rho V_{t 1} \\
\rho V_{t 2} \\
P
\end{array}\right)
$$

\section{Viscous flux}

For the viscous fluxes, we have to give appropriate values for both the entropy variables $\mathbf{W}^{\star}$ and the viscous numerical fluxes $\overrightarrow{\mathbf{F}}_{v}^{\star}$ at the boundaries. However, only the way the 
velocities in $\mathbf{W}^{\star}$ are specified is important, since the viscous fluxes (the stress tensor (4.9)) are independent of the pressure gradient.

For the free-slip wall, we use a Neumann boundary condition: we take the entropy variables from the interior $\mathbf{W}^{\star}=\mathbf{W}$ and set viscous fluxes to zero $\overrightarrow{\mathbf{F}}_{v}^{\star} \cdot \vec{n}=\mathbf{0}$.

For the no-slip wall, we use a Dirichlet boundary condition: we use zeroed entropy variables $\mathbf{W}^{\star}=(0,0,0,0, P)$ (recall that the pressure is not relevant), and we take the viscous fluxes from the interior $\overrightarrow{\mathbf{F}}_{v}^{\star}=\overrightarrow{\mathbf{F}}_{v}$.

\section{Second artificial compressibility model}

For the second artificial compressibility model, the homogeneous Neumann boundary condition for the pressure time derivative is enforced using $\vec{G}_{p, t}^{\star} \cdot \vec{n}=0$.

\subsubsection{Equivalence of the two-point flux to a split form equation}

The discrete entropy-stability requires the use of the entropy conserving two-point volume flux (4.39). The two-point divergence is needed because in the continuous entropy analysis we used the product rule (which does not hold discretely) in momentum (4.39), and also because the metric terms need additional de-aliasing to satisfy a discrete version of (4.24).

The divergence at a point is approximated by the two-point formula (2.75), which we repeat here,

$$
\begin{aligned}
\mathbb{D}\left(\overrightarrow{\tilde{\mathbf{F}}}_{e}\right)_{i j k}^{\mathrm{EC}}=2 \sum_{m=0}^{N} & D_{i m} \overrightarrow{\mathbf{F}}_{e}^{\mathrm{EC}}\left(Q_{i j k}, Q_{m j k}\right) \cdot\left\{\left\{\mathscr{J} \vec{a}^{1}\right\}\right\}_{(i m) j k} \\
+ & D_{j m} \overrightarrow{\mathbf{F}}_{e}^{\mathrm{EC}}\left(Q_{i j k}, Q_{i m k}\right) \cdot\left\{\left\{\mathscr{J} \vec{a}^{2}\right\}\right\}_{i(j m) k} \\
& +D_{k m} \overrightarrow{\mathbf{F}}_{e}^{\mathrm{EC}}\left(Q_{i j k}, Q_{i j m}\right) \cdot\left\{\left\{\mathscr{J} \vec{a}^{3}\right\}\right\}_{i j(k m)} .
\end{aligned}
$$

Note that the approximation contains products of the two-point ( $i$ and $m$ ) averages of the fluxes and metric terms. The fluxes themselves, as seen in (4.39), also contain products of two-point averages.

Two-point approximations are algebraically equivalent to the approximation of the divergence written in a more recognizable split form that contains averages of conservative and nonconservative forms, C.F. Gassner et al. [2016a].

To show the equivalent PDEs approximations, we first show the equivalence for a split flux that depends only on a single average vector,

$$
\vec{F}^{\# 1}=\left(\left\{\left\{A_{1}\right\}\right\}\left\{\left\{A_{2}\right\}\right\}\left\{\left\{A_{3}\right\}\right\}\right)
$$


for a (space) vector flux

$$
\vec{F}=\left(A_{1} A_{2} A_{3}\right),
$$

whose components are polynomials of the reference space variables. Then (4.53) becomes

$$
\begin{aligned}
\mathbb{D}(\vec{F})_{i j k}^{\# 1} & =2 \sum_{m=0}^{N}\left\{D_{i m}\left\{\left\{A_{1}\right\}\right\}_{(i m) j k}+D_{j m}\left\{\left\{A_{2}\right\}\right\}_{i(j m) k}+D_{k m}\left\{\left\{A_{3}\right\}\right\}_{i j(k m)}\right\} \\
& =\sum_{m=0}^{N}\left\{D_{i m}\left(A_{1}\right)_{m j k}+D_{j m}\left(A_{2}\right)_{i m k}+D_{k m}\left(A_{3}\right)_{i j m}\right\} \\
& +\sum_{m=0}^{N}\left\{D_{i m}\left(A_{1}\right)_{i j k}+D_{j m}\left(A_{2}\right)_{i j k}+D_{k m}\left(A_{3}\right)_{i j k}\right\}
\end{aligned}
$$

Since $\sum_{m=0}^{N} D_{i m}=0$, the discrete derivative of a constant function is zero. As a result, the second sum in (4.56) vanishes so that

$$
\mathbb{D}(\vec{F})_{i j k}^{\# 1}=\sum_{m=0}^{N}\left\{D_{i m}\left(A_{1}\right)_{m j k}+D_{j m}\left(A_{2}\right)_{i m k}+D_{k m}\left(A_{3}\right)_{i j m}\right\}=\left(\nabla_{\xi} \cdot \vec{F}\right)_{i j k}
$$

Thus, the divergence approximation using a simple two-point average is equivalent to the divergence of the original flux.

Next in complexity is if the flux is the product of two quantities

$$
\vec{F}=\left(A_{1} B A_{2} B A_{3} B\right) .
$$

and the associated two-point flux replaces the product with the product of two averages

$$
\vec{F}^{\# 2}=\left(\left\{\left\{A_{1}\right\}\right\}\{\{B\}\}\left\{\left\{A_{2}\right\}\right\}\{\{B\}\}\left\{\left\{A_{3}\right\}\right\}\{\{B\}\}\right) .
$$

Since

$$
\begin{aligned}
4 & \sum_{m=0}^{N} D_{i m}\left\{\left\{A_{1}\right\}\right\}\{\{B\}\} \\
& =\sum_{m=0}^{N} D_{i m}\left(\left(A_{1}\right)_{i j k}+\left(A_{1}\right)_{m j k}\right)\left(B_{i j k}+B_{m j k}\right) \\
& =\sum_{m=0}^{N} D_{i m}\left(A_{1}\right)_{m j k} B_{m j k}+\left(A_{1}\right)_{i j k} \sum_{m=0}^{N} D_{i m} B_{m j k}+B_{i j k} \sum_{m=0}^{N} D_{i m}\left(A_{1}\right)_{m j k},
\end{aligned}
$$

it follows that

$$
\mathbb{D}(\vec{F})_{i j k}^{\# 2}=\frac{1}{2}\left(\nabla_{\xi} \cdot \vec{F}\right)_{i j k}+\frac{1}{2}\left(\vec{A} \cdot \nabla_{\xi} B+B \nabla_{\xi} \cdot \vec{A}\right)_{i j k}
$$


which is the average of the conservative form of the divergence and the product rule applied to it.

Finally, we follow the same steps to find the equivalent approximation for a triple product flux of the form

$$
\vec{F}=\left(C A_{1} B C A_{2} B C A_{3} B\right) .
$$

approximated by

$$
\vec{F}^{\# 3}=\left(\{\{C\}\}\left\{\left\{A_{1}\right\}\right\}\{\{B\}\}\{\{C\}\}\left\{\left\{A_{2}\right\}\right\}\{\{B\}\}\{\{C\}\}\left\{\left\{A_{3}\right\}\right\}\{\{B\}\}\right) \text {. }
$$

The result is a specific application Kennedy and Gruber [2008] of the product rule to the product of three polynomials,

$$
\begin{aligned}
\mathbb{D}(\vec{F})_{i j k}^{\# 3}=\frac{1}{4}\left(\nabla_{\xi} \cdot \vec{F}\right)_{i j k} & +\frac{1}{4}\left\{(C \vec{A})_{i j k} \cdot \nabla_{\xi} B_{i j k}+(C B)_{i j k} \nabla_{\xi} \cdot \vec{A}+C_{i j k} \nabla_{\xi} \cdot \mathbb{I}^{N}(\vec{A} B)_{i j k}\right. \\
& \left.+(B \vec{A})_{i j k} \cdot \nabla_{\xi} C_{i j k}+\vec{A}_{i j k} \cdot \nabla_{\xi} \mathbb{I}^{N}(C B)_{i j k}+B_{i j k} \nabla_{\xi} \cdot \mathbb{I}^{N}(C \vec{A})_{i j k}\right\} .
\end{aligned}
$$

The second form, (4.61), can be used to assess the influence of the metric terms in (4.53). Replacing $\vec{A}$ by the matrix $\overline{\bar{M}}$ whose columns are $\mathscr{J} \vec{a}^{i}, i=1,2,3$, the contravariant vector form of the divergence becomes

$$
\mathbb{D}(\overrightarrow{\tilde{F}})_{i j k}^{\# 1}=\frac{1}{2}\left(\vec{\nabla}_{\xi} \cdot \overrightarrow{\tilde{F}}\right)_{i j k}+\frac{1}{2}\left(\mathscr{J} \vec{a}^{1} \cdot \frac{\partial \vec{F}}{\partial \xi}+\mathscr{J} \vec{a}^{2} \cdot \frac{\partial \vec{F}}{\partial \eta}+\mathscr{J} \vec{a}^{3} \cdot \frac{\partial \vec{F}}{\partial \zeta}\right)_{i j k}
$$

by virtue of the discrete metric identities

$$
\sum_{m=0}^{N}\left(D_{i m} \mathscr{J} \vec{a}_{m j k}^{1}+D_{j m} \mathscr{J} \vec{a}_{i m k}^{2}+D_{k m} \mathscr{J} \vec{a}_{i j m}^{3}\right)=\left(\vec{\nabla}_{\xi} \cdot \bar{M}\right)_{i j k}=\left(\sum_{n=1}^{3} \frac{\partial \mathscr{J} \vec{a}^{n}}{\partial \xi^{n}}\right)_{i j k}=0 .
$$

In light of (4.65), we simplify the discussion below and examine the approximation (4.53) using the point-wise values of the metric terms rather than the averages.

From these three forms (4.57), (4.61) and (4.64), we can derive the split form approximation equivalent to the two-point fluxes given by (4.39) with the two choices for the momentum approximation, (4.40). Notice that with the first momentum average, the entropy conserving fluxes are single or product averages. Using the second momentum average the flux is made up of double or triple averages.

Option 1: $\rho \tilde{u}_{i}^{(1)}=\left\{\left\{\rho u_{i}\right\}\right\}$ 
When the momentum is implemented by the average, the continuity equation is in single average form and hence by (4.57),

$$
\vec{\nabla} \cdot(\rho \vec{u})_{i j k} \approx \vec{\nabla}_{\xi} \cdot \mathbb{I}^{N}(\rho \overrightarrow{\tilde{U}})_{i j k},
$$

where $\overrightarrow{\tilde{u}}=\bar{M}^{T} \vec{u}$ is the contravariant velocity.

The momentum equation is approximated using the product of two averages for the momentum and the average for the pressure so that it includes forms (4.61) and (4.57). The two-point flux form therefore approximates

$$
\begin{aligned}
\vec{\nabla}_{\xi} \cdot\left(\rho \overrightarrow{\tilde{u}} u_{l}+p \overline{\bar{M}}^{T} \vec{e}_{l}\right) \approx & \frac{1}{2} \vec{\nabla}_{\xi} \cdot \mathbb{I}^{N}\left(\rho U_{l} \overrightarrow{\tilde{U}}\right)_{i j k}+\frac{1}{2} U_{l, i j k} \vec{\nabla}_{\xi} \cdot \mathbb{I}^{N}(\rho \overrightarrow{\tilde{U}})_{i j k} \\
& +\frac{1}{2}(\rho \vec{U})_{i j k} \cdot\left(\vec{\nabla}_{\xi} \cdot \mathbb{I}^{N}\left(U_{l} \overline{\bar{M}}\right)\right)_{i j k}+\vec{\nabla}_{\xi} \cdot \mathbb{I}^{N}\left(P \overline{\bar{M}}^{T} \vec{e}_{l}\right)_{i j k} .
\end{aligned}
$$

Finally, the two-point flux form of the artificial compressibility equation, like the continuity equation, is equivalent to using standard DG since it is linear in the velocities.

Option 2: $\rho \tilde{u}_{i}^{(2)}=\{\{\rho\}\}\left\{\left\{u_{i}\right\}\right\}$

Approximating the momentum as the product of two averages (vs. the average of the product) leads to the approximation of a different form of the equations by increasing the number of products in each equation.

Under the second approximation, the continuity equation now has the product of two averages and hence is the equivalent to the approximation

$$
\vec{\nabla}_{\xi} \cdot(\rho \overrightarrow{\tilde{u}})_{i j k} \approx \frac{1}{2} \vec{\nabla}_{\xi} \cdot \mathbb{I}^{N}(\rho \overrightarrow{\tilde{U}})_{i j k}+\frac{1}{2} \rho_{i j k} \vec{\nabla}_{\xi} \cdot(\overrightarrow{\tilde{U}})_{i j k}+\frac{1}{2}(\vec{U})_{i j k} \cdot\left(\vec{\nabla}_{\xi} \cdot \mathbb{I}^{N}(\rho \overline{\bar{M}})\right)_{i j k} \cdot
$$

The momentum equation is approximated with the triple product for the momentum, and still the standard approximation in the pressure,

$$
\begin{aligned}
\vec{\nabla}_{\xi} & \cdot\left(\rho \overrightarrow{\tilde{u}} u_{l}+p \overline{\bar{M}}^{T} \vec{e}_{l}\right) \approx \frac{1}{4} \vec{\nabla}_{\xi} \cdot \mathbb{I}^{N}\left(\rho U_{l} \overrightarrow{\tilde{U}}\right)_{i j k}+\frac{1}{4} \rho_{i j k} U_{l, i j k}\left(\vec{\nabla}_{\xi} \cdot \overrightarrow{\tilde{U}}\right)_{i j k} \\
& +\frac{1}{4}(\rho \overrightarrow{\tilde{U}})_{i j k} \cdot\left(\vec{\nabla}_{\xi} \cdot \mathbb{I}^{N}\left(U_{l} \overline{\bar{M}}\right)\right)_{i j k}+\frac{1}{4} \rho_{i j k} \vec{\nabla}_{\xi} \cdot \mathbb{I}^{N}\left(U_{l} \overrightarrow{\tilde{U}}\right)_{i j k} \\
& +\frac{1}{4}\left(u_{l} \overrightarrow{\tilde{U}}\right)_{i j k} \cdot\left(\vec{\nabla}_{\xi} \cdot \mathbb{I}^{N}(\rho \overline{\bar{M}})\right)_{i j k}+\frac{1}{4} U_{l, i j k} \vec{\nabla}_{\xi} \cdot \mathbb{I}^{N}(\rho \overrightarrow{\tilde{U}})_{i j k} \\
& +\frac{1}{4}(\overrightarrow{\tilde{U}})_{i j k} \cdot\left(\vec{\nabla}_{\xi} \cdot \mathbb{I}^{N}\left(\rho U_{l} \overline{\bar{M}}\right)\right)_{i j k} \cdot
\end{aligned}
$$

The scheme that results from the use of the two-point divergence is algebraically equivalent to the split-form schemes. However, the former results in a simpler, more 
efficient, and maintainable implementation, as one only needs to change the twopoint volume flux to switch from different split-forms.

\subsection{Stability analysis}

In Sec. 3.5, we show that the split-form scheme (4.38) is entropy-stable for a general conservation law. However, in that general case, we assumed that inviscid terms are discretely contracted (3.93), and that the approximation of the boundary terms is stable (IBT $\geqslant 0, \mathrm{PBT} \geqslant 0$ ). In this section, we discuss the particularities of the incompressible NSE system, to fill the details of the general entropy analysis and prove its discrete stability.

\section{Contraction of the time coefficients}

For the time coefficients, we need to write them as in the general form (3.88),

$$
\sum_{e}\left\langle\mathscr{J} \mathrm{L}(\mathbf{Q})_{t}, \mathbf{W}\right\rangle_{E, N}=\overline{\mathscr{E}}_{t}^{N}
$$

where $\overline{\mathscr{E}}^{N}$ is the total discrete entropy. The case of the first artificial compressibility model is immediate, since $\mathrm{L}(\mathbf{Q})=\mathbf{Q}$, and we can use the chain rule as in (3.82) to get

$$
\sum_{e}\left\langle\mathscr{J} \mathbf{Q}_{t}, \mathbf{W}\right\rangle_{E, N}=\overline{\mathscr{E}}_{\mathrm{AC} 1, t}^{N}, \quad \overline{\mathscr{E}}_{\mathrm{AC} 1}^{N}=\sum_{e}\left\langle\mathscr{J}\left(\mathscr{K}+\frac{1}{2 \rho_{0} c_{0}^{2}} P^{2}\right)\right\rangle_{E, N} \geqslant 0 .
$$

For the second artificial compressibility model, we write the time coefficients inner product, where the terms associated to the kinetic energy do not change from the first artificial compressibility model,

$$
\left\langle\mathscr{J} \mathrm{L}(\mathbf{Q})_{t}, \mathbf{W}\right\rangle_{E, N}=\left\langle\mathscr{J} \mathscr{K}_{t}\right\rangle_{E, N}+\frac{1}{\rho_{0} c_{0}^{2}}\left\langle\overrightarrow{\tilde{G}}_{p, t}, \vec{\nabla}_{\xi} P\right\rangle_{E, N}-\frac{1}{\rho_{0} c_{0}^{2}} \int_{\partial e, N} P \vec{G}_{p, t}^{\star} \cdot \mathrm{d} \vec{S}
$$

From the definition of the discrete gradients in (4.38), we get the last equation, which defines $\vec{G}_{p}$, use the discrete Gauss law, take its time derivative, and set $\vec{\varphi}=\vec{G}_{p}$, to get

$$
\left\langle\mathscr{J} \vec{G}_{p, t}, \vec{G}_{t}\right\rangle_{E, N}=\int_{\partial e, N}\left(P_{t}^{\star}-P_{t}\right)\left(\vec{G}_{p} \cdot \vec{n}\right) \mathrm{d} \vec{S}+\left\langle\vec{\nabla}_{\xi} P_{t}, \overrightarrow{\tilde{G}}_{p}\right\rangle_{E, N}
$$


We replace the last inner product in the pressure terms of (4.73),

$$
\begin{aligned}
& \frac{1}{\rho_{0} c_{0}^{2}}\left\langle\overrightarrow{\tilde{G}}_{p, t}, \vec{\nabla}_{\xi} P\right\rangle_{E, N}-\frac{1}{\rho_{0} c_{0}^{2}} \int_{\partial e, N} P \vec{G}_{p, t}^{\star} \cdot \mathrm{d} \vec{S} \\
& =\frac{1}{\rho_{0} c_{0}^{2}}\left\langle\mathscr{J} \vec{G}_{p, t}, \vec{G}_{t}\right\rangle_{E, N}+\frac{1}{\rho_{0} c_{0}^{2}} \int_{\partial e, N}\left(P_{t} \vec{G}_{p}-P_{t}^{\star} \vec{G}_{p}-P_{t} \vec{G}_{p}^{\star}\right) \cdot \mathrm{d} \vec{S} \\
& =\left\langle\mathscr{J}\left(\frac{1}{2 \rho_{0} c_{0}^{2}}\left|\vec{G}_{p}\right|^{2}\right)_{t}\right\rangle_{E, N}+\frac{1}{\rho_{0} c_{0}^{2}} \int_{\partial e, N} \vec{H}^{\mathscr{E}} \cdot \vec{S} \\
& =\left\langle\mathscr{J} \mathscr{P}_{\mathrm{AC} 2, t}\right\rangle_{E, N}+\frac{1}{\rho_{0} c_{0}^{2}} \int_{\partial e, N} \vec{H}^{\mathscr{E}} \cdot \vec{S},
\end{aligned}
$$

with $\mathscr{P}_{\mathrm{AC} 2}=\frac{1}{2 \rho_{0} c_{0}^{2}}\left|\vec{G}_{p}\right|^{2}$. We have been able to write the contraction of the time coefficients as in the general form (3.83), with the surface term in the time contraction $\vec{H}_{\mathrm{AC} 2}^{\mathscr{E}}$,

$$
\vec{H}_{\mathrm{AC} 2}^{\mathscr{E}}=P_{t} \vec{G}_{p}-P_{t}^{\star} \vec{G}_{p}-P_{t} \vec{G}_{p}^{\star}
$$

The last step is to assess if the surface term $\vec{H}_{\mathrm{AC} 2}^{\mathscr{E}}$ can be written as the time derivative of positive quantities at the interior and physical boundaries. With the numerical fluxes given in (4.49) and (4.50), we get for interior boundaries

$$
\llbracket-\vec{H}^{\mathscr{E}} \rrbracket_{\mathrm{AC} 2} \cdot \vec{n}_{L}=\left\{\left\{P_{t}\right\}\right\} \llbracket \vec{G}_{p} \rrbracket+\llbracket P_{t} \rrbracket\left\{\left\{\vec{G}_{p}\right\}\right\}-\llbracket P_{t} \vec{G}_{p} \rrbracket+\beta_{p} \llbracket P \rrbracket \llbracket P_{t} \rrbracket=\frac{\beta_{p}}{2} \frac{\mathrm{d}}{\mathrm{d} t} \llbracket P \rrbracket^{2},
$$

the time derivative of a positive inter-element entropy $\mathscr{P}_{i, \mathrm{AC} 2}^{S}=\frac{1}{2} \beta_{p} \llbracket P \rrbracket^{2}$. For physical boundaries,

$$
\vec{H}^{\mathscr{E}} \cdot \vec{n}=P_{t} \vec{G}_{p}-P_{t} \vec{G}_{p}=0,
$$

and the surface entropy $\mathscr{E}_{\mathrm{AC} 2}^{S}=0$, which is consistent with the physical estimate. Then we sum (4.75) for all the elements to get the contraction of the time coefficients associated to pressure,

$$
\begin{aligned}
& \sum_{e} \frac{1}{\rho_{0} c_{0}^{2}}\left\langle\overrightarrow{\tilde{G}}_{p, t}, \vec{\nabla}_{\xi} P\right\rangle_{E, N}-\sum_{e} \frac{1}{\rho_{0} c_{0}^{2}} \int_{\partial e, N} P \vec{G}_{p, t}^{\star} \cdot \mathrm{d} \vec{S} \\
& =\sum_{e}\left\langle\mathscr{J} \mathscr{P}_{\mathrm{AC} 2, t}\right\rangle_{E, N}+\sum_{\substack{\text { interior } \\
\text { faces }}} \int_{f, N} \llbracket-\vec{H}^{\mathscr{E}} \rrbracket \cdot \vec{n}_{L} \mathrm{~d} S+\sum_{\substack{\text { boundary } \\
\text { faces }}} \int_{f, N} \vec{H}^{\mathscr{E}} \cdot \mathrm{d} \vec{S} \\
& =\frac{\mathrm{d}}{\mathrm{d} t}\left(\sum_{e}\left\langle\mathscr{J} \mathscr{P}_{\mathrm{AC} 2}\right\rangle_{E, N}+\sum_{\substack{\text { interior } \\
\text { faces }}} \int_{f, N} \frac{\beta_{p}}{2} \frac{1}{\rho_{0} c_{0}^{2}} \llbracket P \rrbracket^{2} \mathrm{~d} S\right)=\overline{\mathscr{P}}_{\mathrm{AC} 2, t}^{N} \cdot
\end{aligned}
$$


The total time coefficients contraction is then

$$
\sum_{e}\left\langle\mathscr{J} \mathrm{L}(\mathbf{Q})_{t}, \mathbf{W}\right\rangle_{E, N}=\frac{\mathrm{d}}{\mathrm{d} t}\left(\sum_{e}\left\langle\mathscr{J} \mathscr{E}_{\mathrm{AC} 2}\right\rangle_{E, N}+\sum_{\substack{\text { interior } \\ \text { faces }}} \frac{\beta_{p}}{2} \frac{1}{\rho_{0} c_{0}^{2}} \llbracket P \rrbracket^{2} \mathrm{~d} S\right)=\overline{\mathscr{E}}_{\mathrm{AC} 2, t},
$$

with

$$
\overline{\mathscr{E}}_{\mathrm{AC} 2}^{N}=\sum_{e}\left\langle\mathscr{J}\left(\mathscr{K}+\mathscr{P}_{\mathrm{AC} 2}\right)\right\rangle_{E, N}+\sum_{\substack{\text { interior } \\ \text { faces }}} \int_{f, N} \frac{\beta_{p}}{2} \frac{1}{\rho_{0} c_{0}^{2}} \llbracket P \rrbracket^{2} \mathrm{~d} S \geqslant 0 .
$$

In summary, for both artificial compressibility models, we can simply write,

$$
\sum_{e}\left\langle\mathscr{J} \mathrm{L}(\mathbf{Q})_{t}, \mathbf{W}\right\rangle_{E, N}=\overline{\mathscr{E}}_{t}^{N}
$$

\section{Contraction of the inviscid fluxes}

For the inviscid fluxes discrete contraction (3.93), it suffices to show that the twopoint volume flux used in the two-point divergence satisfies Tadmor's jump condition (3.91). For the incompressible NSE, we replace the entropy conserving flux (4.39) and the entropy variables (4.16) in (3.91), to see that

$$
\begin{aligned}
\llbracket \mathbf{W}^{T} \rrbracket \mathbf{F}^{e c, l}-\llbracket \mathbf{W}^{T} \mathbf{F}^{l} \rrbracket= & -\frac{1}{2} \llbracket V_{\text {tot }}^{2} \rrbracket \rho \tilde{U}_{l}+\rho \tilde{U}_{l}(\{\{U\}\} \llbracket U \rrbracket+\{\{V\}\} \llbracket V \rrbracket+\{\{W\}\} \llbracket W \rrbracket) \\
& +\{\{P\}\} \llbracket U_{l} \rrbracket+\left\{\left\{U_{l}\right\}\right\} \llbracket P \rrbracket+\frac{1}{2} \llbracket \rho U_{l} V_{\text {tot }}^{2} \rrbracket \\
& -\llbracket \rho\left(U^{2}+V^{2}+W^{2}\right) U_{l} \rrbracket-\llbracket U_{l} p \rrbracket-\llbracket U_{l} P \rrbracket \\
= & -\frac{1}{2} \rho \tilde{U}_{l} \llbracket U^{2}+V^{2}+W^{2} \rrbracket+\frac{1}{2} \rho \tilde{U}_{l} \llbracket U^{2}+V^{2}+W^{2} \rrbracket+\llbracket P U_{l} \rrbracket \\
& -\llbracket\left(\frac{1}{2} \rho\left(U^{2}+V^{2}+W^{2}\right)+P\right) U_{l} \rrbracket-\llbracket P U_{l} \rrbracket \\
= & -\llbracket\left(\frac{1}{2} \rho\left(U^{2}+V^{2}+W^{2}\right)+P\right) U_{l} \rrbracket=-\llbracket F_{e}^{\mathscr{E}, l} \rrbracket,
\end{aligned}
$$

where $F_{e}^{\mathscr{E}, l}$ is the entropy flux (4.28). In the process, we used the two arithmetic properties of the average and jump operators (1.28). Note that (4.83) holds independently of which momentum approximation $\rho \tilde{u}_{l}$ (4.40) is used, so from the point of view of stability, either approximation in (4.40) is acceptable. 


\section{Positive semi-definiteness of the viscous fluxes}

We bound the viscous flux volume contribution using the viscous positive semi-definiteness property (4.33),

$$
\langle\mathscr{J} \overrightarrow{\mathbf{G}}, \underline{\mathbf{B}} \overrightarrow{\mathbf{G}}\rangle_{E, N} \geqslant \min _{E, N}(\mathscr{J})\langle\overrightarrow{\mathbf{G}}, \underline{\mathbf{B}} \overrightarrow{\mathbf{G}}\rangle_{E, N} \geqslant 0,
$$

since all nodal values of the Jacobian, $\mathscr{J}_{i j k}$, are strictly positive in an admissible quality mesh. Now, (4.84) is the discrete version of (4.33), therefore, it can be written as

$$
\langle\mathscr{J} \overrightarrow{\mathbf{G}}, \underline{\mathbf{B}} \overrightarrow{\mathbf{G}}\rangle_{E, N}=\frac{2}{\operatorname{Re}}\left\langle\mathscr{J}|\overline{\bar{S}}|^{2}\right\rangle_{E, N} \geqslant 0
$$

where $\overline{\bar{S}}$ is the strain tensor computed from the approximated entropy variable gradient $\overline{\bar{S}}=\overline{\bar{s}}(\overrightarrow{\mathbf{G}})$.

Once we have studied the time coefficients, inviscid fluxes and viscous fluxes as a particular case of the general conservation law in Sec. 3.5, we can write the discrete entropy equation of the incompressible NSE,

$$
\overline{\mathscr{E}}_{t}^{N}+\mathrm{PBT}=-\mathrm{IBT}-\sum_{e} \frac{2}{\operatorname{Re}}\left\langle\mathscr{J}|\overline{\bar{S}}|^{2}\right\rangle_{E, N} \leqslant 0,
$$

where IBT is the contribution of the interior faces to the surface integral (3.96),

$$
\begin{aligned}
\mathrm{IBT}= & -\sum_{\substack{\text { interior } \\
\text { faces }}} \int_{N}\left(\llbracket \mathbf{W}^{T} \rrbracket \overrightarrow{\mathbf{F}}_{e}^{\star}+\llbracket \vec{F}^{\mathscr{E}} \rrbracket-\llbracket \mathbf{W}^{T} \overrightarrow{\mathbf{F}}_{e} \rrbracket\right) \cdot \vec{n}_{L} \mathrm{~d} S \\
& +\sum_{\substack{\text { interior } \\
\text { faces }}} \int_{N}\left(\llbracket \mathbf{W}^{T} \rrbracket \overrightarrow{\mathbf{F}}_{v}^{\star}+\mathbf{W}^{\star, T} \llbracket \overrightarrow{\mathbf{F}}_{v} \rrbracket-\llbracket \mathbf{W}^{T} \overrightarrow{\mathbf{F}}_{v} \rrbracket\right) \cdot \vec{n}_{L} \mathrm{~d} S \\
= & \mathrm{IBT}_{e}+\mathrm{IBT}_{v},
\end{aligned}
$$

and PBT is the physical boundary contribution (3.98),

$$
\begin{aligned}
\mathrm{PBT}= & \sum_{\substack{\text { boundary } \\
\text { faces }}} \int_{N}\left(\mathbf{W}^{T}\left(\overrightarrow{\mathbf{F}}_{e}^{\star}-\overrightarrow{\mathbf{F}}_{e}\right)+\vec{F}^{\mathscr{E}}\right) \cdot \vec{n} \mathrm{~d} S \\
& -\sum_{\substack{\text { boundary } \\
\text { faces }}} \int_{N}\left(\mathbf{W}^{T}\left(\overrightarrow{\mathbf{F}}_{v}^{\star}-\overrightarrow{\mathbf{F}}_{v}\right)+\mathbf{W}^{\star, T} \overrightarrow{\mathbf{F}}_{v}\right) \cdot \vec{n} \mathrm{~d} S \\
= & \mathrm{PBT}_{e}+\mathrm{PBT}_{v} .
\end{aligned}
$$

In (4.87), as in Sec. 3.5, we chose to write the contributions of the elements on the left and the right of the surface integrals taking the left face normal $\vec{n}_{L}$ as a reference. Thus, the right side face terms are added with opposing sign since $\vec{n}_{R}=-\vec{n}_{L}$. As a result, we get the jump in the argument as in (3.96). Note that the numerical fluxes $\overrightarrow{\mathbf{F}}_{e}^{\star}, \overrightarrow{\mathbf{F}}_{v}^{\star}$ and $\mathbf{W}^{\star}$ can factor out of the jump operators since they are shared (single valued) by both left and right states. 


\subsubsection{Stability of the Interior Boundary Terms (IBT)}

According to (4.86), interior boundary terms (4.87) are stable if IBT $\geqslant 0$. For inviscid fluxes we consider two possibilities: the two-point entropy conserving flux (4.39), or the exact Riemann solver (4.47).

\section{Inviscid fluxes: two-point entropy conserving numerical flux}

As demonstrated in (3.97), if we use the two-point entropy conserving flux (4.39) as the numerical flux $\overrightarrow{\mathbf{F}}_{e}^{\star}=\overrightarrow{\mathbf{F}}_{e}^{e c}$, we get $\mathrm{IBT}_{e}=0$ as a result of Tadmor's jump condition (3.91),

$$
\mathrm{IBT}_{e}=-\sum_{\substack{\text { interior } \\ \text { faces }}} \int_{N}\left(\llbracket \mathbf{W}^{T} \rrbracket \overrightarrow{\mathbf{F}}_{e}^{e c}+\llbracket \vec{F}^{\mathscr{E}} \rrbracket-\llbracket \mathbf{W}^{T} \overrightarrow{\mathbf{F}}_{e} \rrbracket\right) \cdot \vec{n}_{L} \mathrm{~d} S=0 .
$$

\section{Inviscid fluxes: exact Riemann solver}

The stability of the interior boundary terms rests on the positivity of $\mathrm{IBT}_{e}$ when using the exact Riemann solver (4.47). The positivity of $\mathrm{IBT}_{e}$ is ensured if at every face degree of freedom the argument

$$
\Delta_{e}=-\llbracket \mathbf{W}^{T} \rrbracket \overrightarrow{\mathbf{F}}_{e}^{\star} \cdot \vec{n}_{L}-\llbracket \vec{F}^{\mathscr{E}} \rrbracket \cdot \vec{n}_{L}+\llbracket \mathbf{W}^{T} \overrightarrow{\mathbf{F}}_{e} \rrbracket \cdot \vec{n}_{L} \geqslant 0 .
$$

We use the rotational invariance (4.42) to transform (4.90) to the face oriented system,

$$
\begin{aligned}
\Delta_{e}= & -\llbracket \mathbf{W}^{T} \rrbracket \underline{\mathrm{T}}^{T} \mathbf{F}_{e}\left(\mathbf{Q}_{n}^{\star}\right)-\llbracket F^{\mathscr{E}}\left(\mathbf{Q}_{n}\right) \rrbracket+\llbracket \mathbf{W}^{T} \underline{\mathrm{T}}^{T} \mathbf{F}_{e}\left(\mathbf{Q}_{n}\right) \rrbracket \\
& -\llbracket \mathbf{W}_{n}^{T} \rrbracket \mathbf{F}_{e}\left(\mathbf{Q}_{n}^{\star}\right)-\llbracket F^{\mathscr{E}}\left(\mathbf{Q}_{n}\right) \rrbracket+\llbracket \mathbf{W}_{n}^{T} \mathbf{F}_{e}\left(\mathbf{Q}_{n}\right) \rrbracket,
\end{aligned}
$$

where for the entropy flux

$$
\vec{F}^{\mathscr{E}} \cdot \vec{n}_{L}=\left(\frac{1}{2} \rho V_{t o t}^{2}+P\right) \vec{U} \cdot \vec{n}_{L}=\left(\frac{1}{2} \rho\left(U_{n}^{2}+V_{t 1}^{2}+V_{t 2}^{2}\right)+P\right) U_{n}=F^{\mathscr{E}}\left(\mathbf{Q}_{n}\right)
$$

Replacing the inviscid and entropy flux expressions in (4.91), we arrive at the 
condition to be satisfied by the exact Riemann problem solution,

$$
\begin{aligned}
\Delta_{e}= & -\left(\llbracket-\frac{1}{2} V_{t o t}^{2} \rrbracket, \llbracket U_{n} \rrbracket, \llbracket V_{t 1} \rrbracket, \llbracket V_{t 2} \rrbracket, \frac{1}{\rho_{0} c_{0}^{2}} \llbracket P \rrbracket\right)\left(\begin{array}{c}
\rho^{\star} U_{n}^{\star} \\
\rho^{\star}\left(U_{n}^{\star}\right)^{2}+P^{\star} \\
\rho^{\star} U_{n}^{\star} V_{t 1}^{\star} \\
\rho^{\star} U_{n}^{\star} V_{t 2}^{\star} \\
\rho_{0} c_{0}^{2} U_{n}^{\star}
\end{array}\right) \\
& -\llbracket\left(\frac{1}{2} \rho V_{t o t}^{2}+P\right) U_{n} \rrbracket+\llbracket-\frac{1}{2} \rho V_{t o t}^{2} U_{n}+\rho V_{t o t}^{2} U_{n}+2 P U_{n} \rrbracket \\
= & \frac{1}{2} \rho^{\star} \llbracket V_{t o t}^{2} \rrbracket U_{n}^{\star}-\rho^{\star}\left(U_{n}^{\star}\right)^{2} \llbracket U_{n} \rrbracket-P^{\star} \llbracket U_{n} \rrbracket-\rho^{\star} U_{n}^{\star} V_{t 1}^{\star} \llbracket V_{t 1} \rrbracket \\
& -\rho^{\star} U_{n}^{\star} V_{t 2}^{\star} \llbracket V_{t 2} \rrbracket-\llbracket P \rrbracket U_{n}^{\star}+\llbracket P U_{n} \rrbracket .
\end{aligned}
$$

Next, we use (1.28) to write,

$\frac{1}{2} \llbracket V_{\text {tot }}^{2} \rrbracket=\left\{\left\{U_{n}\right\}\right\} \llbracket U_{n} \rrbracket+\left\{\left\{V_{t 1}\right\}\right\} \llbracket V_{t 1} \rrbracket+\left\{\left\{V_{t 2}\right\}\right\} \llbracket V_{t 2} \rrbracket, \llbracket P U_{n} \rrbracket=\{\{P\}\} \llbracket U_{n} \rrbracket+\llbracket P \rrbracket\left\{\left\{U_{n}\right\}\right\}$,

which implies that

$$
\begin{aligned}
\Delta_{e}= & \left(\rho^{\star}\left(\left\{\left\{U_{n}\right\}\right\}-U_{n}^{\star}\right) U_{n}^{\star}+\{\{P\}\}-P^{\star}\right) \llbracket U_{n} \rrbracket+\left(\left\{\left\{U_{n}\right\}\right\}-U_{n}^{\star}\right) \llbracket P \rrbracket \\
+ & \rho^{\star}\left(\left\{\left\{V_{t 1}\right\}\right\}-V_{t 1}^{\star}\right) U_{n}^{\star} \llbracket V_{t 1} \rrbracket+\rho^{\star}\left(\left\{\left\{V_{t 2}\right\}\right\}-V_{t 2}^{\star}\right) U_{n}^{\star} \llbracket V_{t 2} \rrbracket .
\end{aligned}
$$

Now we replace the star region solution (4.47). To do so, without loss of generality we assume that $U_{n}^{\star} \geqslant 0$, where $\rho^{\star}=\rho_{L}^{\star}$ and $V_{t i}^{\star}=V_{t i L}$,

$$
\begin{aligned}
\Delta_{e}= & \left(\rho_{L}^{\star}\left(\left\{\left\{U_{n}\right\}\right\}-U_{n}^{\star}\right) U_{n}^{\star}+\{\{P\}\}-P^{\star}\right) \llbracket U_{n} \rrbracket+\left(\left\{\left\{U_{n}\right\}\right\}-U_{n}^{\star}\right) \llbracket P \rrbracket \\
+ & \rho_{L}^{\star}\left(\left\{\left\{V_{t 1}\right\}\right\}-V_{t 1 L}\right) U_{n}^{\star} \llbracket V_{t 1} \rrbracket+\rho_{L}^{\star}\left(\left\{\left\{V_{t 2}\right\}\right\}-V_{t 2 L}\right) U_{n}^{\star} \llbracket V_{t 2} \rrbracket .
\end{aligned}
$$

The last part, which involves tangential velocities, is stable since

$$
\rho_{L}^{\star}\left(\frac{V_{t i L}+V_{t i R}}{2}-V_{t i L}\right) U_{n}^{\star} \llbracket V_{t i} \rrbracket=\frac{1}{2} \rho_{L}^{\star} U_{n}^{\star} \llbracket V_{t i} \rrbracket^{2} \geqslant 0
$$

We write all pressures involved in (4.96) in terms of the velocities. First,

$$
U_{n}^{\star}=-\frac{\llbracket P \rrbracket+\rho_{R} U_{n R} \lambda_{R}^{-}-\rho_{L} U_{n L} \lambda_{L}^{+}}{\rho_{L} \lambda_{L}^{+}-\rho_{R} \lambda_{R}^{-}}, \llbracket P \rrbracket=-\Lambda U_{n}^{\star}-\rho_{R} U_{n R} \lambda_{R}^{-}+\rho_{L} U_{n L} \lambda_{L}^{+},
$$

where,

$$
\Lambda=\rho_{L} \lambda_{L}^{+}-\rho_{R} \lambda_{R}^{-}>0
$$

Next, the averaged pressure minus the star region pressure is

$$
\begin{aligned}
\{\{P\}\}-P^{\star} & =\frac{P_{L}+P_{R}}{2}-P_{L}-\rho_{L} \lambda_{L}^{+}\left(U_{n L}-U_{n}^{\star}\right)=\frac{1}{2} \llbracket P \rrbracket-\rho_{L} \lambda_{L}^{+}\left(U_{n L}-U_{n}^{\star}\right) \\
& =-\frac{1}{2} \Lambda U_{n}^{\star}-\frac{1}{2} \rho_{R} U_{n R} \lambda_{R}^{-}+\frac{1}{2} \rho_{L} U_{n L} \lambda_{L}^{+}-\rho_{L} \lambda_{L}^{+}\left(U_{n L}-U_{n}^{\star}\right) \\
& =-\frac{1}{2} \Lambda U_{n}^{\star}-\frac{1}{2} \rho_{R} U_{n R} \lambda_{R}^{-}-\frac{1}{2} \rho_{L} \lambda_{L}^{+} U_{n L}+\rho_{L} \lambda_{L}^{+} U_{n}^{\star},
\end{aligned}
$$


which replaced in (4.96), and replacing the star region density $\rho_{L}^{\star}$ from (4.47) gives,

$$
\begin{aligned}
\Delta_{e}= & \left(\rho_{L}^{\star}\left(\frac{U_{n L}+U_{n R}}{2}-U_{n}^{\star}\right) U_{n}^{\star}-\frac{1}{2} \Lambda U_{n}^{\star}-\frac{1}{2} \rho_{R} U_{n R} \lambda_{R}^{-}-\frac{1}{2} \rho_{L} \lambda_{L}^{+} U_{n L}+\rho_{L} \lambda_{L}^{+} U_{n}^{\star}\right)\left(U_{n R}-U_{n L}\right) \\
& +\left(\frac{U_{n L}+U_{n R}}{2}-U_{n}^{\star}\right)\left(-\Lambda U_{n}^{\star}-\rho_{R} U_{n R} \lambda_{R}^{-}+\rho_{L} U_{n L} \lambda_{L}^{+}\right)+\frac{1}{2} \rho_{L}^{\star} U_{n}^{\star}\left(\llbracket V_{t 1} \rrbracket^{2}+\llbracket V_{t 2} \rrbracket^{2}\right) \\
= & \frac{1}{U_{n}^{\star}-\lambda_{L}^{-}}\left\{\left[\frac{1}{2} \rho_{L} \lambda_{L}^{+}\left(U_{n L}+U_{n R}-2 U_{n}^{\star}\right) U_{n}^{\star}\right.\right. \\
& \left.-\left(\frac{1}{2} \Lambda U_{n}^{\star}+\frac{1}{2} \rho_{R} U_{n R} \lambda_{R}^{-}+\frac{1}{2} \rho_{L} \lambda_{L}^{+} U_{n L}-\rho_{L} \lambda_{L}^{+} U_{n}^{\star}\right)\left(U_{n}^{\star}-\lambda_{L}^{-}\right)\right]\left(U_{n R}-U_{n L}\right) \\
& +\frac{1}{2}\left(U_{n L}+U_{n R}-2 U_{n}^{\star}\right)\left(-\Lambda U_{n}^{\star}-\rho_{R} U_{n R} \lambda_{R}^{-}+\rho_{L} U_{n L} \lambda_{L}^{+}\right)\left(U_{n}^{\star}-\lambda_{L}^{-}\right) \\
& \left.+\frac{1}{2} \rho_{L} \lambda_{L}^{+} U_{n}^{\star}\left(\llbracket V_{t 1} \rrbracket^{2}+\llbracket V_{t 2} \rrbracket^{2}\right)\right\} .
\end{aligned}
$$

Next, we define $\hat{U}_{L}=U_{n L}-U_{n}^{\star}$ and $\hat{U}_{R}=U_{n R}-U_{n}^{\star}$ and use them to write $\Delta_{e}$,

$$
\begin{aligned}
\Delta_{e}= & \frac{1}{U_{n}^{\star}-\lambda_{L}^{-}}\left\{\left[\frac{1}{2} \rho_{L} \lambda_{L}^{+}\left(\hat{U}_{L}+\hat{U}_{R}\right) U_{n}^{\star}\right.\right. \\
& \left.-\left(\frac{1}{2} \Lambda U_{n}^{\star}+\frac{1}{2} \rho_{R}\left(\hat{U}_{R}+U_{n}^{\star}\right) \lambda_{R}^{-}+\frac{1}{2} \rho_{L} \lambda_{L}^{+}\left(\hat{U}_{L}+U_{n}^{\star}\right)-\rho_{L} \lambda_{L}^{+} U_{n}^{\star}\right)\left(U_{n}^{\star}-\lambda_{L}^{-}\right)\right]\left(\hat{U}_{R}-\hat{U}_{L}\right) \\
& +\frac{1}{2}\left(\hat{U}_{L}+\hat{U}_{R}\right)\left(-\Lambda U_{n}^{\star}-\rho_{R}\left(\hat{U}_{R}+U_{n}^{\star}\right) \lambda_{R}^{-}+\rho_{L}\left(\hat{U}_{L}+U_{n}^{\star}\right) \lambda_{L}^{+}\right)\left(U_{n}^{\star}-\lambda_{L}^{-}\right) \\
& \left.+\frac{1}{2} \rho_{L} \lambda_{L}^{+} U_{n}^{\star}\left(\llbracket V_{t 1} \rrbracket^{2}+\llbracket V_{t 2} \rrbracket^{2}\right)\right\} \\
= & \frac{1}{U_{n}^{\star}-\lambda_{L}^{-}}\left\{\left[\frac{1}{2} \rho_{L} \lambda_{L}^{+}\left(\hat{U}_{L}+\hat{U}_{R}\right) U_{n}^{\star}-\frac{1}{2}\left(\rho_{R} \hat{U}_{R} \lambda_{R}^{-}+\rho_{L} \lambda_{L}^{+} \hat{U}_{L}\right)\left(U_{n}^{\star}-\lambda_{L}^{-}\right)\right]\left(\hat{U}_{R}-\hat{U}_{L}\right)\right. \\
& \left.+\frac{1}{2}\left(\hat{U}_{L}+\hat{U}_{R}\right)\left(\rho_{L} \hat{U}_{L} \lambda_{L}^{+}-\rho_{R} \hat{U}_{R} \lambda_{R}^{-}\right)\left(U_{n}^{\star}-\lambda_{L}^{-}\right)+\frac{1}{2} \rho_{L} \lambda_{L}^{+} U_{n}^{\star}\left(\llbracket V_{t 1} \rrbracket^{2}+\llbracket V_{t 2} \rrbracket^{2}\right)\right\} \\
= & \frac{1}{U_{n}^{\star}-\lambda_{L}^{-}}\left\{\frac{1}{2} \rho_{L} \lambda_{L}^{+}\left(\hat{U}_{R}^{2}-\hat{U}_{L}^{2}\right) U_{n}^{\star}+\left(\rho_{L} \lambda_{L}^{+} \hat{U}_{L}^{2}-\rho_{R} \lambda_{R}^{-} \hat{U}_{R}^{2}\right)\left(U_{n}^{\star}-\lambda_{L}^{-}\right)\right. \\
& \left.+\frac{1}{2} \rho_{L} \lambda_{L}^{+} U_{n}^{\star}\left(\llbracket V_{t 1} \rrbracket^{2}+\llbracket V_{t 2} \rrbracket^{2}\right)\right\} \\
= & \frac{1}{U_{n}^{\star}-\lambda_{L}^{-}}\left(\frac{1}{2} \rho_{L} \lambda_{L}^{+}\left(U_{n}^{\star}-2 \lambda_{L}^{-}\right) \hat{U}_{L}^{2}+\left(\frac{1}{2} \rho_{L} \lambda_{L}^{+} U_{n}^{\star}-\rho_{R} \lambda_{R}^{-}\left(U_{n}^{\star}-\lambda_{L}^{-}\right)\right) \hat{U}_{R}^{2}\right. \\
& \left.+\frac{1}{2} \rho_{L} \lambda_{L}^{+} U_{n}^{\star}\left(\llbracket V_{t 1} \rrbracket^{2}+\llbracket V_{t 2} \rrbracket^{2}\right)\right) \geqslant 0 .
\end{aligned}
$$


Therefore,

$$
\begin{aligned}
\Delta_{e}= & \frac{1}{U_{n}^{\star}-\lambda_{L}^{-}}\left(\frac{1}{2} \rho_{L} \lambda_{L}^{+}\left(U_{n}^{\star}-2 \lambda_{L}^{-}\right) \hat{U}_{L}^{2}+\left(\frac{1}{2} \rho_{L} \lambda_{L}^{+} U_{n}^{\star}-\rho_{R} \lambda_{R}^{-}\left(U_{n}^{\star}-\lambda_{L}^{-}\right)\right) \hat{U}_{R}^{2}\right. \\
& \left.+\frac{1}{2} \rho_{L} \lambda_{L}^{+} U_{n}^{\star}\left(\llbracket V_{t 1} \rrbracket^{2}+\llbracket V_{t 2} \rrbracket^{2}\right)\right) \geqslant 0,
\end{aligned}
$$

which confirms that $\Delta_{e}$ is always positive since $U_{n}^{\star} \geqslant 0, \lambda_{L}^{+}>0, \lambda_{L}^{-}<0$, and $\lambda_{R}^{-}<0$.

The magnitude of the dissipation introduced depends on the square of the tangential speed jumps and the square of the jumps between the star solution $U_{n}^{\star}$ and the left and right states,

$$
\begin{aligned}
\Delta_{e}=\frac{1}{U_{n}^{\star}-\lambda_{L}^{-}} & \left(\frac{1}{2} \rho_{L} \lambda_{L}^{+}\left(U_{n}^{\star}-2 \lambda_{L}^{-}\right)\left(U_{n L}-U_{n}^{\star}\right)^{2}+\left(\frac{1}{2} \rho_{L} \lambda_{L}^{+} U_{n}^{\star}-\rho_{R} \lambda_{R}^{-}\left(U_{n}^{\star}-\lambda_{L}^{-}\right)\right)\left(U_{n R}-U_{n}^{\star}\right)^{2}\right. \\
& \left.+\frac{1}{2} \rho_{L} \lambda_{L}^{+} U_{n}^{\star}\left(\llbracket V_{t 1} \rrbracket^{2}+\llbracket V_{t 2} \rrbracket^{2}\right)\right) \geqslant 0 .
\end{aligned}
$$

In the other possible case, $U_{n}^{\star}<0$, the exact Riemann solver is also dissipative and

$$
\begin{gathered}
\Delta_{e}=\frac{1}{U_{n}^{\star}-\lambda_{R}^{+}}\left(-\frac{1}{2} \rho_{R} \lambda_{R}^{-}\left(U_{n}^{\star}-2 \lambda_{R}^{+}\right)\left(U_{n R}-U_{n}^{\star}\right)^{2}+\left(-\frac{1}{2} \rho_{R} \lambda_{R}^{-} U_{n}^{\star}+\rho_{L} \lambda_{L}^{+}\left(U_{n}^{\star}-\lambda_{R}^{+}\right)\right)\left(U_{n L}-U_{n}^{\star}\right)^{2}\right. \\
\left.-\frac{1}{2} \rho_{R} \lambda_{R}^{-} U_{n}^{\star}\left(\llbracket V_{t 1} \rrbracket^{2}+\llbracket V_{t 2} \rrbracket^{2}\right)\right) \geqslant 0 .
\end{gathered}
$$

Therefore, the positivity of $\mathrm{IBT}_{e}$ in the split-form DGSEM with the exact Riemann solver derived in Bassi et al. [2017] is established.

\section{Viscous fluxes: BR1 method}

For the viscous fluxes we write the viscous contribution in IBT by replacing the numerical values using the BR1 scheme (4.49),

$$
\mathrm{IBT}_{v}=\sum_{\substack{\text { interior } \\ \text { faces }}} \int_{N}\left(\llbracket \mathbf{W}^{T} \rrbracket\left\{\left\{\overrightarrow{\mathbf{F}}_{v}\right\}\right\}+\left\{\left\{\mathbf{W}^{T}\right\}\right\} \llbracket \overrightarrow{\mathbf{F}}_{v} \rrbracket-\llbracket \mathbf{W}^{T} \overrightarrow{\mathbf{F}}_{v} \rrbracket\right) \cdot \vec{n}_{L} \mathrm{~d} S=0,
$$

since the algebraic identity (1.28) holds.

\subsubsection{Interior Boundary Terms (IBT): summary}

We conclude that interior boundary terms are stable, IBT $\geqslant 0$. Moreover, we get an entropy conserving scheme using the two-point entropy flux (4.39) as the numerical flux, IBT $=0$, and an entropy-stable scheme if we use the exact Riemann solver, 
IBT $\geqslant 0$. Neutral stability usually leads to undesirable, oscillating solutions and evenodd error behaviors (see Gassner et al. [2016a]; Liu et al. [2020]). On the contrary, the exact Riemann solver provides numerical dissipation so that $\mathrm{IBT}_{e} \geqslant 0$, which usually enhances the accuracy of the scheme. A different systematic procedure to add controlled dissipation is to augment the two-point entropy flux at the boundaries with a matrix dissipation term Bohm et al. [2018]; Gassner et al. [2018]. However, we have not explored how the latter should be performed for the set of equations studied.

\subsubsection{Physical Boundary Terms (PBT): wall boundary conditions}

Like at interior boundaries, boundary condition prescriptions are stable if $\mathrm{PBT} \geqslant 0$. In this section we follow Hindenlang et al. [2019] and show that the two approaches described in Sec. 4.4.3 stably impose both free- and no-slip wall boundary conditions.

\section{Inviscid fluxes}

The stability condition for the inviscid boundary flux is

$$
\operatorname{PBT}_{e}=\sum_{\substack{\text { boundary } \\ \text { faces }}} \int_{N}\left(\mathbf{W}^{T}\left(\overrightarrow{\mathbf{F}}_{e}^{\star}-\overrightarrow{\mathbf{F}}_{e}\right) \cdot \vec{n}+\vec{F}^{\mathscr{E}} \cdot \vec{n}\right) \mathrm{d} S \geqslant 0
$$

A sufficient condition for (4.107) to hold is that the argument

$$
\Delta_{e}=\mathbf{W}^{T}\left(\overrightarrow{\mathbf{F}}_{e}^{\star}-\overrightarrow{\mathbf{F}}_{e}\right) \cdot \vec{n}+\vec{F}^{\mathscr{E}} \cdot \vec{n} \geqslant 0
$$

We presented two choices by which to enforce the wall boundary condition through the numerical flux $\overrightarrow{\mathbf{F}}_{e}^{\star}$, and examine their stability in turn.

1. If we do not to use a Riemann solver at the boundaries, we write (4.108) replacing the fluxes,

$$
\Delta_{e}=\left(-\frac{1}{2} V_{\text {tot }}^{2}, \vec{U}, \frac{1}{\rho_{0} c_{0}^{2}} P\right)\left[\left(\begin{array}{c}
0 \\
P \vec{n} \\
0
\end{array}\right)-\left(\begin{array}{c}
\rho U_{n} \\
\rho U_{n} \vec{U}+P \vec{n} \\
\rho_{0} c_{0}^{2} U_{n}
\end{array}\right)\right]+\left(\frac{1}{2} \rho V_{\text {tot }}^{2}+P\right) U_{n}=0 .
$$

Therefore, enforcing the wall boundary condition through direct prescription of the numerical flux is neutrally stable $\mathrm{PBT}=0$.

2. If we use the exact Riemann solver at the physical boundaries and we construct the external state (4.52), we construct the star region solution (4.47) for the 
particular states $\mathbf{Q}_{n}^{e}$,

$$
U^{\star}=\frac{P-P+\rho \lambda^{+} U_{n}-\rho\left(-\lambda^{+}\right)\left(-U_{n}\right)}{\rho \lambda^{+}-\rho\left(-\lambda^{+}\right)}=0, \quad P^{\star}=P+\rho \lambda^{+} U_{n},
$$

where,

$$
\lambda_{L}^{+}=\frac{U_{n}+a}{2}=\lambda^{+} \geq 0, \quad \lambda_{R}^{-}=\frac{-U_{n}-a}{2}=-\lambda^{+}<0 .
$$

Therefore,

$$
\mathbf{F}_{e}\left(\mathbf{Q}_{n}^{\star}\right)=\left(\begin{array}{c}
\rho^{\star} U_{n}^{\star} \\
\rho^{\star}\left(U_{n}^{\star}\right)^{2}+P^{\star} \\
\rho^{\star} U_{n}^{\star} V_{t 1}^{\star} \\
\rho^{\star} V_{n}^{\star} v_{t 2}^{\star} \\
\rho_{0} c_{0}^{2} U_{n}^{\star}
\end{array}\right)=\left(\begin{array}{c}
0 \\
P+\rho \lambda^{+} U_{n} \\
0 \\
0 \\
0
\end{array}\right) .
$$

Now we write (4.108) using the rotational invariance (4.42), $\overrightarrow{\mathbf{F}}_{e}^{\star} \cdot \vec{n}=\underline{\mathrm{T}}^{T} \mathbf{F}_{e}\left(\mathbf{Q}_{n}^{\star}\right)$, and $\overrightarrow{\mathbf{F}}_{e} \cdot \vec{n}=\underline{\mathrm{T}}^{T} \mathbf{F}_{e}\left(\mathbf{Q}_{n}\right)$,

$$
\begin{aligned}
\Delta_{e} & =\mathbf{W}^{T} \underline{\mathrm{T}}^{T}\left(\mathbf{F}_{e}\left(\mathbf{Q}_{n}^{\star}\right)-\mathbf{F}_{e}\left(\mathbf{Q}_{n}^{i}\right)\right)+\vec{F}^{\mathscr{E}} \cdot \vec{n} \\
& =\mathbf{W}_{n}^{T}\left(\mathbf{F}_{e}\left(\mathbf{Q}_{n}^{\star}\right)-\mathbf{F}_{e}\left(\mathbf{Q}_{n}^{i}\right)\right)+\vec{F}^{\mathscr{E}} \cdot \vec{n} \\
& =\left(-\frac{1}{2} V_{t o t}^{2}, U_{n}, V_{t 1}, V_{t 2}, P\right)\left(\begin{array}{c} 
\\
P+\rho \lambda^{+} U_{n}-\rho U_{n}^{2}-P \\
-\rho U_{n} V_{t 1} \\
-\rho U_{n} V_{t 2} \\
-\rho_{0} c_{0}^{2} U_{n}
\end{array}\right)+\frac{1}{2} \rho V_{t o t}^{2} U_{n}+P U_{n} \\
& =\frac{1}{2} \rho V_{t o t}^{2} U_{n}+\rho \lambda^{+} U_{n}^{2}-\rho U_{n}\left(U_{n}^{2}+V_{t 1}^{2}+V_{t 2}^{2}\right)-P U_{n}+\frac{1}{2} \rho V_{t o t}^{2} U_{n}+P U_{n} \\
& =\rho \lambda^{+} U_{n}^{2} \geqslant 0,
\end{aligned}
$$

which is always dissipative (for positive density) since $\lambda^{+} \geqslant 0$. The dissipation introduced at the boundaries increases with the square of the velocity normal to the wall (which vanishes when the boundary condition is exactly satisfied, $\left.U_{n}=0\right)$.

We conclude that wall boundary conditions can be stably enforced either by direct imposition through the numerical flux, or using the exact Riemann solver constructing an external state. The former is neutrally stable and does not add any dissipation, the latter introduces numerical dissipation that vanishes as the normal velocity converges (weakly). 


\section{Viscous fluxes}

The difference between free- and no-slip boundary condition rests on the implementation of the entropy variables and viscous numerical flux. We will see the effect of the choices given in Sec. 4.4 .3 on viscous fluxes stability

$$
\mathrm{PBT}_{v}=-\sum_{\substack{\text { boundary } \\ \text { faces }}} \int_{N}\left(\mathbf{W}^{T} \overrightarrow{\mathbf{F}}_{v}^{\star}+\mathbf{W}^{\star, T} \overrightarrow{\mathbf{F}}_{v}-\mathbf{W}^{T} \overrightarrow{\mathbf{F}}_{v}\right) \cdot \vec{n} \mathrm{~d} S .
$$

For the free-slip wall, the choice $\mathbf{W}^{\star}=\mathbf{W}$ and $\overrightarrow{\mathbf{F}}_{v}^{\star} \cdot \vec{n}=\mathbf{0}$ implies that the viscous contribution is zero,

$$
\begin{aligned}
\mathrm{PBT}_{v} & =-\sum_{\substack{\text { boundary } \\
\text { faces }}} \int_{N}\left(\mathbf{W}^{T} \overrightarrow{\mathbf{F}}_{v}^{\star}+\mathbf{W}^{\star, T} \overrightarrow{\mathbf{F}}_{v}-\mathbf{W}^{T} \overrightarrow{\mathbf{F}}_{v}\right) \cdot \vec{n} \mathrm{~d} S \\
& =-\sum_{\substack{\text { boundary } \\
\text { faces }}} \int_{N}\left(\mathbf{W}^{T} \overrightarrow{\mathbf{F}}_{v}-\mathbf{W}^{T} \overrightarrow{\mathbf{F}}_{v}\right) \cdot \vec{n} \mathrm{~d} S=0 .
\end{aligned}
$$

The same result holds for the no-slip wall choice $\mathbf{W}^{\star}=(0,0,0,0, P)$ and $\overrightarrow{\mathbf{F}}_{v}^{\star} \cdot \vec{n}=\overrightarrow{\mathbf{F}}_{v} \cdot \vec{n}$.

\subsubsection{Final remarks}

We have proved that the split-form DG scheme with the two-point entropy conserving flux (4.39) satisfies the discrete entropy law (4.86). On the modelling side, we have studied two artificial compressibility models (4.2) and (4.3) that share the same entropy equation, for different mathematical entropy functions. The boundary terms are identically zero if the two-point entropy conserving flux is used as the numerical flux at interior boundaries and if the wall boundary condition is enforced via direct prescription, and stable if we use the exact Riemann solver (4.47). The former yields an entropy conserving scheme (where all the dissipation is due to the physical viscosity),

$$
\overline{\mathscr{E}}_{t}^{N}=-\sum_{e} \frac{2}{\operatorname{Re}}\left\langle\mathscr{J}|\overline{\bar{S}}|^{2}\right\rangle_{E, N} \leqslant 0
$$

while the latter produces an entropy-stable (dissipative) scheme, where the physical viscosity is complemented with numerical dissipation at the element boundaries,

$$
\overline{\mathscr{E}}_{t}^{N} \leqslant-\sum_{e} \frac{2}{\operatorname{Re}}\left\langle\mathscr{J}|\overline{\bar{S}}|^{2}\right\rangle_{E, N} \leqslant 0 .
$$




\subsection{Numerical experiments}

The purpose of this section is to address the accuracy and robustness of the method. For the former, we perform a convergence analysis in three-dimensions using the manufactured solution method, and in two-dimensions using the Kovasznay flow problem Kovasznay [1948]. For the latter, we solve a two-dimensional lid driven cavity at three Reynolds numbers, a three-dimensional inviscid Taylor-Green vortex, and a two-dimensional Rayleigh-Taylor instability Guermond and Quartapelle [2000]. Unless specified otherwise, we use the exact Riemann solver at the interior and physical (using the ghost state) boundaries.

Since it results in a simpler implementation, we only consider the first artificial compressibility model (4.3) for the numerical experiments. The choice of the artificial compressibility sound speed, $c_{0}$, depends on the nature of the problem. For steadystate problems, since the purpose is to arrive at the stationary solution with $p_{t}=$ $-\rho_{0} c_{0}^{2} \vec{\nabla} \cdot \vec{u}=0$, the choice of the artificial compressibility solely considers achieving the best system conditioning and convergence rate to steady-state. Apart from the density transport equation, which is highly influenced by the choice of this parameter, we have found that the impact of the artificial compressibility sound speed on the accuracy in steady-state problems is not remarkable.

On the side of stability, the artificial compressibility sound speed $c_{0}$ controls the size of pressure fluctuations on the entropy (4.12). As a result, a solution computed with a very low value of $c_{0}$ features large oscillations in space in the state variables, which eventually could lead to negative densities, causing numerical instabilities.

For transient simulations, however, the choice of the artificial compressibility sound speed $c_{0}$ is influenced by temporal accuracy. Typical values used in this work are encompassed in the region $\rho_{0} c_{0}^{2} \in\left[10^{3}, 10^{4}\right]$, so that they keep pressure waves low throughout, while not inducing a severe time-step restriction for an explicit time integrator. 


\subsubsection{Convergence study}

In this section, we address the accuracy of the scheme by considering a three dimensional manufactured solution with a divergence free velocity field,

$$
\begin{aligned}
& \rho_{0}=1 \\
& u_{0}=\cos (\pi(x+y+z-2 t)) \\
& v_{0}=-2 \cos (\pi(x+y+z-2 t)) \\
& w_{0}=\cos (\pi(x+y+z-2 t)) \\
& p_{0}=2 \cos (\pi(x+y+z-2 t))-3 \frac{1}{\mathrm{Re}} \pi \sin (\pi(x+y+z-2 t))
\end{aligned}
$$

the Reynolds number being $\operatorname{Re}=1000$, and the corresponding source term,

$$
\begin{aligned}
& q_{\rho}=0 \\
& q_{\rho u}=0 \\
& q_{\rho v}=-6 \pi \sin (\pi(x+y+z-2 t))-\frac{9}{\operatorname{Re}} \pi^{2} \cos (\pi(x+y+z-2 t)), \\
& q_{\rho w}=0 \\
& q_{p}=4 \pi \sin (\pi(x+y+z-2 t))+\frac{6}{\operatorname{Re}} \pi^{2} \cos (\pi(x+y+z-2 t)),
\end{aligned}
$$

solved in a fully periodic box with size $[-1,1]^{3}$. The stability analysis holds for periodic boundary conditions. Periodic boundary points are included in IBT as interior points, while $\mathrm{PBT}=0$.

The artificial compressibility sound speed is $\rho_{0} c_{0}^{2}=10^{3}$. In these simulations, we use a CFL type condition, using the local maximum wave speed of the advective fluxes and the relative grid size $\Delta x /(N+1)$. We use $\mathrm{CFL}=0.5$ for all computations, and the end time is set to $t_{F}=10$. After time integration, we compute the $\mathrm{L}^{2}$ error as

$$
\left\|u-u_{0}\right\|_{L^{2}}=\sqrt{\sum_{e} \sum_{i, j, k=0}^{N} w_{i} w_{j} w_{k}\left(u_{i j k}-u_{0, i j k}\right)^{2} \mathscr{J}_{i j k}}
$$

We first perform a polynomial order study on a regular a $4^{3}$ mesh, with the polynomial order ranging from $N=1$ to $N=11$. We solve the incompressible NSE with the three schemes presented in this work: split-form with both average of the product and product of the averages in momentum, and standard DG. We show the $\mathrm{L}^{2}$ errors computed for the five state variables in Figs. 4.1(a)-4.1(d). (Because of the symmetry, the $\rho u$ and $\rho w L^{2}$ errors are identical, so both have been represented in Fig. 4.1(b).)

We find that the $\mathrm{L}^{2}$ errors are systematically smaller with the split-form, and the convergence is smoother than standard DG. Both remain stable in this smooth, wellresolved problem. The slight non-optimality found in the convergence rates is a result 


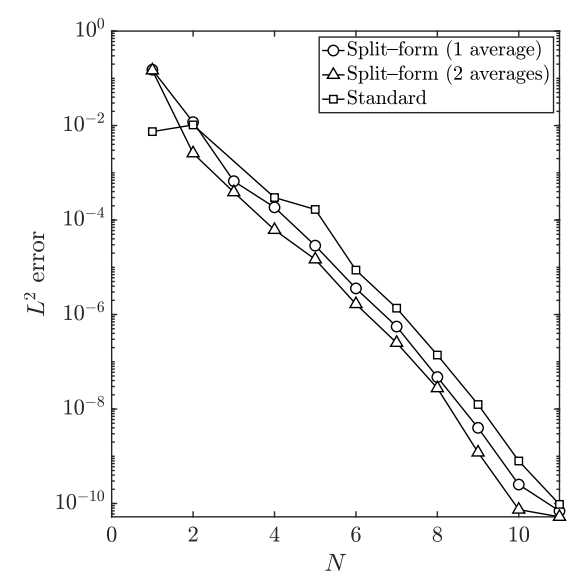

(a) $\rho$ error

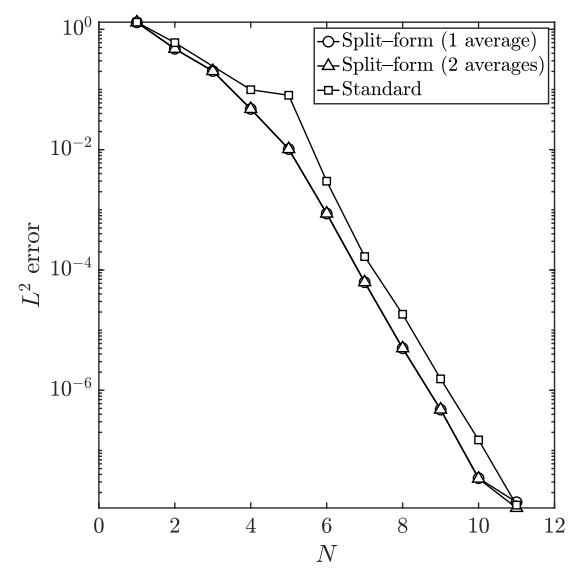

(c) $\rho v$ error

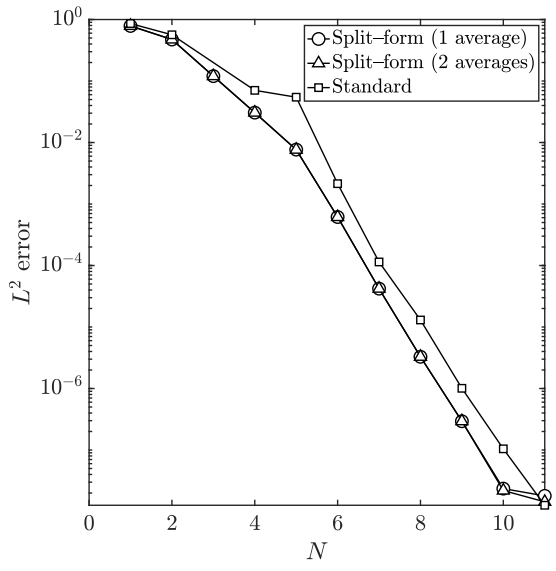

(b) $\rho u$ and $\rho w$ errors

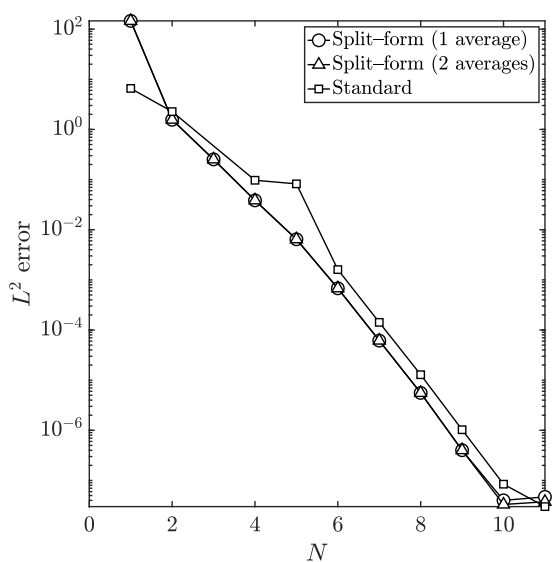

(d) $p$ error

Figure 4.1: Polynomial order $N$ convergence study using the manufactured solution (4.118) on a $4^{3}$ Cartesian grid. We consider three schemes: split-form DG with average of the product (one average) and product of the averages (two averages) in the momentum, and standard DG. All three schemes show spectral convergence behavior. The uneven rate of convergence is an effect of the BR1 scheme 
of the BR1 scheme even-odd behavior (note that the Reynolds number is moderate, $\operatorname{Re}=1000$ ), which we have not seen when solving the purely inviscid problem.

Next, we address the mesh convergence and consider different meshes: from $4^{3}$ to $16^{3}$, and polynomial orders: from $N=2$ to $N=5$. For this experiment, we use the split-form with the average of the product, and maintain the same parameters used in the polynomial order study. The results are reported in Table 4.1, with the $\mathrm{L}^{2}$ errors computed for each of the five state variables, and the estimated order of convergence, showing agreement with the reference Bassi et al. [2017]. As in Bassi et al. [2017], we note that the pressure convergence order is systematically smaller than the others.

Table 4.1: Manufactured solution (4.118) convergence analysis: we use $4^{3}, 6^{3}, 8^{3}, 12^{3}$ and $16^{3}$ meshes, and $N=2,3,4$ and 5 . We select the split-form DG with the average of the momentum, and run all simulations until $t_{F}=10$ using the explicit RK3 scheme with $C F L=0.5$

\begin{tabular}{llllllllll}
\hline & Mesh & $\rho$ error & order & $\rho u, w$ error & order & $\rho v$ error & order & $p$ error & order \\
\hline $\mathrm{N}=2$ & $4^{3}$ & $1.18 \mathrm{E}-2$ & - & $4.73 \mathrm{E}-1$ & - & $4.75 \mathrm{E}-1$ & - & $1.56 \mathrm{E}-0$ & - \\
& $6^{3}$ & $3.29 \mathrm{E}-3$ & 3.16 & $2.12 \mathrm{E}-1$ & 1.98 & $2.16 \mathrm{E}-1$ & 1.94 & $7.23 \mathrm{E}-1$ & 1.89 \\
& $8^{3}$ & $1.55 \mathrm{E}-3$ & 2.61 & $9.98 \mathrm{E}-2$ & 2.62 & $1.11 \mathrm{E}-1$ & 2.32 & $4.26 \mathrm{E}-1$ & 1.84 \\
& $12^{3}$ & $5.44 \mathrm{E}-4$ & 2.58 & $2.88 \mathrm{E}-2$ & 3.07 & $3.32 \mathrm{E}-2$ & 2.98 & $1.91 \mathrm{E}-1$ & 1.98 \\
& $16^{3}$ & $2.39 \mathrm{E}-4$ & 2.86 & $1.24 \mathrm{E}-2$ & 2.92 & $1.56 \mathrm{E}-2$ & 2.63 & $9.90 \mathrm{E}-2$ & 2.29 \\
$\mathrm{~N}=3$ & $4^{3}$ & $6.65 \mathrm{E}-4$ & - & $1.20 \mathrm{E}-1$ & - & $2.03 \mathrm{E}-1$ & - & $2.53 \mathrm{E}-1$ & - \\
& $6^{3}$ & $1.58 \mathrm{E}-4$ & 3.55 & $2.57 \mathrm{E}-2$ & 3.80 & $4.72 \mathrm{E}-2$ & 3.60 & $5.82 \mathrm{E}-2$ & 3.62 \\
& $8^{3}$ & $6.79 \mathrm{E}-5$ & 2.92 & $9.59 \mathrm{E}-3$ & 3.42 & $1.70 \mathrm{E}-2$ & 3.54 & $2.51 \mathrm{E}-2$ & 2.92 \\
& $12^{3}$ & $1.45 \mathrm{E}-5$ & 3.80 & $2.24 \mathrm{E}-3$ & 3.58 & $3.13 \mathrm{E}-3$ & 4.18 & $6.79 \mathrm{E}-3$ & 3.23 \\
& $16^{3}$ & $4.44 \mathrm{E}-6$ & 4.12 & $8.03 \mathrm{E}-4$ & 3.57 & $1.13 \mathrm{E}-3$ & 3.55 & $2.56 \mathrm{E}-3$ & 3.39 \\
$\mathrm{~N}=4$ & $4^{3}$ & $1.86 \mathrm{E}-4$ & - & $3.06 \mathrm{E}-2$ & - & $4.75 \mathrm{E}-2$ & - & $3.85 \mathrm{E}-2$ & - \\
& $6^{3}$ & $3.04 \mathrm{E}-5$ & 4.47 & $3.00 \mathrm{E}-3$ & 5.73 & $4.87 \mathrm{E}-3$ & 5.62 & $6.78 \mathrm{E}-3$ & 4.28 \\
& $8^{3}$ & $8.02 \mathrm{E}-6$ & 4.63 & $7.66 \mathrm{E}-4$ & 4.74 & $1.20 \mathrm{E}-3$ & 4.88 & $1.90 \mathrm{E}-3$ & 4.43 \\
& $12^{3}$ & $9.92 \mathrm{E}-7$ & 5.16 & $8.34 \mathrm{E}-5$ & 5.47 & $1.40 \mathrm{E}-4$ & 5.29 & $2.76 \mathrm{E}-4$ & 4.76 \\
& $16^{3}$ & $2.02 \mathrm{E}-7$ & 5.54 & $1.79 \mathrm{E}-5$ & 5.35 & $3.07 \mathrm{E}-5$ & 5.27 & $6.62 \mathrm{E}-5$ & 4.96 \\
$\mathrm{~N}=5$ & $4^{3}$ & $2.88 \mathrm{E}-5$ & - & $7.67 \mathrm{E}-3$ & - & $1.02 \mathrm{E}-2$ & - & $6.44 \mathrm{E}-3$ & - \\
& $6^{3}$ & $2.29 \mathrm{E}-6$ & 6.24 & $4.59 \mathrm{E}-4$ & 6.94 & $7.10 \mathrm{E}-4$ & 6.58 & $5.42 \mathrm{E}-4$ & 6.11 \\
& $8^{3}$ & $4.59 \mathrm{E}-7$ & 5.59 & $6.67 \mathrm{E}-5$ & 6.70 & $1.06 \mathrm{E}-4$ & 6.61 & $1.10 \mathrm{E}-4$ & 5.54 \\
& $12^{3}$ & $3.78 \mathrm{E}-8$ & 6.15 & $5.57 \mathrm{E}-6$ & 6.13 & $8.89 \mathrm{E}-6$ & 6.11 & $1.11 \mathrm{E}-5$ & 5.66 \\
& $16^{3}$ & $6.14 \mathrm{E}-9$ & 6.32 & $1.07 \mathrm{E}-6$ & 5.73 & $1.77 \mathrm{E}-6$ & 5.61 & $2.09 \mathrm{E}-6$ & 5.81 \\
\hline
\end{tabular}

In conclusion, the scheme and its implementation show the expected convergence behavior. 


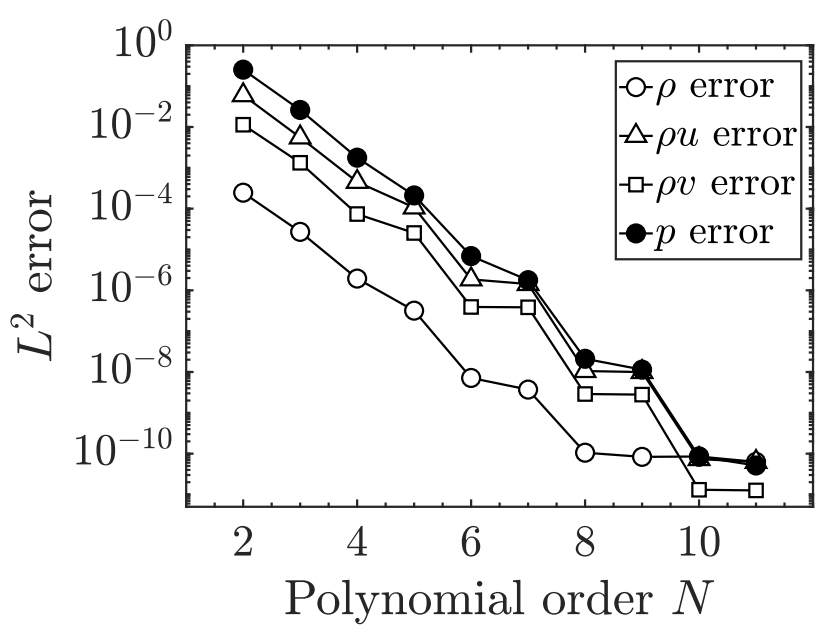

Figure 4.2: $\mathrm{L}^{2}$ error of the Kovasznay test case solution with an $8^{2}$ mesh, for different polynomial orders. We use the split-form scheme with the product of the averages, and the residual threshold in the time integration is $10^{-9}$

\subsubsection{Kovasznay test case}

We investigate the accuracy of the scheme on the Kovasznay two dimensional steady flow problem Kovasznay [1948], with Reynolds number $\operatorname{Re}=40$ on the domain $\Omega=$ $[-0.5,1.5] \times[0,2]$. At the four boundaries (weakly through the exact Riemann solver), we apply the analytical solution,

$$
\begin{aligned}
& \rho_{0}=1, \\
& u_{0}=1-e^{\lambda x} \cos (2 \pi y), \\
& v_{0}=\frac{\lambda}{2 \pi} e^{\lambda x} \sin (2 \pi y), \\
& p_{0}=\frac{1-e^{2 \lambda x}}{2},
\end{aligned}
$$

where $\lambda$ is a parameter related to the Reynolds number $\lambda=\frac{\mathrm{Re}}{2}-\sqrt{\frac{\mathrm{Re}^{2}}{4}+4 \pi^{2}}$. We use a uniform flow with uniform pressure as the initial condition. The mesh is an $8^{2}$ Cartesian grid, and the polynomial order ranges from $N=2$ to $N=11$. The artificial compressibility sound speed is $\rho_{0} c_{0}^{2}=10^{3}$. We use the split-form scheme with the product of the averages, $\mathrm{CFL}=0.75$, and we integrate in time for a residual threshold of $10^{-9}$. The $\mathrm{L}^{2}$ error computed is represented in Fig. 4.2. Although the solution converges to the residual threshold, the convergence rate is non-optimal. This is because the problem is viscous dominant, and the BR1 scheme suffers from even-odd behavior Gassner et al. [2018]. As mentioned in Manzanero et al. [2020d, 2018b], this can be 


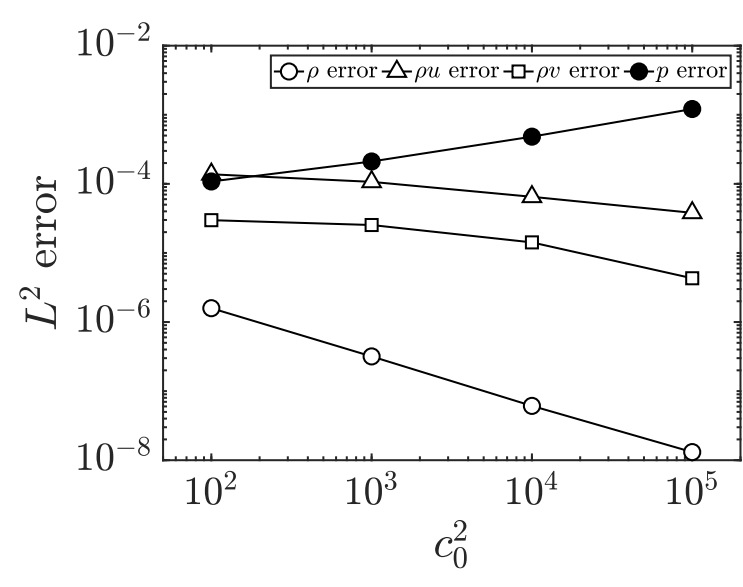

(a) $N=5$

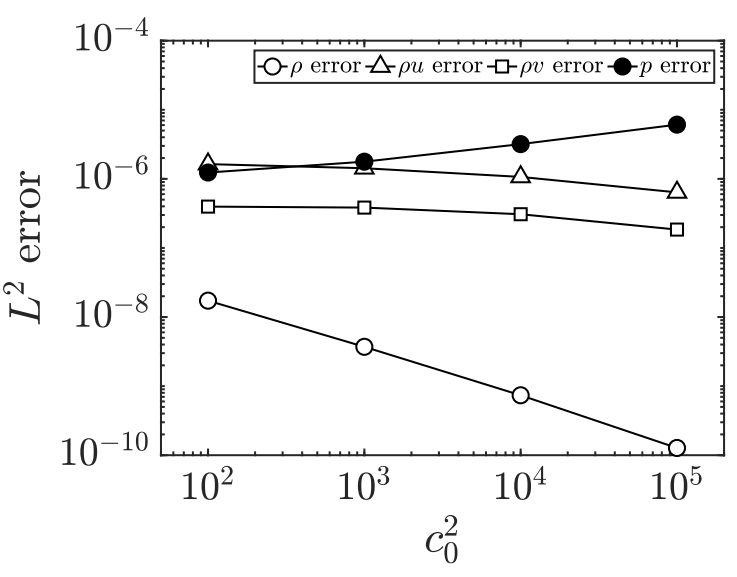

(b) $N=7$

Figure 4.3: $\mathrm{L}^{2}$ error of the Kovasznay test case solution with an $8^{2}$ mesh, for different values of the artificial compressibility sound speed $c_{0}$. We use the split-form scheme with the product of the averages, and the residual threshold in the time integration is $10^{-9}$. Two polynomial orders are considered: $N=5$ and $N=7$

solved by adding interface penalization to the BR1 inter-element fluxes, which, for simplicity, we are not considering here. However, the even-odd effect is minimal for higher Reynolds number flows (e.g. as in the convergence analysis, $R e=1000$ ).

We chose the artificial compressibility sound speed after a study of the errors, shown in Fig. 4.3 for the two polynomial orders $N=5$ and $N=7$. For transient problems, the temporal accuracy is enhanced by maintaining the artificial compressibility sound speed large enough to control velocity divergence errors. For steady-state problems, however, the artificial compressibility sound speed affects the conditioning of the system, which has an impact on the steady-state errors, where $\vec{\nabla} \cdot \vec{u}=0$. For this problem, and for the two polynomial orders studied, we see that increasing $c_{0}$ decreases the errors in the density and the momentum, but increases the errors in the pressure. Therefore, for this problem, the errors are minimized for the artificial compressibility sound speed approximately in the range $\rho_{0} c_{0}^{2} \in\left[10^{2}, 10^{3}\right]$.

\subsubsection{Lid driven cavity}

In this section, we solve the lid driven cavity flow Ghia et al. [1982]; Schranner et al. [2013] at three Reynolds numbers, $R e=1000,5000$, and 7500. We discretize the domain $\Omega=[0,1]^{2}$ into a $20 \times 20$ Cartesian grid, and we use a polynomial order $N=$ 10. This produces a total of $220^{2}$ degrees of freedom, which are fewer than the $257^{2}$ points used in the DNS Ghia et al. [1982]; Schranner et al. [2013]. We apply no-slip 


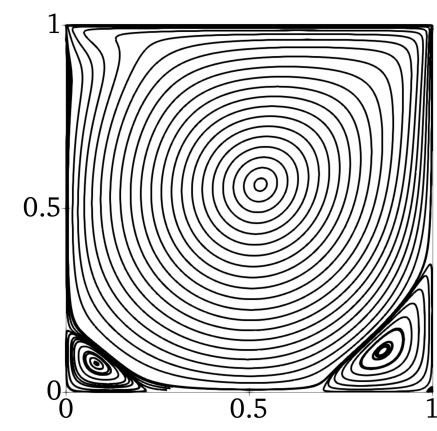

(a) $\operatorname{Re}=1000$

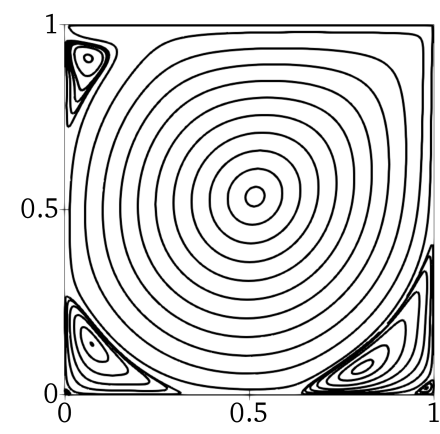

(b) $\operatorname{Re}=5000$

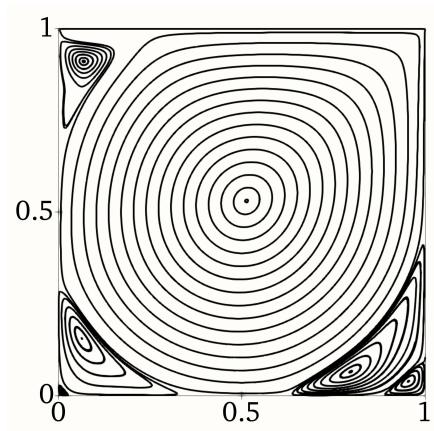

(c) $\operatorname{Re}=7500$

Figure 4.4: Lid driven cavity: streamlines of the solution for the three Reynolds numbers considered.

wall boundary conditions at the four boundaries, but at the top boundary $(y=1)$ the ghost state is modified to impose the lid velocity,

$$
\mathbf{Q}_{n}^{i, \text { lid }}=\left(\begin{array}{c}
\rho \\
\rho U_{n} \\
\rho V_{t 1} \\
\rho V_{t 2} \\
P
\end{array}\right), \quad \mathbf{Q}_{n}^{e, \text { lid }}=\left(\begin{array}{c}
\rho \\
-\rho U_{n} \\
\rho\left(-V_{t 1}+2 \vec{u}_{\text {lid }} \cdot \vec{t}_{1}\right) \\
\rho\left(-V_{t 2}+2 \vec{u}_{\text {lid }} \cdot \vec{t}_{2}\right) \\
P
\end{array}\right)
$$

with $\vec{u}_{\text {lid }}=\vec{e}_{1}$. The artificial compressibility sound speed is $\rho_{0} c_{0}^{2}=10^{3}$, and we use the split-form scheme that takes the product of the averages in the momentum equation.

We use the RK3 method to reach the steady-state solution, with $\Delta t=2.5 \cdot 10^{-5}$, and following Ghia et al. [1982], we stop when the residuals are lower than $10^{-3}$. The final solution is represented in Fig. 4.4 for the three Reynolds numbers studied. In Tables 4.2, 4.3, and 4.4, we tabulate the results corresponding to the center location, size, and vorticities of the different vortical regions. Following the naming used in Ghia et al. [1982], we consider the primary vortex, the top-left vortex (TL), the bottom-left vortex region (BL), and the bottom-right vortex region (BR). Although

Table 4.2: Lid driven cavity with $\mathrm{Re}=1000$

\begin{tabular}{lccc|ccc} 
& \multicolumn{3}{c|}{ This work } & \multicolumn{3}{c}{ Ghia et al. [1982] } \\
& $\left(x_{c}, y_{c}\right)$ & $\omega_{v c}$ & $(H, V)$ & $\left(x_{c}, y_{c}\right)$ & $\omega_{v c}$ & $(H, V)$ \\
\hline Primary & $(0.531,0.565)$ & 2.067 & & $(0.5313,0.5625)$ & 2.04968 \\
$\mathrm{BL}_{1}$ & $(0.083,0.078)$ & -0.350 & $(0.219,0.168)$ & $(0.0859,0.0781)$ & -0.36175 & $(0.2188,0.1680)$ \\
$\mathrm{BR}_{1}$ & $(0.865,0.112)$ & -1.107 & $(0.299,0.354)$ & $(0.8594,0.1094)$ & -1.15465 & $(0.3034,0.3536)$ \\
$\mathrm{BR}_{2}$ & $(0.993,0.007)$ & $6.34 \cdot 10^{-3}$ & $(0.015,0.015)$ & $(0.9922,0.0078)$ & $8.52782 \cdot 10^{-3}$ & $(0.0078,0.0078)$
\end{tabular}


Table 4.3: Lid driven cavity with $\mathrm{Re}=5000$

\begin{tabular}{lccc|cccc} 
& \multicolumn{3}{c|}{ This work } & \multicolumn{3}{c}{ Ghia et al. [1982] } \\
& $\left(x_{c}, y_{c}\right)$ & $\omega_{v c}$ & $(H, V)$ & $\left(x_{c}, y_{c}\right)$ & $\omega_{v c}$ & $(H, V)$ \\
\hline Primary & $(0.515,0.535)$ & 1.835 & & $(0.5117,0.5352)$ & 1.86016 & \\
TL & $(0.063,0.909)$ & -2.135 & $(0.1205,0.256)$ & $(0.0625,0.9102)$ & -2.08843 & $(0.1211,0.2693)$ \\
$\mathrm{BL}_{1}$ & $(0.073,0.137)$ & -1.512 & $(0.313,0.266)$ & $(0.0703,0.1367)$ & -1.53055 & $(0.3184,0.2643)$ \\
$\mathrm{BL}_{2}$ & $(0.007,0.007)$ & 0.007 & $(0.0158,0.0165)$ & $(0.0117,0.0078)$ & 0.0188395 & $(0.0156,0.0163)$ \\
$\mathrm{BR}_{1}$ & $(0.806,0.073)$ & -2.728 & $(0.358,0.416)$ & $(0.8086,0.0742)$ & -2.66354 & $(0.3565,0.4180)$ \\
$\mathrm{BR}_{2}$ & $(0.979,0.018)$ & 0.032 & $(0.052,0.041)$ & $(0.9805,0.0195)$ & 0.0319311 & $(0.0528,0.0417)$
\end{tabular}

Table 4.4: Lid driven cavity with $\mathrm{Re}=7500$

\begin{tabular}{lccc|ccc} 
& \multicolumn{3}{c|}{ This work } & \multicolumn{3}{c}{ Ghia et al. [1982] } \\
& $\left(x_{c}, y_{c}\right)$ & $\omega_{v c}$ & $(H, V)$ & $\left(x_{c}, y_{c}\right)$ & $\omega_{v c}$ & $(H, V)$ \\
\hline Primary & $(0.513,0.531)$ & 1.58886 & & $(0.5117,0.5322)$ & 1.87987 & \\
TL & $(0.068,0.911)$ & -2.32587 & $(0.145,0.297)$ & $(0.0664,0.941)$ & -2.15507 & $(0.1445,0.2993)$ \\
$\mathrm{BL}_{1}$ & $(0.064,0.153)$ & -1.90179 & $(0.335,0.283)$ & $(0.0645,0.1504)$ & -1.78511 & $(0.3339,0.2793)$ \\
$\mathrm{BL}_{2}$ & $(0.011,0.0125)$ & 0.0158559 & $(0.0246,0.0275)$ & $(0.0117,0.0117)$ & 0.0172980 & $(0.0234,0.0254)$ \\
$\mathrm{BR}_{1}$ & $(0.7935,0.0655)$ & -3.17717 & $(0.365,0.4300)$ & $(0.7813,0.0625)$ & -3.49312 & $(0.3779,0.4375)$ \\
$\mathrm{BR}_{2}$ & $(0.953,0.041)$ & 0.154345 & $(0.115,0.091)$ & $(0.9492,0.0430)$ & 0.141058 & $(0.1270,0.0938)$ \\
$\mathrm{BR}_{3}$ & NA & NA & NA & $(0.9961,0.0039)$ & NA & $(0.0039,0.0039)$
\end{tabular}

the methods and equations solved differ considerably (the authors use finite differences with multigrid and they solve a vorticity-stream function formulation) we find an overall good agreement with the reference Ghia et al. [1982] and with other incompressible NSE solvers Schranner et al. [2013], with an absolute difference less than $5 \times 10^{-2}$ in all quantities except for the vorticity in the highest Reynolds number case because of the under-resolution. In very small vortices (e.g. $\mathrm{BL}_{2}$ for $\mathrm{Re}=7500$ ) the errors are higher since the resolution in those regions is not sufficient.

\subsubsection{Inviscid Taylor-Green vortex}

The high-order community has being using the three-dimensional inviscid TaylorGreen Vortex (TGV) as the reference problem to assess the robustness of different methods when computing under-resolved transitional/turbulent flows. The configuration is a three-dimensional periodic box $[-1,1]^{3}$, with the initial condition,

$$
\begin{aligned}
& \rho_{0}=1, \\
& u_{0}=\sin \pi x \cos \pi y \cos \pi z, \\
& v_{0}=-\cos \pi x \sin \pi y \cos \pi z, \\
& w_{0}=0 \\
& p_{0}=\frac{1}{16}(\cos 2 \pi x+\cos 2 \pi y)(2+\cos 2 \pi z),
\end{aligned}
$$




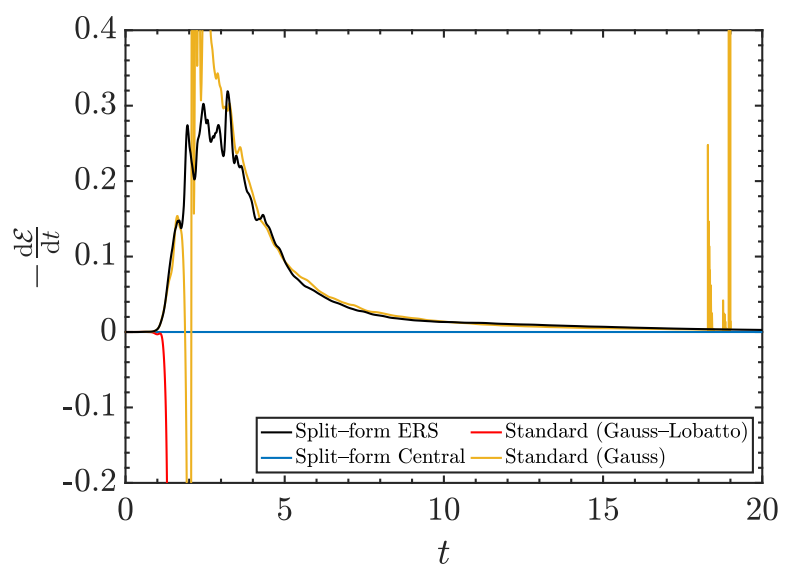

Figure 4.5: Evolution of the entropy time derivative for the four schemes considered

and we keep the artificial compressibility sound speed to $\rho_{0} c_{0}^{2}=10^{3}$.

Since the viscosity is zero, the mathematical entropy $\mathscr{E}$ should be constant according to the continuous bound (4.36), when periodic boundary conditions are applied. The method is stable in the sense that the mathematical entropy, $\mathscr{E}$ (4.12), is bounded.

The purpose of this example is only to show that the method is entropy preserving in under-resolved conditions. We construct a Cartesian $8^{3}$ mesh, approximate the solution with $N=4$ order polynomials, and integrate in time until $t_{F}=20$ using the explicit RK3 scheme and CFL $=0.75$. Using the exact Riemann solver (ERS), we consider the split-form scheme, the standard scheme, and the standard scheme with Gauss points. The first scheme should remain entropy-stable $\left(\mathscr{E}_{t} \leqslant 0\right)$, while the standard DG does not satisfy an entropy law. Additionally, we consider the splitform scheme using the two-point entropy conserving numerical flux (Central), which is entropy conserving $\mathscr{E}_{t}=0$.

The evolution of the (negative) entropy time derivative is represented in Fig. 4.5 for the four schemes considered. On the one hand, the standard scheme is unstable and crashes for both Gauss $(t \approx 19.0)$ and Gauss-Lobatto $(t \approx 1.4)$ points. On the other hand, the split-form scheme is stable using both the ERS and the two-point entropy conserving numerical flux. While the former dissipates entropy at the interelement boundaries, thus $\overline{\mathscr{E}}_{t}^{N} \leqslant 0$, the latter is entropy conserving and $\overline{\mathscr{E}}_{t}^{N}$ is machine precision order at all time instants. We are aware that to get an accurate solution to this problem it is not enough to be entropy preserving, but to dissipate entropy at the appropriate rate. However, we confirm with these findings that using an entropy preserving scheme can be a suitable baseline scheme to which add additional artificial viscosity or LES models as subgrid-scale models Manzanero et al. [2020d]; Flad and Gassner [2017]; Fernandez et al. [2017], which we study in Chapter 9. 


\subsubsection{Rayleigh-Taylor instability}

To assess the robustness of the approximation in more challenging conditions, we solve the (surface tension free) two-dimensional Rayleigh-Taylor instability with Reynolds numbers $\mathrm{Re}=1000$ and $\mathrm{Re}=5000$, and compare our results to those in Bassi et al. [2017] (where the authors used an incompressible solver with artificial viscosity).

The initial condition is a heavy fluid $\left(\rho_{2}=3\right.$ ) placed on top of a lighter one $\left(\rho_{1}=1\right)$. We follow the same configuration used in Guermond and Quartapelle [2000], where the domain is a rectangle $\Omega=[0,0.5] \times[0,4]$, which we discretize following Bassi et al. [2017] using $16 \times 128$ equally-spaced elements with polynomial order $N=6$. The initial position of the interface is regularized following Guermond and Quartapelle [2000],

$$
\rho=2+\tanh \left(\frac{y-\eta(x)}{0.01}\right), \eta(x)=2-0.01 \cos (2 \pi x) .
$$

The boundary conditions are free-slip walls at left and right boundaries, and no-slip walls at bottom and top boundaries. We set the parameter $\rho_{0} c_{0}^{2}=5 \cdot 10^{3}$, the Froude number $\mathrm{Fr}=1$, we use the split-form scheme with the product of the averages, and a fixed time-step $\Delta t=1.5 \cdot 10^{-5}$ for the explicit RK3 scheme.

The approximation should remain stable even for under-resolved flows. Thus, we should not need to include artificial viscosity to stabilize it (although is desirable to enhance the accuracy Manzanero et al. [2020d]). However, the analysis was performed assuming density positivity, which might not be the case if a sharp discontinuity in density is encountered. If the continuity equation is not regularized using a multiphase method (e.g. the Cahn-Hilliard equation Cahn and Hilliard [1958]; Manzanero et al. [2020b], see Chapter 5), the flow around the discontinuity suffers from Gibbs phenomena that eventually leads to negative density values, thus leading to a code crash.

To tackle this problem without increasing the complexity of the underlying physics (i.e. without additional regularization of the density), we enforce a density limiter which is mass and momentum conserving. Leaving the state vector unaltered, we modify the fluxes and instead of dividing by the density to get the velocities (which can be close to zero and lead to divergence), we divide by the limited densities $\tilde{\rho}=f(\rho)$,

$$
\frac{\partial}{\partial t}\left\{\begin{array}{c}
\rho \\
\rho u \\
\rho v \\
\rho w \\
p
\end{array}\right\}+\vec{\nabla} \cdot\left(\begin{array}{ccc}
\rho u & \rho v & \rho w \\
\frac{(\rho u)^{2}}{\tilde{\rho}}+p & \frac{\rho u \cdot \rho v}{\tilde{\rho}} & \frac{\rho u \cdot \rho w}{\tilde{\rho}} \\
\frac{\rho u \cdot \rho v}{\tilde{\rho}} & \frac{(\rho v)^{2}}{\tilde{\rho}}+p & \frac{\rho v \cdot \rho w}{\tilde{\rho}} \\
\frac{\rho u \cdot \rho w}{\tilde{\rho}} & \frac{\rho w \cdot \rho v}{\tilde{\rho}} & \frac{(\rho w)^{2}}{\tilde{\rho}}+p \\
\rho_{0} c_{0}^{2} \frac{\rho u}{\tilde{\rho}} & \rho_{0} c_{0}^{2} \frac{\rho v}{\tilde{\rho}} & \rho_{0} c_{0}^{2} \frac{\rho w}{\tilde{\rho}}
\end{array}\right)=\vec{\nabla} \cdot \overrightarrow{\mathbf{f}}_{v} \cdot
$$




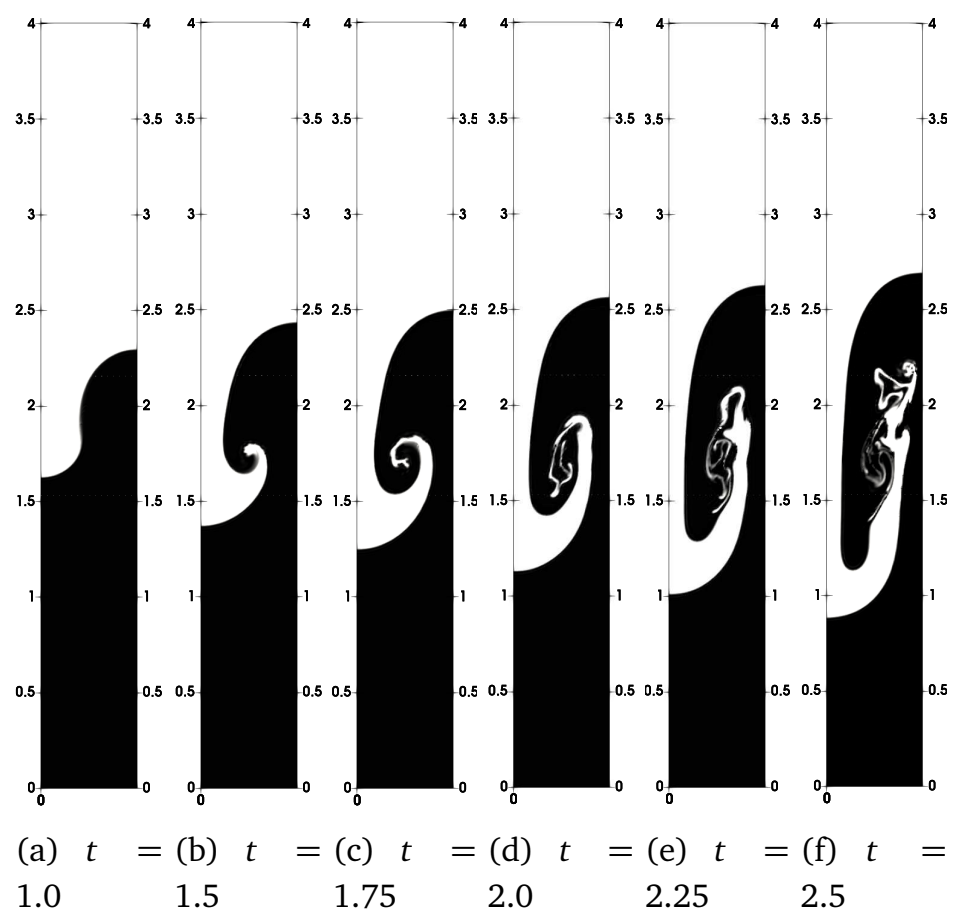

Figure 4.6: Rayleigh-Taylor instability with $\mathrm{Re}=1000$ : density contours. The artificial compressibility sound speed is $\rho_{0} c_{0}^{2}=5 \cdot 10^{3}$

The limited density $\tilde{\rho}$ can be any positive continuous function of the density. In this work, we use

$$
\tilde{\rho}=f(\rho)=\left\{\begin{array}{ccc}
\rho & \text { if } \rho \geqslant \rho_{0} \\
\rho_{0} & \text { if } \rho<\rho_{0}
\end{array} .\right.
$$

With this approach, the density acts as a working variable whose overshoots are controlled using the auxiliary value $\rho_{0}$ in troubled cells. In this work, we set $\rho_{0}=0.9$.

In Fig. 4.6 we represent the density contours for $\mathrm{Re}=1000$ at the same time snapshots shown in Bassi et al. [2017] (in Trygvarsson's time scale $t=\sqrt{2} t_{\text {Tryg }}$ ). In this moderate Reynolds number simulation, the solutions look the same as Bassi et al. [2017], although once the flow becomes under-resolved, the small structures patterns are different due to the artificial viscosity effect. Nonetheless, we find the differences subtle in this lower Reynolds number configuration.

The artificial compressibility sound speed $\rho_{0} c_{0}^{2}=5 \cdot 10^{3}$ was selected after a convergence study. In Fig. 4.7 we represent the isocontour $\rho=2$ for three values of $\rho_{0} c_{0}^{2}$ : 200 (blue), $10^{3}$ (red), and $5 \cdot 10^{3}$ (black). We see that for the well-resolved parts (i.e. the rising and falling vortices), there are large differences only between the smaller $c_{0}$ value (blue) and the rest, and that the differences are subtle between the two large values (red and black). In the badly-resolved regions, there are large differences be- 


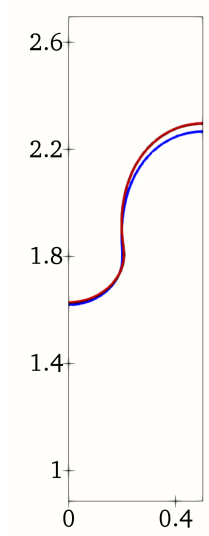

(a) $t=1.0$

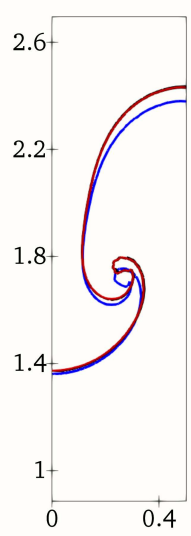

(b) $t=1.5$

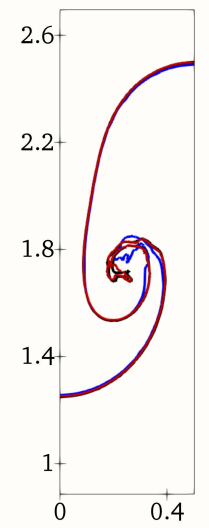

(c) $t=1.75$

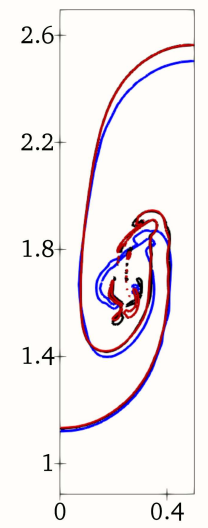

(d) $t=2.0$
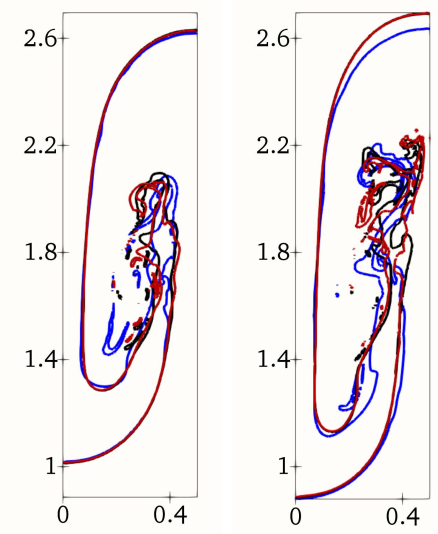

(e) $t=2.25$

(f) $t=2.5$

Figure 4.7: Rayleigh-Taylor instability with $\mathrm{Re}=1000$ : effect of the artificial compressibility $M_{0}$ parameter. We studied the temporal convergence comparing the solution with three different values: $\rho_{0} c_{0}^{2}=200$ (blue), $10^{3}$ (red), $5 \cdot 10^{3}$ (black). We see that $5 \cdot 10^{3}$ represents a converged value in the well-resolved regions, while where the flow is under-resolved, the solution is highly dependent on $c_{0}$

tween the three cases. Due to the low resolution, the small structures unlocked are differently shaped for each $c_{0}$ value, and there is no convergence expected. On general lines, we can confirm that the value $\rho_{0} c_{0}^{2}=5 \cdot 10^{3}$ is small enough to provide accurate transient solutions for this problem.

We represent the same density contours for $\mathrm{Re}=5000$ in Fig. 4.8. The solution presented here looks the same as that presented in Bassi et al. [2017]. However when the solution is under-resolved, $t>1.5$, differences are visible. This is because we have not introduced any artificial dissipation, and thus we can represent smaller scales. By not introducing artificial dissipation, we also confirm the robustness of the method.

Finally, we solve the $\mathrm{Re}=5000$ Rayleigh-Taylor instability, also using the standard DG scheme, and compare the entropy evolution in Fig. 4.9. First we note that the entropy increases due to gravitational force work. While the split-form is stable until the final time (extended here to $t=10.0$ ), the standard scheme crashes close to $t=2.4$, when the flow gets severely under-resolved. Even in the laminar stage of the problem $(t<1.2)$, the standard scheme starts to show minor noise. Not only is the standard scheme less robust, but the amplitude of the oscillations at the under-resolved stages is higher in the standard scheme, as a result of the uncontrolled creation of small structures without any stabilizing mechanism. 


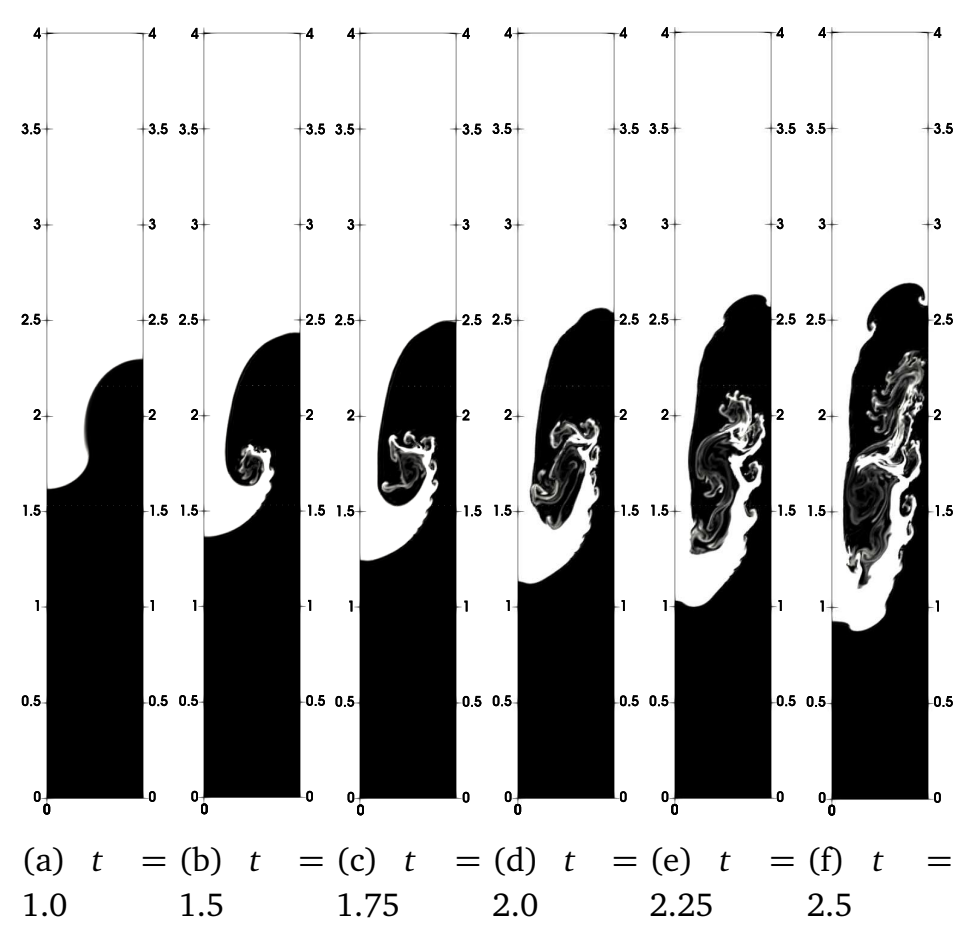

Figure 4.8: Rayleigh-Taylor instability with $\mathrm{Re}=5000$ : density contours. The artificial compressibility sound speed is $\rho_{0} c_{0}^{2}=5 \cdot 10^{3}$

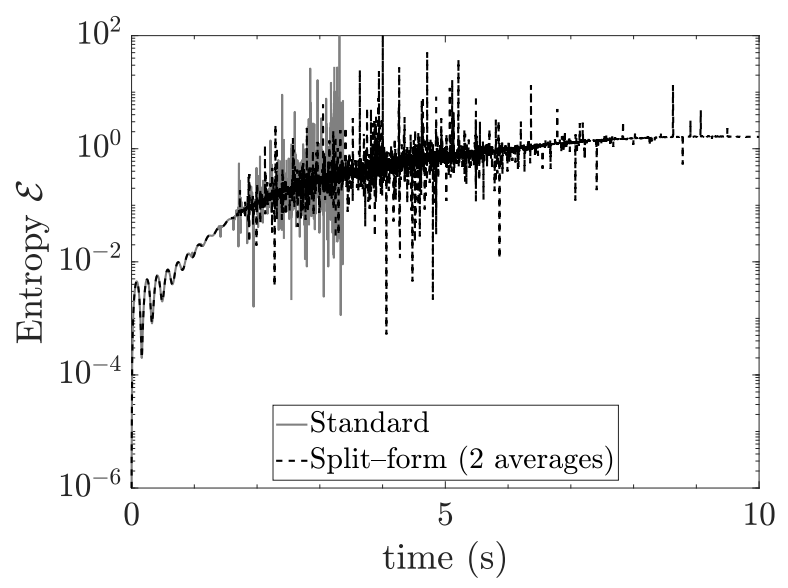

Figure 4.9: Rayleigh-Taylor instability with $\mathrm{Re}=5000$ : time evolution of the entropy (4.12) for the split-form scheme (with the product of the averages in momentum) and the standard DG scheme. We find that not only the standard scheme is unstable and crashes, but also that the amplitude of the oscillations in the under-resolved stages are higher. Although the entropy of the under-resolved split form solution is oscillatory, it is more robust 


\subsection{Summary and conclusions}

We have developed an entropy-stable DG approximation for the incompressible NSE with variable density and artificial compressibility. To do so, we first performed the continuous entropy analysis on a novel entropy function that includes both kinetic energy and artificial compressibility effects. We showed that the particular mathematical entropy for this set of equations is bounded in time, not including gravitational effects. Next, we constructed a DG scheme using Gauss-Lobatto points and the SBP-SAT property that mimics the continuous entropy analysis discretely. This was achieved using a two-point entropy conserving flux, with two options to discretize momentum, and the exact Riemann solver Bassi et al. [2017] at both interior and physical boundaries. For viscous fluxes, we use the Bassi-Rebay 1 (BR1) scheme, which results in a parameterfree numerical discretization. The analysis was completed with the study of the solid wall boundary condition.

Lastly, we tested the numerical convergence of the scheme using manufactured solutions and the Kovasznay flow, and the robustness by solving the inviscid TaylorGreen Vortex (TGV) and the Rayleigh-Taylor Instability (RTI) with Re $=1000$ and $\mathrm{Re}=5000$. We show that the split-form scheme remains entropy-stable (and entropy conserving if we use the two-point entropy conserving flux as the numerical flux) in severely under-resolved conditions, while the standard scheme can solve accurately the $\mathrm{Re}=1000$ problem, but it is unstable and crashes for the inviscid TGV and RTI with $\operatorname{Re}=5000$.

We conclude that:

1. The DGSEM with two-point entropy conserving fluxes and wall boundary conditions is entropy-stable with the exact Riemann solver.

2. Stability is reflected in robustness: the original scheme can be unstable and crashes without the enhancements.

3. Numerical experiments show that the scheme is spectrally accurate, with exponential convergence for smooth flows.

4. Even when they were both stable, the entropy-stable scheme was more accurate.

5. The splitting of the nonlinear terms in the two-point fluxes is not unique. Two splittings were studied. Writing the momentum as the product of two averages was slightly more accurate than using the average of the momentum. Both are entropy-stable, however, with similar computational cost. 



\section{Chapter 5}

\section{The Cahn-Hilliard equation (CHE)}

\section{Contents}

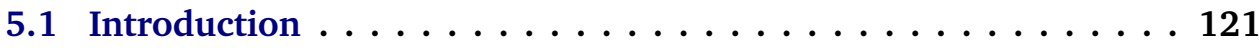

5.2 Cahn-Hilliard equation $\ldots \ldots \ldots \ldots \ldots \ldots \ldots \ldots \ldots \ldots$

5.3 Continuous free-energy stability . . . . . . . . . . . . . 124

5.4 Discontinuous Galerkin spectral element approximation of the

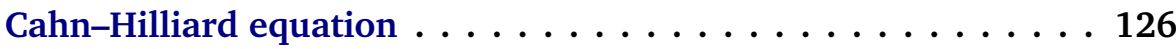

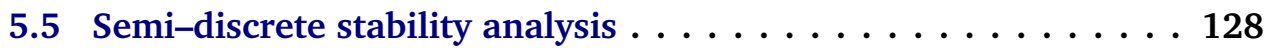

5.6 Fully-discrete stability using an IMEX time integrator $\ldots \ldots$. . 132

5.7 Numerical experiments . . . . . . . . . . . . . . . . . . . 142

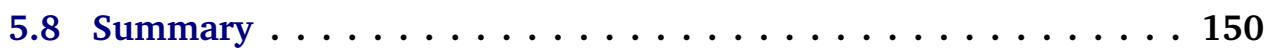

\subsection{Introduction}

Phase field models describe the phase separation dynamics of two immiscible liquids by minimizing a chosen free-energy. For an arbitrary free-energy function, it is possible to construct different phase field models. Among the most popular, one can find the Cahn-Hilliard Cahn and Hilliard [1958] and the Allen-Cahn Allen and Cahn [1972] models. The popularity of the first, despite being a fourth order operator in space, comes from its ability to conserve the phases Lee and Kim [2016].

We present a nodal Discontinuous Galerkin (DG) Spectral Element Method (DGSEM) for the Cahn-Hilliard equation Cahn and Hilliard [1959]. In particular, this work 
uses the Gauss-Lobatto version of the DGSEM, which makes it possible to construct energy-stable schemes using the summation-by-parts simultaneous-aproximationterm (SBP-SAT) property. Moreover, it handles arbitrary three dimensional curvilinear hexahedral meshes while maintaining high-order spectral accuracy and free-energy stability. An alternative, which is not considered in this work, but has been studied in Xia et al. [2007], is to use consistent integration in all quadratures used in the weak-formulation.

We present a free-energy stable approximation for the Cahn-Hilliard equation. The stability analysis presented here is both semi-discrete (assuming exact integration in time) and fully-discrete (i.e. considering the discrete approximation of space and time). Since the Cahn-Hilliard equation is fourth order in space, the numerical stiffness of the scheme leads to impractical time step limitations when using explicit methods. Therefore, we study the stability of a first order IMplicit-EXplicit (IMEX) approximation in time, which has been previously used for the Cahn-Hilliard equation Dong [2018a], and whose efficiency is similar to that of explicit methods. This is possible since the scheme is designed to have a constant (in time) coefficient matrix of the linear system. Without loss of generality, we use LU factorization and Gauss elimination, but iterative solvers could be used.

The rest of this chapter is organised as follows: In Sec. 5.2, we introduce the CahnHilliard equation, and describe the continuous energy estimate in Sec. 5.3. Next, in Sec. 5.4, we construct the DG approximation. We perform the energy analysis in a semi-discrete fashion in Sec. 5.5 and fully-discrete in Sec. 5.6. Lastly, we provide numerical experiments in Sec. 5.7 that assess the capabilities of the method.

\subsection{Cahn-Hilliard equation}

In this section we give a brief description of the Cahn-Hilliard equation and its properties. Among the different Cahn-Hilliard settings, we solve the constant mobility model, with polynomic double-well chemical free-energy, and we restrict ourselves to two-phase flows Dong [2014a]; Yang et al. [2019]. The Cahn-Hilliard equation describes the phase separation dynamics of binary alloys or two phase flows. The concentration of phase $1, c$, satisfies the evolution equation

$$
c_{t}=\vec{\nabla} \cdot\left(M_{0} \vec{\nabla} \mu\right), \quad \text { in } \Omega,
$$

where $M_{0}$ is a positive parameter named mobility, $\Omega$ is the physical domain (with boundaries $\partial \Omega$ ), and $\mu$ is a scalar field called the chemical potential. The chemical energy is designed to minimize an arbitrary free-energy functional, $\overline{\mathscr{F}}(c, \vec{\nabla} c)$, which 
depends on the phase field and its gradient,

$$
\mu=\frac{\delta \bar{F}}{\delta c}
$$

For the chemical potential, $\mu$, we apply a homogeneous Neumann boundary condition that guarantees the mass conservation,

$$
\left.\vec{\nabla} \mu \cdot \vec{n}\right|_{\partial \Omega}=0
$$

The free-energy is constructed so that two opposing effects balance: the chemical free-energy, $f_{0}(c)$, which favors phase separation, and the interfacial energy $\frac{3}{4} \sigma \varepsilon|\vec{\nabla} c|^{2}$, which favors homogenization,

$$
\overline{\mathscr{F}}(c, \vec{\nabla} c)=\langle\mathscr{F}(c, \vec{\nabla} c)\rangle+\int_{\partial \Omega} \mathscr{F}^{S}(c) \mathrm{d} S=\int_{\Omega}\left(f_{0}(c)+\frac{3}{4} \sigma \varepsilon|\vec{\nabla} c|^{2}\right) \mathrm{d} \vec{x}+\int_{\partial \Omega} f_{w}(c) \mathrm{d} S .
$$

In (5.4) $\mathscr{F}$ is the free-energy, $\mathscr{F}^{S}$ is the surface free-energy, and $\mathscr{F}$ is the total freeenergy. Moreover, $f_{w}(c)$ represents a boundary free-energy that will also be minimized with appropriate boundary conditions, $\sigma$ is the interface tension coefficient, and $\varepsilon$ is a positive constant that controls the thickness of the (diffuse) interface.

To perform the minimization, one linearizes the free-energy (5.4) around an equilibrium solution,

$$
\delta \bar{F}=\int_{\Omega}\left(\frac{\mathrm{d} f_{0}}{\mathrm{~d} c} \delta c+\frac{3}{2} \sigma \varepsilon \vec{\nabla} c \cdot \vec{\nabla}(\delta c)\right) \mathrm{d} \vec{x}+\int_{\partial \Omega} \frac{\mathrm{d} f_{w}}{\mathrm{~d} c} \delta c \mathrm{~d} S
$$

where $\delta c$ is a small perturbation. Since we will also apply Neumann boundary conditions for $c$, the perturbation $\delta c$ is not restricted to vanish at the boundaries $\partial \Omega$. We integrate the second term of the first integral in (5.5) by parts,

$$
\delta \bar{F}=\int_{\Omega}\left(\frac{\mathrm{d} f_{0}}{\mathrm{~d} c}-\frac{3}{2} \sigma \varepsilon \vec{\nabla}^{2} c\right) \delta c \mathrm{~d} \vec{x}+\int_{\partial \Omega}\left(\frac{\mathrm{d} f_{w}}{\mathrm{~d} c}+\frac{3}{2} \sigma \varepsilon \vec{\nabla} c \cdot \vec{n}\right) \delta c \mathrm{~d} S,
$$

which infers both the chemical potential definition from the volume term,

$$
\mu=\frac{\mathrm{d} f_{0}}{\mathrm{~d} c}-\frac{3}{2} \sigma \varepsilon \vec{\nabla}^{2} c=\frac{24 \sigma}{\varepsilon}\left(c(1-c)^{2}-c^{2}(1-c)\right)-\frac{3}{2} \sigma \varepsilon \vec{\nabla}^{2} c,
$$

and the appropriate Neumann boundary conditions prescription from the surface term,

$$
-\left.\frac{3}{2} \sigma \varepsilon \vec{\nabla} c \cdot \vec{n}\right|_{\partial \Omega}=\frac{\mathrm{d} f_{w}}{\mathrm{~d} c}
$$


In this chapter we use the polynomial double-well function for the chemical freeenergy Cahn and Hilliard [1958],

$$
f_{0}(c)=\frac{12 \sigma}{\varepsilon} c^{2}(1-c)^{2},
$$

and a linear function for the boundary energy,

$$
f_{w}(c)=\beta c,
$$

since they represent standard choices in the literature, but other choices that are not covered here exist (e.g. the logarithmic chemical free-energy Debussche and Dettori [1995]).

\subsection{Continuous free-energy stability}

The Cahn-Hilliard is a non-linear conservative system, with $\mathbf{q}=c, \overrightarrow{\mathbf{f}}_{e}(\mathbf{q})=0$, and $\overrightarrow{\mathbf{f}}_{v}(\mathbf{q}, \vec{\nabla} \mathbf{w})=M_{0} \vec{\nabla} \mu$. Therefore, the entropy analysis presented in this section is a particularization of the general stability analysis studied in Sec. 3.5, where the inviscid fluxes are not involved. The stability analysis of the Cahn-Hilliard equation features a mathematical entropy function that depends on the state gradient, which entails the need of an surface entropy (free-energy) $\mathscr{F}^{S}=f_{w}$ to contract the time coefficients (3.71). Following Cahn and Hilliard [1958], we call free-energy $\mathscr{F}(c, \vec{\nabla} c)$ the mathematical entropy.

We first show that the free-energy is bounded by that computed with a given initial condition. The minimization of the free-energy (5.6) suggests the use of the chemical potential $\mu$ as entropy variable, $\mathbf{w}=\mu$, since $\delta \mathscr{F}=\langle\mu, \delta c\rangle$, ignoring surface terms. Therefore, we construct the inner product of the Cahn-Hilliard equation (5.1) with the chemical potential,

$$
\left\langle c_{t}, \mu\right\rangle=\left\langle\vec{\nabla} \cdot\left(M_{0} \vec{\nabla} \mu\right), \mu\right\rangle=\int_{\partial \Omega} M_{0} \mu \vec{\nabla} \mu \cdot \mathrm{d} \vec{S}-\left\langle M_{0}|\vec{\nabla} \mu|^{2}\right\rangle,
$$

which is the particular version of (3.76) for the Cahn-Hilliard equation.

We show that the Cahn-Hilliard equations satisfies the three properties of the general entropy analysis: time coefficients contraction (3.71), inviscid terms contraction (3.72) (not needed in this case since there are no inviscid fluxes involved), and viscous fluxes positive definiteness (3.74). 


\subsubsection{Time coefficients contraction}

We derive the particular version of (3.71) for the Cahn-Hilliard equation. To transform (5.11) similarly to (3.71), so that we get the time derivative of the free-energy, and an additional time entropy flux, we construct a second inner product from the definition of the chemical potential (5.7) and the concentration time derivative,

$$
\begin{aligned}
\left\langle\mu, c_{t}\right\rangle & =\left\langle\frac{\mathrm{d} f_{0}}{\mathrm{~d} c}-\frac{3}{2} \sigma \varepsilon \vec{\nabla}^{2} c, c_{t}\right\rangle=\left\langle\frac{\mathrm{d} f_{0}}{\mathrm{~d} c}, c_{t}\right\rangle+\left\langle\frac{3}{2} \sigma \varepsilon \vec{\nabla} c, \vec{\nabla} c_{t}\right\rangle-\int_{\partial \Omega} \frac{3}{2} \sigma \varepsilon c_{t} \vec{\nabla} c \cdot \mathrm{d} \vec{S} \\
& =\frac{\mathrm{d}}{\mathrm{d} t}\left\langle f_{0}+\frac{3}{4} \sigma \varepsilon|\vec{\nabla} c|^{2}\right\rangle+\int_{\partial \Omega} c_{t} \frac{\mathrm{d} f_{w}}{\mathrm{~d} c} \mathrm{~d} S=\frac{\mathrm{d}}{\mathrm{d} t}\left(\langle\mathscr{F}\rangle+\int_{\partial \Omega} f_{w} \mathrm{~d} S\right)=\overline{\mathscr{F}}_{t} .
\end{aligned}
$$

In the second identity, we have integrated the interfacial energy term by parts, to get the surface integral that is shaped to the surface free-energy. In the third identity, we have used the chain rule in time for the chemical free-energy and the interfacial energy, and applied the non-homogeneous Neumann boundary condition for the concentration gradient (5.8) of the surface integral. In the last identity, we have again used the chain rule in time for the surface integral. As a result, we find that the contraction of the time derivative coefficients is transformed to the time derivative of the total free-energy $\mathscr{F}$ (5.4). Eq. (5.12) is the particular version of (3.71) for the Cahn-Hilliard equation.

\subsubsection{Inviscid terms contraction}

The Cahn-Hilliard equation does not feature an inviscid flux, so

$$
\vec{f}_{e}^{\mathscr{E}}=0
$$

\subsubsection{Viscous fluxes positive definiteness}

We easily see that the viscous flux $\vec{f}_{v}(c, \vec{\nabla} \mu)=M_{0} \vec{\nabla} \mu$ is positive definite, since $\underline{\mathbf{B}}=M_{0}>0$. Therefore, the particular version of (3.74) for the Cahn-Hilliard equation is

$$
\langle\underline{\mathbf{B}} \vec{\nabla} \mathbf{w}, \vec{\nabla} \mathbf{w}\rangle=\left\langle M_{0}|\vec{\nabla} \mu|^{2}\right\rangle \geqslant 0 .
$$

The viscous entropy flux for the Cahn-Hilliard equation is

$$
\vec{f}_{v}^{\mathscr{E}}=\mathbf{w}^{T} \overrightarrow{\mathbf{f}}_{v}=M_{0} \mu \vec{\nabla} \mu=0,
$$


which vanishes at the boundaries as a result of the homogeneous Neumann boundary condition for the chemical potential (5.3).

Then, the free-energy equation for the Cahn-Hilliard equation is the general entropy equation for a conservation law (3.77), with the particular choice of the entropy (free-energy (5.4)), and the resulting inviscid (5.13) and viscous (5.15) entropy fluxes and volume terms (5.15),

$$
\overline{\mathscr{F}}_{t}=-\left\langle M_{0}|\vec{\nabla} \mu|^{2}\right\rangle \leqslant 0,
$$

which we can integrate in time to show that

$$
\overline{\mathscr{F}}(T)=\overline{\mathscr{F}}(0)-\int_{0}^{T}\left\langle M_{0}|\vec{\nabla} \mu|^{2}\right\rangle \mathrm{d} t \leqslant \overline{\mathscr{F}}(0) .
$$

As a result, the Cahn-Hilliard equation (5.1) with chemical potential (5.7) and Neumann boundary conditions (5.3) and (5.8) guarantees that the free-energy $\mathscr{F}$, defined in (5.4), is bounded in time, and is the property to be mimicked by the subsequent approximation.

\subsection{Discontinuous Galerkin spectral element approximation of the Cahn-Hilliard equation}

We construct the Cahn-Hilliard discrete approximation. Note that the Cahn-Hilliard equation solves only one scalar equation $\left(N_{E}=1\right)$. Thus, state vectors (bold) are scalar fields, and block vectors (bold and arrow) are simply space vectors (with an arrow). The Cahn-Hilliard equation is a particular conservation law. Thus, we only need to write the general DG scheme (2.66) with $\mathbf{Q}=C, \mathbf{W}=\mu, \overrightarrow{\mathbf{f}}_{e}=0$, and $\overrightarrow{\mathbf{f}}_{v}=M_{0} \overrightarrow{\mathbf{G}}$ (recall that $\overrightarrow{\mathbf{G}}$ is the gradient of the entropy variables, i.e. the chemical potential),

$$
\begin{aligned}
& \left\langle\mathscr{J} C_{t}, \phi\right\rangle_{E, N}-\int_{\partial E, N} \phi^{T}\left(M_{0} \overrightarrow{\tilde{G}}\right)^{\star} \cdot \hat{n} \mathrm{~d} \hat{S}=-\left\langle M_{0} \overrightarrow{\tilde{G}}, \vec{\nabla}_{\xi} \phi\right\rangle_{E, N}, \\
& \langle\mathscr{J} \vec{G}, \vec{\varphi}\rangle_{E, N}=\int_{\partial E, N} \mu^{\star, T} \overrightarrow{\tilde{\varphi}} \cdot \hat{n} \mathrm{~d} \hat{S}-\left\langle\mu, \vec{\nabla} \vec{\nabla}_{\xi} \cdot \overrightarrow{\tilde{\varphi}}\right\rangle_{E, N} .
\end{aligned}
$$

For the Cahn-Hilliard equation, however, the discretization (5.18) is incomplete, as it assumes that the discrete approximation of the chemical potential is known. To compute the approximation of the chemical potential $\mu$, we regard the chemical potential definition equation,

$$
\mu=\frac{\mathrm{d} f_{0}}{\mathrm{~d} c}-\vec{\nabla} \cdot\left(\frac{3}{2} \sigma \varepsilon \vec{\nabla} c\right)
$$


as a conservative system too. For this equation there is no state vector, $\mathbf{q}=0$ (since it does not involve time derivatives), the inviscid flux is zero $\overrightarrow{\mathbf{f}}_{e}=0$, the gradient variable is $\mathbf{w}=c$, whose gradient is $\vec{g}_{c}=\vec{\nabla} c$, and the chemical potential and chemical free-energy terms are simply treated as source terms. Thus, the DG approximation of (5.19) is adapted from the general DG scheme (2.66),

$$
\begin{aligned}
\left\langle\mathscr{J} \mu, \phi_{\mu}\right\rangle_{E, N} & =\left\langle\mathscr{J} \frac{\mathrm{d} F_{0}}{\mathrm{~d} c}, \phi_{\mu}\right\rangle_{E, N}-\frac{3}{2} \sigma \varepsilon \int_{\partial e, N} \phi_{\mu} \vec{G}_{c}^{\star} \cdot \mathrm{d} \vec{S}+\frac{3}{2} \sigma \varepsilon\left\langle\overrightarrow{\tilde{G}}_{c}, \vec{\nabla}_{\xi} \phi_{\mu}\right\rangle_{E, N}, \\
\left\langle\mathscr{J} \vec{G}_{c}, \vec{\phi}_{G_{c}}\right\rangle_{E, N} & =\int_{\partial e, N} C^{\star} \vec{\phi}_{G_{c}} \cdot \mathrm{d} \vec{S}-\left\langle C, \vec{\nabla}_{\xi} \cdot \overrightarrow{\tilde{\phi}}_{G_{c}}\right\rangle_{E, N} .
\end{aligned}
$$

For the gradient interface numerical fluxes, we use the Bassi-Rebay 1 scheme (BR1) Bassi and Rebay [1997],

$$
\mu^{\star}=\{\{\mu\}\}, C^{\star}=\{\{C\}\},
$$

whereas for the divergence weak forms, we propose to augment the averages used in the BR1 scheme with additional interface dissipation,

$$
\begin{aligned}
\left(M_{0} \vec{G}\right)^{\star} & =\left\{\left\{M_{0} \vec{G}\right\}\right\}-\beta_{\mu} M_{0}\left(\mu_{L} \vec{n}_{L}+\mu_{R} \vec{n}_{R}\right), \\
\vec{G}_{c}^{\star} & =\left\{\left\{\vec{G}_{c}\right\}\right\}-\beta_{c}\left(C_{L} \vec{n}_{L}+C_{R} \vec{n}_{R}\right),
\end{aligned}
$$

where $\beta_{\mu}$ and $\beta_{c}$ are positive penalty parameters $\left(\beta_{i} \geqslant 0\right)$.

For the penalty parameters $\beta_{i}$, we propose to use the estimate introduced for the DGSEM-GL variant in Manzanero et al. [2018b],

$$
\beta_{i}=\kappa_{\beta i} \frac{N(N+1)}{2}\left|\mathscr{J}_{f}\right|\left\{\left\{\mathscr{J}^{-1}\right\}\right\}
$$

which has only one dimensionless free parameter, $\kappa_{\beta i}$ (also positive), and the dependency of $\beta_{i}$ on the mesh and the polynomial order are taken into account explicitly. In (5.23), $N$ is the polynomial order, $\left|\mathscr{J}_{f}\right|$ is the surface Jacobian of the face, and $\left\{\left\{\mathscr{J}^{-1}\right\}\right\}$ is the average of the inverse of the Jacobians of the elements that share the face evaluated at the face. It will be shown that the scheme is stable without interface stabilization. Nevertheless, the advantage of adding interface stabilization will be demonstrated with a numerical example.

For the Neumann physical boundary conditions, we use the adjacent element interior value to compute the gradients in (5.18) and (5.20),

$$
\mu^{\star}=\left.\mu\right|_{\partial e}, \quad C^{\star}=\left.C\right|_{\partial e}
$$


and directly impose Neumann boundary values for the fluxes,

$$
\left(M_{0} \vec{G}\right)^{\star} \cdot \vec{n}=0,-\frac{3}{2} \sigma \varepsilon \vec{G}_{c}^{\star} \cdot \vec{n}=\frac{\mathrm{d} f_{w}\left(\left.C\right|_{\partial e}\right)}{\mathrm{d} c}=\beta .
$$

With (5.18) and (5.20), and the numerical fluxes, the semi-discrete approximation is completed. The DG approximation used solves a system of four first order equations, where we first compute the gradient of the concentration and the chemical potential in (5.20), and then the gradient of the chemical potential and then we compute the Cahn-Hilliard equation in (5.18).

\subsection{Semi-discrete stability analysis}

We have identified the Cahn-Hilliard equation as a conservative system that is provably stable. In Sec. 5.3 we have demonstrated its entropy (free-energy) stability showing that it satisfies the three properties: time coefficients contraction (3.71), inviscid terms contraction (3.72), and viscous fluxes positive definiteness (3.74). We now show that we can formulate these steps discretely.

From the general entropy weak form (3.81), we get the particular form for the Cahn-Hilliard equation scheme (5.18), replacing $\phi=\mathbf{W}=\mu$ and $\vec{\varphi}=\overrightarrow{\mathbf{F}}_{v}=M_{0} \vec{G}$,

$$
\left\langle\mathscr{J} C_{t}, \mu\right\rangle_{E, N}+\int_{\partial E, N}\left(\mu\left(M_{0} \overrightarrow{\tilde{G}}-\left(M_{0} \overrightarrow{\tilde{G}}\right)^{\star}\right)-\mu^{\star} M_{0} \overrightarrow{\tilde{G}}\right) \cdot \hat{n} \mathrm{~d} \hat{S}=-\left\langle\mathscr{J} \vec{G}, M_{0} \vec{G}\right\rangle_{E, N}
$$

The first volume integral is the discrete version of (5.12), which has to be shaped into the time derivative of the free-energy following (3.83). The boundary integral will yield the particular version of interior boundary terms (3.96) and physical boundary terms (3.96) for the Cahn-Hilliard equation. The last volume integral is the discrete version of (5.14) and it is positive as a result of the viscous fluxes positive definiteness.

\subsubsection{Contraction of the time coefficients}

We get a discrete equivalent of (5.12), which is a particular version of the general contraction (3.83). Precisely, we will show how to get the surface term in the time contraction $\vec{H}^{\mathscr{E}}$, as a result of the dependency of the free-energy with solution gradients.

In the continuous analysis, we constructed the inner product of the chemical potential definition, and the concentration time derivative. Discretely, we replace $\phi_{\mu}=C_{t}$ 
on the first weak form of (5.20),

$$
\left\langle\mathscr{J} \mu, C_{t}\right\rangle_{E, N}=\left\langle\mathscr{J} \frac{\mathrm{d} F_{0}}{\mathrm{~d} c}, C_{t}\right\rangle_{E, N}-\frac{3}{2} \sigma \varepsilon \int_{\partial e, N} C_{t} \vec{G}_{c}^{\star} \cdot \mathrm{d} \vec{S}+\frac{3}{2} \sigma \varepsilon\left\langle\overrightarrow{\tilde{G}}_{c}, \vec{\nabla}_{\xi} C_{t}\right\rangle_{E, N} .
$$

The first volume integral is the contraction of the time coefficients we want to compute. The second volume integral can use the chain rule in time to get the time derivative of the chemical free-energy $\left\langle\mathscr{J}\left(F_{0}\right)_{t}\right\rangle_{E, N}$. We need to transform the last volume integral into the time derivative of the interfacial energy. To do so, we take the time derivative of the second weak form in (5.20), that defines the concentration gradient, and also we use the discrete Gauss law to get

$$
\left\langle\mathscr{J} \vec{G}_{c, t}, \vec{\phi}_{G_{c}}\right\rangle_{E, N}=\int_{\partial e, N}\left(C_{t}^{\star}-C_{t}\right) \vec{\phi}_{G_{c}} \cdot \mathrm{d} \vec{S}+\left\langle\vec{\nabla}_{\xi} C_{t}, \overrightarrow{\tilde{\phi}}_{G_{c}}\right\rangle_{E, N} .
$$

In (5.28), if we use $\phi_{G_{c}}=\vec{G}_{c}$, we identify the last volume integral as the last integral from (5.27) that we want to compute,

$$
\left\langle\vec{\nabla}_{\xi} C_{t}, \overrightarrow{\tilde{G}}_{c}\right\rangle_{E, N}=\left\langle\mathscr{J} \vec{G}_{c, t}, \vec{G}_{c}\right\rangle_{E, N}-\int_{\partial e, N}\left(C_{t}^{\star}-C_{t}\right) \vec{G}_{c} \cdot \mathrm{d} \vec{S}
$$

We replace (5.29) in (5.27) to get the discrete contraction of the time coefficients for the Cahn-Hilliard equation,

$$
\begin{aligned}
\left\langle\mathscr{J} \mu, C_{t}\right\rangle_{E, N} & =\left\langle\mathscr{J} F_{0, t}\right\rangle_{E, N}+\frac{3}{2} \sigma \varepsilon\left\langle\mathscr{J} \vec{G}_{c, t}, \vec{G}_{c}\right\rangle_{E, N}+\frac{3}{2} \sigma \varepsilon \int_{\partial e, N}\left(C_{t} \vec{G}_{c}-C_{t}^{\star} \vec{G}_{c}-C_{t} \vec{G}_{c}^{\star}\right) \cdot \mathrm{d} \vec{S} \\
& =\frac{\mathrm{d}}{\mathrm{d} t}\left\langle\mathscr{J}\left(F_{0}+\frac{3}{4} \sigma \varepsilon\left|\vec{G}_{c}\right|^{2}\right)\right\rangle_{E, N}+\frac{3}{2} \sigma \varepsilon \int_{\partial e, N}\left(C_{t} \vec{G}_{c}-C_{t}^{\star} \vec{G}_{c}-C_{t} \vec{G}_{c}^{\star}\right) \cdot \mathrm{d} \vec{S} \\
& =\frac{\mathrm{d}}{\mathrm{d} t}\langle\mathscr{J} \mathscr{F}\rangle_{E, N}+\int_{\partial e, N} \vec{H}^{\mathscr{E}} \cdot \mathrm{d} \vec{S} .
\end{aligned}
$$

Eq. (5.30) is the particular version of the general time contraction (3.83) for the CahnHilliard equation. We identify the surface term in the time contraction

$$
\vec{H}^{\mathscr{E}}=\frac{3}{2} \sigma \varepsilon\left(C_{t} \vec{G}_{c}-C_{t}^{\star} \vec{G}_{c}-C_{t} \vec{G}_{c}^{\star}\right) .
$$

We sum all the element contributions of (5.30) to find

$$
\sum_{e}\left\langle\mathscr{J} \mu, C_{t}\right\rangle_{E, N}=\frac{\mathrm{d}}{\mathrm{d} t} \sum_{e}\langle\mathscr{J} \mathscr{F}\rangle_{E, N}+\sum_{\substack{\text { interior } \\ \text { faces }}} \int_{f, N} \llbracket-\vec{H}^{\mathscr{E}} \rrbracket \cdot \vec{n}_{L} \mathrm{~d} S+\sum_{\substack{\text { boundary } \\ \text { faces }}} \int_{f} \vec{H}^{\mathscr{E}} \cdot \mathrm{d} \vec{S} .
$$


To arrive at the time derivative of the total free-energy (3.89), we have to transform the interior faces integral following (3.86), and the physical boundary faces integral as in (3.87). For the interior faces sum, we consider the numerical fluxes (5.21) and (5.22),

$$
\begin{aligned}
\llbracket \overrightarrow{-H}^{\mathscr{E}} \rrbracket \cdot \vec{n}_{L} & \left.=\frac{3}{2} \sigma \varepsilon\left(\left(\left\{C_{t}\right\}\right\} \llbracket \vec{G}_{c} \rrbracket+\llbracket C_{t} \rrbracket\left\{\left\{\vec{G}_{c}\right\}\right\}-\llbracket C_{t} \vec{G}_{c} \rrbracket\right) \cdot \vec{n}_{L}+\beta_{c} \llbracket C_{t} \rrbracket \llbracket C \rrbracket\right) \\
& =\frac{3}{2} \sigma \varepsilon \beta_{c} \llbracket C_{t} \rrbracket \llbracket C \rrbracket=\frac{\mathrm{d}}{\mathrm{d} t}\left(\frac{3}{4} \sigma \varepsilon \beta_{c} \llbracket C \rrbracket^{2}\right)=\left(\mathscr{F}_{i}^{S}\right)_{t}
\end{aligned}
$$

thus, we identify the positive interior faces free-energy $\mathscr{F}_{i}^{S}=\frac{3}{4} \sigma \varepsilon \beta_{c} \llbracket C \rrbracket^{2}$. The first three terms in (5.33) vanish as a result of the algebraic relationship between the average and jump operators (1.27).

For the physical boundary faces sum, we apply the non-homogeneous Neumann boundary condition (5.24) and (5.25),

$$
\vec{H}^{\mathscr{E}} \cdot \vec{n}=\frac{3}{2} \sigma \varepsilon\left(C_{t} \vec{G}_{c}-C_{t} \vec{G}_{c}\right)+C_{t} \frac{\mathrm{d} F_{w}}{\mathrm{~d} C}=\left(F_{w}\right)_{t}=\mathscr{F}_{t}^{S} .
$$

Therefore, we infer the total discrete free-energy $\overline{\mathscr{F}}^{N}$,

$$
\mathscr{F}^{N}=\sum_{e}\langle\mathscr{J} \mathscr{F}\rangle_{E, N}+\sum_{\substack{\text { interior } \\ \text { faces }}} \int_{f, N} \frac{3}{4} \sigma \varepsilon \beta_{c} \llbracket C \rrbracket^{2} \mathrm{~d} S+\sum_{\substack{\text { boundary } \\ \text { faces }}} \int_{f, N} F_{w} \mathrm{~d} S \geqslant 0,
$$

which satisfies the contraction of the time coefficients,

$$
\sum_{e}\left\langle\mathscr{J} \mathbf{Q}_{t}, \mathbf{W}\right\rangle_{E, N}=\sum_{e}\left\langle\mathscr{J} C_{t}, \mu\right\rangle_{E, N}=\mathscr{F}_{t}^{N} .
$$

\subsubsection{Contraction of the inviscid fluxes}

The Cahn-Hilliard does not involve inviscid fluxes, so $\overrightarrow{\mathbf{F}}_{e}=0$.

\subsubsection{Positive semi-definiteness of the viscous fluxes}

We study the contribution of viscous fluxes to the free-energy equation (5.26). Thus, the particular form of (3.93) for the Cahn-Hilliard equation is

$$
\langle\mathscr{J} \overrightarrow{\mathbf{G}}, \underline{\mathbf{B}} \overrightarrow{\mathbf{G}}\rangle_{E, N}=\left\langle\mathscr{J} M_{0}|\vec{G}|^{2}\right\rangle_{E, N} \geqslant 0 .
$$

The positive definiteness of the viscous fluxes dissipates the free-energy in the interior of the elements. 
As a result of the contraction of time coefficients (5.36) and the positive definiteness of viscous fluxes (5.36), we get the discrete free-energy equation, which is the particular form of (3.99),

$$
\mathscr{\mathscr { F }}_{t}^{N}+\mathrm{PBT}=-\mathrm{IBT}-\sum_{e}\left\langle\mathscr{J} M_{0}|\vec{G}|^{2}\right\rangle_{E, N}
$$

and where the interior and physical boundary terms are (5.26) (which coincide with their particular version of the general analysis (3.96) and (3.98)),

$$
\begin{aligned}
& \text { IBT }=\sum_{\substack{\text { interior } \\
\text { faces }}} \int_{f, N}\left(\llbracket \mu \rrbracket\left(M_{0} \vec{G}\right)^{\star}+\mu^{\star} \llbracket M_{0} \vec{G} \rrbracket-\llbracket \mu M_{0} \vec{G} \rrbracket\right) \cdot \vec{n}_{L} \mathrm{~d} S, \\
& \mathrm{PBT}=\sum_{\substack{\text { boundary } \\
\text { faces }}} \int_{f, N}\left(\mu M_{0} \vec{G}-\mu\left(M_{0} \vec{G}\right)^{\star}-\mu^{\star} M_{0} \vec{G}\right) \cdot \mathrm{d} \vec{S} .
\end{aligned}
$$

We insert the numerical fluxes (5.21) and (5.22),

$$
\begin{aligned}
\mathrm{IBT} & =\sum_{\substack{\text { interior } \\
\text { faces }}} \int_{f, N}\left(\llbracket \mu \rrbracket\left\{\left\{M_{0} \vec{G}\right\}\right\}+\{\{\mu\}\} \llbracket M_{0} \vec{G} \rrbracket-\llbracket \mu M_{0} \vec{G} \rrbracket\right) \cdot \mathrm{d} \vec{S}+\int_{f, N} \beta_{\mu} M_{0} \llbracket \mu \rrbracket^{2} \mathrm{~d} S \\
& =\sum_{\substack{\text { interior } \\
\text { faces }}} \int_{f, N} \beta_{\mu} M_{0} \llbracket \mu \rrbracket^{2} \mathrm{~d} S \geqslant 0, \\
\mathrm{PBT} & =\sum_{\substack{\text { boundary } \\
\text { faces }}} \int_{f, N}\left(\mu M_{0} \vec{G}-\mu M_{0} \vec{G}\right) \cdot \mathrm{d} \vec{S}=0 .
\end{aligned}
$$

We get zero dissipation at the inter-element faces if $\beta_{\mu}=0$, and otherwise the approximation is stable IBT $\geqslant 0$. The interface dissipation is not needed for stability, but with the help of the numerical experiments, we will see that it enhances the accuracy. The physical boundary terms are zero, $\mathrm{PBT}=0$.

Therefore, the semi-discrete DG scheme (5.18) is stable, in the sense that the discrete total free-energy (5.35) is bounded in time by its initial value,

$$
\overline{\mathscr{F}}^{N}(T) \leqslant \overline{\mathscr{F}}^{N}(0)-\sum_{e}\left\langle\mathscr{J} M_{0}|\vec{G}|^{2}\right\rangle_{E, N} \leqslant \overline{\mathscr{F}}^{N}(0) .
$$

The discrete free-energy includes the effect of the the interface stabilization $\beta_{c}$ in $\vec{G}_{c}^{\star}$. Note $\beta_{c}$ is a free parameter that we can choose, and that if $\beta_{c}=0$ the interelement dissipation does not contribute to the free-energy, and neither does a continuous solution (i.e. $\llbracket C \rrbracket=0$ ). The physical meaning behind the inter-element free-energy is that the free-energy measures the fluid interfaces using the solution 
gradients $\frac{3}{4} \sigma \varepsilon\left|\vec{G}_{c}\right|^{2}$. However, if the discrete solution is allowed to be discontinuous at the inter-elements faces, it enables the creation of a fluid interface, which is not reflected in the continuous free-energy. The addition of this penalization allows us to account for inter-element discontinuities as part of the fluid interface, so that their associated energy is also weighted in the free-energy. In practice, we have found that we only need interface dissipation to enhance the accuracy in some under-resolved simulations of the coupled system with the Navier-Stokes equations, and is not used in any of the numerical experiments presented in this chapter. In any case, the use of interface stabilization does not compromise the stability of the scheme.

\subsection{Fully-discrete stability analysis using an IMEX time integrator}

Because of the stiffness due to the high order derivatives in the Cahn-Hilliard equation, we introduce a first order IMplicit-EXplicit (IMEX) time integrator. We consider equally-spaced time steps $\Delta t$ and we use the superscript $n$ to represent state values in $t_{n}=n \Delta t$,

$$
\vartheta^{n}=\vartheta\left(t_{n}\right)=\vartheta(n \Delta t)
$$

In particular, this IMEX solver combines a forward and backward first order Euler scheme. We split the equations into implicit and explicit parts to make the algebraic system that needs to be solved linear, despite the original equation being non-linear, so that its solution can be found with a fast, efficient direct linear solver.

We look at the spatially continuous PDE to emphasize the time discretization now

$$
c_{t}=\vec{\nabla} \cdot\left(M_{0} \vec{\nabla} \mu\right)=\vec{\nabla} \cdot\left(M_{0} \vec{\nabla}\left(f_{0}(c)-\frac{3}{2} \sigma \varepsilon \vec{\nabla}^{2} c\right)\right) .
$$

Integrating over one time-step $t \in\left[t_{n}, t_{n+1}\right]$, we find,

$$
\int_{t_{n}}^{t_{n+1}} c_{t} \mathrm{~d} t=c^{n+1}-c^{n}=\int_{t_{n}}^{t_{n+1}} \vec{\nabla} \cdot\left(M_{0} \vec{\nabla}\left(f_{0}(c)-\frac{3}{2} \sigma \varepsilon \vec{\nabla}^{2} c\right)\right) \mathrm{d} t .
$$

Depending on whether we evaluate the right hand side of (5.44) at $t_{n}$ or $t_{n+1}$ we identify forward (explicit) and backward (implicit) Euler schemes respectively.

On the one hand, the interface energy term $\frac{3}{2} \sigma \varepsilon \vec{\nabla}^{2} c$ needs to be implicit, since otherwise explicit time integration requires an impractical time-step restriction. On the other hand, the chemical free-energy term $f_{0}(c)$ is nonlinear and is more easily 
treated explicitly. For these reasons, we evaluate (5.44) in time as

$$
c^{n+1}-c^{n}=\Delta t \vec{\nabla} \cdot\left(M_{0} \vec{\nabla}\left(f_{0}\left(c^{n}\right)-\frac{3}{2} \sigma \varepsilon \vec{\nabla}^{2} c^{n+1}\right)\right) .
$$

The time integration scheme (5.45) is not provably stable because of the nonlinearities in the chemical free-energy. Following Dong [2018b], we modify the chemical potential adding numerical stabilization proportional to solution time jumps $\Delta c=$ $c^{n+1}-c^{n}$,

$$
c^{n+1}-c^{n}=\Delta t \vec{\nabla} \cdot\left(M_{0} \vec{\nabla}\left(f_{0}\left(c^{n}\right)+\frac{12 \sigma}{\varepsilon} S_{0}\left(c^{n+1}-c^{n}\right)-\frac{3}{2} \sigma \varepsilon \vec{\nabla}^{2} c^{n+1}\right)\right),
$$

where $S_{0}$ is a constant, which maintains first order accuracy and makes it possible to construct a stable scheme.

We introduce the time discretization (5.46) into the semi-discrete Cahn-Hilliard equation (5.18) and the definition of the discrete chemical potential (5.20) to construct the fully-discrete discontinuous Galerkin approximation,

$$
\begin{aligned}
\left\langle\mathscr{J} \frac{C^{n+1}-C^{n}}{\Delta t}, \phi\right\rangle_{E, N}= & \int_{\partial e, N} \phi\left(M_{0} \vec{G}\right)^{\star, \theta} \cdot \mathrm{d} \vec{S}-\left\langle M_{0} \overrightarrow{\tilde{G}}^{\theta}, \vec{\nabla}_{\xi} \phi\right\rangle_{E, N}, \\
\left\langle\mathscr{J} \vec{G}^{\theta}, \vec{\varphi}\right\rangle_{E, N}= & \int_{\partial e, N}\left(\mu^{\star, \theta}-\mu^{\theta}\right) \vec{\varphi} \cdot \mathrm{d} \vec{S}+\left\langle\vec{\nabla}_{\xi} \mu^{\theta}, \overrightarrow{\tilde{\varphi}}\right\rangle_{E, N}, \\
\left\langle\mathscr{J} \mu^{\theta}, \phi_{\mu}\right\rangle_{E, N}= & \left\langle\left(\frac{\mathrm{d} F_{0}}{\mathrm{~d} C}\right)^{n}+\frac{12 \sigma}{\varepsilon} S_{0}\left(c^{n+1}-c^{n}\right), \mathscr{J} \phi_{\mu}\right\rangle_{E, N} \\
& -\frac{3}{2} \sigma \varepsilon \int_{\partial e, N} \phi_{\mu} \vec{G}_{c}^{\star, n+1} \cdot \mathrm{d} \vec{S}+\frac{3}{2} \sigma \varepsilon\left\langle\overrightarrow{\tilde{G}}_{c}^{n+1}, \vec{\nabla}_{\xi} \phi_{\mu}\right\rangle_{E, N}, \\
\left\langle\mathscr{J} \vec{G}_{c}^{n+1}, \vec{\phi}_{G_{c}}\right\rangle_{E, N}= & \int_{\partial e, N}\left(C^{\star, n+1}-C^{n+1}\right) \vec{\phi}_{G_{c}} \cdot \mathrm{d} \vec{S}+\left\langle\vec{\nabla}_{\xi} C^{n+1}, \overrightarrow{\tilde{\phi}}_{G_{c}}\right\rangle_{E, N},
\end{aligned}
$$

where we use the superscript $\theta$ for variables (e.g. $\vec{G}^{\theta}$ or $\mu^{\theta}$ ) that are not directly evaluated at $t_{n}$ or $t_{n+1}$ with the IMEX strategy, but on a combination of those depending on the different terms involved in (5.47c). Moreover, following the semi-discrete analysis, we have already applied the discrete Gauss law in (5.47b) and (5.47d).

The stability analysis of the fully-discrete system is not a particular case of the general semi-discrete analysis performed in Sec. 3.5. Therefore, we have to extend the semi-discrete analysis of the Cahn-Hilliard equation in Sec. 5.5 to the fully discrete scheme. We start from the fully discrete equivalent of the Cahn-Hilliard product with the entropy variables. As the entropy variable, we use $\mu^{\theta}$. Thus, we replace $\phi=\mu^{\theta}$, 
and also $\vec{\varphi}=M_{0} \vec{G}^{\theta}$ in (5.47), to get the fully discrete equivalent of (5.26),

$$
\begin{aligned}
&\left\langle\mathscr{J} \frac{\Delta C}{\Delta t}, \mu^{\theta}\right\rangle_{E, N}=\int_{\partial e, N} \mu^{\theta}\left(M_{0} \vec{G}\right)^{\star, \theta} \cdot \mathrm{d} \vec{S}-\left\langle M_{0} \overrightarrow{\tilde{G}}^{\theta}, \vec{\nabla}_{\xi} \mu^{\theta}\right\rangle_{E, N}, \\
&\left\langle\mathscr{J} \vec{G}^{\theta}, M_{0} \vec{G}^{\theta}\right\rangle_{E, N}=\int_{\partial e, N}\left(\mu^{\star, \theta}-\mu^{\theta}\right) M_{0} \vec{G}^{\theta} \cdot \mathrm{d} \vec{S}+\left\langle\vec{\nabla}_{\xi} \mu^{\theta}, M_{0} \overrightarrow{\tilde{G}}^{\theta}\right\rangle_{E, N} \cdot
\end{aligned}
$$

We have defined the jump in time $\Delta \phi=\phi^{n+1}-\phi^{n}$. Now, we add both equations,

$$
\left\langle\mathscr{J} \frac{\Delta C}{\Delta t}, \mu^{\theta}\right\rangle_{E, N}+\int_{\partial e, N}\left(\mu^{\theta}\left(M_{0} \vec{G}^{\theta}-\left(M_{0} \vec{G}\right)^{\star, \theta}\right)-\mu^{\star, \theta} M_{0} \vec{G}^{\theta}\right) \cdot \mathrm{d} \vec{S}=-\left\langle\mathscr{J} M_{0}\left|\vec{G}^{\theta}\right|^{2}\right\rangle_{E, N} .
$$

Eq. (5.49), is the fully-discrete version of (5.26). Instead of the product $\left\langle\mathscr{J} C_{t}, \mu\right\rangle_{E, N}$, we have its semi-discrete version $\left\langle\mathscr{J} \Delta C / \Delta t, \mu^{\theta}\right\rangle_{E, N}$, and similarly on the viscous volume fluxes and the surface integral.

We perform the same analysis: study of the time coefficients contraction to the time derivative (increment on the fully-discrete analysis) of the discrete free-energy, the contraction of inviscid fluxes, and the positive definiteness of viscous fluxes.

\subsubsection{Contraction of the time coefficients}

We extend the study in Sec. 5.5.1 to the fully discrete approximation (5.47). Thus, we need to transform the inner product of the concentration time increment $\left\langle\mathscr{J} \Delta C / \Delta t, \mu^{\theta}\right\rangle_{E, N}$ to the increment of the discrete free-energy $\Delta \overline{\mathscr{F}}^{N}$.

In the semi-discrete analysis, we made use of the discrete chemical potential to get an inner product with the concentration time derivative $C_{t}$. Now, we use similar arguments to get its inner product with $\Delta C / \Delta t$. Thus, we let $\phi_{\mu}=\Delta C / \Delta t$ in (5.47a),

$$
\begin{aligned}
\left\langle\mathscr{J} \mu^{\theta}, \frac{\Delta C}{\Delta t}\right\rangle_{E, N}= & \left\langle\left(\frac{\mathrm{d} F_{0}}{\mathrm{~d} C}\right)^{n}+\frac{12 \sigma}{\varepsilon} S_{0}\left(C^{n+1}-C^{n}\right), \mathscr{J} \frac{\Delta C}{\Delta t}\right\rangle_{E, N} \\
& -\frac{3}{2} \sigma \varepsilon \int_{\partial e, N} \frac{\Delta C}{\Delta t} \vec{G}_{c}^{\star, n+1} \cdot \mathrm{d} \vec{S}+\frac{3}{2} \sigma \varepsilon\left\langle\overrightarrow{\tilde{G}}_{c}^{n+1}, \vec{\nabla}_{\xi} \frac{\Delta C}{\Delta t}\right\rangle_{E, N} .
\end{aligned}
$$

Eq. (5.50) is the fully discrete approximation of (5.27). Although we find similarities, we see that now we cannot apply the chain rule in time to the chemical free-energy, which features the stabilization in time term. We will focus on the chemical freeenergy later. Besides the chemical free-energy term, the rest maintains parallelisms with the semi-discrete version, where the time derivatives are approximated with time increments, and the other quantities are evaluated at an appropriate time instant. 
In the semi-discrete analysis, we computed the interfacial energy from the weak form that defines the concentration gradient (5.28): we took its time derivative, and used the discrete Gauss law. Now in the fully discrete analysis, we take the time increment of (5.47d) (i.e. we write the equation for $n$ and $n+1$, and compute the difference), divide by $\Delta t$, and use the discrete Gauss law,

$$
\left\langle\mathscr{J} \frac{\Delta \vec{G}_{c}}{\Delta t}, \vec{\phi}_{G_{c}}\right\rangle_{E, N}=\int_{\partial e, N}\left(\frac{\Delta C^{\star}}{\Delta t}-\frac{\Delta C}{\Delta t}\right) \vec{\phi}_{G_{c}} \cdot \mathrm{d} \vec{S}+\left\langle\vec{\nabla}_{\xi} \frac{\Delta C}{\Delta t}, \overrightarrow{\tilde{\phi}}_{G_{c}}\right\rangle_{E, N},
$$

to arrive at the fully-discrete version of (5.28). Now, as in the semi-discrete analysis, we find that the last inner product of (5.51) is the same as the last volume inner product of (5.50) if we use $\vec{\phi}_{G_{c}}=\vec{G}_{c}^{n+1}$ (note that in the semi-discrete analysis we simply used $\vec{\phi}_{G_{c}}=\vec{G}_{c}$ ),

$$
\left\langle\vec{\nabla}_{\xi} \frac{\Delta C}{\Delta t}, \vec{G}_{c}^{n+1}\right\rangle_{E, N}=\left\langle\mathscr{J} \frac{\Delta \vec{G}_{c}}{\Delta t}, \vec{G}_{c}^{n+1}\right\rangle_{E, N}-\int_{\partial e, N}\left(\frac{\Delta C^{\star}}{\Delta t}-\frac{\Delta C}{\Delta t}\right) \vec{G}_{c}^{n+1} \cdot \mathrm{d} \vec{S} .
$$

We replace (5.52) in (5.50) to get the time coefficients contraction, which is the fully-discrete equivalent of (5.30)

$$
\begin{aligned}
\left\langle\mathscr{J} \mu^{\theta}, \frac{\Delta C}{\Delta t}\right\rangle_{E, N}= & \left\langle\left(\frac{\mathrm{d} F_{0}}{\mathrm{~d} C}\right)^{n}+\frac{12 \sigma}{\varepsilon} S_{0} \Delta C, \mathscr{J} \frac{\Delta C}{\Delta t}\right\rangle_{E, N}+\frac{3}{2} \sigma \varepsilon\left\langle\mathscr{J} \frac{\Delta \vec{G}_{c}}{\Delta t}, \vec{G}_{c}^{n+1}\right\rangle_{E, N} \\
& +\frac{3}{2} \sigma \varepsilon \int_{\partial e, N}\left(\frac{\Delta C}{\Delta t} \vec{G}_{c}^{n+1}-\frac{\Delta C^{\star}}{\Delta t} \vec{G}_{c}^{n+1}-\frac{\Delta C}{\Delta t} \vec{G}_{c}^{\star, n+1}\right) \cdot \mathrm{d} \vec{S} .
\end{aligned}
$$

Comparing (5.53) to its semi-discrete counterpart (5.30), we see that the only difference is the evaluation of the variables at their fully-discrete time instant (e.g. $\mu^{\theta}$, $\vec{G}_{c}^{n+1}$, or $\left.\left(\mathrm{d} F_{0} / \mathrm{d} C\right)^{n}\right)$, the change of exact time derivatives by increments, and the additional dissipation in time. In its semi-discrete fashion, we used the chain rule in time to transform the two first volume integrals to the chemical free-energy and interfacial energy time derivatives. We cannot perform that in the fully discrete analysis, and we have to proceed differently to transform (5.53) into $\Delta \bar{F}^{N}$.

\section{Chemical free-energy}

For the chemical free-energy $F_{0}$ terms, we perform the Taylor expansion of $F_{0}(C)$ centered on $C^{n}$,

$$
F_{0}^{n+1}=F_{0}^{n}+\left(\frac{\mathrm{d} F_{0}}{\mathrm{~d} C}\right)^{n} \Delta C+\frac{1}{2}\left(\frac{\mathrm{d}^{2} F_{0}}{\mathrm{~d} C^{2}}\right)^{n} \Delta C^{2}+\frac{1}{6}\left(\frac{\mathrm{d}^{3} F_{0}}{\mathrm{~d} C^{3}}\right)^{n} \Delta C^{3}+\frac{1}{24}\left(\frac{\mathrm{d}^{4} F_{0}}{\mathrm{~d} C^{4}}\right)^{n} \Delta C^{4}
$$


For the polynomic chemical free-energy (5.9) the coefficients are

$$
\begin{aligned}
& \left(d^{2} F_{0} / d C^{2}\right)^{n}=\frac{12 \sigma}{\varepsilon}\left(12\left(C^{n}\right)^{2}-12 C^{n}+2\right) \\
& \left(d^{3} F_{0} / d C^{3}\right)^{n}=\frac{12 \sigma}{\varepsilon}\left(24 C^{n}-12\right) \\
& \left(d^{4} F_{0} / d C^{4}\right)^{n}=\frac{12 \sigma}{\varepsilon} 24
\end{aligned}
$$

and it follows exactly that

$$
F_{0}^{n+1}=F_{0}^{n}+\left(\frac{\mathrm{d} F_{0}}{\mathrm{~d} C}\right)^{n} \Delta C+\frac{12}{\varepsilon}\left(\left(6\left(C^{n}\right)^{2}-6 C^{n}+1\right)+\left(4 C^{n}-2\right) \Delta C+\Delta C^{2}\right) \Delta C^{2}
$$

We use (5.56) to write the first right hand side volume quadrature in (5.53) as

$$
\left\langle\left(\frac{\mathrm{d} F_{0}}{\mathrm{~d} C}\right)^{n}+\frac{12 \sigma}{\varepsilon} S_{0} \Delta C, \mathscr{J} \Delta C\right\rangle_{E, N}=\left\langle\mathscr{J} F_{0}^{n+1}\right\rangle_{E, N}-\left\langle\mathscr{J} F_{0}^{n}\right\rangle_{E, N}+\langle\mathscr{J} \Pi\rangle_{E, N}
$$

where the remainder $\Pi\left(C^{n+1}, C^{n}\right)$ is the polynomial function

$\Pi=\frac{12 \sigma}{\varepsilon} S_{0} \Delta C^{2}-\frac{12 \sigma}{\varepsilon}\left(\left(6\left(C^{n}\right)^{2}-6 C^{n}+1\right)+\left(4 C^{n}-2\right) \Delta C+\Delta C^{2}\right) \Delta C^{2}=\frac{12 \sigma}{\varepsilon} \Delta C^{2} \Pi^{\star}$,

with

$$
\Pi^{\star}=S_{0}-\left(\left(6\left(C^{n}\right)^{2}-6 C^{n}+1\right)+\left(4 C^{n}-2\right)\left(C^{n+1}-C^{n}\right)+\left(C^{n+1}-C^{n}\right)^{2}\right) .
$$

The quantity $\Pi^{\star}$ is a second order concave polynomial (an elliptic paraboloid, with center in $C_{0}^{n+1}=C_{0}^{n}=\frac{1}{2}$ ). For the time integration to be stable, it suffices that $\Pi^{\star}$ remains positive, as will be inferred from the stability analysis. Since $\Pi^{\star}$ is a concave function, it will always shift negative for sufficiently large values of $C^{n+1}$ or $C^{n}$. However, acceptable solutions of the Cahn-Hilliard equation remain close to the range $C \in[0,1]$. Thus, we can choose the value $S_{0}$ so that $\Pi^{\star}$ remains positive in a reasonably large neighborhood of $\left(C^{n+1}, C^{n}\right) \in[0,1]^{2}$.

The elliptical isocontours $\Pi^{\star}=0$ are shown in Fig. 5.1 for several $S_{0}$ values (as labeled on each contour line). A sufficient condition for positivity is that every pair $\left(C^{n}, C^{n+1}\right)$ remains inside the $\Pi^{\star}=0$ isoline for the given $S_{0}$ value. For instance, given the range $C \in[0,1]$, the scheme is stable for $S_{0} \geqslant 1$, and for $C \in$ $\left[\frac{1}{2}(1-\sqrt{5 / 3}), \frac{1}{2}(1+\sqrt{5 / 3})\right] \simeq[-0.15,1.15]$, the scheme remains stable for $S_{0} \geqslant 2$. 


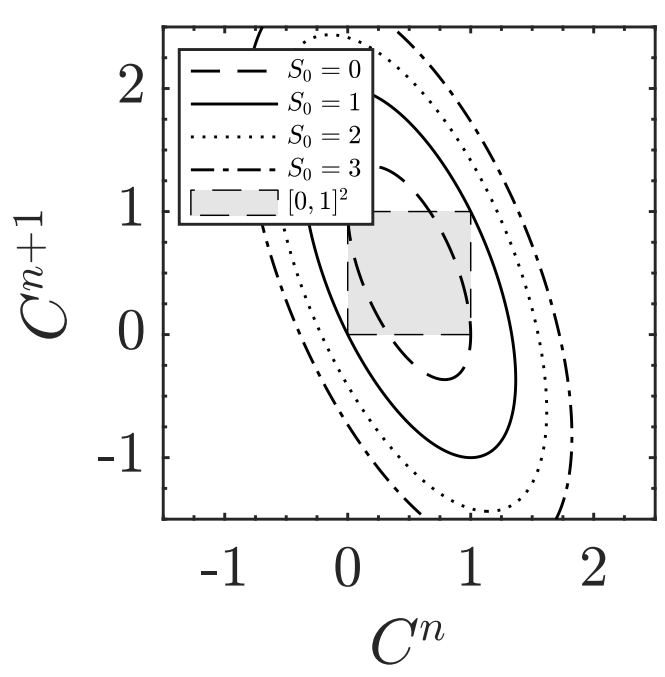

(a) $\Pi^{\star}=0$ isolines

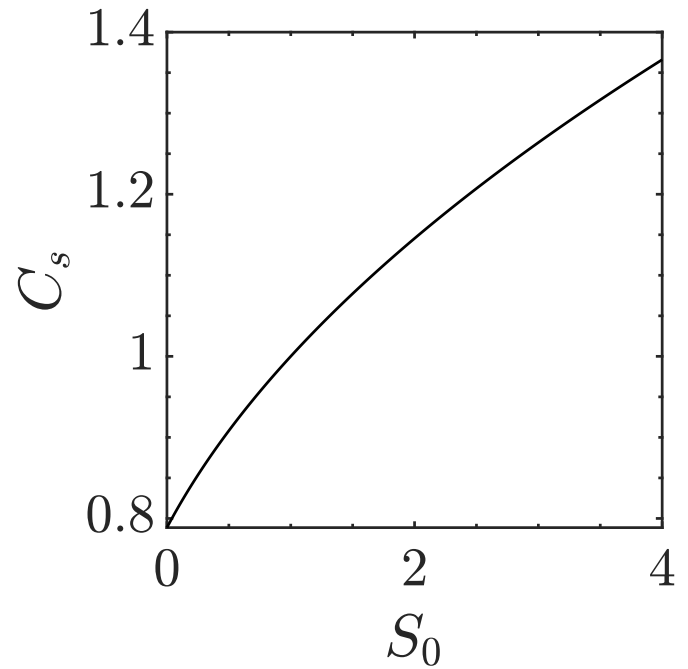

(b) Range $C \in\left[1-C_{s}, C_{s}\right]^{2}$ in which nonlinear terms are stable

Figure 5.1: Graphical representation of the elliptical isoline $\Pi^{\star}=0$ for several $S_{0}$ values (indicated by the contour labels). The box $[0,1]^{2}$ has also been represented. A sufficient condition for positiveness is that the pair $\left(C^{n}, C^{n+1}\right)$ stays inside the ellipse for a given $S_{0}$ value. In 5.1(b) we have represented for each $S_{0}$ the interval $\left[1-C_{s}, C_{s}\right]^{2}$ in which the non-linear terms are stable, which corresponds to the formula $C_{s}=\frac{1}{2}\left(1+\sqrt{\frac{2 S_{0}+1}{3}}\right)$

\section{Interfacial energy}

For the interface energy in (5.53), we complete the square,

$$
\begin{aligned}
\left\langle\mathscr{J} \Delta \vec{G}_{c}, \vec{G}_{c}^{n+1}\right\rangle_{E, N} & =\frac{1}{2}\left\langle\mathscr{J} \vec{G}_{c}^{n+1}, \vec{G}_{c}^{n+1}\right\rangle_{E, N}-\frac{1}{2}\left\langle\mathscr{J} \vec{G}_{c}^{n}, \vec{G}_{c}^{n}\right\rangle_{E, N}+\frac{1}{2}\left\langle\mathscr{J} \Delta \vec{G}_{c}, \Delta \vec{G}_{c},\right\rangle_{E, N} \\
& =\frac{1}{2} \Delta\left\langle\mathscr{J}\left|\vec{G}_{c}\right|^{2}\right\rangle+\frac{1}{2}\left\langle\mathscr{J} \Delta \vec{G}_{c}, \Delta \vec{G}_{c},\right\rangle_{E, N} .
\end{aligned}
$$

With (5.57) and (5.60), we transform (5.53) to

$$
\begin{aligned}
\left\langle\mathscr{J} \mu^{\theta}, \frac{\Delta C}{\Delta t}\right\rangle_{E, N}= & \frac{\Delta}{\Delta t}\left\langle\mathscr{J}\left(F_{0}+\frac{3}{4} \sigma \varepsilon\left|\vec{G}_{c}\right|^{2}\right)\right\rangle_{E, N}+\frac{1}{\Delta t}\left\langle\mathscr{J}\left(\Pi+\frac{3}{4} \sigma \varepsilon\left|\Delta \vec{G}_{c}\right|^{2}\right)\right\rangle_{E, N} \\
& +\frac{3}{2} \sigma \varepsilon \int_{\partial e, N}\left(\frac{\Delta C}{\Delta t} \vec{G}_{c}^{n+1}-\frac{\Delta C^{\star}}{\Delta t} \vec{G}_{c}^{n+1}-\frac{\Delta C}{\Delta t} \vec{G}_{c}^{\star, n+1}\right) \cdot \mathrm{d} \vec{S},
\end{aligned}
$$

which now looks more similar to (5.30), but with the discrete time derivatives, and 
two additional terms as a result of the time integrator errors. We identify the fullydiscrete surface term in the time contraction

$$
\vec{H}^{\mathscr{E}}=\frac{3}{2} \sigma \varepsilon\left(\frac{\Delta C}{\Delta t} \vec{G}_{c}^{n+1}-\frac{\Delta C^{\star}}{\Delta t} \vec{G}_{c}^{n+1}-\frac{\Delta C}{\Delta t} \vec{G}_{c}^{\star, n+1}\right),
$$

and define the additional errors as a result of the discrete time integration,

$$
\operatorname{diss}_{\mathrm{IMEX}}^{N}=\sum_{e}\left\langle\mathscr{J}\left(\Pi+\frac{3}{4} \sigma \varepsilon\left|\Delta \vec{G}_{c}\right|^{2}\right)\right\rangle_{E, N} \geqslant 0
$$

We sum (5.61) for all the elements,

$$
\begin{aligned}
\sum_{e}\left\langle\mathscr{J} \mu^{\theta}, \frac{\Delta C}{\Delta t}\right\rangle_{E, N}= & \sum_{e} \frac{\Delta}{\Delta t}\langle\mathscr{J} \mathscr{F}\rangle_{E, N}+\sum_{\substack{\text { interior } \\
\text { faces }}} \int_{f, N} \llbracket-\vec{H}^{\mathscr{E}} \rrbracket \cdot \mathrm{d} \vec{S} \\
& +\sum_{\substack{\text { boundary } \\
\text { faces }}} \int_{f, N} \vec{H}^{\mathscr{E}} \cdot \mathrm{d} \vec{S}+\frac{1}{\Delta t} \operatorname{diss}_{\mathrm{IMEX}}^{N}
\end{aligned}
$$

In the semi-discrete analysis, we rewrote the interior terms (5.33) as the time derivative of a positive inter-element entropy $\mathscr{E}_{i}^{S}$. For the fully-discrete analysis,

$$
\begin{aligned}
\llbracket-\vec{H}^{\mathscr{E}} \rrbracket \cdot \vec{n}_{L} & =\frac{3}{2} \sigma \varepsilon\left(\left(\left\{\left\{\frac{\Delta C}{\Delta t}\right\}\right\} \llbracket \vec{G}_{c}^{n+1} \rrbracket+\llbracket \frac{\Delta C}{\Delta t} \rrbracket\left\{\left\{\vec{G}_{c}^{n+1}\right\}\right\}-\llbracket \frac{\Delta C}{\Delta t} \vec{G}_{c}^{n+1} \rrbracket\right) \cdot \vec{n}_{L}\right. \\
& \left.+\beta_{c} \llbracket \frac{\Delta C}{\Delta t} \rrbracket \llbracket C^{n+1} \rrbracket\right) \\
& =\frac{\Delta}{\Delta t}\left(\frac{3}{4} \sigma \varepsilon \beta_{c} \llbracket C \rrbracket^{2}\right)+\frac{3}{4 \Delta t} \sigma \varepsilon \beta_{c} \llbracket \Delta C \rrbracket^{2}=\frac{\Delta \mathscr{F}_{i}^{S}}{\Delta t}+\frac{3}{4 \Delta t} \sigma \varepsilon \beta_{c} \llbracket \Delta C \rrbracket^{2},
\end{aligned}
$$

which is the discrete time derivative of a positive inter-element surface free-energy $\mathscr{F}_{i}^{S}=\frac{3}{4} \sigma \varepsilon \beta_{c} \llbracket C \rrbracket^{2}$, and it also adds a positive numerical dissipation which depends on both time $(\Delta C)$ and space $(\llbracket \Delta C \rrbracket)$ errors. To get (5.65), we completed the squares for the term $\llbracket \Delta C \rrbracket \llbracket C^{n+1} \rrbracket$ in a similar fashion to (5.60).

Next, for the physical boundary terms we apply the boundary conditions (5.24) and (5.25) to get

$$
\vec{H}^{\mathscr{E}} \cdot \vec{n}=\frac{3}{2} \sigma \varepsilon\left(\frac{\Delta C}{\Delta t} \vec{G}_{c}^{n+1}-\frac{\Delta C}{\Delta t} \vec{G}_{c}^{n+1}\right)+\frac{\Delta C}{\Delta t} \beta=\frac{\Delta F_{w}}{\Delta t}=\frac{\Delta \mathscr{F}^{S}}{\Delta t} .
$$

We now finish the fully-discrete study of time coefficients contraction. We intro- 
duce (5.65) and (5.66) in (5.64) to get

$$
\begin{aligned}
\sum_{e}\left\langle\mathscr{J} \mu^{\theta}, \frac{\Delta C}{\Delta t}\right\rangle_{E, N}= & \frac{\Delta}{\Delta t}\left(\sum_{e}\langle\mathscr{J} \mathscr{F}\rangle_{E, N}+\sum_{\substack{\text { interior } \\
\text { faces }}} \int_{f, N} \frac{3}{4} \sigma \varepsilon \beta_{c} \llbracket C \rrbracket^{2} \mathrm{~d} S+\sum_{\substack{\text { boundary } \\
\text { faces }}} \int_{f, N} F_{w} \mathrm{~d} S\right) \\
& +\frac{1}{\Delta t} \operatorname{diss}_{\mathrm{IMEX}}^{N}+\sum_{\substack{\text { interior } \\
\text { faces }}} \int_{f, N} \frac{3}{4 \Delta t} \sigma \varepsilon \beta_{c} \llbracket \Delta C \rrbracket^{2} \mathrm{~d} S \\
= & \frac{\Delta \bar{F}^{N}}{\Delta t}+\frac{1}{\Delta t} \operatorname{diss}_{\mathrm{IMEX}}^{N}+\sum_{\substack{\text { interior } \\
\text { faces }}} \int_{f, N} \frac{3}{4 \Delta t} \sigma \varepsilon \beta_{c} \llbracket \Delta C \rrbracket^{2} \mathrm{~d} S,
\end{aligned}
$$

which is the fully-discrete version of (5.36), where the definition of the discrete total free-energy (5.35) holds, the exact time derivative is a discrete increment, and there are two additional stable (positive) dissipative terms as a result of the inexact integration. The fully-discrete contraction of time coefficients is the part of the fullydiscrete analysis that requires more details, as it involves time derivatives that now are approximated. The rest of the analysis will easily translate from the semi-discrete analysis.

\subsubsection{Contraction of the inviscid fluxes}

The Cahn-Hilliard equation does not involve inviscid fluxes.

\subsubsection{Positive semi-definiteness of the viscous fluxes}

The study of the viscous fluxes now include the evaluation of the gradients at the IMEX state $\vec{G}^{\theta}$. Nonetheless, the approximation of positive definite viscous fluxes is stable fully-discretely,

$$
\left\langle\mathscr{J} \overrightarrow{\mathbf{G}}^{\theta}, \underline{\mathbf{B}}^{\theta}\right\rangle_{E, N}=\left\langle\mathscr{J} M_{0}\left|\vec{G}^{\theta}\right|^{2}\right\rangle_{E, N} \geqslant 0
$$

\subsubsection{Stability of the boundary terms}

As a result, we get the fully-discrete free-energy equation,

$$
\frac{\Delta \overline{\mathscr{F}}^{N}}{\Delta t}+\mathrm{PBT}=-\mathrm{IBT}-\sum_{e}\left\langle\mathscr{J} M_{0}\left|\vec{G}^{\theta}\right|^{2}\right\rangle_{E, N}-\frac{1}{\Delta t} \operatorname{diss}_{\mathrm{IMEX}}^{N}-\sum_{\substack{\text { interior } \\ \text { faces }}} \int_{f, N} \frac{3}{4 \Delta t} \sigma \varepsilon \beta_{c} \llbracket \Delta C \rrbracket^{2} \mathrm{~d} S
$$


where the interior and physical boundary terms are the fully-discrete version of (5.39)

$$
\begin{aligned}
& \mathrm{IBT}=\sum_{\substack{\text { interior } \\
\text { faces }}} \int_{f, N}\left(\llbracket \mu^{\theta} \rrbracket\left(M_{0} \vec{G}\right)^{\star, \theta}+\mu^{\star, \theta} \llbracket M_{0} \vec{G}^{\theta} \rrbracket-\llbracket \mu^{\theta} M_{0} \vec{G}^{\theta} \rrbracket\right) \cdot \vec{n}_{L} \mathrm{~d} S, \\
& \mathrm{PBT}=\sum_{\substack{\text { boundary } \\
\text { faces }}} \int_{f, N}\left(\mu^{\theta} M_{0} \vec{G}^{\theta}-\mu^{\theta}\left(M_{0} \vec{G}\right)^{\star, \theta}-\mu^{\star, \theta} M_{0} \vec{G}^{\theta}\right) \cdot \mathrm{d} \vec{S},
\end{aligned}
$$

which arrive to a very similar result to the semi-discrete analysis, with the introduction of the numerical fluxes (all the terms are identical, but with the superscript $\theta$ ),

$$
\begin{aligned}
\mathrm{IBT} & =\sum_{\substack{\text { interior } \\
\text { faces }}} \int_{f, N} \beta_{\mu} M_{0} \llbracket \mu^{\theta} \rrbracket^{2} \mathrm{~d} S \geqslant 0, \\
\mathrm{PBT} & =\sum_{\substack{\text { boundary } \\
\text { faces }}} \int_{f, N}\left(\mu^{\theta} M_{0} \vec{G}^{\theta}-\mu^{\theta} M_{0} \vec{G}^{\theta}\right) \cdot \mathrm{d} \vec{S}=0 .
\end{aligned}
$$

We get the change of the free-energy in each time-step,

$$
\begin{aligned}
& \mathscr{F}^{N, n+1}= \bar{F}^{N, n}-\Delta t \sum_{e}\left\langle\mathscr{J} M_{0}\left|\vec{G}^{\theta}\right|^{2}\right\rangle_{E, N}-\operatorname{diss}_{\text {IMEX }}^{N} \\
&-\sum_{\substack{\text { interior } \\
\text { faces }}} \int_{f, N}\left(\frac{3}{4} \sigma \varepsilon \beta_{c} \llbracket \Delta C \rrbracket^{2}+\Delta t \beta_{\mu} M_{0} \llbracket \mu^{\theta} \rrbracket^{2}\right) \mathrm{d} S \\
& \leqslant \mathscr{F}^{N, n}-\Delta t \sum_{e}\left\langle\mathscr{J} M_{0}\left|\vec{G}^{\theta}\right|^{2}\right\rangle_{E, N},
\end{aligned}
$$

and we can sum $n=0, \ldots, T-1$ time-steps to get,

$$
\overline{\mathscr{F}}^{N, T} \leqslant \overline{\mathscr{F}}^{N, 0}-\Delta t \sum_{e, n}\left\langle\mathscr{J} M_{0}\left|\vec{G}^{\theta}\right|^{2}\right\rangle_{E, N} \leqslant \overline{\mathscr{F}}^{0} .
$$

Eq. (5.73) assumes that $\langle\mathscr{J} \Pi\rangle \geqslant 0$. We do not provide here a proof of positivity besides the sufficient condition given in Fig. 5.1. In practice we have not seen any violation of the positivity in all the numerical experiments presented herein when $S_{0} \geqslant 1$. The $\leqslant$ sign is a result of numerical dissipation from the inexactness of the approximation. There is dissipation in space proportional to $\beta_{\mu} \llbracket \mu^{\theta} \rrbracket^{2}$, dissipation in space and time proportional to $\beta_{c} \llbracket \Delta C \rrbracket^{2}$, and dissipation in time as a result of the non-linear terms in the chemical free-energy, $\Pi$, and proportional to $\left|\Delta \vec{G}_{c}\right|^{2}$. The terms that belong to the interface dissipation $\left(\beta_{c}\right.$ and $\left.\beta_{\mu}\right)$ can be removed taking $\beta_{c}=$ $\beta_{\mu}=0$, since this stabilization is not required for the stability, but because it enhances the accuracy. We conclude that the DG-IMEX approximation of the Cahn-Hilliard equation (5.47) is free-energy stable. 


\subsubsection{Extension of the fully-stability analysis to the Crank-Nicolson scheme}

It has been shown in (5.63) that the implicit Euler scheme used for $\vec{G}_{c}$ in (5.47c) adds numerical dissipation that is proportional to the jumps in time $\Delta \vec{G}_{c}$ squared,

$$
\frac{3}{4} \sigma \varepsilon\left\langle\mathscr{J}\left|\Delta \vec{G}_{c}\right|^{2}\right\rangle_{E, N} \geqslant 0
$$

This dissipation can be effectively controlled (and removed) using a linear combination of $\vec{G}_{c}^{n+1}$ and $\vec{G}_{c}^{n}$. To show this, we define an intermediate state $\vec{G}_{c}^{\theta}$,

$$
\vec{G}_{c}^{\theta}=K_{0} \vec{G}_{c}^{n+1}+\left(1-K_{0}\right) \vec{G}_{c}^{n},
$$

so that $K_{0}=1$ recovers backward Euler, $K_{0}=1 / 2$ is Crank-Nicolson, and $K_{0}=0$ is forward (explicit) Euler.

We revisit the fully-discrete stability of the interfacial energy terms (5.60), where now $\vec{G}_{c}$ is evaluated at the $\theta$ state,

$$
\begin{aligned}
\left\langle\mathscr{J} \Delta \vec{G}_{c}, \vec{G}_{c}^{\theta}\right\rangle_{E, N}= & \left\langle\mathscr{J}\left(\vec{G}_{c}^{n+1}-\vec{G}_{c}^{n}\right), K_{0} \vec{G}_{c}^{n+1}+\left(1-K_{0}\right) \vec{G}_{c}^{n},\right\rangle_{E, N} \\
= & \frac{1}{2}\left\langle\mathscr{J}\left|\vec{G}_{c}^{n+1}\right|^{2}\right\rangle_{E, N}-\frac{1}{2}\left\langle\mathscr{J}\left|\vec{G}_{c}^{n}\right|^{2}\right\rangle_{E, N} \\
& +\left(K_{0}-\frac{1}{2}\right)\left\langle\mathscr{J}\left|\Delta \vec{G}_{c}\right|^{2}\right\rangle_{E, N} .
\end{aligned}
$$

Following the rest of the steps in the fully-discrete analysis, which remain the same, we arrive to the same bound in (5.72), but with new IMEX dissipation, $\operatorname{diss}_{\text {IMEX }}^{K_{0}, N}$ in terms of $K_{0}$

$$
\operatorname{diss}_{\mathrm{IMEX}}^{K_{0}, N}=\sum_{e}\left\langle\mathscr{J}\left(\Pi+\frac{3}{2} \sigma \varepsilon\left(K_{0}-\frac{1}{2}\right)\left|\Delta \vec{G}_{c}\right|^{2}\right)\right\rangle_{E, N} \geqslant 0 .
$$

Thus, the amount of dissipation related to $\vec{G}_{c}$ added by the implicit scheme varies linearly with $K_{0}$, and vanishes with $K_{0}=1 / 2$ (Crank-Nicolson scheme).

Note that, with this approach, we cannot confirm that the explicit Euler is stable, which does not necessarily mean that it is unstable, as a low $\Delta t$ can balance spatial and temporal errors (i.e. explicit Euler needs a spatially-dissipative scheme). The stability of the explicit Euler scheme is not analyzed in detail in this work as we consider its use impractical due to the time step limitation imposed by the fourth order spatial derivative of the Cahn-Hilliard equation. In the numerical experiments, we set $K_{0}$ to both 1 (implicit Euler) and 1/2 (Crank-Nicolson) to see whether it is enough with the dissipation provided by $\langle\mathscr{J} \Pi\rangle_{E, N}$ and the physical dissipation for the approximation to be stable. 


\subsection{Numerical experiments}

In this section we present numerical experiments to address the capabilities and robustness of the fully discrete approximation. First, we perform a convergence study using the method of manufactured solutions. The experiment performed here is two dimensional in a Cartesian mesh with straight sides. Second, we compare our results with two-dimensional results available in the literature, using a Cartesian mesh, a distorted mesh, a distorted mesh with curvilinear faces, and a fully unstructured mesh forming a "T" domain. Lastly, we explore the spinodal-decomposition in a three dimensional cylindrical geometry to address the robustness of the scheme to solve three-dimensional meshes with curvilinear elements.

\subsubsection{Convergence study}

We now address the convergence of the fully discrete scheme. To do so, we follow Kästner et al. [2016] and consider a two-dimensional unit square, $[-1,1]^{2}$, and impose the solution,

$$
c_{0}(x, y, t)=\frac{1}{2}(1+\cos (\pi \alpha x) \cos (\pi \alpha y) \cos (t)) .
$$

For (5.78) to be solution of (5.1), we add a source term to the latter,

$$
\frac{\partial c_{0}}{\partial t}=\vec{\nabla} \cdot\left(M_{0} \vec{\nabla}\left(\frac{\mathrm{d} f_{0}\left(c_{0}\right)}{\mathrm{d} c}-\frac{3}{2} \sigma \varepsilon \vec{\nabla} c_{0}^{2}\right)\right)+q(\vec{x}, t),
$$

whose expression is

$$
\begin{aligned}
q(x, y, t)= & 3 M_{0} \pi^{4} \alpha^{4} \sigma \varepsilon \cos (t) \cos (\pi \alpha x) \cos (\pi \alpha y) \\
& +18 \frac{\sigma}{\varepsilon} M_{0} \pi^{2} \alpha^{2} \cos (t)^{3} \cos (\pi \alpha x)^{3} \cos (\pi \alpha y)^{3} \\
& -36 \frac{\sigma}{\varepsilon} M_{0} \pi^{2} \alpha^{2} \cos (t)^{3} \cos (\pi \alpha x) \cos (\pi \alpha y)^{3} \sin (\pi \alpha x)^{2} \\
& -6 \frac{\sigma}{\varepsilon} M_{0} \pi^{2} \alpha^{2} \cos (t) \cos (\pi \alpha x) \cos (\pi \alpha y) \\
& +18 \frac{\sigma}{\varepsilon} M_{0} \pi^{2} \alpha^{2} \cos (t)^{3} \cos (\pi \alpha x)^{3} \cos (\pi \alpha y)^{3} \\
& -36 \frac{\sigma}{\varepsilon} M_{0} \pi^{2} \alpha^{2} \cos (t)^{3} \cos (\pi \alpha x)^{3} \cos (\pi \alpha y) \sin (\pi \alpha y)^{2} \\
& -6 \frac{\sigma}{\varepsilon} M_{0} \pi^{2} \alpha^{2} \cos (t) \cos (\pi \alpha x) \cos (\pi \alpha y) \\
& -\frac{1}{2} \sin (t) \cos (\pi \alpha x) \cos (\pi \alpha y)
\end{aligned}
$$


We solve the Cahn-Hilliard equation to a final time $t_{F}=0.1$, varying the polynomial order, the element spacing, and the time step size. We check the $L^{2}$ norm of the error, defined as

$$
\text { error }=\left\|C-C_{0}\right\|_{\mathscr{J}, N}=\sqrt{\sum_{e}\left\langle\mathscr{J}\left(C-C_{0}\right),\left(C-C_{0}\right)\right\rangle_{E, N}} .
$$

The physical parameters are set to $M_{0}=1$ and $k=0.01$, and we will vary $\alpha$ to analyse space under-resolved and time under-resolved solutions.

\section{Polynomial order convergence study (p-refinement)}

In this test the mesh is a fixed $4 \times 4$ Cartesian mesh, and the polynomial order (represented in the $x$-axis of the figures) ranges from $N=3$ to $N=8$, with three time steps $\Delta t=10^{-3}, 5 \cdot 10^{-4}$, and $10^{-4}$. In all these tests we take $\alpha=1$, so that the solution is well-resolved in space with relatively coarse meshes. The results are shown in Fig. 5.2, where we performed the study for two penalty parameter coefficient values

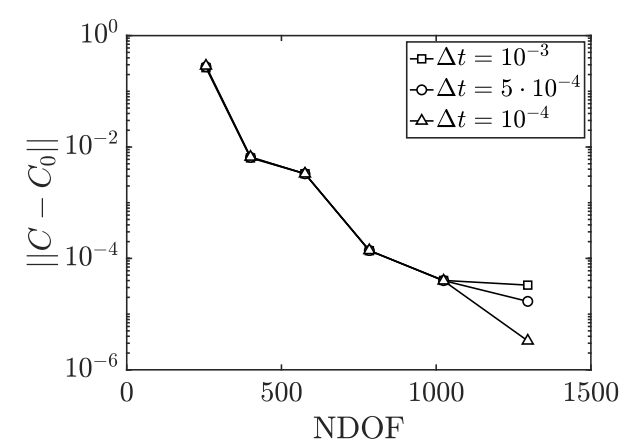

(a) Without interface stabilization $\left(\kappa_{\beta \mu}=0\right)$

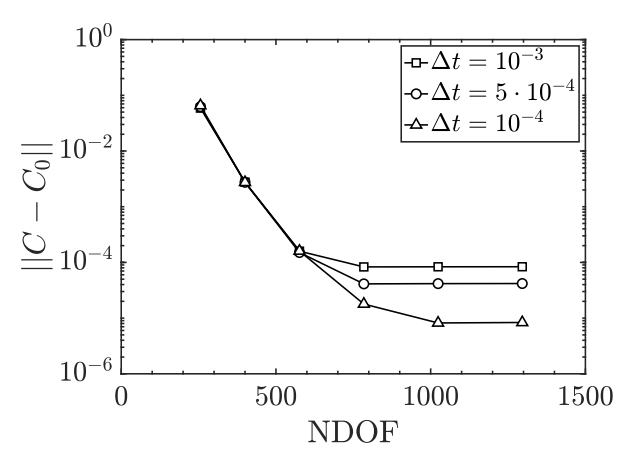

(b) With interface stabilization $\left(\kappa_{\beta \mu}=3\right)$

Figure 5.2: Polynomial order convergence study without and with interface stabilization. We show that the convergence is faster and smoother with interface stabilization, and that the convergence rate is exponential only with interface stabilization

$\kappa_{\beta \mu}$, which was defined in (5.23): without stabilization $\left(\kappa_{\beta \mu}=0\right)$ in Fig. 5.2(a) and with stabilization $\left(\kappa_{\beta \mu}=3\right)$ in Fig. 5.2(b). Moreover, we use the IMEX Euler scheme with $K_{0}=1$ and $S_{0}=1$.

We find that the convergence is slower without interface stabilization $\left(\kappa_{\beta \mu}=0\right)$, and also that it is uneven. This even-odd phenomena has been also reported in Gassner et al. [2016a] in the context of the compressible Euler equations, where it was tackled by adding interface stabilization. 
With interface stabilization (Fig. 5.2(b)), we find not only that the convergence is smoother, but errors are always lower. The curve shows the typical pattern of a polynomial order convergence study: on the one hand, for low polynomial orders the solution is under-resolved in space, and errors decrease exponentially with the polynomial order (linear decay in semi-logarithmic plot). In this region, errors are not affected by the time step $\Delta t$. On the other hand, for high polynomial orders, the solution is under-resolved in time, and thus it reaches a stagnation with further increase of the polynomial order. Now, the error is controlled by the time step $\Delta t$, as the different plots in Fig. 5.2(b) show. We have represented in Fig. 5.3 the different

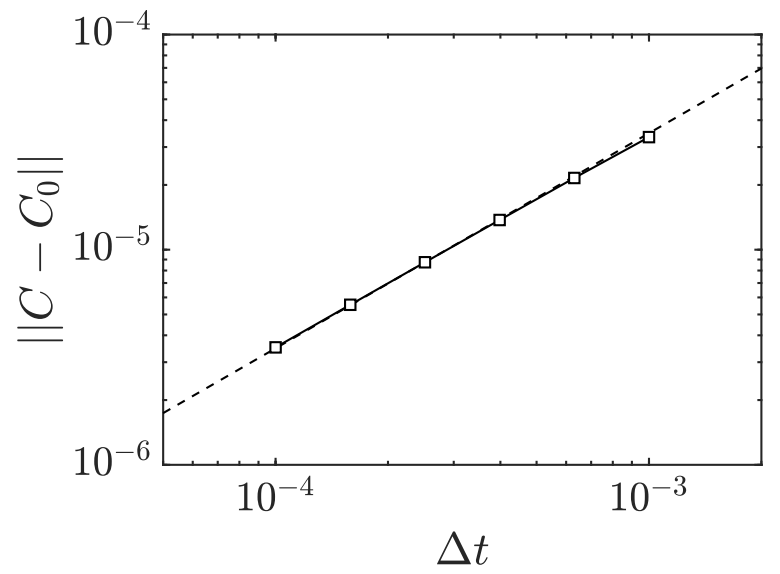

Figure 5.3: Temporal convergence study. The dashed line represents the theoretical linear convergence rate. We confirm that the scheme is first order accurate in time, as designed

errors computed once the stagnation is reached to show that the scheme is first order accurate in time, as designed.

\section{Mesh convergence study (h-refinement)}

In this test, we increase the manufactured solution's wavenumber to $\alpha=8$, so that we extend the region with spatial under-resolution. We vary the number of elements from a $16 \times 16$ mesh to a $64 \times 64$ mesh, and we consider four polynomial orders, from $N=2$ to $N=5$. The rest of the parameters remain the same as in Sec. 5.7.1, and we use the scheme with interface stabilization $\left(\kappa_{\beta \mu}=3\right)$. The time-step used is $\Delta t=10^{-3}$ for $N=2,3$, and $\Delta t=10^{-4}$ for $N=4,5$. The results are represented in Fig. 5.4, where we have drawn the theoretical convergence rates for each polynomial order 
Hesthaven and Warburton [2007],

$$
\left\|C-C_{0}\right\| \propto \Delta x^{N+1}
$$

We find that despite adding the interface stabilization, there still remains some slight even-odd effect, as the convergence is faster than the theoretical for even polynomial orders, and slower otherwise. Similar behavior was noted in Ainsworth [2004] and the reason behind this effect in this particular scheme still remains an open question.

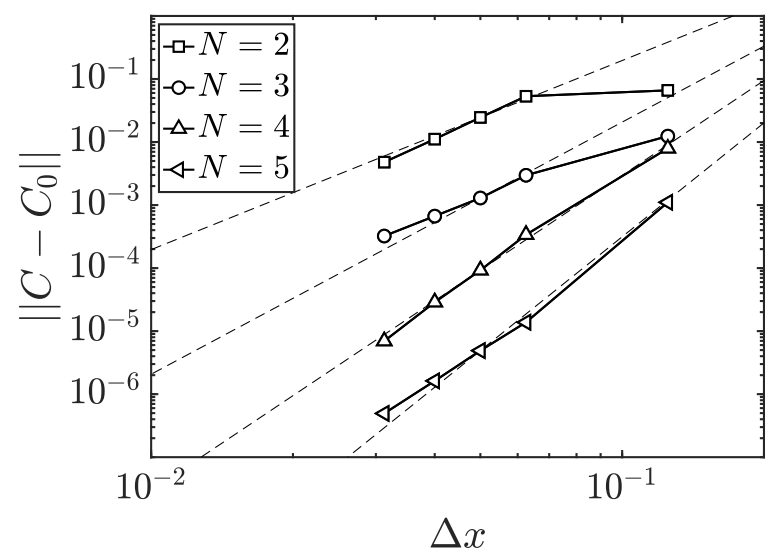

Figure 5.4: Spatial convergence study for different polynomial orders. We vary the mesh spacing from $\Delta x=0.125$ (16x16 mesh) to $\Delta x=0.03125$ (64x64 mesh). Dashed lines represent the theoretical convergence rate, $\Delta x^{N+1}$, for each polynomial

\subsubsection{Two dimensional spinodal decomposition}

In this section, we compare the scheme when solving a two dimensional spinodal decomposition. The spinodal decomposition describes the phase separation process from an initial mixed state. To trigger the separation, we use the initial condition proposed in Jokisaari et al. [2017],

$$
\begin{aligned}
c_{0}(x, y)= & 0.5+0.025\left(\cos (0.105 x) \cos (0.11 y)+(\cos (0.13 x) \cos (0.087 y))^{2}\right. \\
& +\cos (0.025 x-0.15 y) \cos (0.07 x-0.02 y)) .
\end{aligned}
$$

The domain is "T"-shaped, which we mesh using four strategies: a Cartesian mesh (Fig. 5.5(a)), the Cartesian mesh distorted with straight sided elements (Fig. 5.5(b)), the Cartesian mesh distorted with curved elements (Fig. 5.5(c)), and an unstructured quad mesh (Fig. 5.5(d)). 


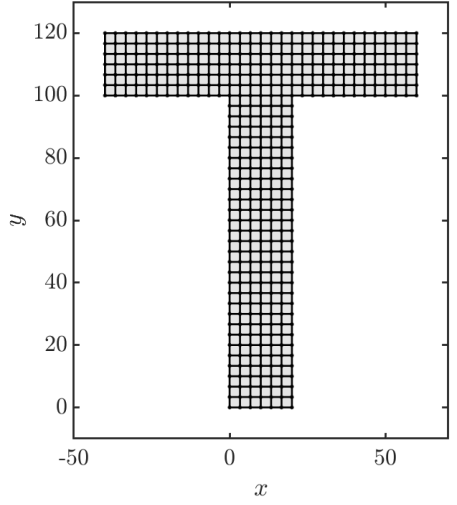

(a) Cartesian mesh

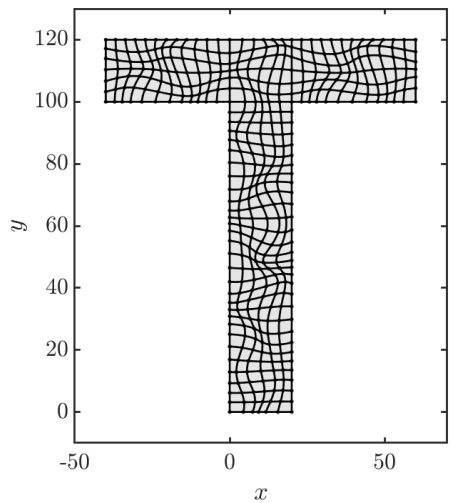

(c) Curved mesh (curvilinear edges)

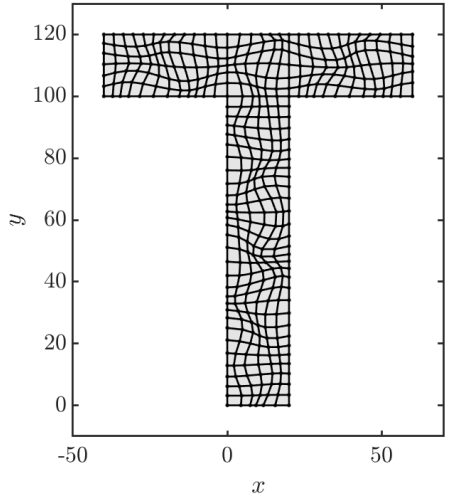

(b) Distorted mesh (straight edges)

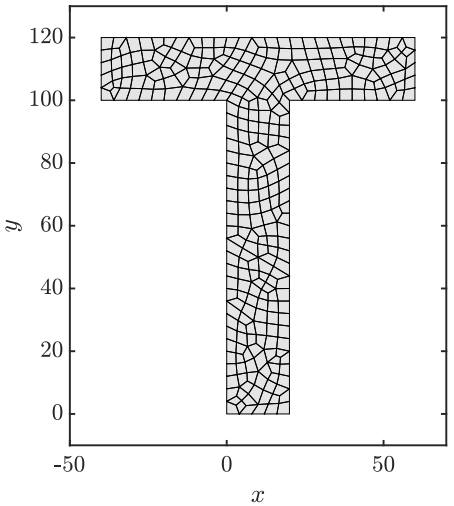

(d) Unstructured quad mesh

Figure 5.5: Four meshes used for the spinodal decomposition test. The distorted mesh has straight faces, while the curved mesh edges are curvilinear

The parameters of the Cahn-Hilliard equation used are that of Jokisaari et al. [2017], but adapted to the concentration $c$ that ranges from 0 to 1 (in Jokisaari et al. [2017] it ranges from 0.3 to 0.7),

$$
M_{0}=0.25,3 \sigma=\varepsilon=8.944, \quad \beta=0, \Delta t=0.1,
$$

and we use a polynomial order $N=4$. We monitor the free-energy evolution with time and compare with the results provided in Jokisaari et al. [2017].

We first study the effect of the IMEX parameters $K_{0}$ and $S_{0}$ in the curved (Fig. 5.5(c)) and the unstructured (Fig. 5.5(d)) meshes. For $K_{0}$ we consider two scenarios: the Crank-Nicolson scheme, $K_{0}=1 / 2$, and the backward Euler scheme $K_{0}=1$. For $S_{0}$ we study three values: $S_{0}=0$, which does not guarantee stability for the non-linear terms, $S_{0}=1$, which guarantees non-linear term stability for $C \in[0,1]$, and $S_{0}=2$, 


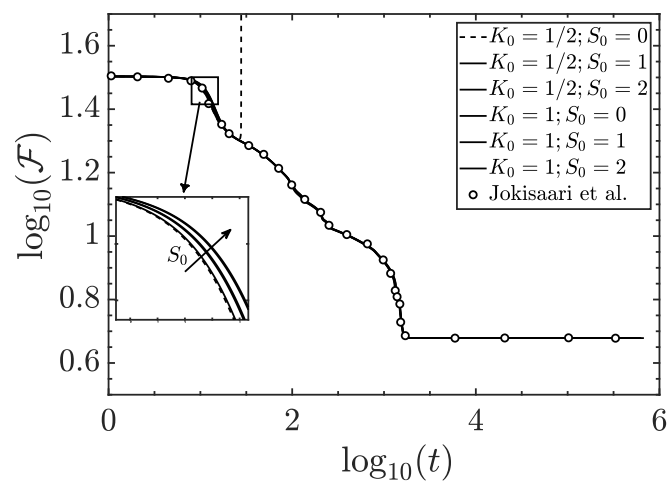

(a) Curved mesh

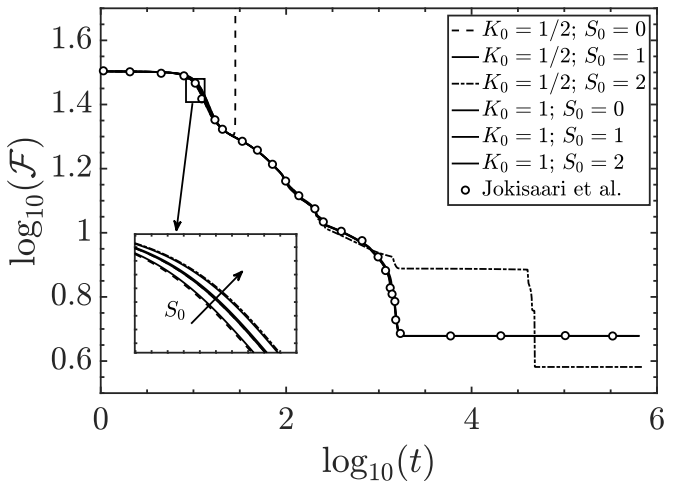

(b) Unstructured mesh

Figure 5.6: Evolution of the free-energy $\overline{\mathscr{F}}^{N}$ for the "T" domain. We consider two $K_{0}$ cases: Crank-Nicolson $\left(K_{0}=1 / 2\right)$ and backward Euler $\left(K_{0}=1\right)$. We find that the solution depends on $S_{0}$ only if we use the Crank-Nicolson scheme, producing an unstable scheme if $S_{0}=0$ (dashed line), and a different solution for $S_{0}=2$ (dot-dash line in the unstructured mesh). For the rest of the cases (solid lines), the solution agrees with Jokisaari et al. [2017], represented with circles

which guarantees the non-linear term stability for $C \in[-0.15,1.15]$ (see Fig. 5.1).

The evolution of the free-energy is shown in Fig. 5.6 for all parameter combinations and the two meshes (curvilinear in Fig. 5.6(a), and unstructured in Fig. 5.6(b)). The circles represent the reference solution from Jokisaari et al. [2017]. On the one hand, for the backward Euler scheme $\left(K_{0}=1\right.$, solid lines in Fig. 5.6), there is no high impact of $S_{0}$ on the free-energy. The scheme is stable even for the lowest $S_{0}$ value. Thus, the physical dissipation and the dissipation introduced by backward Euler scheme are enough to balance non-linear instabilities in this case. On the other hand, for the Crank-Nicolson scheme $\left(K_{0}=1 / 2\right)$, the solution depends highly on $S_{0}$. For $S_{0}=0$ (represented with a dashed line), the scheme is unstable. The physical dissipation is not sufficient to counteract the instabilities that arise from the non-linear terms. For $S_{0}=1$ (solid line), we get approximately the same solution as the backward Euler scheme on both meshes. However, for $S_{0}=2$ (dot-dash line), and only for the unstructured mesh, the final solution is different from the others, leading to lower free-energy values in the steady-state. Furthermore, in the detailed view (Fig. 5.6) we find small differences in the free-energy evolution when varying $S_{0}$. In a non-intuitive way, the free-energy decreases at a slower rate for higher $S_{0}$ values. Nonetheless, this result is still in agreement with the theoretical bound (5.73).

Overall, we recommend the use of the backward Euler scheme to remove the final solution dependency on $S_{0}$, and to use $S_{0}=1$ to avoid instabilities from non-linear 
terms, although they have not appeared in this experiment (with $K_{0}=1$ ).

With this settings $\left(S_{0}=1\right.$ and $K_{0}=1$ ), we represent the differences in the freeenergy evolution for the rest of the meshes considered in Fig. 5.5. First, in Fig. 5.7 we

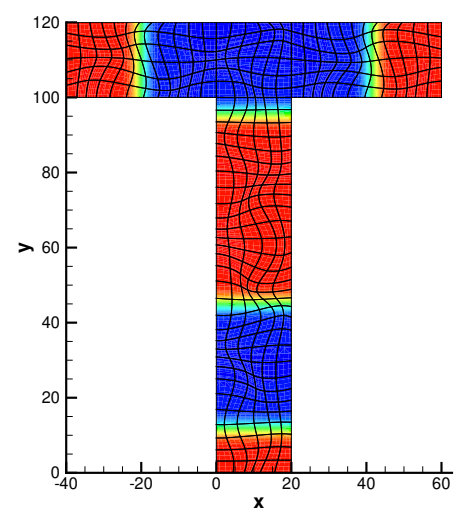

(a) Curved mesh

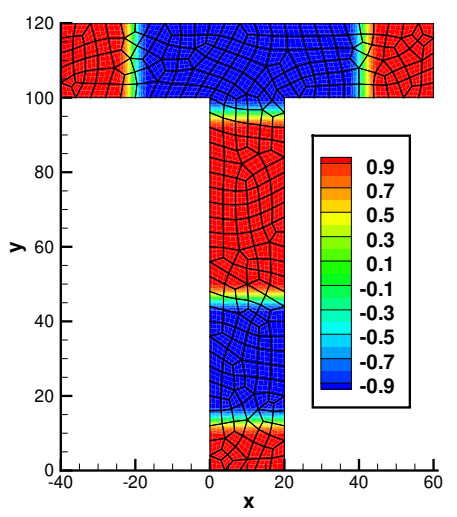

(b) Unstructured mesh

Figure 5.7: Representation of the final equilibrium solution in the curved and unstructured mesh. We find that the impact from the mesh on the solution is minimal, since interfaces are not aligned with the elements faces

represent the final state for the curvilinear and unstructured meshes, where we find that the mesh has no visual impact on the solution, since the resulting interfaces are not aligned with the mesh.

To quantify the differences due to the mesh, we consider the Cartesian mesh (Fig. 5.5(a)) as the reference solution, and represent the free-energy difference of the other meshes with that. The result is represented in Fig. 5.8. In the $y$-axis we represent the difference $\log _{10}\left(\overline{\mathscr{F}}_{\text {cartesian }}^{N}\right)-\log _{10}\left(\overline{\mathscr{F}}_{1}^{N}\right)$, between the Cartesian mesh and $\overline{\mathscr{F}}_{1}^{N}$ stands for the free-energy of the distorted (solid line), curved (dashed line) and unstructured (dash-dot line). In any case, the errors (and more substantially, in the initial and final states) remain low $\left(<10^{-3}\right)$, and all meshes considered were stable and accurate once the IMEX parameters were set-up appropriately.

\subsubsection{Three dimensional spinodal decomposition}

We also show the method's capability to solve the spinodal decomposition in three dimensions. We consider the interior of a cylinder $(L=D=1)$, which we divide into 920 elements. A representation of the cylinder and the mesh is provided in Fig. 5.9. We use order $N=3$ polynomials, and the following values for the rest of the parameters: 


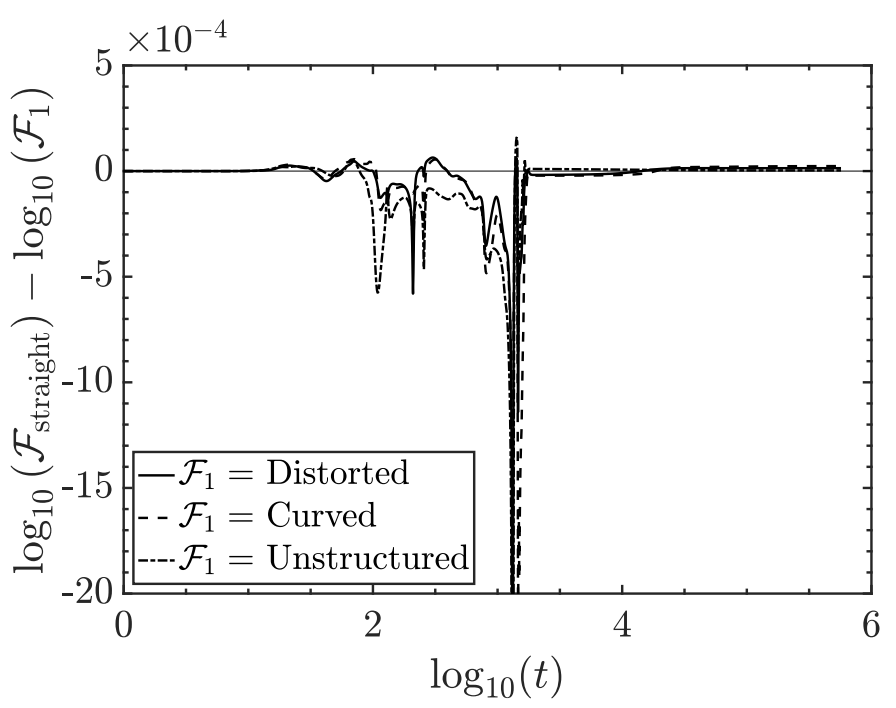

Figure 5.8: Free-energy evolution comparison of the distorted (solid line), curved (dashed line), and unstructured (dash-dot line) meshes with the Cartesian mesh. We find that, despite the initial and final values being approximately the same, in the evolution the errors are maintained low (of order $10^{-3}$ ) for the same number of degrees of freedom

$$
M_{0}=0.25, \quad 3 \sigma=\varepsilon=0.2828, \quad \beta=0, \Delta t=10^{-4}, K_{0}=1, S_{0}=1.0 .
$$

Initially, as in the two dimensional example, the phases are mixed, and we trigger the decomposition with the initial condition,

$$
\begin{aligned}
c_{0}(x, y, z)=0.5 & +0.0075 \cos (5 x-10 z) \cos (7 x+10 z y+1) \\
& +0.01 \cos \left(20 y^{2}+15 x^{2}\right) \sin (5 x+2 y+3 x) \\
& +0.01 \cos \left(10 \sqrt{y^{2}+z^{2}}\right) \cos (15 x y) \sin (20 x+10 z) \\
& +0.005 \cos (3 x) \cos (3 z) \cos (4 y) .
\end{aligned}
$$

We run the simulation until the steady-state is reached. We show the evolution of the phases in Fig. 5.10, where we have represented the interfaces and coloured the rest with blue $(C=0)$ and red $(C=1)$. Each plot in Fig. 5.10 represents a different time instant, one for each power of 10 . The initial condition corresponds to Fig. 5.10(a), and the final steady-state is Fig. $5.10(\mathrm{~g})$. Note that the final state is achieved with a flat interface separating both phases. Additionally, in Fig. 5.11 we depict the evolution of the free-energy $\overline{\mathscr{F}}^{N}$, showing that like in two dimensional simulations, it decreases monotonically from the initial condition to the steady state. 


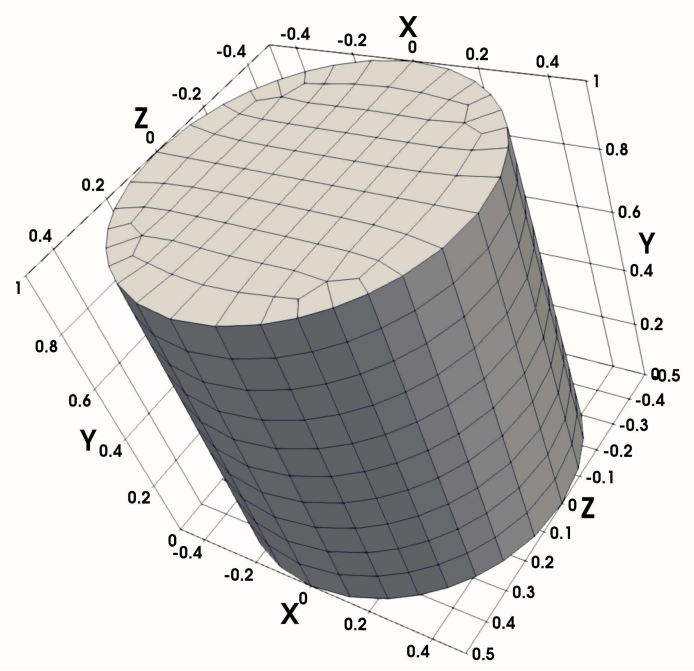

Figure 5.9: Representation of the mesh used for the three dimensional spinodal decomposition simulation

\subsection{Summary}

We have developed a nodal Discontinuous Galerkin (DG) spectral element method (DGSEM) approximation to solve the Cahn-Hilliard equation. We used the GaussLobatto variant of the DG method to use the summation-by-parts simultaneous-approximationterm (SBP-SAT) property and showed that it is discretely stable. The spatial discretization uses the Bassi-Rebay 1 (BR1) method to couple inter-element fluxes, and the time discretization uses an efficient IMEX scheme. We first show the semi-discrete stability analysis (i.e. continuous in time), and later we show the fully-discrete one using the IMEX scheme. Both analyses show that the discrete free-energy is bounded in time by the initial value, which is in accordance with the continuous energy estimate. Fullydiscrete stability was found to depend on a positivity condition for the phase field. Lastly, we perform a convergence study of the scheme, we compare the scheme with previous results from the literature, and perform a simulation in three-dimensional curvilinear geometries, showing that the scheme is stable (i.e. its free-energy decreases) as proved under all conditions tested herein. 


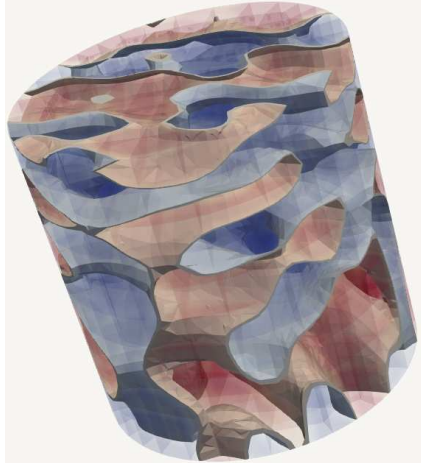

(a) $t=0$

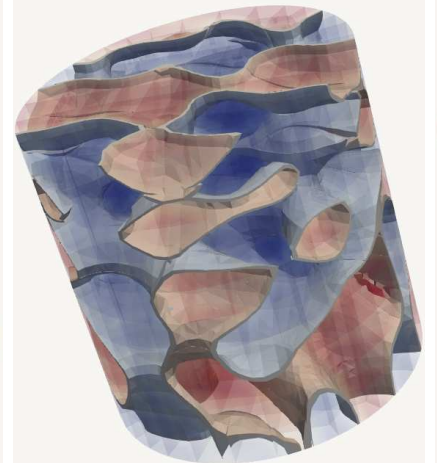

(b) $t=10^{-4}$

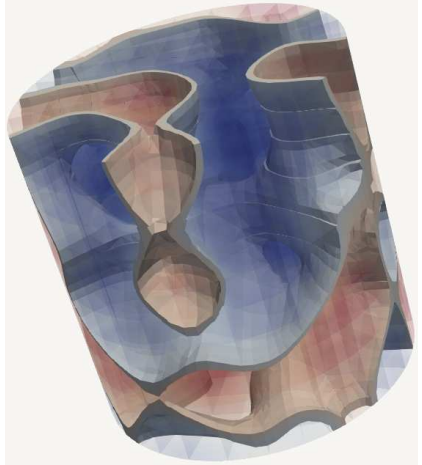

(c) $t=10^{-3}$

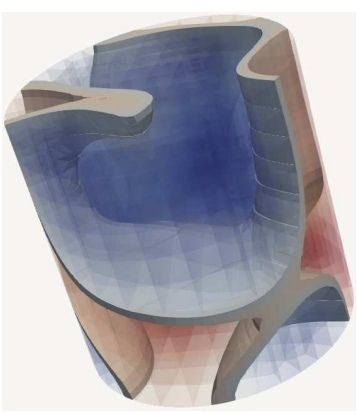

(d) $t=10^{-2}$

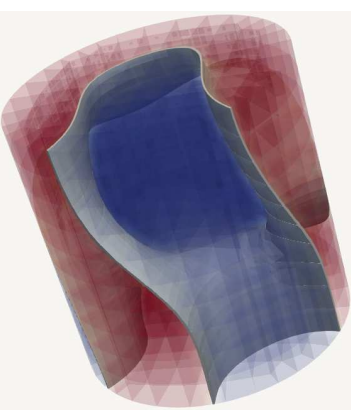

(e) $t=0.1$

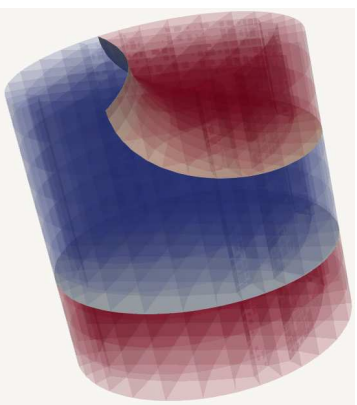

(f) $t=1.0$

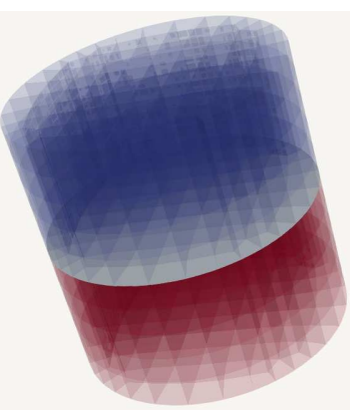

(g) $t=10.0$

Figure 5.10: Evolution of the phases with time for the three dimensional spinoidal decomposition. Blue and red contours represent the equilibrium phases $C=0$ and $C=1$ respectively

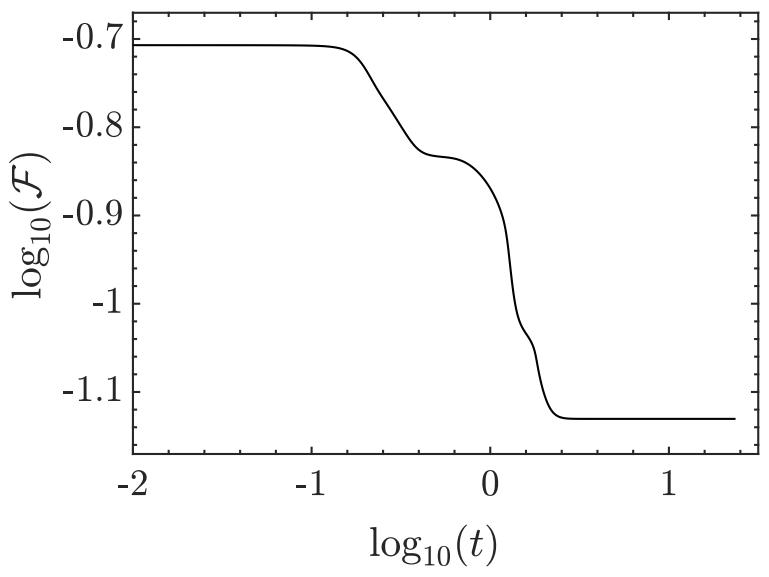

Figure 5.11: Evolution of the free-energy $\overline{\mathscr{F}}^{N}$ with time for the three-dimensional spinodal decomposition simulation 



\section{Chapter 6}

\section{Entropy-stable two-phase}

incompressible Navier-Stokes/Cahn-Hilliard (iNS/CH) solver

\section{Contents}

6.1 Introduction $\ldots \ldots \ldots \ldots \ldots \ldots \ldots \ldots \ldots \ldots \ldots \ldots \ldots$

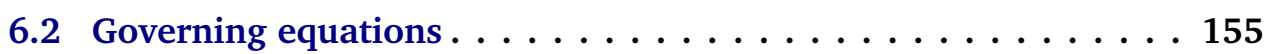

6.3 Entropy analysis of the iNS/CH system . . . . . . . . 160

6.4 Space and time discretization . . . . . . . . . . . 166

6.5 Semi-discrete stability analysis . . . . . . . . . . . . . . 177

6.6 Numerical experiments . . . . . . . . . . . . . . . . . . . 185

6.7 Summary and conclusions . . . . . . . . . . . . . . . . 199

\subsection{Introduction}

In this chapter we combine the diffuse interface model of Cahn-Hilliard Cahn and Hilliard [1958, 1959] of Chapter 5 with the incompressible Navier-Stokes equations with variable density and artificial (also called pseudo) compressibility Shen [1996] of Chapter 4. A review of alternate Navier-Stokes/Cahn-Hilliard models can be found in Hosseini et al. [2017]. 
This chapter uses the major part of the findings of the standalone version of the Cahn-Hilliard equation presented in Chapter 5 and of the incompressible NSE in Chapter 4. We combine the developments in those previous chapters to construct an entropy-stable DG approximation of the incompressible Navier-Stokes/Cahn-Hilliard system for two-phases.

The continuous system and its discrete approximation are entropy-stable (see Chapter 3). As opposed to the more traditional Model $\mathrm{H}$ Hohenberg and Halperin [1977] with constant density, when the density is allowed to vary between the two fluids in a multiphase flow the design of an entropy-stable scheme requires care. The variable density compromises the formulation used for the momentum equation Shen and Yang [2010]. Two main approaches to the design of a variable density entropystable approximation are commonly adopted: to use a skew-symmetric version of the momentum equation Guermond and Quartapelle [2000], or to augment the momentum equation with an additional diffusive flux that depends on the density difference Dong [2018b]; Ding et al. [2007]. The first option is adopted in this work.

The addition of the artificial compressibility model, where a divergence-free velocity is not strictly enforced, completes the formulation used for the body interfacial forces. Without proper choices, terms proportional to $\vec{\nabla} \cdot \vec{u}$, which cannot be neglected nor bounded, might appear in the entopy equation. With the proper choices, we confirm that the entropy of the Navier-Stokes/Cahn-Hilliard system obeys the Onsager principle; the total entropy is the sum of the entropy of the incompressible Navier-Stokes equations, plus the free-energy of the Cahn-Hilliard equation Cahn and Hilliard [1958]. In Chapter 4 we derived a mathematical entropy of the incompressible Navier-Stokes equations with artificial compressibility as the sum of the traditional kinetic energy, plus an additional energy term that accounts for compressibility effects. As a result, the total entropy becomes the sum of the kinetic energy $(\mathscr{K})$, the artificial compressibility energy $(\mathscr{P})$, and the free-energy $(\mathscr{F})$.

In this chapter we construct a multiphase approximation that is appropriate to solve typical applications in the oil industry. An example is the transport of crude oil in pipes, where the flow of oil and water is solved under high Reynolds number conditions. Since one desires to keep the mesh and degrees of freedom as low as possible, the flow configuration is often under-resolved. Under these circumstances, a robust method that is provably stable is attractive, as it avoids aliasing driven numerical instabilities that might lead to numerical divergence Manzanero et al. [2018a].

The rest of this chapter is organized as follows: in Sec. 6.2 we describe the incompressible Navier-Stokes/Cahn-Hilliard model, with the continuous entropy analysis in Sec. 6.3. The construction of the discrete DG approximation is described in Sec. 6.4. Then, the semi-discrete stability analysis of the approximation is performed 
in Sec. 6.5, for which a summary can be found in Sec. 6.5.5. Lastly, we provide numerical experiments in Sec. 6.6 that assess both the accuracy and robustness of the method. We solve a manufactured solution convergence analysis in Sec. 6.6.1, and an assessment of the robustness solving a random initial condition in Sec. 6.6.2. Then, we solve classic static and rising bubble test problems in Sec. 6.6.3 and Sec. 6.6.4 respectively. Lastly, in Sec. 6.6.5 we challenge the technique by solving a multiphase pipe in the annular flow regime. Conclusions and discussions can be found in Sec. 6.7.

\subsection{Governing equations}

In this section we describe a model that combines the incompressible Navier-Stokes and the Cahn-Hilliard equations, which we will refer to as iNS/CH. In the single phase variable density incompressible Navier-Stokes equations, the density $\rho(\vec{x}, t)$ is an independent variable that satisfies the continuity equation (see Manzanero et al. [2020a]; Bassi et al. [2017]; Guermond and Quartapelle [2000]),

$$
\rho_{t}+\vec{\nabla} \cdot(\rho \vec{u})=0
$$

where $\vec{u}(\vec{x}, t)=(u, v, w)$ is the velocity field. In the two-phase iNS/CH system, however, the density is computed from the concentration $c(\vec{x}, t)$ of the two fluids Lowengrub and Truskinovsky [1998],

$$
\rho=\rho(c)=\rho_{1} c+\rho_{2}(1-c)
$$

where $\rho_{1,2}$ are the densities of fluids 1 and 2 , respectively, which are constant in space and time.

In phase field methods, an advection-diffusion equation drives the concentration. Among the model choices, we pick the Cahn-Hilliard equation Cahn and Hilliard [1958, 1959]. The Cahn-Hilliard equation has already been introduced in this work in (5.1), and it is now extended following Han and Wang [2015] to include an advective term (in conservative form),

$$
c_{t}+\vec{\nabla} \cdot(c \vec{u})=M_{0} \vec{\nabla}^{2} \mu
$$

In (6.3), $\mu$ is the chemical potential (5.7), $f_{0}(c)$ is the chemical free-energy, (5.9), $\sigma$ is the coefficient of interface tension between the fluids, $\varepsilon$ is the interface width, and $M_{0}$ is the mobility, computed in this chapter from the chemical characteristic time, $t_{\mathrm{CH}}$, as

$$
M_{0}=\frac{\varepsilon}{\sigma t_{\mathrm{CH}}}
$$


The parameters $\sigma, \varepsilon, M_{0}$, and $t_{\mathrm{CH}}$ are positive constants. As discussed in Sec. 5.2, the Cahn-Hilliard equation (6.3), with the chemical potential definition (5.7), has an associated free-energy

$$
\mathscr{F}(c, \vec{\nabla} c)=f_{0}(c)+\frac{3}{4} \sigma \varepsilon|\vec{\nabla} c|^{2}
$$

Moreover, it was described in Chapter 5 that the Cahn-Hilliard equation is complemented with the Neumann boundary conditions (5.3) and (5.8), that define the surface free-energy $\mathscr{F}^{S}=f_{w}(c)$.

The velocity field is computed from the momentum equation. In the single phase incompressible Navier-Stokes solver (Chapter 4), we adopted the conservative version of the momentum equation (4.1). This choice needs to be revisited for the two-phase solver. If one assumes that the continuity equation (6.1) holds, the conservative, nonconservative, and skew-symmetric forms of the momentum advection are identical in the continuous setting, that is,

$$
(\rho \vec{u})_{t}+\vec{\nabla} \cdot(\rho \vec{u} \vec{u})=\rho(\vec{u})_{t}+\rho \vec{u} \cdot \vec{\nabla} \vec{u}=\frac{1}{2}(\rho \vec{u})_{t}+\frac{1}{2} \rho(\vec{u})_{t}+\vec{\nabla} \cdot\left(\frac{1}{2} \rho \vec{u} \vec{u}\right)+\frac{1}{2} \rho \vec{u} \cdot \vec{\nabla} \vec{u} .
$$

However, since the density does not satisfy the continuity equation (6.1), but rather the compatibility condition (6.2), the three forms (6.6) are no longer equivalent.

The momentum equation is frozen as one of the three forms. Following Guermond and Quartapelle [2000]; Shen and Yang [2010], we choose the skew-symmetric version of the momentum (i.e. the last form in (6.6)) since is the only one that satisfies an entropy inequality for any positive density field. (An alternative that produces an entropy-stable scheme for the iNS/CH system is to include a relative flux in the momentum equation that models the diffusion of the components, Abels et al. [2012].)

We use the chain rule in time to perform an additional manipulation on the time derivative coefficients of the skew-symmetric form (6.6),

$$
\frac{1}{2}(\rho \vec{u})_{t}+\frac{1}{2} \rho \vec{u}_{t}=\frac{1}{2} \rho_{t} \vec{u}+\rho \vec{u}_{t}=\sqrt{\rho}(\sqrt{\rho})_{t} \vec{u}+\sqrt{\rho} \sqrt{\rho} \vec{u}_{t}=\sqrt{\rho}(\sqrt{\rho} \vec{u})_{t},
$$

and derive the momentum form by Guermond et al. Guermond and Quartapelle [2000],

$\sqrt{\rho}(\sqrt{\rho} \vec{u})_{t}+\vec{\nabla} \cdot\left(\frac{1}{2} \rho \vec{u} \vec{u}\right)+\frac{1}{2} \rho \vec{u} \cdot \vec{\nabla} \vec{u}=-\vec{\nabla} p_{s}-\vec{\nabla} \cdot\left(\vec{\nabla} c \otimes \frac{\partial \mathscr{F}}{\partial \vec{\nabla} c}\right)+\vec{\nabla} \cdot\left(\eta\left(\vec{\nabla} \vec{u}+\vec{\nabla} \vec{u}^{T}\right)\right)+\rho \vec{g} \cdot$

In (6.8), $p_{s}(\vec{x}, t)$ is the static pressure, and the second term is the body force approximation for the capillary pressure Lowengrub and Truskinovsky [1998],

$$
\vec{p}_{c}=-\vec{\nabla} \cdot\left(\vec{\nabla} c \otimes \frac{\partial \mathscr{F}}{\partial \vec{\nabla} c}\right)=-\frac{3}{2} \sigma \varepsilon \vec{\nabla} \cdot(\vec{\nabla} c \otimes \vec{\nabla} c)
$$


Finally, $\eta$ is the (non-constant) viscosity, computed from the concentration $c$ and the (constant) viscosities of fluids 1 and $2, \eta_{1,2}$,

$$
\eta=\eta(c)=\eta_{1} c+\eta_{2}(1-c)
$$

As in Lowengrub and Truskinovsky [1998], we write the capillary pressure more conveniently. Applying the derivative product rule,

$$
\vec{p}_{c}=-\frac{3}{2} \sigma \varepsilon\left(\vec{\nabla}^{2} c \vec{\nabla} c+\vec{\nabla}\left(\frac{1}{2}|\vec{\nabla} c|^{2}\right)\right),
$$

and adding and subtracting the term $\vec{\nabla} f_{0}=f_{0}^{\prime}(c) \vec{\nabla} c$, we can rewrite the expression for the body force as

$$
\begin{aligned}
\vec{p}_{c}=\left(f_{0}^{\prime}(c)-\frac{3}{2} \sigma \varepsilon \vec{\nabla}^{2} c\right) \vec{\nabla} c-\vec{\nabla}\left(f_{0}(c)+\frac{3}{4} \sigma \varepsilon|\vec{\nabla} c|^{2}\right) & =\mu \vec{\nabla} c-\vec{\nabla} \mathscr{F} \\
& =-c \vec{\nabla} \mu-\vec{\nabla}(\mathscr{F}-\mu c)
\end{aligned}
$$

In the last form, an additional application of the product derivative rule for the first term has been performed. Eq. (6.12) represents equivalent expressions of the capillary pressure (6.9), the last form being the one adopted here. The first term of the last form, $-c \vec{\nabla} \mu$, is a non-conservative term, and the second, $-\vec{\nabla}(\mathscr{F}-\mu c)$, is combined with the static pressure gradient to define an auxiliary pressure

$$
p=p_{s}+\mathscr{F}-\mu c
$$

With all of these manipulations, we get the final expression of the momentum equation,

$$
\sqrt{\rho}(\sqrt{\rho} \vec{u})_{t}+\vec{\nabla} \cdot\left(\frac{1}{2} \rho \vec{u} \vec{u}\right)+\frac{1}{2} \rho \vec{u} \cdot \vec{\nabla} \vec{u}+c \vec{\nabla} \mu=-\vec{\nabla} p+\vec{\nabla} \cdot\left(\eta\left(\vec{\nabla} \vec{u}+\vec{\nabla} \vec{u}^{T}\right)\right)+\rho \vec{g} .
$$

Many authors have explored how to relax the incompressibility constraint $\vec{\nabla} \cdot \vec{u}=0$ in Navier-Stokes and Navier-Stokes/Cahn-Hilliard systems by using the artificial (or pseudo) compressibility method Shen [1997, 1996]; Feng et al. [2018]; Zhu et al. [2019]. We recover here the two approaches studied in Chapter 4,

$$
p_{t}+\rho_{0} c_{0}^{2} \vec{\nabla} \cdot \vec{u}=0,-\vec{\nabla}^{2} p_{t}+\rho_{0} c_{0}^{2} \vec{\nabla} \cdot \vec{u}=0,\left.\quad \vec{\nabla} p_{t} \cdot \vec{n}\right|_{\partial \Omega}=0
$$

where $c_{0}$ is the artificial sound speed, and $\rho_{0}=\max \left(\rho_{1}, \rho_{2}\right)$. We can compactly write (6.16) as

$$
\mathrm{L}_{p}(p)_{t}+\rho_{0} c_{0}^{2} \vec{\nabla} \cdot \vec{u}=0
$$


where $\mathrm{L}_{p, \mathrm{AC} 1}=p$ for the first model, and $\mathrm{L}_{p, \mathrm{AC} 2}=-\vec{\nabla}^{2} p$ for the second model. As in the incompressible Navier-Stokes chapter, we analyze both artificial compressibility models, but we only implement and validate the multiphase solver that uses the first model.

The combination of (6.3), (6.14) and (6.16) is the iNS/CH system studied in this chapter. The coupling between the Cahn-Hilliard equation and the incompressible Navier-Stokes equations is two-way, through the compatibility conditions (6.2), (6.10), and the capillary pressure. We note that even in the continuous setting, the momentum is not conserved because of the choice of the skew-symmetric form (6.14). Although one can argue about the physical implications, we note that the capillary force is a non-conservative term as well, so momentum conservation is not guaranteed in any of the forms (6.6). Alternative incompressible Navier-Stokes/Cahn-Hilliard discretizations using the skew-symmetric form of the momentum equation can be found in Shen and Yang [2010]; Boyer et al. [2010].

To simplify the notation, we write the iNS/CH system as a general advectiondiffusion equation,

$$
\begin{aligned}
& \left(\begin{array}{ccccc}
1 & 0 & 0 & 0 & 0 \\
0 & \sqrt{\rho} & 0 & 0 & 0 \\
0 & 0 & \sqrt{\rho} & 0 & 0 \\
0 & 0 & 0 & \sqrt{\rho} & 0 \\
0 & 0 & 0 & 0 & 1
\end{array}\right)\left(\begin{array}{c}
c \\
\sqrt{\rho} u \\
\sqrt{\rho} v \\
\sqrt{\rho} w \\
L_{p}(p)
\end{array}\right)+\vec{\nabla} \cdot\left(\begin{array}{c}
c \vec{u} \\
\frac{1}{2} \rho \vec{u} u+p \vec{e}_{1} \\
\frac{1}{2} \rho \vec{u} v+p \vec{e}_{2} \\
\frac{1}{2} \rho \vec{u} w+p \vec{e}_{3} \\
0
\end{array}\right) \\
& +\left(\begin{array}{c}
\frac{1}{2} \rho \vec{u} \cdot \vec{\nabla} u+c \vec{e}_{1} \cdot \vec{\nabla} \mu \\
\frac{1}{2} \rho \vec{u} \cdot \vec{\nabla} v+c \vec{e}_{2} \cdot \vec{\nabla} \mu \\
\frac{1}{2} \rho \vec{u} \cdot \vec{\nabla} w+c \vec{e}_{3} \cdot \vec{\nabla} \mu \\
\rho_{0} c_{0}^{2}\left(\vec{e}_{1} \cdot \vec{\nabla} u+\vec{e}_{2} \cdot \vec{\nabla} v+\vec{e}_{3} \cdot \vec{\nabla} w\right)
\end{array}\right)=\vec{\nabla} \cdot\left(\begin{array}{c}
M_{0} \vec{\nabla} \mu \\
2 \eta \bar{s} \cdot \vec{e}_{1} \\
2 \eta \bar{s} \cdot \vec{e}_{2} \\
2 \eta \bar{s} \cdot \vec{e}_{3} \\
\overrightarrow{0}
\end{array}\right)+\left(\begin{array}{c}
0 \\
\rho \vec{g} \cdot \vec{e}_{1} \\
\rho \vec{g} \cdot \vec{e}_{2} \\
\rho \vec{g} \cdot \vec{e}_{3} \\
0
\end{array}\right),
\end{aligned}
$$

where,

$$
\overline{\bar{s}}=\operatorname{sym}(\vec{\nabla} \vec{u})=\frac{1}{2}\left(\vec{\nabla} \vec{u}+\vec{\nabla} \vec{u}^{T}\right),
$$

is the strain tensor, and $\vec{e}_{i}$ are the space unit vectors. Note that we have grouped the velocity divergence in the artificial compressibility equation (6.16) into the nonconservative terms. Although treating the velocity divergence as conservative or nonconservative is equivalent, the latter makes it easier to show the stability.

We now adopt the block vector notation (see Sec. 1.7) to write the iNS/CH system (6.17) compactly in the form of a general non-conservative system (2.77). Since the gradient variables in the non-conservative terms and the viscous fluxes are the same, instead of using the symbol $\lambda_{n}$ to refer to those in the non-conservative terms, we use the letter $w_{n}$ for both. 
The iNS/CH system written as a general non-conservative system is

$$
\underline{h L}(\mathbf{q})_{t}+\vec{\nabla} \cdot \overrightarrow{\mathbf{f}}_{e}(\mathbf{q})+\sum_{m=1}^{5} \overrightarrow{\boldsymbol{\phi}}_{m}(\mathbf{q}) \cdot \vec{\nabla} w_{m}=\vec{\nabla} \cdot \overrightarrow{\mathbf{f}}_{v}(\mathbf{q}, \vec{\nabla} \mathbf{w})+\mathbf{s}(\mathbf{q}),
$$

with state vector $\mathbf{q}=(c, \sqrt{\rho} \vec{u}, p)$, time derivative operator $\mathrm{L}(\mathbf{q})=\left(c, \sqrt{\rho} \vec{u}, \mathrm{~L}_{p}(p)\right)$, gradient variables vector $\mathbf{w}=\left(w_{1}, w_{2}, \ldots, w_{5}\right)=(\mu, \vec{u}, p)$, mass matrix $\underline{h}$,

$$
\underline{h}=\left(\begin{array}{ccc}
1 & 0 & 0 \\
0 & \sqrt{\rho} \overline{\bar{I}}_{3} & 0 \\
0 & 0 & 1
\end{array}\right)
$$

inviscid fluxes $\overrightarrow{\mathbf{f}}_{e}(\mathbf{q})$,

$$
\mathbf{f}_{e, 1}=\mathbf{f}_{e}=\left(\begin{array}{c}
c u \\
\rho u^{2}+p \\
\rho u v \\
\rho u w \\
0
\end{array}\right), \mathbf{f}_{e, 2}=\mathbf{g}_{e}=\left(\begin{array}{c}
c v \\
\rho u v \\
\rho v^{2}+p \\
\rho v w \\
0
\end{array}\right), \mathbf{f}_{e, 3}=\mathbf{h}_{e}=\left(\begin{array}{c}
c w \\
\rho u w \\
\rho v w \\
\rho w^{2}+p \\
0
\end{array}\right)
$$

non-conservative term coefficients $\overrightarrow{\boldsymbol{\phi}}_{m}(\mathbf{q})$,

$$
\begin{aligned}
& \overrightarrow{\boldsymbol{\phi}}_{1}=\Upsilon\left(\begin{array}{c}
0 \\
c \vec{e}_{1} \\
c \vec{e}_{2} \\
c \vec{e}_{3} \\
0
\end{array}\right), \overrightarrow{\boldsymbol{\phi}}_{2}=\Upsilon\left(\begin{array}{c}
0 \\
\frac{1}{2} \rho \vec{u} \\
0 \\
0 \\
\rho_{0} c_{0}^{2} \vec{e}_{1}
\end{array}\right), \overrightarrow{\boldsymbol{\phi}}_{3}=\Upsilon\left(\begin{array}{c}
0 \\
0 \\
\frac{1}{2} \rho \vec{u} \\
0 \\
\rho_{0} c_{0}^{2} \vec{e}_{2}
\end{array}\right), \\
& \overrightarrow{\boldsymbol{\phi}}_{4}=\Upsilon\left(\begin{array}{c}
0 \\
0 \\
0 \\
0 \\
\frac{1}{2} \rho \vec{u} \\
\rho_{0} c_{0}^{2} \vec{e}_{3}
\end{array}\right), \overrightarrow{\boldsymbol{\phi}}_{5}=\Upsilon\left(\begin{array}{c}
0 \\
0 \\
0
\end{array}\right),
\end{aligned}
$$

viscous fluxes $\overrightarrow{\mathbf{f}}_{v}(\vec{\nabla} \mathbf{w})$,

$$
\mathbf{f}_{v, 1}=\mathbf{f}_{v}=\left(\begin{array}{c}
M_{0} \mu_{x} \\
2 \eta \bar{s}_{11} \\
2 \eta \bar{s}_{21} \\
2 \eta \bar{s}_{31} \\
0
\end{array}\right), \mathbf{f}_{v, 2}=\mathbf{g}_{v}=\left(\begin{array}{c}
M_{0} \mu_{y} \\
2 \eta \bar{s}_{12} \\
2 \eta \bar{s}_{22} \\
2 \eta \bar{s}_{32} \\
0
\end{array}\right), \mathbf{f}_{v, 3}=\mathbf{h}_{v}=\left(\begin{array}{c}
M_{0} \mu_{z} \\
2 \eta \bar{s}_{13} \\
2 \eta \bar{s}_{23} \\
2 \eta \bar{s}_{33} \\
0
\end{array}\right)
$$

and source term $\mathbf{s}(\mathbf{q})=(0, \rho \vec{g}, 0)$. Recall that the operator $\Upsilon$ defined in (1.13) transforms a $5 \times 3$ matrix in a block vector. 
An attractive property of the iNS/CH system from the point of view of computational efficiency is that the same gradient variables $\mathbf{w}$ are used in both the nonconservative terms and the viscous fluxes. Moreover, these gradient variables will be shown to be the entropy variables associated with the mathematical entropy in Section 6.3. Additionally, recall that the velocity divergence from the artificial compressibility equation (6.16) was grouped into the non-conservative terms since it is beneficial when proving stability.

\subsubsection{Boundary conditions}

We describe the imposition of free- and no-slip wall boundary conditions. For the Cahn-Hilliard equation, we use a non-homogeneous Neumann condition for the concentration Dong [2012], and a homogeneous Neumann condition for the chemical potential,

$$
-\left.\frac{3}{2} \sigma \varepsilon \vec{\nabla} c \cdot \vec{n}\right|_{\partial \Omega}=f_{w}^{\prime}(c),\left.\quad \vec{\nabla} \mu \cdot \vec{n}\right|_{\partial \Omega}=0
$$

where $f_{w}(c)$ is the boundary free-energy function that controls the wall contact angle. In this chapter we use the function Dong [2012]

$$
f_{w}(c)=\frac{1}{2} \sigma \cos \theta_{w}(2 c-1)\left(1+2 c-2 c^{2}\right), f_{w}^{\prime}(c)=6 \sigma \cos \theta_{w} c(1-c),
$$

where $\theta_{w}$ is the imposed contact angle with the wall. In most simulations performed in this work we use a $90^{\circ}$ angle, which simplifies $f_{w}(c)=0$.

For free-slip boundary conditions, we impose a zero normal velocity $\vec{u} \cdot \vec{n}=0$ and zero normal stress $\overline{\bar{\tau}} \cdot \vec{n}=0$ for momentum, while for no-slip walls, all velocity components are zero $(\vec{u}=0)$, and we do not impose any conditions on the stress tensor.

\subsection{Entropy analysis of the iNS/CH system}

We study the stability of the iNS/CH system as a particular case of the general theory developed for non-conservative systems in Sec. 3.6. As described in Sec. 3.5 and Sec. 3.6, the entropy analysis rests on the existence of a pair $\mathscr{E}(\mathbf{q})$ (mathematical entropy) and $\mathbf{w}$ (entropy variables) that contract the system of equations (6.19) into 
a conservation law Tadmor [2003],

$$
\begin{gathered}
\left\langle\underline{h} \mathrm{~L}(\mathbf{q})_{t}, \mathbf{w}\right\rangle+\left\langle\vec{\nabla} \cdot \overrightarrow{\mathbf{f}}_{e}, \mathbf{w}\right\rangle+\sum_{m=1}^{N_{c}}\left\langle\overrightarrow{\boldsymbol{\phi}}_{m} \cdot \vec{\nabla} w_{m}, \mathbf{w}\right\rangle-\left\langle\vec{\nabla} \cdot \overrightarrow{\mathbf{f}}_{v}, \mathbf{w}\right\rangle \\
=\overline{\mathscr{E}}_{t}+\int_{\partial \Omega} \vec{f}^{\mathscr{E}} \cdot \mathrm{d} \vec{S}+\left\langle\overrightarrow{\mathbf{f}}_{v}, \vec{\nabla} \mathbf{w}\right\rangle=0,
\end{gathered}
$$

with an entropy flux $\vec{f}^{\mathscr{E}}$, and a positive viscous contribution $\left\langle\overrightarrow{\mathbf{f}}_{v}, \vec{\nabla} \mathbf{w}\right\rangle \geqslant 0$.

To arrive at the entropy of the iNS/CH system, we add the Cahn-Hilliard freeenergy $\mathscr{F}$ (5.4), to the entropy of the incompressible NSE (4.12)/(4.13),

$$
\begin{aligned}
\mathscr{E}_{\mathrm{AC} 1}(\mathbf{q}, \vec{\nabla} \mathbf{q}) & =\mathscr{F}(c, \vec{\nabla} c)+\mathscr{K}(\sqrt{\rho} \vec{u})+\mathscr{P}_{\mathrm{AC} 1}(p) \\
& =f_{0}(c)+\frac{3}{4} \sigma \varepsilon|\vec{\nabla} c|^{2}+\frac{1}{2} \rho v_{t o t}^{2}+\frac{p^{2}}{2 \rho_{0} c_{0}^{2}} \geqslant 0,
\end{aligned}
$$

for the first artificial compressibility model, and

$$
\begin{aligned}
\mathscr{E}_{\mathrm{AC} 2}(\mathbf{q}, \vec{\nabla} \mathbf{q}) & =\mathscr{F}(c, \vec{\nabla} c)+\mathscr{K}(\sqrt{\rho} \vec{u})+\mathscr{P}_{\mathrm{AC} 2}(\vec{\nabla} p) \\
& =f_{0}(c)+\frac{3}{4} \sigma \varepsilon|\vec{\nabla} c|^{2}+\frac{1}{2} \rho v_{t o t}^{2}+\frac{|\vec{\nabla} p|^{2}}{2 \rho_{0} c_{0}^{2}} \geqslant 0,
\end{aligned}
$$

for the second artificial compressibility model. In the kinetic energy $\mathscr{K}, v_{\text {tot }}^{2}=u^{2}+$ $v^{2}+w^{2}$ is the square of the total speed. Eq. (6.27) assumes positivity on the density $\rho$, which is ensured here by limiting the maximum and minimum density values used in the momentum equation with a simple cutoff,

$$
\rho(\tilde{c})=\rho_{1} \tilde{c}+\rho_{2}(1-\tilde{c}), \quad \tilde{c}=\min (\max (c, 0), 1)
$$

We evaluate the three properties for the entropy-stabilty of the non-conservative iNS/CH system: contraction of time coefficients (3.71), contraction of inviscid fluxes (3.102), and positive definiteness of viscous fluxes (3.74). As in the incompressible NSE solver, only the contraction of the time coefficients depends on the choice of the artificial compressibility model, whereas the demonstration of the other two property is shared between both models.

None of the entropies depend solely on the state vector q. Thus, we cannot compute the entropy variables directly using differentiation (3.82). For this system, we extract the entropy variables directly from the time coefficients contraction. 


\subsubsection{Contraction of the time coefficients}

From all the steps required for entropy-stability, the contraction of time coefficients (3.71),

$$
\left\langle\underline{h} \mathrm{~L}(\mathbf{q})_{t}, \mathbf{w}\right\rangle=\left\langle\mathscr{E}_{t}\right\rangle+\frac{\mathrm{d}}{\mathrm{d} t} \int_{\partial \Omega} \mathscr{E}^{S} \mathrm{~d} S=\overline{\mathscr{E}}_{t},
$$

is the only one that depends on the artificial compressibility model. However, we will see that the entropy variables $\mathbf{w}$ are the same for both models.

\section{First artificial compressibility model}

We look for a set of entropy variables $\mathbf{w}$ that contracts the time coefficients (6.30). To do so, we write the time derivative of the entropy,

$$
\begin{aligned}
\left\langle\mathscr{E}_{\mathrm{AC} 1, t}\right\rangle & =\left\langle f_{0}^{\prime}(c) c_{t}+\frac{3}{2} \sigma \varepsilon \vec{\nabla} c \cdot \vec{\nabla} c_{t}+\sqrt{\rho \vec{u}} \cdot(\sqrt{\rho} \vec{u})_{t}+\frac{p p_{t}}{\rho_{0} c_{0}^{2}}\right\rangle \\
& =\left\langle c_{t}, f_{0}^{\prime}(c)-\frac{3}{2} \sigma \varepsilon \vec{\nabla}^{2} c\right\rangle+\int_{\partial \Omega} \frac{3}{2} \sigma \varepsilon c_{t} \vec{\nabla} c \cdot \mathrm{d} \vec{S}+\left\langle\sqrt{\rho}(\sqrt{\rho} \vec{u})_{t}, \vec{u}\right\rangle+\left\langle p_{t}, \frac{p}{\rho_{0} c_{0}^{2}}\right\rangle \\
& =\left\langle c_{t}, \mu\right\rangle-\frac{\mathrm{d}}{\mathrm{d} t} \int_{\partial \Omega} f_{w} \mathrm{~d} S+\left\langle\sqrt{\rho}(\sqrt{\rho} \vec{u})_{t}, \vec{u}\right\rangle+\left\langle p_{t}, \frac{p}{\rho_{0} c_{0}^{2}}\right\rangle .
\end{aligned}
$$

In the second identity, we used the Gauss law for the interfacial energy, and in the third identity we used the definition of the chemical potential (5.7) and the physical boundary condition for $\vec{\nabla} c$ (5.8) plus the chain rule in time.

Now we compute the left hand side of (6.30) (with $\mathrm{L}(\mathbf{q})=\mathbf{q}$ for the first artificial compressibility model), and equate it to the total entropy time derivative

$$
\begin{aligned}
\left\langle\underline{h} \mathbf{q}_{t}, \mathbf{w}\right\rangle & =\left\langle c_{t}, w_{1}\right\rangle+\left\langle\sqrt{\rho}(\sqrt{\rho} \vec{u})_{t},\left(w_{2}, w_{3}, w_{4}\right)\right\rangle+\left\langle p_{t}, w_{5}\right\rangle \\
& =\left\langle c_{t}, \mu\right\rangle-\frac{\mathrm{d}}{\mathrm{d} t} \int_{\partial \Omega} f_{w} \mathrm{~d} S+\left\langle\sqrt{\rho}(\sqrt{\rho} \vec{u})_{t}, \vec{u}\right\rangle+\left\langle p_{t}, \frac{p}{\rho_{0} c_{0}^{2}}\right\rangle+\frac{\mathrm{d}}{\mathrm{d} t} \int_{\partial \Omega} \mathscr{E}^{S} \mathrm{~d} S,
\end{aligned}
$$

so that reordering,

$$
\begin{aligned}
\left\langle c_{t},\left(w_{1}-\mu\right)\right\rangle+ & \left\langle\sqrt{\rho}(\sqrt{\rho} \vec{u})_{t},\left(w_{2}-u, w_{3}-v, w_{4}-w\right)\right\rangle+\left\langle p_{t}, w_{5}-\frac{p}{\rho_{0} c_{0}^{2}}\right\rangle \\
& =\frac{\mathrm{d}}{\mathrm{d} t} \int_{\partial \Omega}\left(\mathscr{E}^{S}-f_{w}\right) \mathrm{d} S,
\end{aligned}
$$


we identify the entropy variables and the surface entropy,

$$
\mathbf{w}=\left(\mu, \vec{u}, \frac{p}{\rho_{0} c_{0}^{2}}\right), \mathscr{E}^{S}=\mathscr{F}^{S}=f_{w} .
$$

Note that the entropy variables are indeed the gradient variables vector $\mathbf{w}$ that we used to write viscous fluxes and non-conservative terms.

Note that the minimum of the entropy is found when all the entropy variables are zero. For the first artificial compressibility model, we define the total entropy as

$$
\overline{\mathscr{E}}_{\mathrm{AC} 1}=\left\langle\mathscr{E}_{\mathrm{AC} 1}\right\rangle+\int_{\partial \Omega} \mathscr{E}^{S} \mathrm{~d} S=\left\langle f_{0}+\frac{3}{4} \sigma \varepsilon|\vec{\nabla} c|^{2}+\frac{1}{2} \rho v_{t o t}^{2}+\frac{p^{2}}{2 \rho_{0} c_{0}^{2}}\right\rangle+\int_{\partial \Omega} f_{w} \mathrm{~d} S .
$$

Moreover, a very interesting propoperty is that we see that the contraction of the time coefficients is isolated for the Cahn-Hilliard, momentum, and pressure terms, so that individually they satisfy,

$$
\begin{aligned}
\left\langle c_{t}, \mu\right\rangle & =\left\langle\mathscr{F}_{t}\right\rangle+\frac{\mathrm{d}}{\mathrm{d} t} \int_{\partial \Omega} f_{w} \mathrm{~d} S, \\
\left\langle\sqrt{\rho}(\sqrt{\rho} \vec{u})_{t}, \vec{u}\right\rangle & =\left\langle\mathscr{K}_{t}\right\rangle \\
\left\langle p_{t}, \frac{p}{\rho_{0} c_{0}^{2}}\right\rangle & =\left\langle\mathscr{P}_{\mathrm{AC} 1, t}\right\rangle .
\end{aligned}
$$

\section{Second artificial compressibility model}

We show that the entropy variables (6.34) are also valid for the second artificial compressibility model. We only write the pressure dependent part of the time coefficients contraction, since the rest is retained from the study of the first artificial compressibility model (6.36),

$$
\left\langle\mathrm{L}_{p, \mathrm{AC} 2}(p)_{t}, \frac{p}{\rho_{0} c_{0}^{2}}\right\rangle=\left\langle-\vec{\nabla}^{2} p_{t}, \frac{p}{\rho_{0} c_{0}^{2}}\right\rangle=\frac{\mathrm{d}}{\mathrm{d} t}\left\langle\frac{|\vec{\nabla} p|^{2}}{2 \rho_{0} c_{0}^{2}}\right\rangle-\int_{\partial \Omega} \frac{p \vec{\nabla} p_{t}}{\rho_{0} c_{0}^{2}} \cdot \mathrm{d} \vec{S}=\left\langle\mathscr{P}_{\mathrm{AC} 2, t}\right\rangle .
$$

In the second equality we used the Gauss law and the chain rule in time, and in the third we applied the Neumann boundary condition for $\vec{\nabla} p_{t}$. As in the incompressible NSE, despite the second artificial compressibility involves gradients in the definition of the entropy function, it does not contribute to the surface entropy because of the boundary condition $\vec{\nabla} p_{t} \cdot \vec{n}=0$.

We define the total entropy for the second artificial compressibility model as

$$
\overline{\mathscr{E}}_{\mathrm{AC} 2}=\left\langle\mathscr{E}_{\mathrm{AC} 2}\right\rangle+\int_{\partial \Omega} \mathscr{E}^{S} \mathrm{~d} S=\left\langle f_{0}+\frac{3}{4} \sigma \varepsilon|\vec{\nabla} c|^{2}+\frac{1}{2} \rho v_{t o t}^{2}+\frac{|\vec{\nabla} p|^{2}}{2 \rho_{0} c_{0}^{2}}\right\rangle+\int_{\partial \Omega} f_{w} \mathrm{~d} S .
$$




\section{Summary}

The contraction of the time coefficients arrives at the same results for the two artificial compressibility models, which we can simply write as

$$
\left\langle\underline{h} \mathrm{~L}(\mathbf{q})_{t}, \mathbf{w}\right\rangle=\overline{\mathscr{E}}_{t}
$$

\subsubsection{Contraction of the inviscid fluxes}

The entropy variables contract the inviscid fluxes and non-conservative terms (3.102). In other solvers, e.g. the compressible Navier-Stokes or the single phase incompressible Navier-Stokes equations in Chapter 4, the contraction involves the use of the product rule, which does not translate discretely. When the continuous analysis requires the use of the product rule, then the discrete approximation needs the use of split-forms and special treatment of metric terms to be stable (see Sec. 3.6). However, for non-conservative systems, we demonstrated that if the conservative and nonconservative inviscid fluxes satisfy Prop. 1 (3.109) and Prop. 2 (3.110) the demonstration of the inviscid part contraction (3.102) only involves the Gauss law (which makes the standard DG scheme with Gauss-Lobatto nodes entropy-stable). We demonstrate that the iNS/CH system satisfies these two properties, which automatically demonstrates the inviscid part contraction (3.102).

For Prop. 1 (3.109), we have already seen that the non-conservative gradient variables $\lambda_{n}$ are the entropy variables, $\lambda_{m}=w_{m}$. For Prop. 2 (3.110), we write the condition (3.110) for the five equations,

$$
\begin{aligned}
& \mathbf{e}_{1}^{T} \overrightarrow{\mathbf{f}}_{e}=c \vec{u}=\mathbf{w}^{T} \overrightarrow{\boldsymbol{\phi}}_{1}, \\
& \mathbf{e}_{2}^{T \overrightarrow{\mathbf{f}}_{e}}=\frac{1}{2} \rho u \vec{u}+p \vec{e}_{1}=\mathbf{w}^{T} \overrightarrow{\boldsymbol{\phi}}_{2}, \\
& \mathbf{e}_{3}^{T \overrightarrow{\mathbf{f}}_{e}}=\frac{1}{2} \rho v \vec{u}+p \vec{e}_{2}=\mathbf{w}^{T} \overrightarrow{\boldsymbol{\phi}}_{3}, \\
& \mathbf{e}_{4}^{T} \overrightarrow{\mathbf{f}}_{e}=\frac{1}{2} \rho w \vec{u}+p \vec{e}_{3}=\mathbf{w}^{T} \overrightarrow{\boldsymbol{\phi}}_{4}, \\
& \mathbf{e}_{5}^{T} \overrightarrow{\mathbf{f}}_{e}=0=\mathbf{w}^{T} \overrightarrow{\boldsymbol{\phi}}_{5} .
\end{aligned}
$$

The inviscid entropy flux for the iNS/CH system therefore follows from (3.111) and is

$$
\vec{f}_{e}^{\mathscr{E}}=\left(\frac{1}{2} \rho v_{t o t}^{2}+p+\mu c\right) \vec{u} .
$$

The satisfaction of Prop. 1 (3.109) and Prop. 2 (3.110) is a sufficient but not necessary condition for the inviscid terms contraction (3.102). For completeness, we show 
how the inviscid terms are actually contracted. To do so, we replace the entropy variables, fluxes, and non-conservative terms in (3.102),

$$
\begin{aligned}
\left\langle\vec{\nabla} \cdot \overrightarrow{\mathbf{f}}_{e}, \mathbf{w}\right\rangle+\sum_{m=1}^{5}\left\langle\overrightarrow{\boldsymbol{\phi}}_{m} \cdot \vec{\nabla} w_{m}, \mathbf{w}\right\rangle= & \langle\vec{\nabla} \cdot(c \vec{u}), \mu\rangle+\frac{1}{2}\langle\vec{\nabla} \cdot(\rho \vec{u} \vec{u}), \vec{u}\rangle+\langle\vec{\nabla} p, \vec{u}\rangle \\
& +\frac{1}{2}\langle\rho \vec{u} \vec{u}, \vec{\nabla} \vec{u}\rangle+\langle c \vec{\nabla} \mu, \vec{u}\rangle+\langle\vec{\nabla} \cdot \vec{u}, p\rangle \\
= & \int_{\partial \Omega}\left(\left(\frac{1}{2} \rho v_{t o t}^{2}+p+c \mu\right) \vec{u}\right) \cdot \mathrm{d} \vec{S} .
\end{aligned}
$$

Thus, we see that the term $\vec{\nabla} \cdot(c \vec{u})$ in the Cahn-Hilliard equation balances the capillary pressure $c \vec{\nabla} \mu$ from the momentum non-conservative terms when multiplied by their respective entropy variables. Similarly, it shows that momentum terms from the conservative and non-conservative parts cancel each other, and that the pressure term in the momentum equation cancels the velocity divergence term by way of the artificial compressibility equation.

\subsubsection{Positive semi-definiteness of the viscous fluxes}

The viscous fluxes are positive definite (3.74). We compute the inner product,

$$
\begin{aligned}
\left\langle\overrightarrow{\mathbf{f}}_{v}, \vec{\nabla} \mathbf{w}\right\rangle & =\left\langle M_{0} \vec{\nabla} \mu, \vec{\nabla} \mu\right\rangle+\left\langle\eta\left(\vec{\nabla} \vec{u}+\vec{\nabla} \vec{u}^{T}\right), \vec{\nabla} \vec{u}\right\rangle=\left\langle\overrightarrow{\mathbf{f}}_{v}, \vec{\nabla} \mathbf{w}\right\rangle_{\mathrm{CH}}+\left\langle\overrightarrow{\mathbf{f}}_{v}, \vec{\nabla} \mathbf{w}\right\rangle_{\mathrm{iNS}} \\
& =\left\langle M_{0} \vec{\nabla} \mu, \vec{\nabla} \mu\right\rangle+\frac{1}{2}\left\langle\eta\left(\vec{\nabla} \vec{u}+\vec{\nabla} \vec{u}^{T}\right), \vec{\nabla} \vec{u}+\vec{\nabla} \vec{u}^{T}\right\rangle \\
& =\left\langle M_{0}|\vec{\nabla} \mu|^{2}+2 \eta|\bar{s}|^{2}\right\rangle \geqslant 0,
\end{aligned}
$$

and the viscous entropy flux that follows the general analysis (3.74),

$$
\vec{f}_{v}^{\mathscr{E}}=\mathbf{w}^{T} \overrightarrow{\mathbf{f}}_{v}=M_{0} \mu \vec{\nabla} \mu+2 \eta \overline{\bar{s}} \cdot \vec{u},
$$

which is the sum of the dissipation in the incompressible solver (4.33), and in the Cahn-Hilliard equation (5.14). In (6.43), we replaced $\vec{\nabla} \vec{u}$ by its symmetric part (6.18) since it multiplies a symmetric tensor.

With these results, we confirm that the iNS/CH system, (6.17), with entropy, (6.27)/(6.28), satisfies a particular version of the general entropy equation (3.77) law

$$
\overline{\mathscr{E}}_{t}+\int_{\partial \Omega}\left(\left(\frac{1}{2} \rho v_{t o t}^{2}+p+c \mu\right) \vec{u}-M_{0} \mu \vec{\nabla} \mu-2 \eta \overline{\bar{s}} \cdot \vec{u}\right) \cdot \mathrm{d} \vec{S}=-\left\langle M_{0}|\vec{\nabla} \mu|^{2}+2 \eta|\bar{s}|^{2}\right\rangle \leqslant 0,
$$


with the entropy flux $\vec{f}^{\mathscr{E}}$,

$$
\vec{f}^{\mathscr{E}}=\vec{f}_{e}^{\mathscr{E}}+\vec{f}_{v}^{\mathscr{E}}=\left(\frac{1}{2} \rho v_{t o t}^{2}+p+\mu c\right) \vec{u}+2 \eta \overline{\bar{s}} \cdot \vec{u}+M_{0} \mu \vec{\nabla} \mu .
$$

Eq. (6.45) shows that the entropy (6.27) is always dissipated in the interior of the domain, and it can only increase due to boundary exchanges. The discrete approximation will be constructed so that it mimics (6.45), which will guarantee that the discrete entropy of the approximation also remains bounded by the boundary and initial data.

We finally apply the wall boundary conditions described in Sec. 6.2.1. In free-slip walls we enforce $\vec{u} \cdot \vec{n}=0$ and $\overline{\bar{s}} \cdot \vec{u}=0$, while in no-slip walls is $\vec{u}=0$. Either way, $\vec{\nabla} \mu \cdot \vec{n}=0$ and

$$
\vec{f}_{e}^{\mathscr{E}}=\left(\frac{1}{2} \rho v_{\text {tot }}^{2}+p+\mu c\right) \vec{u} \cdot \vec{n}+2 \eta \vec{n} \cdot \overline{\bar{s}} \cdot \vec{u}+M_{0} \mu \vec{\nabla} \mu \cdot \vec{n}=0 .
$$

As a result, the entropy equation with wall boundary conditions produces non-growth for the total entropy,

$$
\overline{\mathscr{E}}_{t}=\left\langle\mathscr{F}_{t}+\mathscr{K}_{t}+\mathscr{P}_{t}\right\rangle+\frac{\mathrm{d}}{\mathrm{d} t} \int_{\partial \Omega} f_{w} \mathrm{~d} S=-\left\langle M_{0}|\vec{\nabla} \mu|^{2}+2 \eta|\overline{\bar{s}}|^{2}\right\rangle \leqslant 0 .
$$

\subsection{Space and time discretization}

We now construct the entropy-stable DGSEM approximation. As in the rest of the entropy-stable works in this thesis, we restrict ourselves to the tensor product DGSEM with Gauss-Lobatto (GL) points, since it satisfies the Summation-By-Parts SimultaneousApproximation-Term (SBP-SAT) property Carpenter et al. [2014]. The SBP-SAT property allows us to discretely follow the continuous stability steps to construct a discrete entropy law.

In Sec. 2.6 we derived the steps to construct a DG approximation for a general non-conservative systems, written in the same fashion as (6.19). Thus, we simply copy the standard DG scheme here,

$$
\begin{gathered}
\left\langle\mathscr{J} \underline{H} \mathrm{~L}(\mathbf{Q})_{t}, \boldsymbol{\phi}\right\rangle_{E, N}+\int_{\partial E, N} \boldsymbol{\phi}^{T}\left(\overrightarrow{\mathbf{F}}_{e}^{\star}-\overrightarrow{\mathbf{F}}_{e}+\sum_{m=1}^{N_{c}}\left(\overrightarrow{\boldsymbol{\Phi}}_{m} W_{m}\right)^{\diamond}-\left(\overrightarrow{\boldsymbol{\Phi}}_{m} W_{m}\right)-\overrightarrow{\mathbf{F}}_{v}^{\star}\right) \cdot \mathrm{d} \vec{S} \\
+\left\langle\vec{\nabla}_{\xi} \cdot \overrightarrow{\tilde{\mathbf{F}}}_{e}, \boldsymbol{\phi}\right\rangle_{E, N}+\sum_{n=1}^{N_{c}}\left\langle\overrightarrow{\tilde{\boldsymbol{\Phi}}}_{n} \cdot \vec{\nabla}_{\xi} W_{n}, \boldsymbol{\phi}\right\rangle_{E, N}+\left\langle\overrightarrow{\mathbf{F}}_{v}, \vec{\nabla}_{\xi} \boldsymbol{\phi}\right\rangle_{E, N}=\langle\mathscr{J} \mathbf{S}, \boldsymbol{\phi}\rangle_{E, N}, \\
\langle\mathscr{J} \overrightarrow{\mathbf{G}}, \vec{\varphi}\rangle_{E, N}=\int_{\partial E, N}\left(\mathbf{W}^{\star, T}-\mathbf{W}^{T}\right) \vec{\varphi} \cdot \mathrm{d} \vec{S}+\left\langle\vec{\nabla}_{\xi} \mathbf{W}, \overrightarrow{\tilde{\varphi}}\right\rangle_{E, N}
\end{gathered}
$$


where we specified the entropy variables as the gradient variables $\mathbf{W}$ and non-conservative gradient variables $\Lambda_{m}=W_{m}$. We have used the discrete Gauss law for viscous fluxes, and for the gradient equation (recall that the use of the discrete Gauss law does not modify the scheme). The reason for doing so is that in this particular system, nonconservative gradients and entropy variables gradients coincide.

The final configuration after the application of the discrete Gauss law (6.49) enhances the algorithm efficiency by the use of single calculation of the local gradient of the entropy variables, $\vec{\nabla}_{\xi} \mathbf{W}$, for both non-conservative terms in (6.49a) and gradients in (6.49b). As a result, the non-conservative terms are as computationally expensive as source terms (i.e. no additional matrix multiplications are required). After the non-conservative terms are computed, for the gradients $\overrightarrow{\mathbf{G}}$ we only augment the precomputed local gradients $\vec{\nabla}_{\xi} \mathbf{W}$ with the surface integral in (6.49).

As in the Cahn-Hilliard solver (Chapter 5), the discretization (6.49) is incomplete, as it depends on the approximation of the chemical potential (5.20), which is directly copied here from Chapter 5 ,

$$
\begin{aligned}
\left\langle\mathscr{J} \mu, \phi_{\mu}\right\rangle_{E, N} & =\left\langle\mathscr{J} \frac{\mathrm{d} F_{0}}{\mathrm{~d} c}, \phi_{\mu}\right\rangle_{E, N}-\frac{3}{2} \sigma \varepsilon \int_{\partial e, N} \phi_{\mu} \vec{G}_{c}^{\star} \cdot \mathrm{d} \vec{S}+\frac{3}{2} \sigma \varepsilon\left\langle\overrightarrow{\tilde{G}}_{c}, \vec{\nabla}_{\xi} \phi_{\mu}\right\rangle_{E, N}, \\
\left\langle\mathscr{J} \vec{G}_{c}, \vec{\phi}_{G_{c}}\right\rangle_{E, N} & =\int_{\partial e, N} C^{\star} \vec{\phi}_{G_{c}} \cdot \mathrm{d} \vec{S}-\left\langle C, \vec{\nabla}_{\xi} \cdot \overrightarrow{\tilde{\phi}}_{G_{c}}\right\rangle_{E, N} .
\end{aligned}
$$

Eq. (6.50) regards the chemical potential definition (5.7) as an additional second order conservation law. Now, the discretization is complete: from the state $(C)$, we compute the chemical potential $\mu$ (6.50), which completes the entropy variables $\mathbf{W}$, and finally we compute the PDE (6.49) from $\overrightarrow{\mathbf{G}}$ and $\mathbf{Q}$.

The Euler conservative fluxes and the viscous fluxes have inter-element coupling and physical boundary conditions enforced by numerical fluxes at the element boundary quadratures in (6.49a),

$$
\begin{aligned}
& \overrightarrow{\mathbf{F}}_{e} \approx \overrightarrow{\mathbf{F}}_{e}^{\star}\left(\mathbf{Q}_{L}, \mathbf{Q}_{R}\right), \overrightarrow{\mathbf{F}}_{v} \approx \overrightarrow{\mathbf{F}}_{v}^{\star}\left(\overrightarrow{\mathbf{G}}_{L}, \overrightarrow{\mathbf{G}}_{R}\right), \quad \mathbf{W} \approx \mathbf{W}^{\star}\left(\mathbf{Q}_{L}, \mathbf{Q}_{R}\right), \\
& \vec{G}_{c} \approx \vec{G}_{c}^{\star}\left(\vec{G}_{c L}, \vec{G}_{c R}\right), \quad C \approx C^{\star}\left(C_{L}, C_{R}\right) .
\end{aligned}
$$

Whereas for non-conservative terms, we follow Bohm et al. [2018] and use diamond fluxes at the boundaries, which are also functions of two states,

$$
\overrightarrow{\mathbf{\Phi}}_{m} W_{m} \approx\left(\overrightarrow{\boldsymbol{\Phi}}_{m} W_{m}\right)^{\diamond}\left(\mathbf{Q}_{L}, \mathbf{Q}_{R}\right)
$$

Both numerical and diamond flux functions are defined below in Sec. 6.4.1. In (6.51) and (6.52), $L$ and $R$ represent the values from the left and right adjacent elements. 
Although numerical fluxes are single valued at each interface, diamond fluxes are not so constrained and their value can jump from one side to the other.

To get the point-wise values, we replace the test function by the Lagrange polynomials $l_{i}(\xi) l_{j}(\eta) l_{k}(\zeta)$. We sketch the algorithm:

1. Compute the entropy variables, W. The first entropy variable is the chemical potential, $\mu$, which is computed from (6.50),

$$
\begin{aligned}
\mathscr{J}_{i j k} \vec{G}_{c, i j k}= & \left.\left(C^{\star}-C\right)_{i j k}\left(\frac{\delta_{i m}}{w_{i}} \mathscr{J} \vec{a}_{i j k}^{1}+\frac{\delta_{j m}}{w_{j}} \mathscr{J} \vec{a}_{i j k}^{2}+\frac{\delta_{k m}}{w_{k}} \mathscr{J} \vec{a}_{i j k}^{3}\right)\right|_{m=0} ^{m=N} \\
& +\mathscr{J} \vec{a}_{i j k}^{1} \sum_{m=0}^{N} D_{i m} C_{m j k}+\mathscr{J} \vec{a}_{i j k}^{2} \sum_{m=0}^{N} D_{j m} C_{i m k}+\mathscr{J} \vec{a}_{i j k}^{3} \sum_{m=0}^{N} D_{k m} C_{i j m} \\
\mathscr{J}_{i j k} \mu_{i j k}= & \mathscr{J}_{i j k} F_{0}^{\prime}\left(C_{i j k}\right)-\left.\frac{3}{2} \sigma \varepsilon\left(\frac{\delta_{i m}}{w_{i}} \tilde{G}_{c, i j k}^{1, \star}+\frac{\delta_{j m}}{w_{j}} \tilde{G}_{c, i j k}^{2, \star}+\frac{\delta_{k m}}{w_{k}} \tilde{G}_{c, i j k}^{3, \star}\right)\right|_{m=0} ^{m=N} \\
& +\frac{3}{2} \sigma \varepsilon \sum_{m=0}^{N}\left(\frac{w_{m}}{w_{i}} D_{m i} \tilde{G}_{c, m j k}^{1}+\frac{w_{m}}{w_{j}} D_{m j} \tilde{G}_{c, i m k}^{2}+\frac{w_{m}}{w_{k}} D_{m k} \tilde{G}_{c, i j m}^{3}\right),
\end{aligned}
$$

where $D_{i j}=l_{j}^{\prime}\left(\xi_{i}\right)$ is the derivative matrix, and recall the definition of contravariant components (2.18). We then get the entropy variables from the current values of the state vector, $\mathbf{Q}$, and the chemical potential, $\mu$ computed by (6.53),

$$
\mathbf{W}_{i j k}=\left(\mu, \frac{Q_{2}}{\sqrt{\rho}}, \frac{Q_{3}}{\sqrt{\rho}}, \frac{Q_{4}}{\sqrt{\rho}}, \frac{Q_{5}}{\rho_{0} c_{0}^{2}}\right)_{i j k}, \rho=\rho_{1} \tilde{C}+\rho_{2}(1-\tilde{C}),
$$

with $\tilde{C}=\min (\max (C, 0), 1)$.

2. Compute the gradient of the entropy variables $\overrightarrow{\mathbf{G}}$ from (6.49),

$$
\begin{aligned}
\mathscr{J}_{i j k} \overrightarrow{\mathbf{G}}_{i j k}= & \left.\left(\mathbf{W}^{\star, T}-\mathbf{W}^{T}\right)_{i j k}\left(\frac{\delta_{i m}}{w_{i}} \mathscr{J} \vec{a}_{i j k}^{1}+\frac{\delta_{j m}}{w_{j}} \mathscr{J} \vec{a}_{i j k}^{2}+\frac{\delta_{k m}}{w_{k}} \mathscr{J} \vec{a}_{i j k}^{3}\right)\right|_{m=0} ^{m=N} \\
& +\mathscr{J} \vec{a}_{i j k}^{1} \sum_{m=0}^{N} D_{i m} \mathbf{W}_{m j k}+\mathscr{J} \vec{a}_{i j k}^{2} \sum_{m=0}^{N} D_{j m} \mathbf{W}_{i m k}+\mathscr{J} \vec{a}_{i j k}^{3} \sum_{m=0}^{N} D_{k m} \mathbf{W}_{i j m} .
\end{aligned}
$$


3. Compute the state vector time derivative,

$$
\begin{aligned}
\mathscr{J}_{i j k} \underline{H}_{i j k} \frac{\mathrm{dL}(\mathbf{Q})_{i j k}}{\mathrm{~d} t}+ & \left(\overrightarrow{\mathbf{F}}_{e}^{\star}-\overrightarrow{\mathbf{F}}_{e}+\sum_{m=1}^{5}\left(\overrightarrow{\mathbf{\Phi}}_{m} W_{m}\right)^{\diamond}-\overrightarrow{\mathbf{\Phi}}_{m} W_{m}\right)_{i j k} \\
& \left.\cdot\left(\frac{\delta_{i m}}{w_{i}} \mathscr{J} \vec{a}_{i j k}^{1}+\frac{\delta_{j m}}{w_{j}} \mathscr{J} \vec{a}_{i j k}^{2}+\frac{\delta_{k m}}{w_{k}} \mathscr{J} \vec{a}_{i j k}^{3}\right)\right|_{m=0} ^{m=N} \\
& +\sum_{m=0}^{N}\left(D_{i m} \tilde{\mathbf{F}}_{e, m j k}^{1}+D_{j m} \tilde{\mathbf{F}}_{e, i m k}^{2}+D_{k m} \tilde{\mathbf{F}}_{e, i j m}^{3}\right) \\
& +\sum_{p=1}^{N} \sum_{m=1}^{5}\left(\tilde{\mathbf{\Phi}}_{m, i j k}^{1} D_{i p} W_{m, p j k}+\tilde{\mathbf{\Phi}}_{m, i j k}^{2} D_{j p} W_{m, i p k}+\tilde{\mathbf{\Phi}}_{m, i j k}^{3} D_{k p} W_{m, i j p}\right) \\
= & \left.\left(\frac{\delta_{i m}}{w_{i}} \tilde{\mathbf{F}}_{v, i j k}^{1, \star}+\frac{\delta_{j m}}{w_{j}} \tilde{\mathbf{F}}_{v, i j k}^{2, \star}+\frac{\delta_{k m}}{w_{k}} \tilde{\mathbf{F}}_{v, i j k}^{3, \star}\right)\right|_{m=0} ^{m=N} \\
& +\sum_{m=0}^{N}\left(\frac{w_{m}}{w_{i}} D_{m i} \tilde{\mathbf{F}}_{v, m j k}^{1}+\frac{w_{m}}{w_{j}} D_{m j} \tilde{\mathbf{F}}_{v, i m k}^{2}+\frac{w_{m}}{w_{k}} D_{m k} \tilde{\mathbf{F}}_{v, i j m}^{3}\right)+\mathscr{J}_{i j k} \mathbf{S}_{i j k} .
\end{aligned}
$$

\section{Split-form scheme}

It is also possible to use a split-form scheme. The split-form scheme is the most general procedure to construct an entropy-stable scheme, as it encapsulates all the possible split-forms with the same operator (2.75). For this set of equations, that satisfies Prop. 1 (3.109) and Prop. 2 (3.110), the use of a split-form scheme is not encouraged. Since the standard scheme, which is simpler and more efficient, is stable by itself, there is no practical need to use the split-form scheme. Nonetheless, we provide the split-form scheme for completeness, which we also borrow from Sec. 2.6

$$
\begin{aligned}
\left\langle\mathscr{J} \underline{H} \mathrm{~L}(\mathbf{Q})_{t}, \boldsymbol{\phi}\right\rangle_{E, N} & +\int_{\partial E, N} \boldsymbol{\phi}^{T}\left(\overrightarrow{\mathbf{F}}_{e}^{\star}-\overrightarrow{\mathbf{F}}_{e}+\sum_{m=1}^{N_{c}}\left(\overrightarrow{\mathbf{\Phi}}_{m} W_{m}\right)^{\diamond}-\left(\overrightarrow{\boldsymbol{\Phi}}_{m} W_{m}\right)-\overrightarrow{\mathbf{F}}_{v}^{\star}\right) \cdot \mathrm{d} \vec{S} \\
& +\left\langle\mathbb{D}\left(\overrightarrow{\tilde{\mathbf{F}}}_{e}\right)^{\mathrm{EC}}, \boldsymbol{\phi}\right\rangle_{E, N}+\sum_{m=1}^{N_{c}}\left\langle\overrightarrow{\tilde{\mathbf{\Phi}}}_{m} \cdot \mathbb{D}_{\mathrm{grad}}^{\mathrm{DM}} W_{m}, \boldsymbol{\phi}\right\rangle_{E, N}+\left\langle\overrightarrow{\mathbf{F}}_{v}, \vec{\nabla}_{\xi} \boldsymbol{\phi}\right\rangle_{E, N}=0, \\
\langle\mathscr{J} \overrightarrow{\mathbf{G}}, \vec{\varphi}\rangle_{E, N} & =\int_{\partial E, N}\left(\mathbf{W}^{\star, T}-\mathbf{W}^{T}\right) \vec{\varphi} \cdot \mathrm{d} \vec{S}+\left\langle\vec{\nabla}_{\xi} \mathbf{W}, \overrightarrow{\tilde{\varphi}}\right\rangle_{E, N},
\end{aligned}
$$


with the two-point entropy conserving flux $\overrightarrow{\mathbf{F}}^{\mathrm{EC}}=\left\{\left\{\overrightarrow{\mathbf{F}}_{e}\right\}\right\}$ computed simply as the average of the inviscid fluxes.

\section{Second artificial compressibility discrete time coefficients}

The DG discretization of the iNS/CH system (6.49) treats time coefficients as source terms, which is valid for the first artificial compressibility model, where $L_{A C 1}(\mathbf{Q})=\mathbf{Q}$, and valid for all the equations of the second artificial compressibility model, except the pressure, since $\mathrm{L}_{\mathrm{AC} 2}(\mathbf{Q})=\left(C, \sqrt{\rho} \vec{U},-\vec{\nabla}^{2} P\right)$. For the pressure terms of the second artificial compressibility model, we follow the discretization developed for the incompressible solver (4.41), which constructs an additional weak-form for the pressure Laplacian,

$$
-\left\langle J \vec{\nabla}^{2} p_{t}, \phi\right\rangle_{E} \approx\left\langle\overrightarrow{\tilde{G}}_{p, t}, \vec{\nabla}_{\xi} \phi\right\rangle_{E, N}-\int_{\partial E, N} \phi \overrightarrow{\tilde{G}}_{p, t}^{\star} \cdot \hat{n} \mathrm{~d} \hat{S}
$$

\subsubsection{Numerical and diamond fluxes}

The approximation (6.49) is completed with the specification of numerical fluxes $\mathbf{F}_{e}^{\star}$, $\mathbf{F}_{v}^{\star}, \mathbf{W}^{\star}, \vec{G}_{c}^{\star}, C^{\star}$, and diamond fluxes $\left(\overrightarrow{\boldsymbol{\Phi}}_{m} W_{m}\right)^{\diamond}$. For the inviscid fluxes we propose two possibilities: an entropy conserving option using central fluxes, or an entropy-stable approximation using the exact Riemann solver derived in Bassi et al. [2017]. For the viscous fluxes and concentration gradient, we use the Bassi-Rebay 1 (BR1) scheme Bassi and Rebay [1997].

Before we write the numerical fluxes, we first describe the rotational invariance property satisfied by the iNS/CH system inviscid fluxes and non-conservative terms. The rotational invariance of the flux Toro [2013] allows us to write the normal flux $\overrightarrow{\mathbf{F}}_{e} \cdot \vec{n}$ from a rotated version of the inviscid flux $x$-component $\mathbf{F}_{e}$,

$$
\overrightarrow{\mathbf{F}}_{e} \cdot \vec{n}=\underline{\mathrm{T}}^{T} \mathbf{F}_{e}(\underline{\mathrm{T}} \mathbf{Q})=\underline{\mathrm{T}}^{T} \mathbf{F}_{e}\left(\mathbf{Q}_{n}\right), \quad \underline{\mathrm{T}}=\left(\begin{array}{ccccc}
1 & 0 & 0 & 0 & 0 \\
0 & n_{x} & n_{y} & n_{z} & 0 \\
0 & t_{1, x} & t_{1, y} & t_{1, z} & 0 \\
0 & t_{2, x} & t_{2, y} & t_{2, z} & 0 \\
0 & 0 & 0 & 0 & 1
\end{array}\right),
$$

where $\underline{\mathrm{T}}$ is a rotation matrix that only affects velocities, $\vec{n}=\left(n_{x}, n_{y}, n_{z}\right)$ is the normal unit vector to the face, and $\vec{t}_{1}$ and $\vec{t}_{2}$ are two tangent unit vectors to the face. When the rotation matrix $\underline{T}$ multiplies the state vector $\mathbf{Q}$, we get the face normal state vector $\mathbf{Q}_{n}$,

$$
\mathbf{Q}_{n}=\underline{\mathrm{T}} \mathbf{Q}=\left(C, \sqrt{\rho} U_{n}, \sqrt{\rho} V_{t 1}, \sqrt{\rho} V_{t 2}, P\right)
$$


where $U_{n}=\vec{U} \cdot \vec{n}$ the normal velocity, and $V_{t i}=\vec{U} \cdot \vec{t}_{i}(i=1,2)$ are the two tangent velocities. Note that the reference system rotation does not affect the total speed

$$
V_{t o t}^{2}=U^{2}+V^{2}+W^{2}=U_{n}^{2}+V_{t 1}^{2}+V_{t 2}^{2}
$$

The non-conservative terms,

$$
\sum_{m=1}^{5} \overrightarrow{\boldsymbol{\Phi}}_{m} W_{m}=\Upsilon\left(\begin{array}{ccc}
0 & 0 & 0 \\
\frac{1}{2} \rho U^{2}+\mu C & \frac{1}{2} \rho U V & \frac{1}{2} \rho U W \\
\frac{1}{2} \rho U V & \frac{1}{2} \rho V^{2}+\mu C & \frac{1}{2} \rho V W \\
\frac{1}{2} \rho U W & \frac{1}{2} \rho V W & \frac{1}{2} \rho W^{2}+\mu C \\
\rho_{0} c_{0}^{2} U & \rho_{0} c_{0}^{2} V & \rho_{0} c_{0}^{2} W
\end{array}\right)
$$

are also rotationally invariant,

$$
\sum_{m=1}^{5}\left(\overrightarrow{\boldsymbol{\Phi}}_{m} W_{m}\right) \cdot \vec{n}=\underline{\mathrm{T}}^{T}\left(\begin{array}{c}
0 \\
\frac{1}{2} \rho U_{n}^{2}+\mu C \\
\frac{1}{2} \rho U_{n} V_{t 1} \\
\frac{1}{2} \rho U_{n} V_{t 2} \\
\rho_{0} c_{0}^{2} U_{n}
\end{array}\right)=\underline{\mathrm{T}}^{T} \mathbf{F}_{\Phi W}\left(\mathbf{Q}_{n}\right)
$$

with its equivalent $x$-component,

$$
\mathbf{f}_{\phi w}(\mathbf{q})=\left(\begin{array}{c}
0 \\
\frac{1}{2} \rho u^{2}+\mu c \\
\frac{1}{2} \rho u v \\
\frac{1}{2} \rho u w \\
\rho_{0} c_{0}^{2} u
\end{array}\right)
$$

\section{Inviscid fluxes: entropy conserving central fluxes}

The first choice for the inviscid numerical and diamond fluxes is to use central fluxes (3.115), which was shown in the general analysis to lead to an entropy conserving approximation (3.116). We adapt the approach used for the resistive MHD in Bohm et al. [2018] for the iNS/CH system, so that

$$
\overrightarrow{\mathbf{F}}_{e}^{\star} \cdot \vec{n}=\overrightarrow{\mathbf{F}}_{e}^{\mathrm{EC}} \cdot \vec{n}=\left\{\left\{\overrightarrow{\mathbf{F}}_{e}\right\}\right\} \cdot \vec{n}=\underline{\mathrm{T}}^{T}\left(\begin{array}{c}
\left\{\left\{C U_{n}\right\}\right\} \\
\left\{\left\{\frac{1}{2} \rho U_{n}^{2}+p\right\}\right\} \\
\left\{\left\{\frac{1}{2} \rho U_{n} V_{t 1}\right\}\right\} \\
\left\{\left\{\frac{1}{2} \rho U_{n} V_{t 2}\right\}\right\} \\
0
\end{array}\right)
$$


and

$$
\sum_{m=1}^{5}\left(\overrightarrow{\boldsymbol{\Phi}}_{m} W_{m}\right)^{\diamond} \cdot \vec{n}=\sum_{m=1}^{5} \overrightarrow{\boldsymbol{\Phi}}_{m}\left\{\left\{W_{m}\right\}\right\} \cdot \vec{n}=\underline{\mathrm{T}}^{T}\left(\begin{array}{c}
0 \\
\frac{1}{2} \rho U_{n}\left\{\left\{U_{n}\right\}\right\}+C\{\{\mu\}\} \\
\frac{1}{2} \rho U_{n}\left\{\left\{V_{t 1}\right\}\right\} \\
\frac{1}{2} \rho U_{n}\left\{\left\{V_{t 2}\right\}\right\} \\
\rho_{0} c_{0}^{2}\left\{\left\{U_{n}\right\}\right\}
\end{array}\right) .
$$

where $\overrightarrow{\boldsymbol{\Phi}}_{m}$ (without average) represents the element local state at the face.

\section{Inviscid fluxes: entropy-stable exact Riemann solver (ERS)}

The second option uses the solution of the exact Riemann problem derived in Bassi et al. [2017], and described in Sec. 4.4.2. The star region solution of the incompressible NSE (i.e. without the Cahn-Hilliard equation) is (4.47),

$$
\begin{aligned}
U_{n}^{\star} & =\frac{P_{L}-P_{R}+\rho_{L} U_{n L} \lambda_{L}^{+}-\rho_{R} U_{n R} \lambda_{R}^{-}}{\rho_{L} \lambda_{L}^{+}-\rho_{R} \lambda_{R}^{-}}, P^{\star}=P_{L}+\rho_{L} \lambda_{L}^{+}\left(U_{n L}-U_{n}^{\star}\right), \\
\rho^{\star} & =\left\{\begin{array}{lll}
\rho_{L}^{\star} & \text { if } \quad U_{n}^{\star} \geqslant 0 \\
\rho_{R}^{\star} & \text { if } \quad U_{n}^{\star}<0
\end{array}, \rho_{L}^{\star}=\frac{\rho_{L} \lambda_{L}^{+}}{U_{n}^{\star}-\lambda_{L}^{-}}, \quad \rho_{R}^{\star}=\frac{\rho_{R} \lambda_{R}^{-}}{U_{n}^{\star}-\lambda_{R}^{+}}, \quad V_{t i}^{\star}=\left\{\begin{array}{lll}
V_{t i L} & \text { if } & U_{n}^{\star} \geqslant 0 \\
V_{t i R} & \text { if } & U_{n}^{\star}<0
\end{array},\right.\right.
\end{aligned}
$$

with eigenvalues (4.48),

$$
\lambda_{L}^{ \pm}=\frac{U_{n L} \pm a_{L}}{2}, \quad \lambda_{R}^{ \pm}=\frac{U_{n R} \pm a_{R}}{2}, a=\sqrt{U_{n}^{2}+\frac{4 \rho_{0} c_{0}^{2}}{\rho}}
$$

We evaluate the numerical flux $\overrightarrow{\mathbf{F}}_{e}^{\star}$ with the star region solution with the normal state $\mathbf{Q}_{n}$ in (6.59) for the momentum, and perform the average for the Cahn-Hilliard part,

$$
\overrightarrow{\mathbf{F}}_{e}^{\star} \cdot \vec{n}=\underline{\mathrm{T}}^{T}\left(\begin{array}{c}
\left\{\left\{C U_{n}\right\}\right\} \\
\frac{1}{2} \rho^{\star} U_{n}^{\star, 2}+p^{\star} \\
\frac{1}{2} \rho^{\star} U_{n}^{\star} V_{t 1}^{\star} \\
\frac{1}{2} \rho^{\star} U_{n}^{\star} V_{t 2}^{\star} \\
0
\end{array}\right)
$$


For the non-conservative terms, we choose the following diamond fluxes,

$$
\begin{aligned}
& \sum_{m=1}^{5}\left(\overrightarrow{\mathbf{\Phi}}_{m} W_{m}\right)^{\diamond} \cdot \vec{n}=\overrightarrow{\mathbf{\Phi}}_{1}\left\{\left\{W_{1}\right\}\right\} \cdot \vec{n}+\sum_{m=2}^{5}\left(\overrightarrow{\mathbf{\Phi}}_{m}^{\star} W_{m}^{\star}+\overrightarrow{\mathbf{\Phi}}_{m} W_{m}-\overrightarrow{\mathbf{\Phi}}_{m}^{\star} W_{m}\right) \cdot \vec{n} \\
& 0 \\
&=\underline{\mathrm{T}}^{T}\left(\begin{array}{c}
\frac{1}{2} \rho^{\star} U_{n}^{\star, 2}+\frac{1}{2} \rho U_{n}^{2}-\frac{1}{2} \rho^{\star} U_{n}^{\star} U_{n}+C\{\{\mu\}\} \\
\frac{1}{2} \rho^{\star} U_{n}^{\star} V_{t 1}^{\star}+\frac{1}{2} \rho U_{n} V_{t 1}-\frac{1}{2} \rho^{\star} U_{n}^{\star} V_{t 1} \\
\frac{1}{2} \rho^{\star} U_{n}^{\star} V_{t 2}^{\star}+\frac{1}{2} \rho U_{n} V_{t 2}-\frac{1}{2} \rho^{\star} U_{n}^{\star} V_{t 2} \\
\rho_{0} c_{0}^{2} U_{n}^{\star}
\end{array}\right)=\underline{\mathrm{T}}^{T} \mathbf{F}_{\Phi W}^{\mathrm{ERS}},
\end{aligned}
$$

where $\overrightarrow{\boldsymbol{\Phi}}_{m}^{\star}$ and $W_{m}^{\star}$ refer to non-conservative coefficients and entropy variables evaluated with the star region solution (6.67). This choice is motivated and justified by the stability analysis.

\section{Viscous and chemical potential fluxes: Bassi-Rebay 1 (BR1) method}

For the viscous fluxes and the chemical potential we use the Bassi-Rebay 1 (BR1) scheme Bassi and Rebay [1997], which averages entropy variables and fluxes between the adjacent elements. However, for the Cahn-Hilliard equation, we include the possibility to add interface stabilization. Although it is not a requirement for stability, we have found it enhances accuracy when the flow configuration is under-resolved Manzanero et al. [2020b]. The viscous and Cahn-Hilliard fluxes are

$$
\begin{aligned}
\mathbf{W}^{\star} & =\{\{\mathbf{W}\}\}, \quad \overrightarrow{\mathbf{F}}_{v}^{\star}=\left\{\left\{\overrightarrow{\mathbf{F}}_{v}\right\}\right\}-\beta_{\mu}\left(\mu_{L} \vec{n}_{L}+\mu_{R} \vec{n}_{R}\right) \mathbf{e}_{1}, \\
C^{\star} & =\{\{C\}\}, \quad \vec{G}_{c}^{\star}=\left\{\left\{\vec{G}_{c}\right\}\right\}-\beta_{c}\left(C_{L} \vec{n}_{L}+C_{R} \vec{n}_{R}\right),
\end{aligned}
$$

where $\beta_{i}$ is the penalty parameter, which uses the same estimate Manzanero et al. [2020b, 2018b] as in the Cahn-Hilliard solver (5.23). Note that the interface stabilization can be disabled with $\kappa_{\beta_{i}}=0$, and enabled with $\kappa_{\beta_{i}}=1$. It will be demonstrated that this dissipation is not required for the stability of the scheme, but it does enhance its accuracy.

\section{Second artificial compressibility model}

The second artificial compressibility model includes the pressure gradient numerical flux $\vec{G}_{p}^{\star}$ at the inter-element boundaries (4.41). We follow the discretization of the incompressible NSE solver, and use (4.50),

$$
\vec{G}_{p}^{\star}=\left\{\left\{\vec{G}_{p}\right\}\right\}-\beta_{p}\left(P_{L} \vec{n}_{L}+P_{R} \vec{n}_{R}\right) .
$$




\subsubsection{Boundary conditions}

The approximation is completed with the addition of boundary conditions. Here we show how we impose free- and no-slip wall boundary conditions. The boundary conditions are imposed through the numerical and diamond fluxes at the physical boundary faces. We consider the inviscid and viscous fluxes individually.

\section{Inviscid flux}

The inviscid numerical flux controls the normal velocity $\vec{u} \cdot \vec{n}=0$ for both free- and no-slip boundary conditions. Again, as at interior faces, we provide an entropy conserving option with central fluxes, and an entropy-stable version with the exact Riemann problem solution. Either way, we apply central fluxes (6.65)-(6.66) or the ERS (6.69)-(6.70) to the interior state $\mathbf{Q}_{n}^{i}$ and a mirrored ghost state $\mathbf{Q}_{n}^{e}$,

$$
\mathbf{Q}_{n}^{i}=\left(\begin{array}{c}
C \\
\sqrt{\rho} U_{n} \\
\sqrt{\rho} V_{t 1} \\
\sqrt{\rho} V_{t 2} \\
P
\end{array}\right), \mathbf{Q}_{n}^{e}=\left(\begin{array}{c}
C \\
-\sqrt{\rho} U_{n} \\
\sqrt{\rho} V_{t 1} \\
\sqrt{\rho} V_{t 2} \\
P
\end{array}\right)
$$

The fluxes differ depending on which numerical flux is used.

1. The entropy conserving approximation with central fluxes. The numerical (6.65) and diamond (6.66) central fluxes for (6.73) are

$$
\overrightarrow{\mathbf{F}}_{e}^{\star} \cdot \vec{n}=\underline{\mathrm{T}}^{T}\left(\begin{array}{c}
0 \\
\frac{1}{2} \rho U_{n}^{2}+P \\
0 \\
0 \\
0
\end{array}\right), \sum_{m=1}^{5}\left(\overrightarrow{\boldsymbol{\Phi}}_{m} W_{m}\right)^{\diamond} \cdot \vec{n}=\underline{\mathrm{T}}^{T}\left(\begin{array}{c}
0 \\
C \mu \\
\frac{1}{2} \rho U_{n} V_{t 1} \\
\frac{1}{2} \rho U_{n} V_{t 2} \\
0
\end{array}\right) .
$$

2. The entropy-stable approximation with the exact Riemann solver. The star region values computed from the states (6.73) are

$$
U_{n}^{\star}=0, \quad P^{\star}=P+\rho \lambda^{+} U_{n}, \quad \lambda^{+}=\frac{U_{n}+a}{2} .
$$

With these star values the numerical (6.69) and diamond (6.70) fluxes become

$$
\overrightarrow{\mathbf{F}}_{e}^{\star} \cdot \vec{n}=\underline{\mathrm{T}}^{T}\left(\begin{array}{c}
0 \\
P+\rho \lambda^{+} U_{n} \\
0 \\
0 \\
0
\end{array}\right), \sum_{m=1}^{5}\left(\overrightarrow{\boldsymbol{\Phi}}_{m} W_{m}\right)^{\diamond} \cdot \vec{n}=\underline{\mathrm{T}}^{T}\left(\begin{array}{c}
0 \\
\frac{1}{2} \rho U_{n}^{2}+C \mu \\
\frac{1}{2} \rho U_{n} V_{t 1} \\
\frac{1}{2} \rho U_{n} V_{t 2} \\
0
\end{array}\right) .
$$




\section{Viscous and Cahn-Hilliard fluxes}

For the free-slip wall boundary condition, we impose zero normal stress $\overline{\bar{S}} \cdot \vec{n}=0$ (Neumann), whereas for the no-slip wall boundary condition, we impose zero velocity $\vec{u}=0$ (Dirichlet). Furthermore, we implement the discrete version of the homogeneous and non-homogeneous Neumann boundary conditions (6.24) for the CahnHilliard equation. The concentration, concentration gradient, entropy variables, and viscous fluxes at the boundaries are:

1. Free-slip wall boundary condition.

$$
C^{\star}=C,-\frac{3}{2} \sigma \varepsilon \vec{G}_{c}^{\star} \cdot \vec{n}=f_{w}^{\prime}(C), \quad \mathbf{W}^{\star}=\mathbf{W}, \quad \overrightarrow{\mathbf{F}}_{v}^{\star} \cdot \vec{n}=0
$$

2. No-slip wall boundary condition.

$$
C^{\star}=C,-\frac{3}{2} \sigma \varepsilon \vec{G}_{c}^{\star} \cdot \vec{n}=f_{w}^{\prime}(C), \mathbf{W}^{\star}=(\mu, 0,0,0, P), \overrightarrow{\mathbf{F}}_{v}^{\star} \cdot \vec{n}=\left(\begin{array}{c}
0 \\
2 \eta \overline{\bar{S}} \\
0
\end{array}\right) \cdot \vec{n} .
$$

\section{Second artificial compressibility model}

The second artificial compressibility model follows (4.4.3) and uses the homogeneous Neumann boundary condition for the pressure time derivative which is enforced using $\vec{G}_{p, t}^{\star} \cdot \vec{n}=0$.

\subsubsection{Implicit-Explicit (IMEX) time discretization}

The fully-discrete scheme is completed with the discretization of the time derivatives of the semi-discrete scheme (6.49). Since only an implementation of the first artificial compressibility model is presented in this work, we only consider the development of a time discretization for the first model, and leave that of the second model for future work. The numerical stiffness induced by the fourth order derivatives present in the Cahn-Hilliard equation prevents us from practically using fully explicit time marching. Therefore, we use the IMplicit-EXplicit (IMEX) Backward Differentiation Formula (BDF) time integrator described in Dong [2018b]. We revisit the continuous 
setting (6.17)

$$
\begin{aligned}
& c_{t}+\vec{\nabla} \cdot(c \vec{u})=M_{0} \vec{\nabla}^{2}\left(f_{0}^{\prime}(c)-\frac{3}{2} \sigma \varepsilon \vec{\nabla}^{2} c\right), \\
& \sqrt{\rho}(\sqrt{\rho} \vec{u})_{t}+\vec{\nabla} \cdot\left(\frac{1}{2} \rho \vec{u} \vec{u}\right)+\frac{1}{2} \rho \vec{u} \cdot \vec{\nabla} \vec{u}+c \vec{\nabla} \mu=-\vec{\nabla} p+2 \eta \bar{s}+\rho \vec{g}, \\
& p_{t}+\rho_{0} c_{0}^{2} \vec{\nabla} \cdot \vec{u}=0,
\end{aligned}
$$

to describe the approximation in time.

The IMEX version of the BDF integrator for a time derivative $\mathrm{d} y / \mathrm{d} t$ is

$$
\left(\gamma_{0}, \hat{y}, y^{n+1, e}\right)=\left\{\begin{array}{ll}
\left(1, y^{n}, y^{n}\right) & \text { if } J=1 \\
\left(\frac{3}{2}, 2 y^{n}-\frac{1}{2} y^{n-1}, 2 y^{n}-y^{n-1}\right) & \text { if } J=2
\end{array},\right.
$$

where $y^{n}=y\left(t_{n}\right), \gamma_{0}$ and $\hat{y}$ yield a $J$-th order approximation of the time derivative,

$$
y_{t}^{n+1} \approx \frac{\gamma_{0} y^{n+1}-\hat{y}}{\Delta t}
$$

and $y^{n+1, e}$ is a $J$-th order explicit approximation of $y^{n+1}$. The IMEX scheme used in Dong [2018b] was constructed to solve the fourth order derivative implicitly, and the remaining terms explicitly,

$$
\begin{aligned}
& \frac{\gamma_{0} c^{n+1}-\hat{c}}{\Delta t}+\vec{\nabla} \cdot(c \vec{u})^{n+1, e}=M_{0} \vec{\nabla}^{2}\left(f_{0}^{\prime}\left(c^{n+1, e}\right)-\frac{3}{2} \sigma \varepsilon \vec{\nabla}^{2} c^{n+1}\right), \\
& \sqrt{\rho^{n+1, e}} \frac{\gamma_{0} \sqrt{\rho} \vec{u}^{n+1}-\widehat{\sqrt{\rho \vec{u}}}}{\Delta t}+\left(\vec{\nabla} \cdot\left(\frac{1}{2} \rho \vec{u} \vec{u}\right)+\frac{1}{2} \rho \vec{u} \cdot \vec{\nabla} \vec{u}+c \vec{\nabla} \mu\right)^{n+1, e} \\
&=(-\vec{\nabla} p+2 \eta \overline{\bar{s}}+\rho \vec{g})^{n+1, e}, \\
& \frac{\gamma_{0} p^{n+1}-\hat{p}}{\Delta t}+\rho_{0} c_{0}^{2} \vec{\nabla} \cdot \vec{u}^{n+1, e}=0 .
\end{aligned}
$$

As in the vast majority of BDF implementations, the first step is first order $(J=1)$, and the following steps can be maintained first or use second order $(J=2)$. Note that the Jacobian matrix associated with the fourth order derivative is constant in time. Hence, only one computation of its solution is required. In this work, we perform a single LU decomposition, and afterwards use Gauss substitution to solve the linear system at each time step, but other direct or iterative solvers could be used.

One can use a fully explicit time integrator if the time step restriction allows it (e.g. when the chemical characteristic time is large enough). We have also implemented a low-storage third-order Runge-Kutta method to explicitly solve the system Williamson [1980]. 


\subsection{Semi-discrete stability analysis}

In Sec. 3.6 we studied the entropy-stability of the DG discretization of the general non-conservative system, and in Sec. 6.3 we studied the particularities of the continuous analysis for the iNS/CH system. As sketched in Sec. 3.6, the translation of the continuous stability to the discrete stability rests on the validity of the assumptions made for the continuous analysis in the discrete framework. As described in Sec. 3.6, the entropy equation is made from the scheme weak-forms (6.49), with $\phi=\mathbf{W}$ and $\vec{\varphi}=\overrightarrow{\mathbf{F}}_{v}$,

$$
\begin{aligned}
\left\langle\mathscr{J} \underline{H} L(\mathbf{Q})_{t}, W\right\rangle_{E, N}+\int_{\partial E, N} & \left(W^{T}\left(\overrightarrow{\mathbf{F}}_{e}^{\star}-\overrightarrow{\mathbf{F}}_{e}+\sum_{m=1}^{N_{c}}\left(\left(\overrightarrow{\boldsymbol{\Phi}}_{m} W_{m}\right)^{\diamond}-\left(\overrightarrow{\boldsymbol{\Phi}}_{m} W_{m}\right)\right)-\overrightarrow{\mathbf{F}}_{v}^{\star}\right)\right. \\
& \left.+\left(\mathbf{W}^{T}-\mathbf{W}^{\star, T}\right) \overrightarrow{\mathbf{F}}_{v}\right) \cdot \mathrm{d} \vec{S} \\
& +\left\langle\vec{\nabla}_{\xi} \cdot \overrightarrow{\tilde{\mathbf{F}}}_{e}, \boldsymbol{W}\right\rangle_{E, N}+\sum_{m=1}^{N_{c}}\left\langle\overrightarrow{\tilde{\mathbf{\Phi}}}_{m} \cdot \vec{\nabla}_{\xi} W_{m}, W\right\rangle_{E, N}=-\left\langle\mathscr{J} \overrightarrow{\mathbf{G}}, \overrightarrow{\mathbf{F}}_{v}\right\rangle_{E, N} .
\end{aligned}
$$

As with the rest of the solvers, we only need to validate three properties: time coefficients contraction (3.71), inviscid conservative and non-conservative terms simultaneous contraction (3.105), and viscous fluxes positive definiteness (3.74). Then, we study the stability of the boundary terms.

\subsubsection{Contraction of the time coefficients}

Time coefficients are stable if we can write them as in the general form (3.88),

$$
\sum_{e}\left\langle\mathscr{g} \underline{H} \mathrm{~L}(\mathbf{Q})_{t}, \mathbf{W}\right\rangle_{E, N}=\overline{\mathscr{E}}_{t}^{N},
$$

where $\overline{\mathscr{E}}_{t}^{N}$ is the time derivative of a total discrete entropy. We first study the simpler first artificial compressibility model, and then extend the results to the second artificial compressibility model.

\section{First artificial compressibility model}

For the first artificial compressibility model, $\mathrm{L}(\mathbf{Q})=\mathbf{Q}$, then,

$$
\sum_{e}\left\langle\mathscr{J} \underline{H} \mathbf{Q}_{t}, \mathbf{W}\right\rangle_{E, N}=\sum_{e}\left\langle\mathscr{J} C_{t}, \mu\right\rangle_{E, N}+\sum_{e}\left\langle\mathscr{J} \sqrt{\rho}(\sqrt{\rho} \vec{U})_{t}, \vec{U}\right\rangle_{E, N}+\sum_{e}\left\langle\mathscr{J} P_{t}, \frac{P}{\rho_{0} c_{0}^{2}}\right\rangle_{E, N} \text {. }
$$


For the first term, we demonstrated in (5.36) that,

$$
\sum_{e}\left\langle\mathscr{J} C_{t}, \mu\right\rangle_{E, N}=\mathscr{F}_{t}^{N}
$$

with the total discrete free-energy $\overline{\mathscr{F}}^{N}$

$$
\overline{\mathscr{F}}^{N}=\sum_{e}\langle\mathscr{J} \mathscr{F}\rangle_{E, N}+\sum_{\substack{\text { interior } \\ \text { faces }}} \int_{f, N} \frac{3}{4} \sigma \varepsilon \beta_{c} \llbracket C \rrbracket^{2} \mathrm{~d} S+\sum_{\substack{\text { boundary } \\ \text { faces }}} \int_{f, N} F_{w} \mathrm{~d} S \geqslant 0 .
$$

We use the chain rule in time for the the second term,

$$
\sum_{e}\left\langle\mathscr{J} \sqrt{\rho}(\sqrt{\rho} \vec{U})_{t}, \vec{U}\right\rangle_{E, N}=\sum_{e}\left\langle\mathscr{J}\left(\frac{1}{2} \rho V_{t o t}^{2}\right)_{t}\right\rangle_{E, N}=\frac{\mathrm{d}}{\mathrm{d} t}\langle\mathscr{J} \mathscr{K}\rangle_{E, N}=\overline{\mathscr{K}}_{t}^{N},
$$

and for the third term,

$$
\sum_{e}\left\langle\mathscr{J} P_{t}, \frac{P}{\rho_{0} c_{0}^{2}}\right\rangle_{E, N}=\sum_{e}\left\langle\mathscr{J}\left(\frac{P^{2}}{2 \rho_{0} c_{0}^{2}}\right)_{t}\right\rangle_{E, N}=\frac{\mathrm{d}}{\mathrm{d} t}\left\langle\mathscr{J} \mathscr{P}_{\mathrm{AC} 1}\right\rangle_{E, N}=\overline{\mathscr{P}}_{\mathrm{AC} 1, t}^{N}
$$

Adding (6.86), (6.88) and (6.89), we get the contraction of the time coefficients to the time derivative of the total entropy,

$$
\sum_{e}\left\langle\mathscr{J} \underline{H} \mathrm{~L}(\mathbf{Q})_{t}, \mathbf{W}\right\rangle_{E, N}=\overline{\mathscr{F}}_{t}^{N}+\overline{\mathscr{K}}_{t}^{N}+\overline{\mathscr{P}}_{\mathrm{AC} 1, t}^{N}=\overline{\mathscr{E}}_{\mathrm{AC} 1, t}^{N},
$$

with

$$
\begin{aligned}
\overline{\mathscr{E}}_{\mathrm{AC} 1}^{N}= & \left\langle\mathscr{J}\left(F_{0}+\frac{3}{4} \sigma \varepsilon\left|\vec{G}_{c}\right|^{2}+\frac{1}{2} \rho V_{\text {tot }}^{2}+\frac{P^{2}}{2 \rho_{0} c_{0}^{2}}\right)\right\rangle_{E, N} \\
& +\sum_{\substack{\text { interior } \\
\text { faces }}} \int_{f, N} \frac{3}{4} \sigma \varepsilon \beta_{c} \llbracket C \rrbracket^{2} \mathrm{~d} S+\sum_{\substack{\text { boundary } \\
\text { faces }}} \int_{f, N} F_{w} \mathrm{~d} S .
\end{aligned}
$$

\section{Second artificial compressibility model}

For the second artificial compressibility model, we only change the pressure associated terms. Now we have,

$$
\begin{aligned}
\sum_{e}\left\langle\mathscr{J} \underline{H} \mathrm{~L}(\mathbf{Q})_{t}, \mathbf{W}\right\rangle_{E, N}= & \sum_{e}\left\langle\mathscr{J} C_{t}, \mu\right\rangle_{E, N}+\sum_{e}\left\langle\mathscr{J} \sqrt{\rho}(\sqrt{\rho} \vec{U})_{t}, \vec{U}\right\rangle_{E, N} \\
& +\sum_{e} \frac{1}{\rho_{0} c_{0}^{2}}\left\langle\overrightarrow{\tilde{G}}_{p, t}, \vec{\nabla}_{\xi} P\right\rangle_{E, N}-\sum_{e} \frac{1}{\rho_{0} c_{0}^{2}} \int_{\partial e, N} P \vec{G}_{p, t}^{\star} \cdot \mathrm{d} \vec{S} .
\end{aligned}
$$


The first two terms are unaltered from the first artificial compressibility model (6.86) and (6.88), and the last two terms have already been studied in Sec. 4.5 for the incompressible NSE (4.79),

$$
\sum_{e} \frac{1}{\rho_{0} c_{0}^{2}}\left\langle\overrightarrow{\tilde{G}}_{p, t}, \vec{\nabla}_{\xi} P\right\rangle_{E, N}-\sum_{e} \frac{1}{\rho_{0} c_{0}^{2}} \int_{\partial e, N} P \vec{G}_{p, t}^{\star} \cdot \mathrm{d} \vec{S}=\overline{\mathscr{P}}_{\mathrm{AC} 2, t}^{N},
$$

with

$$
\overline{\mathscr{P}}_{\mathrm{AC} 2}^{N}=\sum_{e}\left\langle\mathscr{J} \frac{\left|\vec{G}_{p}\right|^{2}}{2 \rho_{0} c_{0}^{2}}\right\rangle_{E, N}+\sum_{\substack{\text { interior } \\ \text { faces }}} \int_{f, N} \frac{\beta_{p}}{2 \rho_{0} c_{0}^{2}} \llbracket P \rrbracket^{2} \mathrm{~d} S .
$$

Therefore, for the second artificial compressibility model, the time coefficients contraction holds,

$$
\sum_{e}\left\langle\mathscr{J} \underline{H} \mathrm{~L}(\mathbf{Q})_{t}, \mathbf{W}\right\rangle_{E, N}=\overline{\mathscr{F}}_{t}^{N}+\overline{\mathscr{K}}_{t}^{N}+\overline{\mathscr{P}}_{\mathrm{AC} 2, t}^{N}=\overline{\mathscr{E}}_{\mathrm{AC} 2, t}^{N},
$$

with

$$
\begin{aligned}
\overline{\mathscr{E}}_{\mathrm{AC} 2}^{N}= & \left\langle\mathscr{J}\left(F_{0}+\frac{3}{4} \sigma \varepsilon\left|\vec{G}_{c}\right|^{2}+\frac{1}{2} \rho V_{\text {tot }}^{2}+\frac{\left|\vec{G}_{p}\right|^{2}}{2 \rho_{0} c_{0}^{2}}\right)\right\rangle_{E, N} \\
& +\sum_{\substack{\text { interior } \\
\text { faces }}} \int_{f, N}\left(\frac{3}{4} \sigma \varepsilon \beta_{c} \llbracket C \rrbracket^{2}+\frac{\beta_{p}}{2 \rho_{0} c_{0}^{2}} \llbracket P \rrbracket^{2}\right) \mathrm{d} S+\sum_{\substack{\text { boundary } \\
\text { faces }}} \int_{f, N} F_{w} \mathrm{~d} S \geqslant 0 .
\end{aligned}
$$

\section{Summary}

The entropy variables $\mathbf{W}=(\mu, \vec{U}, P)$ semi-discretely contract the time coefficients for both the first and second artificial compressibility models,

$$
\sum_{e}\left\langle\mathscr{g} \underline{H} \mathrm{~L}(\mathbf{Q})_{t}, \mathbf{W}\right\rangle_{E, N}=\overline{\mathscr{E}}_{t}^{N},
$$

where we get the discrete total entropy of the first (6.91) and the second (6.96) artificial compressibility models.

\subsubsection{Simultaneous contraction of the inviscid conservative and non-conservative fluxes}

For non-conservative systems, the entropy variables simultaneously contract the inviscid conservative and non-conservative terms (3.102). This was proven for the DG 
scheme of the general non-conservative system in Sec. 3.6.1, and also for the particular case of a non-conservative system that satisfies Prop. 1 (3.109) and Prop. 2 (3.110) in Sec. 3.6.2, as is the case for the iNS/CH system.

Then, since the iNS/CH system satisfies Prop. 1 (3.109) and Prop. 2 (3.110), the standard DG scheme automatically satisfies the inviscid terms contraction (3.105),

$$
\left\langle\vec{\nabla}_{\xi} \cdot \overrightarrow{\tilde{\mathbf{F}}}_{e}, \mathbf{W}\right\rangle_{E, N}+\sum_{m=1}^{5}\left\langle\overrightarrow{\tilde{\mathbf{\Phi}}}_{m} \cdot \vec{\nabla}_{\xi} W_{m}, \mathbf{W}\right\rangle_{E, N}=\int_{\partial e, N} \overrightarrow{\tilde{F}}_{e}^{\mathscr{E}} \cdot \hat{n} \mathrm{~d} \hat{S},
$$

since the demonstration of (6.98) only used the discrete Gauss law and the two properties.

For completeness, we also show the stability of the split-form scheme (6.57). For the split-form, it suffices that the two-point volume flux satisfies Tadmor's jump condition (3.106). We first write the potential (3.107) for the iNS/CH system,

$$
\begin{aligned}
\Psi & =\mu C \vec{U}+\frac{1}{2} \rho V_{t o t}^{2} \vec{U}+P \vec{U}+U \vec{P}+\frac{1}{2} \rho V_{t o t}^{2} \vec{U}+\mu C \vec{U}-\frac{1}{2} \rho V_{t o t}^{2} \vec{U}-P \vec{U}-C \mu \vec{U} \\
& =\mu C \vec{U}+\frac{1}{2} \rho \vec{U} \vec{U} \cdot \vec{U}+P \vec{U}
\end{aligned}
$$

and Tadmor's jump condition for $\overrightarrow{\mathbf{F}}_{e}^{\mathrm{EC}}=\left\{\left\{\overrightarrow{\mathbf{F}}_{e}\right\}\right\}$,

$$
\begin{aligned}
& \llbracket \mu \rrbracket\{\{C \vec{U}\}\}+\llbracket \vec{U} \rrbracket \cdot\left\{\left\{\frac{1}{2} \rho \vec{U} \vec{U}\right\}\right\}+\llbracket \vec{U} \rrbracket\{\{P\}\} \\
& =\llbracket \mu C \vec{U}+\frac{1}{2} \rho \vec{U} \vec{U} \cdot \vec{U}+P \vec{U} \rrbracket-\frac{1}{2} \llbracket \rho \vec{U} \vec{U} \rrbracket \cdot\{\{\vec{U}\}\}-\llbracket \vec{U} C \rrbracket\{\{\mu\}\}-\{\{P\}\} \llbracket \vec{U} \rrbracket,
\end{aligned}
$$

which is satisfied, after the use of the average and jump algebraic relation (1.28). We confirm that the split-form scheme also successfully contracts the inviscid terms.

\subsubsection{Positive semi-definiteness of the viscous fluxes}

Finally, the study of volume terms finishes with the viscous flux stability (3.93). For the iNS/CH system,

$$
\begin{aligned}
\left\langle\mathscr{J} \overrightarrow{\mathbf{G}}, \overrightarrow{\mathbf{F}}_{v}\right\rangle_{E, N} & =\left\langle\mathscr{J} \vec{G}_{\mu}, M_{0} \vec{G}_{\mu}\right\rangle_{E, N}+\left\langle\mathscr{J} \overline{\bar{G}}_{\vec{u}}, \eta\left(\overline{\bar{G}}_{\vec{u}}+\overline{\bar{G}}_{\vec{u}}\right)\right\rangle_{E, N} \\
& =\left\langle\mathscr{J} \vec{G}_{\mu}, M_{0} \vec{G}_{\mu}\right\rangle_{E, N}+\left\langle\mathscr{J} \frac{\overline{\bar{G}}_{u}+\overline{\bar{G}}_{u}^{T}}{2}, 2 \eta \frac{\bar{G}_{\vec{u}}+\overline{\bar{G}}_{\vec{u}}}{2}\right\rangle_{E, N} \\
& =\left\langle\mathscr{J}\left(M_{0}\left|\vec{G}_{\mu}\right|^{2}+2 \eta|\overline{\bar{S}}|^{2}\right)\right\rangle_{E, N} \geqslant 0,
\end{aligned}
$$


and we get the sum of the incompressible NSE (4.85) and the Cahn-Hilliard (5.68) physical dissipation.

All the volume terms have been studied, and are shown to produce a stable scheme. Now we study the boundary terms, where we propose two options: the entropy conserving scheme with central fluxes, and the entropy-stable scheme with the exact Riemann solver.

\subsubsection{Boundary terms}

We sum the entropy equation for each element (6.83), to get the discrete entropy equation (3.99),

$$
\overline{\mathscr{E}}_{t}^{N}+\mathrm{PBT}=-\mathrm{IBT}-\left\langle\mathscr{J}\left(M_{0}\left|\overrightarrow{\mathbf{G}}_{\mu}\right|^{2}+2 \eta|\overline{\bar{S}}|^{2}\right)\right\rangle_{E, N} \leqslant 0,
$$

with the interior boundary terms in (3.115) and physical boundary terms in (3.118). We prove the positivity of the interior and boundary terms in the next section, which have to be non-negative for the entropy-stability.

\section{Inviscid interior boundary terms: entropy conserving scheme with central fluxes}

The case with central fluxes was studied in the general stability section (3.117), which get IBT $=0$. Note that in the iNS/CH system the entropy conserving two-point flux is the average of the inviscid fluxes, $\overrightarrow{\mathbf{F}}_{e}^{\mathrm{EC}}=\left\{\left\{\overrightarrow{\mathbf{F}}_{e}\right\}\right\}$.

\section{Inviscid interior boundary terms: entropy-stable scheme with the exact Riemann solver}

The exact Riemann solver was proven to be stable in (4.90) for the incompressible Navier-Stokes equations. Now we extend the proof to the iNS/CH system, which requires a consistent choice of the diamond fluxes, $\overrightarrow{\mathbf{F}}_{\Phi W}^{\mathrm{ERS}}$. The proof rests on the exact Riemann solver star solution satisfying

$$
\begin{aligned}
\Delta_{e}^{\mathrm{ERS}}= & \frac{1}{2} \rho^{\star} \llbracket V_{t o t}^{2} \rrbracket U_{n}^{\star}-\rho^{\star} U_{n}^{\star}\left(U_{n}^{\star} \llbracket U_{n} \rrbracket+V_{t 1}^{\star} \llbracket V_{t 1} \rrbracket+V_{t 2}^{\star} \llbracket V_{t 2} \rrbracket\right) \\
& -P^{\star} \llbracket U_{n} \rrbracket-\llbracket P \rrbracket U_{n}^{\star}+\llbracket P U_{n} \rrbracket \geqslant 0 .
\end{aligned}
$$

Eq. (6.103) implies that the ERS is dissipative for the incompressible NSE. We use the same star region solution for the inviscid numerical fluxes (6.69) and the choice for the diamond fluxes given in (6.70). The result is that the argument of the inviscid 
interior boundary terms is

$$
\begin{aligned}
\Delta_{\mathrm{e}}^{\mathrm{IBT}} & =\left(-\llbracket \mathbf{W}^{T} \rrbracket \overrightarrow{\mathbf{F}}_{e}^{\star}-\sum_{m=1}^{5} \llbracket \mathbf{W}^{T}\left(\overrightarrow{\mathbf{\Phi}}_{m} W_{m}\right)^{\diamond} \rrbracket+\llbracket \mathbf{W}^{T} \overrightarrow{\boldsymbol{\Phi}}_{m} W_{m} \rrbracket\right) \cdot \vec{n}_{L} \\
& =-\llbracket \mathbf{W}^{T} \rrbracket \underline{\mathrm{T}}^{T} \mathbf{F}_{e}^{\star}-\llbracket \mathbf{W}^{T} \underline{\mathrm{T}}^{T} \mathbf{F}_{\Phi W}^{\mathrm{ERS}} \rrbracket+\llbracket \mathbf{W}^{T} \underline{\mathrm{T}}^{T} \mathbf{F}_{\Phi W} \rrbracket \\
& =-\llbracket \mathbf{W}_{n}^{T} \rrbracket \mathbf{F}_{e}^{\star}-\llbracket \mathbf{W}_{n}^{T}\left(\mathbf{F}_{\Phi W}^{\mathrm{ERS}}-\mathbf{F}_{\Phi W}\right) \rrbracket .
\end{aligned}
$$

We write the first term in the last line of (6.104) as

$$
-\llbracket \mathbf{W}_{n}^{T} \rrbracket \mathbf{F}_{e}^{\star}=-\llbracket \mu \rrbracket\left\{\left\{C U_{n}\right\}\right\}-\frac{1}{2} \rho^{\star} U_{n}^{\star}\left(U_{n}^{\star} \llbracket U_{n} \rrbracket+V_{t 1}^{\star} \llbracket V_{t 1} \rrbracket+V_{t 2}^{\star} \llbracket V_{t 2} \rrbracket\right)-P^{\star} \llbracket U_{n} \rrbracket,
$$

and the second term as

$$
\begin{aligned}
-\llbracket \mathbf{W}_{n}^{T}\left(\mathbf{F}_{\Phi W}^{\mathrm{ERS}}-\mathbf{F}_{\Phi W}\right) \rrbracket= & -\frac{1}{2} \rho^{\star} U_{n}^{\star, 2} \llbracket U_{n} \rrbracket+\frac{1}{2} \rho^{\star} U_{n}^{\star} \llbracket U_{n}^{2} \rrbracket-\{\{\mu\}\} \llbracket C U_{n} \rrbracket+\llbracket C U_{n} \mu \rrbracket \\
& -\frac{1}{2} \rho^{\star} U_{n}^{\star} V_{t 1}^{\star} \llbracket V_{t 1} \rrbracket+\frac{1}{2} \rho^{\star} U_{n}^{\star} \llbracket V_{t 1}^{2} \rrbracket \\
& -\frac{1}{2} \rho^{\star} U_{n}^{\star} V_{t 2}^{\star} \llbracket V_{t 2} \rrbracket+\frac{1}{2} \rho^{\star} U_{n}^{\star} \llbracket V_{t 2}^{2} \rrbracket \\
& -U_{n}^{\star} \llbracket P \rrbracket+\llbracket P U_{n} \rrbracket \\
= & \frac{1}{2} \rho^{\star} U_{n}^{\star} \llbracket V_{t o t}^{2} \rrbracket+\llbracket \mu \rrbracket\left\{\left\{C U_{n}\right\}\right\} \\
& -\frac{1}{2} \rho^{\star} U_{n}^{\star}\left(U_{n}^{\star} \llbracket U_{n} \rrbracket+V_{t 1}^{\star} \llbracket V_{t 1} \rrbracket+V_{t 2}^{\star} \llbracket V_{t 2} \rrbracket\right) \\
& -U_{n}^{\star} \llbracket P \rrbracket+\llbracket P U_{n} \rrbracket,
\end{aligned}
$$

so that when it is inserted into (6.104),

$$
\begin{aligned}
\Delta_{\mathrm{e}}^{\mathrm{IBT}}= & \frac{1}{2} \rho^{\star} U_{n}^{\star} \llbracket V_{t o t} \rrbracket^{2}-\rho^{\star} U_{n}^{\star}\left(U_{n}^{\star} \llbracket U_{n} \rrbracket+V_{t 1}^{\star} \llbracket V_{t 1} \rrbracket+V_{t 2}^{\star} \llbracket V_{t 2} \rrbracket\right) \\
& -P^{\star} \llbracket U_{n} \rrbracket-U_{n}^{\star} \llbracket P \rrbracket+\llbracket P U_{n} \rrbracket=\Delta_{e}^{\mathrm{ERS}} \geqslant 0 .
\end{aligned}
$$

The non-negativity of $\Delta_{\mathrm{e}}^{\mathrm{IBT}}$ confirms that the incompressible NSE exact Riemann solver (6.69), with the associated diamond flux choice (6.70) derived in this work produces an entropy-stable interface approximation for the iNS/CH system.

\section{Viscous terms: the Bassi-Rebay 1 method}

The use of the standard BR1 method identically cancels the boundary integrals (see Gassner et al. [2018] for the compressible NSE, Manzanero et al. [2020a] for the incompressible NSE, Bohm et al. [2018] for the MHD equations, and Manzanero et al. 
[2020b] for the Cahn-Hilliard equation). We have to prove that the extra interface stabilization terms are dissipative. In this work, we get the same integral approximation as in Manzanero et al. [2020a] for the incompressible NSE, and as in Manzanero et al. [2020b] for the Cahn-Hilliard part,

$$
\begin{aligned}
\mathrm{IBT}_{v} & =\sum_{\substack{\text { interior } \\
\text { faces }}} \int_{f, N}\left(\llbracket \mathbf{W}^{T} \rrbracket \overrightarrow{\mathbf{F}}_{v}^{\star}-\llbracket \mathbf{W}^{T} \overrightarrow{\mathbf{F}}_{v} \rrbracket+\mathbf{W}^{\star, T} \llbracket \overrightarrow{\mathbf{F}}_{v} \rrbracket\right) \cdot \vec{n}_{L} \mathrm{~d} S \\
& =\sum_{\substack{\text { interior } \\
\text { faces }}} \int_{f, N}\left(\llbracket \mathbf{W}^{T} \rrbracket\left\{\left\{\overrightarrow{\mathbf{F}}_{v}\right\}\right\}+\beta_{\mu} \llbracket \mu \rrbracket^{2} \vec{n}_{L}-\llbracket \mathbf{W}^{T} \overrightarrow{\mathbf{F}}_{v} \rrbracket+\{\{\mathbf{W}\}\}^{T} \llbracket \overrightarrow{\mathbf{F}}_{v} \rrbracket\right) \cdot \vec{n}_{L} \mathrm{~d} S \\
& =\sum_{\substack{\text { interior } \\
\text { faces }}} \int_{f, N} \beta_{\mu} \llbracket \mu \rrbracket^{2} \mathrm{~d} S \geqslant 0 .
\end{aligned}
$$

The interface stabilization is not a requirement for stability, but it is added to enhance the accuracy. We conclude that viscous interior boundary terms are stable.

\section{Interior boundary terms: Summary}

The contribution to the interior boundary terms from viscous terms are identically zero when using the BR1 method without interface stabilization $\left(\beta_{\mu}=0\right)$, and dissipative otherwise $\left(\beta_{\mu}>0\right)$. As for the inviscid terms, interior boundary terms are conservative with central fluxes ( $\mathrm{IBT}_{e}=0$ ), and dissipative with the exact Riemann solver $\left(\mathrm{IBT}_{e} \geqslant 0\right)$.

\section{Physical boundary terms: free- and no-slip walls}

We now address the stability of the physical boundary terms (3.118) for the solid wall boundary condition. For inviscid fluxes $\mathrm{PBT}_{e}$, we use the entropy conserving approach with central fluxes (6.74),

$$
\begin{aligned}
\operatorname{PBT}_{e} & =\sum_{\substack{\text { boundary } \\
\text { faces }}} \int_{f, N}\left(\mathbf{W}^{T} \overrightarrow{\mathbf{F}}_{e}^{\star}+\sum_{m=1}^{5} \mathbf{W}^{T}\left(\left(\overrightarrow{\boldsymbol{\Phi}}_{m} W_{m}\right)^{\diamond}-\overrightarrow{\boldsymbol{\Phi}}_{m} W_{m}\right)\right) \cdot \vec{n} \mathrm{~d} S \\
& =\sum_{\substack{\text { boundary } \\
\text { faces }}} \int_{f, N}\left(U_{n}\left(\frac{1}{2} \rho U_{n}^{2}+P\right)+U_{n}\left(\mu C-\frac{1}{2} \rho U_{n}^{2}-\mu C\right)-P U_{n}\right) \mathrm{d} S=0,
\end{aligned}
$$


or the entropy-stable counterpart with the exact Riemann solver (6.76),

$$
\begin{aligned}
\operatorname{PBT}_{e} & =\sum_{\substack{\text { boundary } \\
\text { faces }}} \int_{f, N}\left(\mathbf{W}^{T} \overrightarrow{\mathbf{F}}_{e}^{\star}+\sum_{m=1}^{5} \mathbf{W}^{T}\left(\left(\overrightarrow{\boldsymbol{\Phi}}_{m} W_{m}\right)^{\diamond}-\overrightarrow{\boldsymbol{\Phi}}_{m} W_{m}\right)\right) \cdot \vec{n} \mathrm{~d} S \\
& =\sum_{\substack{\text { boundary } \\
\text { faces }}} \int_{f, N}\left(U_{n}\left(\frac{1}{2} \rho \lambda^{+} U_{n}+P\right)-P U_{n}\right) \mathrm{d} S=\sum_{\substack{\text { boundary } \\
\text { faces }}} \int_{f, N}\left(\frac{1}{2} \rho \lambda^{+} U_{n}^{2}\right) \mathrm{d} S \geqslant 0 .
\end{aligned}
$$

Either way, the inviscid physical boundary contribution is stable. Furthermore, for the viscous terms, the free-slip wall boundary condition (6.77),

$$
\begin{aligned}
\operatorname{PBT}_{v}= & -\sum_{\substack{\text { boundary } \\
\text { faces }}} \int_{f, N}\left(\mathbf{W}^{T}\left(\overrightarrow{\mathbf{F}}_{v}^{\star}-\overrightarrow{\mathbf{F}}_{v}\right)+\mathbf{W}^{\star, T} \overrightarrow{\mathbf{F}}_{v}\right) \cdot \vec{n} \mathrm{~d} S \\
& -\sum_{\substack{\text { boundary } \\
\text { faces }}} \int_{f, N}\left(\mathbf{W}^{T}\left(0-\overrightarrow{\mathbf{F}}_{v}\right)+\mathbf{W}^{T} \overrightarrow{\mathbf{F}}_{v}\right) \cdot \vec{n} \mathrm{~d} S=0,
\end{aligned}
$$

and for the no-slip wall boundary condition (6.78),

$$
\begin{aligned}
\operatorname{PBT}_{v}= & -\sum_{\substack{\text { boundary } \\
\text { faces }}} \int_{f, N}\left(\mathbf{W}^{T}\left(\overrightarrow{\mathbf{F}}_{v}^{\star}-\overrightarrow{\mathbf{F}}_{v}\right)+\mathbf{W}^{\star, T} \overrightarrow{\mathbf{F}}_{v}\right) \cdot \vec{n} \mathrm{~d} S \\
& -\sum_{\substack{\text { boundary } \\
\text { faces }}} \int_{f, N}\left(-\mu \vec{G}_{\mu}+\mu \vec{G}_{\mu}+\vec{U} \overline{\bar{S}}-\vec{U} \overline{\bar{S}}\right) \cdot \vec{n} \mathrm{~d} S=0,
\end{aligned}
$$

are dissipative.

\subsubsection{Semi-discrete stability: Summary}

We have constructed a DG approximation of the iNS/CH system (6.49) whose analysis mimics the continuous stability analysis of Sec. 6.3 semi-discretely. As a result, we have shown that the discrete entropy satisfies a balance law,

$$
\overline{\mathscr{E}}_{t}^{N} \leqslant-\sum_{e}\left\langle\mathscr{J}\left(M_{0}\left|\vec{G}_{\mu}\right|^{2}+2 \eta|\overline{\bar{S}}|^{2}\right)\right\rangle_{E, N} \leqslant 0
$$

for both free- and no-slip wall boundary conditions. The total entropy $\overline{\mathscr{E}}_{t}^{N}$ depends on the choice of the first (6.91) or the second (6.96) artificial compressibility method.

The total entropy contains a stable inter-element surface entropy term proportional to $\beta_{c} \llbracket C \rrbracket^{2}$. A physical argument to include this term in the entropy is that 
the free-energy measures the fluid interfaces using the solution gradients $\frac{3}{4} \sigma \varepsilon\left|\vec{G}_{c}\right|^{2}$. However, if the discrete solution is allowed to be discontinuous at the inter-elements faces, it enables the creation of a fluid interface, which is not reflected in the original free-energy. The addition of this penalization, allows us to account for inter-element discontinuities as part of the fluid interface, so that their associated energy is also weighted in the entropy. In practice, we have found that if the flow is under-resolved and $\beta_{c}=0$, the solution tends to minimize the entropy allowing sharp discontinuities at the inter-elements interface. Since that effect has not been taken into account in the entropy with $\beta_{c}=0$, the flow wrongly enables the discontinuity mechanism to minimize the entropy. This adverse effect (from the point of view of the accuracy) is mitigated when $\beta_{c}>0$ Gassner et al. [2016a]; Manzanero et al. [2020b]. Since now the inter-element discontinuities belong to the entropy, their size can be kept bounded and controlled. In any case, the use of interface stabilization does not compromise the stability of the scheme. Also, note that the continuous analysis assumes a continuous solution, and that a continuous solution would have the same discrete and continuous entropies, since $\llbracket C \rrbracket=0$.

Finally, the form of (6.113) depends on the choice of the inviscid numerical and diamond fluxes, and the Cahn-Hilliard interface stabilization: an equality for central fluxes and $\beta_{\mu}=0$, and an inequality for the exact Riemann solver and $\beta_{\mu}>0$. For either choice, the discrete entropy plus the surface free-energy remains bounded by the initial condition.

\subsection{Numerical experiments}

We now explore the capabilities of the new DGSEM scheme with numerical simulations. We only use the first artificial compressibility model for the numerical experiments. First, we validate the implementation with a convergence study on a manufactured solution. Second, we explore the robustness of the scheme by integrating in time from a random initial condition, where we compare an entropy-stable scheme with a Gauss point formulation that is not provably stable. Third, we solve a static bubble as a benchmark for steady-state accuracy, and a transient rising bubble. Finally, we present solutions for a three-dimensional pipe flow in the annular regime.

\subsubsection{Convergence study}

We first assess the spatial and temporal accuracy of the implementation of the approximation. We test with the same two-dimensional manufactured solution and config- 
uration used in other Navier-Stokes/Cahn-Hilliard works, specifically Dong [2018b],

$$
\begin{aligned}
& c_{0}(x, y ; t)=\frac{1}{2}(1+\cos (\pi x) \cos (\pi y) \sin (t)), \\
& u_{0}(x, y ; t)=2 \sin (\pi x) \cos (\pi z) \sin (t), \\
& v_{0}(x, y ; t)=-2 \cos (\pi x) \sin (\pi y) \sin (t), \\
& p_{0}(x, y ; t)=2 \sin (\pi x) \sin (\pi z) \cos (t),
\end{aligned}
$$

on the domain $(x, y) \in[-1,1]^{2}$. The final time is $t_{F}=0.1$, and all physical parameters are presented in Table 6.1.

Table 6.1: List of the parameter values used with the manufactured solution (6.114)

\begin{tabular}{llllllll}
\hline$\rho_{1}$ & $\rho_{2}\left(\mathrm{~kg} / \mathrm{m}^{3}\right)$ & $\eta_{1}$ & $\eta_{2}(\mathrm{~Pa} \cdot \mathrm{s})$ & $\varepsilon(\mathrm{m})$ & $t_{C H}(\mathrm{~s})$ & $c_{0}^{2}\left(\mathrm{~m} / \mathrm{s}^{2}\right)^{2}$ & $\sigma(\mathrm{N} / \mathrm{m})$ \\
\hline 1.0 & 2.0 & $1.0 \mathrm{E}-3$ & $1.0 \mathrm{E}-3$ & $1 / \sqrt{2}$ & $1.0 \mathrm{E} 3$ & $1.0 \mathrm{E} 3$ & $6.236 \mathrm{E}-3$ \\
\hline
\end{tabular}

We measure the $\mathrm{L}^{2}$ errors using the discrete inner product,

$$
\text { error }=\left\|\phi-\phi_{0}\right\|_{\mathscr{J}, N}=\sqrt{\sum_{e}\left\langle\mathscr{J}\left(\Phi-\Phi_{0}\right),\left(\Phi-\Phi_{0}\right)\right\rangle_{E, N}} .
$$

Regarding the configuration of the tests, we use the exact Riemann solver, and we vary the polynomial order $N$, mesh size (we use an equally spaced Cartesian mesh $M^{2}$ ), and time-step size $\Delta t$ of the BDF2 scheme $(J=2)$ to evaluate the convergence rates as the resolution is increased either by increasing the polynomial order or decreasing the mesh size.

We first use a Cartesian $4^{2}$ element mesh and vary the polynomial order from $N=3$ to 10 . The $\mathrm{L}^{2}$ errors (6.115) are presented in Fig. 6.1, using $\Delta t=10^{-4}$ in Fig. 6.1(a) and $\Delta t=10^{-5}$ in Fig. 6.1(b).

Figs. 6.1(a) and 6.1(b) show typical and expected error behavior: an approximately linear semi-log convergence for lower polynomial orders implying exponential convergence of the error (i.e. under-resolved solution in space), and error stagnation for higher orders (under-resolved solution in time). The stagnation error threshold can be controlled with $\Delta t$, as shown in Fig. 6.1(b).

For the next test configuration, we use polynomial orders $N=2,3,4$ and 5, and meshes with $4^{2}, 6^{2}, 8^{2}, 12^{2}$ and $16^{2}$ elements for an $h$-type convergence study. The time-step is $\Delta t=5 \cdot 10^{-5}$. We solve the manufactured solution problem (6.114), and summarize the errors in Table 6.2. An estimate of the scheme's order of convergence is provided there. We note that for the momentum components, the convergence orders 


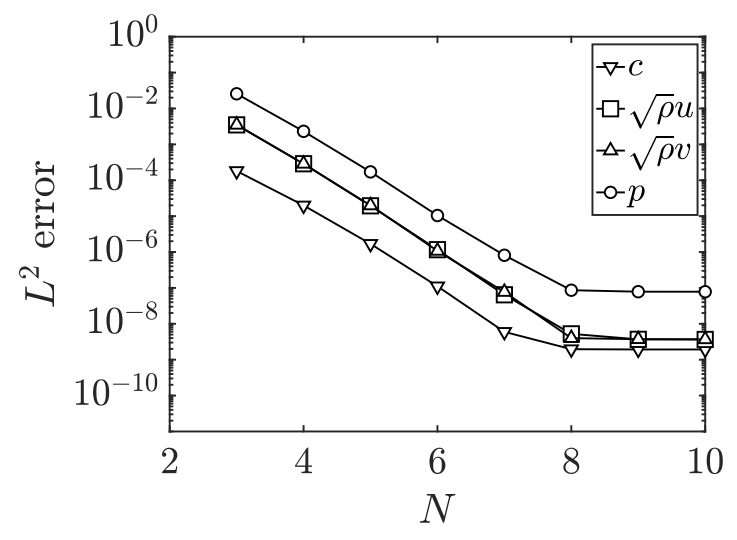

(a) $\Delta t=10^{-4}$

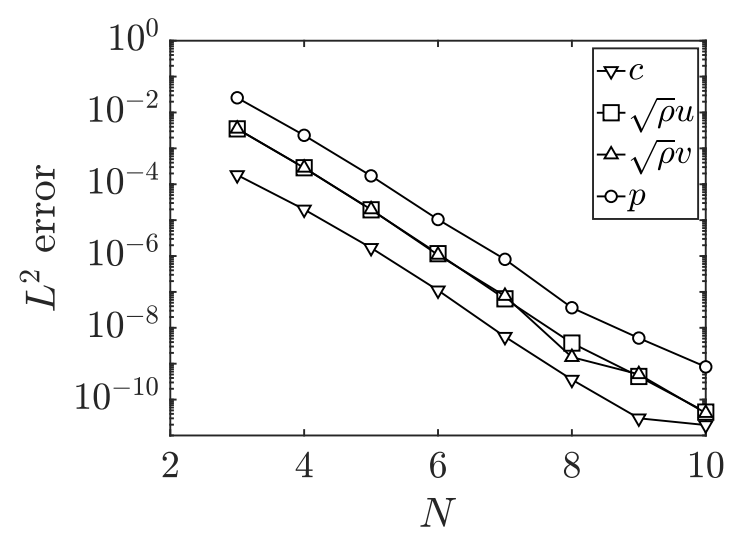

(b) $\Delta t=10^{-5}$

Figure 6.1: Exponential convergence of the manufactured solution (6.114) as the polynomial order increases. We represent the $\mathrm{L}^{2}$ errors in concentration, $x$ - and $y-$ momentum, and pressure. The polynomial order ranges from 3 to 10, and we integrate in time until $t_{F}=0.1$ with two time step sizes: $\Delta t=10^{-4}$ and $10^{-5}$. All physical parameters are given in Table 6.1

are as expected, as they are close to $N+1$. For the pressure, the order of convergence is somewhat smaller (closer to $N$ ), which was also experienced in Manzanero et al. [2020a]; Bassi et al. [2017]. The convergence is also not optimal for the concentration; for even polynomials, the convergence can surpass the theoretical $N+1$ value, while for odd polynomials, the convergence rate is roughly $N$ in most of the cases. This effect was also noticed in Chapter 5 and Manzanero et al. [2020b], whose nature remains unclear.

\subsubsection{Assessing robustness: random initial condition}

We also assess the robustness of the scheme by introducing a random initial condition. Because the scheme developed here is entropy-stable even for severely under-resolved flows, the numerical implementation should not crash, as long as the time-step is small enough to stay within the time integrator stability region.

As a point of reference, and only in this section, we also consider the DG scheme using Gauss points. The standard DG method with Gauss points does not satisfy the SAT property Kopriva [2017] and the proofs developed for semi-discrete stability do not carry over. As a result, the standard scheme is not provably stable. To make the comparison even more stark, we compare robustness of the Gauss scheme with upwind flux dissipation to the new Gauss-Lobatto entropy-conserving method with 
Table 6.2: Manufactured solution (6.114) convergence analysis: we use $4^{2}, 8^{2}$, and $16^{2}$ meshes, and $N=2,3,4$ and 5 . The final time is $t_{F}=0.1$, and we use the IMEX BDF2 scheme with $\Delta t=5 \cdot 10^{-5}$

\begin{tabular}{llllllllll}
\hline $\mathrm{N}=2$ & Mesh & $c$ error & order & $\sqrt{\rho} u$ error & order & $\sqrt{\rho} v$ error & order & $p$ error & order \\
\hline & $4^{2}$ & $1.35 \mathrm{E}-03$ & - & $3.39 \mathrm{E}-02$ & - & $3.39 \mathrm{E}-02$ & - & $2.22 \mathrm{E}-01$ & - \\
& $6^{2}$ & $8.27 \mathrm{E}-04$ & 1.21 & $1.24 \mathrm{E}-02$ & 2.47 & $1.24 \mathrm{E}-02$ & 2.47 & $9.02 \mathrm{E}-02$ & 2.22 \\
& $8^{2}$ & $4.40 \mathrm{E}-04$ & 2.19 & $5.96 \mathrm{E}-03$ & 2.56 & $5.95 \mathrm{E}-03$ & 2.56 & $4.68 \mathrm{E}-02$ & 2.28 \\
& $12^{2}$ & $1.30 \mathrm{E}-04$ & 3.01 & $2.04 \mathrm{E}-03$ & 2.65 & $2.03 \mathrm{E}-03$ & 2.65 & $1.78 \mathrm{E}-02$ & 2.39 \\
& $16^{2}$ & $5.35 \mathrm{E}-05$ & 3.08 & $9.23 \mathrm{E}-04$ & 2.75 & $9.23 \mathrm{E}-04$ & 2.75 & $8.67 \mathrm{E}-03$ & 2.50 \\
$\mathrm{~N}=3$ & $4^{2}$ & $1.81 \mathrm{E}-04$ & - & $3.49 \mathrm{E}-03$ & - & $3.50 \mathrm{E}-03$ & - & $2.57 \mathrm{E}-02$ & - \\
& $6^{2}$ & $5.71 \mathrm{E}-05$ & 2.85 & $8.28 \mathrm{E}-04$ & 3.55 & $8.29 \mathrm{E}-04$ & 3.55 & $6.81 \mathrm{E}-03$ & 3.27 \\
& $8^{2}$ & $2.76 \mathrm{E}-05$ & 2.53 & $2.90 \mathrm{E}-04$ & 3.65 & $2.91 \mathrm{E}-04$ & 3.65 & $2.57 \mathrm{E}-03$ & 3.39 \\
& $12^{2}$ & $9.53 \mathrm{E}-06$ & 2.62 & $6.34 \mathrm{E}-05$ & 3.75 & $6.37 \mathrm{E}-05$ & 3.74 & $6.17 \mathrm{E}-04$ & 3.52 \\
& $16^{2}$ & $4.36 \mathrm{E}-06$ & 2.72 & $2.10 \mathrm{E}-05$ & 3.83 & $2.12 \mathrm{E}-05$ & 3.83 & $2.17 \mathrm{E}-04$ & 3.64 \\
$\mathrm{~N}=4$ & $4^{2}$ & $1.99 \mathrm{E}-05$ & - & $2.89 \mathrm{E}-04$ & - & $2.87 \mathrm{E}-04$ & - & $2.31 \mathrm{E}-03$ & - \\
& $6^{2}$ & $5.27 \mathrm{E}-06$ & 3.28 & $4.40 \mathrm{E}-05$ & 4.64 & $4.40 \mathrm{E}-05$ & 4.62 & $3.94 \mathrm{E}-04$ & 4.36 \\
& $8^{2}$ & $7.50 \mathrm{E}-07$ & 6.78 & $1.12 \mathrm{E}-05$ & 4.75 & $1.12 \mathrm{E}-05$ & 4.75 & $1.09 \mathrm{E}-04$ & 4.47 \\
& $12^{2}$ & $8.46 \mathrm{E}-08$ & 5.38 & $1.55 \mathrm{E}-06$ & 4.89 & $1.55 \mathrm{E}-06$ & 4.89 & $1.70 \mathrm{E}-05$ & 4.59 \\
& $16^{2}$ & $1.94 \mathrm{E}-08$ & 5.12 & $3.61 \mathrm{E}-07$ & 5.05 & $3.61 \mathrm{E}-07$ & 5.05 & $4.42 \mathrm{E}-06$ & 4.68 \\
$\mathrm{~N}=5$ & $4^{2}$ & $1.69 \mathrm{E}-06$ & - & $1.94 \mathrm{E}-05$ & - & $1.98 \mathrm{E}-05$ & - & $1.71 \mathrm{E}-04$ & - \\
& $6^{2}$ & $1.91 \mathrm{E}-07$ & 5.38 & $1.96 \mathrm{E}-06$ & 5.65 & $1.97 \mathrm{E}-06$ & 5.70 & $1.87 \mathrm{E}-05$ & 5.46 \\
& $8^{2}$ & $4.67 \mathrm{E}-08$ & 4.89 & $3.76 \mathrm{E}-07$ & 5.74 & $3.77 \mathrm{E}-07$ & 5.74 & $3.78 \mathrm{E}-06$ & 5.57 \\
& $12^{2}$ & $6.43 \mathrm{E}-09$ & 4.89 & $3.64 \mathrm{E}-08$ & 5.76 & $3.65 \mathrm{E}-08$ & 5.76 & $3.79 \mathrm{E}-07$ & 5.67 \\
& $16^{2}$ & $1.63 \mathrm{E}-09$ & 4.77 & $7.08 \mathrm{E}-09$ & 5.69 & $7.11 \mathrm{E}-09$ & 5.69 & $7.47 \mathrm{E}-08$ & 5.64 \\
\hline
\end{tabular}

central fluxes.

We solve the problem on the domain $\Omega=[0,1]^{3}$, divided into a three-dimensional $4^{3}$ element Cartesian mesh. We initialize the solution using uniform random numbers in $[0,1]$ for concentration, and in $[-1,1]$ for the velocities and the pressure. We use a high density ratio $\left(\rho_{1} / \rho_{2}=1000\right)$, and the Reynolds number $R e=10^{6}$ so that physical viscosity will have only a weak stabilizing effect. The rest of the parameters are given in Table 6.3.

Table 6.3: Physical parameters for the random initial condition test problem

\begin{tabular}{llllllll}
\hline$\rho_{1}$ & $\rho_{2}\left(\mathrm{~kg} / \mathrm{m}^{3}\right)$ & $\eta_{1}$ & $\eta_{2}(\mathrm{~Pa} \cdot \mathrm{s})$ & $\varepsilon(\mathrm{m})$ & $t_{C H}(\mathrm{~s})$ & $c_{0}^{2}\left(\mathrm{~m} / \mathrm{s}^{2}\right)^{2}$ & $\sigma(\mathrm{N} / \mathrm{m})$ \\
\hline 1000.0 & 1.0 & $10^{-3}$ & $10^{-4}$ & 0.75 & 10.0 & $1.0 \mathrm{E} 2$ & 1.0 \\
\hline
\end{tabular}

With Gauss-Lobatto points, the discrete entropy balance (6.113) shows that the free-energy time derivative $\overline{\mathscr{E}}_{t}^{N}$ should be always negative as a result of the viscous 


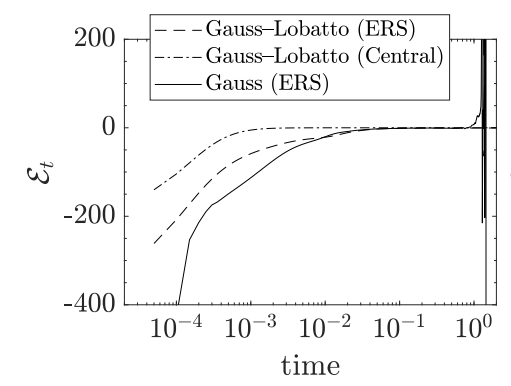

(a) $N=2$

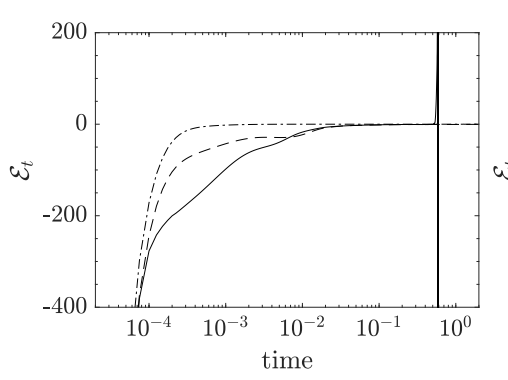

(b) $N=3$

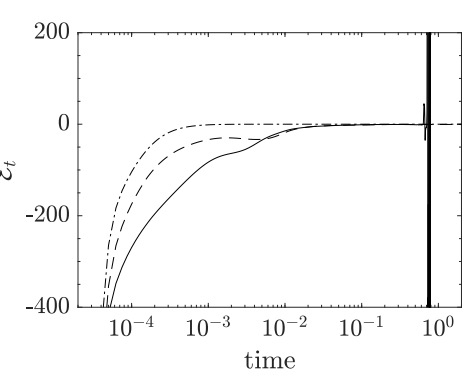

(c) $N=4$

Figure 6.2: Random chosen initial condition: evolution of the entropy time derivative $\overline{\mathscr{E}}_{t}^{N}$ for three polynomial orders $N=2,3,4$. We consider three schemes: GaussLobatto points with the ERS and central fluxes, and Gauss points with the ERS. For all the polynomial orders, using Gauss-Lobatto points is an entropy preserving scheme. The dissipation is a result of physical viscosity and chemical potential for central fluxes, to which the dissipation by the exact Riemann solver can added. On the contrary, the Gauss approximation entropy time derivative is not always negative, which numerically diverges as a result

and chemical potential dissipation, for either central fluxes or the ERS. Moreover, the entropy remainder, defined as

$$
\mathscr{R}(\mathscr{E})=\overline{\mathscr{E}}_{t}^{N}+\sum_{e}\left\langle\mathscr{J}\left(M_{0}\left|\vec{G}_{\mu}\right|^{2}+2 \eta|\overline{\bar{S}}|^{2}\right)\right\rangle_{E, N} \leqslant 0,
$$

should be zero at each time step for central fluxes, and negative for the ERS. None of the statements regarding $\overline{\mathscr{E}}_{t}^{N}$ or $\mathscr{R}(\mathscr{E})$ being negative are guaranteed to hold for Gauss points by theory.

The entropy time derivative evolution is presented in Fig. 6.2, and the entropy remainder (6.116) in Fig. 6.3. Both are presented for three polynomial orders $(N=2,3,4)$, and for the three configurations described: Gauss-Lobatto points with the ERS, Gauss-Lobatto points with central fluxes, and Gauss points with ERS. We find that the solutions follow the same pattern for the different polynomial orders studied. Gauss-Lobatto points are always stable, and the ERS enhances the stability (i.e. the entropy time derivative is always smaller or equal when compared to central fluxes). This is confirmed by looking at the entropy remainder in Fig. 6.3, which is machine precision for central fluxes, and negative for the ERS. Whereas for Gauss points, the solution is divergent (i.e. crashes) when $t \geq 1.53$. We see that for this case, Gauss points are more dissipative than Gauss-Lobatto points for the first steps, but then the opposite occurs when $t>10^{-2}$.

With a random initial condition, the Gauss approximation is prone to diverge, but 


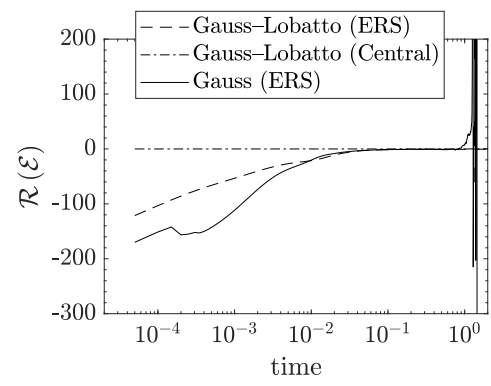

(a) $N=2$

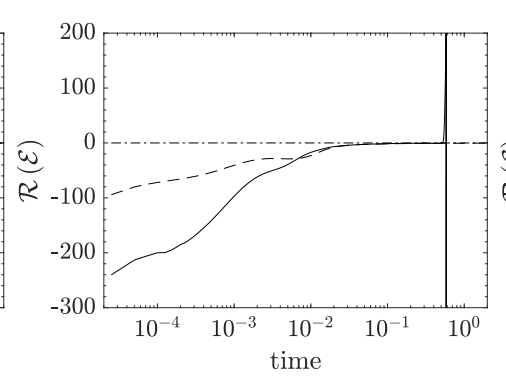

(b) $N=3$

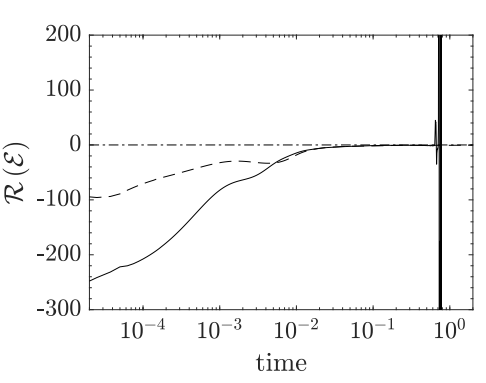

(c) $N=4$

Figure 6.3: Random initial condition: evolution of the entropy remainder (6.116) for three polynomial orders $N=2,3,4$. We consider three schemes: Gauss-Lobatto points with the ERS and central fluxes, and Gauss points with the ERS. The remainder studies the dissipation introduced by the scheme without considering physical viscosity and chemical potential. Thus, the Gauss-Lobatto scheme with the ERS is dissipative (always negative), the Gauss-Lobatto scheme with central fluxes is conserving (always zero), and the scheme with Gauss points is unstable and crashes

it might not. We ran another 100 random initial conditions with different seeds until a final time $t_{F}=10.0$, of which $30 / 100$ crashed. For the 70 simulations that did not crash, most of them experienced growth of their entropy at some points in time. We represent in Fig. 6.4 the number of simulations that crashed before the physical time on the horizontal axis. Most often crashes occur early, i.e. times $t \approx 1.0$, after which the simulation is less likely to crash. None of the simulations crashed if they stayed stable for more than 4.0 seconds. In other words, if it can be computed beyond the initial stages, it is more likely to continue.

We repeated the experiment for the entropy-stable Gauss-Lobatto variant, to confirm that none of them crashed. There may be advantages to use Gauss points, because of higher accuracy per degree of freedom Gassner and Kopriva [2011]; Manzanero et al. [2018c], and indeed the scheme presented herein allows us to use Gauss points in practice. However, as it is not entropy-stable, it might crash under certain conditions, perhaps severely under-resolved simulations like high Reynolds turbulent flows, etc. Moreover, Gauss-Lobatto points based schemes allow larger time-step sizes Klose et al. [2019], they do not require the projection of the solution to the faces, and they can be statically condensed Rueda-Ramírez et al. [2019b]. 


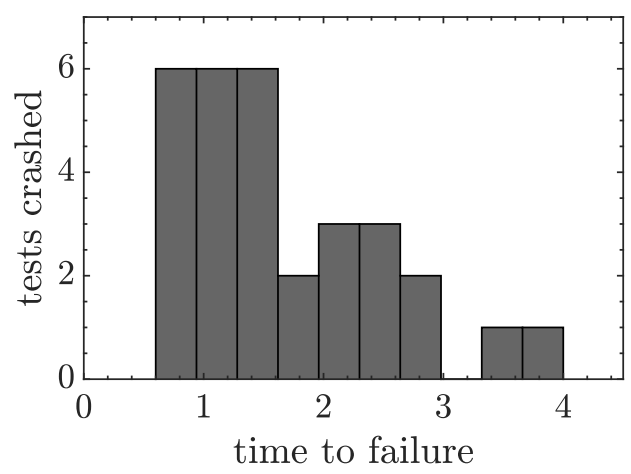

Figure 6.4: Random initial condition: we run 100 simulations modifying the random numbers sequence, of which 30 crashed with Gauss points. In this figure, the physical time in which the simulation diverged is represented. Although the other test passed the final time $t_{F}=10.0$, their entropy time derivative was found to be positive in several time-steps. None of the 100 simulations crashed with Gauss-Lobatto

\subsubsection{Static bubble}

In this test problem we solve a steady two-dimensional bubble and validate the pressure jump that results from surface tension. In the domain $\Omega=[0,1] \times[0,1]$, the initial condition for the concentration is

$$
c(x, y ; 0)=\frac{1-\tanh \left(-\frac{r-0.25}{\zeta}\right)}{2}, r=\sqrt{(x-0.5)^{2}+(y-0.5)^{2}}, \quad \zeta=2.5 \cdot 10^{-3},
$$

which approximates a circle with radius $R_{0}=0.25$. Since we project the initial condition into our polynomial ansatz, the computed radius, $R_{e}$, subtly differs to $R_{0}$. The analytical pressure jump between outside and inside of the bubble $\Delta p_{a}$ is given by the Poisson law,

$$
\Delta p_{a}=p_{i}-p_{e}=p(0.5,0.5)-p(1.0,0.5)=\frac{\sigma}{R_{e}} .
$$

Note that we used the radius $R_{e}$, which is estimated from the final solution contour $c(x, y)=0.5$, to compute the analytical solution. The rest of the parameters, including the surface tension $\sigma=1 \mathrm{~N} / \mathrm{m}$, are given in Table 6.4. We apply periodic boundary conditions at the four physical boundaries, and use the first order IMEX scheme $(J=1)$ until the residuals are kept lower than $10^{-7}$.

The results are summarized in Table 6.5, which are computed with polynomial order $N=2$ and three meshes $16^{2}, 32^{2}$, and $64^{2}$. For all meshes, we compute the solution radius $R_{e}$, the analytical pressure jump $\Delta p_{a}$, which are compared to the numerical solution interior pressure, $p_{i}$, exterior pressure $p_{e}$, and pressure jump $\Delta p$. 
Table 6.4: Parameters of the Static bubble test problem

\begin{tabular}{llllllll}
\hline Grid & $\rho_{1,2}\left(\mathrm{~kg} / \mathrm{m}^{3}\right)$ & $\eta_{1,2}(\mathrm{~Pa} \cdot \mathrm{s})$ & $\varepsilon(\mathrm{m})$ & $t_{C H}(\mathrm{~s})$ & $c_{0}^{2}\left(\mathrm{~m} / \mathrm{s}^{2}\right)^{2}$ & $\sigma(\mathrm{N} / \mathrm{m})$ & $\Delta t(\mathrm{~s})$ \\
\hline $16^{2}$ & 1.0 & 1.0 & 0.16 & 7.0 & $1.0 \mathrm{E} 3$ & 1.0 & $10^{-5}$ \\
$32^{2}$ & 1.0 & 1.0 & 0.08 & 7.0 & $1.0 \mathrm{E} 3$ & 1.0 & $5 \cdot 10^{-6}$ \\
$64^{2}$ & 1.0 & 1.0 & 0.05 & 7.0 & $1.0 \mathrm{E} 3$ & 1.0 & $2.5 \cdot 10^{-6}$ \\
\hline
\end{tabular}

Here we refer to the static pressure, and not the auxiliary pressure (6.13). The results show that the pressure jump converges to that given by the Poisson law (6.118) as we refine the grid.

In the last column of Table 6.5, we represent the norm of the velocity, which is of the size of the residuals. This implies that no parasitic currents are produced using this formulation, which are common in alternative Navier-Stokes/Cahn-Hilliard formulations Lee [2009]. Usually, the size of the parasitic currents is $10^{-3}$ for this problem, and since they do not disappear as the grid is refined, they can dominate in low speed simulations.

Table 6.5: Results of the Static bubble test problem

\begin{tabular}{llllllll}
\hline Grid & $R_{e}(\mathrm{~m})$ & $\Delta p_{a}$ & $p_{i}$ & $p_{e}$ & $\Delta p(\mathrm{~Pa})$ & $\left|\Delta p-\Delta p_{a}\right| / \Delta p_{a}$ & $\|\vec{u}\|$ \\
\hline $16^{2}$ & 0.220 & 4.546 & 0.033 & -4.571 & 4.604 & $1.27 \cdot 10^{-2}$ & $4.0 \cdot 10^{-10}$ \\
$32^{2}$ & 0.240 & 4.175 & 0.029 & -4.120 & 4.150 & $6.09 \cdot 10^{-3}$ & $2.4 \cdot 10^{-8}$ \\
$64^{2}$ & 0.244 & 4.100 & 0.173 & -4.094 & 4.111 & $2.75 \cdot 10^{-3}$ & $3.7 \cdot 10^{-9}$ \\
\hline
\end{tabular}

\subsubsection{Rising bubble}

The rising bubble problem has been widely used in the multiphase flow community to assess the space-time accuracy and robustness of the methods Hysing et al. [2009]; Hosseini et al. [2017]; Ding et al. [2007]. The problem follows the trajectory of a bubble submerged in a heavier fluid as it rises. We consider the domain $(x, y) \in$ $[0,1] \times[0,2]$, and a circular bubble with center in $(0.5,0.5)$ and diameter 0.5 . The bubble is approximated with the initial condition,

$$
c(x, y ; 0)=1-\frac{1}{2}\left(\tanh \left(-\frac{2(r-0.25)}{\varepsilon}\right)+1\right), \quad r=\sqrt{(x-0.5)^{2}+(y-0.5)^{2}},
$$

and the rest of the variables are initialized to zero. We consider a $16 \times 32$ Cartesian mesh with element size $h=2^{-4}$, and a polynomial order $N=10$. The physical parameters are taken from Hysing et al. [2009] and are summarized in Table 6.6. We 
Table 6.6: Physical parameters of the Rising bubble test problem

\begin{tabular}{llllllllll}
\hline Test & $\rho_{1}$ & $\rho_{2}\left(\mathrm{~kg} / \mathrm{m}^{3}\right)$ & $\eta_{1}$ & $\eta_{2}(\mathrm{~Pa} \cdot \mathrm{s})$ & $\varepsilon(\mathrm{m})$ & $t_{C H}(\mathrm{~s})$ & $c_{0}^{2}\left(\mathrm{~m} / \mathrm{s}^{2}\right)^{2}$ & $\sigma(\mathrm{N} / \mathrm{m})$ & $g\left(\mathrm{~m} / \mathrm{s}^{2}\right)$ \\
\hline 1 & 1000.0 & 100.0 & 10.0 & 1.0 & 0.03 & $1.0 \mathrm{E} 3$ & $1.0 \mathrm{E} 3$ & 24.5 & 0.98 \\
2 & 1000.0 & 1.0 & 10.0 & 0.1 & 0.04 & $1.0 \mathrm{E} 4$ & $1.0 \mathrm{E} 3$ & 1.96 & 0.98 \\
\hline
\end{tabular}

consider two tests: the first with moderate density ratio $\rho_{1} / \rho_{2}=10$, the second with large density ratio $\rho_{1} / \rho_{2}=1000$. The boundary conditions are free-slip walls in $x=0$ and $x=1$, and no-slip walls in $y=0$ and $y=2$. For the Test 1 , the chemical characteristic time is not big enough to use the explicit Runge-Kutta, and we use the second order IMEX BDF method, with time-step $\Delta t=7.2 \cdot 10^{-6}$. For the Test 2, we use the explicit third order Runge-Kutta, with $\Delta t=7.2 \cdot 10^{-7}$. Although we use fixed time-stepping, in both cases the CFL number $(\mathrm{CFL}=16(N+1)(|u|+a) \Delta t)$ is maintained close to 0.2 .

Although the model presented in this Chapter is diffuse interface, we compare it to a sharp interface model. Moreover, in this test case we compare the artificial compressibility method to a pressure-correction method that enforces the incompressible constraint in a transient simulation. Thus, we can assess the validity of the model in the incompressible limit and its implementation.

The shape of the bubble at the final time $t=3.0$ is represented in Fig. 6.5. First, in Fig. 6.5(a), we represent the final shape of the interface by drawing three contour lines: $c=0.1$ in dash-dot, $c=0.5$ as a solid line, and $c=0.9$ as a dashed line. The sharp interface reference solution is represented with black dots. We find good agreement in both the position and the shape of the bubble, as the $c=0.5$ contour follows the shape of the bubble, and the dots are always found inside the $c=0.1$ and $c=0.9$ contours. Next, in Fig. 6.5(b) we represent the concentration contour, with the velocity vectors on top. The solution is also in agreement with other diffuse interface works Hosseini et al. [2017].

In Fig. 6.6 we represent the evolution of the center of gravity (Fig. 6.6(a)) and the bubble rise velocity (Fig. 6.6(b)),

$X_{c}=\frac{1}{A} \sum_{e}\langle\mathscr{J}(1-C), X\rangle_{E, N}, \quad V_{c}=\frac{1}{A} \sum_{e}\langle\mathscr{J}(1-C), U\rangle_{E, N}, \quad A=\sum_{e}\langle\mathscr{J}(1-C), 1\rangle_{E, N}$.

Both center of gravity position and rise velocity show good agreement with the sharp interface reference. As for the rise velocity in Fig. 6.6(b), the oscillations are a result of the artificial compressibility pressure waves. One can reduce the amplitude of these oscillations by increasing the artificial sound speed $c_{0}$, but we have only found small differences in the evolution when increasing this parameter above the value used here. 


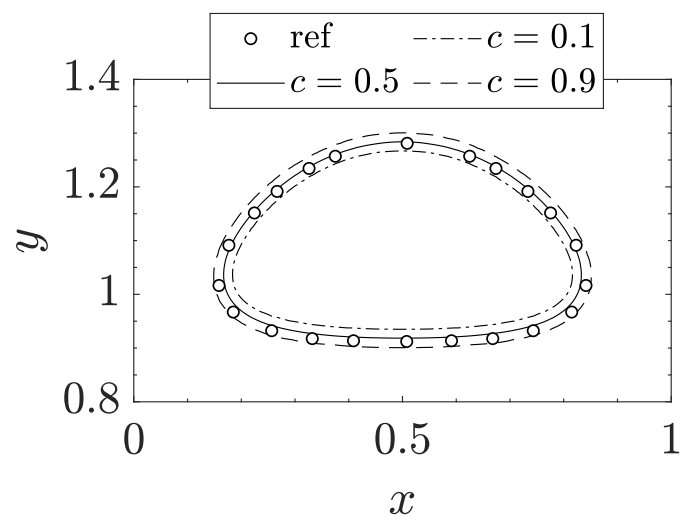

(a) Final shape of the bubble. We represent the interface with three contours: $c=0.1, c=$ 0.5 , and $c=1.0$. The dots represent the sharp interface reference Hysing et al. [2009]

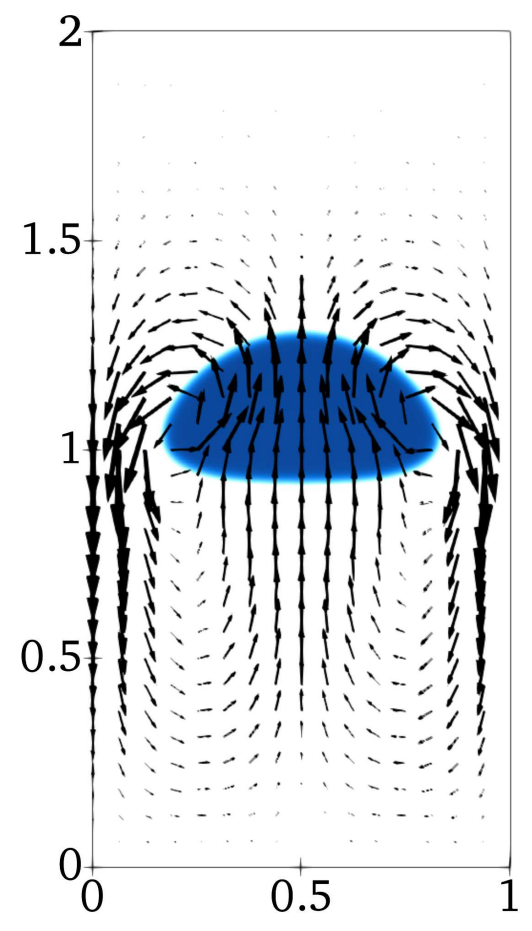

(b) Concentration contour at the final time $t=3$. The velocity field is also represented, showing the currents that raise the bubble

Figure 6.5: Rising bubble Test 1: position of the bubble and flow configuration at the final time $t=3.0$

Next, we solve the more challenging rising bubble Test 2 (see Table 6.6) with higher density $\left(\rho_{1} / \rho_{2}=1000\right)$ and viscosity $\left(\eta_{1} / \eta_{2}=100\right)$ ratios. The evolution of the bubble and the flow configuration are represented in Fig. 6.7 at each 0.5 second. Both the shape and position of the bubble are in agreement with the sharp interface method Hysing et al. [2009], and other diffuse interface Cahn-Hilliard solvers Hosseini et al. [2017]. Contrary to the rising bubble Test 1, the bubble now leaves behind an elongated skirt, which influences the velocity field.

For completeness, we represent the center of gravity position and rise velocity as a function of time in Fig. 6.8, compared to the sharp interface solution provided in Hysing et al. [2009]. Compared to the sharp interface reference, there are visible differences. However, it has been noted in Hysing et al. [2009]; Hosseini et al. [2017] that the solution with different sets of equations might differ for this more challenging test case. Nonetheless, the evolution of the center of gravity and the rise velocity presented here agree with other diffuse interface solvers Hosseini et al. [2017]. 


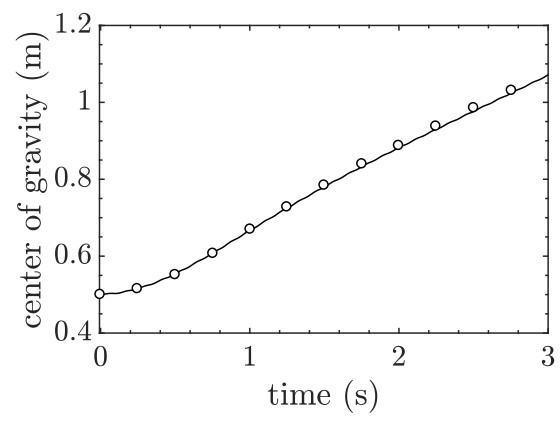

(a) Center of gravity

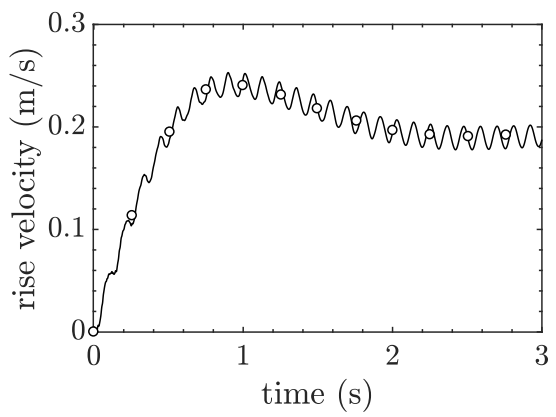

(b) Rise velocity

Figure 6.6: Rising bubble Test 1: evolution of the bubble center of gravity and rise velocity. The dots represent the reference solution from Hysing et al. [2009], while the solid line represents the solution obtained in this work

\subsubsection{Three-dimensional annular flow simulation}

The last numerical experiment we present in this chapter is a three-dimensional flow in a straight pipe $(L=10)$ with circular section $(D=1)$. Depending on the flow rates of fluid 1 and 2, the flow regime can be of different nature: stratified, slug, dispersed bubble, and annular. The last is the one we compute here, where one of the fluids behaves as a coating around the pipe surface. More details of the flow can be found in Taitel and Dukler [1976]; Manzanero et al. [2019].

We construct a z-oriented mesh of the pipe with 8200 elements, and use a polynomial order $N=3$ (for both solutions and physical boundary representation). Note that this is an unstructured curvilinear mesh. The cross section of the mesh is represented in Fig. 6.10(c). The physical parameters are taken from Taitel and Dukler [1976] and given in Table 6.7.

Table 6.7: Physical parameters of the annular flow

\begin{tabular}{|c|c|c|c|c|c|c|c|c|c|}
\hline$\rho_{1}$ & $\rho_{2}\left(\mathrm{~kg} / \mathrm{m}^{3}\right)$ & $\eta_{1}$ & $\eta_{2}(\mathrm{~Pa} \cdot \mathrm{s})$ & $\varepsilon(\mathrm{m})$ & $t_{C H}(\mathrm{~s})$ & $c_{0}^{2}\left(\mathrm{~m} / \mathrm{s}^{2}\right)^{2}$ & $\sigma(\mathrm{N} / \mathrm{m})$ & $g\left(\mathrm{~m} / \mathrm{s}^{2}\right)$ & $\theta_{w}(\mathrm{deg})$ \\
\hline 1.0 & 5.0 & $5 \cdot 10^{-3}$ & $10^{-2}$ & 0.0424 & 900.0 & $1.0 \mathrm{E} 3$ & $2.5 \cdot 10^{-4}$ & 1.0 & $45^{\circ}$ \\
\hline
\end{tabular}

For the inflow and outflow boundary conditions, we construct auxiliary ghost states,

$$
\mathbf{Q}^{i n}=\left(\begin{array}{c}
c_{i n} \\
\sqrt{\rho\left(c_{i n}\right)} \vec{u}_{i n} \\
p
\end{array}\right), \quad \mathbf{Q}^{\text {out }}=\left(\begin{array}{c}
c \\
\sqrt{\rho} \vec{u} \\
0
\end{array}\right)
$$




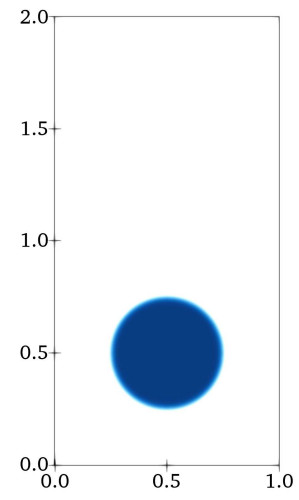

(a) $t=0 \mathrm{~s}$

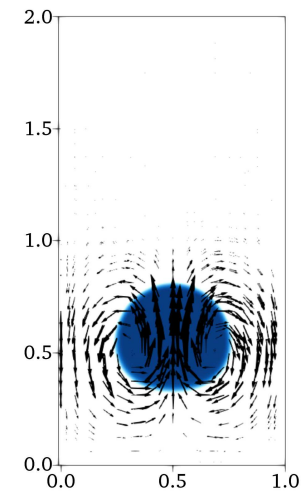

(b) $t=0.5 \mathrm{~s}$

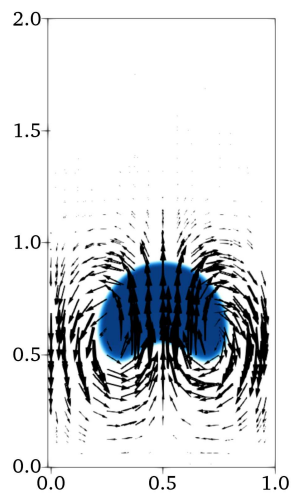

(c) $t=1.0 \mathrm{~s}$

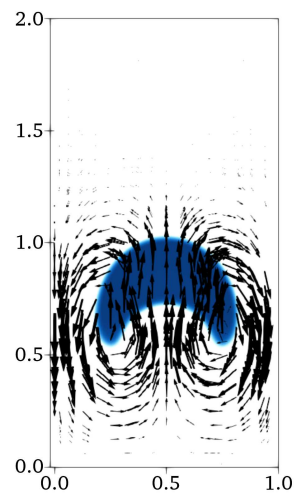

(d) $t=1.5 \mathrm{~s}$

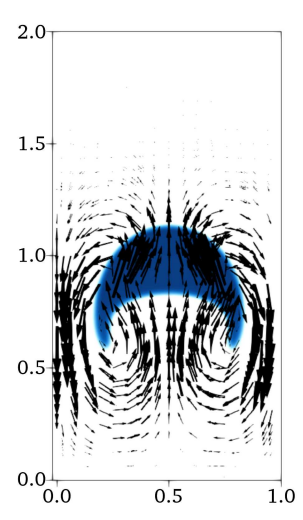

(e) $t=2.0 \mathrm{~s}$

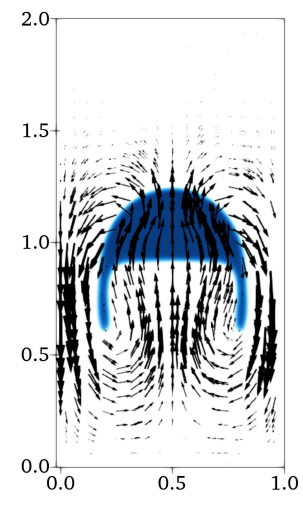

(f) $t=2.5 \mathrm{~s}$

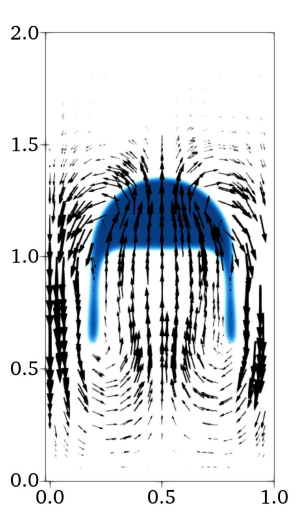

(g) $t=3.0 \mathrm{~s}$

Figure 6.7: Rising bubble Test 2: different snapshots showing the position of the bubble and the flow configuration

with

$$
\begin{aligned}
c_{i n} & =\frac{1}{2}+\frac{1}{2} \tanh \left(\frac{x-x_{0}}{\varepsilon}+\frac{1}{10} \sin (10 z)+\frac{1}{10} \sin (20 y)\right), \\
u_{i n} & =0 \\
v_{i n} & =0, \\
w_{i n} & =\left(V_{1} c_{i n}+V_{2}\left(1-c_{i n}\right)\right)\left(1-4 \frac{x^{2}+y^{2}}{D^{2}}\right), \\
p_{i n} & =0 .
\end{aligned}
$$

Furthermore, we use $\mathbf{Q}^{\text {in }}$ as the initial condition. The velocities $V_{1}$ and $V_{2}$, and interface position $x_{0}$ are set so that the superficial velocities, defined as

$$
V_{s 1}=\frac{1}{\pi R^{2}} \int_{\text {inlet }} V_{1} c_{i n}\left(1-4 \frac{x^{2}+y^{2}}{D^{2}}\right) \mathrm{d} S, V_{s 2}=\frac{1}{\pi R^{2}} \int_{\text {inlet }} V_{2}\left(1-c_{i n}\right)\left(1-4 \frac{x^{2}+y^{2}}{D^{2}}\right) \mathrm{d} S \text {, }
$$




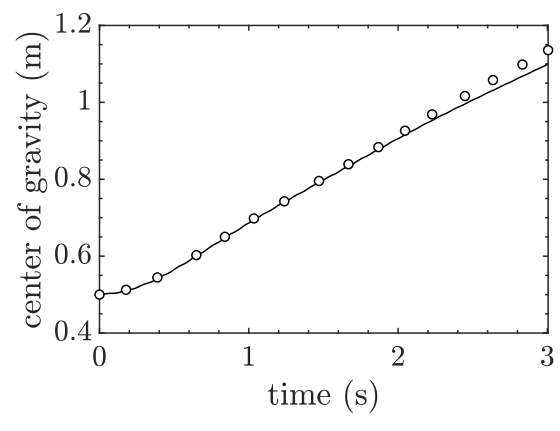

(a) Center of gravity

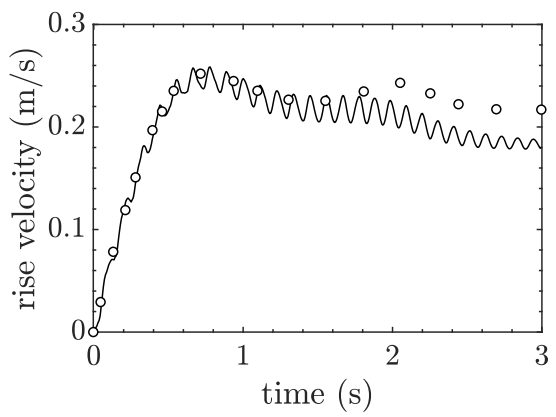

(b) Rise velocity

Figure 6.8: Rising bubble Test 2: evolution of the bubble center of gravity and rise velocity. The dots represent the reference solution from Hysing et al. [2009], while the solid line represents the solution obtained in this work

are those found in Taitel and Dukler [1976]. Precisely, we chose the velocities $V_{s 1}=4.9$ $\mathrm{m} / \mathrm{s}$ and $V_{s 2}=0.06 \mathrm{~m} / \mathrm{s}$, which produce the annular flow regime. If we place the interface between both fluids at $x_{0}=-0.262 \mathrm{~m}$ (which yields a slip velocity $V_{1}-$ $V_{2}=10 \mathrm{~m} / \mathrm{s}$ ), the inlet velocities are $V_{1}=11.06 \mathrm{~m} / \mathrm{s}$ and $V_{2}=1.06 \mathrm{~m} / \mathrm{s}$. More details on inflow configurations for multiphase flows can be found in Chapter 8 of this work. At the walls, we also impose a contact angle $\theta_{w}=45^{\circ}$ (see Fig. 7.1). With this configuration, we can use the third order Runge-Kutta scheme as time integrator with $\Delta t=10^{-5}$. In this simulation, we take into account the effect of gravity, which points in the negative $x$-direction.

The annular flow configuration is reached through an unstable mode, which makes the transient problem under-resolved until this mode is non-linearly damped and the final annular flow configuration is found. For that reason, we use a non-zero penalty parameter, $\beta_{c}$, on the Cahn-Hilliard equation. Precisely, we use the definition (5.23) with $\kappa_{\beta c}=1$. Otherwise, we have found the scheme to be stable, but the solution is mostly noise (i.e. it is not accurate). The introduction of the penalty parameter enhances the accuracy when the flow is under-resolved Ferrer [2017].

We represent snapshots of the initial stages of the flow in Fig. 6.9. The fluid 2 is represented $(c \leqslant 0.5)$, and colored by the vertical velocity (along the $x$-axis, $u$ ). We see that in the initial steps there is an unstable mode that grows until it changes the flow configuration. The vertical velocity contour shows that when fluid 2 rises above the interface it is dragged and accelerated by the quicker fluid 1, and the contrary occurs when it is confined below the interface because of the wall. This produces a wavy pattern at $t=0.3 \mathrm{~s}$, which eventually wraps the fluid to the wall and produces 
the annular flow. In the early stages, the flow is highly under-resolved.

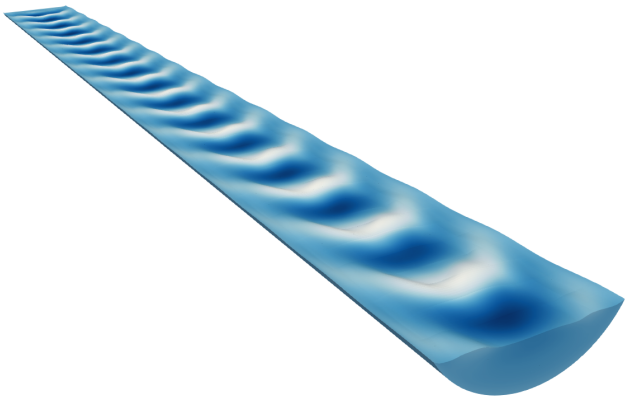

(a) $t=0.1 \mathrm{~s}$

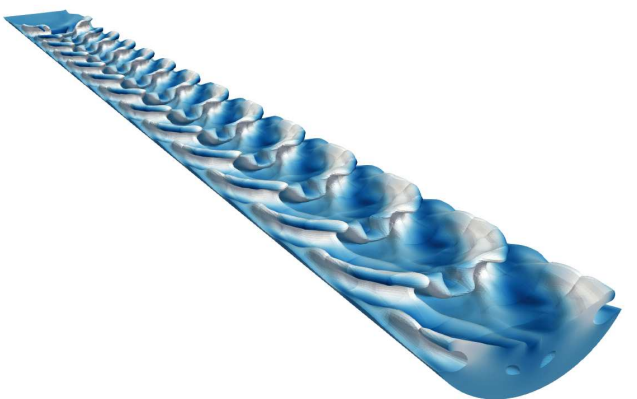

(c) $t=0.5 \mathrm{~s}$

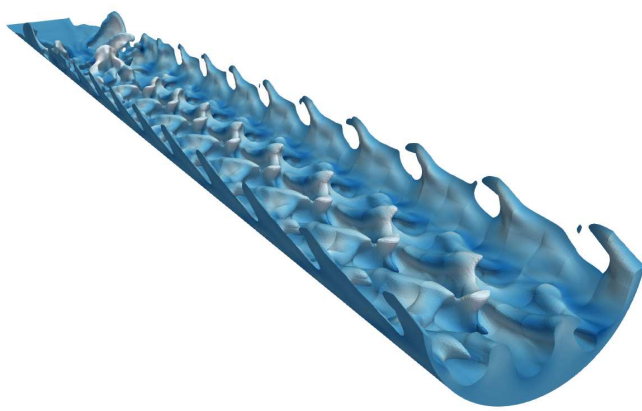

(e) $t=0.9 \mathrm{~s}$

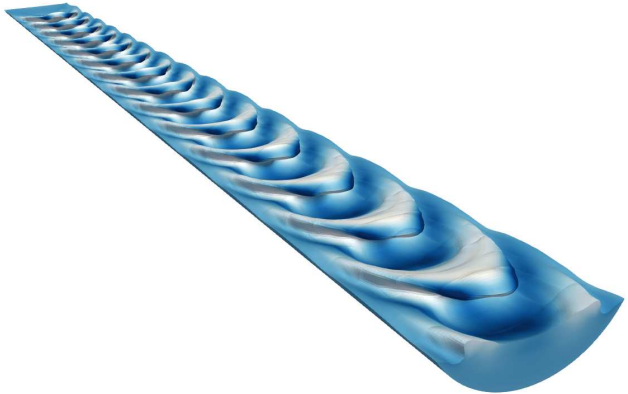

(b) $t=0.3 \mathrm{~s}$

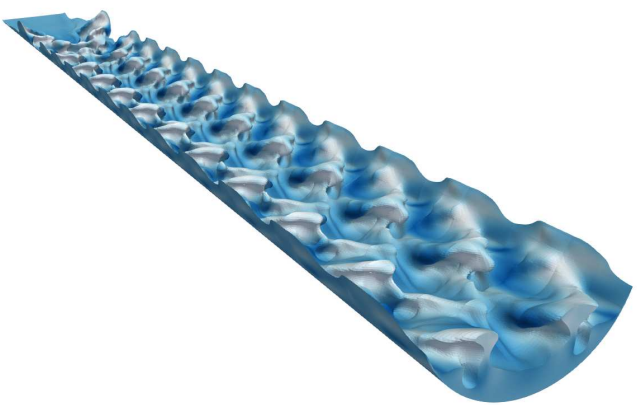

(d) $t=0.7 \mathrm{~s}$

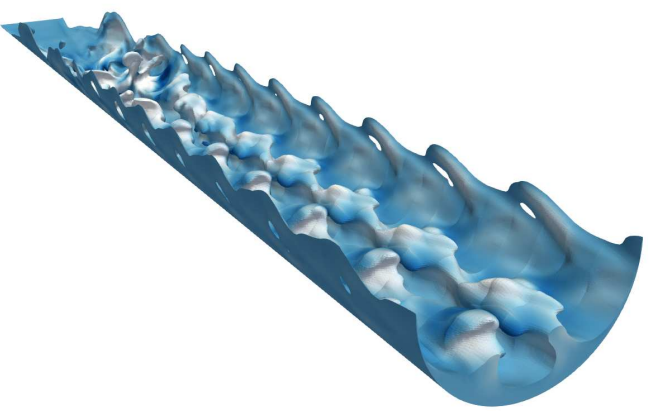

(f) $t=1.1 \mathrm{~s}$

Figure 6.9: Evolution of the physical mode that configures the annular flow. We have represented fluid 2 (i.e. $c \leqslant 0.5$ ), colored with vertical velocity, $u$. We see that during early times, there is an unstable physical mode that triggers the annular flow. When fluid 2 rises above the interface, it is dragged and accelerated by the quicker fluid 1. When it is confined below the interface, it is slowed down by the wall. This evolves non-linearly to the final state

We represent a final snapshot for $t=4.0 \mathrm{~s}$ in Fig. 6.10, showing the contour $c \leqslant 0.5$ 
(i.e. the region occupied by fluid 2), colored by the longitudinal velocity (i.e. along the $z$-axis, $w$ ). Although the interface is flat at the inlet, the flow is unstable and breaks

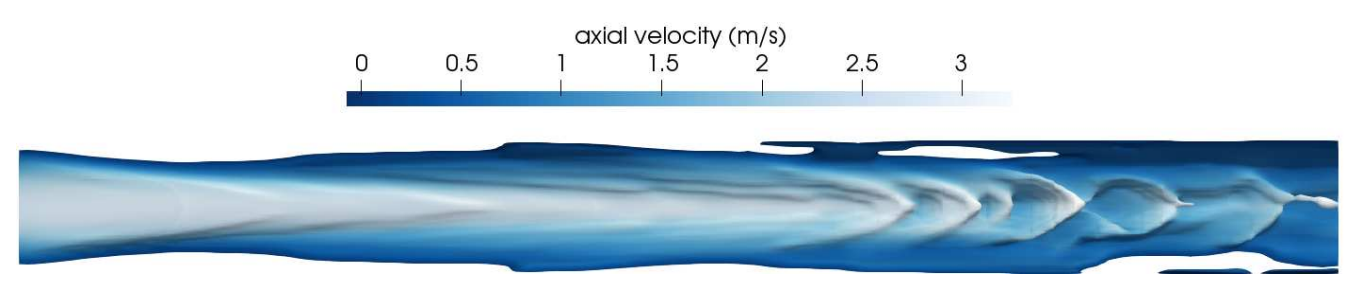

(a) Top view

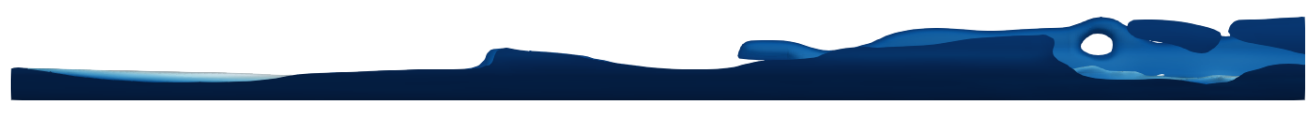

(b) Side view

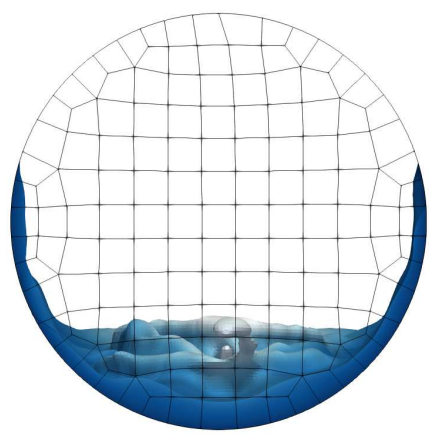

(c) Front view

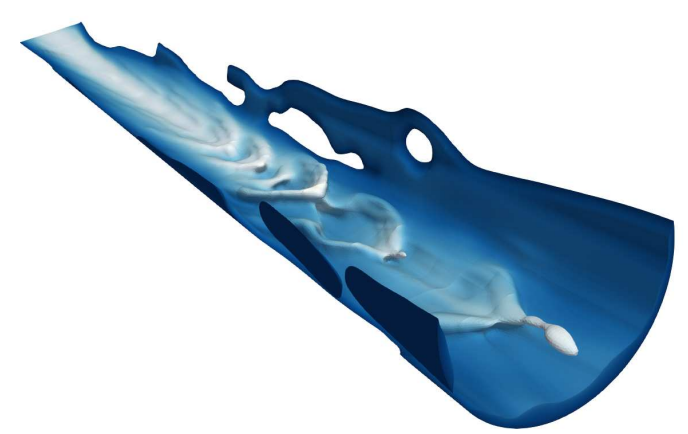

(d) Three dimensional view

Figure 6.10: Annular flow simulation. We present four view angles to the simulation at $t=4.0 \mathrm{~s}$. In the front view, the mesh cross section is also represented

down into the annular flow configuration. The heavy fluid (fluid 2) is confined to the wall by the light fluid, which is introduced 82 times faster at the inlet. In Fig. 6.10(c) we represent the front view of the pipe, with the mesh on top of it. We see in Fig. 6.10 that the flow gets smoother after the numerical and physical dissipation take over the mode breakdown after the under-resolved stages.

\subsection{Summary and conclusions}

We have derived a two-phase flow model that combines the Cahn-Hilliard equation (6.3), a skew-symmetric version of the momentum equation (6.14), and an artificial compressibility method (6.16) to get the pressure. Among the many available options, the versions were chosen to satisfy an entropy inequality, (6.48). The entropy 
inequality bounds the mathematical entropy inside a finite domain $\Omega$ limited with wall boundary conditions by the entropy of the initial condition. As time marches, the mathematical entropy decreases as a result of the physical and the chemical potential dissipation.

We then constructed a DG approximation that satisfies the SBP-SAT property, which allowed us to mimic the continuous entropy analysis discretely. As is usual in a DG approximation, we had choices for the fluxes at inter-element and physical boundaries. We studied two options:

1. Entropy conserving, using central fluxes for the advective fluxes and the BR1 scheme for the diffusion. This choice gives a bound that discretely mimics the continuous entropy bound (6.48). This scheme is not of practical use, since some amount of numerical dissipation is required to provide accurate solutions when non-linear terms are present, Gassner et al. [2016a]; Manzanero et al. [2020d]; Flad and Gassner [2017]; Manzanero et al. [2018c]. However, it serves as a baseline model to verify the stability proofs and to construct a dissipation-free scheme.

2. Entropy-stable, with an exact Riemann solver for the advective fluxes and the BR1 scheme for diffusive, with additional interface dissipation for the CahnHilliard equation. This scheme transforms the entropy balance to an entropy inequality in the discrete version of (6.48), as a result of the numerical dissipation at the inter-element faces. This scheme uses the exact Riemann solver presented in Bassi et al. [2017] for the incompressible Navier-Stokes equations, with an appropriate choice of the diamond fluxes that arise from the non-conservative terms, and a modification of the discrete entropy to account for solution discontinuities in the concentration as interfacial energy in the numerical solution.

The scheme is designed for and the stability proofs hold for three-dimensional unstructured meshes with hexahedral curvilinear elements.

We selected two choices to march the scheme in time: an explicit third-order Runge-Kutta method, and an implicit-explicit BDF with first or second order or accuracy. The former is used when the mobility is small enough to not severely restrict the time-step size, and the latter otherwise.

We test the scheme, addressing its accuracy with a manufactured solution convergence analysis, and its robustness by initializing the flow with random initial conditions. We showed that the scheme converges as expected, and that the scheme is robust in the sense that none of one hundred simulations crashed from random initial conditions, a high density ratio $\left(\rho_{2} / \rho_{1}=1000\right)$, and a high Reynolds number 
$\left(\operatorname{Re}=10^{6}\right)$. We compared the entropy-stable scheme with the more accurate Gauss counterpart (which is not provably entropy stable) to find that the latter crashes in $30 \%$ of the simulations. We also solved commonly used static and rising bubble test problems to assess the steady state and transient accuracy of the solver. Finally we challenged the method by solving a three-dimensional pipe flow in the annular regime. 



\section{Part III}

\section{Development of a three-phase solver for industrial applications}

Summary Three-phase flows can be found in the nature (e.g. natural gas, oil, and water that coexist in crude reservoirs), nuclear plants (e.g. the reactivity initiated accident, which involves liquid water and its vapor combined with hot fission gases), industrial processes (e.g. froth flotation), and chemical engineering plants (e.g. the oil and gas industry, and immiscible condensate-vapor flows in steam/hydrocarbon condensing systems).

In this part we develop a three-phase incompressible Navier-Stokes/Cahn-Hilliard model to solve three-phase flows. The solver is then applied to solve oil transport problems, such as those found in the oil and gas industry. Precisely, this solver has been developed for the oil and gas company Repsol, during a one-year project. The threephase model adopted in this work is a Cahn-Hilliard diffuse interface model, which was derived in Boyer and Lapuerta [2006]. The Cahn-Hilliard model is coupled to the entropy-stable incompressible Navier-Stokes equations model derived in Chapter 4 of this work.

As a result of parasitic terms that depend on the divergence of the velocity field, the combined solver is not entropy-stable. A study, which has not been performed herein, similar to that performed in Chapter 6 to modify the current model appropriately and construct an entropy-stable model, should follow. Nevertheless, we have found that the DG scheme, with the split-form scheme derived in Chapter 4 is more robust than the standard scheme for the solution of three-phase flows.

This part is organized as follows: in Chapter 7, we present a novel DGSEM scheme for the three-phase Cahn-Hilliard model of Boyer and Lapuerta [2006] (i.e. without the flow dynamics), and in Chapter 8 we present the three-phase DGSEM multiphase solver that has been used for industrial applications. 



\section{Chapter 7}

\section{Discontinuous Galerkin approximation of a three-phase Cahn-Hilliard model}

Contents

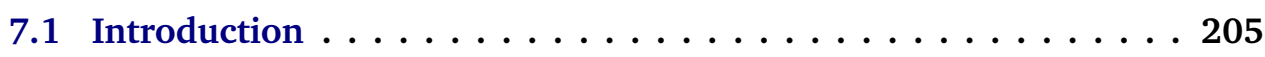

7.2 Model description . . . . . . . . . . . . 206

7.3 Discontinuous Galerkin approximation . . . . . . . . 211

7.4 Numerical experiments . . . . . . . . . . . . . . . 214

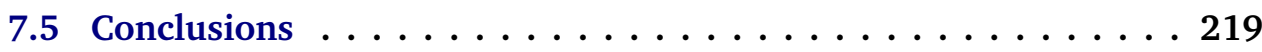

\subsection{Introduction}

In this chapter we study the dynamics of three dissimilar coexisting phases, e.g. oil water and air. As in the rest of this work, we use a Cahn-Hilliard phase field model. The Cahn-Hilliard model for two-phase flows is the simpler, and it has been widely studied in the past Manzanero et al. [2020b,c]; Shen and Yang [2010]; Xia et al. [2009], and also in Chapters 5 and 6 of this work.

Extending the two-phase model to three phases is not immediate, since new physical considerations should be taken into account: the model has to be consistent, i.e., if one of the three phases is not present initially, it cannot show up at later times. Furthermore, it is desirable that the model reduces to the two-phase Cahn-Hilliard model when one of the phases is not present. In this chapter, we use the model presented in Boyer and Lapuerta [2006], which solves the consistency problem by considering 
a particular choice for the chemical free-energy. A more general approach, which enables the consistent simulation of $N$-phases flows, was developed in Dong [2018a, 2014b]. The author addressed the consistency of the model using a non-constant degenerate mobility.

The three-phase model is augmented with the boundary condition developed in Shi and Wang [2014], which permits the prescription of the contact angle between the phases and the wall by solving a non-homogeneous Neumann boundary condition.

The three-phase Cahn-Hilliard flow model has been discretized by means of the finite element method Boyer and Minjeaud [2011], local discontinuous Galerkin method Xia et al. [2007] or spectral element method Dong [2014b], and in this work we provide a DGSEM approximation. To the best of our knowledge this is the first implementation of the three-phase Cahn-Hilliard flow model in a discontinuous Galerkin Spectral Element method framework.

Finally, we use a first order IMplicit-EXplicit (IMEX) time integrator. We use an IMEX method since the Cahn-Hilliard equation features a linear fourth order spatial operator (which is solved implicitly), and a non-linear second order spatial operator (which is solved explicitly). Therefore the solution of the fully-discrete system requires the solution of one linear system for each of the Cahn-Hilliard equations. The two linear systems, however, are decoupled so that the Jacobian matrices are constant in time and identical for both Cahn-Hilliard equations. This method is efficient as only one LU factorization is performed for the two equations.

The rest of this chapter is organized as follows: in Sec. 7.2 we describe the threephase Cahn-Hilliard model. The construction of the discrete DG approximation is described in Sec. 7.3. Lastly, we provide numerical experiments in Sec. 7.4 that assess the accuracy of the method. Final conclusions and discussions can be found in Sec. 7.5.

\subsection{Model description}

The phase field approach to multiphase flows introduces one scalar field $c_{j}$ per fluid, which represents the relative concentration of phase $j$ at each space-time point. The phase conservation condition, then, reads as

$$
\sum_{j=1}^{N_{\text {phases }}} c_{j}=1 .
$$

In this chapter, we restrict ourselves to three phase flows, $N_{\text {phases }}=3$.

We revisit in this section the three-phase model derived in Boyer and Lapuerta 
[2006]. As usual in phase field methods, each of the concentration fields is subjected to a Cahn-Hilliard diffusion equation. The Cahn-Hilliard equation evolves the concentrations to minimize a free-energy. In Boyer and Lapuerta [2006], the free-energy function is

$$
\mathscr{\mathscr { F }}_{\varepsilon}^{[\boldsymbol{\sigma}]}(\mathbf{c}, \vec{\nabla} \mathbf{c})=\int_{\Omega}\left(\frac{12}{\varepsilon} F_{0}^{[\boldsymbol{\sigma}]}(\mathbf{c})-\frac{3}{8} \varepsilon(\vec{\nabla} \mathbf{c})^{T} \overline{\overline{\boldsymbol{\sigma}}} \vec{\nabla} \mathbf{c}\right) \mathrm{d} \mathbf{x} .
$$

The free-energy contains two terms. The first term contains the chemical free-energy, $F_{0}^{[\sigma]}$, which in this model is a polynomial function of the three concentrations $\mathbf{c}=$ $\left(c_{1}, c_{2}, c_{3}\right)$. The second term is the interfacial energy, where $\overline{\bar{\sigma}}$ is the symmetric interface tension coefficients tensor, whose entries are the surface tension coefficients of all the possible interfaces, $\sigma_{i j}$,

$$
\overline{\overline{\boldsymbol{\sigma}}}=\left(\begin{array}{ccc}
0 & \sigma_{12} & \sigma_{13} \\
\sigma_{12} & 0 & \sigma_{23} \\
\sigma_{13} & \sigma_{23} & 0
\end{array}\right)
$$

The interface tension coefficients $\sigma_{i j}$ are positive constants. Finally, $\varepsilon$ is the interface width parameter, which controls the thickness of the diffuse interfaces.

The Cahn-Hilliard equation is constructed so that the concentration time derivatives are proportional to the Laplacian of additional scalar fields called chemical potentials, $\boldsymbol{\mu}=\left(\mu_{1}, \mu_{2}, \mu_{3}\right)$,

$$
c_{i, t}=\frac{M_{0}}{\Sigma_{i}} \vec{\nabla}^{2} \mu_{i}, \quad \Sigma_{i}=\sigma_{i j}+\sigma_{i k}-\sigma_{j k}
$$

where the positive constant $M_{0}$ is the mobility, and the $\Sigma_{i}$ are constant coefficients called spreading factors. Although the authors in Boyer and Lapuerta [2006] show an extension that allows negative spreading factors, this chapter only considers positive spreading factors.

For the three-phase model, the chemical potentials are defined as

$$
\mu_{i}=\frac{4 \Sigma_{T}}{\varepsilon} \sum_{\substack{j=1 \\ j \neq i}}^{3}\left(\frac{1}{\Sigma_{j}}\left[\frac{\partial F_{0}^{\sigma}}{\partial c_{i}}-\frac{\partial F_{0}^{\sigma}}{\partial c_{j}}\right]\right)-\frac{3}{4} \varepsilon \Sigma_{i} \vec{\nabla}^{2} c_{i}, \frac{3}{\Sigma_{T}}=\frac{1}{\Sigma_{1}}+\frac{1}{\Sigma_{2}}+\frac{1}{\Sigma_{3}} .
$$

For simplicity, we define $f_{i}$ as

$$
f_{i}=\frac{\Sigma_{T}}{3 \Sigma_{i}} \sum_{\substack{j=1 \\ j \neq i}}^{3}\left(\frac{1}{\Sigma_{j}}\left[\frac{\partial F_{0}^{\boldsymbol{\sigma}}}{\partial c_{i}}-\frac{\partial F_{0}^{\boldsymbol{\sigma}}}{\partial c_{j}}\right]\right), i=1,2,3
$$

to write the chemical potential as $\mu_{i}=\frac{12}{\varepsilon} \Sigma_{i} f_{i}-\frac{3}{4} \varepsilon \Sigma_{i} \vec{\nabla}^{2} c_{i}$.

The model is completed with the definition of the chemical free-energy $F_{0}^{[\sigma]}$. In Boyer and Lapuerta [2006] the free energy is defined to satisfy: 
Property 3 Conservative model. If the initial condition satisfies $c_{1}(\mathbf{x}, 0)+c_{2}(\mathbf{x}, 0)+$ $c_{3}(\mathbf{x}, 0)=1$, then, for all further times a conservative model maintains

$$
c_{1}(\mathbf{x}, t)+c_{2}(\mathbf{x}, t)+c_{3}(\mathbf{x}, t)=1, \quad \forall t \geqslant 0 .
$$

This property is ensured by an appropriate construction of the chemical potentials (7.5). We sum the three Cahn-Hilliard equations to find that

$$
\left(c_{1}+c_{2}+c_{3}\right)_{t}=M_{0} \vec{\nabla}^{2}\left(\frac{\mu_{1}}{\Sigma_{1}}+\frac{\mu_{2}}{\Sigma_{2}}+\frac{\mu_{3}}{\Sigma_{3}}\right)=0 .
$$

Therefore, it suffices that the chemical potentials satisfy

$$
\frac{\mu_{1}}{\Sigma_{1}}+\frac{\mu_{2}}{\Sigma_{2}}+\frac{\mu_{3}}{\Sigma_{3}}=0
$$

which they do by construction. The relation (7.9) allows us to compute the chemical potential of one of the phases (typically the third phase) as a function of the other twophase,

$$
\mu_{3}=-\frac{\Sigma_{3}}{\Sigma_{1}} \mu_{1}-\frac{\Sigma_{3}}{\Sigma_{2}} \mu_{2}
$$

Property 4 Consistent model. If in the initial condition $c_{j}(\mathbf{x}, 0)=0$, then

$$
c_{j}(\mathbf{x}, t)=0, \quad \forall t \geqslant 0 .
$$

As shown in Boyer and Lapuerta [2006], this property is guaranteed with the chemical potential construction (7.5), and an appropriate choice of the chemical free-energy. The chemical potential must satisfy

$$
\left.\mu_{j}\right|_{\substack{c_{j}=0 \\ c_{i}+c_{k}=1}}=0 .
$$

We are left with two properties to be satisfied by the chemical free-energy to construct a consistent model. First, the chemical free-energy of a two-phase system (when one of the phases is not present in the flow, e.g. phase three) is

$$
F_{0}^{[\sigma]}\left(c_{1}, c_{2}, 0\right)=F_{0}^{\sigma_{12}}\left(c_{1}, c_{2}\right)=\sigma_{12} c_{1}^{2} c_{2}^{2} .
$$

A consistent chemical free-energy must satisfy the fact that if one of the phases is not present, the resulting chemical free-energy is equivalent to that of a two-phase flow for the other two fluids. A natural approach that satisfies this property is to add the chemical free-energies for the three possible pairs,

$$
\begin{aligned}
F_{0, N C}^{\sigma} & =F_{0}^{\sigma_{12}}\left(c_{1}, c_{2}\right)+F_{0}^{\sigma_{13}}\left(c_{1}, c_{3}\right)+F_{0}^{\sigma_{23}}\left(c_{2}, c_{3}\right) \\
& =\sigma_{12} c_{1}^{2} c_{2}^{2}+\sigma_{13} c_{1}^{2} c_{3}^{2}+\sigma_{23} c_{2}^{2} c_{3}^{2} .
\end{aligned}
$$


Second, the reduction to a two-phase chemical free-energy is not enough to guarantee the consistency of the model. If $c_{3}(\mathbf{x}, 0)=0$, then, to ensure $c_{3, t}=0$, the chemical potential $\mu_{3}$ must be algebraically zero. This implies that the chemical free-energy must satisfy that

$$
\left(\frac{1}{\Sigma_{1}}+\frac{1}{\Sigma_{2}}\right) \frac{\partial F_{0}^{\sigma}}{\partial c_{3}}-\frac{1}{\Sigma_{1}} \frac{\partial F_{0}^{\sigma}}{\partial c_{1}}-\frac{1}{\Sigma_{2}} \frac{\partial F_{0}^{\sigma}}{\partial c_{2}}=0 \text {, if } c_{3}=0 .
$$

Eq. (7.15) is automatically satisfied if an additional term is added to the non-consistent chemical free-energy (7.14),

$$
\begin{aligned}
F_{0}^{[\sigma]} & =F_{0, N C}^{[\sigma]}+c_{1} c_{2} c_{3}\left(\Sigma_{1} c_{1}+\Sigma_{2} c_{2}+\Sigma_{3} c_{3}\right) \\
& =\sigma_{12} c_{1}^{2} c_{2}^{2}+\sigma_{13} c_{1}^{2} c_{3}^{2}+\sigma_{23} c_{2}^{2} c_{3}^{2}+c_{1} c_{2} c_{3}\left(\Sigma_{1} c_{1}+\Sigma_{2} c_{2}+\Sigma_{3} c_{3}\right) .
\end{aligned}
$$

The chemical free-energy (7.16) completes the three-phase model. Note that the additional term also cancels when one of the phases is not present, and the chemical free-energy still reduces to that of a two-phase model (7.13). As a result, one CahnHilliard equation (7.4) per phase is solved, with the chemical potential defined in (7.5) and the chemical free-energy in (7.16). In practice, since (7.7) holds, we only solve two Cahn-Hilliard equations (7.4), and the concentration of the third phase is computed from the constraint (7.1), $c_{3}=1-c_{1}-c_{2}$. Its associated chemical potential, if needed, is also computed from the other two-phase using (7.10).

Finally, to borrow the DG scheme directly from Chapter 2, we write a block vector representation of (7.4). The number of equations is $N_{E}=2$, and the state vector contains the two concentrations $\mathbf{c}=\left(c_{1}, c_{2}\right)$. We define the chemical potential (divided by the spreading factors) vector $\mu_{\Sigma}=\left(\mu_{1} / \Sigma_{1}, \mu_{2} / \Sigma_{2}\right)$, and the vector that contains the chemical free-energy derivatives $\mathbf{f}=\left(f_{1}, f_{2}\right)$. Thus, we pack the Cahn-Hilliard equations as

$$
\mathbf{c}_{t}=\vec{\nabla} \cdot\left(M_{0} \vec{\nabla} \mu_{\Sigma}\right),
$$

and the definition of the chemical potentials as

$$
\mu_{\Sigma}=\frac{12}{\varepsilon} \mathbf{f}-\vec{\nabla} \cdot\left(\frac{3}{4} \varepsilon \vec{\nabla} \mathbf{c}\right)
$$

\subsubsection{Boundary conditions}

The Cahn-Hilliard equation is complemented with an appropriate choice of the boundary conditions. Here we adopt a wall boundary condition model with non-zero contact angle for the three-phase Cahn-Hilliard equations. 
Since the equation is fourth order in space, two boundary conditions must be specified, for both the chemical potentials and the concentration Cahn and Hilliard [1958, 1959]. To guarantee the phase conservation, we enforce a homogeneous Neumann boundary condition for the chemical potential,

$$
\left.\vec{\nabla} \mu_{i} \cdot \vec{n}\right|_{\partial \Omega}=0 .
$$

For two-phase flows, a wall boundary condition with contact angle can be found in Dong [2012], which was used in Chapter 6 of this work. The latter is achieved with a non-homogeneous Neumann boundary condition for the concentration,

$$
\begin{aligned}
& \left.\vec{\nabla} c_{1} \cdot \vec{n}\right|_{\partial \Omega}=-\frac{4}{\varepsilon} \cos \theta_{12}^{w} c_{1} c_{2}, \\
& \left.\vec{\nabla} c_{2} \cdot \vec{n}\right|_{\partial \Omega}=-\frac{4}{\varepsilon} \cos \theta_{21}^{w} c_{1} c_{2}=\frac{4}{\varepsilon} \cos \theta_{12}^{w} c_{1} c_{2} .
\end{aligned}
$$

The contact angle convention is represented in Fig. 7.1, where $\theta_{i j}^{w}$ represents the angle

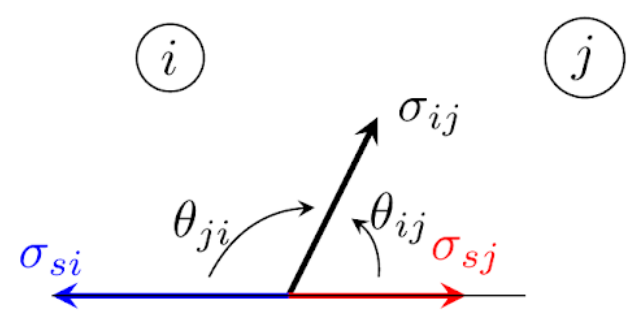

Figure 7.1: Convention used to designate the wall contact angle with respect to twophase $i$ and $j$. Note that $\theta_{i j}^{w}+\theta_{j i}^{w}=\pi$

of an interface between fluids $i$ and $j$ with fluid $j$, and $\theta_{j i}^{w}$ is that with fluid $i$. The relation between both angles that was applied in (7.20) is $\theta_{i j}^{w}+\theta_{j i}^{w}=\pi$. The stress equilibrium at the wall contact point implies that

$$
\sigma_{s i}=\sigma_{s j}+\sigma_{i j} \cos \theta_{i j}^{w}
$$

For three-phase flows, one cannot freely choose the three contact angles with the wall, since a constraint relates them. We write the three wall stress equilibrium equations as

$$
\begin{aligned}
& \sigma_{s 1}=\sigma_{s 2}+\sigma_{12} \cos \theta_{12}^{w}, \\
& \sigma_{s 1}=\sigma_{s 3}+\sigma_{13} \cos \theta_{13}^{w}, \\
& \sigma_{s 2}=\sigma_{s 3}+\sigma_{23} \cos \theta_{23}^{w} .
\end{aligned}
$$


Now we subtract the second equation from the first, and then add the third equation to the result, to find that wall contact angles and interface tension coefficients are constrained by the relation

$$
\sigma_{12} \cos \theta_{12}^{w}+\sigma_{23} \cos \theta_{23}^{w}=\sigma_{13} \cos \theta_{13}^{w}
$$

Lastly, in Shi and Wang [2014]; Dong [2017], the non-homogeneous Neumann boundary condition was extended to three phases, preserving the consistency property by

$$
\begin{aligned}
& \left.\vec{\nabla} c_{1} \cdot \vec{n}\right|_{\partial \Omega}=-\frac{4}{\varepsilon}\left[\cos \theta_{12}^{w} c_{1} c_{2}\left(c_{1}+c_{2}\right)+\cos \theta_{13}^{w} c_{1} c_{3}\left(c_{1}+c_{3}\right)\right]=f_{w, 1}, \\
& \left.\vec{\nabla} c_{2} \cdot \vec{n}\right|_{\partial \Omega}=-\frac{4}{\varepsilon}\left[-\cos \theta_{12}^{w} c_{1} c_{2}\left(c_{1}+c_{2}\right)+\cos \theta_{23}^{w} c_{2} c_{3}\left(c_{2}+c_{3}\right)\right]=f_{w, 2} \\
& \left.\vec{\nabla} c_{3} \cdot \vec{n}\right|_{\partial \Omega}=\frac{4}{\varepsilon}\left[\cos \theta_{13}^{w} c_{1} c_{3}\left(c_{1}+c_{3}\right)+\cos \theta_{23}^{w} c_{2} c_{3}\left(c_{2}+c_{3}\right)\right]=f_{w, 3} .
\end{aligned}
$$

Eq. (7.24) allows us to conveniently set the wall equilibrium contact angles. We write the block vector form of (7.24) with the definition $\overrightarrow{\mathbf{f}}_{w}=\left(f_{w, 1}, f_{w, 2}\right)$,

$$
\left.\vec{\nabla} \mathbf{c} \cdot \vec{n}\right|_{\partial \Omega}=\mathbf{f}_{w} .
$$

\subsection{Discontinuous Galerkin approximation}

We construct the DG approximation of the Cahn-Hilliard equation using the standard DG scheme for conservation laws (see Sec. 2.5). Thus, we adapt (2.66) for the CahnHilliard equations (7.17), with $\mathbf{Q}=\mathbf{C}, \overrightarrow{\mathbf{F}}_{e}=0$, and $\overrightarrow{\mathbf{F}}_{v}=M_{0} \overrightarrow{\mathbf{G}}$, and $\mathbf{W}=\mu_{\Sigma}$,

$$
\begin{aligned}
& \left\langle\mathscr{J} \mathbf{C}_{t}, \boldsymbol{\phi}\right\rangle_{E, N}=\int_{\partial E, N} \phi^{T}\left(M_{0} \overrightarrow{\tilde{\mathbf{G}}}^{\star}\right) \cdot \hat{n} \mathrm{~d} \hat{S}-\left\langle M_{0} \overrightarrow{\tilde{\mathbf{G}}}, \vec{\nabla}_{\xi} \boldsymbol{\phi}\right\rangle_{E, N}, \\
& \langle\mathscr{J} \overrightarrow{\mathbf{G}}, \vec{\varphi}\rangle_{E, N}=\int_{\partial E, N} \mu_{\Sigma}^{\star T} \overrightarrow{\tilde{\varphi}} \cdot \hat{n} \mathrm{~d} \hat{S}-\left\langle\mu_{\Sigma}, \vec{\nabla}_{\xi} \cdot \overrightarrow{\tilde{\varphi}}\right\rangle_{E, N} .
\end{aligned}
$$

Next, the definition of the chemical potential (7.18) is also discretized following the scheme of the general conservation law (2.66). The equation does not involve a time derivative $(\mathbf{Q}=0)$, and we treat the chemical potential as a source term, $\mathbf{s}=\frac{12}{\varepsilon} \mathbf{f}-\boldsymbol{\mu}_{\Sigma}$. The inviscid fluxes are $\overrightarrow{\mathbf{F}}_{e}=0$, and the viscous fluxes are $\overrightarrow{\mathbf{F}}_{v}=-\frac{3}{4} \varepsilon \overrightarrow{\mathbf{G}}_{c}$, with the entropy 
variables, $\mathbf{W}=\mathbf{C}$,

$$
\begin{aligned}
\left\langle\mathscr{J} \boldsymbol{\mu}_{\Sigma}, \boldsymbol{\phi}_{\mu}\right\rangle_{E, N} & =\left\langle\mathscr{J} \frac{12}{\varepsilon} \mathbf{F}, \boldsymbol{\phi}_{\mu}\right\rangle_{E, N}-\frac{3}{4} \varepsilon \int_{\partial E, N} \boldsymbol{\phi}_{\mu}^{T}\left(\overrightarrow{\tilde{\mathbf{G}}}_{c}^{\star}\right) \cdot \hat{n} \mathrm{~d} \hat{S}+\frac{3}{4} \varepsilon\left\langle\overrightarrow{\tilde{\mathbf{G}}}_{c}, \vec{\nabla}_{\xi} \boldsymbol{\phi}_{\mu}\right\rangle_{E, N}, \\
\left\langle\mathscr{J} \overrightarrow{\mathbf{G}}_{c}, \vec{\varphi}_{G_{c}}\right\rangle_{E, N} & =\int_{\partial E, N} \mathbf{C}^{\star, T} \overrightarrow{\tilde{\varphi}}_{G_{c}} \cdot \hat{n} \mathrm{~d} \hat{S}-\left\langle\mathbf{C}, \vec{\nabla}_{\xi} \cdot \overrightarrow{\tilde{\varphi}}_{G_{c}}\right\rangle_{E, N} \cdot
\end{aligned}
$$

The inter-element solution and fluxes are responsible for coupling adjacent elements and the enforcement of boundary conditions at the physical boundaries. In this chapter, we use the symmetric interior penalty method (IP),

$$
\begin{array}{rlrl}
\mathbf{C}^{\star} & =\{\{\mathbf{C}\}\}, & \overrightarrow{\mathbf{G}}_{c}^{\star}=\{\{\vec{\nabla} \mathbf{C}\}\}-\sigma\left(\mathbf{C}_{L} \vec{n}_{L}+\mathbf{C}_{R} \vec{n}_{R}\right), \\
\mu_{\Sigma}^{\star}=\left\{\left\{\boldsymbol{\mu}_{\Sigma}\right\}\right\}, & \overrightarrow{\mathbf{G}}^{\star}=\left\{\left\{\vec{\nabla} \boldsymbol{\mu}_{\Sigma}\right\}\right\}-\sigma\left(\boldsymbol{\mu}_{\Sigma, L} \vec{n}_{L}+\boldsymbol{\mu}_{\Sigma, R} \vec{n}_{R}\right),
\end{array}
$$

where the gradients are locally computed using (5.7), and $\sigma$ is the penalty parameter computed with the estimation by Shahbazi [2005],

$$
\sigma=\frac{(N+1)(N+2)}{2} \max \left(\left|\mathscr{J}_{f}\right|\left\{\left\{\mathscr{J}^{-1}\right\}\right\}\right)
$$

We arrive at the evolution equation for the coefficients by replacing the test functions $\phi$ and $\vec{\varphi}$ with the Lagrange polynomials $l_{i}(\xi) l_{j}(\eta) l_{k}(\zeta)$ as in Sec. 2.5.

\subsubsection{Boundary conditions}

The boundary condition enforcement is performed through the numerical fluxes at the physical boundaries. For the chemical potential gradient, we use a homogeneous Neumann boundary condition. Thus, the chemical potential is taken from the interior element, and the normal gradient is set to zero,

$$
\mu_{\Sigma}^{\star}=\mu_{\Sigma}, \quad \overrightarrow{\mathrm{G}}^{\star} \cdot \vec{n}=0 .
$$

For the concentration, we apply the non-homogeneous Neumann boundary condition that accounts for arbitrary wall contact angle (7.24). Thus, we use the interior value for the concentration, and the normal gradients are set to

$$
\mathbf{C}^{\star}=\mathbf{C}, \quad \overrightarrow{\mathbf{G}}_{c}^{\star} \cdot \vec{n}=\mathbf{F}_{w} .
$$

As in the continuous boundary condition, the wall contact angles $\theta_{i j}^{w}$ are subjected to the constitutive constraint (7.23). 


\subsubsection{Time discretization}

The semi-discrete scheme (7.26) is complemented with the numerical integration of the left hand side time derivative coefficients. Looking at the continuous equation (7.4), with chemical potential (7.5), one finds a linear bi-Laplacian operator for the concentration $c_{i}$, and a non-linear Laplacian term corresponding to the chemical free-energy derivatives. The bi-Laplacian operator includes fourth order derivatives, which translates to severe numerical stiffness that restricts the time-step size in explicit solvers. Since the bi-Laplacian operator is linear, but the Laplacian of the chemical potential is non-linear, a commonly adopted technique (see Chapters 5 and 6) is to use an IMplicit-EXplicit (IMEX) method Manzanero et al. [2020b,c]; Dong [2018b].

We revisit the continuous setting (7.4),

$$
c_{i, t}=M_{0} \vec{\nabla}^{2}\left(\frac{12}{\varepsilon} f_{i}-\frac{3}{4} \varepsilon \vec{\nabla}^{2} c_{i}\right),
$$

to describe the approximation in time. We use the IMEX version of the first order Euler method described in Dong [2018b]. We define the coefficients $c^{n}=c\left(t_{n}\right)$ as the solution evaluated at $t_{n}$, so that we evaluate the chemical free-energy at the old time step, $t_{n}$, and the interfacial energy at the new time step, $t_{n+1}$,

$$
\frac{c_{i}^{n+1}-c_{i}^{n}}{\Delta t}=M_{0} \vec{\nabla}^{2}\left(\frac{12}{\varepsilon} f_{i}\left(c_{1}^{n}, c_{2}^{n}, c_{3}^{n}\right)+S_{0}\left(c_{i}^{n+1}-c_{i}^{n}\right)-\frac{3}{4} \varepsilon \vec{\nabla}^{2} c_{i}^{n+1}\right) .
$$

The term $S_{0}\left(c_{i}^{n+1}-c_{i}^{n}\right)$ stabilizes the non-linear terms, where $S_{0}$ is a positive constant, while retaining first order accuracy, as described in Chapter 5. Note that the addition of the stabilization term modifies the Jacobian of the implicit solver, but maintains the linearity in $c_{i}^{n+1}$.

This implementation has two advantages:

1. The implicit system is decoupled: for each of the two-phase, an individual linear system only involving $c_{i}^{n+1}$ is solved.

2. The Jacobian matrix of the implicit solver is identical for the two Cahn-Hilliard equations. Hence, only one Jacobian matrix needs to be computed and stored. This matrix is constant in time due to the linearity of the operator.

In this chapter, and as in Chapters 5 and 6, the linear system of equations is solved with an LU factorization and Gauss substitution. Since the Jacobian matrix is constant in time, and the LU factorization is done only one time at the beginning of the computations, the resulting implementation is very efficient. However, the algorithm is not restricted to other techniques, e.g. iterative solvers. 
Introducing the IMEX scheme (7.33) in the semi-discrete DG formulation (7.26), we get the fully-discrete system,

$$
\begin{gathered}
\left\langle\mathscr{J} \frac{\mathbf{C}^{n+1}-\mathbf{C}^{n}}{\Delta t}, \boldsymbol{\phi}\right\rangle_{E, N}=\int_{\partial E, N} \boldsymbol{\phi}^{T}\left(M_{0} \overrightarrow{\tilde{\mathbf{G}}}^{\star, \theta}\right) \cdot \hat{n} \mathrm{~d} \hat{S}-\left\langle M_{0} \overrightarrow{\tilde{\mathbf{G}}}^{\theta}, \vec{\nabla}_{\xi} \boldsymbol{\phi}\right\rangle_{E, N}, \\
\left\langle\mathscr{J} \overrightarrow{\mathbf{G}}^{\theta}, \vec{\varphi}\right\rangle_{E, N}=\int_{\partial E, N} \boldsymbol{\mu}_{\Sigma}^{\star, \theta, T} \overrightarrow{\tilde{\varphi}} \cdot \hat{n} \mathrm{~d} \hat{S}-\left\langle\boldsymbol{\mu}_{\Sigma}^{\theta}, \vec{\nabla}_{\xi} \cdot \overrightarrow{\tilde{\varphi}}\right\rangle_{E, N},
\end{gathered}
$$

where $\theta$ refers to the IMEX state, computed as

$$
\begin{aligned}
\left\langle\mathscr{J} \mu_{\Sigma}^{\theta}, \boldsymbol{\phi}_{\mu}\right\rangle_{E, N}= & \left\langle\mathscr{J}\left(\frac{12}{\varepsilon} \mathbf{F}^{n}+S_{0}\left(\mathbf{C}^{n+1}-\mathbf{C}^{n}\right)\right), \boldsymbol{\phi}_{\mu}\right\rangle_{E, N} \\
& -\frac{3}{4} \varepsilon \int_{\partial E, N} \boldsymbol{\phi}_{\mu}^{T}\left(\overrightarrow{\tilde{\mathbf{G}}}_{c}^{\star, n+1}\right) \cdot \hat{n} \mathrm{~d} \hat{S}+\frac{3}{4} \varepsilon\left\langle\overrightarrow{\tilde{\mathbf{G}}}_{c}^{n+1}, \vec{\nabla}_{\xi} \boldsymbol{\phi}_{\mu}\right\rangle_{E, N}, \\
\left\langle\mathscr{J} \overrightarrow{\mathbf{G}}_{c}^{n+1}, \vec{\varphi}_{G_{c}}\right\rangle_{E, N}= & \int_{\partial E, N} \mathbf{C}^{\star, n+1, T} \overrightarrow{\tilde{\varphi}}_{G_{c}} \cdot \hat{n} \mathrm{~d} \hat{S}-\left\langle\mathbf{C}^{n+1}, \vec{\nabla}_{\xi} \cdot \overrightarrow{\tilde{\varphi}}_{G_{c}}\right\rangle_{E, N} \cdot
\end{aligned}
$$

\subsection{Numerical experiments}

In this section we perform numerical experiments to evaluate the scheme (7.34) and (7.35) and its numerical implementation. We first study the accuracy of the scheme with a convergence analysis, which solves for a manufactured solution. Then, we study the captive bubble test, where a bubble is immersed between the two other phases. Lastly, we test the wall contact angle boundary condition by solving two bubbles of two phases immersed in the third phase.

\subsubsection{Convergence analysis}

We perform a two-dimensional convergence analysis with an adaptation to three phases of the the manufactured solution used in Dong [2018b] to solve four-phase flows,

$$
\begin{aligned}
& c_{1,0}(x, y ; t)=\frac{1}{3}(1+\cos (4 \pi x) \sin (4 \pi y) \sin (t)), \\
& c_{2,0}(x, y ; t)=\frac{1}{3}(1+\cos (4 \pi x) \sin (4 \pi y) \sin (1.2 t)),
\end{aligned}
$$

with the physical parameters given in Table 7.1. The physical domain is $\Omega=[-1,1]^{2}$, and we enforce periodic boundary conditions at the four physical boundaries. We solve 
Table 7.1: List of the parameter values used with the manufactured solution (7.36)

\begin{tabular}{lllllll}
\hline$\sigma_{12}$ & $\sigma_{13}$ & $\sigma_{23}$ & $M_{0}$ & $\varepsilon$ & $S_{0}$ & $\Delta t$ \\
\hline $6.236 \mathrm{E}-3$ & $7.265 \mathrm{E}-3$ & $8.165 \mathrm{E}-3$ & $1.0 \mathrm{E}-3$ & 0.1 & 0.0 & $1.0 \mathrm{E}-4$ \\
\hline
\end{tabular}

the fully-discrete system (7.34) into a Cartesian $2^{2}$ grid until a final time $t_{F}=0.1$, to then measure the $\mathrm{L}^{2}$ errors as

$$
\left\|C_{i}-C_{i, 0}\right\|=\sqrt{\sum_{e} \sum_{i, j, k=0}^{N} w_{i j k} \mathscr{J}_{i j k}\left(C_{i}-C_{i, 0}\right)^{2}}
$$

The $\mathrm{L}^{2}$ errors for both fluid concentrations are represented in Fig. 7.2. The polynomial

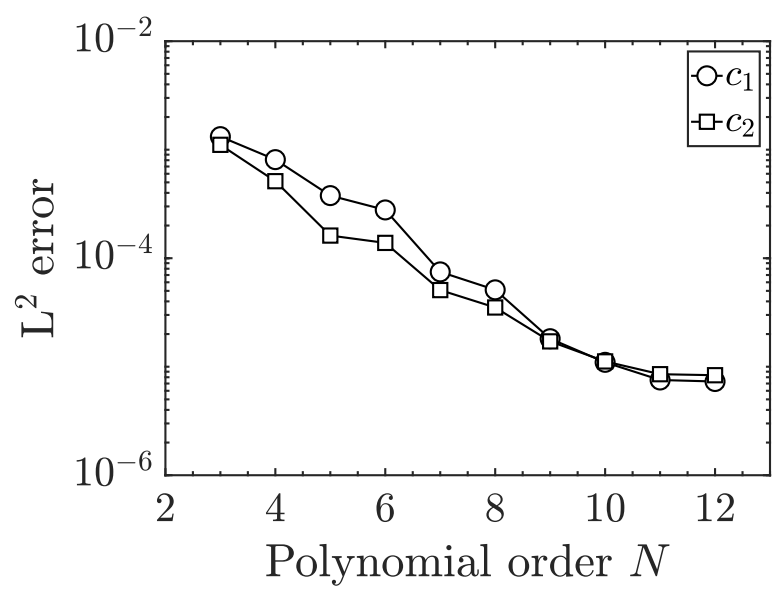

Figure 7.2: Convergence study: $\mathrm{L}^{2}$ errors as a function of the polynomial order $N$ for the manufactured solution (7.36)

order ranges from $N=3$ to $N=12$. The error configuration is the typical: a region with under-resolution in space $(N<11)$ where the solution converges as the grid is refined, and a region with under-resolution in time $(N>11)$, where the error stagnates. We see that the error is higher for $c_{1}$ than for $c_{2}$, and that the behavior is not regular, with systematically higher convergence rates for odd polynomial orders. We also see that for $c_{2}$, odd polynomial orders yield smaller errors than the theoretical linear behavior, while for $c_{1}$, on the contrary, even polynomial orders yield higher errors than expected. We do not have an answer for this behavior, although it has been seen in other works that particular choices of the manufactured solution might lead to this even-odd phenomena Gassner et al. [2016a]; Manzanero et al. [2020b]; Liu et al. [2020]; Rubio et al. [2013]. 
Table 7.2: Captive bubble simulation: interface tension coefficient values and equilibrium angles studied

\begin{tabular}{lllllll}
\hline Test & $\sigma_{12}$ & $\sigma_{13}$ & $\sigma_{23}$ & $\theta_{1}$ & $\theta_{2}$ & $\theta_{3}$ \\
\hline 1 & 1 & 0.8 & 1.4 & $130.54^{\circ}$ & $111.80^{\circ}$ & $117.66^{\circ}$ \\
2 & 1 & 1 & 1 & $120^{\circ}$ & $120^{\circ}$ & $120^{\circ}$ \\
3 & 1 & 0.6 & 0.6 & $130.54^{\circ}$ & $111.80^{\circ}$ & $117.66^{\circ}$ \\
\hline
\end{tabular}

\subsubsection{Captive bubble simulation}

In the second test, we study a Phase 3 bubble immersed in two layers of the other two phases. As a result of the interface tension, the equilibrium is reached when the angles between the phases at the triple point $\left(c_{1}=c_{2}=c_{3}=1 / 3\right)$ satisfy Boyer and Lapuerta [2006],

$$
\frac{\sigma_{23}}{\cos \theta_{1}}=\frac{\sigma_{13}}{\cos \theta_{2}}=\frac{\sigma_{12}}{\cos \theta_{3}}
$$

We consider the three cases for the interface tension coefficients described in Boyer and Lapuerta [2006], which are summarized in Table 7.2 along with the equilibrium triple point angles computed with (7.38). For all the three cases, the initial condition is

$$
\begin{aligned}
& c_{1}(x, y, 0)=\frac{1}{2}\left(1+\tanh \left(\frac{2 \min (\|\mathbf{x}\|-0.1, y)}{\varepsilon}\right)\right), \\
& c_{2}(x, y, 0)=\frac{1}{2}\left(1-\tanh \left(\frac{2 \max (0.1-\|\mathbf{x}\|, y)}{\varepsilon}\right)\right),
\end{aligned}
$$

which is represented in Fig. 7.3(a), where we have represented Phase 1 in white, Phase 2 in black, and Phase 3 in gray. The domain is $\Omega=[-0.3,0.3] \times[-0.15,0.15]$, divided into a Cartesian mesh with $40 \times 20$ elements, and we approximate the solution with $N=8$ polynomials. We apply a wall boundary condition at all physical boundaries (with $\theta_{i j}^{w}=90^{\circ}$ wall contact angles). The rest of the physical and numerical parameters are given in Table 7.3.

Table 7.3: Captive bubble simulation: physical and numerical parameters

\begin{tabular}{llllllll}
\hline$N$ & $M_{0}$ & $\varepsilon$ & $S_{0}$ & $\Delta t$ & $\theta_{12}^{w}$ & $\theta_{13}^{w}$ & $\theta_{23}^{w}$ \\
\hline 8 & $1.0 \mathrm{E}-4$ & 0.01 & 8.0 & 0.01 & $90^{\circ}$ & $90^{\circ}$ & $90^{\circ}$ \\
\hline
\end{tabular}

The final equilibrium solution is presented in Fig. 7.3 for the three cases studied. In the first case (Fig. 7.3(b)), given $\sigma_{13}<\sigma_{23}$, the bubble rises from the initial position 


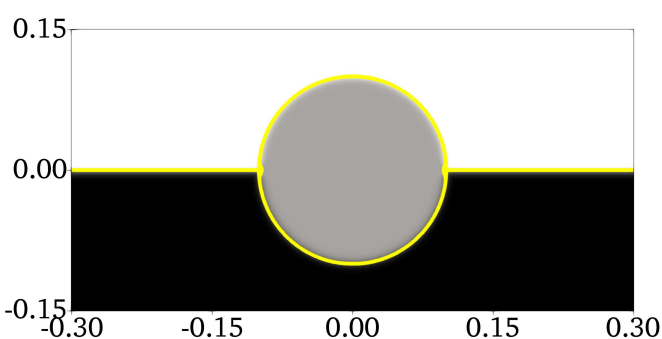

(a) Initial condition

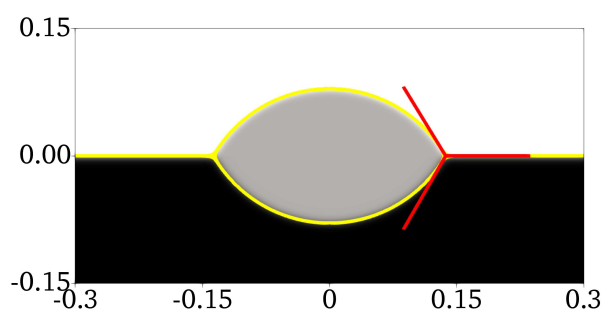

(c) $\sigma_{12}=1, \sigma_{13}=1, \sigma_{23}=1$

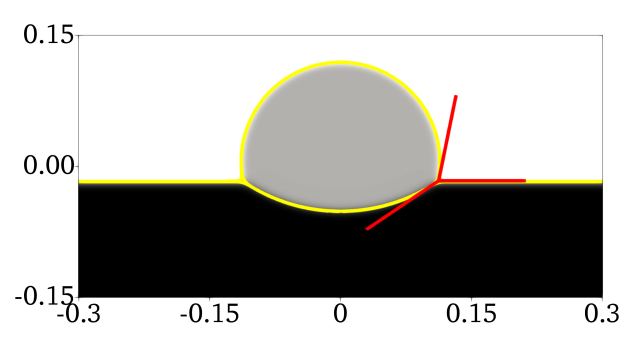

(b) $\sigma_{12}=1, \sigma_{13}=0.8, \sigma_{23}=1.4$

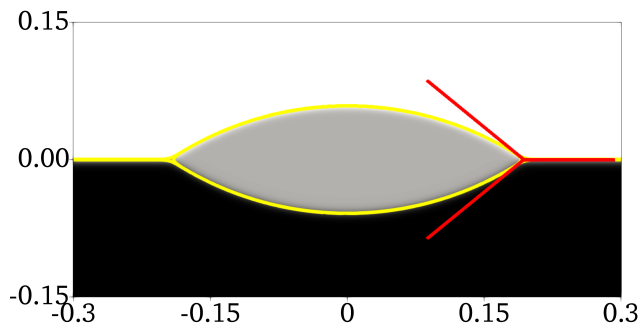

(d) $\sigma_{12}=1, \sigma_{13}=0.6, \sigma_{23}=0.6$

Figure 7.3: Captive bubble simulation. Phase 1 is white, Phase 2 is black, and Phase 3 is gray

as a result of a higher interfacial tension of the bubble with Phase 2 than compared with Phase 1.

In the other two cases (Figs. 7.3(c),7.3(d)), the conditions are symmetric as $\sigma_{13}=\sigma_{23}$, and we get a lenticular shape. We observe that when $\sigma_{12}>\sigma_{13}=\sigma_{23}$, the lens flattens. These results are in agreement with those in Boyer and Lapuerta [2006]. Moreover, at the right triple point, we have represented the angles estimated from the theoretical equilibrium configuration (7.38) with red lines. We confirm that all three solutions show good agreement with the theory (7.38) and with the reference Boyer and Lapuerta [2006].

\subsubsection{Wall contact angle simulation}

The last test case assesses the accuracy of the wall contact angle boundary condition (7.24). We consider two bubbles of Phases 1 and 2 in contact with the inferior and immersed in Phase 3. We specify the equilibrium wall contact angles $\theta_{13}^{w}$ and $\theta_{23}^{w}$, and compute the remaining third angle $\theta_{12}^{w}$ from (7.23). We maintain the same domain and mesh (with polynomial order $N=8$ ) used for the captive bubble simulation. The 
Table 7.4: Wall contact angle simulation: physical and numerical parameters

\begin{tabular}{lllllll}
\hline$M_{0}$ & $\varepsilon$ & $S_{0}$ & $\Delta t$ & $\sigma_{12}$ & $\sigma_{13}$ & $\sigma_{23}$ \\
\hline $1.0 \mathrm{E}-4$ & 0.01 & 8.0 & 0.01 & 2.0 & 1.0 & 2.0 \\
\hline
\end{tabular}

new initial condition is

$$
\begin{aligned}
& c_{1}(x, y, 0)=\frac{1}{2}-\frac{1}{2} \tanh \left(\frac{2\left(\sqrt{(x+0.05)^{2}+(y+0.15)^{2}}-0.1\right)}{\varepsilon}\right), \\
& \hat{c}_{2}(x, y, 0)=\frac{1}{2}-\frac{1}{2} \tanh \left(\frac{2\left(\sqrt{(x-0.1)^{2}+(y+0.12)^{2}}-0.1\right)}{\varepsilon}\right), \\
& c_{2}(x, y, 0)=\min \left(\hat{c}_{2}(x, y, 0), 1-c_{1}(x, y, 0)\right),
\end{aligned}
$$

represented in Fig. 7.4(a). The rest of the physical parameters are given in Table 7.4.

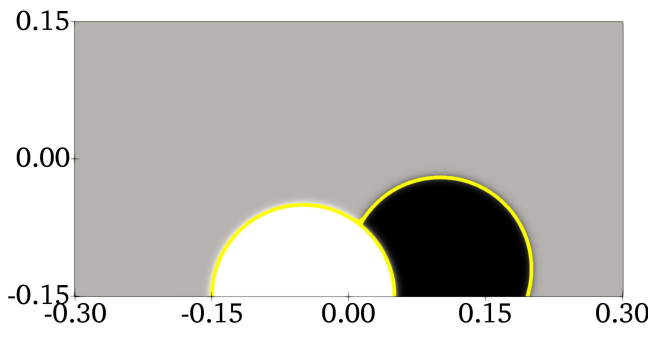

(a) Initial condition

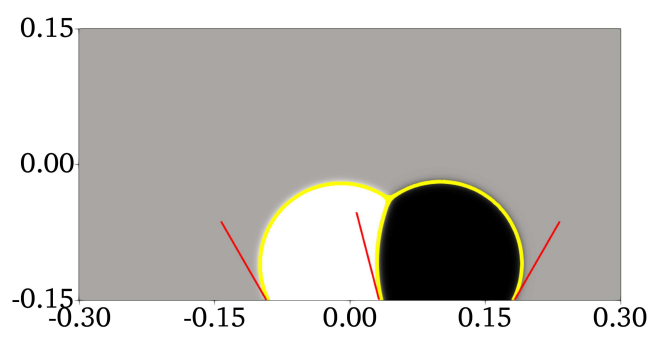

(c) $\theta_{12}=104.48^{\circ}, \theta_{13}=60^{\circ}, \theta_{23}=60^{\circ}$

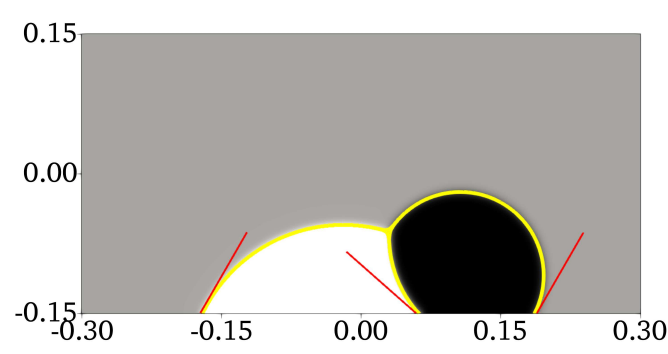

(b) $\theta_{12}=138.59^{\circ}, \theta_{13}=120^{\circ}, \theta_{23}=60^{\circ}$

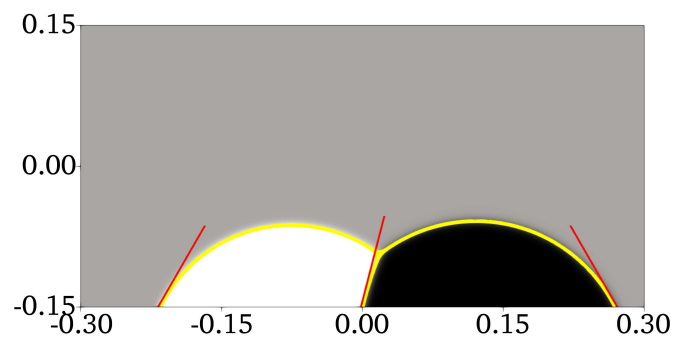

(d) $\theta_{12}=75.52^{\circ}, \theta_{13}=120^{\circ}, \theta_{23}=120^{\circ}$

Figure 7.4: Wall contact angle simulation

We consider three cases for the wall contact angles, given in Table 7.5, so that each one produces a different configuration of the bubbles in equilibrium.

The results are represented in Fig. 7.4. In red line, we have represented the specified angles, following the convention described in Fig. 7.1. The first case, Fig. 7.4(b), represents a configuration where Phase 1 is hydrophilic (wets the wall) and Phase 
Table 7.5: Wall contact angle simulation: wall contact angles specified for the three tests studied

\begin{tabular}{llll}
\hline Test & $\theta_{12}^{w}$ & $\theta_{13}^{w}$ & $\theta_{23}^{w}$ \\
\hline 1 & $138.60^{\circ}$ & $120^{\circ}$ & $60^{\circ}$ \\
2 & $104.48^{\circ}$ & $60^{\circ}$ & $60^{\circ}$ \\
3 & $75.52^{\circ}$ & $120^{\circ}$ & $120^{\circ}$ \\
\hline
\end{tabular}

2 is hydrophobic (rejects the wall). In the second case, Fig. 7.4(c), both phases are hydrophobic, whereas in the third case, Fig. 7.4(c), both phases are hydrophilic. All in all, the contact angles with the wall are in agreement with those imposed by the boundary condition.

\subsection{Conclusions}

In this chapter, we have developed and implemented a high-order DG scheme for the three-phase Cahn-Hilliard equation. The model developed in Boyer and Lapuerta [2006], complemented with the wall boundary condition developed in Shi and Wang [2014] (which allows the prescription of the wall contact angle for each of the three phases) has been chosen. The continuous system of equations is approximated with a discontinuous Galerkin spectral element scheme that uses the symmetric interior penalty method to compute the diffusive fluxes. We use a first order IMplicit-EXplicit (IMEX) method to integrate in time, where non-linear terms are solved explicitly, and linear terms are solved implicitly. The solution of the fully-discrete system requires the solution of one linear system for each of the Cahn-Hilliard equations. The two linear systems, however, are decoupled and the Jacobian matrices are constant in time and identical for both Cahn-Hilliard equations. This implementation is efficient in that only one Jacobian matrix is needed and only one LU factorization is performed for the two equations. Finally, numerical experiments have been performed to evaluate the scheme presented and its numerical implementation. The method exhibits spectral convergence and correctly reproduces the contact angles between the different phases (both in the bulk and in the wall). 



\section{Chapter 8}

\section{A three-phase multiphase solver for the oil and gas industry}

Contents

8.1 Introduction . . . . . . . . . . . . . 221

8.2 Governing equations. . . . . . . . . . . . 223

8.3 Discontinuous Galerkin method and IMEX scheme $\ldots \ldots \ldots 226$

8.4 Two-phase simulations . . . . . . . . . . . . . . 232

8.5 Three-phase simulations . . . . . . . . . . . 238

8.6 Conclusions . . . . . . . . . . . . . . . . . 248

\subsection{Introduction}

In Chapter 6, we presented continuous and discrete formulations to solve two-phases flows. We now augment the complexity of the physics by adding a third phase in the flow. This three-phase flow solver solver was entirely developed in a one-year project with the oil and gas company Repsol. Alongside the solver, the project encompasses automatic mesh generation for pipe flows, and a one way particle tracking module, with the final objective of predicting erosion and corrosion rates. Further details on the project can be found in Gómez-Álvarez et al. [2019]. In this chapter, we describe the construction of the three-phase solver, with the solution of pipe flows as its main application.

Although the solver has been designed for industrial applications, this solver has 
not been constructed to be entropy-stable, as opposed to the two-phase solver presented in Chapter 6. The derivation of an entropy-stable model is possible however, and it is left as future work. Yet, we still use the findings in Chapters 4, 5, and 7, and the model and its discretization have proven to be robust for the applications sought. With these enhancements, the scheme is more robust than an standard discretization.

Previous studies on $\mathrm{N}$-phase incompressible Navier-Stokes/Cahn-Hilliard flows can be found in Dong [2018a, 2014b]; Boyer and Minjeaud [2014]. However, in those works we see that the solution of three-phase flows with Cahn-Hilliard models are obtained for academic two-dimensional simulations that are somewhat far in complexity from the larger scale simulations that were expected for the industrial project. In this project Repsol sought the simulation in complex three-dimensional geometries, the robustness for high-Reynolds numbers, and the simplicity in the user interface for users who are not experts in numerical methods. To decrease the solver complexity (compared to the aforementioned works) and obtain approximated, but realistic three-phase flow solutions, we relax the incompressibility constraint by an artificial (or pseudo) compressibility model as in Chapters 4 and 6. By doing so, we can take advantage of the foundation in the nodal discontinuous Galerkin methods for hyperbolic/parabolic systems, as well as decreasing the complexity of the solver and its implementation.

Here we present the continuous model, and the discontinuous Galerkin approximation of a three-phase flow model suitable for industrial applications. The method chosen in this work couples the continuous and discrete frameworks for the incompressible Navier-Stokes equations with artificial compressibility developed in Chapter 4, and the discretization of the three-phase Cahn-Hilliard model performed in Chapter 7. The connection of both methods is performed as in Chapter 6: we replace the continuity equation of the incompressible Navier-Stokes equations by the two Cahn-Hilliard equations of the three-phase model of Chapter 7. The Cahn-Hilliard equations are then augmented with an advective term, and the momentum equation includes a capillary pressure term, which completes the model.

Similarly, the DG implementation uses features from Chapter 4 and Chapter 7. Precisely, we adapt the two-point fluxes of the incompressible Navier-Stokes model (4.40), and we use the symmetric Interior Penalty (IP) method for the Cahn-Hilliard equation and viscous terms. As the Cahn-Hilliard equation can cause a large timestep restriction, we present a time-discretization that combines a third-order explicit Runge-Kutta with a first order implicit-explicit method. Finally, we consider the implementation of inflow, outflow, and solid wall boundary conditions. The first is designed to automatically compute the concentration and velocity inflow profiles to reproduce different pipe-flow regimes. 
The rest of this chapter is organized as follows: we write the governing equations in Sec. 8.2, and we construct its DG approximation and the IMEX time discretization in Sec. 8.3. Finally we present numerical experiments for the two-phase version of the model in Sec. 8.4, and for the three-phase model in Sec. 8.5.

\subsection{Governing equations}

We combine the three-phase model of Chapter 7 with the incompressible NavierStokes equation solver of Chapter 4. Thus, we solve the two Cahn-Hilliard equations (7.4), which we augment with the advective term as in the two-phases solver of Chapter 6 ,

$$
c_{i, t}+\vec{\nabla} \cdot\left(\vec{u} c_{i}\right)=\frac{M_{0}}{\Sigma_{i}} \vec{\nabla} \cdot\left(\vec{\nabla} \mu_{i}\right), \quad i=1,2,
$$

with the definition of the chemical potentials $\mu_{i}$ given in (7.5). The concentration of the third phase follows the conservation condition (7.1). Now the density is not computed from the continuity equation (4.4) but from the concentration of the three phases,

$$
\rho\left(c_{1}, c_{2}, c_{3}\right)=\rho_{1} c_{1}+\rho_{2} c_{2}+\rho_{3} c_{3}=\rho_{1} c_{1}+\rho_{2} c_{2}+\rho_{3}\left(1-c_{1}-c_{2}\right),
$$

where $\rho_{1,2,3}$ are the densities of fluids 1,2 and 3, respectively, which are constant in space and time. We solve the momentum equation (4.1) used in Chapter 4, which is augmented with a capillary pressure term following Boyer and Lapuerta [2006],

$$
(\rho \vec{u})_{t}+\vec{\nabla} \cdot(\rho \vec{u} \vec{u})=-\vec{\nabla} p+\sum_{m=1}^{3} \mu_{m} \nabla c_{m}+\vec{\nabla} \cdot\left(\eta\left(\vec{\nabla} \vec{u}+\vec{\nabla} \vec{u}^{T}\right)\right)+\rho \vec{g} .
$$

The chemical potential of the third phase is easily computed using (7.9), whereas for the gradient of the concentration we use the constraint (7.1) that gives $\vec{\nabla} c_{3}=$ $-\vec{\nabla} c_{1}-\vec{\nabla} c_{2}$. We maintain the first artificial compressibility model (6.16) to compute the pressure,

$$
p_{t}+\rho_{0} c_{0}^{2} \vec{\nabla} \cdot \vec{u}=0
$$

with $\rho_{0}=\max \left(\rho_{1}, \rho_{2}, \rho_{3}\right)$ and $c_{0}$ the artificial compressibility sound speed.

We write the system built from (8.1), (8.3) and (8.4) in compact form using the block vector notation of Sec. 1.7. We write the equations as a non-conservative system,

$$
\mathbf{q}_{t}+\vec{\nabla} \cdot \overrightarrow{\mathbf{f}}_{e}(\mathbf{q})+\sum_{n=1}^{N_{c}} \overrightarrow{\boldsymbol{\phi}}_{n}(\mathbf{q}) \cdot \vec{\nabla} \lambda_{n}(\mathbf{q})=\vec{\nabla} \cdot \overrightarrow{\mathbf{f}}_{v}(\mathbf{q}, \vec{\nabla} \mathbf{w})+\mathbf{s}(\mathbf{q})
$$


with the state vector $\mathbf{q}=\left(c_{1}, c_{2}, \rho \vec{u}, p\right)$, gradient variables $\mathbf{w}=\left(\mu_{1} / \Sigma_{1}, \mu_{2} / \Sigma_{2}, \vec{u}, p\right)$, inviscid fluxes,

$$
\mathbf{f}_{e}=\left(\begin{array}{c}
c_{1} u \\
c_{2} u \\
\rho u^{2}+p \\
\rho u v \\
\rho u w \\
\rho_{0} c_{0}^{2} u
\end{array}\right), \mathbf{g}_{e}=\left(\begin{array}{c}
c_{1} v \\
c_{2} v \\
\rho u v \\
\rho v^{2}+p \\
\rho v w \\
\rho_{0} c_{0}^{2} v
\end{array}\right), \mathbf{h}_{e}=\left(\begin{array}{c}
c_{1} w \\
c_{2} w \\
\rho u w \\
\rho v w \\
\rho w^{2}+p \\
\rho_{0} c_{0}^{2} w
\end{array}\right)
$$

viscous fluxes,

$$
\mathbf{f}_{v}=\left(\begin{array}{c}
\frac{M_{0}}{\Sigma_{1}} \mu_{1, x} \\
\frac{M_{0}}{\Sigma_{2}} \mu_{2, x} \\
2 \eta \overline{\bar{s}}_{11} \\
2 \eta \overline{\bar{s}}_{21} \\
2 \eta \overline{\bar{s}}_{31} \\
0
\end{array}\right), \mathbf{g}_{v}=\left(\begin{array}{c}
\frac{M_{0}}{\Sigma_{1}} \mu_{1, y} \\
\frac{M_{0}}{\Sigma_{2}} \mu_{2, y} \\
2 \eta \overline{\bar{s}}_{12} \\
2 \eta \overline{\bar{s}}_{22} \\
2 \eta \overline{\bar{s}}_{32} \\
0
\end{array}\right), \mathbf{h}_{v}=\left(\begin{array}{c}
\frac{M_{0}}{\Sigma_{1}} \mu_{1, z} \\
\frac{M_{0}}{\Sigma_{2}} \mu_{2, z} \\
2 \eta \overline{\bar{s}}_{13} \\
2 \eta \overline{\bar{s}}_{23} \\
2 \eta \overline{\bar{s}}_{33} \\
0
\end{array}\right)
$$

the three non-conservative terms,

$$
\overrightarrow{\boldsymbol{\phi}}_{i}=-\Upsilon\left(\begin{array}{c}
0 \\
\mu_{i} \overline{\bar{I}}_{3} \\
0
\end{array}\right), \quad \lambda_{i}=c_{i}, \quad i=1,2,3
$$

and the source term $\mathbf{s}=(0,0, \rho \vec{g}, 0)$. In (8.7), $\overline{\bar{s}}$ is the strain tensor (6.18).

The system (8.5) studied in this chapter is not entropy-stable. If one performs an entropy analysis of (8.5) it contains parasitic terms that depend on $\vec{\nabla} \cdot \vec{u}$, which cannot be bounded, nor ignored. Therefore, we do not construct the approximation with stability in mind as in Chapters 4-6, as we do not have a continuous entropy analysis to mimic.

\subsubsection{Reduction of the model to a two-phases flow model}

The three-phase model reduces to a two-phase model if one of the concentrations is not present initially. For instance, we consider that $c_{2}(\vec{x}, 0)=0$, and as a result $c_{1}(\vec{x}, 0)=c=1-c_{3}(\vec{x}, 0)$. The second Cahn-Hilliard equation is

$$
c_{2, t}=-\vec{\nabla} \cdot\left(c_{2} \vec{u}\right)+\frac{M_{0}}{\Sigma_{2}} \vec{\nabla}^{2} \mu_{2}=0
$$


since $\mu_{2}=0$ when $c_{2}=0$, as described in (7.12). Therefore, $c_{2}(\vec{x}, t)=0$ for $t>0$. We compute the chemical potential of the three-phase model, $\mu_{1}^{3 p h}$, with $c_{2}=0$, and $c_{1}=c=1-c_{3}$. To do so, we compute the free-energy derivatives,

$$
\begin{aligned}
& \frac{\partial F_{0}^{\sigma}}{\partial c_{1}}=2 \sigma_{13} c_{1} c_{3}^{2}=\Sigma_{1} c_{1} c_{3}^{2}+\Sigma_{3} c_{1} c_{3}^{2}, \\
& \frac{\partial F_{0}^{\sigma}}{\partial c_{2}}=c_{1} c_{3}\left(\Sigma_{1} c_{1}+\Sigma_{3} c_{3}\right), \\
& \frac{\partial F_{0}^{\sigma}}{\partial c_{3}}=2 \sigma_{13} c_{1}^{2} c_{3}=\Sigma_{1} c_{1}^{2} c_{3}+\Sigma_{3} c_{1}^{2} c_{3},
\end{aligned}
$$

to obtain the chemical potential,

$$
\mu_{1}^{3 \mathrm{ph}}=\frac{12 \Sigma_{1}}{\varepsilon}\left(c_{1} c_{3}^{2}-c_{1}^{2} c_{3}\right)-\frac{3}{4} \Sigma_{1} \varepsilon \nabla^{2} c_{1}=\frac{12 \Sigma_{1}}{\varepsilon}\left(c(1-c)^{2}-c^{2}(1-c)\right)-\frac{3}{4} \Sigma_{1} \varepsilon \nabla^{2} c .
$$

From a direct comparison with the two-phase model (5.7), we see that the chemical potentials in the two models differ by a constant,

$$
\mu_{1}^{3 p h}=\frac{\Sigma_{1}}{2 \sigma_{13}} \mu^{2 p h} .
$$

However, the diffusive terms in the Cahn-Hilliard equation are identical if we use different choices for the mobility,

$$
\frac{M_{0}^{3 p h}}{\Sigma_{1}} \vec{\nabla}^{2} \mu_{1}^{3 p h}=M_{0}^{2 p h} \vec{\nabla}^{2} \mu^{2 p h}, \quad M_{0}^{3 p h}=2 \sigma_{13} M_{0}^{2 p h} .
$$

Thus, for equivalent simulations between the two-phase Cahn-Hilliard model of Chapter 5, and the reduction to two-phase of the three-phase model of Chapter 7, we must relate the mobility parameters following (8.13).

Lastly, we check that the capillary pressure term of the three-phase flow is equivalent to the capillary term of the two-phase flow. For three phases,

$$
\begin{aligned}
\vec{p}_{c} & =\mu_{1}^{3 p h} \vec{\nabla} c_{1}+\mu_{2}^{3 p h} \vec{\nabla} c_{2}+\mu_{3}^{3 p h} \vec{\nabla} c_{3}=\mu_{1}^{3 p h} \vec{\nabla} c_{1}+\frac{\Sigma_{3}}{\Sigma_{1}} \mu_{1}^{3 p h} \vec{\nabla} c_{1} \\
& =\frac{\Sigma_{1}+\Sigma_{3}}{\Sigma_{1}} \mu_{1}^{3 p h} \vec{\nabla} c_{1}=\frac{2 \sigma_{13}}{\Sigma_{1}} \mu_{1}^{3 p h} \vec{\nabla} c_{1}=\mu^{2 p h} \vec{\nabla} c .
\end{aligned}
$$

Therefore, we confirm that the approximation for the capillary pressure is identical in both models. In the second line, we used the property $\Sigma_{i}+\Sigma_{j}=2 \sigma_{i j}$ of the spreading factors.

We conclude that the three-phase model is a valid two-phase model if one of the three phases is not present initially. 


\subsection{Discontinuous Galerkin method and IMEX scheme}

The evolution of the three-phase flow is driven by the two Cahn-Hilliard equations, (8.1), the momentum equation (8.3), and the artificial compressibility model (8.4). The discretization of the spatial differential operators is performed using a nodal Discontinuous Galerkin Spectral Element Method (DGSEM), and the discretization of the time derivatives is performed using an EXplicit-IMplicit (IMEX) method. The latter combines a third order low-storage explicit Runge-Kutta RK3 method, and a first order backward and forward Euler method.

\subsubsection{Spatial discretization using the DGSEM}

We approximate the three-phase system (8.5) with split-form DG scheme (2.85),

$$
\begin{aligned}
\left\langle\mathscr{J} \mathbf{Q}_{t}, \boldsymbol{\phi}\right\rangle_{E, N} & +\int_{\partial E, N} \boldsymbol{\phi}^{T}\left(\overrightarrow{\tilde{\mathbf{F}}}_{e}^{\star}-\overrightarrow{\tilde{\mathbf{F}}}_{e}+\sum_{m=1}^{N_{c}}\left(\overrightarrow{\tilde{\mathbf{\Phi}}}_{m} \Lambda_{m}\right)^{\diamond}-\left(\overrightarrow{\tilde{\mathbf{\Phi}}}_{m} \Lambda_{m}\right)-\overrightarrow{\tilde{\mathbf{F}}}_{v}^{\star}+\overrightarrow{\tilde{\mathbf{F}}}_{v}\right) \cdot \hat{n} \mathrm{~d} \hat{S} \\
& +\left\langle\mathbb{D}\left(\overrightarrow{\tilde{\mathbf{F}}}_{e}\right)^{\#}, \boldsymbol{\phi}\right\rangle_{E, N}+\sum_{m=1}^{N_{c}}\left\langle\overrightarrow{\tilde{\mathbf{\Phi}}}_{m} \cdot \vec{\nabla}_{\xi} \Lambda_{m}, \boldsymbol{\phi}\right\rangle_{E, N}=\left\langle\vec{\nabla}_{\xi} \cdot \overrightarrow{\tilde{\mathbf{F}}}_{v}, \boldsymbol{\phi}\right\rangle_{E, N}+\langle\mathscr{J} \mathbf{S}, \boldsymbol{\phi}\rangle_{E, N}, \\
\langle\mathscr{J} \overrightarrow{\mathbf{G}}, \vec{\varphi}\rangle_{E, N} & =\int_{\partial E, N} \mathbf{W}^{\star, T} \overrightarrow{\tilde{\varphi}} \cdot \hat{n} \mathrm{~d} \hat{S}-\left\langle\mathbf{W}, \vec{\nabla}_{\xi} \cdot \overrightarrow{\tilde{\varphi}}\right\rangle_{E, N} \cdot
\end{aligned}
$$

The two-point volume flux $\overrightarrow{\mathbf{F}}_{e}^{\#}$ does not satisfy a Tadmor jump condition, hence, we cannot call it entropy conserving flux (EC). For this chapter, we use

$$
\mathbf{F}_{e}^{\#}=\left(\begin{array}{c}
\left\{\left\{c_{1}\right\}\right\}\{\{u\}\} \\
\left\{\left\{c_{2}\right\}\right\}\{\{u\}\} \\
\{\{\rho\}\}\{\{u\}\}^{2}+\{\{p\}\} \\
\{\{\rho\}\}\{\{u\}\}\{\{v\}\} \\
\{\{\rho\}\}\{\{u\}\}\{\{w\}\} \\
\rho_{0} c_{0}^{2}\{\{u\}\}
\end{array}\right), \mathbf{G}_{e}^{\#}=\left(\begin{array}{c}
\left\{\left\{c_{1}\right\}\right\}\{\{v\}\} \\
\left\{\left\{c_{2}\right\}\right\}\{\{v\}\} \\
\{\{\rho\}\}\{\{u\}\}\{\{v\}\} \\
\{\{\rho\}\}\{\{v\}\}^{2}+\{\{p\}\} \\
\{\{\rho\}\}\{\{v\}\}\{\{w\}\} \\
\rho_{0} c_{0}^{2}\{\{v\}\}
\end{array}\right), \mathbf{H}_{e}^{\#}=\left(\begin{array}{c}
\left\{\left\{c_{1}\right\}\right\}\{\{w\}\} \\
\left\{\left\{c_{2}\right\}\right\}\{\{w\}\} \\
\{\{\rho\}\}\{\{u\}\}\{\{w\}\} \\
\{\{\rho\}\}\{\{v\}\}\{\{w\}\} \\
\{\{\rho\}\}\{\{w\}\}^{2}+\{\{p\}\} \\
\rho_{0} c_{0}^{2}\{\{w\}\}
\end{array}\right),
$$

which combines the split-form scheme used for the incompressible Navier-Stokes equations in Chapter 4 for the last four equations, and then performs the product of the average for the first two equations. Since the two-point volume flux is not designed to satisfy Tadmor's jump condition, we dropped the dealiased metrics gradient $\mathbb{D}_{\text {grad }}^{\mathrm{NC}}$ from the general scheme to the standard gradient operator. Since the scheme is not provably stable either way, we chose to keep a simpler and lighter scheme. 
The approximation of the equations is completed with the discrete equation for the chemical potentials. We borrow the standard DG scheme from Chapter 7, which is simply copied here,

$$
\begin{aligned}
\left\langle\mathscr{J} \mu_{i}, \phi_{\mu}\right\rangle_{E, N} & =\left\langle\mathscr{J} \frac{12}{\varepsilon} \Sigma_{i} F_{i}, \phi_{\mu}\right\rangle_{E, N}-\frac{3}{4} \varepsilon \Sigma_{i} \int_{\partial E, N} \phi_{\mu}^{T}\left(\overrightarrow{\tilde{G}}_{c, i}^{\star}\right) \cdot \hat{n} \mathrm{~d} \hat{S}+\frac{3}{4} \varepsilon \Sigma_{i}\left\langle\overrightarrow{\tilde{G}}_{c, i}, \vec{\nabla}_{\xi} \phi_{\mu}\right\rangle_{E, N}, \\
\left\langle\mathscr{J} \vec{G}_{c, i}, \overrightarrow{\tilde{\varphi}}_{G_{c}}\right\rangle_{E, N} & =\int_{\partial E, N} C_{i}^{\star, T} \overrightarrow{\tilde{\varphi}}_{G_{c}} \cdot \hat{n} \mathrm{~d} \hat{S}-\left\langle C_{i}, \vec{\nabla}_{\xi} \cdot \overrightarrow{\tilde{\varphi}}_{G_{c}}\right\rangle_{E, N} \cdot
\end{aligned}
$$

From the concentration field $\left(C_{1}, C_{2}\right)$, we compute the chemical potentials $\left(\mu_{1}, \mu_{2}\right)$, which are then introduced in the entropy variables $\mathbf{W}$, to compute their gradient $\overrightarrow{\mathbf{G}}$, and the state vector time derivative $\mathbf{Q}_{t}$. In the next sections we describe the computation of the numerical fluxes for inter-element and physical boundary faces.

\section{Numerical fluxes}

For the inviscid Riemann solver $\vec{F}_{e}^{\star}$ we use solution of the exact Riemann problem derived in Bassi et al. [2017], and used in Chapters 4 and 6, for the normal velocity and the pressure,

$$
\begin{aligned}
& u^{\star}=\frac{p_{R}-p_{L}+\rho_{R} u_{R} \lambda_{R}^{-}-\rho_{L} u_{L} \lambda_{L}^{+}}{\rho_{R} \lambda_{R}^{-}-\rho_{L} \lambda_{L}^{+}}, p^{\star}=p_{R}+\rho_{R} \lambda_{R}^{-}\left(u_{R}-u^{\star}\right), \\
& c_{1}^{\star}, c_{2}^{\star}, v^{\star}, w^{\star}=\left\{\begin{array}{lll}
c_{1 L}, c_{2 L}, v_{L}, w_{L} & \text { if } \quad u^{\star} \geqslant 0 \\
c_{1 R}, c_{2 R}, v_{R}, w_{R} & \text { if } \quad u^{\star}<0
\end{array}\right.
\end{aligned}
$$

and we compute the tangential velocities and concentrations from the appropriate element depending on the sign of $u^{\star}$. Amongst the beneficial properties of this Riemann solver that leads to this choice, we highlight that it is physical (the exact solution of the Riemann problem), efficient (e.g. does not need any iterative solution), and parameter-free.

For the diamond fluxes, we simply take the interior values,

$$
\left(\overrightarrow{\boldsymbol{\Phi}}_{m} \Lambda_{m}\right)^{\diamond}=\overrightarrow{\boldsymbol{\Phi}}_{m} \Lambda_{m}
$$

For viscous fluxes and gradient variables, $\overrightarrow{\mathbf{F}}_{v}^{\star}$ and $\mathbf{W}^{\star}$, we use the symmetric Interior Penalty (IP) method,

$$
\mathbf{W}^{\star}=\{\{\mathbf{W}\}\}, \quad \overrightarrow{\mathbf{F}}_{v}^{\star}=\left\{\left\{\overrightarrow{\mathbf{F}}_{v}(\mathbf{Q}, \vec{\nabla} \mathbf{W})\right\}\right\}+\beta\left(\begin{array}{c}
\frac{M_{0}}{\Sigma_{1}} \llbracket \mu_{1} \rrbracket \\
\frac{M_{0}}{\Sigma_{2}} \llbracket \mu_{2} \rrbracket \\
\mu \llbracket \rho \vec{u} \rrbracket \\
\mu \llbracket p \rrbracket
\end{array}\right) \vec{n}_{L},
$$


where the definition of the local gradients is (2.89), and we take the penalty parameter from Shahbazi [2005]

$$
\beta=\frac{(N+1)(N+2)}{2 \bar{h}}, \bar{h}=\frac{\min \left(V_{L}, V_{R}\right)}{S},
$$

where $\bar{h}$ is an approximated measure of the minimum element size normal to the wall, $V_{L}$ and $V_{R}$ are the volumes of the neighbouring elements, and $S$ is the area of the face. Finally, for the concentration and its gradient, as in Chapter 7 we also use the IP method,

$$
C_{i}^{\star}=\left\{\left\{C_{i}\right\}\right\}, \vec{G}_{c, i}^{\star}=\left\{\left\{\vec{\nabla} C_{i}\right\}\right\}-\beta\left(C_{i, L} \vec{n}_{L}+C_{i, R} \vec{n}_{R}\right) .
$$

\subsubsection{Boundary conditions}

In this section we describe the imposition of inflow, outflow, and no-slip wall boundary conditions. In this work, we prescribe the boundary conditions weakly. Hence, we create a ghost (exterior) state with the appropriate boundary information, and then compute the interface fluxes between the interior $\mathbf{Q}^{i}$ and exterior $\mathbf{Q}^{e}$ states.

\section{Inflow boundary condition}

For the inflow boundary condition, we specify the inflow concentration $c_{i, \text { in }}(\vec{x} ; t)$ and the velocity $\vec{u}_{i n}(\vec{x} ; t)$. For the inviscid fluxes, we construct a ghost state,

$$
\mathbf{Q}^{e}=\left(\begin{array}{c}
C_{1, i n} \\
C_{2, i n} \\
\rho\left(C_{i n}\right) \vec{U}_{i n} \\
P
\end{array}\right)
$$

where we take the pressure $P$ from the interior, and compute the interface flux from the exact Riemann problem solution (8.18), $\overrightarrow{\mathbf{F}}_{e}^{\star}\left(\mathbf{Q}_{e}^{i}, \mathbf{Q}_{e}^{e}\right)$.

For the viscous fluxes, we use

$$
\mathbf{W}^{\star}=\frac{\mathbf{W}^{i}+\mathbf{W}^{e}}{2}, \quad \mathbf{F}_{v}^{\star} \cdot \vec{n}=\left(\begin{array}{c}
0 \\
0 \\
\eta\left(\nabla \vec{U}+\nabla \vec{U}^{T}\right) \cdot \vec{n} \\
0
\end{array}\right),
$$

where we apply the Neumann boundary conditions to the chemical potential, and take the interior values for viscous stresses. In (8.24), we compute the gradient variables from the ghost state, $\mathbf{W}^{e}=\mathbf{W}\left(\mathbf{Q}^{e}\right)$. 


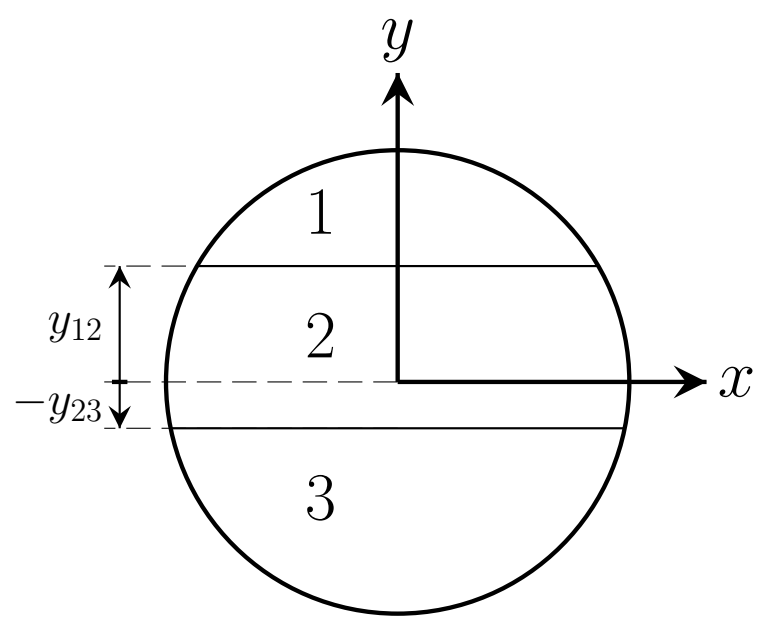

Figure 8.1: Configuration of a layered inflow. The position of the two interfaces is provided by the values $y_{12}$ and $y_{23}$

The implementation features an automatic method for the distribution of the phases in a circular section, given the superficial velocities and the slip velocities. The superficial velocity of each phase is, for a given flow rate, the equivalent velocity obtained if the phase occupies the entire section,

$$
v_{s, i}=\frac{1}{A_{\text {inflow }}} \int_{\text {inflow }} \vec{u}_{i} \cdot \mathrm{d} \vec{S} .
$$

Furthermore, because real flow configurations feature a large ratio of the superficial velocities that confine one of the phases to the near wall region, we allow the velocity to be discontinuous at the interface between the phases. By doing so, we can use lower velocities for the phases with smaller superficial velocities, so that they occupy a larger region of the cross section.

The problem with a phase being confined to the near wall region is that it might not be well captured by the numerical method. For the flow configuration given in Fig. 8.1, the concentration inflow boundary condition is

$$
\begin{aligned}
& c_{1, \text { in }}(x, y)=\frac{1}{2}+\frac{1}{2} \tanh \left(\frac{y-y_{12}}{\varepsilon}\right), \\
& c_{2, \text { in }}(x, y)=\frac{1}{2} \tanh \left(\frac{y-y_{23}}{\varepsilon}\right)-\frac{1}{2} \tanh \left(\frac{y-y_{12}}{\varepsilon}\right),
\end{aligned}
$$

and the velocities are computed from a Poiseuille flow,

$$
u_{\text {in }}=\left(V_{1, \max } c_{1, \text { in }}+V_{2, \max } c_{2, \text { in }}+V_{3, \max } c_{3, \text { in }}\right)\left(1-\left(\frac{r}{R}\right)^{2}\right),
$$

with the two slip velocities, which are user-input,

$$
V_{s, 12}=V_{1, \max }-V_{2, \max }, V_{s, 23}=V_{2, \max }-V_{3, \max } .
$$


Therefore, there are five unknowns $\left(V_{1, \max }, V_{2, \max }, V_{3, \max }, y_{12}\right.$, and $\left.y_{23}\right)$, and five equations: the two slip-velocities definitions (8.28), and the superficial velocities,

$$
v_{s, i}=\frac{V_{i, \text { max }}}{A_{\text {inflow }}} \int_{\text {inflow }} c_{i, \text { in }}\left(1-\left(\frac{r}{R}\right)^{2}\right) \mathrm{d} S
$$

that are solved using a Newton-Rhapson method.

\section{Outflow boundary condition}

The outflow boundary condition specifies the ambient pressure at the exit of the domain $P_{o}$, and applies a Neumann boundary condition to the rest of the variables. Therefore, for the inviscid fluxes the exterior state is

$$
\mathbf{Q}^{e}=\left(\begin{array}{c}
C_{1} \\
C_{2} \\
\rho \vec{U} \\
P_{o}
\end{array}\right)
$$

whereas for viscous fluxes we simply use a Neumann boundary condition for all the variables,

$$
\mathbf{W}^{\star}=\mathbf{W}_{i}, \quad \mathbf{F}_{v}^{\star} \cdot \vec{n}=0 .
$$

\section{No-slip wall boundary condition}

We construct a ghost state with the same variables as the inside, but changing the sign of the normal velocity,

$$
\mathbf{Q}^{e}=\left(\begin{array}{c}
c_{1} \\
c_{2} \\
\rho(\vec{U}-2(\vec{U} \cdot \vec{n}) \vec{n}) \\
p
\end{array}\right)
$$

For the viscous numerical fluxes, we apply Neumann boundary conditions in all variables except velocities, which take the interior values,

$$
\mathbf{W}^{\star}=\frac{\mathbf{W}^{i}+\mathbf{W}^{e}}{2}, \overrightarrow{\mathbf{F}}_{v}^{\star} \cdot \vec{n}=\left(\begin{array}{c}
0 \\
0 \\
\eta\left(\nabla \vec{U}+\nabla \vec{U}^{T}\right) \cdot \vec{n} \\
0
\end{array}\right)
$$


Finally, for the gradient of the concentrations $\vec{G}_{c, i}^{\star}$ the Neumann boundary condition is non-homogeneous if one wants to solve for arbitrary wall contact angles. Thus, we follow Sec. 7.3.1 and use

$$
C_{i}^{\star}=C_{i}, \quad \vec{G}_{c, i}^{\star} \cdot \vec{n}=F_{w, i},
$$

where the boundary coefficients $F_{w, i}$ were defined in (7.24). For $90^{\circ}$ angles, the coefficients are zero.

\subsubsection{Time discretization}

The fourth order spatial derivative in the Cahn-Hilliard equation is too stiff to be solved explicitly. Unless the mobility parameter is low enough to allow reasonable time-steps, we use an IMplicit-EXplicit (IMEX) method to integrate in time: NavierStokes terms are solved using a third-order explicit Runge-Kutta (RK3) method, CahnHilliard chemical free-energy is solved using forward Euler, and Cahn-Hilliard interface energy is solved using backward Euler.

The IMEX procedure has two steps, which we describe in a semi-discrete fashion (continuous in space, discrete in time):

1. We perform the explicit RK3 step for the Navier-Stokes terms, without the contribution from the chemical-free energy and interfacial energy in the Cahn-Hilliard equation,

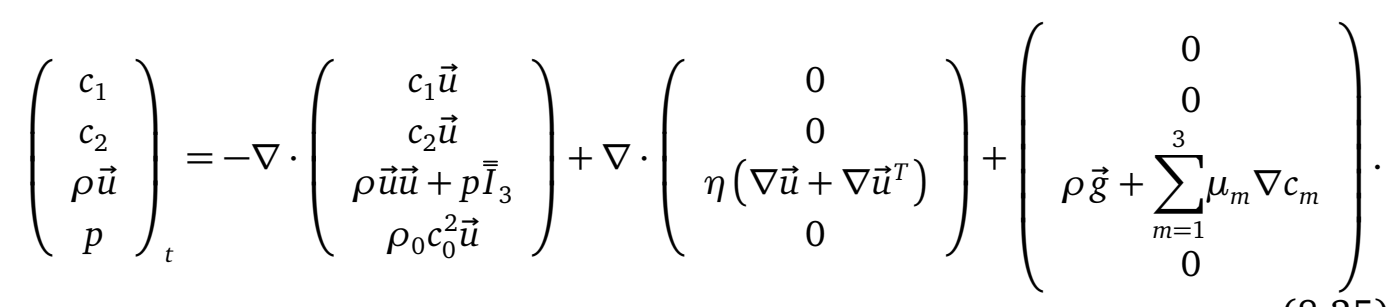

After the RK3 time-step, the variables are $\left(\hat{c}_{1}, \hat{c}_{2}, \rho \vec{u}^{n+1}, p^{n+1}\right)$, since the concentrations need a correction step to include the chemical potential.

2. Perform the time-step on the two concentrations to solve the Cahn-Hilliard equations. To do so, we use the IMEX scheme (7.33) derived in Sec. 7.3.2, with $\hat{c}_{i}$ as $c_{i}^{n}$ only in the time-derivative operator,

$$
\frac{c_{i}^{n+1}-\hat{c}_{i}}{\Delta t}=M_{0} \vec{\nabla}^{2}\left(\frac{12}{\varepsilon} f_{i}\left(c_{1}^{n}, c_{2}^{n}, c_{3}^{n}\right)+S_{0}\left(c_{i}^{n+1}-c_{i}^{n}\right)-\frac{3}{4} \varepsilon \vec{\nabla}^{2} c_{i}^{n+1}\right) .
$$




\subsection{Two-phase simulations}

We perform the validation of the solver in the particular case of solving a two-phase flow (i.e. as described in Sec. 8.2.1). We first solve a manufactured solution in Sec. 8.4.1, and then we solve a two-phase horizontal pipe in Sec. 8.4.2. The enhancement of the robustness provided by the split-form scheme is addressed in Sec. 8.4.3.

\subsubsection{Two-phase simulations: manufactured solution}

We use an identical manufactured solution as that used for the entropy-stable twophase solver of Chapter 6 . The manufactured solution is

$$
\begin{aligned}
c_{1,0}(x, y ; t) & =\frac{1}{2}(1+\cos (\pi x) \cos (\pi y) \sin (t)), \\
c_{2,0}(x, y ; t) & =0 \\
u_{0}(x, y ; t) & =2 \sin (\pi x) \cos (\pi z) \sin (t), \\
v_{0}(x, y ; t) & =-2 \cos (\pi x) \sin (\pi y) \sin (t), \\
p_{0}(x, y ; t) & =2 \sin (\pi x) \sin (\pi z) \cos (t),
\end{aligned}
$$

which we solve on the domain $(x, y) \in[-1,1]^{2}$. The final time is $t_{F}=0.1$, and all physical parameters are presented in Table 8.1.

Table 8.1: List of the parameter values used with the manufactured solution (8.37)

\begin{tabular}{llllllll}
\hline$\rho_{1}$ & $\rho_{3}\left(\mathrm{~kg} / \mathrm{m}^{3}\right)$ & $\eta_{1}$ & $\eta_{2}(\mathrm{~Pa} \cdot \mathrm{s})$ & $\varepsilon(\mathrm{m})$ & $M_{0}$ & $c_{0}^{2}\left(\mathrm{~m} / \mathrm{s}^{2}\right)^{2}$ & $\sigma(\mathrm{N} / \mathrm{m})$ \\
\hline 1.0 & 2.0 & $1.0 \mathrm{E}-3$ & $1.0 \mathrm{E}-3$ & $1 / \sqrt{2}$ & $1.134 \mathrm{E}-2$ & $1.0 \mathrm{E} 3$ & $6.236 \mathrm{E}-3$ \\
\hline
\end{tabular}

We first present the polynomial order convergence analysis in Fig. 8.2 for two values of the $\Delta t$ parameter. We consider a $4^{2}$ mesh, and the polynomial order ranges from $N=2$ to $N=10$. We represent the $\mathrm{L}^{2}$ errors for the concentration, momentum, and pressure. For both time-step values, the errors behave similarly, with a space underresolved region for lower polynomial orders, where the errors decrease exponentially, and a time under-resolved region where the error stagnates with the polynomial order. We see that the use of a first order Euler scheme for the Cahn-Hilliard part impacts its accuracy, as the stagnation is reached for lower polynomial orders (i.e. temporal errors dominate), and the error values once the stagnation is reached are also higher than the entropy-stable scheme with the BDF2 method of Chapter 6.

However, we believe that for an industrial solver, the accuracy of the Cahn-Hilliard part is not critical. The role of the Cahn-Hilliard equation for an industrial simulation 


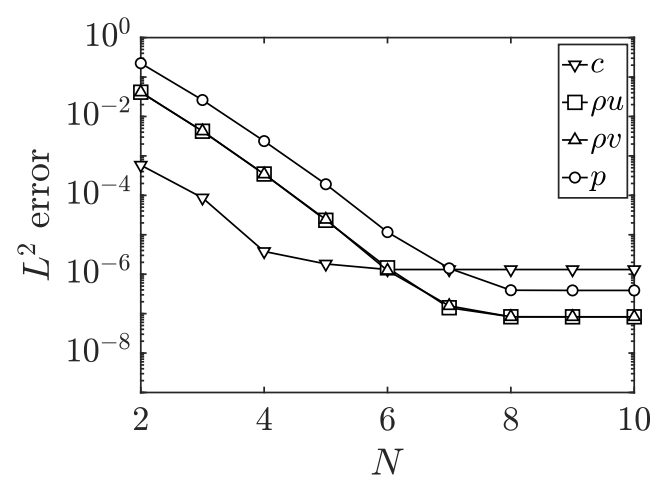

(a) $\Delta t=10^{-4}$

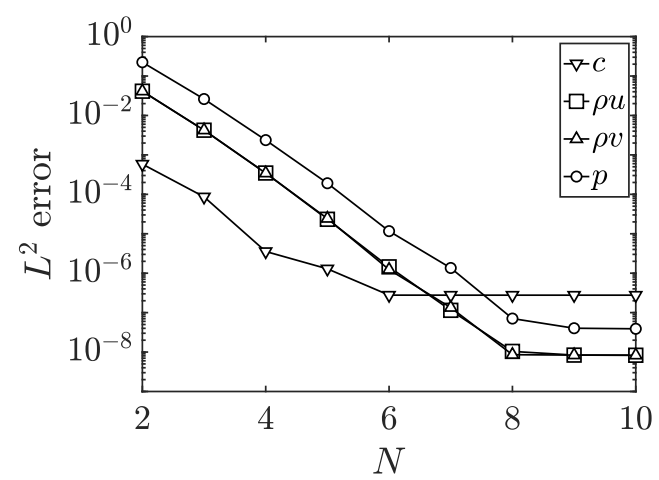

(b) $\Delta t=10^{-5}$

Figure 8.2: Two-phase solver: polynomial order convergence study of the manufactured solution (8.37). We represent the $\mathrm{L}^{2}$ errors in concentration, $x$ - and $y-$ momentum, and pressure. The polynomial order ranges from 2 to 10 , and we integrate in time until $t_{F}=0.1$ with two time step sizes: $\Delta t=10^{-4}$ and $10^{-5}$. All physical parameters are given in Table 8.1

is to advect the phases, to introduce interface regularization that helps to solve underresolved simulations, and to separate the phases, or avoid mixing. In practice, the mobility is usually chosen a-posteriori by trial and error.

We have implemented a second order BDF2 method as in Chapter 6, however, we find the first order Euler more robust for industrial applications. For the flow dynamics we use the third order RK3 method, and we see that we obtain more accurate solutions for the momentum and pressure equations. Overall, we find a satisfactory convergence behavior of the method for smooth solutions.

We also perform a mesh convergence study, presented in Table 8.6. We use meshes with $4^{2}, 6^{2}, 8^{2}, 12^{2}$, and $16^{2}$ elements, and we vary the polynomial order from $N=2$ to $N=5$. For lower polynomial orders, we find the expected order of convergence for the Cahn-Hilliard equation (i.e. even more than $N+1$ ), but then the early stagnation is found as a result of the first-order Euler scheme. For the Navier-Stokes part we find that the order of accuracy is systematically between $N$ and $N+1$, which is a similar error behavior to that seen in Chapter 6 for the BDF2 method and the entropy-stable scheme. However, the final errors are smaller for the Entropy-stable/BDF2 scheme.

The convergence study confirms that the error behavior of the scheme is the expected one for smooth solutions. 
Table 8.2: Two-phase solver: manufactured solution (8.37) convergence analysis: we use five meshes with $4^{2}, 6^{2}, 8^{2}, 12^{2}$ and $16^{2}$ meshes, and $N=2,3,4$ and 5 . The final time is $t_{F}=0.1$, and we use the IMEX scheme with $\Delta t=5 \cdot 10^{-5}$

\begin{tabular}{llllllllll}
\hline & Mesh & $c$ error & order & $\rho u$ error & order & $\rho v$ error & order & $p$ error & order \\
\hline $\mathrm{N}=2$ & $4^{2}$ & $5.85 \mathrm{E}-04$ & - & $4.13 \mathrm{E}-02$ & - & $4.12 \mathrm{E}-02$ & - & $2.24 \mathrm{E}-01$ & - \\
& $6^{2}$ & $2.61 \mathrm{E}-04$ & 1.99 & $1.50 \mathrm{E}-02$ & 2.49 & $1.50 \mathrm{E}-02$ & 2.49 & $9.09 \mathrm{E}-02$ & 2.22 \\
& $8^{2}$ & $9.83 \mathrm{E}-05$ & 3.39 & $7.17 \mathrm{E}-03$ & 2.58 & $7.17 \mathrm{E}-03$ & 2.58 & $4.71 \mathrm{E}-02$ & 2.29 \\
& $12^{2}$ & $2.45 \mathrm{E}-05$ & 3.43 & $2.43 \mathrm{E}-03$ & 2.67 & $2.43 \mathrm{E}-03$ & 2.67 & $1.79 \mathrm{E}-02$ & 2.39 \\
& $16^{2}$ & $9.93 \mathrm{E}-06$ & 3.13 & $1.10 \mathrm{E}-03$ & 2.76 & $1.10 \mathrm{E}-03$ & 2.76 & $8.71 \mathrm{E}-03$ & 2.50 \\
$\mathrm{~N}=3$ & $4^{2}$ & $8.62 \mathrm{E}-05$ & - & $4.23 \mathrm{E}-03$ & - & $4.24 \mathrm{E}-03$ & - & $2.61 \mathrm{E}-02$ & - \\
& $6^{2}$ & $1.43 \mathrm{E}-05$ & 4.44 & $9.90 \mathrm{E}-04$ & 3.58 & $9.90 \mathrm{E}-04$ & 3.59 & $7.04 \mathrm{E}-03$ & 3.23 \\
& $8^{2}$ & $3.52 \mathrm{E}-06$ & 4.87 & $3.40 \mathrm{E}-04$ & 3.72 & $3.40 \mathrm{E}-04$ & 3.72 & $2.69 \mathrm{E}-03$ & 3.34 \\
& $12^{2}$ & $1.41 \mathrm{E}-06$ & 2.26 & $7.11 \mathrm{E}-05$ & 3.86 & $7.12 \mathrm{E}-05$ & 3.86 & $6.55 \mathrm{E}-04$ & 3.48 \\
& $16^{2}$ & $1.39 \mathrm{E}-06$ & 0.05 & $2.27 \mathrm{E}-05$ & 3.97 & $2.27 \mathrm{E}-05$ & 3.97 & $2.31 \mathrm{E}-04$ & 3.62 \\
$\mathrm{~N}=4$ & $4^{2}$ & $3.81 \mathrm{E}-06$ & - & $3.47 \mathrm{E}-04$ & - & $3.43 \mathrm{E}-04$ & - & $2.37 \mathrm{E}-03$ & - \\
& $6^{2}$ & $1.66 \mathrm{E}-06$ & 2.05 & $5.26 \mathrm{E}-05$ & 4.65 & $5.26 \mathrm{E}-05$ & 4.62 & $4.06 \mathrm{E}-04$ & 4.36 \\
& $8^{2}$ & $1.39 \mathrm{E}-06$ & 0.60 & $1.35 \mathrm{E}-05$ & 4.74 & $1.35 \mathrm{E}-05$ & 4.73 & $1.12 \mathrm{E}-04$ & 4.48 \\
& $12^{2}$ & $1.39 \mathrm{E}-06$ & 0.01 & $1.90 \mathrm{E}-06$ & 4.83 & $1.91 \mathrm{E}-06$ & 4.83 & $1.74 \mathrm{E}-05$ & 4.59 \\
& $16^{2}$ & $1.39 \mathrm{E}-06$ & 0.00 & $4.63 \mathrm{E}-07$ & 4.91 & $4.65 \mathrm{E}-07$ & 4.91 & $4.52 \mathrm{E}-06$ & 4.68 \\
$\mathrm{~N}=5$ & $4^{2}$ & $1.87 \mathrm{E}-06$ & - & $2.33 \mathrm{E}-05$ & - & $2.43 \mathrm{E}-05$ & - & $1.91 \mathrm{E}-04$ & - \\
& $6^{2}$ & $1.39 \mathrm{E}-06$ & 0.74 & $2.36 \mathrm{E}-06$ & 5.64 & $2.37 \mathrm{E}-06$ & 5.75 & $2.07 \mathrm{E}-05$ & 5.48 \\
& $8^{2}$ & $1.39 \mathrm{E}-06$ & 0.00 & $4.60 \mathrm{E}-07$ & 5.69 & $4.62 \mathrm{E}-07$ & 5.68 & $4.21 \mathrm{E}-06$ & 5.54 \\
& $12^{2}$ & $1.39 \mathrm{E}-06$ & 0.00 & $6.28 \mathrm{E}-08$ & 4.91 & $6.29 \mathrm{E}-08$ & 4.92 & $4.61 \mathrm{E}-07$ & 5.45 \\
& $16^{2}$ & $1.39 \mathrm{E}-06$ & 0.00 & $4.26 \mathrm{E}-08$ & 1.34 & $4.26 \mathrm{E}-08$ & 1.35 & $2.10 \mathrm{E}-07$ & 2.74 \\
\hline
\end{tabular}

\subsubsection{Two-phase simulations: pipe simulations}

In this section we focus on more practical test cases, relevant for the oil and gas industry. In particular, we simulate the flow in horizontal pipes with different superficial velocities (8.29) at the inflow. Depending on the superficial velocities values, we obtain different flow regimes. This test case follows the one proposed in Xie et al. [2017] and agrees with the experimental results of Taitel and Dukler [1976].

The flow pattern map of Taitel \& Dukler (see Fig. 8.3) classifies the flow regimes as stratified flow, slug flow, dispersed bubble flow and annular flow. In a stratified flow the phases are completely separated with gas in the upper part and liquid in the lower part of the pipe. In a slug flow waves in the flow reach the top of the pipe, eventually closing the gas path in the top. In a dispersed bubble flow, small bubbles are present in the flow, and are dispersed everywhere in the cross section. In an annular flow the liquid forms a coat all around the pipe walls. From all the four regimes, we have 


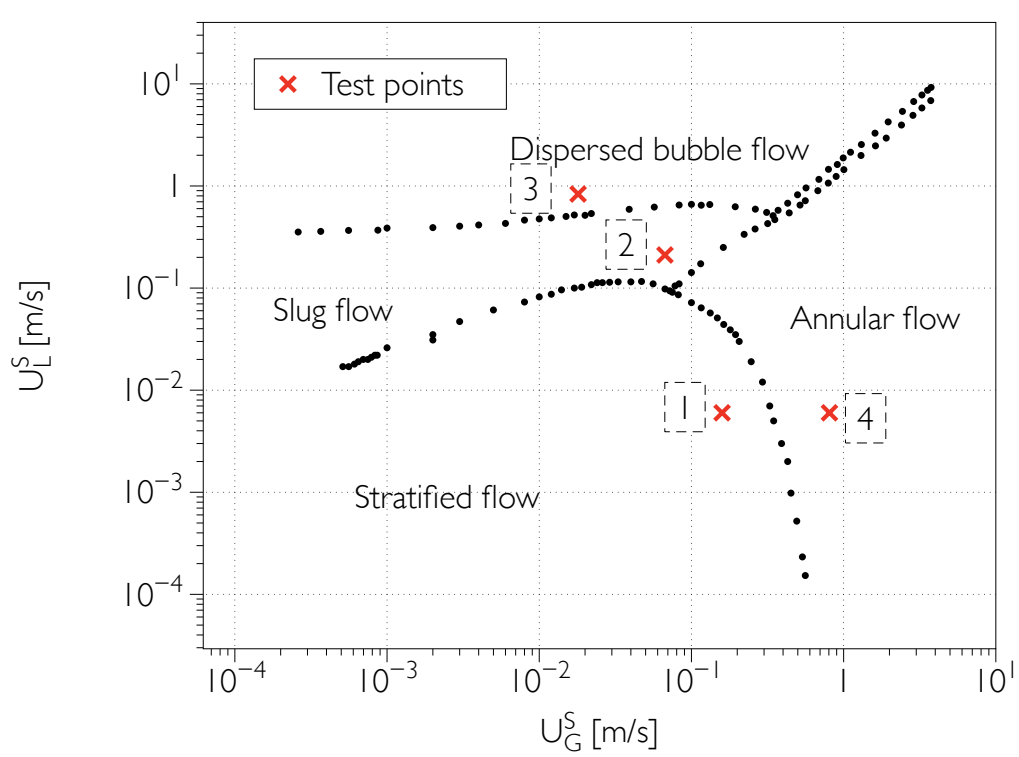

Figure 8.3: Two-phase solver: flow pattern map of the two-phase flow in a horizontal pipe with a diameter of 1 meter. The data has been extracted from Taitel and Dukler [1976]

already reproduced the annular flow in Chapter 6, with the entropy-stable two-phase solver.

Following Xie et al. [2017], we define four test cases that reproduce the four flow regimes, denoted with red crosses in Fig. 8.3, and whose superficial velocities are given in Table 8.3. The domain for the simulations consists of a pipe with length $L=1 \mathrm{~m}$ and diameter $D=0.1 \mathrm{~m}$. The physical domain is discretised using a mesh of 8220 hexahedral elements and the solution is approximated by order $N=3$ polynomials. The physical parameters are summarized in Table 8.4. The mobility is taken from Xie et al. [2017], and the interface width covers approximately three mesh points.

Table 8.3: Two-phase solver: superficial velocities (in $\mathrm{m} / \mathrm{s}$ ) and theoretical flow regime in horizontal pipe test case

\begin{tabular}{llll}
\hline Test case & Flow regime & $U_{G}^{S}$ & $U_{L}^{S}$ \\
\hline 1 & Stratified flow & 0.7 & 0.06 \\
\hline 2 & Slug flow & 0.25 & 2 \\
\hline 3 & Dispersed bubble flow & 0.05 & 8 \\
\hline 4 & Annular flow & 4.9 & 0.06 \\
\hline
\end{tabular}


Table 8.4: Two-phase solver: physical parameters of the pipe flow

\begin{tabular}{lllllllll}
\hline$\rho_{1}$ & $\rho_{2}\left(\mathrm{~kg} / \mathrm{m}^{3}\right)$ & $\eta_{1}$ & $\eta_{2}(\mathrm{~Pa} \cdot \mathrm{s})$ & $\varepsilon(\mathrm{m})$ & $M_{0}(\mathrm{~s})$ & $c_{0}^{2}\left(\mathrm{~m} / \mathrm{s}^{2}\right)^{2}$ & $\sigma(\mathrm{N} / \mathrm{m})$ & $g\left(\mathrm{~m} / \mathrm{s}^{2}\right)$ \\
\hline 1.0 & 5.0 & $5 \cdot 10^{-3}$ & $10^{-2}$ & 0.0424 & 0.1886 & $1.0 \mathrm{E} 3$ & $2.5 \cdot 10^{-4}$ & 1.0 \\
\hline
\end{tabular}

The time step chosen for the simulations is $\Delta t=10^{-5}$. A no-slip boundary condition (8.32) is enforced at the pipe walls (with a contact angle of $90^{\circ}$ ) while a velocity inflow boundary condition (8.23) and a constant pressure outflow boundary condition (8.30) are used. It should be noticed that the flow regime inflow is considered layered (see Fig. 8.1). The initial condition for all the simulations propagates the initial condition along the $Z$ axis, with a small wave-like perturbation with $Z$, to introduce asymmetry.

Fig. 8.4 shows a clip of $c$ values under 0.5 coloured by density for the four test

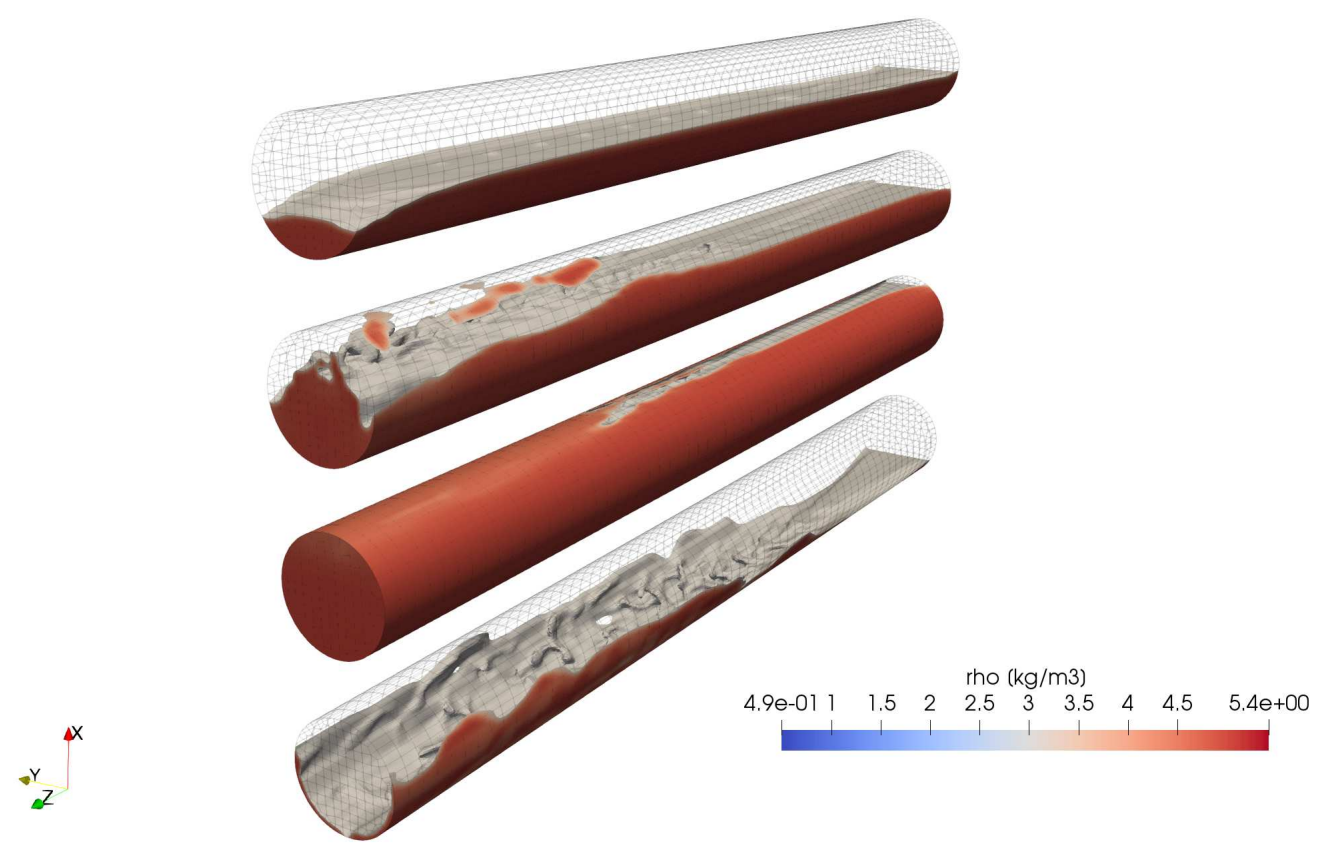

Figure 8.4: Two-phase solver: results of test cases 1-4 (organised top to bottom) at $t=4 \mathrm{~s}$. Stratified, Slug, Dispersed Bubble and Annular Flow regimes can be seen

cases shown in Fig. 8.3 (organised from 1 to 4 top to bottom) at $t=4$ s. In Fig. 8.5 we show the density contour in a $Z$-normal slice at $L / D=8$. As can be seen, the flow regimes are correctly predicted. Note that ignoring the effect of the hydrostatic pressure at the outflow when imposing a constant outlet pressure induces a velocity in the negative $X$ direction that curves the interface. This can be easily seen in the stratified flow regime (Test case 1 ). 


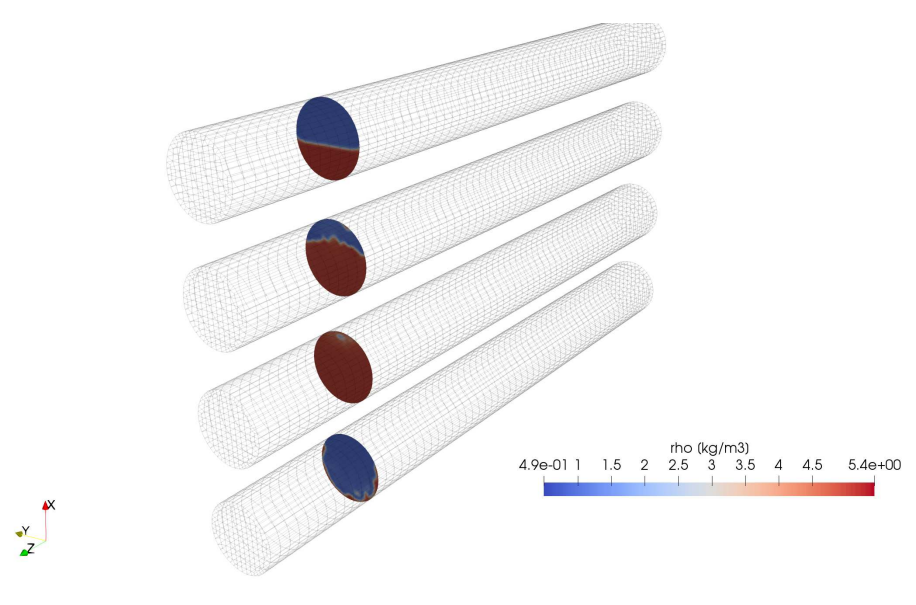

(a) Selection of slices to analyze

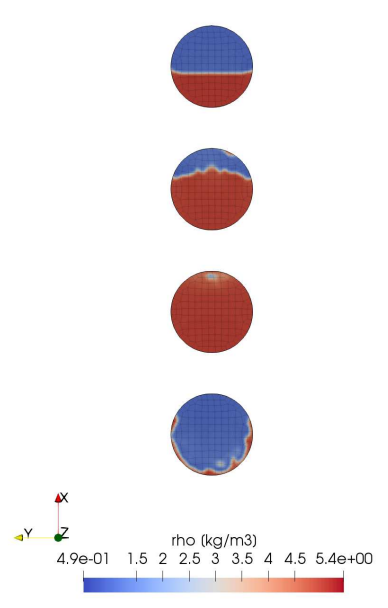

(b) Representation of the density at $z / D=8$

Figure 8.5: Two-phase solver: representation of the density contour in a pipe cross section. The four regimes (stratified, slug, dispersed bubble, and annular) are represented, along with the cross section of the mesh

\subsubsection{Two-phase simulations: enhanced robustness of the discretization}

The simulations of the flow regime prediction in pipes are under-resolved with the mesh used (except the stratified flow). This was seen already in Fig. 6.9 when studying the annular flow. Thus, this numerical experiment can be used to compare the robustness of the discretization developed in this work with the standard DG method (i.e. without the use of split-forms) with the more robust Gauss points. Although none of the two discretizations are entropy-stable (see Chapter 3), we see that the use of the split-form developed in Chapter 4 also enhances the robustness of the multiphase solver.

We solve the four regimes with the two schemes (split-form/Gauss-Lobatto and standard/Gauss). In Fig. 8.6 we represent the maximum residual of the concentration $\left(\max _{e} C_{t}\right)$ with the number of iterations as a representative value of the simulation progress (as a point of reference, the steady-state is $\max _{e} C_{t}=0$ ). Although both methods are stable at the early stages, once the growth of fluid instabilities obtains smaller structures, the standard DG scheme with Gauss points crashes, while the splitform developed in this work is stable and allows us to obtain a final solution. Both approaches are stable for the smoother stratified flow. 


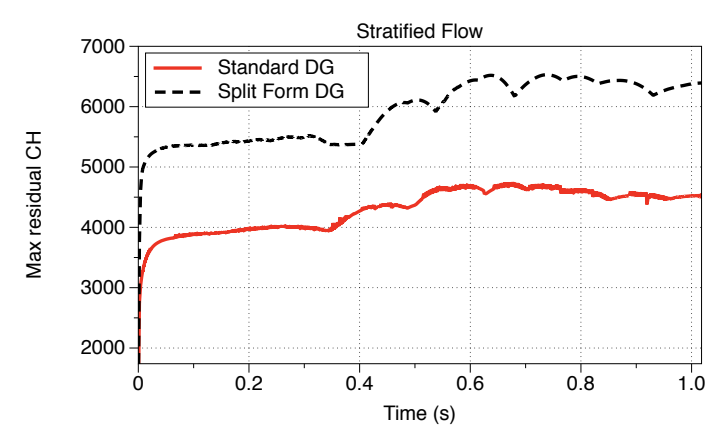

(a) Stratified flow

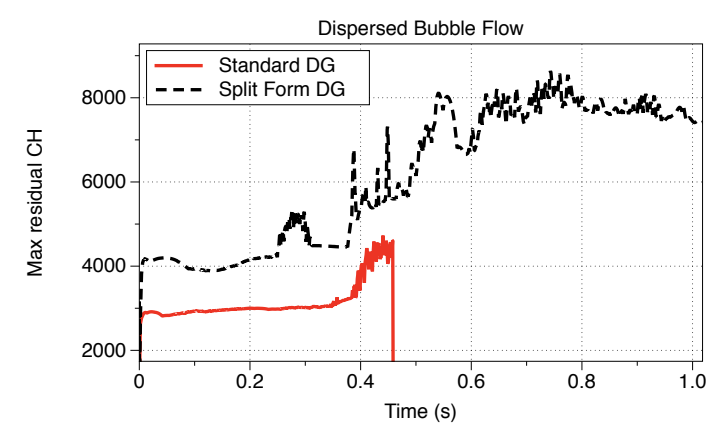

(c) Dispersed bubble flow

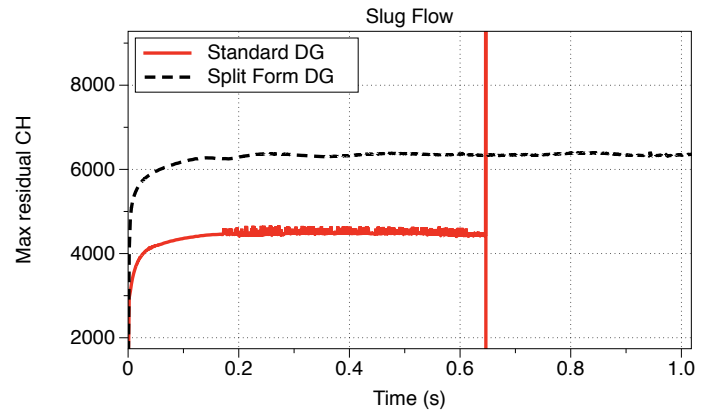

(b) Slug flow

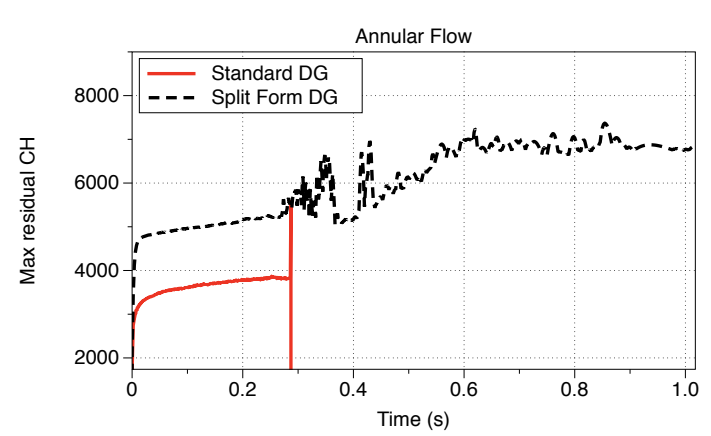

(d) Annular flow

Figure 8.6: Two-phase solver: maximum value of the concentration $c_{1}$ residual $\left(c_{1, t}\right)$ with time, for the four pipe flow regimes studied. We compare the standard DG scheme with Gauss points (solid, red line) to split-form DG (dashed, black), and we see that the standard/Gauss DG scheme is stable only for the smooth stratified flow regime. Otherwise, the standard/Gauss DG scheme crashes after some iterations, while the split-form DG is always stable for the simulations presented here

We conclude that although there is no stability proof, this numerical experiment encourages the use of the split-form scheme.

\subsection{Three-phase simulations}

In this section we validate and test the three-phase solver. We solve a manufactured solution in Sec. 8.5.1, a two-dimensional channel in Sec. 8.5.2, a three-dimensional pipe in annular flow regime in Sec. 8.5.3, and a "T" pipe in Sec. 8.5.4. 


\subsubsection{Three-phase simulations: manufactured solution}

First, we perform a manufactured solution analysis to assess the accuracy of the threephase solver for smooth problems. We extend the two-dimensional manufactured solution used for the two-phase flow in Chapter 6 and Sec. 8.4.1 of this chapter with the manufactured solution of the three-phase solver of Chapter 7.

We seek the solution

$$
\begin{aligned}
c_{1,0}(x, y ; t) & =\frac{1}{3}(1+\cos (\pi x) \sin (\pi y) \sin (t)), \\
c_{2,0}(x, y ; t) & =\frac{1}{3}(1+\cos (\pi x) \sin (\pi y) \sin (1.2 t)), \\
u_{0}(x, y ; t) & =2 \sin (\pi x) \cos (\pi z) \sin (t), \\
v_{0}(x, y ; t) & =-2 \cos (\pi x) \sin (\pi y) \sin (t), \\
p_{0}(x, y ; t) & =2 \sin (\pi x) \sin (\pi z) \cos (t),
\end{aligned}
$$

which requires an appropriate source term to the right-hand side of the equation, not presented here. The configuration is similar to the two-phase manufactured solution, where the domain is $(x, y) \in[-1,1]^{2}$, and the final time is $t_{F}=0.1$. The physical parameters have been adapted from Dong [2018b] and they are given in Table 8.5.

Table 8.5: Three-phase solver: list of the parameter values used with the manufactured solution (8.38)

\begin{tabular}{lllllll}
\hline$\rho_{1}$ & $\rho_{2}$ & $\rho_{3}\left(\mathrm{~kg} / \mathrm{m}^{3}\right)$ & $\eta_{1}$ & $\eta_{2}$ & $\eta_{3}(\mathrm{~Pa} \cdot \mathrm{s})$ & $\varepsilon(\mathrm{m})$ \\
\hline 1.0 & 3.0 & 2.0 & $1.0 \mathrm{E}-3$ & $1.0 \mathrm{E}-3$ & $1.0 \mathrm{E}-3$ & $1 / \sqrt{2}$ \\
\hline$M_{0}$ & $c_{0}\left(\mathrm{~m} / \mathrm{s}^{2}\right)$ & $\sigma_{12}$ & $\sigma_{13}$ & $\sigma_{23}(\mathrm{~N} / \mathrm{m})$ & & \\
\hline $1.134 \mathrm{E}-2$ & $1.0 \mathrm{E} 3$ & $6.236 \mathrm{E}-3$ & $7.265 \mathrm{E}-3$ & $8.165 \mathrm{E}-3$ & & \\
\hline
\end{tabular}

We perform first a polynomial order convergence study with a Cartesian mesh of $4^{2}$ elements, and with the polynomial order ranging from $N=2$ to 10. In Fig. 8.7 we represent the $\mathrm{L}^{2}$ errors on the five variables $\left(c_{1}, c_{2}, \rho u, \rho v\right.$, and $\left.p\right)$, for two time-step sizes, $\Delta t=10^{-4}$ and $10^{-5}$. We find that the error behavior is similar to the twophase solver, where exponential accuracy is obtained for lower polynomial orders, as expected, and then the error stagnation associated to the time discretization is anticipated for the two concentrations, as a result of the first order IMEX scheme.

Finally, we perform the mesh convergence study, where we use meshes with $4^{2}, 6^{2}$, $8^{2}, 12^{2}$ and $16^{2}$ elements, and vary the polynomial order from $N=2$ to $N=5$. The $\mathrm{L}^{2}$ errors and the convergence rates are written in Table 8.6. We observe that for the 


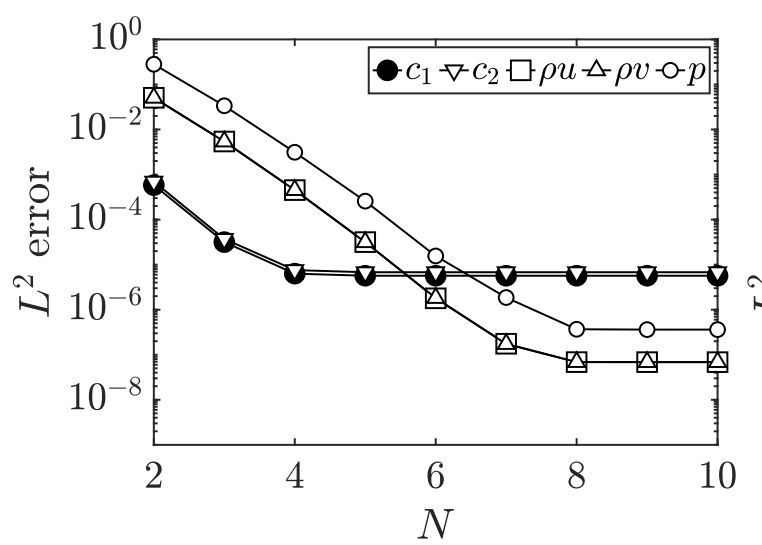

(a) $\Delta t=10^{-4}$

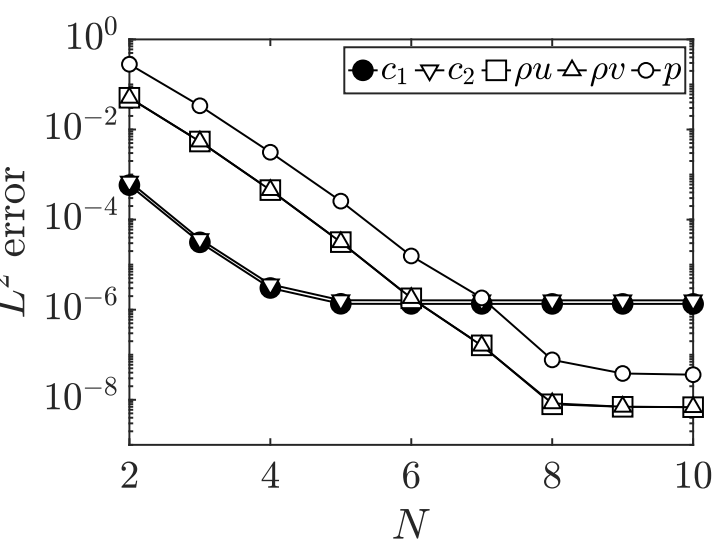

(b) $\Delta t=10^{-5}$

Figure 8.7: Three-phase solver: polynomial order convergence study of the manufactured solution (8.38). We represent the $\mathrm{L}^{2}$ errors of the two concentrations $c_{1}$ and $c_{2}$, $x$ - and $y$-momentum, and pressure. The polynomial order ranges from 2 to 10 , and we integrate in time until $t_{F}=0.1$ with two time step sizes: $\Delta t=10^{-4}$ and $10^{-5}$. All physical parameters are given in Table 8.5

Table 8.6: Three-phase solver: manufactured solution (8.38) convergence analysis: we use $4^{3}, 8^{3}$, and $16^{3}$ meshes, and $N=2,3,4$ and 5 . The final time is $t_{F}=0.1$, and we use the IMEX scheme with $\Delta t=5 \cdot 10^{-5}$

\begin{tabular}{|c|c|c|c|c|c|c|c|c|c|c|c|}
\hline & Mesh & $c_{1}$ error & order & $c_{2}$ error & order & $\rho u$ error & order & $\rho v$ error & order & $p$ error & order \\
\hline \multirow[t]{5}{*}{$\mathrm{N}=2$} & $4^{2}$ & $5.82 \mathrm{E}-04$ & - & $6.97 \mathrm{E}-04$ & - & $5.06 \mathrm{E}-02$ & - & $5.06 \mathrm{E}-02$ & - & $2.81 \mathrm{E}-01$ & - \\
\hline & $6^{2}$ & $1.60 \mathrm{E}-04$ & 3.19 & $1.91 \mathrm{E}-04$ & 3.19 & $1.92 \mathrm{E}-02$ & 2.39 & $1.92 \mathrm{E}-02$ & 2.39 & 1.17E-01 & 2.15 \\
\hline & $8^{2}$ & 5.97E-05 & 3.42 & 7.15E-05 & 3.41 & 9.38E-03 & 2.49 & 9.38E-03 & 2.49 & 6.19E-02 & 2.22 \\
\hline & $12^{2}$ & $1.61 \mathrm{E}-05$ & 3.23 & $1.93 \mathrm{E}-05$ & 3.23 & $3.28 \mathrm{E}-03$ & 2.59 & $3.28 \mathrm{E}-03$ & 2.59 & $2.40 \mathrm{E}-02$ & 2.34 \\
\hline & $16^{2}$ & $6.65 \mathrm{E}-06$ & 3.07 & 7.97E-06 & 3.07 & $1.51 \mathrm{E}-03$ & 2.70 & $1.51 \mathrm{E}-03$ & 2.70 & $1.18 \mathrm{E}-02$ & 2.47 \\
\hline \multirow[t]{5}{*}{$\mathrm{N}=3$} & $4^{2}$ & 3.13E-05 & - & 3.69E-05 & - & $5.41 \mathrm{E}-03$ & - & $5.41 \mathrm{E}-03$ & - & 3.36E-02 & - \\
\hline & $6^{2}$ & 3.67E-06 & 5.29 & $4.38 \mathrm{E}-06$ & 5.26 & $1.30 \mathrm{E}-03$ & 3.52 & $1.30 \mathrm{E}-03$ & 3.52 & $9.41 \mathrm{E}-03$ & 3.14 \\
\hline & $8^{2}$ & $1.49 \mathrm{E}-06$ & 3.13 & $1.79 \mathrm{E}-06$ & 3.11 & $4.51 \mathrm{E}-04$ & 3.68 & $4.50 \mathrm{E}-04$ & 3.68 & 3.65E-03 & 3.30 \\
\hline & $12^{2}$ & $1.35 \mathrm{E}-06$ & 0.25 & $1.62 \mathrm{E}-06$ & 0.25 & 9.55E-05 & 3.83 & 9.55E-05 & 3.83 & 9.01E-04 & 3.45 \\
\hline & $16^{2}$ & $1.35 \mathrm{E}-06$ & 0.00 & $1.61 \mathrm{E}-06$ & 0.00 & $3.08 \mathrm{E}-05$ & 3.94 & 3.08E-05 & 3.94 & $3.20 \mathrm{E}-04$ & 3.60 \\
\hline \multirow[t]{5}{*}{$\mathrm{N}=4$} & $4^{2}$ & 3.03E-06 & - & 3.65E-06 & - & 4.52E-04 & - & 4.52E-04 & - & $3.12 \mathrm{E}-03$ & - \\
\hline & $6^{2}$ & $1.39 \mathrm{E}-06$ & 1.93 & $1.66 \mathrm{E}-06$ & 1.94 & 7.10E-05 & 4.56 & 7.10E-05 & 4.57 & 5.47E-04 & 4.30 \\
\hline & $8^{2}$ & $1.35 \mathrm{E}-06$ & 0.10 & $1.62 \mathrm{E}-06$ & 0.10 & $1.85 \mathrm{E}-05$ & 4.67 & $1.85 \mathrm{E}-05$ & 4.67 & $1.52 \mathrm{E}-04$ & 4.46 \\
\hline & $12^{2}$ & $1.35 \mathrm{E}-06$ & 0.00 & $1.61 \mathrm{E}-06$ & 0.00 & 2.67E-06 & 4.77 & 2.67E-06 & 4.77 & 2.37E-05 & 4.58 \\
\hline & $16^{2}$ & $1.35 \mathrm{E}-06$ & 0.00 & $1.61 \mathrm{E}-06$ & 0.00 & 6.59E-07 & 4.87 & $6.59 \mathrm{E}-07$ & 4.87 & $6.20 \mathrm{E}-06$ & 4.67 \\
\hline \multirow[t]{5}{*}{$\mathrm{N}=5$} & $4^{2}$ & $1.35 \mathrm{E}-06$ & - & $1.62 \mathrm{E}-06$ & - & 3.17E-05 & - & $3.15 \mathrm{E}-05$ & - & $2.57 \mathrm{E}-04$ & - \\
\hline & $6^{2}$ & $1.35 \mathrm{E}-06$ & 0.01 & $1.61 \mathrm{E}-06$ & 0.01 & $3.18 \mathrm{E}-06$ & 5.67 & $3.18 \mathrm{E}-06$ & 5.66 & 2.83E-05 & 5.44 \\
\hline & $8^{2}$ & $1.35 \mathrm{E}-06$ & 0.00 & $1.61 \mathrm{E}-06$ & 0.00 & $6.23 \mathrm{E}-07$ & 5.67 & $6.22 \mathrm{E}-07$ & 5.67 & 5.79E-06 & 5.52 \\
\hline & $12^{2}$ & $1.35 \mathrm{E}-06$ & 0.00 & $1.61 \mathrm{E}-06$ & 0.00 & $6.48 \mathrm{E}-08$ & 5.58 & $6.47 \mathrm{E}-08$ & 5.58 & $5.81 \mathrm{E}-07$ & 5.67 \\
\hline & $16^{2}$ & $1.35 \mathrm{E}-06$ & 0.00 & $1.61 \mathrm{E}-06$ & 0.00 & $1.50 \mathrm{E}-08$ & 5.08 & $1.50 \mathrm{E}-08$ & 5.08 & $1.15 \mathrm{E}-07$ & 5.62 \\
\hline
\end{tabular}


Table 8.7: Three-phase solver: list of the parameter values used for the twodimensional three-phase channel

\begin{tabular}{|c|c|c|c|c|c|c|}
\hline$\rho_{1}$ & $\rho_{2}$ & $\rho_{3}\left(\mathrm{~kg} / \mathrm{m}^{3}\right)$ & $\eta_{1}$ & $\eta_{2}$ & $\eta_{3}(\mathrm{~Pa} \cdot \mathrm{s})$ & $\varepsilon(\mathrm{m})$ \\
\hline 1.0 & 5.0 & 0.8 & $5.0 \mathrm{E}-3$ & $1.0 \mathrm{E}-2$ & $1.0 \mathrm{E}-2$ & 0.0424 \\
\hline$M_{0}$ & $c_{0}\left(\mathrm{~m} / \mathrm{s}^{2}\right)$ & $\sigma_{12}$ & $\sigma_{13}$ & $\sigma_{23}(\mathrm{~N} / \mathrm{m})$ & & \\
\hline $1.0 \mathrm{E}-4$ & $1.0 \mathrm{E} 3$ & $2.5 \mathrm{E}-4$ & $2.5 \mathrm{E}-4$ & $2.5 \mathrm{E}-4$ & & \\
\hline
\end{tabular}

two concentrations, the convergence rates are always between $N$ and $N+2$, for $N=2$ and $N=3$, and due to the early stagnation, as in the two-phase solver, we cannot evaluate the convergence rates for $N=4$ and $N=5$. For the rest of the variables, as in the two-phase solver, we find that the convergence rates are always between $N$ and $N+1$, as expected.

Overall, we confirm that the scheme and its implementation are accurate for the industrial applications.

\subsubsection{Three-phase simulations: two-dimensional channel}

In this section we study the three-phase flow obtained in a two-dimensional channel $\Omega=[0,10] \times[-0.5,0.5]$. The configuration is an extension to three-phase of the two-phase channel studied in Xie et al. [2017]. At the inlet, we introduce a heavy fluid (Phase 2, red) on the top, and a light fluid (Phase 3, black) on the bottom, both immersed in Phase 1, with medium density. The channel features a vertical gravity, such that the heavy fluid tends to fall to the bottom side of the domain, while the light fluid rises. The physical parameters are given in Table 8.7. At the inlet, we impose the inflow boundary condition,

$$
\begin{aligned}
c_{1} & =1-c_{2}-c_{3} \\
c_{2} & =\frac{1}{2}\left(1+\tanh \left(\frac{z-0.3}{\varepsilon}\right)\right), \\
c_{3} & =\frac{1}{2}\left(1-\tanh \left(\frac{z+0.3}{\varepsilon}\right)\right), \\
u & =1-4 z^{2}, \\
v & =w=0, \\
p & =p_{\text {in }},
\end{aligned}
$$

and the initial condition is $c_{1}=1, c_{2}=c_{3}=0, u=1-4 z^{2}$, and $v=w=p=0$. The computational domain $\Omega$ is divided into $15 \times 60$ elements (which is the coarsest 
Table 8.8: Three-phase solver: list of the parameter values used for the three-phase annular flow

\begin{tabular}{lllllll}
\hline$\rho_{1}$ & $\rho_{2}$ & $\rho_{3}\left(\mathrm{~kg} / \mathrm{m}^{3}\right)$ & $\eta_{1}$ & $\eta_{2}$ & $\eta_{3}(\mathrm{~Pa} \cdot \mathrm{s})$ & $\varepsilon(\mathrm{m})$ \\
\hline 0.5 & 1.0 & 5.0 & $1.0 \mathrm{E}-3$ & $5.0 \mathrm{E}-3$ & $1.0 \mathrm{E}-2$ & 0.0424 \\
\hline \multicolumn{7}{c}{} \\
\hline$M_{0}$ & $c_{0}\left(\mathrm{~m} / \mathrm{s}^{2}\right)$ & $\sigma_{12}$ & $\sigma_{13}$ & $\sigma_{23}(\mathrm{~N} / \mathrm{m})$ & & \\
\hline $9.428 \mathrm{E}-5$ & $1.0 \mathrm{E} 3$ & $2.5 \mathrm{E}-4$ & $2.5 \mathrm{E}-4$ & $2.5 \mathrm{E}-4$ \\
\hline & & & & & & \\
\hline$V_{s, 1}$ & $V_{s, 2}$ & $V_{s, 3}$ & $V_{s, 12}$ & $V_{s, 23}(\mathrm{~m} / \mathrm{s})$ & & \\
\hline 1.0 & 3.9 & 0.06 & 0.0 & 10.0 & & \\
\hline
\end{tabular}

configuration described in Xie et al. [2017]), and we approximate the solution with $N=5$ polynomials. We use the split-form scheme, and the IMEX time integrator, with $S_{0}=8$, and $\Delta t=3.0 \cdot 10^{-5}$.

In Fig. 8.8 we have represented 10 snapshots of the fluid configuration, corresponding to intervals of 0.3 time units. In red, we have represented the heavy fluid (Phase 2), and the light fluid (Phase 3) in black, whereas the medium density fluid (Phase 1 ) is white. We see that Phase 2 is prone to fall faster at the early stages because of the higher density ratio $\left(\rho_{2} / \rho_{1}=5\right)$, while the rise of Phase 3 is more subtle (although it is more pronounced in the first snapshot), since the density ratio is lower $\left(\rho_{3} / \rho_{1}=0.8\right)$. As a result, Phase 3 forms a film in the lower part of the channel, which is then broken by droplets of Phase 2 between $x=4$ and $x=6$. These droplets are a result of the Rayleigh-Taylor instability between fluids 1 and 2 in the upper part, which periodically creates droplets of Phase 2 which fall to the lower part of the channel as a result of gravitational forces. As a result, we find that the heavy fluid accumulates in the lower part of the channel, while the light fluid is scattered into bubbles which leave the domain if they are big enough, whereas the smaller ones dissolve into Phase 1.

\subsubsection{Three-phases simulations: three-dimensional annular flow}

In this section, we include a third phase to the annular flow simulation performed for the two-phase flow solver (the entropy-stable version in Sec. 6.6.5, and the splitform version of this chapter in Sec. 8.4.2). Therefore, to the initial configuration with $\rho_{2}=1$ and $\rho_{3}=5$, we add a lighter third phase with $\rho_{1}=0.5$. The inflow configuration follows the description in Fig. 8.1. The physical parameters including superficial and slip velocities are given in Table 8.8. We maintain the mesh used for 

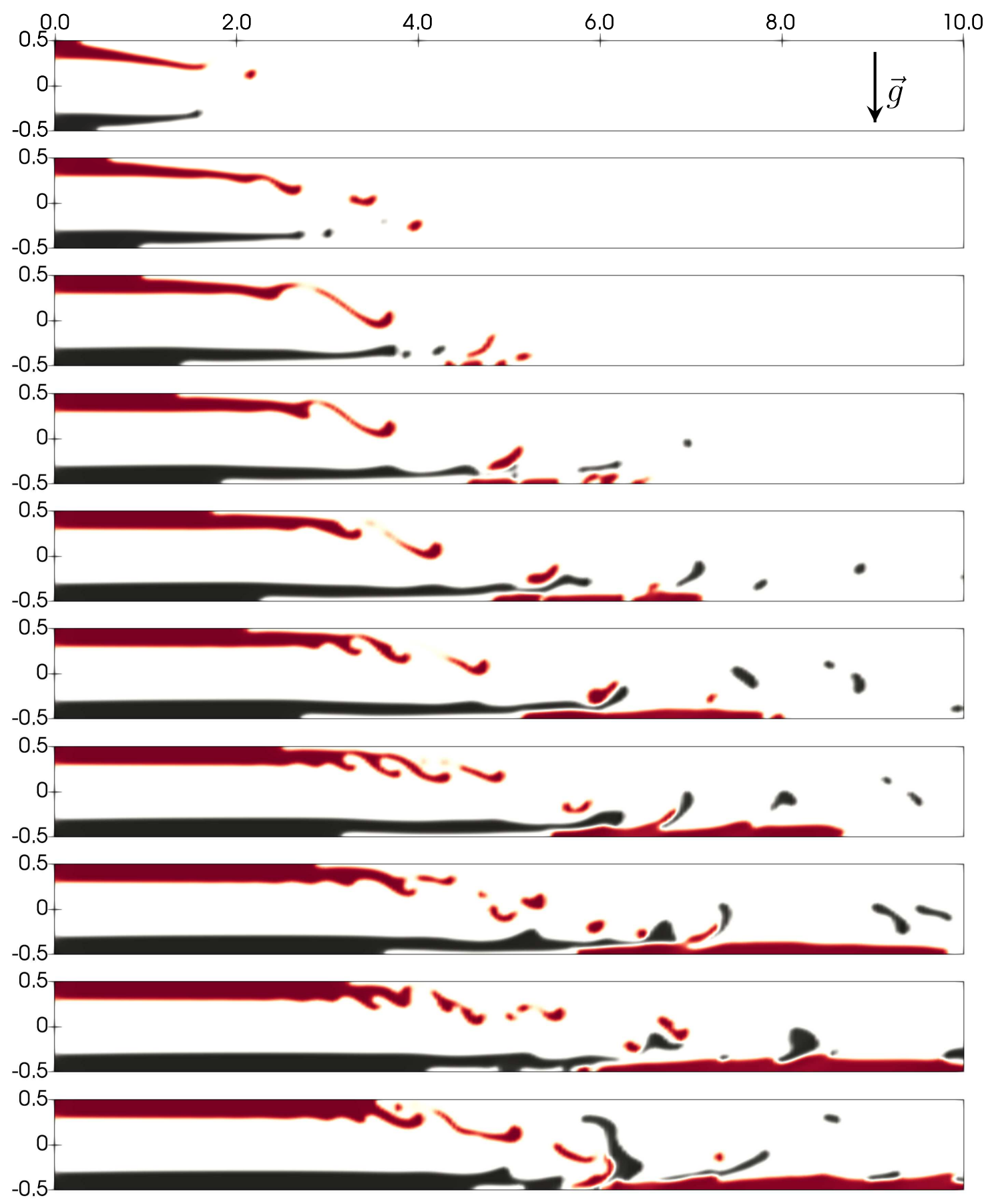

Figure 8.8: Three-phase solver: snapshots of the fluid evolution of the three-phase channel flow. We have represented the heavy fluid (Phase 2) in red, the light fluid (Phase 3) in black, and the medium density fluid (Phase 1) in white 
Table 8.9: Three-phase solver: list of the parameter values used for the three-phase "T" simulation

\begin{tabular}{lllllll}
\hline$\rho_{1}$ & $\rho_{2}$ & $\rho_{3}\left(\mathrm{~kg} / \mathrm{m}^{3}\right)$ & $\eta_{1}$ & $\eta_{2}$ & $\eta_{3}(\mathrm{~Pa} \cdot \mathrm{s})$ & $\varepsilon(\mathrm{m})$ \\
\hline 5.0 & 1.0 & 0.2 & $1.0 \mathrm{E}-5$ & $2.5 \mathrm{E}-5$ & $5.0 \mathrm{E}-5$ & 0.03 \\
\hline & & & & & & \\
\hline$M_{0}$ & $c_{0}\left(\mathrm{~m} / \mathrm{s}^{2}\right)$ & $\sigma_{12}$ & $\sigma_{13}$ & $\sigma_{23}(\mathrm{~N} / \mathrm{m})$ & & \\
\hline $1.8856 \mathrm{E}-2$ & $1.0 \mathrm{E} 3$ & $2.5 \mathrm{E}-4$ & $2.5 \mathrm{E}-4$ & $2.5 \mathrm{E}-4$ & & \\
\hline
\end{tabular}

the two-phase pipe simulations (see Sec. 8.4.2), and we use $N=3$ order polynomials. We use the IMEX time integrator with $S_{0}=8$ and $\Delta t=10^{-5}$.

We represent the flow configuration in $t=3$ in Fig. 8.9. The light fluid (Phase 1 ) on top is represented in gray, and the heavy fluid (Phase 3) is represented in blue, both immersed in Phase 2 (not represented for clarity). We see that Phase 3 describes an annular flow regime very similar to that seen in the two-phase problem. The additional phase fills the upper space, which falls to the lower part because of its lower density, although the length of the pipe is not enough to see large displacements of it.

\subsubsection{Three-phases simulations: three-dimensional "T" pipe}

Finally, we solve a T-pipe configuration, with two inlets and one outlet. The domain features a straight upper inlet whose length is $3 \mathrm{~m}$, which is then coupled to a $90^{\circ}$ bend whose radius is $3 \mathrm{~m}$. Additionally, the second inlet has a straight $5 \mathrm{~m}$ section, and then another $90^{\circ} / 3 \mathrm{~m}$ bend. Finally, the outlet after the "T" junction is a straight pipe whose length is $6 \mathrm{~m}$. The diameter of the pipe is $D=1 \mathrm{~m}$. The computational mesh used, with 1700 elements, is represented in Fig. 8.10.

The physical parameters are given in Table. 8.9. In the upper inlet, we only inject phases 1 and 2 with superficial velocities $V_{s, 1}=V_{s, 2}=4 \mathrm{~m} / \mathrm{s}$. In the lower inlet, we only inject Phase 3 with superficial velocity $V_{s, 3}=2 \mathrm{~m} / \mathrm{s}$. Additionally, the gravity acceleration is $\vec{g}=-1 \vec{i}$. We use $N=3$ order polynomials, and the IMEX scheme uses $S_{0}=8$ and the time-step size $\Delta t=5 \cdot 10^{-5}$. The initial condition is a steady-state with uniform pressure $p=0$, and with the pipe filled with Phase $3\left(c_{1}=c_{2}=0\right)$.

We represent the evolution of the phases in Fig. 8.11, where we represent Phase 2 in blue, and Phase 3 in gray. The space left is occupied by Phase 1. Initially the pipe is filled with Phase 3, which was chosen because it has the minimum density of the three-phase (therefore is easier for the other two phases to displace it). At the initial stages (Fig. 8.11(b)), we see the advancing front at the upper inlet. We see that Phase 


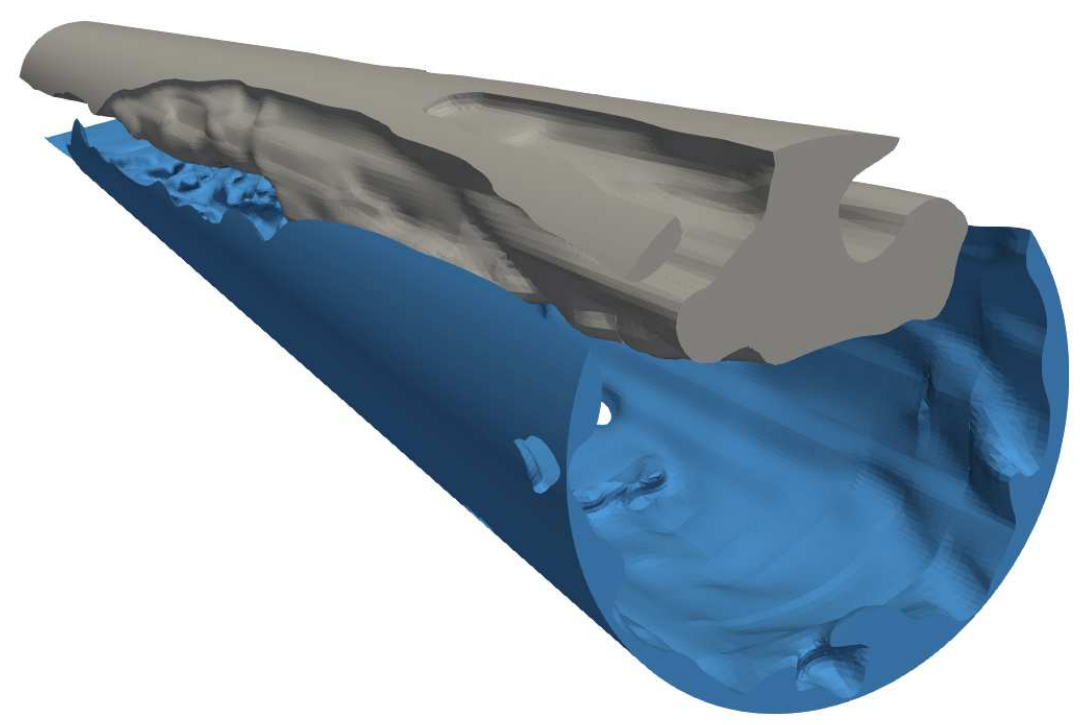

(a) Three-dimensional view

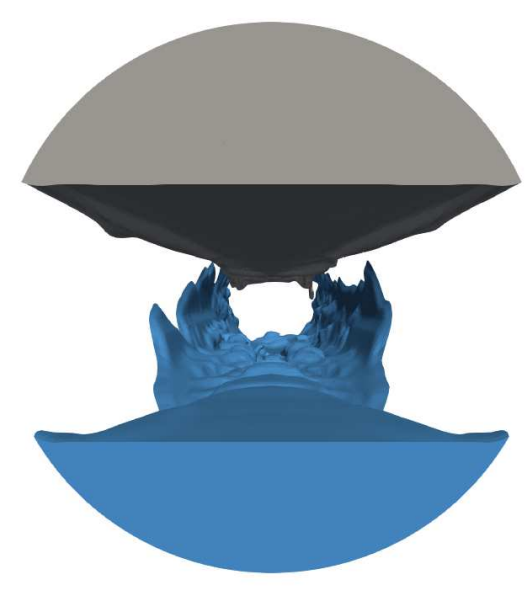

(b) Rear view

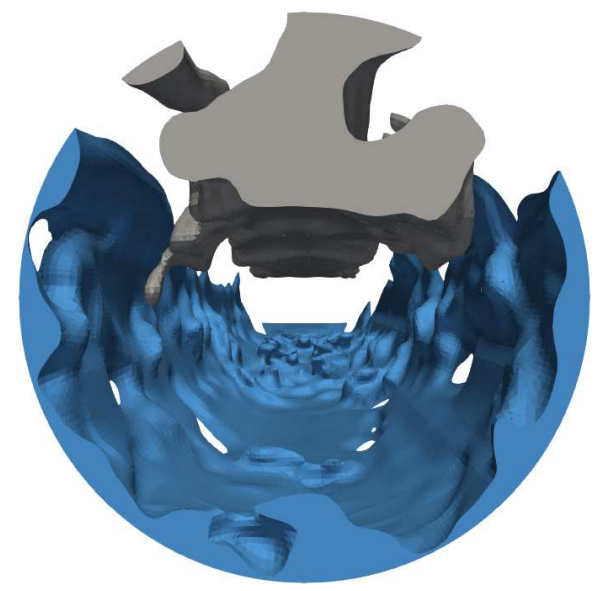

(c) Front view

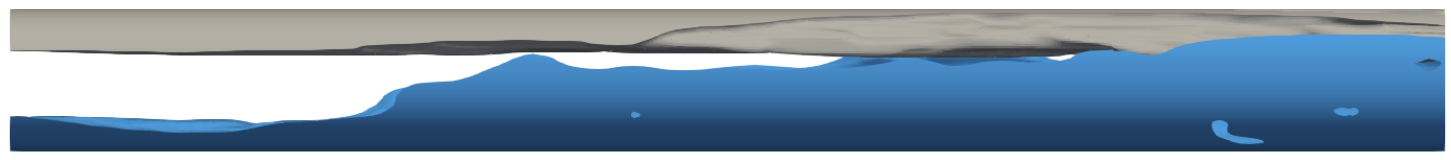

(d) Side view

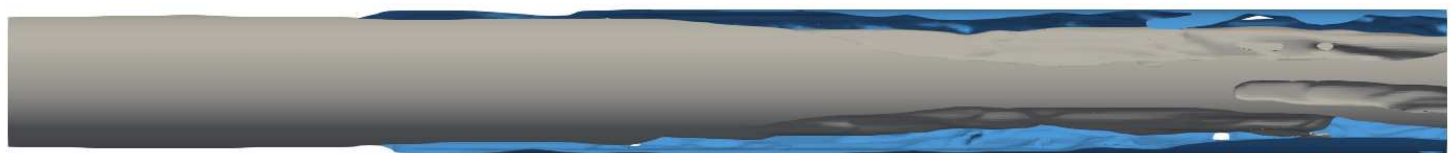

(e) Top view

Figure 8.9: Three-phase solver: three-phase annular flow simulation: the fluid configuration is represented in $t=3$. The heavy fluid (Phase 3 ) is represented in blue, while the light fluid (Phase 1 ) is represented in gray 


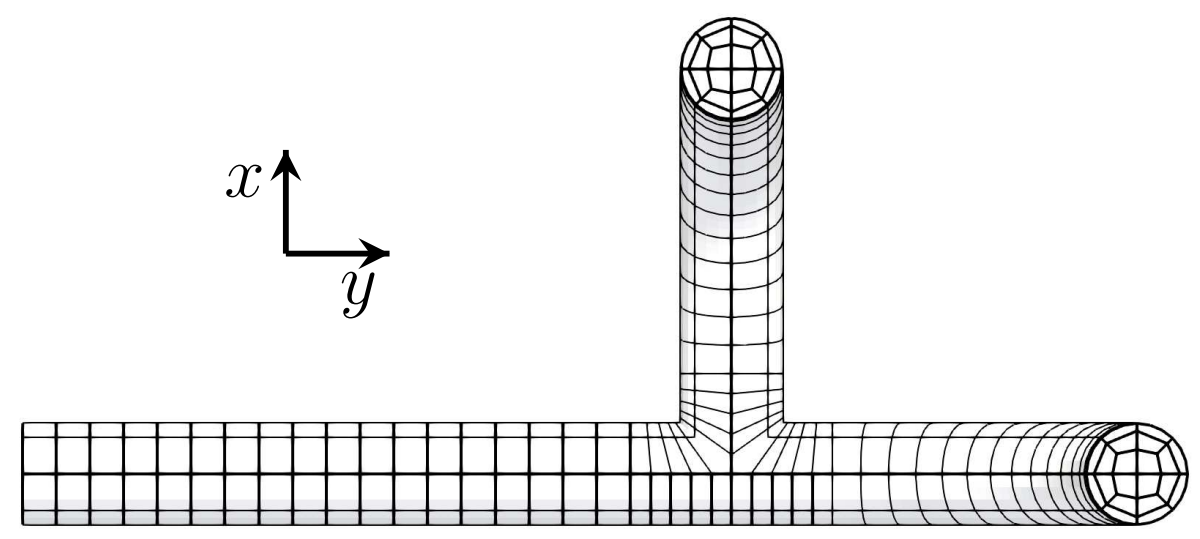

Figure 8.10: Three-phase solver: computational mesh for the T-pipe domain, with 1700 elements

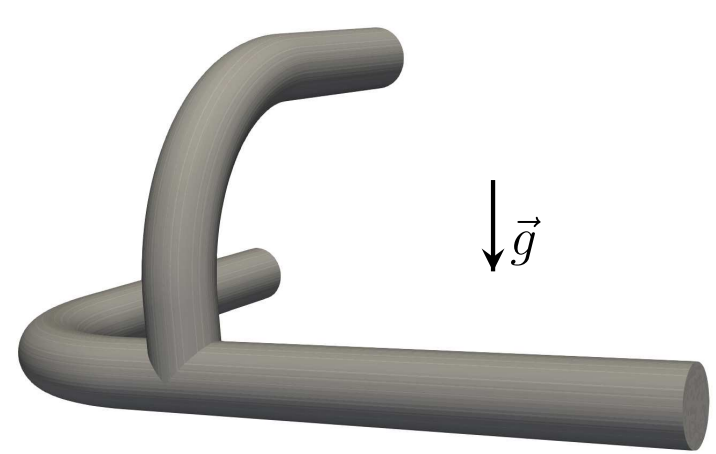

(a) $t=0$

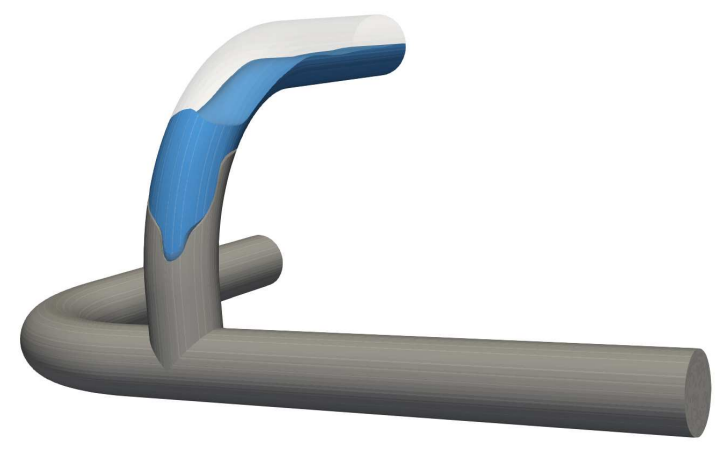

(c) $t=1.0$

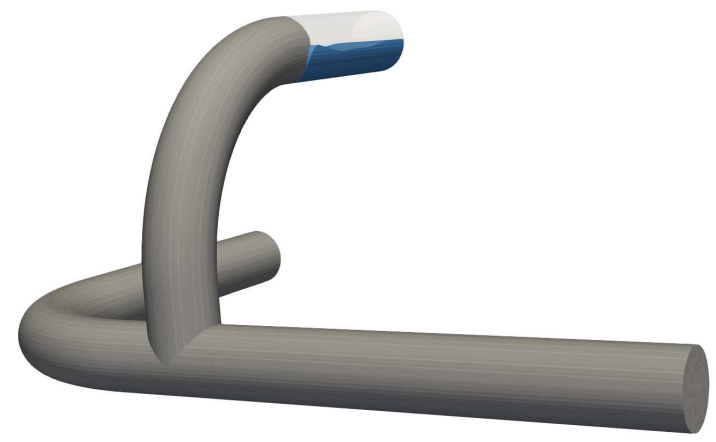

(b) $t=0.5$

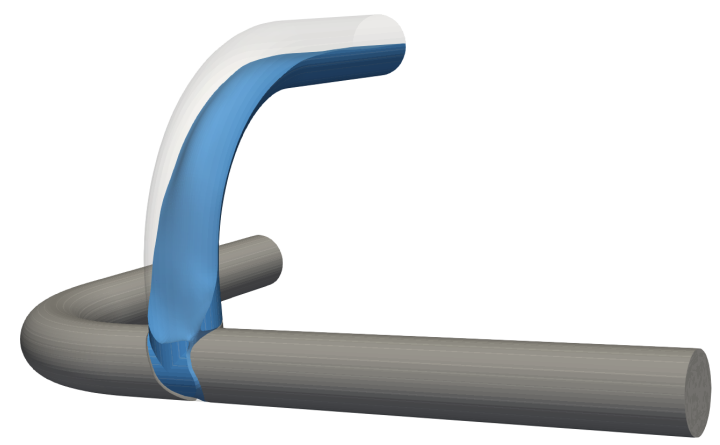

(d) $t=1.5$ 


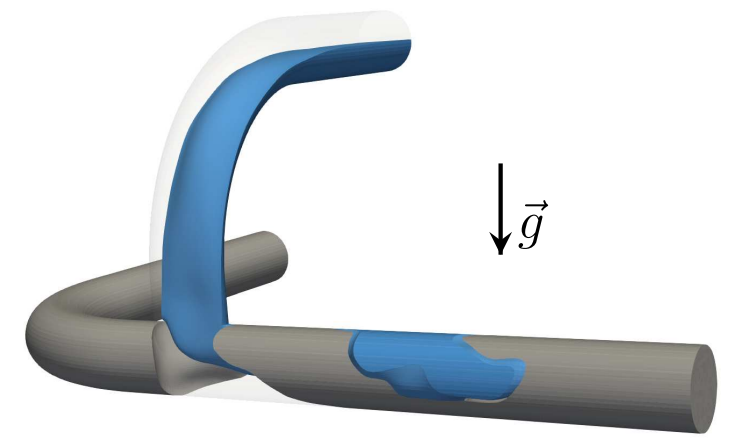

(e) $t=2.0$

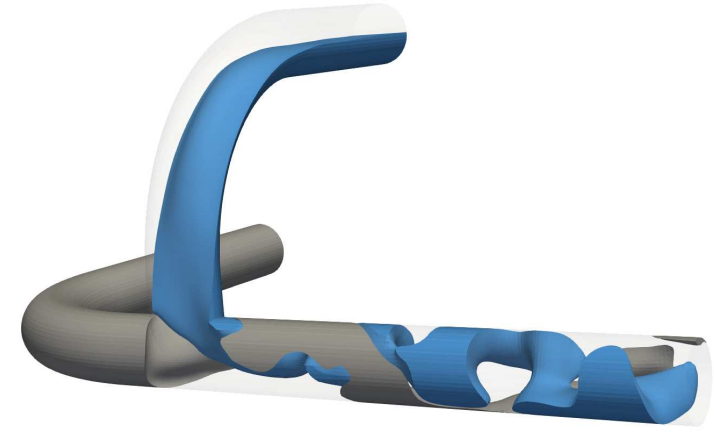

(g) $t=3.0$

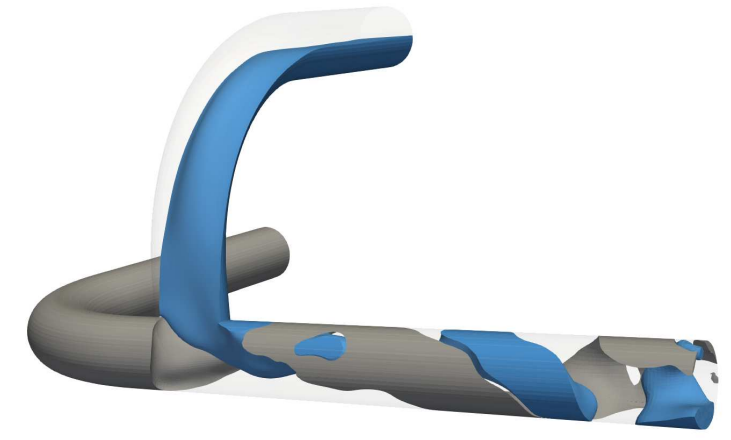

(i) $t=4.0$

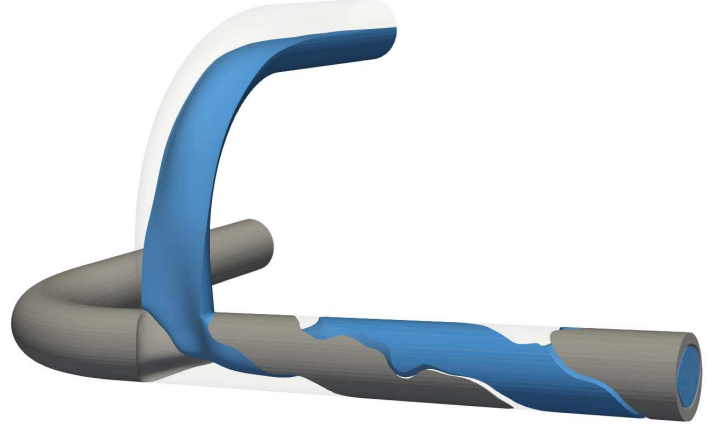

(f) $t=2.5$

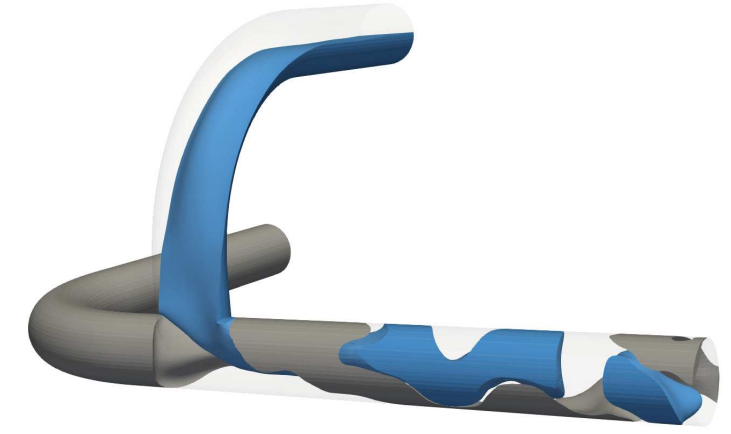

(h) $t=3.5$

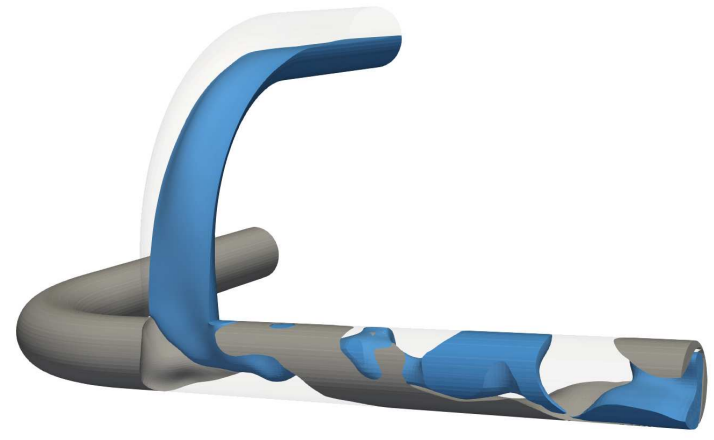

(j) $t=4.5$

Figure 8.11: Three-phase solver: evolution of phase two (blue) and phase three (gray) for the first 4.5 seconds 
2 (blue) overtakes Phase 1 at the elbow, and then both phases arrive at the main pipe at $t \approx 1.5$ (see Fig. 8.11(d)). Then, phases 1 and 2 enter the principal pipe, and they restrict the flow of Phase 3 after the "T" section. The lower density Phase 3 is then confined to the wall, and phases 1 and 2 intermitently occupy the bulk of the pipe (see Fig. (8.11(i))).

In Fig. (8.12) we represent the configuration of the three-phase at the final simulation time, $t=7.5$. Both phases 1 (Fig. 8.12(a)) and 2 (Fig. 8.12(b)) represent the bulk of the pipe, whereas Phase 3 (Fig. 8.12(c)) is forced to coat the pipe walls (similar to an annular flow regime). Due to the rupture of the flow of Phase 3 by the phases 1 and 2, Phase 3 gets a counter-clockwise swirl motion around the pipe.

Finally, we represent the velocity contours at the final time $t=7.5$ in Fig. 8.13. We can see the detachment due to the low viscosity of Phase 2 at the upper elbow, and also in the lower elbow for Phase 3. Then, after the " $\mathrm{T}$ ", the flow becomes under-resolved with large velocity spots as a result of the interaction between the three-phases.

Overall, we confirm the validity of the solver to compute industrial simulations as those found in the oil and gas industry.

\subsection{Conclusions}

We present a three-phase incompressible Navier-Stokes/Cahn-Hilliard system, and its discontinuous Galerkin implementation. The model uses the three-phase CahnHilliard model of Boyer and Lapuerta [2006], and the incompressible Navier-Stokes with artificial compressibility of Chapter 4 . As it is constructed, the continuous system of equations does not satisfy an entropy inequality.

We construct a DG approximation of the equations, where we combine the scheme used for the three-phase Cahn-Hilliard model of Chapter 7 and that used for the entropy-stable incompressible Navier-Stokes equations of Chapter 4.

We validate the solver in the two-phase simplification with a manufactured solution, and solving two-phase pipe regimes. Then, it is used to solve three-phase flows: a two-dimensional channel, a three-dimensional pipe in annular flow regime, and a three-dimensional "T" pipe. We find that the solver has not crashed in any of the simulations once the time-step size has been appropriately chosen. We highlight the ease in the configuration of the solver and the scheme for a user, as it only requires an appropriate choice of the physical parameters and conditions, plus the choice of the polynomial order of the simulation. The rest of the numerical parameters have been proven valid in a vast range of flow conditions, and the boundary conditions 


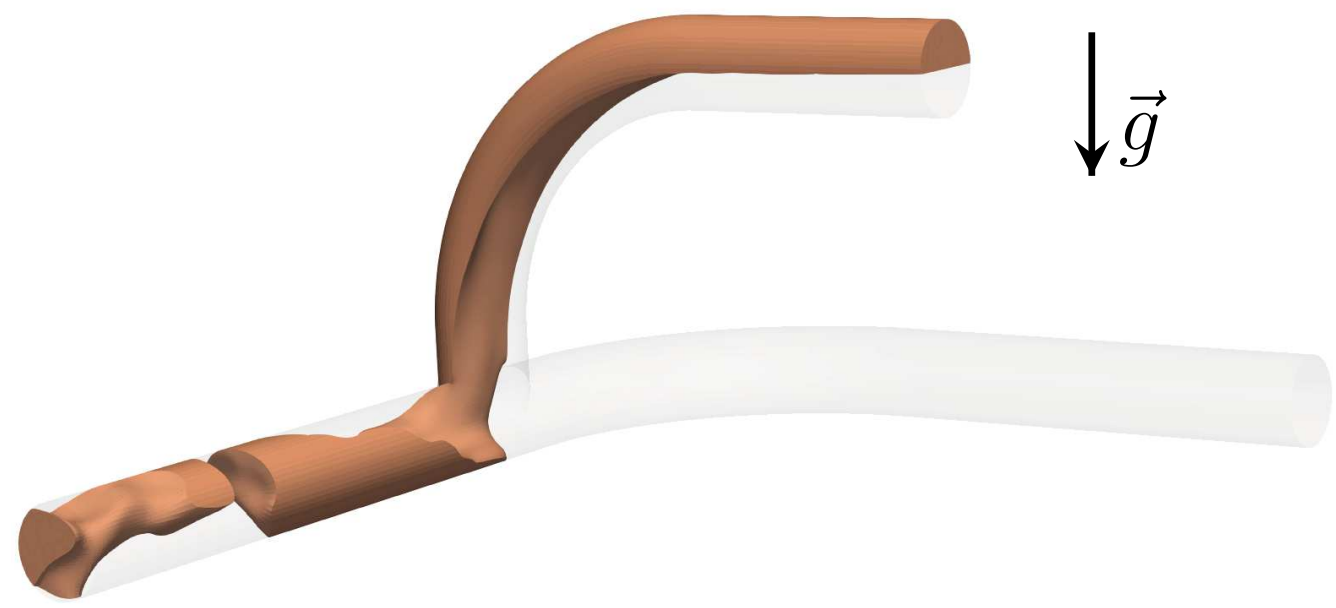

(a) Phase 1

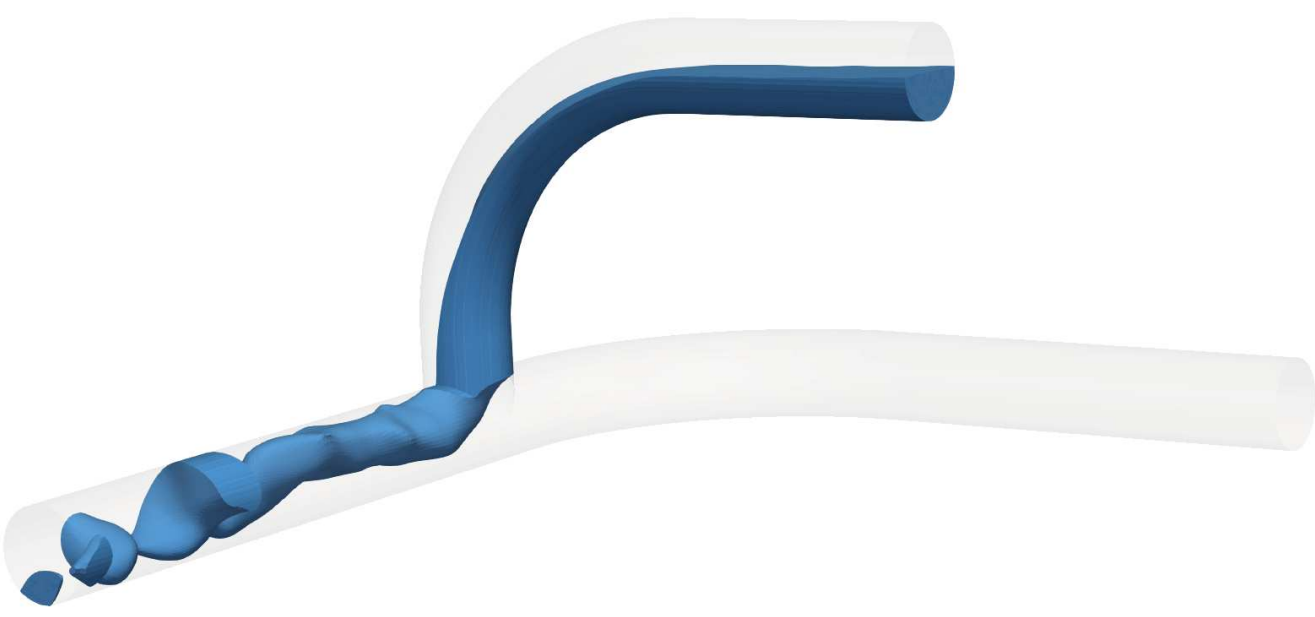

(b) Phase 2

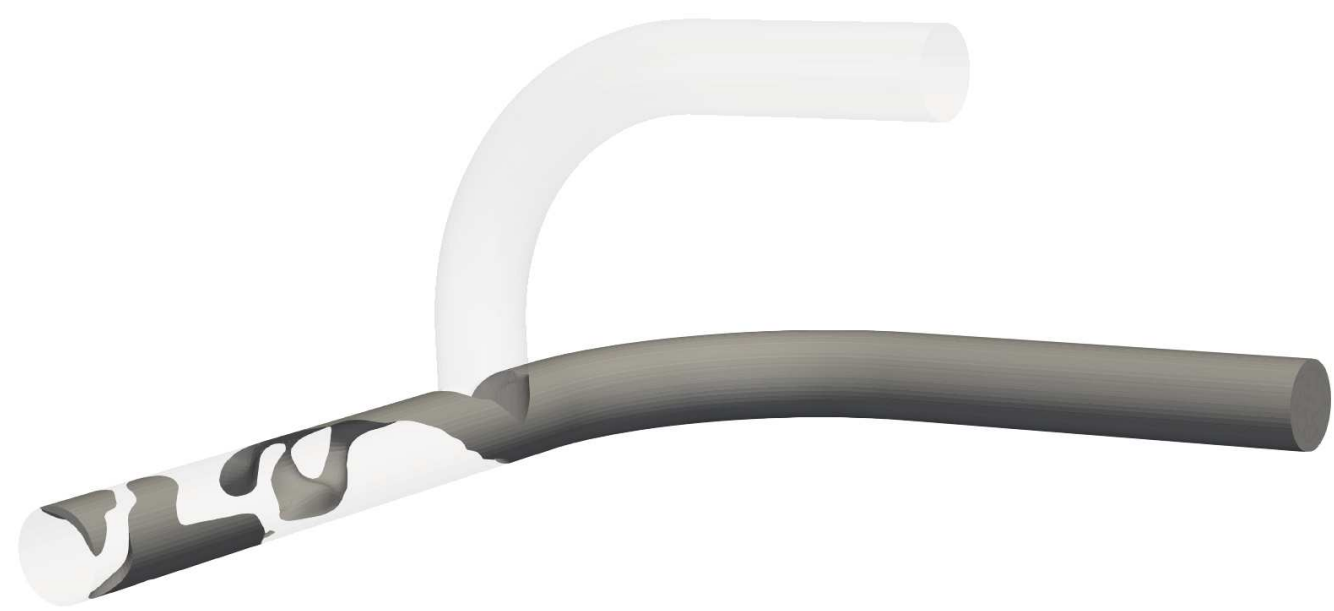

(c) Phase 3

Figure 8.12: Three-phase solver: representation of the three-phase at the final time $t=7.5$ 


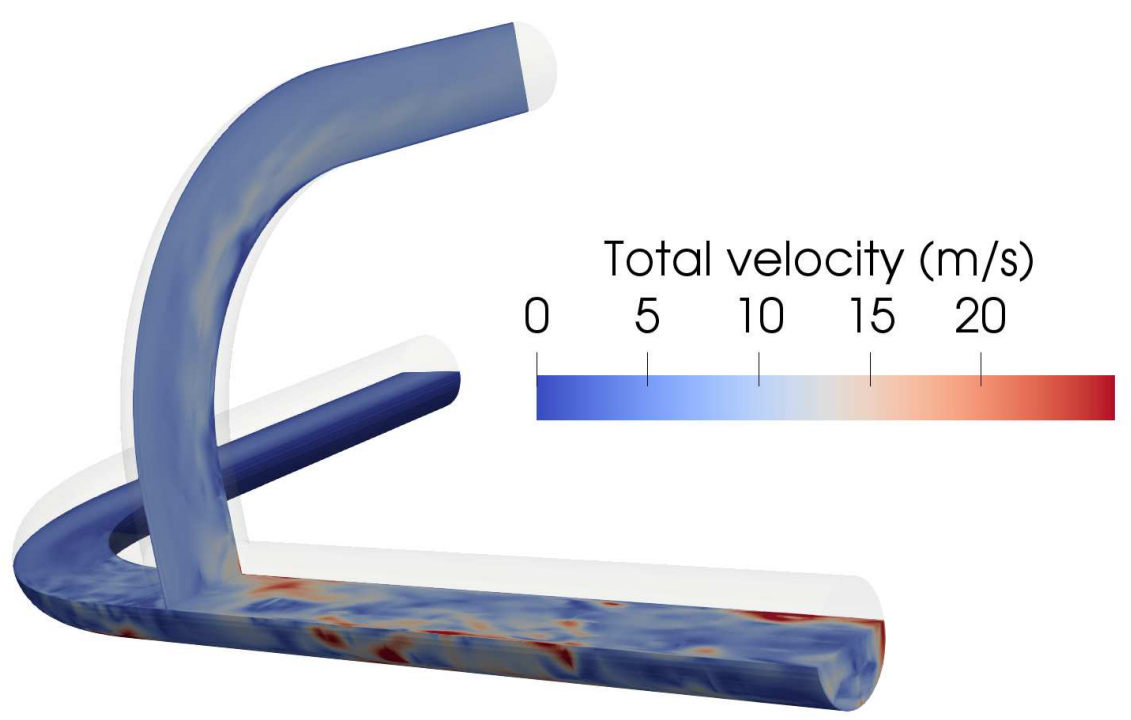

Figure 8.13: Three-phase solver: total velocity contour at the final time $t=7.5$

are automatically set-up by the algorithm that computes the inlet profile for given superficial/slip velocities. The enhancement of the robustness added by the split-form scheme, although formally it does not satisfy a discrete entropy inequality, has been addressed with numerical experiments in Sec. 8.4.3. Although the solutions of the flows presented are under-resolved, a better resolution can be achieved by increasing the polynomial order, which avoids re-meshing the geometry.

The software package belongs to the framework of the Repsol-UPM project, where an automatic mesh generator for pipes, the three-phase solver, and a postprocessor that includes particle tracking, erosion and corrosion computations serve as a R\&D tool for the study of complex multiphase phenomena. 


\section{Part IV}

\section{Accuracy of entropy-stable DG schemes for under-resolved turbulent simulations}

Summary In this thesis, we have studied the entropy-stability of non-linear partial differential equations. The construction of entropy-stable schemes has provided us with robust numerical schemes, to be used in a large variety of problems. Entropystability ensures robustness, but not accuracy. This has already been experienced in Sec. 6.6.5, where some entropy should be dissipated but the scheme, as it conserves the entropy, does not allow it. In this chapter, we perform a thorough study on the numerical dissipation mechanisms available in entropy-stable schemes to obtain not only robust, but more accurate under-resolved turbulent solutions.

We present a new closure model for Large Eddy Simulation to introduce dissipation in under-resolved turbulent simulation using discontinuous Galerkin (DG) schemes applied to the compressible Navier-Stokes equations. The development of the method is based on a thorough analysis of the numerical dissipation mechanisms in DG schemes. In particular, we use upwind Riemann solvers for inter-element dissipation, and a Spectral Vanishing Viscosity (SVV) method for interior dissipation.

These mechanisms are analysed using a linear von Neumann analysis (for a linear advection-diffusion equation) to characterise their properties in wave-number space. Their behaviour is tested using the three-dimensional Taylor-Green Vortex NavierStokes problem to assess the performance on transitional/turbulent flows.

The results of the study are subsequently used to propose a DG-SVV approach that uses a mode-selection Smagorinsky LES model to compute the turbulent viscosity. When the SVV technique is combined with a low dissipation Riemann solver, the scheme is capable of maintaining low dissipation levels for laminar flows, while pro- 
viding the correct dissipation for all wave-number ranges in turbulent regimes. The approach is designed for polynomial orders $N \geqslant 2$ and is specially well suited for high order schemes $(N \geqslant 5)$. This new DG-SVV approach is calibrated with the TaylorGreen test case; to then show its accuracy in an under-resolved $\left(y^{+}>8\right)$ channel flow at Reynolds number $R e_{\tau}=183$. 


\section{Chapter 9}

\section{Insights on the accuracy of entropy-stable DG schemes in under-resolved turbulent simulations}

\section{Contents}

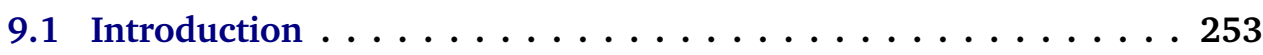

9.2 Methodology . . . . . . . . . . . . . . . 256

9.3 Numerical Results . . . . . . . . . . . . . . . . . 267

9.4 Design of a Smagorisnky-SVV scheme . . . . . . . . . . . . 275

9.5 Conclusions . . . . . . . . . . . . . . . . . . 280

\subsection{Introduction}

In recent years, DG methods have been adapted to solve increasingly complex physics. This popularity may be attributed to two characteristics. First, DG methods provide high accuracy even for distorted unstructured meshes Hesthaven and Warburton [2007], a property difficult to retain when using classic methods (e.g. Finite Differences or Finite Volumes). This property is a result of the compactness and local character of the scheme, enabling high order accuracy with compact stencils.

Second, DG methods have shown to be more robust that their high order continuous Galerkin relatives Kirby and Karniadakis [2003]. This last beneficial property has been often attributed to the use of upwind Riemann solvers, which add controlled dissipation, enhancing robustness.

In recent years, the DG community has exploited the increased robustness provided 
by Riemann solvers to simulate under-resolved turbulent flows, e.g. Gassner and Beck [2013]; Moura et al. [2017a]. This enhanced robustness rests on the localised dissipation provided by upwind Riemann solvers to dissipate small flow structures, which cannot be resolved on coarse meshes.

Methods that rely on the numerics to provide mechanisms for turbulent dissipation are typically known as implicit Large Eddy Simulation (iLES) methods. The term implicit means that the numerical errors (in particular the dissipation) are in charge of dissipating under-resolved flow structures.

An alternative to iLES is provided by explicit LES methods, where flow dissipation at small scales rests on physical arguments and modified flow equations, e.g. Lenormand et al. [2000]; Hughes et al. [2001]. To solve under-resolved turbulent flows using iLES/LES methods, it is necessary to understand and control numerical errors, and especially numerical dissipation introduced by the scheme, which replaces and/or complements explicit subgrid-scale models.

There are alternatives to provide numerical dissipation in DG methods. The most popular choice is to include upwind Riemann solvers, which arise naturally from the integration by parts of the non-linear fluxes and the existence of inter-element discontinuities in DG. These fluxes introduce local dissipation, which scales with the size of the discontinuities in the numerical solution. In under-resolved flows, the size of such discontinuities increases and it has been argued (see for example Ferrer [2017]) that fluxes based on discontinuities may act as an appropriate stabilising mechanism for under-resolved turbulent simulations.

In this chapter, in addition to upwind Riemann solvers we consider the Spectral Vanishing Viscosity (SVV) technique Kirby and Sherwin [2006] to add dissipation when the dissipation resulting from the Riemann solver is insufficient.

The SVV technique was first introduced for Fourier and continuous Galerkin discretizations Tadmor [1989] to regularize the solution (i.e. avoid oscillatory phenomena) in the inviscid Burgers equation, and later in the Navier-Stokes Equations (NSE) Karamanos and Karniadakis [2000]. The SVV provides additional dissipation and complements that provided by the numerical fluxes, enhances stability, vanishes in the laminar limit and provides spectral convergence in high-order discretizations. Let us note that other techniques to introduce localised dissipation in continuous discretizations exist but are not considered in this chapter, e.g. SUPG stabilisation.

A substantial amount of work Ferrer [2017]; Flad and Gassner [2017]; Carton de Wiart et al. [2014]; Fehn et al. [2017]; de la Llave Plata et al. [2018]; Alhawwary and Wang [2018]; Moura et al. [2018] has focused on understanding the stabilising effect of upwind Riemann solvers on under-resolved turbulent flows. However, limited ef- 
forts have been devoted to understanding the combined effect of Riemann solvers (i.e. interface dissipation) and discretised viscous terms (i.e. volume dissipation).

In this chapter, we analyse the individual contribution of each term but also the combined effect. For all dissipative mechanisms, we first analyse their numerical properties in wave-number space, using von Neumann analysis on a linear advectiondiffusion equation. Similar work has been presented for linear advection with constant coefficients Gassner and Kopriva [2011]; Moura et al. [2015] and for non-constant coefficients Manzanero et al. [2018c]. In addition, Fernandez et al. [2019] proposed a non-modal approach as an extension to von Neumann analysis. Second, we correlate these findings with results for DNS and iLES simulations of the 3D Taylor-Green Vortex (TGV) Navier-Stokes problem with transitional/turbulent flow Gassner and Beck [2013]; Moura et al. [2017a].

Even though von Neumann analyses are restricted to the constant advection-diffusion equation, we will confirm, with the help of numerical experiments, that von Neumann results are consistent with observations of under-resolved Navier-Stokes turbulent flows. We show that the dissipation introduced by upwind Riemann solvers affects high wave-numbers (around 75\% of the Nyquist wave-number), while discrete second order derivatives provide dissipation at low and medium wave-numbers (around $25 \%$ and $50 \%$ of the Nyquist wave-number respectively). Additionally, the dissipation introduced by the SVV operator helps to control the amount of dissipation introduced in the low and medium wave-number range, and provides a suitable mechanism to develop new models, like the one proposed in the final section of this chapter.

To perform these studies, we start from a baseline scheme without dissipation (i.e. an energy conserving scheme), and include numerical dissipation through the different stabilisation techniques. In the linear advection equation with constant coefficient it suffices to consider central fluxes (see Manzanero et al. [2018a]; Kopriva and Gassner [2014]), but in the non-linear Navier-Stokes equations, it is required to use split-forms Gassner et al. [2016a, 2018]; Winters et al. [2018], which provide kinetic energy consistency and enhance robustness (See Chapter 3).

Having quantified numerical errors for the above dissipative mechanisms we combine upwind Riemann solvers and SVV in a final section. Following the suggestion of Karamanos and Karniadakis for continuous Galerkin methods Karamanos and Karniadakis [2000]; Kirby and Karniadakis [2002], we modify the classic SVV technique using a Smagorinsky model to adjust the amount of dissipation introduced. This newly proposed DG model is able to maintain low dissipation levels in laminar flows, while modelling small eddies and providing correct dissipation for all wave-number ranges in turbulent regimes. 
The rest of this chapter is organised as follows: we describe the dissipative mechanisms and their inclusion in the one-dimensional advection-diffusion and threedimensional Navier-Stokes equations in Sec. 9.2. In Sec. 9.3, we study two dissipation techniques: Sec. 9.3.2 analyses interface dissipation (upwind Riemann solvers) while Sec. 9.3.3 investigates internal dissipation (discrete second order derivatives and SVV). Finally, we propose and test a Smagorinsky-SVV DG discretization to simulate under-resolved turbulent/transitional flows in Sec. 9.4.

\subsection{Methodology}

We first present, in Sec. 9.2.1, the one-dimensional advection-diffusion equation for the discrete DG approximation and von Neumann stability analysis. In Sec. 9.2.2 we present the DG discretization of the three-dimensional compressible Navier-Stokes equations. Then, in Sec. 9.2.3, we briefly discuss energy conserving schemes. Finally, in Sec. 9.2.4, we describe how the dissipation mechanisms are included both in the Navier-Stokes equations and in the advection-diffusion equation.

\subsubsection{One-dimensional advection-diffusion equation and von Neumann Analysis}

We revisit the one-dimensional linear advection-diffusion equation,

$$
u_{t}+a u_{x}=\left(\eta u_{x}\right)_{x}, \quad 0 \leqslant x \leqslant 1
$$

where $a$ is a constant advection speed and $\eta$ is the viscosity.

To perform von Neumann analysis we introduce an exponential wave solution, with spatial wave-number $k$ and temporal frequency $\omega$,

$$
u(x, t)=e^{i k x-i \omega t}=u_{0}(x) e^{-i \omega t},
$$

with $u_{0}(x)=e^{i k x}$, the part of (9.2) that only depends on the spatial coordinate. The relationship between the temporal frequency and the spatial wavenumber, $\omega(k)$, yields an eigenfunction of the original partial differential equation (9.1).

To get the relationship $\omega(k)$ we introduce (9.2) in (9.1),

$$
-i \omega e^{i k x-i \omega t}+i a k e^{i k x-i \omega t}=-\eta k^{2} e^{i k x-i \omega t}, \quad \omega(k)=a k-i \eta k^{2} .
$$

The original equation (9.1) advects the initial condition (9.2) keeping a constant speed $a$, and decreases its amplitude at a rate given by $\eta k^{2}$. 
Von Neumann analysis uses the same initial condition (9.2) in the discrete version of (9.1) to get the numerical relation $\omega(k)$. As opposed to the continuous equation, the discrete operator will induce errors in the propagation speed and errors in the amplitude of the wave, which depend on the wave-number. The change with time of the wave amplitude of the discrete solution is related to the stability of the numerical scheme. A scheme whose solutions' amplitude grows infinitely $(\operatorname{Im}(w)>0)$ is said to be unstable, while in a dissipative scheme $\operatorname{Im}(w) \leqslant-\eta k^{2}$. Because of the linear relation between spatial and temporal frequencies, without loss of generality, we will only study a constant coefficient $a=1$.

We consider the DG discretization of the linear advection equation (9.1) on a uniform grid that divides the physical domain $[0,1]$ into elements with spacing $h$. We consider the standard DG scheme with one equation and in one dimension, $\mathbf{Q}=\mathbf{W}=u$, $\overrightarrow{\mathbf{F}}_{e}=a U$, and $\overrightarrow{\mathbf{F}}_{v}=\eta G, J=\frac{h}{2}$,

$$
\begin{aligned}
& \frac{h}{2}\left\langle U_{t}, \phi\right\rangle_{E, N}+\int_{\partial e, N} a U^{\star} n \mathrm{~d} S-\left\langle a U, \phi_{\xi}\right\rangle_{E, N}=\int_{\partial e, N} \eta G^{\star} n \mathrm{~d} S-\left\langle\eta G, \phi_{\xi}\right\rangle_{E, N}, \\
& \frac{h}{2}\langle G, \varphi\rangle_{E, N}=\int_{\partial e, N} U^{\star} \varphi n \mathrm{~d} S-\left\langle U, \varphi_{\xi}\right\rangle_{E, N} .
\end{aligned}
$$

Note that in the one dimensional version the integrals are simply,

$$
\langle f, g\rangle_{E, N}=\int_{-1, N}^{N} f g \mathrm{~d} \xi, \quad \int_{\partial e, N} f g n \mathrm{~d} S=\left.f g\right|_{-1} ^{1} .
$$

At the inter-element boundaries, we use numerical fluxes $U^{\star}, G^{\star}$, that take into account the solution from both sides, which couple the solution in adjacent elements. We define $U^{e}=\left(U_{0}^{e}, U_{1}^{e}, \ldots, U_{N}^{e}\right)$ as the vector that contains the $N+1$ nodal values of the polynomial approximation of the solution in the element $e$. As a result, the DG scheme for an element $e$ (9.4) can be compactly written as

$$
\begin{aligned}
& U_{t}^{e}=\left[L_{a}\right] U^{e-1}+\left[C_{a}\right] U^{e}+\left[R_{a}\right] U^{e+1}+\left[L_{g}\right] G^{e-1}+\left[C_{g}\right] G^{e}+\left[R_{g}\right] G^{e+1}, \\
& G^{e}=\left[L_{u}\right] U^{e-1}+\left[C_{u}\right] U^{e}+\left[R_{u}\right] U^{e+1},
\end{aligned}
$$

where, in the linear advection-diffusion equation all the matrices are constant in time. We replace $G^{e}$ from the second equation to the first to get a single equation,

$$
U_{t}^{e}=[L L] U^{e-2}+[L] U^{e-1}+[C] U^{e}+[R] U^{e+1}+[R R] U^{e+2} .
$$

Common choices for the numerical fluxes are central and upwind fluxes for advective terms, and the Bassi-Rebay 1 or the Interior Penalty (IP) method for diffusive 
Table 9.1: Numerical fluxes used in this chapter for advective and diffusive terms

\begin{tabular}{|c|c|c|}
\hline Advective $(a U)^{\star}$ & Diffusive $U^{\star}$ & Diffusive $G^{\star}$ \\
\hline Upwind $\lambda=1$ & BR1 & BR1 \\
\hline$\{\{U\}\}+\frac{1}{2} \mid c$ & $\{\{U\}\}$ & $\{\{\nabla U\}\}-\sigma\left(U_{L}-U_{l}\right.$ \\
\hline
\end{tabular}

terms, summarized in Table 9.1. Insights on the properties of the different options can be found in Arnold et al. [2002].

We describe von Neumann analysis applied to the DG scheme (9.7), which is valid for any other scheme written in matrix-vector form. Further details on the derivation of von Neumann analysis for an advection-diffusion equation can be found in Manzanero et al. [2018c]; Moura et al. [2016].

The choice of the initial condition, and the linearity of the equation, allows us to relate the solution in neighboring elements to one reference element,

$$
U^{e-n}=e^{-i k n h} U^{e}
$$

We introduce (9.8) in the discrete equation (9.7) to get a linear ordinary differential equation system for each individual element,

$$
\frac{h}{2} U_{t}^{e}=\left(e^{-2 i k h}[\boldsymbol{L L}]+e^{-i k h}[\boldsymbol{L}]+[\boldsymbol{C}]+e^{i k h}[\boldsymbol{R}]+e^{2 i k h}[\boldsymbol{R} \boldsymbol{R}]\right) U^{e}=[\boldsymbol{M}(k h)] U^{e} .
$$

The general solution of (9.9) is linearly spanned by the $N+1$ modes of the eigenvalue problem (note that, for simplicity, the index $e$ has been dropped),

$$
-i \omega_{m} \frac{h}{2} V_{m}=[M(k h)] V_{m}, \quad U(t)=\sum_{m=0}^{N} A_{m} V_{m} e^{-i \omega_{m} t},
$$

where the $V_{m}$ are the unitary eigenvectors, and the amplitudes $A_{m}$ are constants computed to recover the initial condition at $t=0$,

$$
U(0)=U_{0}=e^{i k x}=\sum_{m=0}^{N} A_{m} V_{m}
$$

The solution structure (9.11) allows us to classify three numerical error sources, which were already described in Manzanero et al. [2018c]; Moura et al. [2015]. Specifically, only the so-called primary mode (p) propagates with the physical wavespeed and damping (e.g. $\omega_{p}=0$ when $k=0$ ). Hence, we rewrite the solution isolating the primary mode contribution from the remaining modes (called secondary 
modes, $m \neq p$ ),

$$
U=A_{p} V_{p} e^{-i \omega_{p} t}+\sum_{\substack{m=0 \\ m \neq p}}^{N} A_{m} V_{m} e^{-i \omega_{m} t}
$$

Next, the initial condition (9.11) is also separated into the contribution of primary and secondary modes, and introduced in (9.12). As a result, we can regard the numerical solution as the primary mode propagating the initial condition, $U_{0} e^{-i \omega_{p} t}$, and the non-physical errors (which propagate with both primary and secondary mode speeds) as secondary modes, $\Delta U(t)$,

$$
\begin{aligned}
U & =U_{0} e^{-i \omega_{p} t}+\sum_{\substack{m=0 \\
m \neq p}}^{N} A_{m} V_{m}\left(e^{-i \omega_{m} t}-e^{-i \omega_{p} t}\right) \\
& =U_{0} e^{-i \omega_{p} t}+\Delta U(t) .
\end{aligned}
$$

The numerical propagation speed experienced by the initial condition, $U_{0}$, (i.e. the primary mode frequency, $\omega_{p}$ ) will differ from that dictated by the analytical PDE $(\omega=a k)$. The difference between its real part and the theoretical travelling speed, $a k$, will yield a dispersion error (i.e. error in the propagation speed), while its imaginary part, which is generally non-zero, represents numerical dissipation. Following Moura et al. [2015], we define the non-dimensional wave-number, $\hat{k}$, as

$$
\hat{k}=\frac{k h}{N+1},
$$

so that we can distinguish between low wave-numbers $(\hat{k} \lesssim \pi / 4)$, medium wavenumbers $(\hat{k} \sim \pi / 2)$ and high wave-numbers $(\hat{k} \gtrsim 3 \pi / 4)$, with respect to Nyquist wave-number $(\hat{k}=\pi)$. Further details on the discretization and analysis of the onedimensional advection equation and extended von Neumann analysis for advection equations with non-constant coefficients may be found in Manzanero et al. [2018a,c].

\subsubsection{Three-dimensional compressible Navier-Stokes equations}

The three-dimensional compressible Navier-Stokes equations, and its split-form DG approximation were described in Sec. 2.8. In this section, we discuss introducing numerical dissipation on the split-form DG scheme (2.76), through interface and volume dissipation. For the latter, we augment the viscous fluxes $\overrightarrow{\mathbf{F}}_{v}$ with a Spectral Vanishing 
Viscosity (SVV) flux $\overrightarrow{\mathbf{F}}_{S V V}$, that will be described in Sec. (9.2.4) of this chapter,

$$
\overrightarrow{\mathbf{F}}_{v+}=\overrightarrow{\mathbf{F}}_{v}+\overrightarrow{\mathbf{F}}_{\mathrm{SVV}} \text {. }
$$

All Navier-Stokes simulations are time-marched using a three stage Runge-Kutta scheme with a Courant-Friedrichs-Lewy (CFL) number of 0.4 .

\subsubsection{Briefly on energy preserving schemes}

In this chapter we solely use kinetic energy preserving schemes, where the kinetic energy can never increase, and it may decrease as a result of the physical and numerical dissipation Fisher and Carpenter [2013]. Because the schemes are designed to remain stable and to be energy preserving, they do not require extra numerical dissipation for robustness (i.e. they are stable with central fluxes, see Sec. (6.6.2)). Hence, the addition of numerical dissipation to these schemes enables the analysis of dissipation techniques, whose effect will not be masked by other numerical errors inherent to the discretization of non-linear terms. This is useful to understand the requirements on the numerical dissipation, and thus, to design robust dissipation techniques able to achieve accurate solutions. In particular, the dissipation techniques studied herein are the use of upwind Riemann solvers and augmenting the physical (molecular) viscosity with a turbulent viscosity (e.g. LES models and spectral vanishing viscosity).

Because the volume quadratures are exactly computed in the linear advection equation, energy conserving schemes are achieved with an appropriate choice of the numerical flux. Namely, the numerical flux (see Table 9.1) is designed so that the first part (central fluxes) balances volume terms to get an energy conserving scheme (for constant advection speeds). Hence, we will refer to the particular case with $\lambda=0$ as central fluxes (see Table 9.1).

As described in Chapter 3, the extension to the non-constant advection speed and non-linear problems (e.g. the Navier-Stokes equations) needs special treatment of the volume integrals (to use split-forms) and requires the use of Gauss-Lobatto points to cancel out the boundary terms using the Simultaneous-Approximation-Term (SAT) property. The interested reader is referred to Gassner et al. [2016a]; Gassner [2013]; Kopriva and Gassner [2014]; Manzanero et al. [2018c] for the particular split-forms for non-constant advection, Burgers and Euler equations, and Chapter 4 and Chapter 6 for the incompressible NSE and the two-phase iNS/CH system. 


\subsubsection{Description of the dissipation mechanisms}

In this section, we describe two dissipation mechanisms found in DG methods: the interface dissipation through the numerical flux in Sec. 9.2.4 and the internal dissipation through the use of a turbulent viscosity in Sec. 9.2.4. The mechanisms are first studied in the one-dimensional advection-diffusion equation with von Neumann analyses, and then, their effect is correlated to their counterparts in the three-dimensional Navier-Stokes equations. The von Neumann analysis provides useful information, which helps us to understand the effect of the dissipation in transitional/turbulent flows. In Table 9.2 we summarize the two dissipative mechanisms and we describe their effect in the wave-numbers space.

Table 9.2: Summary of dissipative mechanisms: $\hat{k}$ denotes the non-dimensional wave-number, $\hat{k}=k h /(N+1)$

\begin{tabular}{l|cc|c} 
Mechanism & Discretization & Section & Dissipation range \\
\hline Interface dissipation & upwind, Roe, low & 9.2 .4 & High wave-numbers \\
& dissipation Roe Oßwald et al. [2016] & 9.3 .2 & $(\hat{k}>\pi / 2)$ \\
\hline Volume dissipation & LES models, & 9.2 .4 & Low $(\hat{k}<\pi / 2)$ to medium \\
& SVV & 9.3 .3 & $(\hat{k} \sim \pi / 2)$ wave-numbers and \\
& & & tuneable for SVV
\end{tabular}

\section{Interface dissipation through the numerical flux}

Discontinuous Galerkin methods add dissipation through the numerical flux, $F_{e}^{\star}$, (see (2.76)), that concentrates at the interfaces. For the one-dimensional linear advection equation with constant coefficient (9.1), we use the general $\lambda$ flux defined in Table 9.1. As demonstrated in Kopriva and Gassner [2014], and reproduced here in Sec. (3.52), the numerical dissipation introduced by an upwind Riemann solver is proportional to the square of the solution inter-element jumps $\llbracket U \rrbracket=U_{R}-U_{L}$,

$$
\mathscr{E}_{t}=-\lambda \operatorname{diss}=-\lambda \frac{1}{2}|a| \llbracket U \rrbracket^{2} \leqslant 0 .
$$

Additionally, note that $\lambda$ diss vanishes when considering the analytical (smooth) solution (with zero jumps), thus not altering the underlying physics in well resolved simulations. We will investigate the effect of this non-linear dissipation through von Neumann analyses. These non-linearities arise as a result of the penalisation on the size of the interface jumps when increasing $\lambda$.

In the three-dimensional compressible Navier-Stokes equations, inviscid fluxes or Riemann solvers are usually constructed as the average of both adjacent states (which 
in entropy-stable schemes is replaced by the two-point entropy conserving flux), plus an interface dissipation that depends on the two states $\left(\mathbf{Q}_{L}, \mathbf{Q}_{R}\right)$ Toro [2013],

$$
\overrightarrow{\mathbf{F}}_{e}^{\star} \cdot \vec{n}=\overrightarrow{\mathbf{F}}_{e}^{\mathrm{EC}} \cdot \vec{n}-\operatorname{diss}\left(\mathbf{Q}_{L}, \mathbf{Q}_{R}\right) .
$$

In this chapter, we will consider Roe dissipation,

$$
\operatorname{diss}_{\text {Roe }}\left(\mathbf{Q}_{L}, \mathbf{Q}_{R}\right)=\sum_{e=1}^{5} \alpha_{e}\left|\beta_{e}\right| \mathbf{K}_{e},
$$

where the intensities $\alpha_{e}$, eigenvalues $\beta_{e}$ and eigenvectors $\vec{K}_{e}$ are computed from the Roe averaged states Toro [2013]. We introduce the same parameter $\lambda$ used in the advection-diffusion equation (see Table 9.1) for the Navier-Stokes equations. To do so, we modify (9.17) to

$$
\overrightarrow{\mathbf{F}}_{e}^{\star} \cdot \vec{n}=\overrightarrow{\mathbf{F}}_{e}^{\mathrm{EC}} \cdot \vec{n}-\lambda \operatorname{diss}\left(\mathbf{Q}_{L}, \mathbf{Q}_{R}\right),
$$

to control the dissipation added through cell interfaces with the free parameter $\lambda$.

This new expression (9.19) compares to the linear advection form (see Table 9.1). Moreover, this strategy has been already adopted to design low dissipation versions for Roe Riemann solvers that provide an appropriate estimate for $\lambda$ Oßwald et al. [2016]. Nonetheless in the $L^{2} R$ Riemann solver derived in Oßwald et al. [2016] there is a subtle difference, since this atenuation is only applied to the velocities, and not to the pressure, since the pressure atenuatation leads to pressure oscillations (see Flad and Gassner [2017]).

\section{Internal dissipation with spectral vanishing viscosity}

We complement the dissipation provided by the interface numerical flux with additional dissipation in the interior of the elements (i.e. volume dissipation). Precisely, we provide here a framework to study the Spectral Vanishing Viscosity (SVV) method.

The SVV technique was initially introduced to stabilise Fourier spectral methods in Tadmor [1989], and later it was adapted to high-order continuous Galerkin methods in Karamanos and Karniadakis [2000]. This technique considers a spatially-constant viscosity, $\eta_{S V V}$, but it is applied unevenly on the different spectral modes that form the solution. The operator that chooses the intensity of the viscosity on each mode is called the viscous kernel $Q_{\eta}$. To the one-dimensional linear advection-diffusion equation, we add an additional diffusive flux $F_{\mathrm{SVV}}$ to introduce the SVV regularisation,

$$
F_{\mathrm{SVV}}=\eta_{S V V} \bar{G}=\eta_{S V V}\left(Q_{\eta} \star G\right),
$$


where the $\star$ operator denotes the modal convolution operator, applied to the solution gradient $G$ and the SVV viscous kernel.

The application of the modal convolution is trivial when the solution is expanded in a modal basis. We consider the Legendre polynomials $L_{k}(\xi)$, which are the only polynomials that satisfy,

$$
\int_{-1, N}^{1} L_{k}(\xi) \xi^{v} \mathrm{~d} \xi=0, \quad v=0, \ldots, k-1, \quad k=0, \ldots, N .
$$

The Legendre polynomials are (discretely) orthogonal Canuto et al. [2007],

$$
\int_{-1, N}^{1} L_{k}(\xi) L_{n}(\xi) \mathrm{d} \xi=\left\|L_{k}\right\|_{N}^{2} \delta_{k n}
$$

Therefore, the solution can be written in nodal (Lagrange polynomial expansion) and modal (Legendre polynomial expantion) forms,

$$
G=\sum_{i=0}^{N} G_{i} l_{i}(\xi)=\sum_{k=0}^{N} \hat{G}_{k} L_{k}(\xi)
$$

The application of the modal convolution operator in modal space simply multiplies the modal expansion coefficients of the two polynomials,

$$
Q_{\eta} \star G=\sum_{k=0}^{N} Q_{\eta, k} \hat{G}_{k} L_{k}(\xi)
$$

For a nodal polynomial, one first needs to compute the modal coefficients $\hat{G}_{k}$, and then, after the application of the modal convolution, to return the new polynomial to the nodal space. To transform from the nodal to the modal space, we compute the inner product of the solution with the Legendre polynomials,

$$
\begin{aligned}
& \int_{-1, N}^{1} U L_{k} \mathrm{~d} \xi=\sum_{j=0}^{N}\left(\int_{-1, N}^{1} l_{j} L_{k} \mathrm{~d} \xi\right) U_{j}=\sum_{j=0}^{N} w_{j} L_{k}\left(\xi_{j}\right) U_{j}, \\
& \int_{-1, N}^{1} U L_{k} \mathrm{~d} \xi=\sum_{n=0}^{N}\left(\int_{-1, N}^{1} L_{n} L_{k} \mathrm{~d} \xi\right) \hat{U}_{n}=\left\|L_{k}\right\|_{N}^{2} \hat{U}_{k},
\end{aligned}
$$

thus we get transformation matrix $T_{n 2 m}$,

$$
\hat{U}_{k}=\sum_{j=0}^{N} \frac{w_{j} L_{k}\left(\xi_{j}\right)}{\left\|L_{k}\right\|_{N}^{2}} U_{j}=\sum_{j=0}^{N} T_{n 2 m, k j} U_{j}
$$


Proceeding similarly, but computing the inner product with $l_{j}$, we compute the transformation matrix from modal to nodal spaces,

$$
U_{j}=\sum_{k=0}^{N} L_{k}\left(\xi_{j}\right) \hat{U}_{k}=\sum_{k=0}^{N} T_{m 2 n, j k} \hat{U}_{k},
$$

which is simply the evaluation of the Legendre polynomials at the Lagrange interpolation nodes (recall the interpolation operator $\mathrm{I}^{N},(2.24)$ ).

Then, we compute the filtering matrix that performs the convolution of $G$ as a single matrix multiplication,

$$
\bar{G}=Q_{\eta} \star G=T_{m 2 n} \operatorname{diag}\left(Q_{\eta, k}\right) T_{n 2 m} G=\mathscr{F}_{\eta} G .
$$

The resulting approach can be identified as a high-pass filtered dissipation. The spectral distribution of the viscosity is defined ad-hoc in the viscous kernel, $Q_{\eta}$, of the SVV method. In this chapter we adopt the kernel introduced recently by Moura et al. Moura et al. [2016], who considered a power law,

$$
Q_{\eta}(k)=(k / N)^{P_{S V V}}, \quad k=0,1, \ldots, N
$$

In (9.29), the constant $P_{S V V}$ is the kernel power coefficient, and $k$ is the polynomial mode index. This kernel is convenient since a standard viscous discretization is recovered when $P_{S V V}=0$ (since in that case $\bar{G}=G$ ), and the dissipation is restricted to the highest order spectral mode if $P_{S V V} \gg 1$. Therefore, with this framework we can study both the SVV and a standard second order derivative, which serves as a point of reference. A graphical representation of the kernel (9.29) can be found in Figure 9.1.

The SVV has been widely adopted and studied for continuous Galerkin and Fourier spectral methods Ferrer [2017]; Kirby and Sherwin [2006]; Moura et al. [2016]; Guo et al. [2001]. The dispersion-dissipation analysis of the continuous Galerkin SVV was performed in Moura et al. [2016], where the authors found similarities between the numerical dissipation introduced to that using upwind Riemann solvers in discontinuous Galerkin methods.

However, for the discontinuous Galerkin method there are no SVV studies available. One reason would be that the SVV was introduced to achieve similar dissipation behaviour in continuous Galerkin (where the schemes lack numerical dissipation) to that with upwind Riemann solvers in DG. Therefore, it may be naively argued that it is pointless to introduce the SVV method in DG, since the upwind fluxes achieve similar results with a more efficient and simpler implementation. 


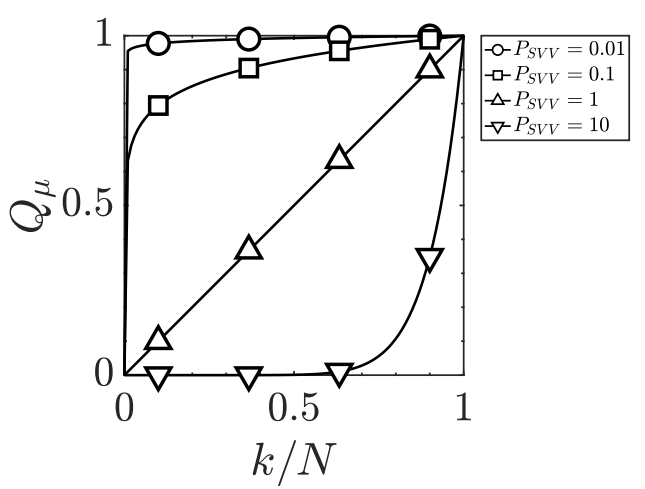

Figure 9.1: Representation of the power law kernel (9.29) for several $P_{S V V}$ values. The higher the kernel power coefficient $P_{S V V}$, the higher the filtering performed on the viscous tensor

In this chapter we show that the SVV method is useful not as a substitute of upwind Riemann solvers, but as a complement of the latter to adjust energy accumulations in medium wave-numbers, which cannot be controlled by the numerical flux.

In the compressible Navier-Stokes equations, the SVV method augments the physical viscous flux (9.15) with,

$$
\overrightarrow{\mathbf{F}}_{\mathrm{SVV}}=\left[\begin{array}{ccc}
0 & 0 & 0 \\
\bar{\tau}_{x x} & \bar{\tau}_{x y} & \bar{\tau}_{x z} \\
\bar{\tau}_{y x} & \bar{\tau}_{y y} & \bar{\tau}_{y z} \\
\bar{\tau}_{z x} & \bar{\tau}_{z y} & \bar{\tau}_{z z} \\
\sum_{j=1}^{3} v_{j} \bar{\tau}_{1 j}+k \bar{T}_{x} & \sum_{j=1}^{3} v_{j} \bar{\tau}_{2 j}+k \bar{T}_{y} & \sum_{j=1}^{3} v_{j} \bar{\tau}_{3 j}+k_{S V V} \bar{T}_{z}
\end{array}\right],
$$

where variables with a bar, e.g., viscous stresses, denotes an application of the SVV operator,

$$
\bar{\tau}_{i j}=\eta_{S V V} Q_{\eta} \star\left[\partial_{j} v_{i}+\partial_{i} v_{j}-\frac{1}{3} \delta_{i j} \partial_{i} v_{i}\right] .
$$

In three dimensions, the SVV operator is applied using a tensor product version of (9.28),

$$
\hat{G}_{i j k}=\sum_{l=0}^{N} \mathscr{F}_{\eta, l} \sum_{n=0}^{N} \mathscr{F}_{\eta, n} \sum_{m=0}^{N} \mathscr{F}_{\eta, m} G_{m n l},
$$

which consists of three one-dimensional matrix-vector products.

We also give a brief introduction to LES models in this section. LES models introduce a solution dependent viscosity, $\eta_{t}$, usually through a second order derivative. The standard Smagorinsky subgrid-scale model for the Navier-Stokes equations Smagorinsky [1963], is used in all studies shown in this chapter. Namely, we modify 
the viscosity in the viscous flux $\overrightarrow{\mathbf{F}}_{v}$ to

$$
\overrightarrow{\mathbf{F}}_{L E S}=\left.\overrightarrow{\mathbf{F}}_{v}\right|_{\eta+\eta_{t}},
$$

The Smagorinsky turbulent viscosity $\eta_{t}$ is defined as

$$
\eta_{t}=C_{S}^{2} \Delta^{2}|S|
$$

The classical value for isotropic turbulence, $C_{S}=0.2$, is selected in this chapter, while the filter width $\Delta$ is computed following Flad and Gassner [2017],

$$
\Delta^{3}=\frac{\text { Cell volume }}{(N+1)^{3}}
$$

which accounts for both the mesh elements size and the polynomial order. The effect of the Smagorinsky model will be analysed in detail in Sec. 9.4.

To summarize, we first study the dissipation introduced by the SVV operator with a constant viscosity $\eta_{S V V}$, using both von Neumann analysis for the linear advection equation, and the Taylor-Green vortex. Then, we construct a Smagorinsky-SVV LES scheme that uses the Smagorinsky model as the SVV viscosity $\eta_{S V V}=\eta_{t}$. In the particular case when $P_{S V V}=0$, the SVV flux is reduced to the viscous flux, and the model is equivalent to the use of the standard Smagorinsky LES model.

\subsubsection{The Taylor-Green Vortex (TGV) problem}

We test the under-resolved capabilities of the strategies studied with von Neumann analysis with the Taylor-Green Vortex (TGV) problem Taylor and Green [1937]. The TGV problem has been widely used to report the subgrid-scale modelling capabilities of iLES approaches and discretizations Gassner et al. [2016a]; Moura et al. [2017b], and also in this thesis (see Sec. 4.6.4).

In this chapter, we assess von Neumann analysis truthfulness to estimate the dissipation introduced by the operators introduced in Sec. 9.2.4. The configuration of the TGV problem is a three dimensional periodic box $\Omega=[-\pi, \pi]^{3}$ with the initial condition,

$$
\begin{aligned}
\rho & =1, \\
u & =\sin x \cos y \cos z, \\
v & =-\cos x \sin y \cos z, \\
w & =0, \\
p & =\frac{1}{\gamma M_{0}^{2}}+\frac{1}{16}(\cos 2 x+\cos 2 y)(\cos 2 z+2) .
\end{aligned}
$$


The Mach number is $M_{0}=0.1$ in all the simulations performed herein. The reported quantities to measure the simulations' accuracy are the kinetic energy rate,

$$
\epsilon=-\frac{\mathrm{d} K}{\mathrm{~d} t}=-\frac{1}{|\Omega|} \frac{\mathrm{d}}{\mathrm{d} t} \int_{\Omega} \frac{1}{2} \rho V^{2} \mathrm{~d} \vec{x},
$$

the enstrophy,

$$
\zeta=\frac{1}{2|\Omega|} \int_{\Omega}|\nabla \times \vec{v}|^{2} \mathrm{~d} \vec{x},
$$

the numerically introduced dissipation estimated with both $\epsilon$ and $\zeta$ Gassner and Beck [2013],

$$
\mu \simeq \frac{\epsilon}{2 \zeta}=-\frac{\mathrm{d} K / \mathrm{d} t}{2 \zeta}
$$

and the kinetic energy spectra, measured at a fixed time snapshot ( $t=8$ to observe transitional flow, and $t=14$ to show the isotropic decay). In this chapter we consider both the inviscid version of the Taylor-Green vortex problem, and the viscous TaylorGreen vortex problem with Reynolds number $R e=1600$.

\subsection{Numerical Results}

We now study the two dissipation mechanisms (interface and internal) introduced in the previous section by two means: performing a linear von Neumann analysis, and solving the Taylor-Green vortex problem. Von Neumann analysis gives a global vision of the behaviour of the linear advection-diffusion equation scheme in the wavenumber domain independent of the initial condition. In contrast, the TGV problem allows us to study the dissipation introduced numerically by inspecting the kinetic energy spectra of the flow. This section is organised with the same structure as Sec. 9.2.4; we first study energy conserving schemes to establish the baseline state, to then analyse the two dissipation techniques introduced in Sections 9.2.4 and 9.2.4.

\subsubsection{Preliminaries on kinetic energy preserving schemes}

We start by considering a kinetic energy preserving scheme. We study the constant speed advection equation with Gauss points and central fluxes (diffusion is not considered here). The choice of Gauss points for this von Neumann analysis is adopted since it represents a more traditional and widely used approach, but all the conclusions hold with Gauss-Lobatto points (see Gassner and Kopriva [2011] for the details). 


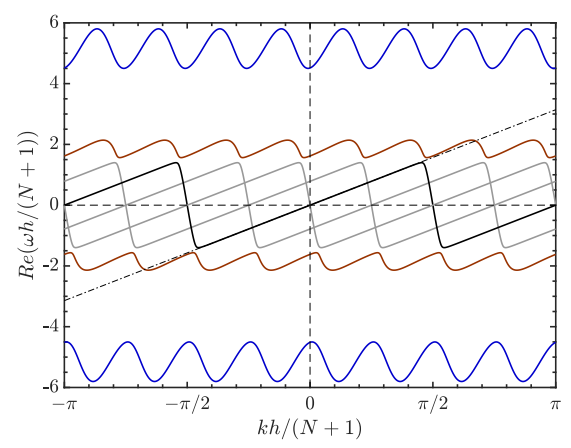

(a) Dispersion error

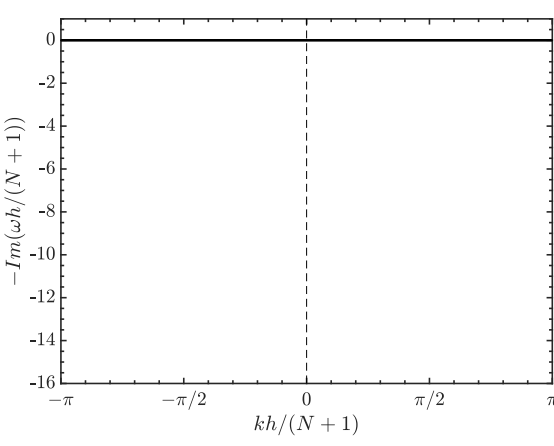

(b) Diffusion error

Figure 9.2: Dispersion-dissipation errors in the advection equation. Figures depict the eigenvalues computed with an energy conserving DG (i.e. with central fluxes, $\lambda=0$ ) and polynomial order $N=7$

\section{Von Neumann analysis}

Numerical dispersion and dissipation errors are depicted in Figures 9.2(a) and 9.2(b) respectively. We have highlighted the mode that satisfies $\operatorname{Re}(\omega)=0$ for $\hat{k}=0$ with a black line. The dash-dot line in Figure 9.2(a) depicts the analytical PDE speed (i.e. the straight line $\operatorname{Re}(\omega)=k$ ). There are three groups of modes: first, grey modes in Figure 9.2(a) are an exact replication of the black mode, therefore, they do not accurately predict the advection speed, and consequently they introduce numerical errors in the solution. Second, brown modes are medium-frequency modes that produce numerical errors in the propagation speed, except in a narrow region close to $k h /(N+1) \simeq$ $\pm \pi / 2$, where these modes follow the analytical propagation speed. Third, blue lines represent non-physical high frequency modes. These results are in agreement with those previously reported in Hesthaven and Warburton [2007].

Figure 9.2(b) shows the dissipation errors, where neither numerical energy decay nor growth are experienced (i.e. the scheme is energy conserving, $\operatorname{Im}(\omega)=0$ ). This result is consistent with numerical energy estimates: the scheme is energy conserving since the discrete energy balance inside the computational domain vanishes (when considering constant advection speeds, Manzanero et al. [2018a]; Kopriva and Gassner [2014]).

\section{Navier-Stokes TGV problem}

We run the inviscid Taylor-Green vortex with the kinetic energy preserving scheme (i.e. with $\lambda=0$ in (9.19)). We consider a coarse Cartesian $8^{3}$ mesh and fourth order 


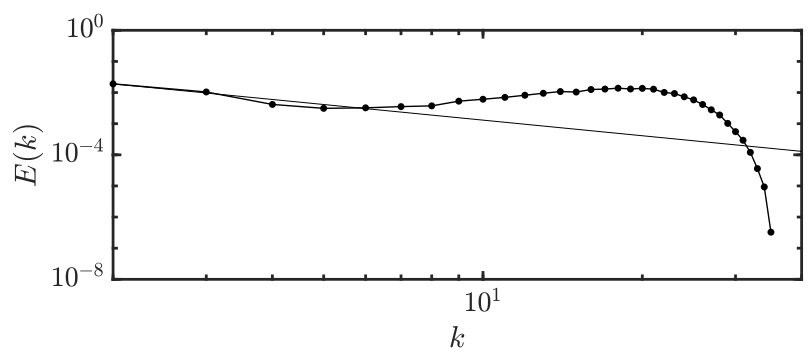

Figure 9.3: Kinetic energy spectra of the inviscid Taylor-Green vortex problem (with Mach number $M_{0}=0.1$ ) in $t=14$, alongside the theoretical Kolmogorov's solution $k^{-5 / 3}$ Pope [2001]. This result has been computed with the energy conserving DG scheme introduced in Gassner et al. [2016a]. We find that the lack of dissipation leads to energy accumulation in high wave-numbers, where the solution is severely under-resolved

$(N=4)$ polynomials.

Figure 9.3 depicts the kinetic energy spectra after 14 time units. We find an undesired accumulation of energy in high wave-numbers as a result of the undissipated kinetic energy transferred from large to small eddies (i.e. the scheme is energy preserving). The solution is severely under-resolved, where high wave-number modes energy (with large dispersion errors) are not dissipated.

Note that this is inferred from von Neumann analyses (see Figure 9.2). A solution that presents a large number of high wave-number spectral components sees an accumulation of energy since, at these wave-numbers, dispersion errors are important in a region where there is no dissipation that drains the energy. Moreover, as a result of the insufficient energy drain, this accumulation is also transferred to lower wave-numbers by the non-linear terms of the Navier-Stokes equations.

Following sections study several techniques to introduce numerical dissipation at appropriate scales, and correct this behaviour. Note that the scheme is stable without numerical dissipation, that is only added in this chapter to enhance the accuracy of the method.

\subsubsection{Interface dissipation through the numerical flux}

In this section, we study the effect on the scheme stability and accuracy of upwind Riemann solvers, by varying $\lambda$ on $(a U)^{\star}$ in Table 9.1 for the linear advection equation and in (9.18) for the compressible Navier-Stokes equations. We consider the inviscid version of both the linear advection $(\eta=0)$, and the compressible Navier-Stokes (Euler equations) $\overrightarrow{\mathbf{F}}_{v+}=0$. 


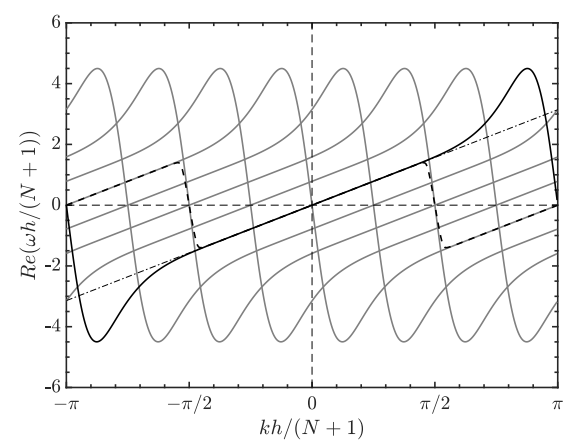

(a) Dispersion error

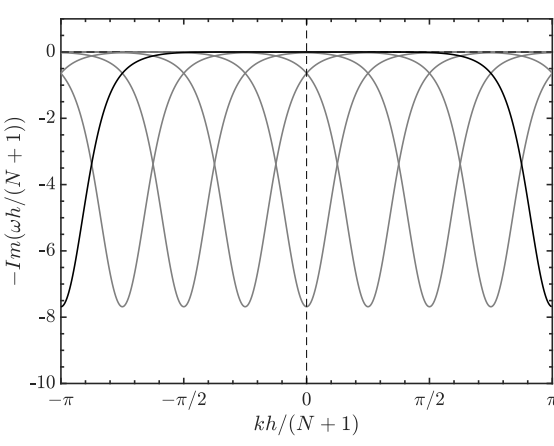

(b) Dissipation error

Figure 9.4: Dispersion and dissipation errors with $\lambda=1.0$

\section{Von Neumann analysis}

We now study the dispersion and dissipation errors for the upwind Riemann solver. In the previous section, we studied a dissipation-free scheme with the central Riemann solver. With an upwind Riemann solver, we start introducing numerical dissipation to the scheme.

The dispersion and dissipation errors for the upwind Riemann solver are represented in Fig. 9.4. This result can also be found in Hesthaven and Warburton [2007]; Gassner and Kopriva [2011]; Alhawwary and Wang [2018]; Moura et al. [2016], and shows the typical DG numerical dissipation: the dissipation concentrates in high wave-numbers, and it vanishes in the small wave-numbers, with a very flat region near $\hat{k}=0$. Moreover, with the upwind Riemann solver all modes describe the same behavior (with a phase shift), which might be regarded as optimal from the point of view of time-stepping limitations in explicit or implicit-iterative solvers.

We now discuss the effect of $\lambda \neq 1$. We reckon $\lambda=1$ as upwind fluxes, but other $\lambda$ values are possible. From (9.16) it can be wrongly inferred that the dissipation is proportional to $\lambda$, and that we can increase and tune the numerical dissipation to the desired levels by modifying $\lambda$ appropriately. This is not the case, however, since the dissipation varies non-linearly with $\lambda$, because the inter-element jumps decrease as $\lambda$ increases.

The maximum dissipation experienced by the different modes as $\lambda$ increases is represented in Fig. 9.5. We see that for lower $\lambda$ values, the dissipation increases approximately linearly. We have represented the dissipation introduced by all the modes, and the mode that recovers $\omega=0$ for $\hat{k}=0$ is colored in black. Next, in the range $\lambda \approx 1$, all the modes are replications of the physical (primary) mode, and we get a configuration similar to Fig. 9.4(b), where the peak of dissipation increases with $\lambda$. 


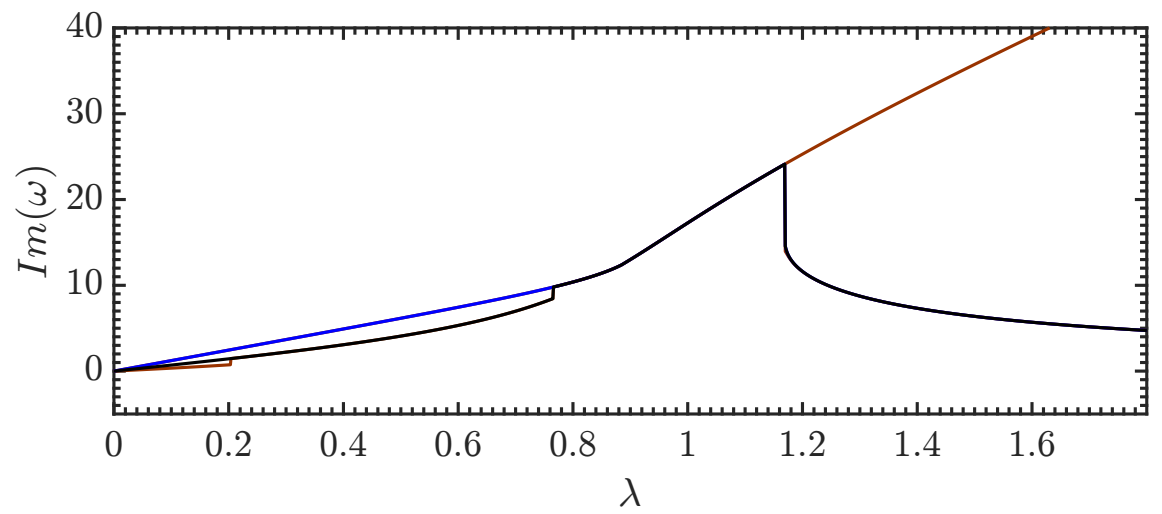

Figure 9.5: Diffusion of the different mode sets, and the effect of the Riemann solver parameter $\lambda$. This representation allows us to follow the different bifurcation points that change the behaviour of the scheme dissipation. The colours represent the different mode groups identified in Figure 9.2(a). The precise values of $\operatorname{Im}(\omega)$ for the cases studied in the TGV (next section) are $0.7038(\lambda=0.1), 17.27(\lambda=1), 0.4065$ $(\lambda=10)$

Finally, for $\lambda>1.2$ the dissipation of all the modes (including the mode that recovers $\omega=0$ for $\hat{k}=0$ ) decreases with $\lambda$. Thus, it is convenient to use a $\lambda$ value below $\lambda \lesssim 1$, so that the numerical flux truly introduces numerical dissipation to the scheme.

\section{Navier-Stokes TGV problem}

In the linear von Neumann analysis, we have found that upwind Riemann solvers concentrate their dissipation in high wave-numbers. This behaviour was already noted in Hesthaven and Warburton [2007]; Gassner and Kopriva [2011]; Alhawwary and Wang [2018]; Moura et al. [2016]. We have also found that the amount of dissipation introduced is non-linearly controlled with $\lambda$, and that values above approximately $\lambda=1$ yield lower dissipation rates.

In this section, we study how this dissipation is introduced in the non-linear Euler equations. To do so, we solve the inviscid TGV problem (with Mach number $M_{0}=0.1$ ) introducing the $\lambda$ stabilisation based on the Roe dissipation described in (9.18).

We construct a Cartesian mesh with $32^{3}$ elements and approximate the solution by order $N=3$ polynomials. We represent the numerical dissipation for $t<20$ in Figure 9.6(a). We have considered three $\lambda$ values: 0.1 (low dissipation Roe), 1 (standard Roe), and 10 (hyper-upwind) Moura et al. [2015]. Recall that the dissipation is only introduced numerically through the Riemann solver, since we consider the inviscid Euler equations (i.e. there is no physical viscosity). From the analysis of Fig. 9.6(a), 


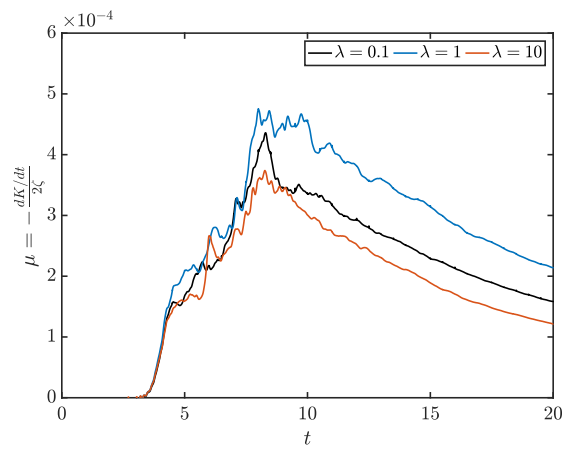

(a) Numerical viscosity

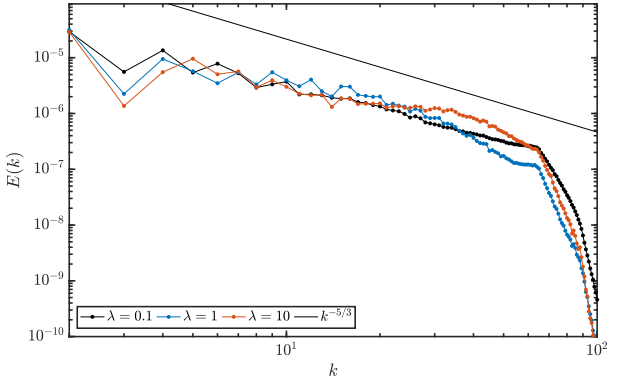

(b) Kinetic energy spectra in $t=14$

Figure 9.6: Inviscid Taylor-Green vortex problem $\left(M_{0}=0.1\right)$. The configuration is a periodic box with $32^{3}$ elements and polynomial order $N=3$. For the interfaces, we have used Roe Riemann solver with the lambda stabilisation $\lambda \operatorname{diss}(u, \phi)$ as defined in (9.19). We have considered the values $\lambda=0.1,1$, and 10 , whose results show clear parallelism with von Neumann analysis performed in Figure 9.5

we find that the maximum dissipation is achieved with $\lambda=1$, which is in agreement with the von Neumann results in the previous section. Therefore, for $\lambda \lesssim 1$ increasing $\lambda$ increases the scheme numerical dissipation, but increasing $\lambda$ for $\lambda \gtrsim 1$ yields the reverse effect, as demonstrated in von Neumann analysis (see Figure 9.5).

Figure 9.6(b) shows the kinetic energy spectra in $t=14$. We find that all three $\lambda$ values behave similarly in the low and medium wave-numbers range. At high wavenumbers, we find that the maximum dissipation is achieved by the standard Roe Riemann solver $(\lambda=1)$, whereas for the other two values there is some energy accumulation in the final part of the spectra. This energy accumulation is common when using central fluxes and it is also a side effect of the hyper-upwinding caused by the Lax-Friedrichs flux with low Mach numbers Winters et al. [2018].

The simulation with $\lambda=1$ can be regarded as an over-dissipated solution, i.e., the dissipation levels are higher than required and the energy spectra does not follow the theoretical $-5 / 3$ slope. This is enhanced with the use of lower numerical dissipation in the $\lambda=0.1$ simulation. Note that $\lambda=0.1$ is similar in spirit to the low dissipation Roe Riemann solver derived in Oßwald et al. [2016] for $M_{0}=0.1$. Finally, the Roe Riemann solver with the high value $\lambda=10$ suffers an accumulation of energy at high wave-numbers, as a result of its lack of dissipation compared to lower $\lambda$ values (see Figure 9.6(a)). 


\subsubsection{Internal dissipation with the Spectral Vanishing Viscosity}

In this section, we study the effect of the spectral vanishing viscosity method applied to discontinuous Galerkin schemes, using the framework introduced in Section 9.2.4, and in the context of turbulence modelling. Only in this section, we use a constant SVV viscosity $\eta_{S V V}$ to emphasize the effect of the filtering operator introduced to the viscous fluxes.

\section{Von Neumann analysis}

This section studies the internal dissipation introduced by the SVV. We perform von Neumann analyses to the linear advection equation, (9.1) with the SVV flux described in (9.20), which uses the power kernel written in (9.29). Note that the SVV method is reduced to a standard second order derivative (a viscous flux) for $P_{S V V}=0$, which is also inluded in the simulations as a point of reference.

We depict von Neumann dissipation curves with $\eta_{S V V}=0.005\left(\operatorname{Re}_{S V V}=a L / \eta_{S V V}=\right.$ 200) and we vary $P_{S V V}$ in Figure 9.7(a), and its detailed view in Figure 9.7(b). This constant value of $\eta_{S V V}$ is only used in this section to provide an example of the behavior of the SVV. As expected, the SVV with $P_{S V V}=0$ represents a second order derivative and behaves in the low wave-numbers range as $-k^{2}$, introducing low wave-number range dissipation. This is basically the impact of a standard Smagorinsky LES model in the discretization. The problem is that methods based on a second order derivative, which were developed in the finite volume community, tend to introduce nonvanishing dissipation even with smooth flows (e.g. laminar regimes), as reported in Fernandez et al. [2017]. The SVV is specially useful to overcome this drawback, since it filters-out low frequency modes and prevents to dissipate smooth solutions.

In the default view of Figure 9.7(a), only a subtle difference is recognised since the effect of the SVV is concentrated in low and medium wave-numbers. Thus, to understand the effectiveness of the SVV, we check the dissipation curve zoomed at low and medium wave-numbers (Figure 9.7(b)). We show that the SVV, with the power kernel (9.29), is able to control the shape of the dissipation curve between a pure viscous discretization $\left(P_{S V V}=0\right.$, labelled as NS) and the inviscid $\left(P_{S V V}>>1\right.$, labelled as Euler), thus enabling a precise control of the numerical dissipation introduced. The impact of the SVV in the dissipation may seem negligible since the overall difference between all the curves is small. Nevertheless, we will show by means of numerical experiments that these small differences are able to control the energy drain in underresolved turbulent flow simulations. 


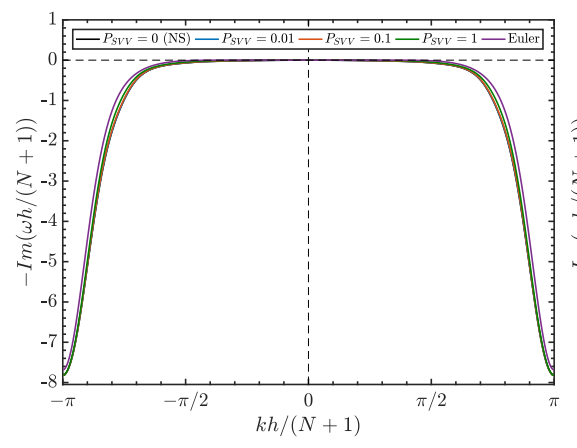

(a) Full view

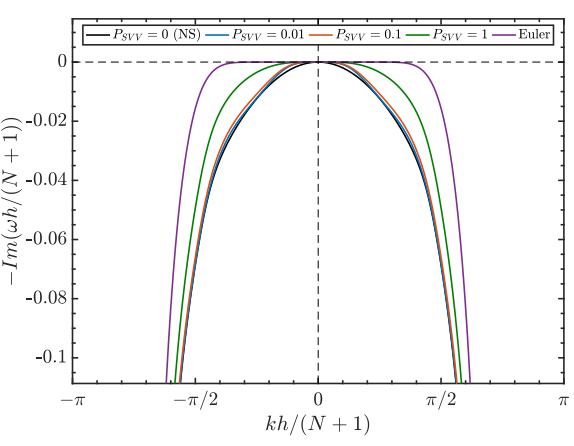

(b) Detailed view

Figure 9.7: Von Neumann dissipation curves using SVV with upwind $(\lambda=1)$. Particularly, the effect of the SVV kernel power, $P_{S V V}$, is studied, where we have also included the particular cases with $P_{S V V}=0$ (standard second order derivative), and the inviscid limit (Euler)

\section{Navier-Stokes equations}

The linear von Neumann analysis shows that the SVV method allows us to modify and reshape the dissipation curve between that with upwind Riemann solvers and that found for second order derivatives $\left(P_{S V V}=0\right)$. The SVV introduces dissipation mostly in low and medium wave-numbers.

To test the SVV capabilities in the Euler equations, we consider the inviscid TGV problem described in Sec. 9.2.5. In this study we use a coarser Cartesian $4^{3}$ mesh with $N=8$ to test the SVV in a severely under-resolved configuration. In this section, we have adopted the low dissipation Roe $\mathrm{L}^{2} \mathrm{R}$ Oßwald et al. [2016] as the Riemann solver (approximately equivalent to $\lambda \approx 0.1$ when $M_{0}=0.1$, although the scaling is performed with the local Mach number, cells with lower velocities will introduce less dissipation) as it was shown before (see Fig. 9.6(b)) that the standard Roe $(\lambda=1)$ produces over-dissipated solutions for this problem. The SVV viscosity is set to $\eta_{S V V}=$ 0.005 (equivalent to a Reynolds number $R e_{S V V}=200$ ), and we study the effect of the kernel power coefficient by evaluating three values: $P_{S V V}=0.1,1$, and 10 . We have chosen this value for the SVV viscosity since preliminary simulations showed an excess of dissipation for the selected mesh. The SVV approach removes the extra dissipation with filtering (controlled through $P_{S V V}$ ), instead of lowering the viscosity $\eta_{S V V}$.

We depict the kinetic energy spectra at $t=8$ in Figure 9.8, for the three SVV kernel power $P_{S V V}$ values considered. We find that the energy decay at high wave-numbers is similar for all simulations, thus supporting von Neumann results (Figure 9.7(a)). At low and medium wave-numbers, we confirm the effectiveness of the SVV to shape 


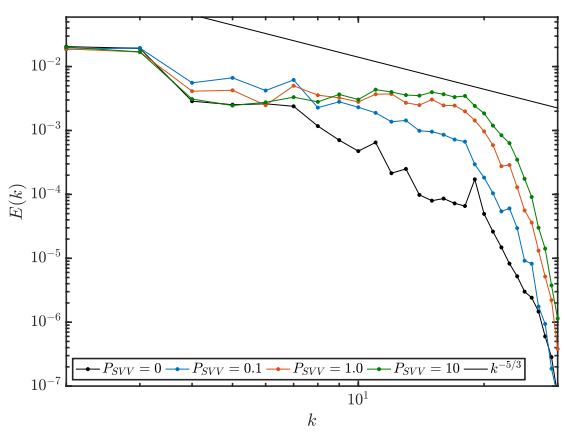

Figure 9.8: Inviscid Taylor-Green vortex problem (with Mach number $M_{0}=0.1$ ) with $4^{3}$ elements and polynomial order $N=8$. We have used the SVV operator as defined in (9.30), where we set the SVV viscosity to $\eta_{S V V}=0.005$ (equivalent to a $R e_{S V V}=$ 200), and we vary the kernel power $P_{S V V}$. Note that the major difference between the standard Navier-Stokes discretization $\left(P_{S V V}=0\right)$ and the $P_{S V V}=0.1$ scheme is on the low wave-numbers

the dissipation curve and adjust the energy spectra. We show that $P_{S V V}=0.1$ yields a favourable result according to Figure 9.8, where the energy decays approximately following Kolmogorov's theoretical $k^{-5 / 3}$ rate Pope [2001], and is dissipated for high wave-numbers without producing energy accumulation. We find that both $P_{S V V}=1$ and $P_{S V V}=10$ present a lack of dissipation at medium wave-numbers, providing a LES configuration with unsatisfactory results, but that $P_{S V V}=0.1$ provide satisfactory results.

Hence, the resulting scheme that combines upwind Riemann solvers and SVV is versatile, but requires an appropriate estimation of two parameters: the SVV viscosity $\eta_{S V V}$ and the kernel power $P_{S V V}$. Besides, a proper LES model (variable value of the turbulent viscosity) has not been included yet. We address these issues in the following section.

\subsection{Design of a Smagorisnky-SVV scheme}

In Sec. 9.3.2 we have assessed the capabilities of upwind Riemann solvers to provide numerical dissipation at high wave-numbers, the capability of second order derivatives to introduce numerical dissipation at low and medium wave-numbers, and the potential of the SVV to shape the dissipation curve between pure elliptical discretizations $\left(P_{S V V}=0\right)$ and the inviscid equation. The latter has been found to effectively introduce dissipation at medium wave-numbers and (if required) also at low wavenumbers. In this section, we combine the strategies presented in the previous section 
to construct a scheme able to provide accurate solutions in turbulent under-resolved flows. Hence, we study the effectiveness for the inviscid TGV problem of a scheme that features a low dissipation Riemann solver to damp high wave-numbers and a SVV to dissipate medium wave-numbers.

One of the drawbacks of the SVV is its requirement to estimate two parameters with a remarkable impact on the final solution (see Figure 9.8). In an attempt to automate the parameter selection, we set the SVV viscosity in (9.31) to that specified by a standard Smagorinsky LES turbulence model as suggested in Karamanos and Karniadakis [2000] and implemented in Kirby and Karniadakis [2002] in the context of continuous Galerkin methods,

$$
\eta_{S V V}=\eta_{t}=C_{S}^{2} \Delta^{2}|S|,
$$

where $S_{i j}=\frac{1}{2}\left(\partial_{j} v_{i}+\partial_{i} v_{j}\right)$ is the laminar strain tensor (i.e. not filtered), and so that only the $P_{S V V}$ parameter remains free. One could argue that we have deleted one parameter $\left(\eta_{S V V}\right)$ to include a new one $\left(C_{S}\right)$. However, we have found that the same value of $C_{S}=0.2$ works in very different configurations, and the same applies to $P_{S V V}$. The Smaroginsky-SVV LES approach works for polynomial orders $N \geq 2$ and is especially well suited for higher orders, where the different scales can be clearly separated.

We consider the inviscid Taylor-Green vortex problem on a Cartesian mesh with $8^{3}$ elements and two polynomial orders, $N=4$ and $N=8$, and we maintain the low dissipation Roe as the Riemann solver. Figure 9.9 depicts the kinetic energy spectra varying the SVV kernel power $P_{S V V}$. It also features the standard Smagorinsky LES model (without SVV) for comparison. We see that the spectra, as in Figure 9.8, shows high dependence on the SVV power kernel, $P_{S V V}$. Precisely, for values $P_{S V V}>0.1$ the dissipation provided by the method is not enough to control the accumulation of energy at high wave-numbers (recall that the higher the kernel power coefficient, the higher is the filtering performed to the viscosity introduced).

The standard Smagorinsky model generates excessive dissipation when the flow is laminar decreasing the overall energy at further times Fernandez et al. [2017]. This has been naturally avoided with the SVV technique, as it filters-out the laminar (smooth) energy components. For completeness, we represent the numerical viscosity introduced by both the Smagorinsky (LES) and the Smagorinsky-SVV LES in Figure 9.10. First, the laminar region (the region without subgrid-scales in the flow, $t<3$ ) produces non-negligible numerical dissipation when using the standard Smagorinsky model, whereas this undesirable dissipation vanishes when considering the Smagorinsky-SVV approach. Second, we confirm the lack of dissipation produced by the SVV method with excessive kernel power $P_{S V V}$.

We conclude that the value $P_{S V V}=0.1$ is appropriate for this test problem. We 

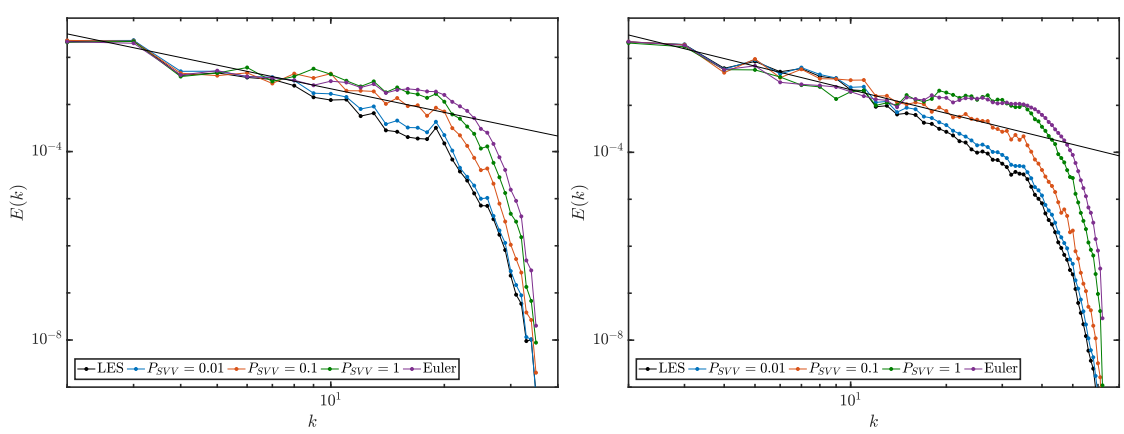

(a) Energy spectra with polynomial (b) Energy spectra with polynomial order $N=4$ order $N=8$

Figure 9.9: Kinetic energy spectra in $t=8$ with the proposed Smagorinsky-SVV strategy for two polynomial orders. Both cases were computed using a $8^{3}$ Cartesian mesh, solving the inviscid Taylor-Green vortex problem with Mach number $M_{0}=0.1$. Different values of the SVV kernel power $P_{S V V}$ were studied
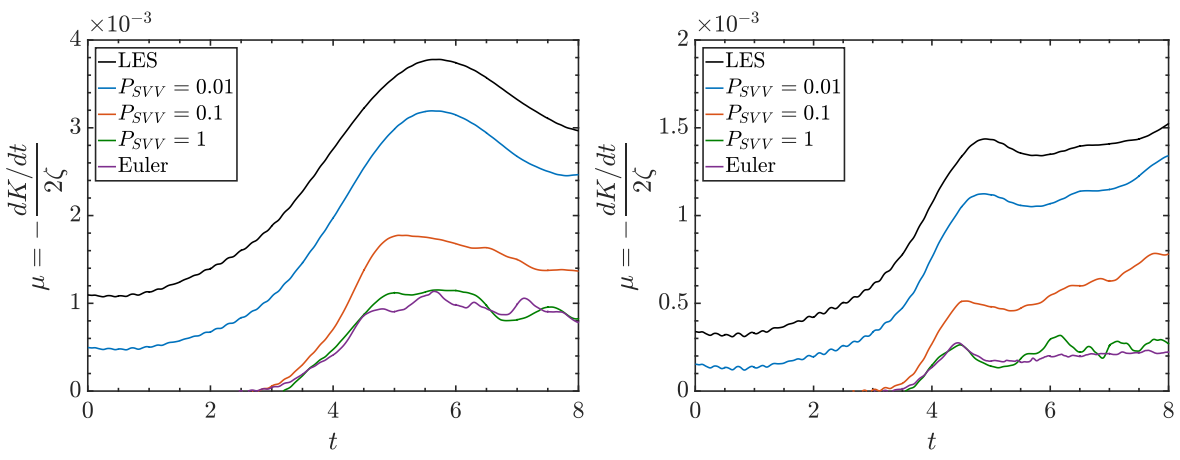

(a) Numerical viscosity with polynomial (b) Numerical viscosity with polynomial order $N=4$ order $N=8$

Figure 9.10: Numerical viscosity introduced by the Smagorinsky-SVV strategy. Both cases were computed using a $8^{3}$ Cartesian mesh. Different values of the SVV kernel power $P_{S V V}$ were studied. Two effects are regarded: the capability of the SVV to remove the dissipation in the laminar region of the TGV problem $(t<3)$, and the lack of dissipation presented by the SVV with high kernel power coefficients 


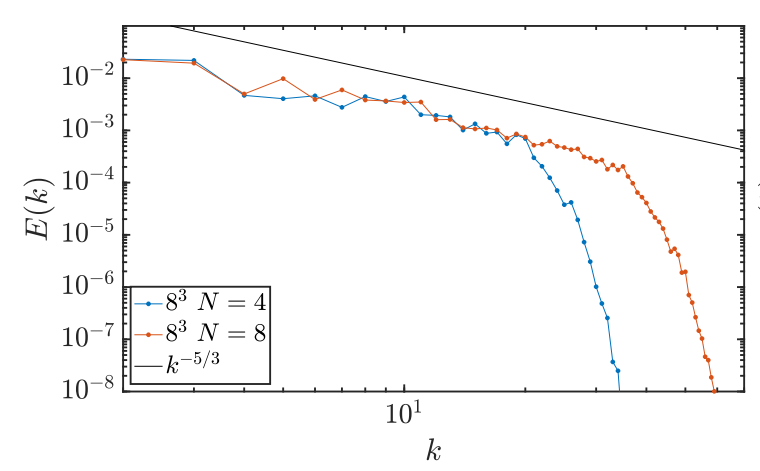

(a) $t=8$

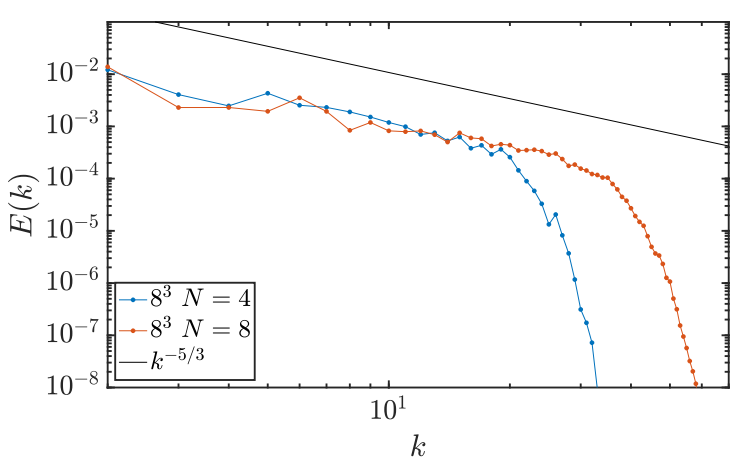

(b) $t=14$

Figure 9.11: Inviscid Taylor-Green vortex problem $\left(M_{0}=0.1\right)$ kinetic energy spectra in $t=8$ and $t=14$ with the Smagorinsky-SVV strategy using two polynomial orders $(N=4$ and $N=8)$. Both cases were computed using a $8^{3}$ Cartesian mesh, and the SVV kernel power is $P_{S V V}=0.1$

show the energy spectra for this configuration in Figure 9.11, showing that the turbulence model is not altered by the polynomial order. For completeness, we have also included the energy spectra for the decay phase $t=14$ in Figure 9.11(b) to confirm that the method still follows the Kolmogorov's slope, and no energy accumulation is found in further times. In summary, the combination of the low dissipation Roe Riemann solver, the spectral vanishing viscosity, and the Smagorinsky model (which has been recently adopted in Flad and Gassner [2017] as a LES model in the context of DG solvers) as an input for $\eta_{S V V}$, with the value $P_{S V V}=0.1$ provides a solid method to simulate turbulent decay.

\subsubsection{Assessment of the Smagorinsky-SVV scheme for a turbulent channel flow at $\operatorname{Re}_{\tau}=183$}

We now test the Smagorinsky-SVV method in a wall-bounded turbulent channel. We maintain the parameters that provided accurate solutions for the TGV problem: the SVV power kernel (9.29) with power coefficient $P_{S V V}=0.1$, and the Smagorinksy model (9.40) with the standard value $C_{S}=0.2$. The Smagorinsky model is complemented with Lilly's near wall treatment Lilly [1992] to avoid non-zero (un-physical) turbulent viscosities near walls. For inviscid fluxes, we retain the $\mathrm{L}^{2} \mathrm{R}$ Riemann solver Oßwald et al. [2016] and Pirozzoli's split form Gassner et al. [2016a]. For time integration, we keep the third order Runge-Kutta scheme with CFL $=0.4$.

The only difference from the previous discretization rests in viscous terms. Due 
to the large velocity gradients experienced in this problem, the turbulent viscosity introduced by Smagorinsky's model is highly discontinuous at element interfaces. This leads to an oscillating LES solution if inter-element dissipation is not enough, which is the case if the Bassi-Rebay 1 scheme is used. Hence, we substitute the latter by the Symmetric Interior Penalty (SIP) method that penalizes inter-element discontinuities, and provides a smoother velocity field (and associated gradients), enabling a smooth LES flow.

The computational domain is a three-dimensional box, the fluid being confined between two planar walls (in the $y$-direction), and periodic boundaries in the remaining two directions. The stream-wise dimension is $L_{x}=2 \pi$, the wall normal direction dimension is $L_{y}=2 \delta$ (with the semi-wall distance $\delta=1$ ), and lateral dimension $L_{z}=\pi$. We construct a mesh with equally spaced divisions $N_{x}=8, N_{y}=12$, and $N_{z}=10$, where the solution is approximated with third order polynomials (which results in a coarse configuration leading to an under-resolved turbulent flow, as described later).

The Reynolds number is fixed to $\operatorname{Re}_{\delta}=U \delta / v=3300$, based on the bulk velocity, or equivalently, $\operatorname{Re}_{\tau}=u_{\tau} \delta / v=183$ based on the friction velocity $u_{\tau}$, whose value is $u_{\tau}=55.5 \cdot 10^{-3} \mathrm{~m} / \mathrm{s}$. The flow is driven by a constant force estimated to achieve a time averaged velocity profile with mean velocity $U=1, F_{x}=\rho u_{\tau}^{2} / \delta=3.08 \cdot 10^{-3} \mathrm{~N}$. The $y^{+}$value for the first cell is $y^{+}=8.34$, which confirms that the simulation is clearly under-resolved. The initial condition is a Poiseuille profile, enriched with Gaussian noise to anticipate the transition onset. We run the simulation for 300 seconds, until the initial transients vanish. Then, we continue the simulation for 650 seconds, and compute averaged statistics in time and homogeneous spatial directions (i.e. only in the $x z$-directions),

$$
\langle\phi\rangle(x)=\frac{1}{N} \sum_{n=1}^{N} \phi\left(x, t_{n}\right), \quad\langle\phi\rangle(y)=\int_{[0,2 \pi] \times[0, \pi]}\langle\phi\rangle(x, y, z) \mathrm{d} x \mathrm{~d} z,
$$

where we get the averaged profiles $\langle\phi\rangle(y)$ as a function of the wall distance $y$. We average both velocities $u_{i}$ and momentum $u_{i} u_{j}$ to get Reynolds stresses $\left\langle u_{i}^{\prime} u_{j}^{\prime}\right\rangle=\left\langle u_{i} u_{j}\right\rangle-$ $\left\langle u_{i}\right\rangle\left\langle u_{j}\right\rangle$.

In Figure 9.12, we show the stream-wise mean velocity $u^{+}=\langle u\rangle / u_{\tau}$ as a function of the wall distance $y^{+}=y u_{\tau} / v$ (Figure 9.12(a)), and the off-diagonal component of the Reynolds stresses $-\left\langle u^{\prime} v^{\prime}\right\rangle / u_{\tau}^{2}$ as a function of the wall distance $y / \delta$ (Figure 9.12(b)). For the mean velocity, we consider the asymptotic law of the wall with $u^{+}=y^{+}$in the viscous sublayer $\left(y^{+}<5\right)$ and $u^{+}=\frac{1}{0.4} \ln y^{+}+5.5$ in the logarithmic region $\left(y^{+}>30\right)$, both represented with dashed lines in Figure 9.12(a). For the Reynolds stresses, we compare with DNS solutions presented in Kim et al. [1987], 


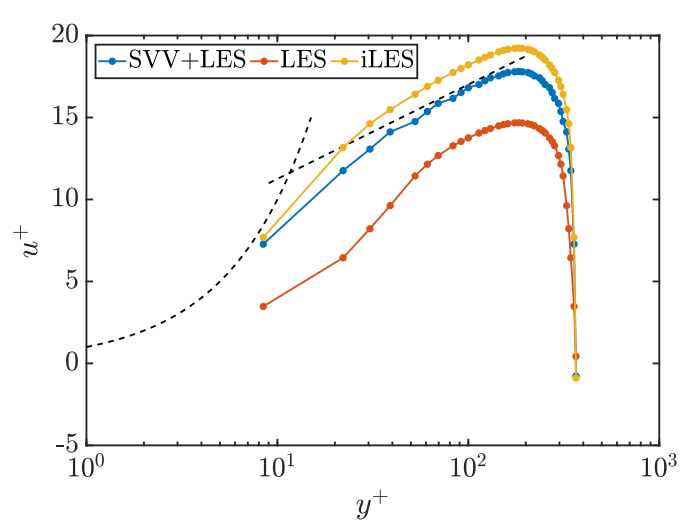

(a) Mean velocity profile $u^{+}=\langle u\rangle / u_{\tau}$

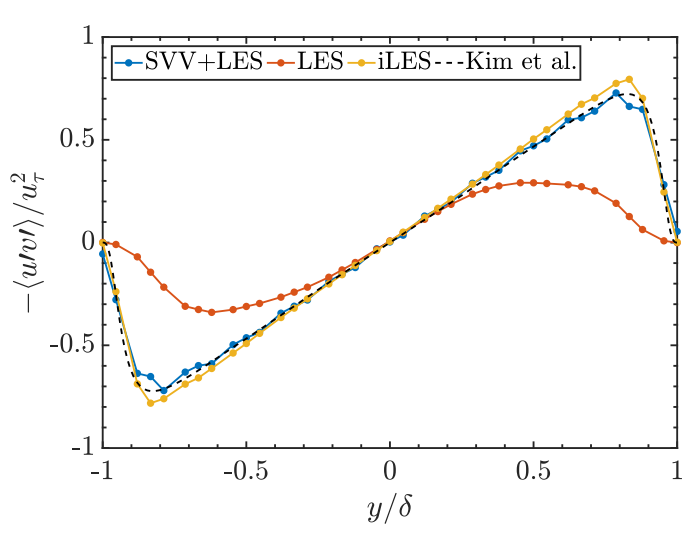

(b) Reynolds stresses $-\left\langle u^{\prime} v^{\prime}\right\rangle / u_{\tau}^{2}$

Figure 9.12: Channel flow statistics as a function of wall distance $y^{+}=y u_{\tau} / v$. We consider three scheme: the Smagorinksy-SVV LES configuration (abbreviated as SVV+LES), the Smagorinsky LES model without SVV (LES), and the implicit LES (iLES). Dashed lines represent the law of the wall, in Figure 9.12(a), and DNS solutions from Kim et al. [1987], in Figure 9.12(b).

also represented with dashed line in Figure 9.12(b).

We consider three schemes, the Smagorinksy-SVV (abbreviated as SVV+LES), the standard Smagorinksy without SVV (LES), and the implicit LES (iLES) where all the dissipation rests on the Riemann solver and the physical viscosity. Whereas the iLES is under-dissipated and the LES is over-dissipated, the Smagorinsky-SVV LES scheme provides a solution comparable to the expected values (for both mean velocity and Reynolds stresses). These favorable results agree with the conclusions found for the TGV problem. Although in the near wall region the results differ from the DNS solution, we did not expect accurate results in this region given the very coarse mesh used near walls $\left(y^{+} \simeq 8.34\right)$.

Overall, we find that the method presented in this chapter provides a remarkable improvement when compared to the standard Smagorinsky method in under-resolved configurations for wall bounded turbulent flows.

\subsection{Conclusions}

We have presented a thorough study on the effect of different dissipation mechanisms for discontinuous Galerkin schemes that can be used to stabilise under-resolved simulations. We have first characterised their properties in wave-number space, by means 
of a linear von Neumann analysis (for a linear advection-diffusion equation), and then validated the model for transitional/turbulent flows in the three-dimensional TaylorGreen Vortex Navier-Stokes problem.

We have studied the dissipation introduced by two strategies: inter-element viscosity (upwind Riemann solvers) and internal viscosity (LES models and spectral vanishing viscosity). We have performed a linear analysis using von Neumann method to discover that the dissipation introduced by upwind Riemann solvers, which penalises the inter-element solution jumps, is not linear with parameter $\lambda$. Instead, the dissipation introduced increases until a critical value is reached, then decreases as the discretization tends to that of a conforming (i.e. continuous Galerkin) method. We have found that an upwind Riemann solver is required to maintain the dissipation introduced at high wave-numbers.

Next, we have studied the dissipation introduced by the Smagorinsky LES model and the spectral vanishing viscosity. On the one hand, we have found that the Smagorinsky LES model introduces an unnecessarily high level of dissipation. On the other hand, we have found that the SVV with an appropriate filtering kernel is able to achieve high control on the dissipation introduced at low and medium wave-number regions, maintaining low dissipation when the flow is laminar.

Combining these ideas, we have proposed a Smagorinsky-SVV LES method, combined with a low dissipation Riemann solver. This Smagorinsky-SVV LES method is able to maintain low dissipation levels in laminar flows, while providing enough dissipation for increased Reynolds numbers. Our method has been calibrated with the Taylor-Green test case, and then challenged for an under-resolved channel flow at Reynolds number $R e_{\tau}=183$, providing accurate statistics.

The Smagorinsky-SVV model has been designed using classic von Neumann analysis. However, new methods based on spatial eigenvalue analyses Mengaldo et al. [2018a,b] may provide further insight into the developed turbulence model, and could complement the presented work. 



\section{Concluding remarks and future work}

In this thesis, we have applied the concept of entropy-stability to construct robust schemes oriented to the solution of multiphase flows. We have derived an entropystable version of the incompressible Navier-Stokes equations with artificial compressibility and variable density, a free-energy stable approximation of the Cahn-Hilliard equation, and an entropy-stable approximation of a two-phase Navier-Stokes/CahnHilliard system. Additionally, we present approximations of the three-phase CahnHilliard model of Boyer and Lapuerta [2006], and a three-phase solver for the prediction of oil and gas transport in pipes. Finally, we study the behavior of the numerical dissipation in the DG method, to provide a novel Smagorinsky-SVV LES model. We conclude that:

- The incompressible Navier-Stokes equations with variable density and artificial compressibility are entropy-stable. We have constructed an entropy-stable DG scheme that uses a split-form scheme (two formulations were derived) and the BR1 scheme. The exact solution of the Riemann problem provides entropystability at the inter-element faces. This solver has been used in this thesis to solve incompressible problems with constant density (e.g. the lid driven cavity), with variable density (e.g. the Rayleigh-Taylor instability), and has been coupled with the Cahn-Hilliard equation to solve multiphase problems.

- The Cahn-Hilliard equation preserves the free-energy, and a DG approximation that discretely preserves the free-energy has been constructed. This scheme uses the BR1 scheme, and we have provided semi- and fully-discrete stability proofs for a first order IMEX scheme. This solver has been used in this thesis to solve the spinodal decomposition in two- and three-dimensional domains.

- We have constructed an entropy-stable two-phase incompressible Navier-Stokes/CahnHilliard system. To get entropy-stability, we have used a conservative form of the Cahn-Hilliard equation, a skew-symmetric form of the momentum equation 
with a specific form of the capillary pressure, and the artificial compressibility model. The system has been constructed such that the standard DG scheme (with Gauss-Lobatto points) is automatically entropy-stable, and there is no need to use split-form schemes. The exact solution of the Riemann problem derived for the incompressible NSE has proven to be entropy-stable also for the two-phase solver if a consistent choice for other additional terms that arise in the multiphase model is provided. The two-phase solver has proven robust and accurate for the test cases solved in this thesis: convergence study (smooth solution), random initial condition, static and rising bubble, and annular flow.

- The last solver constructed in this thesis is a three-phase solver. We use the scheme derived for the incompressible Navier-Stokes equations with artificial compressibility and a newly developed scheme for the three-phase Cahn-Hilliard model derived in Boyer and Lapuerta [2006]. Although the solver uses the splitform scheme, it is not provably stable. However, we have found through the use of numerical experiments that the split-form provides some degree of stabilization, and the resulting scheme is more robust than the standard DG approximation. This solver has been used in this thesis to solve two- and three-phase flows as those found in the oil and gas industry.

- In the last chapter, we design a Smagorinsky-SVV LES model for entropy-stable DG schemes. This model follows from a thorough study of the numerical errors and dissipation techniques available in entropy-stable DG schemes. The model uses the Spectral Vanishing Viscosity method, with the viscosity computed from a standard Smagorinsky model. As a result, the model only has a free-parameter, that controls the SVV filter intensity. The validity of the model has been assessed in the Taylor-Green vortex problem and in the channel flow.

As for the future work, interesting topics that can improve the findings included in this thesis can be:

- The study of a more advanced time integrator (e.g. a velocity-projection scheme Dong [2018a]). In this thesis, all the PDEs are evolved in time using explicit Runge-Kutta methods or simple IMEX schemes. A thorough study of more sophisticated time integration strategies is also essential to fully exploit the capabilities of the solver.

- The study of the stability of alternatives to the BR1 method (e.g. the InteriorPenalty method). The BR1 method has been proven to be stable for the applications used in this thesis, however other alternatives exist (see Arnold et al. 
[2002]). The only drawback we have found to the BR1 method is that the stencil is not compact (i.e. involves the neighbours of the neighbours of the element), which can be avoided using the interior penalty method.

- The study of the Reynolds Averaged Navier-Stokes (RANS) equations. The implementation of a RANS model enables the use of the solver for the stability analysis of complex flows, which is a research topic of the applied mathematics group (see Martinez-Cava et al. [2019]).

- The study of other multiphase models or approximations (e.g. the Allen-Cahn equation). The Cahn-Hilliard equation is attractive since it minimizes the freeenergy and conserves the phases. However, the non-conservative Allen-Cahn equation is also a highly used model, and it would be interesting see how it compares to the Cahn-Hilliard equation to solve multiphase flows.

- The design of an entropy-stable three-phase and N-phase scheme. In this thesis, the three-phase model presented is not entropy-stable, since the entropystability study has not yet been performed. With the entropy-stability analysis, we can introduce modifications to the model and scheme to enhance its robustness. 

Appendices 



\section{Appendix A}

\section{Additional proofs}

\section{A.1 Entropy-stability with non-conservative terms}

In this appendix we show that (3.108) holds if the two-point entropy conserving flux satisfies Tadmor's jump condition (3.106). The DGSEM with Gauss-Lobatto points satisfies the SBP-SAT property, and as a result, the derivative and quadrature mass matrix satisfy,

$$
(M D)+(M D)^{T}=Q+Q^{T}=B
$$

where $B_{i j}=\operatorname{diag}(-1,0, \ldots, 1)$. An important property is that

$$
\sum_{j=0}^{N} Q_{i j} \sum_{j=0}^{N} w_{i} D_{i j}=\sum_{j=0}^{N} w_{i} l_{j}^{\prime}\left(\xi_{i}\right)=0
$$

(i.e. the derivative of a constant is zero). We write the first term in (3.108),

$$
\begin{aligned}
\left\langle\mathbb{D}\left(\overrightarrow{\mathbf{F}}_{e}\right)^{\mathrm{EC}}, \mathbf{W}\right\rangle_{E, N}=2 \sum_{i, j, k=0}^{N} w_{i j k} \mathbf{W}_{i j k}^{T} \sum_{m=0}^{N} & D_{i m} \overrightarrow{\mathbf{F}}_{e}^{\#}\left(Q_{i j k}, Q_{m j k}\right) \cdot\left\{\left\{\mathscr{J} \vec{a}^{1}\right\}\right\}_{(i m) j k} \\
& +D_{j m} \overrightarrow{\mathbf{F}}_{e}^{\#}\left(Q_{i j k}, Q_{i m k}\right) \cdot\left\{\left\{\mathscr{J} \vec{a}^{2}\right\}\right\}_{i(j m) k} \\
& +D_{k m} \overrightarrow{\mathbf{F}}_{e}^{\#}\left(Q_{i j k}, Q_{i j m}\right) \cdot\left\{\left\{\mathscr{J} \vec{a}^{3}\right\}\right\}_{i j(k m)},
\end{aligned}
$$

and we just work on the $\xi$-component, in which we arrange the sums to get the SBPSAT matrix $Q$,

$$
\begin{aligned}
& 2 \sum_{i, j, k=0}^{N} w_{i j k} \mathbf{W}_{i j k}^{T} \sum_{m=0}^{N} D_{i m} \overrightarrow{\mathbf{F}}_{e}^{\#}\left(Q_{i j k}, Q_{m j k}\right) \cdot\left\{\left\{\mathscr{J} \vec{a}^{1}\right\}\right\}_{(i m) j k} \\
= & \sum_{j, k=0}^{N} w_{j k} \sum_{i, m=0}^{N} 2 Q_{i m} \mathbf{W}_{i j k}^{T} \overrightarrow{\mathbf{F}}_{e}^{\#}\left(Q_{i j k}, Q_{m j k}\right) \cdot\left\{\left\{\mathscr{J} \vec{a}^{1}\right\}\right\}_{(i m) j k}
\end{aligned}
$$


we use the SBP-SAT property and switch indexes $i, m$ (all volume terms involved are symmetric),

$$
\begin{aligned}
& \sum_{j, k=0}^{N} w_{j k} \sum_{i, m=0}^{N} 2 Q_{i m} \mathbf{W}_{i j k}^{T} \overrightarrow{\mathbf{F}}_{e}^{\#}\left(Q_{i j k}, Q_{m j k}\right) \cdot\left\{\left\{\mathscr{J} \vec{a}^{1}\right\}\right\}_{(i m) j k} \\
= & \sum_{j, k=0}^{N} w_{j k} \sum_{i, m=0}^{N}\left(Q_{i m}-Q_{m i}+B_{i m}\right) \mathbf{W}_{i j k}^{T} \overrightarrow{\mathbf{F}}_{e}^{\#}\left(Q_{i j k}, Q_{m j k}\right) \cdot\left\{\left\{\mathscr{J} \vec{a}^{1}\right\}\right\}_{(i m) j k} \\
= & \sum_{j, k=0}^{N} w_{j k} \sum_{i, m=0}^{N} Q_{i m}\left(\mathbf{W}_{i j k}^{T}-\mathbf{W}_{m j k}^{T}\right) \overrightarrow{\mathbf{F}}_{e}^{\#}\left(Q_{i j k}, Q_{m j k}\right) \cdot\left\{\left\{\mathscr{J} \vec{a}^{1}\right\}\right\}_{(i m) j k} \\
& +\sum_{j, k=0}^{N} w_{j k} \sum_{i, m=0}^{N} B_{i m} \mathbf{W}_{i j k}^{T} \overrightarrow{\mathbf{F}}_{e}^{\#}\left(Q_{i j k}, Q_{m j k}\right) \cdot\left\{\left\{\mathscr{J} \vec{a}^{1}\right\}\right\}_{(i m) j k}
\end{aligned}
$$

Now we use Tadmor's jump condition (3.106) in the first sum, the potential definition (3.107) in the second (note that $B_{i m} \neq 0$ only if $i=m=0$ or $i=m=N$ ), and we only write the terms inside the $(i, m)$ sum,

$$
\begin{aligned}
= & \sum_{i, m=0}^{N} Q_{i m}\left(\mathbf{W}_{i j k}^{T}-\mathbf{W}_{m j k}^{T}\right) \overrightarrow{\mathbf{F}}_{e}^{\#}\left(Q_{i j k}, Q_{m j k}\right) \cdot\left\{\left\{\mathscr{J} \vec{a}^{1}\right\}\right\}_{(i m) j k} \\
& +\sum_{i, m=0}^{N} B_{i m} \mathbf{W}_{i j k}^{T} \overrightarrow{\mathbf{F}}_{e}^{\#}\left(Q_{i j k}, Q_{m j k}\right) \cdot\left\{\left\{\mathscr{J} \vec{a}^{1}\right\}\right\}_{(i m) j k} \\
= & \sum_{i, m=0}^{N} Q_{i m}\left(\vec{\Psi}_{i j k}-\vec{\Psi}_{m j k}-\sum_{c=1}^{N c} \frac{1}{2}\left(\Lambda_{c, i j k}+\Lambda_{c, m j k}\right)\left(\mathbf{W}_{i j k}^{T} \overrightarrow{\mathbf{\Phi}}_{c, i j k}-\mathbf{W}_{m j k}^{T} \overrightarrow{\mathbf{\Phi}}_{c, m j k}\right)\right) \cdot\left\{\left\{\mathscr{J} \vec{a}^{1}\right\}\right\}_{(i m) j k} \\
& +\sum_{i, m=0}^{N} B_{i m}\left(\vec{\Psi}_{i j k}-\sum_{c=1}^{N_{c}} \mathbf{W}_{i j k}^{T} \overrightarrow{\boldsymbol{\Phi}}_{c, i j k} \Lambda_{c, i j k}+\vec{F}^{\mathscr{E}}\right) \cdot\left\{\left\{\mathscr{J} \vec{a}^{1}\right\}\right\}_{(i m) j k}
\end{aligned}
$$

Next we work on the different terms of the volume integral in order of appearance. In the first, we write the metrics average,

$$
\begin{aligned}
\sum_{i, m=0}^{N} Q_{i m} \vec{\Psi}_{i j k} \cdot\left\{\left\{\mathscr{J} \vec{a}^{1}\right\}\right\}_{(i m) j k} & =\frac{1}{2} \sum_{i=0}^{N} \vec{\Psi}_{i j k} \cdot \mathscr{J} \vec{a}_{i j k}^{1} \sum_{m=0}^{N} Q_{i m}+\frac{1}{2} \sum_{i, m=0}^{N} Q_{i m} \vec{\Psi}_{i j k} \cdot \mathscr{J} \vec{a}_{m j k}^{1} \\
& =\frac{1}{2} \sum_{i, m=0}^{N} Q_{i m} \vec{\Psi}_{i j k} \cdot \mathscr{J} \vec{a}_{m j k}^{1} .
\end{aligned}
$$


In the second, we use the SBP-SAT property and switch the (im) indexes. The volume term associated to $Q_{i m}$ is identical to (A.7),

$$
\begin{aligned}
-\sum_{i, m=0}^{N} Q_{i m} \vec{\Psi}_{m j k}\left\{\left\{\mathscr{J} \vec{a}^{1}\right\}\right\}_{(i m) j k} & =-\sum_{i, m=0}^{N}\left(B_{i m}-Q_{m i}\right) \vec{\Psi}_{m j k}\left\{\left\{\mathscr{J} \vec{a}^{1}\right\}\right\}_{(i m) j k} \\
& =\sum_{i, m=0}^{N} Q_{i m} \vec{\Psi}_{i j k}\left\{\left\{\mathscr{J} \vec{a}^{1}\right\}\right\}_{(i m) j k}-\sum_{i, m=0}^{N} B_{i m} \vec{\Psi}_{i j k} \mathscr{J} \vec{a}_{i j k}^{1} \\
& =\frac{1}{2} \sum_{i, m=0}^{N} Q_{i m} \vec{\Psi}_{i j k} \cdot \mathscr{J} \vec{a}_{m j k}^{1}-\sum_{i, m=0}^{N} B_{i m} \vec{\Psi}_{i j k} \mathscr{J} \vec{a}_{i j k}^{1} .
\end{aligned}
$$

In the third, we span the product, and we define $\vec{\theta}_{c, i j k}=\mathbf{W}_{c, i j k}^{T} \overrightarrow{\boldsymbol{\Phi}}_{c, i j k}$ for simplicity. Then, the first term does not depend on $m$, thus we remove the metrics that do not depend on $m$ as in (A.7), we switch (im) in the third term, and use the SBP-SAT property in the fourth,

$$
\begin{aligned}
& -\frac{1}{2} \sum_{i, m=0}^{N} Q_{i m}\left(\Lambda_{c, i j k} \vec{\theta}_{c, i j k}+\Lambda_{c, m j k} \vec{\theta}_{c, i j k}-\Lambda_{c, i j k} \vec{\theta}_{c, m j k}-\Lambda_{c, m j k} \vec{\theta}_{c, m j k}\right) \cdot\left\{\left\{\mathscr{J} \vec{a}^{1}\right\}\right\}_{(i m) j k} \\
= & -\frac{1}{4} \sum_{i, m=0}^{N} Q_{i m} \Lambda_{c, i j k} \vec{\theta}_{c, i j k} \cdot \mathscr{J} \vec{a}_{m j k}^{1}-\frac{1}{2} \sum_{i, m=0}^{N}\left(Q_{i m}-Q_{m i}\right) \Lambda_{c, m j k} \vec{\theta}_{c, i j k} \cdot\left\{\left\{\mathscr{J} \vec{a}^{1}\right\}\right\}_{(i m) j k} \\
& +\frac{1}{2} \sum_{i, m=0}^{N}\left(B_{i m}-Q_{m i}\right) \Lambda_{c, m j k} \vec{\theta}_{c, m j k} \cdot\left\{\left\{\mathscr{J} \vec{a}^{1}\right\}\right\}_{(i m) j k}
\end{aligned}
$$


Now in the second term we use the SBP-SAT property, we switch (im) in the fourth, and combine boundary and the first and last volume terms,

$$
\begin{aligned}
& -\frac{1}{4} \sum_{i, m=0}^{N} Q_{i m} \Lambda_{c, i j k} \vec{\theta}_{c, i j k} \cdot \mathscr{J} \vec{a}_{m j k}^{1}-\frac{1}{2} \sum_{i, m=0}^{N}\left(Q_{i m}-Q_{m i}\right) \Lambda_{c, m j k} \vec{\theta}_{c, i j k} \cdot\left\{\left\{\mathscr{J} \vec{a}^{1}\right\}\right\}_{(i m) j k} \\
& +\frac{1}{2} \sum_{i, m=0}^{N}\left(B_{i m}-Q_{m i}\right) \Lambda_{c, m j k} \vec{\theta}_{c, m j k} \cdot\left\{\left\{\mathscr{J} \vec{a}^{1}\right\}\right\}_{(i m) j k} \\
= & -\frac{1}{4} \sum_{i, m=0}^{N} Q_{i m} \Lambda_{c, i j k} \vec{\theta}_{c, i j k} \cdot \mathscr{J} \vec{a}_{m j k}^{1}-\frac{1}{2} \sum_{i, m=0}^{N}\left(2 Q_{i m}-B_{i m}\right) \Lambda_{c, m j k} \vec{\theta}_{c, i j k} \cdot\left\{\left\{\mathscr{J} \vec{a}^{1}\right\}\right\}_{(i m) j k} \\
& +\frac{1}{2} \sum_{i, m=0}^{N}\left(B_{i m}-Q_{i m}\right) \Lambda_{c, i j k} \vec{\theta}_{c, i j k} \cdot\left\{\left\{\mathscr{J} \vec{a}^{1}\right\}\right\}_{(i m) j k} \\
= & -\frac{1}{2} \sum_{i, m=0}^{N} Q_{i m} \Lambda_{c, i j k} \vec{\theta}_{c, i j k} \cdot \mathscr{J} \vec{a}_{m j k}^{1}-\sum_{i, m=0} Q_{i m} \Lambda_{c, m j k} \vec{\theta}_{c, i j k} \cdot\left\{\left\{\mathscr{J} \vec{a}^{1}\right\}\right\}_{(i m) j k} \\
& +\sum_{i, m=0}^{N} B_{i m} \Lambda_{c, i j k} \vec{\theta}_{c, i j k} \cdot \mathscr{J} \vec{a}_{i j k}^{1}
\end{aligned}
$$

We replace (A.7), (A.8) and (A.10) in (A.6), and cancel terms out

$$
\begin{aligned}
& \sum_{i, m=0}^{N} Q_{i m}\left(\vec{\Psi}_{i j k}-\vec{\Psi}_{m j k}-\sum_{c=1}^{N c} \frac{1}{2}\left(\Lambda_{c, i j k}+\Lambda_{c, m j k}\right)\left(\mathbf{W}_{i j k}^{T} \overrightarrow{\mathbf{\Phi}}_{c, i j k}-\mathbf{W}_{m j k}^{T} \overrightarrow{\mathbf{\Phi}}_{c, m j k}\right)\right) \cdot\left\{\left\{\mathscr{J} \vec{a}^{1}\right\}\right\}_{(i m) j k} \\
& +\sum_{i, m=0}^{N} B_{i m}\left(\vec{\Psi}_{i j k}-\sum_{c=1}^{N_{c}} \mathbf{W}_{i j k}^{T} \vec{\Phi}_{c, i j k} \Lambda_{c, i j k}+\vec{f}^{\mathscr{E}}\right) \cdot\left\{\left\{\mathscr{J} \vec{a}^{1}\right\}\right\}_{(i m) j k} \\
= & \sum_{i, m=0}^{N} Q_{i m} \vec{\Psi}_{i j k} \cdot \mathscr{J} \vec{a}_{m j k}^{1}-\sum_{i, m=0}^{N} B_{i m} \vec{\Psi}_{i j k} \mathscr{J} \vec{a}_{i j k}^{1} \\
& -\frac{1}{2} \sum_{i, m=0}^{N} Q_{i m} \Lambda_{c, i j k} \vec{\theta}_{c, i j k} \cdot \mathscr{J} \vec{a}_{m j k}^{1}-\sum_{i, m=0} Q_{i m} \Lambda_{c, m j k} \vec{\theta}_{c, i j k} \cdot\left\{\left\{\mathscr{J} \vec{a}^{1}\right\}\right\}_{(i m) j k} \\
& +\sum_{i, m=0}^{N} B_{i m} \Lambda_{c, i j k} \vec{\theta}_{c, i j k} \cdot \mathscr{J} \vec{a}_{i j k}^{1}+\sum_{i, m=0}^{N} B_{i m}\left(\vec{\Psi}_{i j k}-\sum_{c=1}^{N_{c}} \mathbf{W}_{i j k}^{T} \overrightarrow{\mathbf{\Phi}}_{c, i j k} \Lambda_{c, i j k}+\vec{F}^{\mathscr{E}}\right) \cdot\left\{\left\{\mathscr{J} \vec{a}^{1}\right\}\right\}_{(i m) j k} \\
= & \sum_{i, m=0}^{N} Q_{i m} \vec{\Psi}_{i j k} \cdot \mathscr{J} \vec{a}_{m j k}^{1}-\frac{1}{2} \sum_{i, m=0}^{N} Q_{i m} \Lambda_{c, i j k} \vec{\theta}_{c, i j k} \cdot \mathscr{J} \vec{a}_{m j k}^{1} \\
& -\sum_{i, m=0}^{N} Q_{i m} \Lambda_{c, m j k} \vec{\theta}_{c, i j k} \cdot\left\{\left\{\mathscr{J} \vec{a}^{1}\right\}\right\}_{(i m) j k}+\sum_{i, m=0}^{N} B_{i m} \vec{F}^{\mathscr{E}} \cdot \mathscr{J} \vec{a}_{i j k}^{1} \cdot
\end{aligned}
$$


Now, we add the $\eta$ - and $\zeta$ - components,

$$
\begin{aligned}
& \left\langle\mathbb{D}\left(\overrightarrow{\mathbf{F}}_{e}\right)^{\mathrm{EC}}, \mathbf{W}\right\rangle_{E, N}=\sum_{i, j, k=0}^{N} w_{i j k}\left(\Psi_{i j k}-\frac{1}{2} \vec{\theta}_{c, i j k} \Lambda_{c, i j k}\right) \sum_{m=0}^{N}\left(D_{i m} \mathscr{J} \vec{a}_{m j k}^{1}+D_{j m} \mathscr{J} \vec{a}_{i m k}^{2}+D_{k m} \mathscr{J} \vec{a}_{i j m}^{3}\right) \\
& +\sum_{i, j, k=0}^{N} \vec{F}_{i j k}^{\mathscr{E}} \cdot\left(w_{j k} \delta_{i m} \mathscr{J} \vec{a}_{i j k}^{1}+w_{i k} \delta_{j m} \mathscr{J} \vec{a}_{i j k}^{3}+w_{i j} \delta_{k m} \mathscr{J} \vec{a}_{i j k}^{3}\right)_{m=0}^{m=N} \\
& -\sum_{i, j, k=0}^{N} w_{i j k} \mathbf{W}_{i j k}^{T} \overrightarrow{\mathbf{\Phi}}_{c, i j k} \cdot \sum_{m=0}^{N}\left(D_{i m}\left\{\left\{\mathscr{J} \vec{a}^{1}\right\}\right\}_{(i m) j k} \Lambda_{c, m j k}\right. \\
& \left.+D_{j m}\left\{\left\{\mathscr{J} \vec{a}^{2}\right\}\right\}_{i(j m) k} \Lambda_{c, i m k}+D_{k m}\left\{\left\{\mathscr{J} \vec{a}^{3}\right\}\right\}_{i j(k m)} \Lambda_{c, i j m}\right) \\
& =\int_{\partial E, N} \vec{F}^{\mathscr{E}} \cdot \vec{n} \mathrm{~d} S-\left\langle\mathbf{W}^{T}, \overrightarrow{\tilde{\mathbf{\Phi}}}_{c} \cdot \mathbb{D}_{\text {grad }}^{\mathrm{DM}} \Lambda_{c}\right\rangle_{E, N}
\end{aligned}
$$

Thus, we prove (3.108). The first term vanishes since the discrete metric identities hold, the second term is the entropy flux boundary integral, and the third term cancels out the non-conservative term. 



\section{Appendix B}

\section{HORSES3D: A High Order (DG) Spectral Element Solver}

HORSES3D is a 3D parallel code that uses the high-order Discontinuous Galerkin Spectral Element Method (DGSEM) and is written in modern object-oriented Fortran (2008+). The code is currently being developed at ETSIAE-UPM (the School of Aeronautics of the Universidad Politécnica de Madrid), and its the framework where the three entropy-stable solvers presented in this thesis are currently implemented. HORSES3D is built on the NSLITE3D code by David A. Kopriva, which is based on the book "Implementing Spectral Methods for Partial Differential Equations" Kopriva [2009]. The main developers of HORSES3D are Andrés M. Rueda-Ramírez, Gonzalo Rubio, Juan Manzanero, Wojciech Laskowski, and Gerasimos Ntoukas.

HORSES3D provides the following features:

- Hybrid (shared and distributed memory) CPU-based parallelization with OpenMP and MPI.

- A multiphysics environment where the compressible Euler equations of gas dynamics, the compressible Navier-Stokes equations, the incompressible Navier-Stokes equations with artificial compressibility Shen [1997], the CahnHilliard equation, and the entropy-stable two-phases solver are implemented.

- Implementations of the split-form DGSEM.

- Support for arbitrary high-order, p-anisotropic discretizations.

- Implicit time integration schemes such as the Backward Differentiation Formulas (BDFs) and the Rosenbrock type implicit Runge-Kutta methods. 
- Multiple linear solvers for the implicit time-integration schemes: a preconditioned matrix-free GMRES solver, a direct LU solver (the implementation of PARDISO that is present in Intel's Math Kernel Library -MKL), a matrix-based GMRES solver (from the PETSc library Balay et al. [2019]) and static-condensation methods.

- Numerical Jacobian computations with a coloring algorithm.

- Analytical Jacobian computations for nonlinear advection and/or diffusion systems of conservation laws.

- Nonlinear multigrid techniques with explicit (Runge-Kutta schemes) and implicit (BDF + iterative methods) smoothers. Two cycling stratefies are implemented: Full Multigrid (FMG) and V-Cycling.

- Support for curvilinear, hexahedral, conforming meshes in SpecMesh/HOHQMesh format (*.mesh) and HOPR Hindenlang et al. [2015] format (*.h5).

- Static and dynamic $p$-adaptation methods for steady and unsteady simulations that use $p$-anisotropic truncation error estimates.

\section{B.1 Strong Scalability}

The code is equipped with distributed memory parallelization using MPI, and shared memory parallelization using OpenMP. In this section, we present strong scalability tests for HORSES3D.

\section{B.1.1 Shared Memory (OpenMP)}

The shared-memory parallelization distributes the most expensive tasks (i.e. the volume and surface integral computations) to different threads using OpenMP directives.

In this section, a strong scalability test is presented for the flow around a sphere (the details can be found in Rueda-Ramírez [2019]). Roe is used as the inviscid Riemann Solver and the BR1 scheme for the viscous boundary terms.

Two common OpenMP schedules were tested: static and guided. The static schedule divides the loops of elements and faces into equal-sized chunks (or as equal as possible) for the different available threads, i.e.

$$
\text { chunk_size }=\text { num_of_elements/num_of_threads, }
$$


and

$$
\text { chunk_size }=\text { num_of_faces } / \text { num_of_threads, }
$$

respectively. The guided schedule gives a smaller chunk of loop iterations to each thread. When a thread finishes, it retrieves another chunk to compute. The chunk size starts large and decreases as the loop computation is completed.

The computation time needed for taking 100 time steps (RK3) was measured using a 2-socket Intel CPU with $2 \times 20$ cores at $2.2 \mathrm{GHz}$ and 529 GB RAM. Two discretizations with roughly the same number of DOFs $( \pm 5 \%)$ were used:

(a) A uniform order $\left(N_{1}=N_{2}=N_{3}=5\right)$ discretization (411264 DOFs).

(b) The $p$-anisotropic discretization that was obtained with a $\tau$-based $p$-adaptation procedure, which provided an improved accuracy (comparable to the $N=N_{\max }=$ 7 discretization) for a similar number of degrees of freedom (431667 DOFs). The interested reader can find more details in Rueda-Ramírez et al. [2019a,c]

Each of the simulations was run five times and the computation time was averaged. The results are shown in Figure B.1.

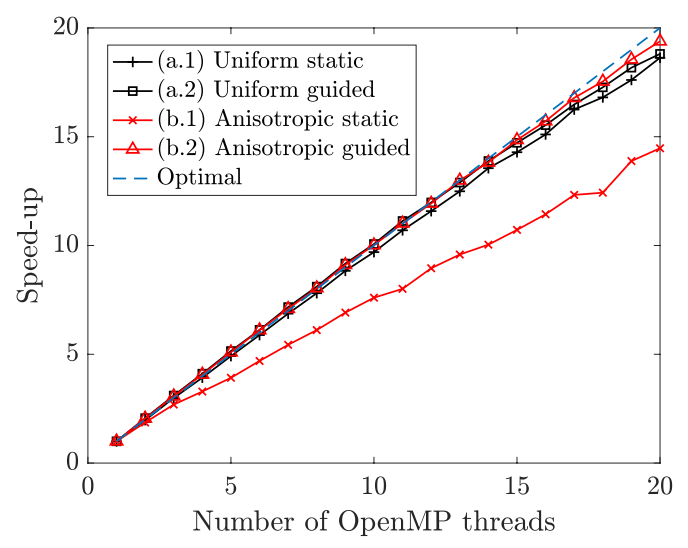

Figure B.1: Strong OpenMP scalability test

As can be seen in Figure B.1, the code has a near-optimal scalability when using OpenMP + guided schedule. Only the anisotropically $p$-adapted discretization performs suboptimally when the OpenMP schedule is static. However, in OpenMP the guided schedule provides an efficient load balancing. 


\section{B.1.2 Distributed Memory (MPI/OpenMP+MPI)}

The benchmark solved herein is the inviscid Taylor-Green Vortex (TGV) problem (see Sec. 9.2.5). We consider a coarse mesh with $32^{3}$, and a fine mesh with $64^{3}$ elements, and we vary the polynomial order from $N=3$ to $N=6$. The solver scalability curves are depicted in Figures B.2 and B.3. We consider both pure MPI, and Hybrid (OpenMP+MPI) strategies. The number of total cores ranges from 1 to 700, and the number of OpenMP threads, when enabled, is 48.

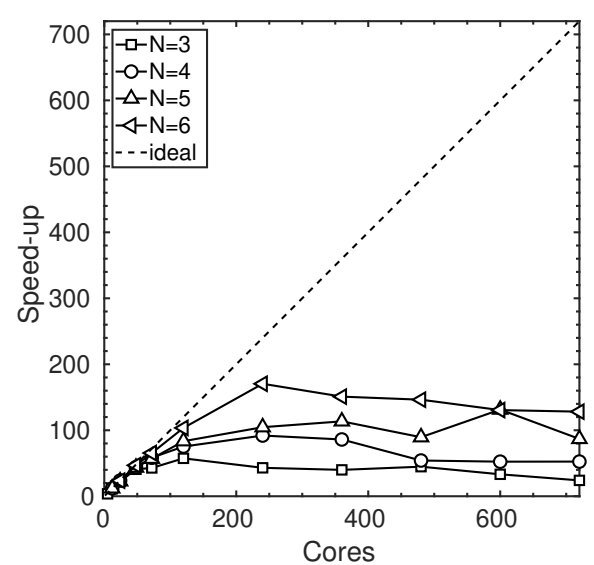

(a) MPI parallelization

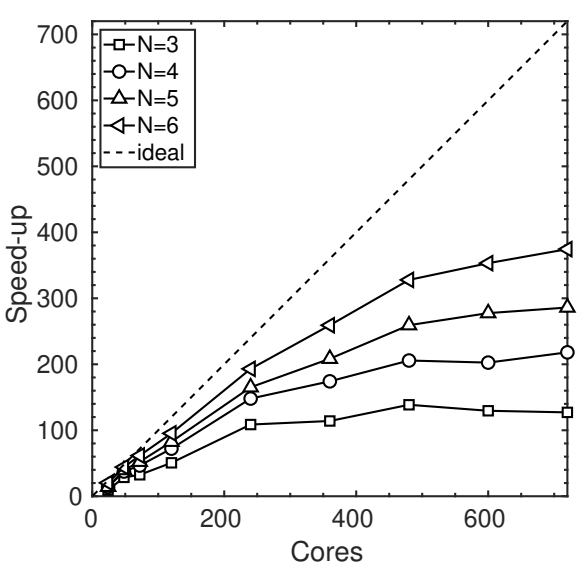

(b) Hybrid MPI+OpenMP parallelization

Figure B.2: Strong scalability solving the TGV problem with a $32^{3}$ mesh

The $32^{3}$ mesh, using MPI (Figure B.2(a)) does not show an increase in the solver speed-up when increasing the number of cores. Since this mesh is coarse (81 elements per core when using 400 cores, 54 elements per core when using 600), it seems normal that communication time is the bottleneck in this simulation. However, with this configuration it is possible to obtain speed-ups of 100 using 100 cores, depending on the polynomial order. The Hybrid strategy B.2(b), on the other hand, is capable to maintain high speed-ups before the stagnation, approximately on 500 cores. The higher the polynomial order, the higher the speed-up since more calculations inside the elements are performed (i.e. they do not involve communication).

The medium mesh is represented in Figure B.3. Regarding the MPI strategy, Figure B.3(a), we observe an improvement when compared to the coarser mesh in Figure B.2(a). We do not observe stagnation for high polynomial orders, and in the rest, the stagnation occurs after using approximately 400 cores. The Hybrid strategy in Figure B.3(b) does not show stagnation in the range considered. Despite being slighly irregular for lower polynomial orders, a straight line is achieved with $N=6$. 


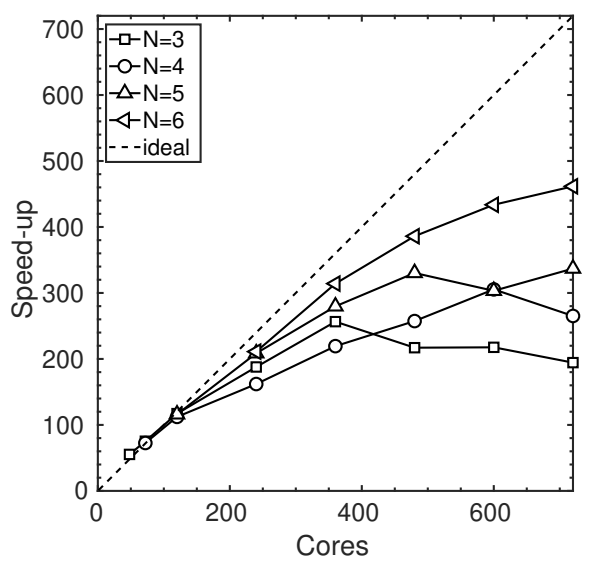

(a) MPI parallelization

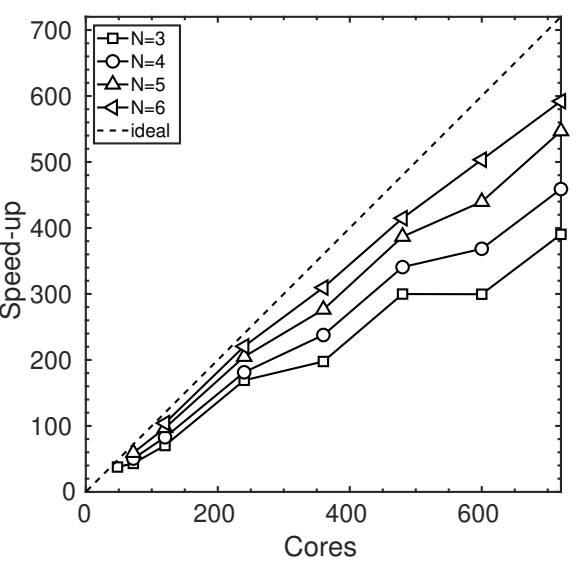

(b) Hybrid MPI+OpenMP parallelization

Figure B.3: Strong scalability solving the TGV problem with a $64^{3}$ mesh 



\section{Bibliography}

A. Jokisaari, P. W. Voorhees, J. E. Guyer, J. Warren, O. Heinonen, Benchmark problems for numerical implementations of phase field models, Computational Materials Science 126 (2017) 139-151.

S.-R. Hysing, S. Turek, D. Kuzmin, N. Parolini, E. Burman, S. Ganesan, L. Tobiska, Quantitative benchmark computations of two-dimensional bubble dynamics, International Journal for Numerical Methods in Fluids 60 (11) (2009) 1259-1288.

Y. Taitel, A. Dukler, A model for predicting flow regime transitions in horizontal and near horizontal gas-liquid flow, AIChE journal 22 (1) (1976) 47-55.

S. B. Pope, Turbulent flows, IOP Publishing, 2001.

G. J. Gassner, A. R. Winters, D. A. Kopriva, Split form nodal discontinuous Galerkin schemes with summation-by-parts property for the compressible Euler equations, Journal of Computational Physics 327 (2016a) 39-66.

J. Kim, P. Moin, R. Moser, Turbulence statistics in fully developed channel flow at low Reynolds number, Journal of fluid mechanics 177 (1987) 133-166.

E. F. Toro, Riemann solvers and numerical methods for fluid dynamics: a practical introduction, Springer Science \& Business Media, 2013.

R. Eymard, T. Gallouët, R. Herbin, Finite volume methods, Handbook of numerical analysis 7 (2000) 713-1018.

R. J. LeVeque, et al., Finite volume methods for hyperbolic problems, vol. 31, Cambridge university press, 2002.

J. Giannakouros, G. E. Karniadakis, A spectral element-FCT method for the compressible Euler equations, Journal of Computational Physics 115 (1) (1994) 65-85. 
D. Funaro, D. Gottlieb, Convergence results for pseudospectral approximations of hyperbolic systems by a penalty-type boundary treatment, Mathematics of Computation 57 (196) (1991) 585-596.

J. S. Hesthaven, D. Gottlieb, A stable penalty method for the compressible NavierStokes equations: I. Open boundary conditions, SIAM Journal on Scientific Computing 17 (3) (1996) 579-612.

D. A. Kopriva, A staggered-grid multidomain spectral method for the compressible Navier-Stokes equations, Journal of computational physics 143 (1) (1998) 125158.

W. H. Reed, T. Hill, Triangular mesh methods for the neutron transport equation, Tech. Rep., Los Alamos Scientific Lab., N. Mex.(USA), 1973.

G. A. Baker, Finite element methods for elliptic equations using nonconforming elements, Mathematics of Computation 31 (137) (1977) 45-59.

F. Bassi, S. Rebay, A high-order accurate discontinuous finite element method for the numerical solution of the compressible Navier-Stokes equations, Journal of computational physics 131 (2) (1997) 267-279.

M. Bohm, A. R. Winters, G. J. Gassner, D. Derigs, F. Hindenlang, J. Saur, An entropy stable nodal discontinuous Galerkin method for the resistive MHD equations. Part I: Theory and numerical verification, Journal of Computational Physics .

F. Bassi, S. Rebay, G. Mariotti, S. Pedinotti, M. Savini, A high-order accurate discontinuous finite element method for inviscid and viscous turbomachinery flows, in: Proceedings of the 2nd European Conference on Turbomachinery Fluid Dynamics and Thermodynamics, Technologisch Instituut, Antwerpen, Belgium, 99-109, 1997.

G. E. Karniadakis, S. J. Sherwin, Spectral/hp element methods for CFD .

W. Klieber, B. Riviere, Adaptive simulations of two-phase flow by discontinuous Galerkin methods, Computer methods in applied mechanics and engineering 196 (1-3) (2006) 404-419.

C. Canuto, M. Y. Hussaini, A. Quarteroni, T. A. Zang, Spectral methods: fundamentals in single domains, Springer Science \& Business Media, 2007.

J. S. Hesthaven, T. Warburton, Nodal discontinuous Galerkin methods: algorithms, analysis, and applications, Springer Science \& Business Media, 2007. 
D. A. Kopriva, Metric Identities and the Discontinuous Spectral Element Method on Curvilinear Meshes, Journal of Scientific Computing 26 (3) (2006) 301, ISSN 15737691.

R. Hartmann, P. Houston, Adaptive discontinuous Galerkin finite element methods for the compressible Euler equations, Journal of Computational Physics 183 (2) (2002) 508-532.

R. Hartmann, Adaptive discontinuous Galerkin methods with shock-capturing for the compressible Navier-Stokes equations, International Journal for Numerical Methods in Fluids 51 (9-10) (2006) 1131-1156.

A. M. Rueda-Ramírez, J. Manzanero, E. Ferrer, G. Rubio, E. Valero, A p-multigrid strategy with anisotropic p-adaptation based on truncation errors for high-order discontinuous Galerkin methods, Journal of Computational Physics 378 (2019a) 209-233.

G. J. Gassner, A skew-symmetric discontinuous Galerkin spectral element discretization and its relation to SBP-SAT finite difference methods, SIAM Journal on Scientific Computing 35 (3) (2013) A1233-A1253.

C. D. Cantwell, D. Moxey, A. Comerford, A. Bolis, G. Rocco, G. Mengaldo, D. De Grazia, S. Yakovlev, J.-E. Lombard, D. Ekelschot, et al., Nektar++: An open-source spectral/hp element framework, Computer physics communications 192 (2015) 205219.

N. Krais, A. Beck, T. Bolemann, H. Frank, D. Flad, G. Gassner, F. Hindenlang, M. Hoffmann, T. Kuhn, M. Sonntag, et al., FLEXI: A high order discontinuous Galerkin framework for hyperbolic-parabolic conservation laws, arXiv preprint arXiv:1910.02858 .

M. Sussman, K. M. Smith, M. Y. Hussaini, M. Ohta, R. Zhi-Wei, A sharp interface method for incompressible two-phase flows, Journal of computational physics 221 (2) (2007) 469-505.

E. Olsson, G. Kreiss, A conservative level set method for two phase flow, Journal of computational physics 210 (1) (2005) 225-246.

J. Lowengrub, L. Truskinovsky, Quasi-incompressible Cahn-Hilliard fluids and topological transitions, Proceedings of the Royal Society of London. Series A: Mathematical, Physical and Engineering Sciences 454 (1978) (1998) 2617-2654.

J. W. Cahn, J. E. Hilliard, Free energy of a nonuniform system. I. Interfacial free energy, The Journal of chemical physics 28 (2) (1958) 258-267. 
J. W. Cahn, J. E. Hilliard, Free energy of a nonuniform system. III. Nucleation in a two-component incompressible fluid, The Journal of chemical physics 31 (3) (1959) 688-699.

A. L. Bertozzi, S. Esedoglu, A. Gillette, Inpainting of binary images using the CahnHilliard equation, IEEE Transactions on image processing 16 (1) (2006) 285-291.

S. Zhou, M. Y. Wang, Multimaterial structural topology optimization with a generalized Cahn-Hilliard model of multiphase transition, Structural and Multidisciplinary Optimization 33 (2) (2007) 89.

E. Khain, L. M. Sander, Generalized Cahn-Hilliard equation for biological applications, Physical Review E 77 (5) (2008) 051129.

B. S. Hosseini, S. Turek, M. Möller, C. Palmes, Isogeometric Analysis of the NavierStokes-Cahn-Hilliard equations with application to incompressible two-phase flows, Journal of Computational Physics 348 (2017) 171-194.

J. Manzanero, G. Rubio, E. Ferrer, E. Valero, D. A. Kopriva, Insights on aliasing driven instabilities for advection equations with application to Gauss-Lobatto discontinuous Galerkin methods, Journal of Scientific Computing 75 (3) (2018a) 1262-1281.

J. Manzanero, G. Rubio, D. A. Kopriva, E. Ferrer, E. Valero, An entropy-stable discontinuous Galerkin approximation for the incompressible Navier-Stokes equations with variable density and artificial compressibility, Journal of Computational Physics (2020a) 109241.

J. Manzanero, G. Rubio, D. A. Kopriva, E. Ferrer, E. Valero, A free-energy stable nodal discontinuous Galerkin approximation with summation-by-parts property for the Cahn-Hilliard equation, Journal of Computational Physics 403 (2020b) 109072.

J. Manzanero, G. Rubio, D. A. Kopriva, E. Ferrer, E. Valero, Entropy-stable discontinuous Galerkin approximation with summation-by-parts property for the incompressible Navier-Stokes/Cahn-Hilliard system, Accepted in Journal of Computational Physics .

J. Manzanero, E. Ferrer, G. Rubio, E. Valero, Design of a Smagorinsky Spectral Vanishing Viscosity turbulence model for discontinuous Galerkin methods, Computers \& Fluids (2020d) 104440.

G. J. Gassner, A. R. Winters, F. J. Hindenlang, D. A. Kopriva, The BR1 scheme is stable for the compressible Navier-Stokes equations, Journal of Scientific Computing 77 (1) (2018) 154-200. 
A. M. Rueda-Ramírez, Efficient Space and Time Solution Techniques for High-Order Discontinuous Galerkin Discretizations of the 3D Compressible Navier-Stokes Equations, Ph.D. thesis, Universidad Politécnica de Madrid, 2019.

D. A. Kopriva, Implementing spectral methods for partial differential equations: Algorithms for scientists and engineers, Springer Science \& Business Media, 2009.

D. A. Kopriva, G. J. Gassner, An energy stable discontinuous Galerkin spectral element discretization for variable coefficient advection problems, SIAM Journal on Scientific Computing 36 (4) (2014) A2076-A2099.

M. H. Carpenter, T. C. Fisher, E. J. Nielsen, S. H. Frankel, Entropy Stable Spectral Collocation Schemes for the Navier-Stokes Equations: Discontinuous Interfaces, SIAM Journal on Scientific Computing 36 (5) (2014) B835-B867.

J. Chan, D. C. Del Rey Fernández, M. H. Carpenter, Efficient entropy stable Gauss collocation methods, SIAM Journal on Scientific Computing 41 (5) (2019) A2938A2966.

E. Tadmor, Entropy stability theory for difference approximations of nonlinear conservation laws and related time-dependent problems, Acta Numerica 12 (2003) 451512.

S. Pirozzoli, Numerical methods for high-speed flows, Annual review of fluid mechanics 43 (2011) 163-194.

P. L. Roe, Approximate Riemann solvers, parameter vectors, and difference schemes, Journal of computational physics 43 (2) (1981) 357-372.

P. D. Lax, Weak solutions of nonlinear hyperbolic equations and their numerical computation, Communications on pure and applied mathematics 7 (1) (1954) 159-193.

K. Oßwald, A. Siegmund, P. Birken, V. Hannemann, A. Meister, $\mathrm{L}^{2}$ Roe: a low dissipation version of Roe's approximate Riemann solver for low Mach numbers, International Journal for Numerical Methods in Fluids 81 (2) (2016) 71-86.

K. Shahbazi, P. F. Fischer, C. R. Ethier, A high-order discontinuous Galerkin method for the unsteady incompressible Navier-Stokes equations, Journal of Computational Physics 222 (1) (2007) 391-407.

E. Ferrer, R. Willden, A high order discontinuous Galerkin finite element solver for the incompressible Navier-Stokes equations, Computers \& Fluids 46 (1) (2011) 224230. 
D. N. Arnold, F. Brezzi, B. Cockburn, L. D. Marini, Unified analysis of discontinuous Galerkin methods for elliptic problems, SIAM journal on numerical analysis 39 (5) (2002) 1749-1779.

E. Ferrer, An interior penalty stabilised incompressible discontinuous Galerkin-Fourier solver for implicit large eddy simulations, Journal of Computational Physics 348 (2017) 754-775.

P.-O. Persson, J. Peraire, Sub-cell shock capturing for discontinuous Galerkin methods, in: 44th AIAA Aerospace Sciences Meeting and Exhibit, 112, 2006.

E. Tadmor, W. Zhong, Entropy stable approximations of Navier-Stokes equations with no artificial numerical viscosity, Journal of Hyperbolic Differential Equations 3 (03) (2006) 529-559.

G. J. Gassner, A. R. Winters, D. A. Kopriva, A well balanced and entropy conservative discontinuous Galerkin spectral element method for the shallow water equations, Applied Mathematics and Computation 272 (2016b) 291-308.

P. J. Schmid, D. S. Henningson, D. Jankowski, Stability and transition in shear flows. Applied mathematical sciences, Vol. 142, Appl. Mech. Rev. 55 (3) (2002) B57-B59.

M. Merriam, An entropy-based approach to nonlinear stability, Tech. Rep., National Aeronautics and Space Administration, Moffett Field, CA (USA). Ames ..., 1989.

D. Flad, G. Gassner, On the use of kinetic energy preserving DG-schemes for large eddy simulation, Journal of Computational Physics 350 (2017) 782-795.

T. C. Fisher, M. H. Carpenter, High-order entropy stable finite difference schemes for nonlinear conservation laws: Finite domains, Journal of Computational Physics 252 (2013) 518 - 557, ISSN 0021-9991.

Y. Liu, C.-W. Shu, M. Zhang, Entropy stable high order discontinuous Galerkin methods for ideal compressible MHD on structured meshes, Journal of Computational Physics 354 (2018) 163-178.

C. W. Hirt, B. D. Nichols, Volume of fluid (VOF) method for the dynamics of free boundaries, Journal of computational physics 39 (1) (1981) 201-225.

B. Nichols, C. Hirt, R. Hotchkiss, SOLA-VOF: A solution algorithm for transient fluid flow with multiple free boundaries, Tech. Rep., Los Alamos Scientific Lab., 1980.

M. Sussman, P. Smereka, S. Osher, A level set approach for computing solutions to incompressible two-phase flow, Journal of Computational physics 114 (1) (1994) 146-159. 
D. Adalsteinsson, J. A. Sethian, A fast level set method for propagating interfaces, Journal of computational physics 118 (2) (1995) 269-277.

D. Jacqmin, Calculation of two-phase Navier-Stokes flows using phase-field modeling, Journal of Computational Physics 155 (1) (1999) 96-127.

V. Badalassi, H. Ceniceros, S. Banerjee, Computation of multiphase systems with phase field models, Journal of computational physics 190 (2) (2003) 371-397.

J. Shen, Pseudo-compressibility methods for the unsteady incompressible NavierStokes equations, in: Proceedings of the 1994 Beijing symposium on nonlinear evolution equations and infinite dynamical systems, 68-78, 1997.

I. Babuška, The finite element method with Lagrangian multipliers, Numerische Mathematik 20 (3) (1973) 179-192.

F. Brezzi, On the existence, uniqueness and approximation of saddle-point problems arising from Lagrangian multipliers, Publications mathématiques et informatique de Rennes (S4) (1974) 1-26.

E. Ferrer, R. H. Willden, A high order discontinuous Galerkin-Fourier incompressible 3D Navier-Stokes solver with rotating sliding meshes, Journal of Computational Physics 231 (21) (2012) 7037-7056.

E. Ferrer, D. Moxey, R. Willden, S. Sherwin, Stability of projection methods for incompressible flows using high order pressure-velocity pairs of same degree: continuous and discontinuous Galerkin formulations, Communications in Computational Physics 16 (3) (2014) 817-840.

C. Cox, C. Liang, M. W. Plesniak, A high-order solver for unsteady incompressible Navier-Stokes equations using the flux reconstruction method on unstructured grids with implicit dual time stepping, Journal of Computational Physics 314 (2016) 414435 .

F. Bassi, F. Massa, L. Botti, A. Colombo, Artificial compressibility Godunov fluxes for variable density incompressible flows, Computers \& Fluids 169 (2017) 186-200.

J. Williamson, Low-storage Runge-Kutta schemes, Journal of Computational Physics 35 (1) (1980) 48 - 56, ISSN 0021-9991.

J. Manzanero, A. M. Rueda-Ramírez, G. Rubio, E. Ferrer, The Bassi Rebay 1 scheme is a special case of the Symmetric Interior Penalty formulation for discontinuous Galerkin discretisations with Gauss-Lobatto points, Journal of Computational Physics 363 (2018b) 1-10. 
C. A. Kennedy, A. Gruber, Reduced aliasing formulations of the convective terms within the Navier-Stokes equations for a compressible fluid, Journal of Computational Physics 227 (3) (2008) 1676-1700.

Y. Liu, C.-W. Shu, M. Zhang, Sub-optimal convergence of discontinuous Galerkin methods with central fluxes for even degree polynomial approximations, arXiv preprint arXiv:2001.03825 .

F. J. Hindenlang, G. J. Gassner, D. A. Kopriva, Stability of Wall Boundary Condition Procedures for Discontinuous Galerkin Spectral Element Approximations of the Compressible Euler Equations, arXiv preprint arXiv:1901.04924 .

L. I. G. Kovasznay, Laminar flow behind a two-dimensional grid, Mathematical Proceedings of the Cambridge Philosophical Society 44 (1) (1948) 58-62.

J.-L. Guermond, L. Quartapelle, A Projection FEM for Variable Density Incompressible Flows, Journal of Computational Physics 165 (1) (2000) 167 - 188.

U. Ghia, K. N. Ghia, C. Shin, High-Re solutions for incompressible flow using the Navier-Stokes equations and a multigrid method, Journal of computational physics 48 (3) (1982) 387-411.

F. S. Schranner, X. Y. Hu, N. A. Adams, A physically consistent weakly compressible high-resolution approach to underresolved simulations of incompressible flows, Computers \& Fluids 86 (2013) 109-124.

P. Fernandez, N.-C. Nguyen, J. Peraire, Subgrid-scale modeling and implicit numerical dissipation in DG-based Large-Eddy Simulation, in: 23rd AIAA Computational Fluid Dynamics Conference, 3951, 2017.

S. Allen, J. Cahn, Ground state structures in ordered binary alloys with second neighbor interactions, Acta Metallurgica 20 (3) (1972) 423 - 433, ISSN 0001-6160.

D. Lee, J. Kim, Comparison study of the conservative Allen-Cahn and the CahnHilliard equations, Mathematics and Computers in Simulation 119 (2016) 35 - 56, ISSN 0378-4754.

Y. Xia, Y. Xu, C.-W. Shu, Local discontinuous Galerkin methods for the Cahn-Hilliard type equations, Journal of Computational Physics 227 (1) (2007) 472 - 491, ISSN 0021-9991.

S. Dong, Multiphase flows of $\mathrm{N}$ immiscible incompressible fluids: A reductionconsistent and thermodynamically-consistent formulation and associated algorithm, Journal of Computational Physics 361 (2018a) 1-49. 
S. Dong, An outflow boundary condition and algorithm for incompressible two-phase flows with phase field approach, Journal of Computational Physics 266 (2014a) 47-73.

Z. Yang, L. Lin, S. Dong, A family of second-order energy-stable schemes for CahnHilliard type equations, Journal of Computational Physics 383 (2019) 24-54.

A. Debussche, L. Dettori, On the Cahn-Hilliard equation with a logarithmic free energy, Nonlinear Analysis: Theory, Methods \& Applications 24 (10) (1995) 1491 - 1514, ISSN 0362-546X.

S. Dong, Multiphase flows of $\mathrm{N}$ immiscible incompressible fluids: A reductionconsistent and thermodynamically-consistent formulation and associated algorithm, Journal of Computational Physics 361 (2018b) 1 - 49, ISSN 0021-9991.

M. Kästner, P. Metsch, R. De Borst, Isogeometric analysis of the Cahn-Hilliard equation-a convergence study, Journal of Computational Physics 305 (2016) 360371.

M. Ainsworth, Dispersive and Dissipative Behaviour of High Order Discontinuous Galerkin Finite Element Methods, J. Comput. Phys. 198 (1) (2004) 106-130, ISSN 0021-9991.

J. Shen, On a new pseudocompressibility method for the incompressible Navier-Stokes equations, Applied numerical mathematics 21 (1) (1996) 71-90.

P. C. Hohenberg, B. I. Halperin, Theory of dynamic critical phenomena, Reviews of Modern Physics 49 (3) (1977) 435.

J. Shen, X. Yang, Energy stable schemes for Cahn-Hilliard phase-field model of twophase incompressible flows, Chinese Annals of Mathematics, Series B 31 (5) (2010) 743-758.

H. Ding, P. D. Spelt, C. Shu, Diffuse interface model for incompressible two-phase flows with large density ratios, Journal of Computational Physics 226 (2) (2007) 2078-2095.

D. Han, X. Wang, A second order in time, uniquely solvable, unconditionally stable numerical scheme for Cahn-Hilliard-Navier-Stokes equation, Journal of Computational Physics 290 (2015) 139-156.

H. Abels, H. Garcke, G. Grün, Thermodynamically consistent, frame indifferent diffuse interface models for incompressible two-phase flows with different densities, Mathematical Models and Methods in Applied Sciences 22 (03) (2012) 1150013. 
X. Feng, J. Kou, S. Sun, A Novel Energy Stable Numerical Scheme for Navier-StokesCahn-Hilliard Two-phase Flow Model with Variable Densities and Viscosities, in: International Conference on Computational Science, Springer, 113-128, 2018.

G. Zhu, H. Chen, J. Yao, S. Sun, Efficient energy-stable schemes for the hydrodynamics coupled phase-field model, Applied Mathematical Modelling 70 (2019) 82-108.

F. Boyer, C. Lapuerta, S. Minjeaud, B. Piar, M. Quintard, Cahn-Hilliard/Navier-Stokes model for the simulation of three-phase flows, Transport in Porous Media 82 (3) (2010) 463-483.

S. Dong, On imposing dynamic contact-angle boundary conditions for wall-bounded liquid-gas flows, Computer Methods in Applied Mechanics and Engineering 247 (2012) 179-200.

D. A. Kopriva, A polynomial spectral calculus for analysis of DG spectral element methods, Springer, 21-40, 2017.

G. Gassner, D. A. Kopriva, A comparison of the dispersion and dissipation errors of Gauss and Gauss-Lobatto discontinuous Galerkin spectral element methods, SIAM Journal on Scientific Computing 33 (5) (2011) 2560-2579.

J. Manzanero, G. Rubio, E. Ferrer, E. Valero, Dispersion-dissipation analysis for advection problems with nonconstant coefficients: Applications to discontinuous Galerkin formulations, SIAM Journal on Scientific Computing 40 (2) (2018c) A747-A768.

B. F. Klose, G. B. Jacobs, D. A. Kopriva, Assessing Standard and Kinetic Energy Conserving Discontinuous Galerkin Formulations for Marginally Resolved Navier-Stokes Flows, arXiv preprint arXiv:1910.13985 .

A. M. Rueda-Ramírez, E. Ferrer, D. A. Kopriva, G. Rubio, E. Valero, A Statically Condensed Discontinuous Galerkin Spectral Element Method on Gauss-Lobatto Nodes for the Compressible Navier-Stokes Equations, arXiv preprint arXiv:1911.02366 .

T. Lee, Effects of incompressibility on the elimination of parasitic currents in the lattice Boltzmann equation method for binary fluids, Computers \& Mathematics with Applications 58 (5) (2009) 987-994.

J. Manzanero, C. Redondo, E. Ferrer, E. Valero, A high-order discontinuous Galerkin solver for multiphase flows, In Lecture notes in computational science and engineering ICOSAHOM 2018. (Accepted for publication) .

F. Boyer, C. Lapuerta, Study of a three component Cahn-Hilliard flow model, ESAIM: Mathematical Modelling and Numerical Analysis 40 (4) (2006) 653-687. 
Y. Xia, Y. Xu, C.-W. Shu, Application of the local discontinuous Galerkin method for the Allen-Cahn/Cahn-Hilliard system, $a \in A$

S. Dong, An efficient algorithm for incompressible N-phase flows, Journal of Computational Physics 276 (2014b) 691-728.

Y. Shi, X.-P. Wang, Modeling and simulation of dynamics of three-component flows on solid surface, Japan Journal of Industrial and Applied Mathematics 31 (3) (2014) 611-631.

F. Boyer, S. Minjeaud, Numerical schemes for a three component Cahn-Hilliard model, ESAIM: Mathematical Modelling and Numerical Analysis 45 (4) (2011) 697-738.

S. Dong, Wall-bounded multiphase flows of $\mathrm{N}$ immiscible incompressible fluids: Consistency and contact-angle boundary condition, Journal of Computational Physics 338 (2017) 21-67.

K. Shahbazi, Short Note: An Explicit Expression for the Penalty Parameter of the Interior Penalty Method, Journal of Computational Physics 205 (2) (2005) 401-407, ISSN 0021-9991.

G. Rubio, F. Fraysse, J. De Vicente, E. Valero, The Estimation of Truncation Error by $\tau$-estimation for Chebyshev Spectral Collocation Method, Journal of Scientific Computing 57 (1) (2013) 146-173.

S. Gómez-Álvarez, A. Rivero-Jiménez, G. Rubio, J. Manzanero, C. Redondo, et al., Novel Coupled Cahn-Hilliard Navier-Stokes Solver for the Evaluation of Oil and Gas Multiphase Flow, in: BHR 19th International Conference on Multiphase Production Technology, BHR Group, 2019.

F. Boyer, S. Minjeaud, Hierarchy of consistent N-component Cahn-Hilliard systems, Mathematical Models and Methods in Applied Sciences 24 (14) (2014) 2885-2928.

F. Xie, X. Zheng, M. S. Triantafyllou, Y. Constantinides, Y. Zheng, G. E. Karniadakis, Direct numerical simulations of two-phase flow in an inclined pipe, Journal of Fluid Mechanics 825 (2017) 189-207.

R. M. Kirby, G. E. Karniadakis, De-aliasing on non-uniform grids: algorithms and applications, Journal of Computational Physics 191 (1) (2003) 249-264.

G. J. Gassner, A. D. Beck, On the accuracy of high-order discretizations for underresolved turbulence simulations, Theoretical and Computational Fluid Dynamics 27 (3-4) (2013) 221-237. 
R. C. Moura, G. Mengaldo, J. Peiró, S. J. Sherwin, On the eddy-resolving capability of high-order discontinuous Galerkin approaches to implicit LES/under-resolved DNS of Euler turbulence, Journal of Computational Physics 330 (2017a) 615-623.

E. Lenormand, P. Sagaut, L. T. Phuoc, P. Comte, Subgrid-scale models for large-eddy simulations of compressible wall bounded flows, AIAA journal 38 (8) (2000) 13401350.

T. J. Hughes, A. A. Oberai, L. Mazzei, Large eddy simulation of turbulent channel flows by the variational multiscale method, Physics of fluids 13 (6) (2001) 1784-1799.

R. M. Kirby, S. J. Sherwin, Stabilisation of spectral/hp element methods through spectral vanishing viscosity: Application to fluid mechanics modelling, Computer methods in applied mechanics and engineering 195 (23-24) (2006) 3128-3144.

E. Tadmor, Convergence of Spectral Methods for Nonlinear Conservation Laws, SIAM Journal on Numerical Analysis 26 (1) (1989) 30-44.

G. Karamanos, G. E. Karniadakis, A spectral vanishing viscosity method for large-eddy simulations, Journal of Computational Physics 163 (1) (2000) 22-50.

C. Carton de Wiart, K. Hillewaert, M. Duponcheel, G. Winckelmans, Assessment of a discontinuous Galerkin method for the simulation of vortical flows at high Reynolds number, International Journal for Numerical Methods in Fluids 74 (7) (2014) 469493.

N. Fehn, W. A. Wall, M. Kronbichler, On the stability of projection methods for the incompressible Navier-Stokes equations based on high-order discontinuous Galerkin discretizations, Journal of Computational Physics 351 (2017) 392-421.

M. de la Llave Plata, V. Couaillier, M.-C. Le Pape, On the use of a high-order discontinuous Galerkin method for DNS and LES of wall-bounded turbulence, Computers \& Fluids 176 (2018) 320-337.

M. Alhawwary, Z. J. Wang, Fourier analysis and evaluation of DG, FD and compact difference methods for conservation laws, Journal of Computational Physics 373 (2018) 835-862.

R. C. Moura, P. Fernandez, G. Mengaldo, S. J. Sherwin, Temporal eigenanalysis of HDG methods for linear advection-diffusion (2018) .

R. C. Moura, S. J. Sherwin, J. Peiró, Linear dispersion-diffusion analysis and its application to under-resolved turbulence simulations using discontinuous Galerkin spectral/hp methods, Journal of Computational Physics 298 (2015) 695-710. 
P. Fernandez, R. C. Moura, G. Mengaldo, J. Peraire, Non-modal analysis of spectral element methods: Towards accurate and robust large-eddy simulations, Computer Methods in Applied Mechanics and Engineering 346 (2019) 43-62.

A. R. Winters, R. C. Moura, G. Mengaldo, G. J. Gassner, S. Walch, J. Peiro, S. J. Sherwin, A comparative study on polynomial dealiasing and split form discontinuous Galerkin schemes for under-resolved turbulence computations, Journal of Computational Physics 372 (2018) 1 - 21, ISSN 0021-9991.

R. M. Kirby, G. E. Karniadakis, Coarse resolution turbulence simulations with spectral vanishing viscosity-large-eddy simulations (SVV-LES), TRANSACTIONS-AMERICAN SOCIETY OF MECHANICAL ENGINEERS JOURNAL OF FLUIDS ENGINEERING 124 (4) (2002) 886-891.

R. C. Moura, S. J. Sherwin, J. Peiró, Eigensolution analysis of spectral/hp continuous Galerkin approximations to advection-diffusion problems: Insights into spectral vanishing viscosity, Journal of Computational Physics 307 (2016) 401-422.

B.-y. Guo, H.-p. Ma, E. Tadmor, Spectral vanishing viscosity method for nonlinear conservation laws, SIAM Journal on Numerical Analysis 39 (4) (2001) 1254-1268.

J. Smagorinsky, General circulation experiments with the primitive equations: I. The basic experiment, Monthly weather review 91 (3) (1963) 99-164.

G. I. Taylor, A. E. Green, Mechanism of the production of small eddies from large ones, Proceedings of the Royal Society of London. Series A-Mathematical and Physical Sciences 158 (895) (1937) 499-521.

R. C. Moura, G. Mengaldo, J. Peiró, S. J. Sherwin, An LES setting for DG-based implicit LES with insights on dissipation and robustness, in: Spectral and High Order Methods for Partial Differential Equations ICOSAHOM 2016, Springer, 161-173, 2017b.

D. K. Lilly, A proposed modification of the Germano subgrid-scale closure method, Physics of Fluids A: Fluid Dynamics 4 (3) (1992) 633-635.

G. Mengaldo, D. De Grazia, R. C. Moura, S. J. Sherwin, Spatial eigensolution analysis of energy-stable flux reconstruction schemes and influence of the numerical flux on accuracy and robustness, Journal of Computational Physics 358 (2018a) 1-20.

G. Mengaldo, R. Moura, B. Giralda, J. Peiró, S. Sherwin, Spatial eigensolution analysis of discontinuous Galerkin schemes with practical insights for under-resolved computations and implicit LES, Computers \& Fluids 169 (2018b) 349-364. 
A. Martinez-Cava, E. Valero, J. De Vicente, G. Paniagua, Coanda Flow Characterization on Base Bleed Configurations Using Global Stability Analysis, in: AIAA Scitech 2019 Forum, 0881, 2019.

S. Balay, S. Abhyankar, M. Adams, J. Brown, P. Brune, K. Buschelman, L. Dalcin, A. Dener, V. Eijkhout, W. Gropp, et al., PETSc users manual .

F. Hindenlang, T. Bolemann, C.-D. Munz, Mesh curving techniques for high order discontinuous Galerkin simulations, in: IDIHOM: Industrialization of high-order methods-a top-down approach, Springer, 133-152, 2015.

A. M. Rueda-Ramírez, G. Rubio, E. Ferrer, E. Valero, Truncation Error Estimation in the p-Anisotropic Discontinuous Galerkin Spectral Element Method, Journal of Scientific Computing 78 (1) (2019c) 433-466. 

